\title{
THE CO-EVOLUTION OF MICROSTRUCTURE FEATURES IN SELF-ION IRRADIATED HT9 AT VERY HIGH DAMAGE LEVELS
}

\author{
by \\ Elizabeth Margaret Getto \\ A dissertation submitted in partial fulfillment \\ of the requirements for the degree of \\ Doctor of Philosophy \\ (Nuclear Engineering and Radiological Sciences) \\ in The University of Michigan \\ 2016
}

Doctoral Committee:

Professor Gary S. Was, Chair

Associate Research Scientist Zhijie Jiao

Professor Amit Misra

Adjunct Professor Roger Stoller

Professor Lumin Wang 
Copyright (C) 2016 Elizabeth Margaret Getto

All rights reserved 
To my family 


\section{ACKNOWLEDGEMENTS}

I would first like to thank my thesis advisor, Dr. Gary Was. His insight, suggestions and input have been instrumental throughout my doctoral program. I would also like to thank my committee members, Dr. Roger Stoller, Dr. Lumin Wang, Dr. Zhijie Jiao and Dr. Amit Misra. Their input has greatly improved the quality of my thesis and has been invaluable.

I would like to thank the members of Was research group including Dr. Janelle Wharry, Dr. Anne Campbell, Dr. Mike McMurtrey, Dr. Cheng Xu, Dr. Gokce Gulsoy, Dr. Tyler Moss, Dr. Kale Stephenson, Dr. Shyam Dwaraknath, Stephen Raiman, Anthony Monterrosa, Justin Hesterberg, Stephen Taller, Gerrit Vancoervering, David Woodley, Drew Johnson, Rigel Hanbury and Rajan Bhambroo. The mentorship, help in the lab, moral support and camaraderie have been the best part of my graduate school experience. I especially would like to thank Gerrit Vancoervering for his aid in the modeling section of my dissertation. I am forever indebted to the staff at the Michigan Ion Beam Laboratory, Dr. Ovidiu Toader, Dr. Fabian Naab, Dr. Prabir Roy, Thomas Kubley and Dr. Ethan Uberseder, for support of many, many hours of ion irradiations and many emergency calls after midnight. I would also like to thank Dr. Kai Sun at the Michigan Center for Material Characterization and Dr. Zhijie Jiao for assistance in the microscopy required for both my research project and dissertation.

Without the support of Dr. Micah Hackett at TerraPower, LLC., this work would have never happened. Dr. Hackett's insights, support and ideas helped shape the irradiation program 
that led to my final dissertation. To Dr. Alexander Barashev and Dr. Stas Golubov at Oak Ridge National Laboratory, I am truly thankful for all your patient assistance helping me with the modeling section of my thesis. A special thanks as well to Dr. Frank Garner for many enjoyable and insightful conversations and discussions about void swelling and radiation effects over the last five years.

I am grateful for the support of the Beering Scholarship at Purdue University, which started me off on my higher education journey. I am also grateful to the National Science Foundation Graduate Research Foundation for the funding of my first three years of graduate school.

To my parents, Anne and David Beckett, thank you for the love and support throughout my whole life. I am forever gratefully for you encouraging me to follow my dreams and for your constant love and prayers. I cannot imagine this journey without your support. Anchors aweigh! To Rob, Lynn, Eleanor and Philip Beckett, thank you for your love and support and making me laugh when I need it the most.

Finally, to my husband Josh: thank you for your love for all these years, always being my biggest fan, knowing just what to say when I'm about to give up, making me a better person and for all those burritos delivered to the lab at all hours of the day or night.

The funding for this work as provided by TerraPower, LLC. 


\section{TABLE OF CONTENTS}

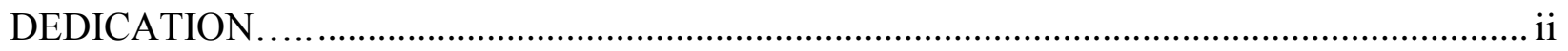

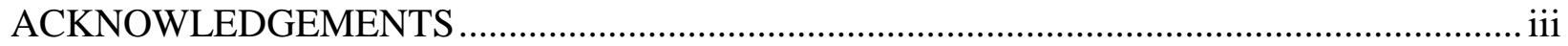

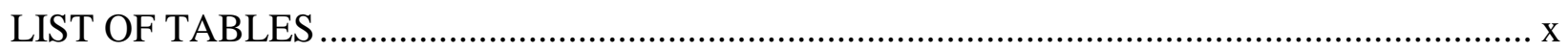

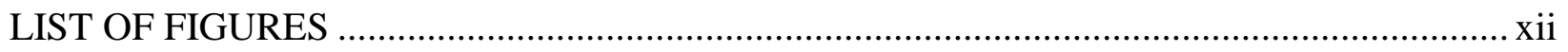

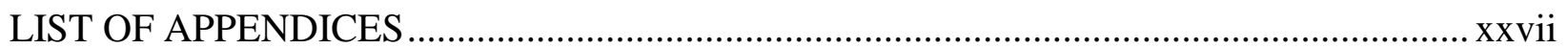

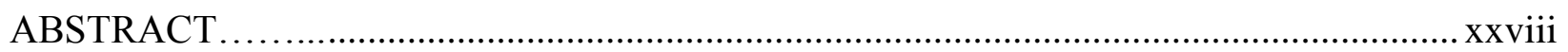

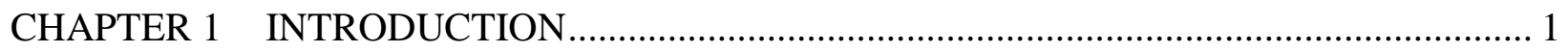

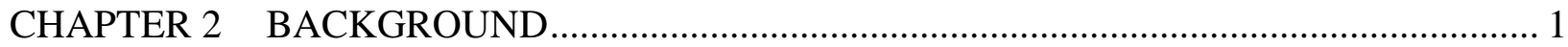

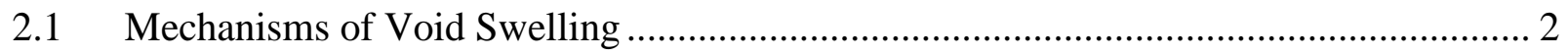

2.1.1 Introduction to Void Swelling ..................................................................... 2

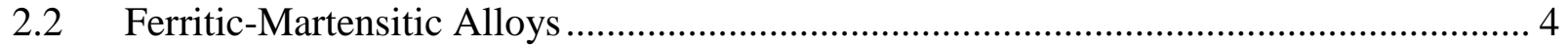

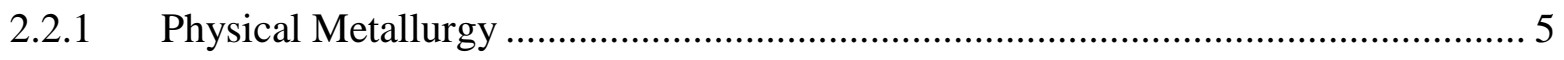

2.3 Void Swelling Experiments in Ferritic-Martensitic Alloys ......................................... 7

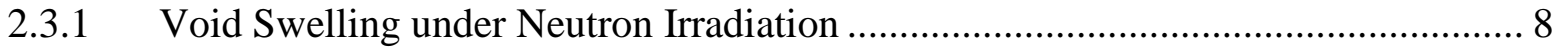

2.3.2 Void Swelling under Ion Irradiation ........................................................... 12

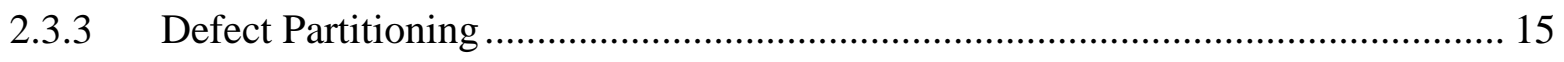

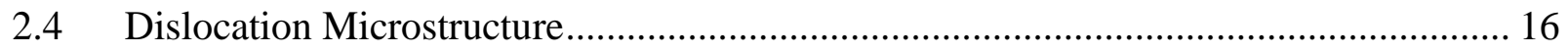

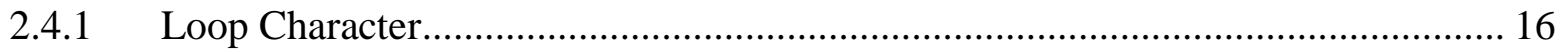

2.4.2 Dislocation Microstructure under Irradiation .................................................. 17

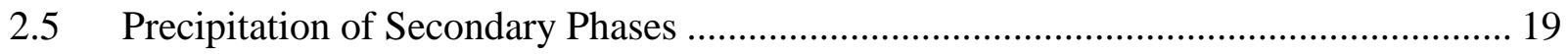

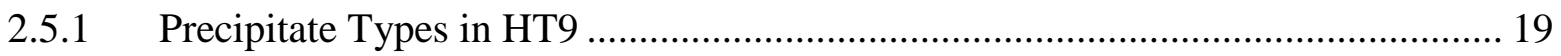

2.5.2 Precipitate Behavior under Irradiation ............................................................... 22

2.6 Other Irradiation Effects................................................................................. 24

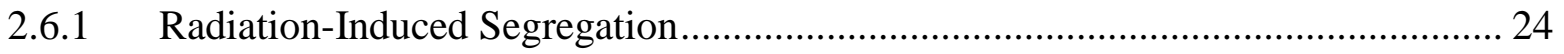

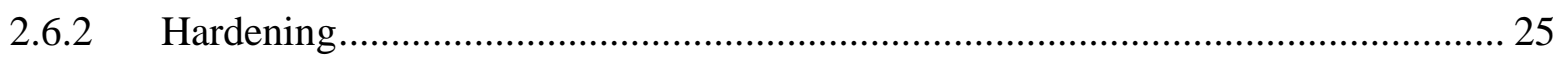




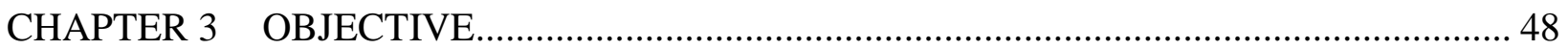

CHAPTER 4 EXPERIMENTAL PROCEDURE ……................................................. 50

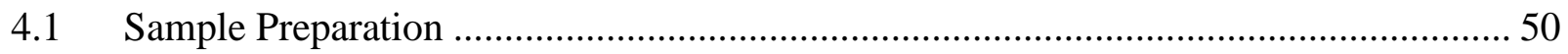

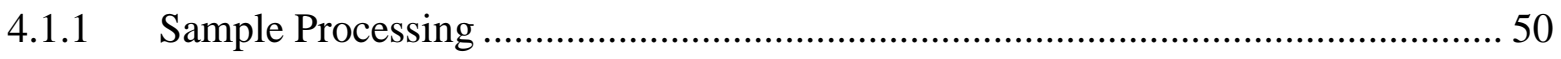

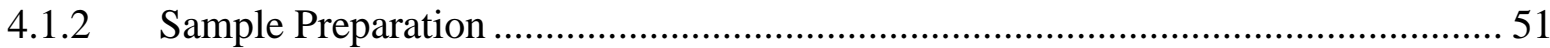

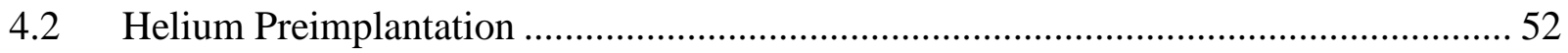

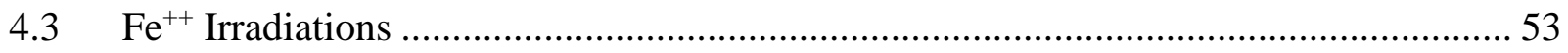

4.3.1 Irradiation Set-up ..................................................................................... 53

4.3.1.1 Copper Stage with Indium ........................................................................ 54

4.3.1.2 Copper Stage with Indifoil ..................................................................... 56

4.3.1.3 Nickel Stage with Copper Foil …………….............................................. 56

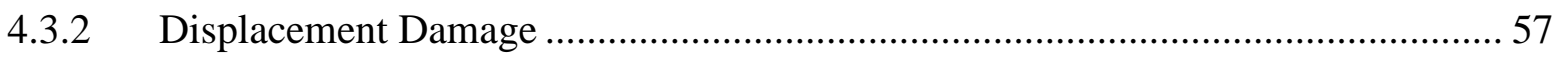

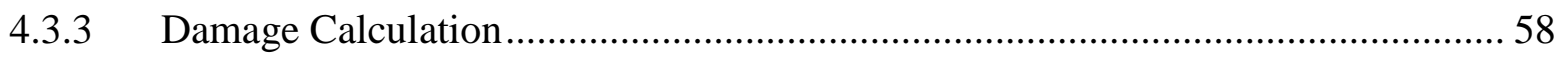

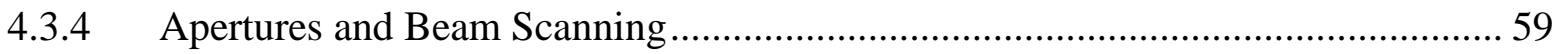

4.3.4.1 Stage Aperture System ……….................................................................. 59

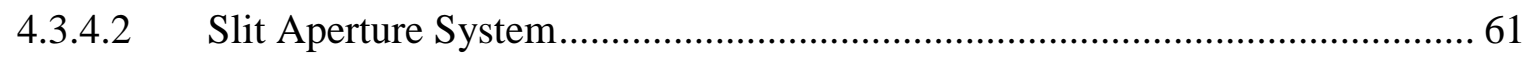

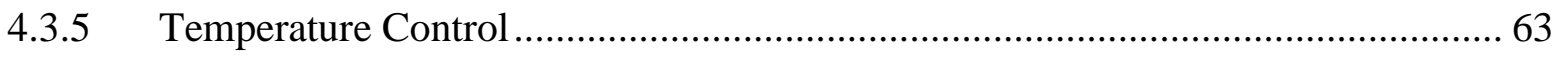

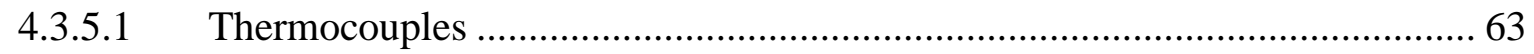

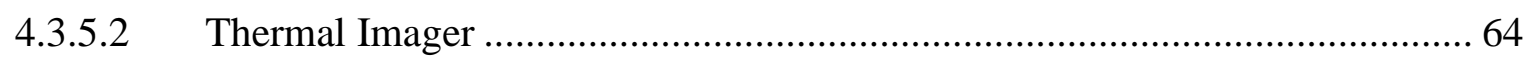

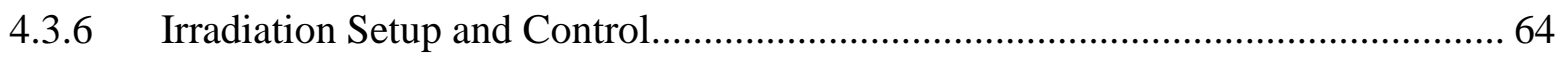

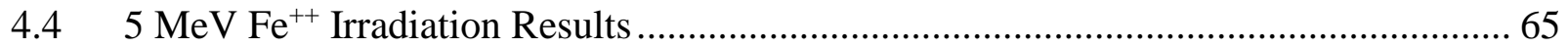

4.5 Post-Irradiation Sample Preparation and Microstructure Characterization .................... 67

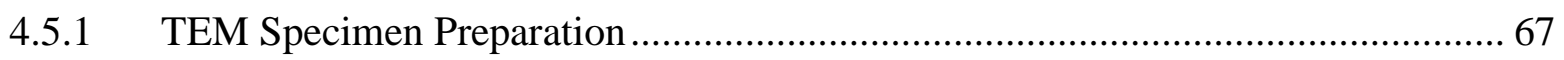

4.5.2 Void Swelling Measurement and Characterization .................................................. 68

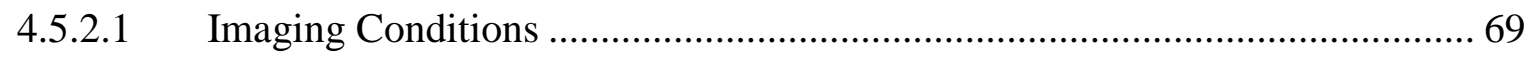

4.5.2.2 Void Counting and Determination of Region of Interest ................................ 71

4.5.2.3 Error Analysis .......................................................................................... 73

4.5.3 Precipitate Measurement, Characterization and Imaging ...................................... 76

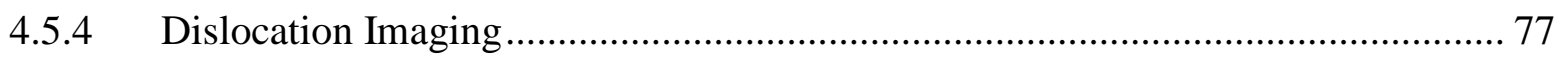

CHAPTER 5 RESULTS OF MICROSTRUCTURE EVOLUTION UNDER ION 
5.1 Unirradiated Microstructure Characterization ........................................................ 111

5.2 Void Swelling Results ......................................................................................... 113

5.2.1 Determination of Valid Region of Interest ................................................... 114

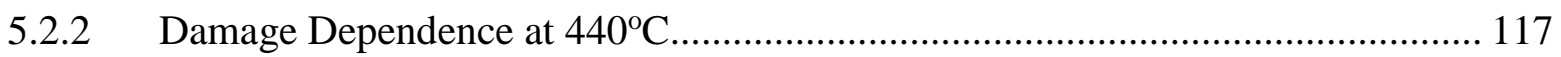

5.2.3 Temperature Dependence at 188 dpa.................................................................. 118

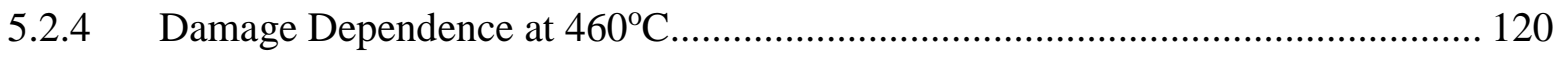

5.2.4.1 Grain to Grain Void Variation.................................................................... 123

5.2.5 High Swelling Correction .............................................................................. 124

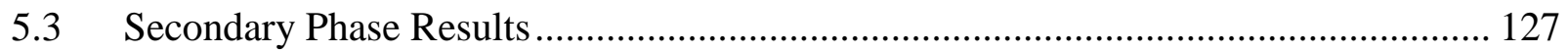

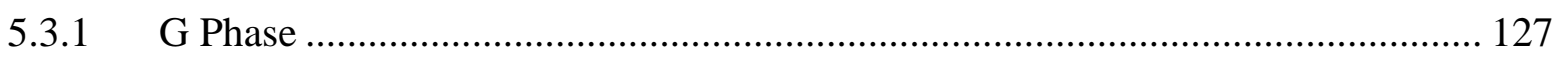

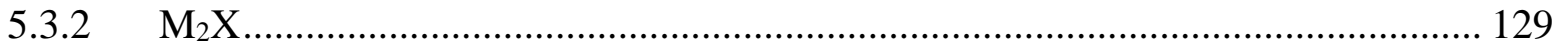

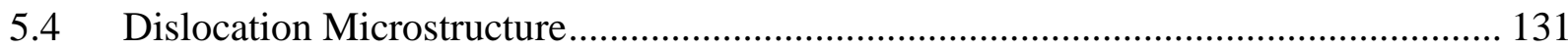

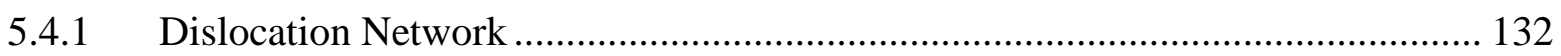

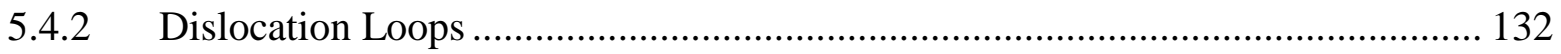

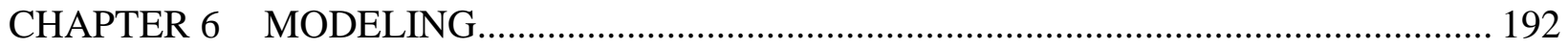

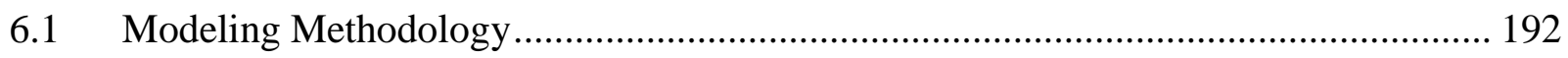

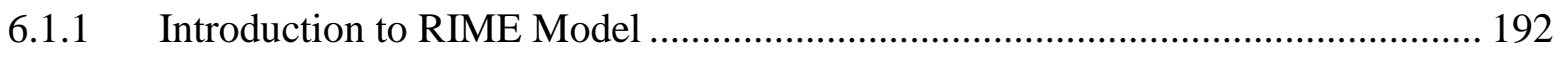

6.1.2 Description of Model Physics ...................................................................... 194

6.1.2.1 Master Equations …………………………........................................... 194

6.1.2.2 Sink Strength Calculations ....................................................................... 197

6.1.2.3 Defect Production Rates .............................................................................. 198

6.1.2.4 Network Dislocation Evolution ................................................................. 198

6.1.2.5 Calculation of Radii/Swelling ................................................................ 200

6.1.2.6 Grouping Scheme ………………………................................................... 201

6.1.2.7 Consideration of Production Bias Model .................................................... 202

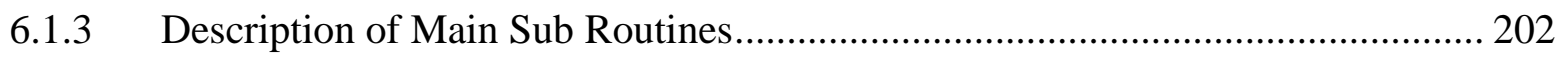

6.1.4 Sensitivity Analysis ....................................................................................... 206

6.1.5 Input Parameter Selection ............................................................................... 208

6.2 Adaptations to RIME Model for Void Growth-Dominated Microstructure ................ 210

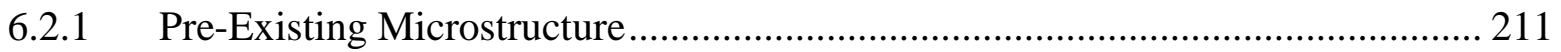

6.2.2 Dislocation Treatment.................................................................................... 213 


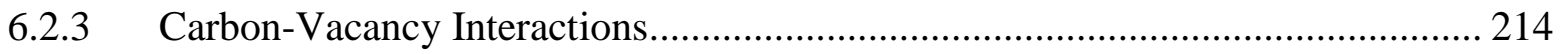

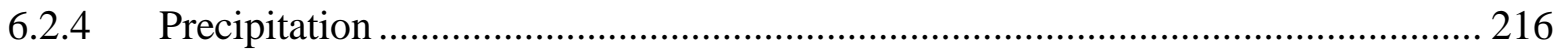

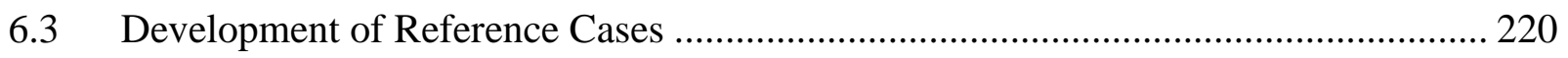

6.3.1 Comparison of Reference Cases to Experimental Data ....................................... 221

6.3.1.1 Ref.0: Constant Network Density................................................................. 221

6.3.1.2 Ref.1: Experimentally Input Dislocation Evolution ...................................... 222

6.3.1.3 Ref.2: Dynamic Dislocation (DD) Evolution ............................................... 223

CHAPTER 7 DISCUSSION: CO-EVOLUTION OF THE IRRADIATED

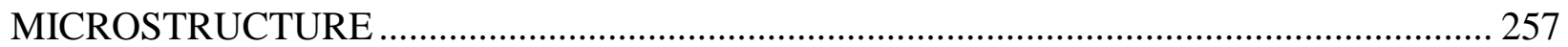

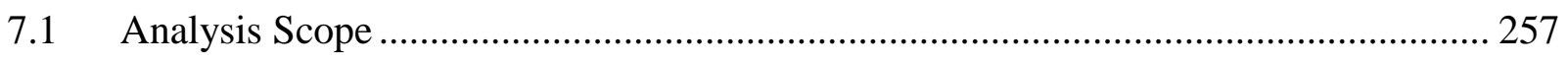

7.1.1 Definitions of Modifications to Reference Cases....................................................... 260

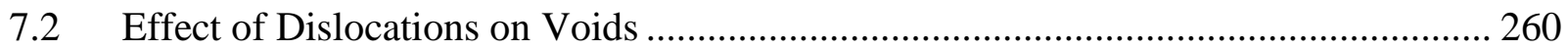

7.2.1 Summary of Dislocation and Void Evolution....................................................... 262

7.2.2 Case Ref.0: Experimentally input network dislocations ...................................... 263

7.2.3 Case Ref.1: Experimentally input network and loops .......................................... 264

7.2.4 Case Ref.2: Dynamic Dislocation Treatment ........................................................... 265

7.2.5 Comparison of Model to Experiment ...................................................................... 267

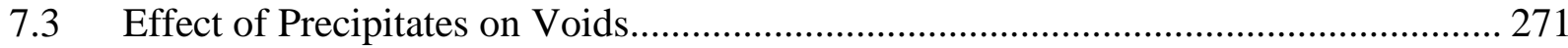

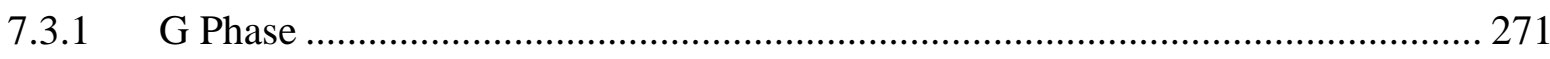

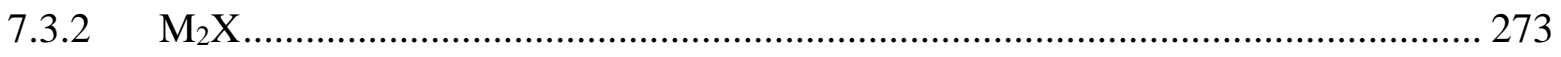

7.4 Effect of Precipitates on Dislocations ...................................................................... 277

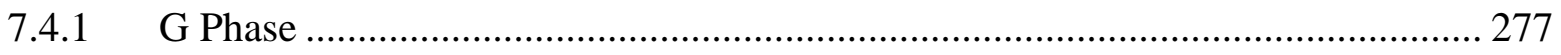

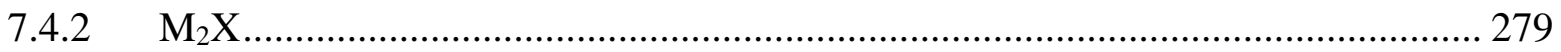

7.5 Carbon Trapping …………….......................................................................... 283

7.6 Combined Impact of Microstructure Features on Voids and Dislocations .................. 289

7.7 Alternate Considerations Regarding Overall Microstructure Co-evolution ................. 292

7.7.1 Discussion of Other Possible Interaction Mechanisms........................................ 292

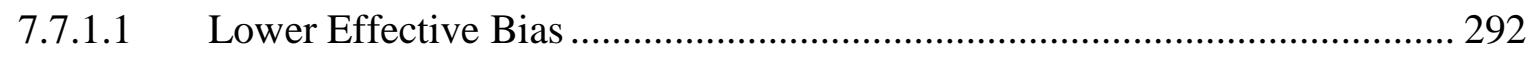

7.7.2 Reconsidering Swelling Rates from Experiment ................................................ 293

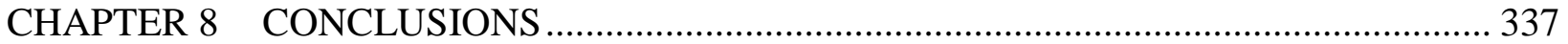

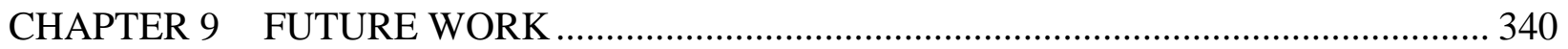




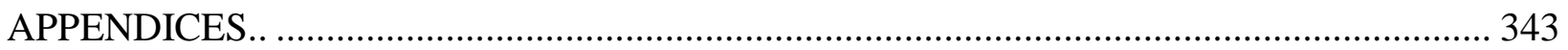

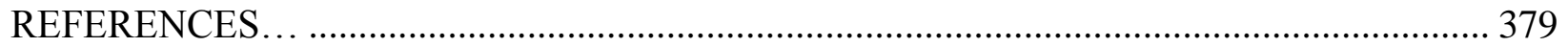




\section{LIST OF TABLES}

Table 2.1: Summary of observed dislocation behavior in FM alloys.................................... 28

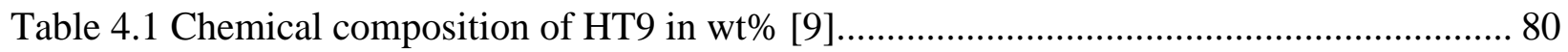

Table 4.2: Variables used in calculation of damage under $5 \mathrm{MeV} \mathrm{Fe}^{++}$irradiation................... 81

Table 4.3: List of samples utilized in this thesis, including target irradiation temperature, damage and date.

Table 4.4: Summary of irradiation data from $5 \mathrm{MeV} \mathrm{Fe}^{++}$irradiations referred to in this thesis. 83

Table 5.1: As-received microstructure characterization of HT9 (84425).

Table 5.2: Void results from damage dependence study at $440^{\circ} \mathrm{C}$ and temperature dependence study at 188 dpa.

Table 5.3: Summary of void data from damage dependence experiment performed at $460^{\circ} \mathrm{C}$. . 139

Table 5.4: Summary of $\mathrm{G}$ phase and $\mathrm{M}_{2} \mathrm{X}$ behavior from damage dependence experiment performed at $460^{\circ} \mathrm{C}$.

Table 5.5: Summary of dislocation microstructure data from damage dependence study performed at $460^{\circ} \mathrm{C}$.

Table 6.1: Summary of sub routines in RIME model. Updates, changes and notes are included in the last column.

Table 6.2: Parameters used in a complete input file. Highlighted boxes in red are not used for the purpose of this work. Highlighted boxes in yellow have been adapted or changed. . 227

Table 6.3: Output file descriptions from RIME Model. 232

Table 6.4: Main input parameters used in sensitivity analysis. These inputs correspond to Ref.2 shown in Table 6.9 . 236

Table 6.5: Significance calculated for swelling and swelling rate at $460^{\circ} \mathrm{C}, 21$ and 100 dpa with and without the addition of unbiased precipitates at experimental levels observed for $\mathrm{G}$

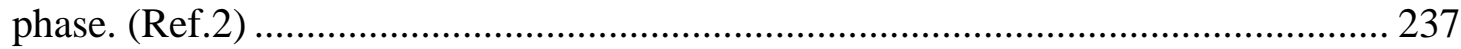

Table 6.6: Significance of key input parameters in RIME model. ......................................... 238 
Table 6.7: Main changes in parameterization from FCC to BCC alloys.

Table 6.8: Concentration [10] and diffusion coefficients at $500^{\circ} \mathrm{C}$ for minor alloying elements in HT9.

Table 6.9: Definition of reference case input. Changes between each reference case highlighted in yellow.

Table 7.1: Description of flags denoting modification of reference cases with various microstructure treatments. 298

Table 7.2: Dislocation biases for interstitials reported in literature using a variety of approaches.

Table 7.3: Description of reference cases with modifications used to match void and dislocation behavior to experiment. 300

Table 7.4: Summary of attempts to match microstructure behavior with precipitate and carbon treatments.

Table 7.5: Comparison of relative effect on final swelling values at $650 \mathrm{dpa}$ of each microstructure treatment. 302

Table 7.6: Comparison of swelling rates calculated in the range of 188 to 650 dpa using nominal or fixed depth method [85] 303 


\section{LIST OF FIGURES}

Figure 2.1: Expected swelling behavior as a function of damage. Adapted from [19].

Figure 2.2: Effect of chromium on the constitution of Fe-Cr-C alloys with $0.1 \%$ carbon.

$(\mathrm{CrFe})_{4} \mathrm{C}$ is the $\mathrm{M}_{23} \mathrm{C}_{6}$ carbide [3]. 30

Figure 2.3: Continuous cooling transformation (CCT) diagram for $12 \mathrm{Cr}-\mathrm{MoVNb}$ martensitic steel where A refers to austenite, $\mathrm{K}$ refers to carbide, $\mathrm{Sp}-\delta$ refers to trace ferrite, $\mathrm{M}$ refers to martensite, $\mathrm{P}=$ pearlite, $\mathrm{A}_{\mathrm{C} 1 \mathrm{~b}}$ is the start of austenite formation upon heating, $\mathrm{A}_{\mathrm{Cle}}$ is the completion of austenite formation upon heating and $\lambda$ is the cooling rate $\left({ }^{\circ} \mathrm{C} / \mathrm{min}\right)$ from $[3]$.

Figure 2.4: a) Schematic [32] and b) micrograph of typical HT9 microstructure prior to irradiation [3]. 32

Figure 2.5: Stress-free swelling of irradiated HT9 up to 165 dpa for 4 heat treatments. Heat 1 and Heat 2 were subjected to the same heat treatment: $1038^{\circ} \mathrm{C} / 5 \mathrm{~min} /$ air followed by $760^{\circ} \mathrm{C} / 30 \mathrm{~min} /$ air cool. Heat 3 and 5 were subjected to $1100^{\circ} \mathrm{C} / 2 \mathrm{~min} /$ air cool followed by $650 \% 120 \mathrm{~min} /$ air cool [4]. 33

Figure 2.6: Swelling in $\mathrm{Fe}-\mathrm{Cr}$ alloys as a function of damage [33]...... 34

Figure 2.7: Adjustment of damage levels in EBR-II results by Garner et al. [8] to data collected by Gelles [33]. 35

Figure 2.8: Summary of HT9 and T91 irradiated in-reactor up to 208 dpa. 36

Figure 2.9: a) Temperature dependence of void swelling in EM12 and HT9 irradiated to 150 dpa

b) Damage dependence of swelling of EM12 and HT9 up to at $500^{\circ} \mathrm{C}$ [14]. 37

Figure 2.10: a) Temperature dependence of swelling in ferritic steel irradiated with $200 \mathrm{keV} \mathrm{C}^{+}$ ions to $150 \mathrm{dpa}$ and b) damage dependence of swelling in ferritic steels irradiated with $200 \mathrm{keV} \mathrm{C}^{+}$ions at $575^{\circ} \mathrm{C}[40]$. 38 
Figure 2.11: a) Damage dependence of commercial alloys up to 600 dpa at 450 or $480^{\circ} \mathrm{C}$. b) Temperature dependence experiment at 100 dpa and 500 dpa on MA957 ODS ferritic steel [15] 39

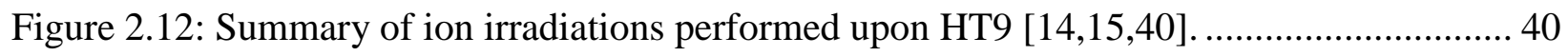

Figure 2.13: Q analysis for various neutron irradiations [34]........................................... 41

Figure 2.14 Temperature dependence of loop number density and diameter in $14 \mathrm{MeV} \mathrm{Ni-}$ irradiated HT9 [35]. Note that loops of size of $800 \mathrm{~nm}$ would typically be classified as an unfaulted network. 42

Figure 2.15: A summary of dislocation loop results as a function of temperature in neutron and ion irradiated HT9 $[7,35]$.

Figure 2.16: HT9 irradiated to a) 4 dpa at $505^{\circ} \mathrm{C} \mathrm{b)} 28 \mathrm{dpa}$ at $384^{\circ} \mathrm{C}$ c) $155 \mathrm{dpa}$ at $443^{\circ} \mathrm{C} \mathrm{[10].} 44$ Figure 2.17: Formation of rod-like precipitates in $13 \mathrm{Cr}-2 \mathrm{Mo}-\mathrm{TiO}_{2}$ after $\mathrm{Cr}$ irradiation at $500^{\circ} \mathrm{C}$ to a) $30 \mathrm{dpa}$ and b) $150 \mathrm{dpa}[38]$. 45

Figure 2.18: Representative stress/strain curves for Mod 9Cr-1Mo after irradiation in spallation

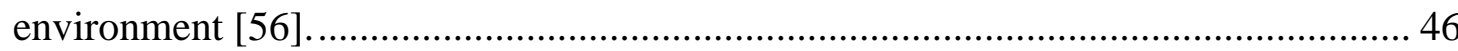

Figure 2.19: Comparison of the behavior of martensitic steels in the tempered and as-quenched conditions [59].

Figure 4.1: Unirradiated HT9 (Heat 84425) imaged in STEM-BF. Sample made via FIB liftout method 84

Figure 4.2 Sample geometry following EDM. Only one face will be irradiated. 85

Figure 4.3: Assembly used for electropolishing of samples. Samples polished in $90 \%$ methanol/10\% perchloric acid solution. 86

Figure 4.4: The helium profile (blue) is overlaid on the damage rate (red) and implanted ion concentration (green) for $5 \mathrm{MeV} \mathrm{Fe}^{++}$in $\mathrm{Fe}-12 \mathrm{Cr}$ calculated from SRIM [64].......... 87

Figure 4.5 Schematic of irradiation stage and position of 2D thermal imager on beamline. ....... 88

Figure 4.6: a) Schematic of hold-down bars and b) view of hold-down bars on the stage [32]... 89

Figure 4.7: a) "First generation stage" schematic where indium is used to ensure good thermal contact and apertures were attached to the stage. b) "Second generation stage" schematic where a combination of indium and graphite foil were utilized to ensure good thermal conductivity and leak protection. 90

Figure 4.8: Stage design as viewed from the top [32]. 
Figure 4.9: Completed stage after irradiation at $460^{\circ} \mathrm{C}$ to $130 \mathrm{dpa}$. 92

Figure 4.10: a) Schematic and b) stage image for temperature uniformity test. c) Results of the 24-hour test on the beamline.

Figure 4.11: Schematic of "third generation" stage configuration with a) stage and b) slit apertures. Copper foil provided sufficient thermal contact and eliminated indium leaks. Slit apertures eliminated danger of aperture shift, which could occur during stage loading onto beamline. 94

Figure 4.12: Top view of stage design with slit aperture system. The aperture feedthrough was replaced with an additional thermocouple feedthrough. 95

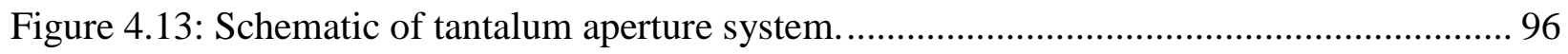

Figure 4.14: Schematic of benchtop alignment setup of stage apertures [32] ........................ 97

Figure 4.15: Path of raster scanned beam center across samples over irradiated area [13,32].... 98

Figure 4.16: a) Schematic of raster scanned beam area after beam sizing procedure with ceramic.

b) Beam intensity profile due to Gaussian beam shape ....................................... 99

Figure 4.17: Irradiation stage a) without and b) with alignment laser................................... 100

Figure 4.18: 2D thermal image from $460^{\circ} \mathrm{C}, 130 \mathrm{dpa}$ irradiation. Two AOIs per samples were placed on 5 samples with two guide bars bookending the samples. ........................ 101

Figure 4.19: Example temperature histogram for $5 \mathrm{MeV} \mathrm{Fe}{ }^{++}$irradiation of ACO3-460C-450dpa10He-021615 showing approximately normal distribution in both areas of interest (top and bottom) on the sample and having $2 \sigma$ well within $\pm 10^{\circ} \mathrm{C}$ 102

Figure 4.20: a) Platinum deposited on the surface of the irradiated sample b) trenching around the platinum protected surface c) under cut of the samples d) attaching the needle to the lamella using platinum e) attaching the lamella to the copper grid f) thinning using the Ga beam. 103

Figure 4.21: Schematic of cross-sectional liftout and resulting image in HAADF STEM. The PT layer and irradiated surface are at the top of the image. Voids are dark circles. Sample irradiated at $460^{\circ} \mathrm{C}$ to 188 dpa with 10 appm He preimplanted. 104

Figure 4.22: Conventional BF (a) and STEM BF (b) images of voids in HT9 irradiated with 5 $\mathrm{MeV} \mathrm{Fe}^{++}$at $460^{\circ} \mathrm{C}$ to 375 dpa with 10 appm He preimplanted. Note that all voids are in focus in (b) while conventional images show some voids in over or under focus. 105 
Figure 4.23: STEM ADF (a) and BF (b) images of HT9 irradiated with $5 \mathrm{MeV} \mathrm{Fe}^{++}$at $440^{\circ} \mathrm{C}$ to 140 dpa with 100 He preimplanted. Voids obscured by diffraction contrast in BF (b) are clearly observed in ADF (a) as black circles 106

Figure 4.24: Example of STEM HAADF and BF images stitched together to show entire liftout. (HT9, 440 $\mathrm{C}, 188$ dpa, 10 appm He) 107

Figure 4.25: Depth profiles for void a) diameter b) number density and c) swelling in HT9 irradiated at $440^{\circ} \mathrm{C}$ to 140 dpa with $100 \mathrm{appm}$ He preimplanted [12]. 108

Figure 4.26: Error for diameter, number density and swelling as a function of average void diameter.

Figure 4.27: Orientation and shapes of dislocation loops in FM alloys imaged along <001> axis [32]. 110

Figure 5.1: As-received HT9 sample prepared via FIB liftout method showing representative microstructure. 142

Figure 5.2: Optical micrograph of as-received HT9 after etching. The etchant used was Vilellas's reagent which includes $1 \mathrm{~g}$ picric acid with $5 \mathrm{~mL}$ of hydrochloric acid in a solution of $100 \mathrm{~mL}$ [63]. 143

Figure 5.3: Indexed diffraction of bcc matrix of HT9. 144

Figure 5.4: Void formation in the a) irradiated area and b) near surface in HT9 preimplanted with 10 appm He then irradiated with $5 \mathrm{MeV} \mathrm{Fe}^{++}$to $188 \mathrm{dpa}$ at $440^{\circ} \mathrm{C}$. 145

Figure 5.5: Void a) diameter b) number density c) and swelling profiles with depth in HT9 preimplanted with 10 appm He then irradiated with $5 \mathrm{MeV} \mathrm{Fe}^{++}$to 188 dpa at $460^{\circ} \mathrm{C}$.

Figure 5.6: Determination of location of onset of swelling increase after swelling peak near the surface (from [12]). 147

Figure 5.7: Determination of location of onset of in swelling decrease at depths of $700 \mathrm{~nm}$ or greater (from [12])..... 148

Figure 5.8: HAADF images of HT9 preimplanted with 10 appm He then irradiated with $5 \mathrm{MeV}$ $\mathrm{Fe}^{++}$at $440^{\circ} \mathrm{C}$ to a) $25 \mathrm{dpa} \mathrm{b)} 50 \mathrm{dpa}$ c) $140 \mathrm{dpa}$ and d) $188 \mathrm{dpa}$. 149

Figure 5.9: a) Diameter and b) number density of voids in HT9 preimplanted with 10 appm He then irradiated with $5 \mathrm{MeV} \mathrm{Fe}^{++}$at $440^{\circ} \mathrm{C}$ up to $188 \mathrm{dpa}$. 150 
Figure 5.10: Void swelling in HT9 preimplanted with 10 appm He then irradiated with $5 \mathrm{MeV}$ $\mathrm{Fe}^{++}$at $440^{\circ} \mathrm{C}$ up to $188 \mathrm{dpa}$. Swelling values are indicated above the data points... 151

Figure 5.11: HAADF images of HT9 preimplanted with 10 appm He then irradiated with $5 \mathrm{MeV}$ $\mathrm{Fe}^{++}$at a) $\left.440{ }^{\circ} \mathrm{C} \mathrm{b}\right) 460{ }^{\circ} \mathrm{C}$ and c) $480^{\circ} \mathrm{C}$ to $188 \mathrm{dpa}$. 152

Figure 5.12: Void a) diameter and b) number density (red) in HT9 preimplanted with 10 appm He then irradiated with $5 \mathrm{MeV} \mathrm{Fe}^{++}$to 188 dpa between 440 to $480^{\circ} \mathrm{C}$. 153

Figure 5.13: Swelling in HT9 preimplanted with 10 appm He then irradiated with $5 \mathrm{MeV} \mathrm{Fe}^{++}$to 188 dpa between 440 and $480^{\circ} \mathrm{C}$. Micrographs of typical voids are included for each temperature

Figure 5.14: a) Diameter and b) number density as a function of damage for $440^{\circ} \mathrm{C}$ (red), $460^{\circ} \mathrm{C}$ (blue) and $480^{\circ} \mathrm{C}$ (green). Dashed lines extrapolated expected damage evolution at $440^{\circ} \mathrm{C}$ and $480^{\circ} \mathrm{C}$ resolving peak diameter and number density observed at $460^{\circ} \mathrm{C}, 188$ dpa. At $300 \mathrm{dpa}$, it is expected that diameter will increase with temperature and number density will decrease as is predicted by theory since all three will be in void growth-dominated regime. 155

Figure 5.15: Void evolution as a function of damage in $\mathrm{HT} 9$ irradiated with $5 \mathrm{MeV} \mathrm{Fe}{ }^{++}$at $460^{\circ} \mathrm{C}$ after preimplantation with 10 appm He. Voids are the dark circles and marked with red arrows. Orange rectangles denote the 500 to $700 \mathrm{~nm}$ region. 156

Figure 5.16: Void diameter as a function of damage in $\mathrm{HT} 9$ irradiated with $\mathrm{Fe}^{++}$at $460^{\circ} \mathrm{C}$ with 10 appm He preimplanted. 157

Figure 5.17: Void number density as a function of damage in HT9 irradiated with $\mathrm{Fe}^{++}$at $460^{\circ} \mathrm{C}$ with 10 appm He preimplanted. 158

Figure 5.18: Void swelling as a function of damage in $\mathrm{HT} 9$ irradiated with $\mathrm{Fe}^{++}$at $460^{\circ} \mathrm{C}$ with 10 appm He preimplanted. 159

Figure 5.19: Comparison of linear or parabolic regression analysis applied to void swelling resulting from $\mathrm{HT} 9$ irradiated with $\mathrm{Fe}^{++}$at $460^{\circ} \mathrm{C}$ with 10 appm He preimplanted... 160

Figure 5.20: Representative images of $\delta$-ferrite (a, c) and martensite (b, d) at 130 and 450 dpa.

Figure 5.21: Comparison of diameter, number density and swelling in ferrite and martensite grains compared at a) $130 \mathrm{dpa}$ and b) $450 \mathrm{dpa}$. 162 
Figure 5.22: Damage rate (dpa/s) curve as a function of increasing damage due to void swelling.

Beam current is assumed to be constant for all irradiations so damage rate can be directly compared. 163

Figure 5.23: a) Effective damage rate calculated from SRIM. b) Swelling vs. damage with fixed depth correction. 164

Figure 5.24: Void diameter, number density and swelling depth profiles as well as void distribution in the 500 to $700 \mathrm{~nm}$ depth region of HT9 irradiated with $5 \mathrm{MeV} \mathrm{Fe}{ }^{++}$ (ACO3) at $460^{\circ} \mathrm{C}$, to $550 \mathrm{dpa}$ with $10 \mathrm{appm} \mathrm{He}$. 165

Figure 5.25: Void diameter, number density and swelling depth profiles as well as void distribution in the 500 to $700 \mathrm{~nm}$ depth region of HT9 irradiated with $5 \mathrm{MeV} \mathrm{Fe}{ }^{++}$ (ACO3) at $460^{\circ} \mathrm{C}$, to 650 dpa with 10 appm He. 166

Figure 5.26: $\mathrm{G}$ phase evolution with increasing damage irradiated at $460^{\circ} \mathrm{C}$ with $5 \mathrm{MeV} \mathrm{Fe}{ }^{++}$with 10 appm He preimplanted. Some G phase precipitates are marked by red arrows.... 167 Figure 5.27: APT analysis of two G phase precipitates observed at HT9 irradiated with $5 \mathrm{MeV}$ $\mathrm{Fe}^{++}$at $440^{\circ} \mathrm{C}$ to 140 dpa with 10 appm preimplanted. APT confirms composition of $\mathrm{Mn}_{6} \mathrm{Ni}_{16} \mathrm{Si}_{7}$ by observation of expected concentrations in at\% $(55 \% \mathrm{Ni}-24 \% \mathrm{Si}-21 \% \mathrm{Mn})$

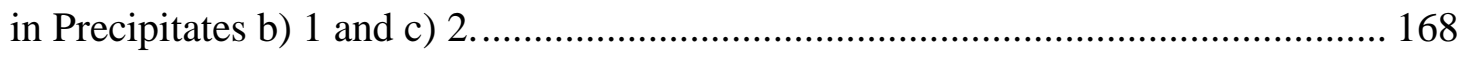

Figure 5.28: STEM BF image of G phase with corresponding XEDS map. Ni is in red and Si in blue.

Figure 5.29: HRTEM BF image of $G$ phase in the matrix. The much larger lattice parameter of $G$ phase $(\mathrm{a}=1.148 \mathrm{~nm})$ relative the matrix $\mathrm{a}=0.286 \mathrm{~nm}$. The incoherent interface is clearly visible. The FFT pattern of the outlined region in the image (red frame) is inset in the top left corner and demonstrates fcc crystallographic structure. 170

Figure 5.30: Evolution of $\mathrm{G}$ phase precipitates in terms of diameter (blue), number density (red) and volume fraction (green) at $460^{\circ} \mathrm{C}$ in $\mathrm{HT} 9$ irradiated with $5 \mathrm{MeV} \mathrm{Fe}{ }^{++}$after preimplantation with 10 appm He.

Figure 5.31: Formation of $\mathrm{M}_{2} \mathrm{X}$ precipitates in irradiated HT9 irradiated with $5 \mathrm{MeV} \mathrm{Fe}{ }^{++}$. (a)-(e) are the low angle annular dark field images of $\mathrm{M}_{2} \mathrm{X}$, shown as light grey rods. (f)-(j) present the corresponding high angle annular dark field images with voids as dark circles and $\mathrm{M}_{2} \mathrm{X}$ can faintly be observed as light grey plates. 
Figure 5.32: Orientation of $\mathrm{M}_{2} \mathrm{X}$ within the matrix. The top view corresponds to what is imaged in Figure 5.31. 173

Figure 5.33: LAADF image of formation of $\mathrm{M}_{2} \mathrm{X}$ precipitates in irradiated HT9 irradiated with 5 $\mathrm{MeV} \mathrm{Fe}^{++}$. Note precipitates form intragranularly. 174

Figure 5.34: Bright field image (a) and corresponding jump ratios for $\mathrm{Cr}$ (b), $\mathrm{C}$ (c) and $\mathrm{N}$ (d) in HT9 irradiated at $460^{\circ} \mathrm{C}$ to 550 dpa with 10 appm He preimplanted. 175

Figure 5.35: EDX spectrum of $\mathrm{M}_{2} \mathrm{X}$ confirming $\mathrm{Cr}_{2} \mathrm{C}$ in $\mathrm{HT} 9$ irradiated at $460^{\circ} \mathrm{C}$ to 550 dpa with 10 appm He preimplanted. 176

Figure 5.36: SAED patterns of $\mathrm{M}_{2} \mathrm{X}$ in HT9 irradiated at $460^{\circ} \mathrm{C}$ to 450 dpa with 10 appm He preimplanted with axis tilted to a) $0^{\circ}$ b) $27^{\circ}$ and c) $43^{\circ}$.....

Figure 5.37: a) HRTEM of $\mathrm{M}_{2} \mathrm{X}$ b) FFT of precipitate from red box in (a), c) nanodiffraction revealing $\mathrm{M}_{2} \mathrm{X}$ (red) and $\mathrm{Fe}$ (green) matrix in $\mathrm{HT} 9$ irradiated at $460^{\circ} \mathrm{C}$ to 650 dpa with 10 appm He preimplanted. 178

Figure 5.38: a) BF b) HR BF and c) HR HAADF images of $\mathrm{M}_{2} \mathrm{X}$ irradiated to $650 \mathrm{dpa}$ at $460^{\circ} \mathrm{C}$. Moiré fringes clearly visible and indicated misfit strain due to slight lattice mismatch, consistent with coherency. 179

Figure 5.39: HR HAADF image of $\mathrm{M}_{2} \mathrm{X}$ (yellow) and matrix (red). FFT show same growth relationship $<1220>M 2 X / /<100>\alpha$, demonstrated by parallel lines. 180

Figure 5.40: a) HR-HAADF image of HT9 irradiated at $460^{\circ} \mathrm{C}$ to $650 \mathrm{dpa}$. b) FFT with simulated SAED pattern overlaid indicating consistency of observed pattern with simulation along [011] direction of matrix, c) FFT with no simulated diffraction pattern overlaid.

Figure 5.41: Evolution of $\mathrm{M}_{2} \mathrm{X}$ precipitates in terms of length (blue), width (red), thickness (green), number density (yellow) and volume fraction (grey) in HT9 irradiated with 5 $\mathrm{MeV} \mathrm{Fe}^{++}$at $460^{\circ} \mathrm{C}$ after preimplantation of 10 appm He. 182

Figure 5.42: Images of representative dislocation network used for characterization of line density in $\mathrm{HT} 9$ irradiated with $5 \mathrm{MeV} \mathrm{Fe}^{++}$at $460^{\circ} \mathrm{C}$ with 10 appm $\mathrm{He}$ preimplanted. Some dislocations pointed out with red arrows. 183

Figure 5.43: High magnification images of dislocation lines/network in HT9 irradiated with 5 $\mathrm{MeV} \mathrm{Fe}^{++}$at $440^{\circ} \mathrm{C}$ with 10 appm He preimplanted to a) 140 and b) $188 \mathrm{dpa}$....... 184 
Figure 5.44: Dislocation network evolution under $5 \mathrm{MeV} \mathrm{Fe}^{++}$irradiation at $460^{\circ} \mathrm{C}$ in $\mathrm{HT} 9 . . .185$ Figure 5.45: Dislocation loop evolution with increasing damage irradiated at $460^{\circ} \mathrm{C}$ with $5 \mathrm{MeV}$ $\mathrm{Fe}^{++}$. Some loops are marked by white circles. 186

Figure 5.46: Quantification of dislocation loop diameter from 130 to $650 \mathrm{dpa}$ at $460^{\circ} \mathrm{C}$ irradiated with $5 \mathrm{MeV} \mathrm{Fe}{ }^{++}$. 187

Figure 5.47: Quantification of dislocation loop number density from 130 to 650 dpa at $460^{\circ} \mathrm{C}$ irradiated with $5 \mathrm{MeV} \mathrm{Fe}{ }^{++}$. 188

Figure 5.48: Dislocation loop line length in HT9 irradiated with $5 \mathrm{MeV} \mathrm{Fe}^{++}$at $460^{\circ} \mathrm{C}$ from 130 to $650 \mathrm{dpa}$. 189

Figure 5.49: Loop (blue), network (red) dislocation line length and total line (green) length in HT9 irradiated with $5 \mathrm{MeV} \mathrm{Fe}{ }^{++}$at $460^{\circ} \mathrm{C}$ up to 650 dpa. 190

Figure 5.50: Summary of evolving microstructure with damage in HT9 irradiated with $5 \mathrm{MeV}$ $\mathrm{Fe}^{++}$at $460^{\circ} \mathrm{C}$ 191

Figure 6.1: Main structure and sub routines in RIME model. 243

Figure 6.2: Effect of changing bias at $20 \mathrm{dpa}$ on swelling at $460^{\circ} \mathrm{C}$ using Ref.2.................. 244

Figure 6.3: Effect of changing grain size at $20 \mathrm{dpa}$ on swelling at $460^{\circ} \mathrm{C}$ using Ref.2. 245

Figure 6.4: Absolute value of significance of damage rate, temperature, grain size and bias.

Treatment including with (Ref.2) and without unbiased G phase (Ref.2.G) are both included as a function of damage in RIME at 21 and 100 dpa.

Figure 6.5: Absolute value of significance of bias, interstitial migration energy, vacancy migration and formation energy, recombination coefficient and cascade efficiency. DD treatment (Ref.2) used with significance reported as a function of damage in RIME at 21 and 100 dpa from the start of the case run. 247

Figure 6.6: Comparison of cascade efficiency and dislocation loop and network bias for interstitials of $1 \%$ (solid lines), $2 \%$ (dashed lines) and 3\% (dotted lines). 248

Figure 6.7: Measured a) void and b) loop fraction in HT9 irradiated with $5 \mathrm{MeV} \mathrm{Fe}^{++}$at $460^{\circ} \mathrm{C}$ to 188 dpa.

Figure 6.8: Experimental void distribution (blue columns) compared with RIME void distribution input (red line) into each case.

Figure 6.9: a) Radius and b) number density of dislocation loops in HT9 irradiated with $5 \mathrm{MeV}$ $\mathrm{Fe}^{++}$from 188 to $650 \mathrm{dpa}$ at $460^{\circ} \mathrm{C}$. Interpolation is from experimental data. 251 xix 
Figure 6.10: a) Radius and b) number density of G phase in HT9 irradiated with $5 \mathrm{MeV} \mathrm{Fe}$ from 188 to $650 \mathrm{dpa}$ at $460^{\circ} \mathrm{C}$. Interpolation is from experimental data.................. 252

Figure 6.11: a) Effective radius and b) number density of $\mathrm{M}_{2} \mathrm{X}$-phase in HT9 irradiated with 5 $\mathrm{MeV} \mathrm{Fe}^{++}$from 188 to $650 \mathrm{dpa}$ at $460^{\circ} \mathrm{C}$. Interpolation is from experimental data. . 253

Figure 6.12: Comparison of Ref.0 treatment (zinet=1.01) with experimental data in terms of a) network sink strength and b) void diameter, number density and swelling.

Figure 6.13: Comparison of Ref.1 treatment (ziloop $=$ zinet=1.01) with experimental data in terms of a) network and loop sink strength and b) void diameter, number density and swelling. 255

Figure 6.14: Comparison of Ref.2 treatment (ziloop $=$ zinet=1.01) with experimental data in terms of a) loop and network sink strength b) loop diameter and number density and c) void diameter, number density and swelling.

Figure 7.1: Schematic of relationships between precipitates, dislocations and voids explored in depth using RIME. 304

Figure 7.2: Comparison of Ref.0 (solid) and Ref.0.N (dashed) treatments (zinet=1.01) with experimental data in terms of a) void and network sink strength and b) void swelling.

Figure 7.3: Comparison of Ref.0 (solid) and Ref.1 (dashed) treatments (zilp = zinet=1.01) with experimental data in terms of a) network, loop and total dislocation sink strength and b) void swelling...... 306

Figure 7.4: Comparison of Ref.1 (solid) and Ref.2 (dashed) treatments (zilp = zinet=1.01) in terms of a) void, network, loop and total dislocation sink strength and b) void swelling. 307

Figure 7.5: Comparison of net vacancies absorbed at voids in Ref.1 (solid) and Ref.2 (dashed). Total dislocation sink strength is included in red. Discontinuity in Ref. 1 case at 550 dpa is due to the manual increase in network sink strength which decreases $d_{i} c_{i}$ and $d_{v} c_{v}$

Figure 7.6: Comparison of swelling response to initial dislocation network density varied from 0.3 to $30 \times 10^{14} \mathrm{~m}^{-2}$ in a) Ref. 2 and b) Ref. 1 309 
Figure 7.7: Comparison of experimentally input loop and network treatment (Ref.1: zilp $=$ zinet=1.01) with experimental data in terms of a) network/loop sink strength and b) void diameter, number density and swelling.

Figure 7.8: Comparison of 1\% (solid lines: Ref.1) and 0.7\% biased (dashed lines: Ref.1.1.007) with experimental data in terms of void diameter, number density and swelling...... 311

Figure 7.9: Comparison of DD treatment (Ref.2: zilp = zinet=1.01) with experimental data in terms of a) loop and network sink strength b) loop diameter and number density and c) void diameter, number density and swelling.

Figure 7.10: Effect of $G$ phase on void a) sink strength and b) swelling when included in experimentally determined levels as an unbiased sink. Dislocations are treated as experimentally input network and loops with (Ref.1.G: dashed lines) and without G phase (Ref.1: solid) with zilp $=$ zinet $=1.01$.

Figure 7.11: Comparison of experimentally input loop and network treatment with $\mathrm{G}$ phase included (Ref.1G: zilp = zinet=1.01) with experimental data in terms of a) G phase, loop and network sink strength and b) void diameter, number density and swelling.314

Figure 7.12: Comparison of evolution of void and $\mathrm{M}_{2} \mathrm{X}$ a) diameter and length and $b$ ) number density and volume fraction/swelling from 188 to $650 \mathrm{dpa}$. All irradiations performed with $5 \mathrm{MeV} \mathrm{Fe}^{++}$at $460^{\circ} \mathrm{C}$ with 10 appm He preimplanted. Lines guide the eye...... 315

Figure 7.13: Comparison of swelling behavior with increasing $E b v$, tr using Ref.1.MR with zilp $=$ zinet $=1.01$ 316

Figure 7.14: Comparison of swelling with $\mathrm{M}_{2} \mathrm{X}$ treated as either variable bias (red) or recombination centers (blue) with experimentally input network and loop with zilp=zinet $=1.01$

Figure 7.15: Comparison of Ref.1.MV (zilp=zinet=1.01) with experimental data in terms of a) $\mathrm{M}_{2} \mathrm{X}$, loop and network sink strength and $\mathrm{b}$ ) void diameter, number density and swelling. All irradiations performed with $5 \mathrm{MeV} \mathrm{Fe}^{++}$at $460^{\circ} \mathrm{C}$ with 10 appm $\mathrm{He}$ preimplanted.

Figure 7.16: Effect of G phase on network and loop a) sink strength and b) loop number density and diameter when included in experimentally determined levels as an unbiased sink. 
Dynamic dislocation treatment used both with (Ref.2.G: dashed lines) and without G phase (Ref.2: solid lines) with zilp $=$ zinet $=1.01$.

Figure 7.17: Comparison of Ref.2.G (zilp = zinet=1.01) with experimental data in terms of a) $\mathrm{G}$ phase, loop and network sink strength and b) dislocation loop diameter and number density. 320

Figure 7.18: Loop and $\mathrm{M}_{2} \mathrm{X}$ diameter/length compared with number density as a function of damage. All irradiations performed with $5 \mathrm{MeV} \mathrm{Fe}^{++}$at $460^{\circ} \mathrm{C}$ with 10 appm $\mathrm{He}$ preimplanted. Lines guide the eye.

Figure 7.19: Effect of $\mathrm{M}_{2} \mathrm{X}$ phase on network and loop a) sink strength and b) loop number density and diameter when included in experimentally determined levels as a variably biased sink. Dynamic dislocation treatment used with (Ref.2.MV: dashed lines) and without $\mathrm{M}_{2} \mathrm{X}$ phase (Ref.2: solid lines) with zilp = zinet=1.01.

Figure 7.20: Effect of changing number of interstitial cluster groups from 60 (solid) to 160 (dashed) with DD treatment (Ref.2.MV: zilp=zinet $=1.01) . \mathrm{M}_{2} \mathrm{X}$ treatment as variable bias sink was used.

Figure 7.21: Comparison of Ref.2.MV (zilp=zinet=1.01) with experimental data in terms of a) $\mathrm{M}_{2} \mathrm{X}$, loop and network sink strength and $\mathrm{b}$ ) dislocation loop diameter and number density. 324

Figure 7.22: Accounting for carbon in HT9 in the as-received condition and at 450 dpa 325 Figure 7.23: Comparison of Ref.2 with Ref.2.C with 0 to 855 appm for a) network and loop sink strength and b) swelling. Case run with 3940 appm terminated at 400 dpa. 0 and 1 appm lines are indistinguishable in $\mathrm{b}$ ). 326

Figure 7.24: Comparison of Ref.1 (blue), Ref.1.C with 100 appm in solution (red) and 100 appm in solution removed stepwise from 188 to $450 \mathrm{dpa}$ (green) representing the precipitation of $\mathrm{M}_{2} \mathrm{X}$.

Figure 7.25: Comparison of Ref.1.C (blue) and Ref.2.C (red) with 100 appm of carbon in solution removed stepwise from 188 to 450 dpa representing the precipitation of $\mathrm{M}_{2} \mathrm{X}$. 328

Figure 7.26: Comparison of void swelling with $\mathrm{M}_{2} \mathrm{X}$ plus 100 appm of carbon in solution removed stepwise from 188 to 450 dpa (Ref.1.MV.C: green), $\mathrm{M}_{2} \mathrm{X}$ (Ref.1.MV: red)

xxii 
and Ref.1 (blue). All $\mathrm{M}_{2} \mathrm{X}$ treated as variably biased with experimentally input dislocations.

Figure 7.27: Comparison of DD treatment (Ref.2.C: zilp = zinet=1.01) with experimental data in terms of a) loop and network sink strength b) loop diameter and number density and c) void diameter, number density and swelling including 100 appm of carbon in solution removed stepwise from 188 to $450 \mathrm{dpa}$. 330

Figure 7.28: Comparison of experimentally input loop and network treatment (Ref.1.G.MV.C: zilp = zinet=1.01) with experimental data in terms of a) network, loop, G phase and $\mathrm{M}_{2} \mathrm{X}$ sink strength and $\mathrm{b}$ ) void diameter, number density and swelling including $\mathrm{M}_{2} \mathrm{X}$ (variable biased), G phase and stepwise removal of 100 appm of carbon in solution from 188 to 450 dpa.

Figure 7.29: Comparison of DD treatment (Ref.2.G.MV.C: zilp = zinet=1.01) with experimental data in terms of a) network, loop, $G$ phase and $M_{2} X$ sink strength b) loop diameter and number density and c) void diameter, number density and swelling including $\mathrm{M}_{2} \mathrm{X}$ (variable biased), $\mathrm{G}$ phase and stepwise removal of $100 \mathrm{appm}$ of carbon in solution from 188 to $450 \mathrm{dpa}$.

Figure 7.30: Comparison of swelling curves with various microstructure treatments. All cases run with experimentally input dislocations (Ref.1: zilp = zinet=1.01).

Figure 7.31: Comparison of experimentally input loop and network treatment with lower bias (Ref.1.1.007.G.MV.C: zilp = zinet=1.01) with experimental data in terms of a) network, loop, $\mathrm{G}$ phase and $\mathrm{M}_{2} \mathrm{X}$ sink strength and $\mathrm{b}$ ) void diameter, number density and swelling including $\mathrm{M}_{2} \mathrm{X}$ (variable biased), $\mathrm{G}$ phase and 100 appm $\mathrm{C}$ in solution removed stepwise from 188 to $450 \mathrm{dpa}$. 334

Figure 7.32: Comparison between swelling rate before and after 450 dpa (Ref.1.G.MV.C: zilp = zinet=1.01). Dashed lines guide the eye to approximate swelling rates calculated from RIME. 335

Figure 7.33: Comparison of instantaneous swelling rate calculated from experiment using parabolic regression before and after 450 dpa using a) uncorrected swelling and b) fixed depth method [85] 336 
Figure A. 1: Temperature histograms for HT9 (heat 84425: ACO3) irradiated at $400^{\circ} \mathrm{C}: 250 \mathrm{dpa}$; $440^{\circ} \mathrm{C}: 25,140,188 ; 460^{\circ} \mathrm{C}: 188 \mathrm{dpa}$ and $480^{\circ} \mathrm{C}: 188 \mathrm{dpa}$ 344

Figure A. 2: Temperature histograms for HT9 (heat 84425: ACO3) irradiated at $440^{\circ} \mathrm{C}: 50$ dpa and $460^{\circ} \mathrm{C}: 75,130,250,350,375 \mathrm{dpa}$. 345

Figure A. 3: Temperature histograms for HT9 (heat 84425: ACO3) irradiated at $460^{\circ} \mathrm{C}: 450,550$, 650 dpa. 346

Figure B. 1: HT9 (ACO3) irradiated with $5 \mathrm{MeV} \mathrm{Fe}^{++}$at $440^{\circ} \mathrm{C}$ up to 140 dpa with 10 appm $\mathrm{He}$ preimplanted. 348

Figure B. 2: HT9 (ACO3) irradiated with $5 \mathrm{MeV} \mathrm{Fe}^{++}$at $440^{\circ} \mathrm{C}$ up to 25 dpa with 10 appm $\mathrm{He}$ preimplanted. 349

Figure B. 4: HT9 (ACO3) irradiated with $5 \mathrm{MeV} \mathrm{Fe}^{++}$at $440^{\circ} \mathrm{C}$ up to 188 dpa with 10 appm $\mathrm{He}$ preimplanted. 350

Figure B. 5: HT9 (ACO3) irradiated with $5 \mathrm{MeV} \mathrm{Fe}^{++}$at $480^{\circ} \mathrm{C}$ up to 188 dpa with 10 appm $\mathrm{He}$ preimplanted. 351

Figure B. 6: HT9 (ACO3) irradiated with $5 \mathrm{MeV} \mathrm{Fe}^{++}$at $400^{\circ} \mathrm{C}$ up to 250 dpa with 0 appm $\mathrm{He}$ preimplanted. 352

Figure B. 7: HT9 (ACO3) irradiated with $5 \mathrm{MeV} \mathrm{Fe}^{++}$at $460^{\circ} \mathrm{C}$ up to 188 dpa with 10 appm $\mathrm{He}$ preimplanted. 353

Figure B. 8: HT9 (ACO3) irradiated with $5 \mathrm{MeV} \mathrm{Fe}^{++}$at $440^{\circ} \mathrm{C}$ up to 50 dpa with $10 \mathrm{appm} \mathrm{He}$ preimplanted. 354

Figure B. 9: HT9 (ACO3) irradiated with $5 \mathrm{MeV} \mathrm{Fe}^{++}$at $460^{\circ} \mathrm{C}$ up to 375 dpa with 10 appm $\mathrm{He}$ preimplanted. 355

Figure B. 10: HT9 (ACO3) irradiated with $5 \mathrm{MeV} \mathrm{Fe}^{++}$at $460^{\circ} \mathrm{C}$ up to 130 dpa with 10 appm $\mathrm{He}$ preimplanted. 356

Figure B. 11: HT9 (ACO3) irradiated with $5 \mathrm{MeV} \mathrm{Fe}^{++}$at $460^{\circ} \mathrm{C}$ up to 75 dpa with 10 appm $\mathrm{He}$ preimplanted. 357

Figure B. 12: HT9 (ACO3) irradiated with $5 \mathrm{MeV} \mathrm{Fe}^{++}$at $460^{\circ} \mathrm{C}$ up to 250 dpa with 10 appm $\mathrm{He}$ preimplanted. 358

Figure B. 13: HT9 (ACO3) irradiated with $5 \mathrm{MeV} \mathrm{Fe}^{++}$at $460^{\circ} \mathrm{C}$ up to 350 dpa with 10 appm $\mathrm{He}$ preimplanted. 359 
Figure B. 14: $\mathrm{HT} 9$ (ACO3) irradiated with $5 \mathrm{MeV} \mathrm{Fe}^{++}$at $460^{\circ} \mathrm{C}$ up to 450 dpa with 10 appm $\mathrm{He}$

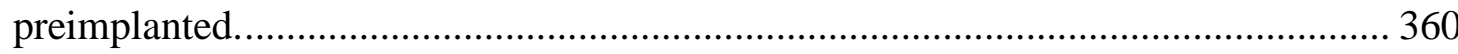

Figure B. 15: $\mathrm{HT} 9$ (ACO3) irradiated with $5 \mathrm{MeV} \mathrm{Fe}^{++}$at $460^{\circ} \mathrm{C}$ up to 550 dpa with 10 appm $\mathrm{He}$

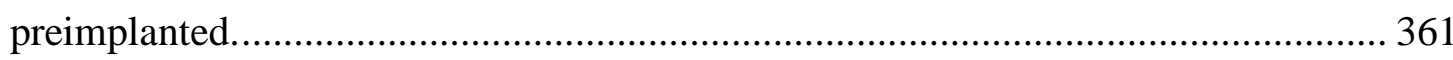

Figure B. 16: HT9 (ACO3) irradiated with $5 \mathrm{MeV} \mathrm{Fe}^{++}$at $460^{\circ} \mathrm{C}$ up to 650 dpa with 10 appm $\mathrm{He}$ preimplanted. 362

Figure C. 1: Void diameter, number density and swelling depth profiles as well as void distribution in the 500 to $700 \mathrm{~nm}$ depth region of HT9 irradiated with $5 \mathrm{MeV} \mathrm{Fe}$ (ACO3) at $440^{\circ} \mathrm{C}$, to $140 \mathrm{dpa}$ with $10 \mathrm{appm} \mathrm{He}$. 364

Figure C. 3: Void diameter, number density and swelling depth profiles as well as void distribution in the 500 to $700 \mathrm{~nm}$ depth region of HT9 irradiated with $5 \mathrm{MeV} \mathrm{Fe}$ (ACO3) at $440^{\circ} \mathrm{C}$, to $25 \mathrm{dpa}$ with 10 appm He. 365

Figure C. 4: Void diameter, number density and swelling depth profiles as well as void distribution in the 500 to $700 \mathrm{~nm}$ depth region of HT9 irradiated with $5 \mathrm{MeV} \mathrm{Fe}$ (ACO3) at $440^{\circ} \mathrm{C}$, to $188 \mathrm{dpa}$ with $10 \mathrm{appm} \mathrm{He}$. 366

Figure C. 5: Void diameter, number density and swelling depth profiles as well as void distribution in the 500 to $700 \mathrm{~nm}$ depth region of HT9 irradiated with $5 \mathrm{MeV} \mathrm{Fe}$ (ACO3) at $480^{\circ} \mathrm{C}$, to $188 \mathrm{dpa}$ with $10 \mathrm{appm} \mathrm{He}$.

Figure C. 6: Void diameter, number density and swelling depth profiles as well as void distribution in the 500 to $700 \mathrm{~nm}$ depth region of HT9 irradiated with $5 \mathrm{MeV} \mathrm{Fe}$ (ACO3) at $400^{\circ} \mathrm{C}$, to $250 \mathrm{dpa}$ with $10 \mathrm{appm} \mathrm{He}$. 368

Figure C. 7: Void diameter, number density and swelling depth profiles as well as void distribution in the 500 to $700 \mathrm{~nm}$ depth region of HT9 irradiated with $5 \mathrm{MeV} \mathrm{Fe}$ (ACO3) at $460^{\circ} \mathrm{C}$, to $188 \mathrm{dpa}$ with $10 \mathrm{appm} \mathrm{He}$.

Figure C. 8: Void diameter, number density and swelling depth profiles as well as void distribution in the 500 to $700 \mathrm{~nm}$ depth region of HT9 irradiated with $5 \mathrm{MeV} \mathrm{Fe}$ (ACO3) at $440^{\circ} \mathrm{C}$, to 50 dpa with 10 appm He. 370

Figure C. 9: Void diameter, number density and swelling depth profiles as well as void distribution in the 500 to $700 \mathrm{~nm}$ depth region of HT9 irradiated with $5 \mathrm{MeV} \mathrm{Fe}^{++}$ (ACO3) at $460^{\circ} \mathrm{C}$, to 375 dpa with $10 \mathrm{appm} \mathrm{He}$. 
Figure C. 10: Void diameter, number density and swelling depth profiles as well as void distribution in the 500 to $700 \mathrm{~nm}$ depth region of HT9 irradiated with $5 \mathrm{MeV} \mathrm{Fe}$ (ACO3) at $460^{\circ} \mathrm{C}$, to $130 \mathrm{dpa}$ with $10 \mathrm{appm} \mathrm{He}$.

Figure C. 11: Void diameter, number density and swelling depth profiles as well as void distribution in the 500 to $700 \mathrm{~nm}$ depth region of HT9 irradiated with $5 \mathrm{MeV} \mathrm{Fe}^{++}$ (ACO3) at $460^{\circ} \mathrm{C}$, to 75 dpa with 10 appm He.

Figure C. 12: Void diameter, number density and swelling depth profiles as well as void distribution in the 500 to $700 \mathrm{~nm}$ depth region of HT9 irradiated with $5 \mathrm{MeV} \mathrm{Fe}$ (ACO3) at $460^{\circ} \mathrm{C}$, to $250 \mathrm{dpa}$ with $10 \mathrm{appm} \mathrm{He}$.

Figure C. 13 Void diameter, number density and swelling depth profiles as well as void distribution in the 500 to $700 \mathrm{~nm}$ depth region of HT9 irradiated with $5 \mathrm{MeV} \mathrm{Fe}$ (ACO3) at $460^{\circ} \mathrm{C}$, to $350 \mathrm{dpa}$ with $10 \mathrm{appm} \mathrm{He}$.

Figure C. 14: Void diameter, number density and swelling depth profiles as well as void distribution in the 500 to $700 \mathrm{~nm}$ depth region of HT9 irradiated with $5 \mathrm{MeV} \mathrm{Fe}^{++}$ (ACO3) at $460^{\circ} \mathrm{C}$, to $450 \mathrm{dpa}$ with $10 \mathrm{appm} \mathrm{He}$.

Figure C. 15: Void diameter, number density and swelling depth profiles as well as void distribution in the 500 to $700 \mathrm{~nm}$ depth region of HT9 irradiated with $5 \mathrm{MeV} \mathrm{Fe}$ (ACO3) at $460^{\circ} \mathrm{C}$, to $550 \mathrm{dpa}$ with $10 \mathrm{appm} \mathrm{He}$.

Figure C. 16: Void diameter, number density and swelling depth profiles as well as void distribution in the 500 to $700 \mathrm{~nm}$ depth region of HT9 irradiated with $5 \mathrm{MeV} \mathrm{Fe}$ (ACO3) at $460^{\circ} \mathrm{C}$, to $650 \mathrm{dpa}$ with $10 \mathrm{appm} \mathrm{He}$. 


\section{LIST OF APPENDICES}

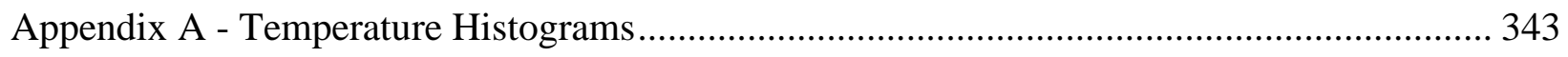

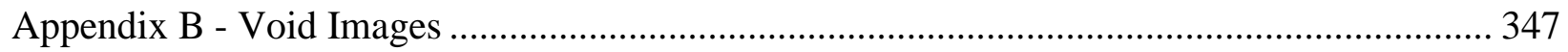

Appendix C - Void Depth Profiles and Distributions .................................................................. 363

xxvii 


\title{
ABSTRACT \\ THE CO-EVOLUTION OF MICROSTRUCTURE FEATURES IN SELF-ION IRRADIATED HT9 AT VERY HIGH DAMAGE LEVELS
}

\author{
by Elizabeth Margaret Getto
}

Chair: Gary S. Was

The objective of this study was to understand the co-evolution of microstructure features in self-ion irradiated HT9 at very high damage levels. HT9 (heat 84425) was pre-implanted with 10 atom parts per million helium and then irradiated with $5 \mathrm{MeV} \mathrm{Fe}^{++}$in the temperature range of $440-480^{\circ} \mathrm{C}$ to $188 \mathrm{dpa}$. A damage dependence study from 75 to $650 \mathrm{dpa}$ was performed at the peak swelling temperature of $460^{\circ} \mathrm{C}$. The swelling, dislocation and precipitate evolution was determined using Analytic Electron Microscopes in both Conventional Transmission electron microscopy (CTEM) and Scanning Transmission Electron Microscopy (STEM) modes. Void swelling reached a nominally linear rate of $0.03 \% / \mathrm{dpa}$ from 188 to 650 dpa at $460^{\circ} \mathrm{C}$. G phase precipitates were observed by 75 dpa and grew linearly up to 650 dpa. $M_{2} X$ was observed by 250 dpa and peaked in volume fraction at $450 \mathrm{dpa}$. Dislocation loop evolution was observed up to $650 \mathrm{dpa}$ including a step change in diameter between 375 and 450 dpa; which correlated with nucleation and growth of $\mathrm{M}_{2} \mathrm{X}$. 
The experimental results were interpreted using a rate theory model, the Radiation Induced Microstructure Evolution (RIME), in the damage range from 188 to 650 dpa. A simple system of voids and dislocations was modeled in which the dislocations measured from experiment were used as input, or the dislocations were allowed to evolve dynamically, resulting in swelling that was overestimated by $63 \%$ relative to that observed experimentally. G phase had limited effect on the void or dislocation behavior. The behavior of $\mathrm{M}_{2} \mathrm{X}$ within the microstructure was characterized as a direct effect as a coherent sink, and as an indirect effect in consuming carbon from the matrix, which had the largest impact on both void and dislocation behavior. A slowly monotonically increasing swelling rate was observed both experimentally and computationally, with swelling rates of $\sim 0.025 \% / \mathrm{dpa}$ and $\sim 0.036 \% / \mathrm{dpa}$ before and after $450 \mathrm{dpa}$. The agreement in void behavior between experiment and model when all effects (loops, network, $\mathrm{G}$ phase, $\mathrm{M}_{2} \mathrm{X}$ formation and growth, and removal of carbon) are accounted for demonstrates the importance of characterizing the evolution of the full microstructure over a large dpa range.

xxix 


\section{CHAPTER 1}

\section{INTRODUCTION}

The rapidly growing world population has increased the demand for natural resources including energy. Growing concerns about climate change have fueled a return to interest in nuclear power as a significant carbon-free base-load energy source. However, uncertainty about nuclear waste policy has led to increased focus on alternative reactor concepts that minimize waste. Generation (Gen) IV reactors are key in meeting the rapidly growing demand for energy now and in the future. The concerns about residual nuclear waste have thus created a demand for greater fuel utilization via increased burnup. Higher burnup will push the limits of reactor structural materials to damage levels not yet reached in the current light water reactor (LWR) fleet or fast reactors used in the past. Thus, understanding the behavior of core and structural materials under irradiation is paramount to the success of new reactor concepts including TerraPower's Traveling Wave Reactor (TWR) concept.

The main differentiator of the TWR from "traditional" breeder reactors is its unique core [1]. Fertile fuel assemblies consisting of depleted uranium (for breeding) and low enriched uranium fuel assemblies will be arranged within the core to enable both burning and breeding of fuel. What presents a unique materials challenge is that the core operates without refueling. Fuel shuffling is utilized to create either a traveling wave from the inside of the core to the outside or alternatively, a "standing wave" of breeding/burning that distinguishes this reactor design and gives it its name. The shuffle would simply move fuel assemblies of high burnup from the middle 
of the core to the outer rim, without reload, a significant deviation from traditional LWR operation leading to a much higher burnup and therefore more radiation damage to core materials. Damage levels are expected to reach at least 200 displacements per atom (dpa) and above in sodium fast reactors [2] and $600 \mathrm{dpa}$ in TWRs after 60 years in operation [1,2], which presents a unique material challenge as this requires highly radiation tolerant materials. Furthermore, there is no existing database at such high damage levels for any type of steel under neutron irradiation. Finally, the push to higher burnup and damage is not unique to TWRs, but also to other Gen IV reactor designs including alternative sodium fast reactor concepts.

Ferritic-martensitic (FM) steel alloys are leading candidates for structural and cladding components in Gen IV designs. FM steels are candidates for fast reactors because of their compatibility with sodium, superior resistance to radiation damage such as void swelling and excellent thermal conductivity and thermal expansion coefficient [3]. It was first determined in the 1970s that austenitic stainless steels, given their susceptibility to both void swelling and creep, were unsuitable for both breeder-type reactors and fusion devices expected to reach very high damage levels. FM alloys, previously developed for applications in high temperature coal plants, have become an alternative to stainless steels. Initial testing at the Fast Flux Test Facility (FFTF), a sodium fast reactor in Hanford, Washington, showed promise in terms of increased radiation tolerance, especially in terms of void swelling resistance, and continued to motivate the transition from advanced stainless steels such as D9 to FM alloys.

FM steels are body centered cubic (bcc) iron alloys with major additions of chromium, typically with $\mathrm{Cr}$ concentrations of $7-15 \mathrm{wt} \%$ with most commercially available alloys having either 9 or $12 \mathrm{Cr} w t \%$ [3]. They have additions of other minor alloying elements, such as $\mathrm{W}, \mathrm{V}$, Mn, Ta, Ti and Mo, to add desirable material properties such as strength or ductility. FM alloys 
are uniquely identified by their complex microstructure including laths, subgrains and secondary phases that form during heat treatment of the alloy or under irradiation, which typically leads to a much higher sink density than conventional austenitic stainless steels.

Under high damage irradiation, a number of microstructure features form, most of which are detrimental to material integrity and safety. Of primary interest to this thesis, formation of voids causes dimensional instability. In addition, formation and evolution of secondary phases and dislocation loops under irradiation can lead to increased hardening and embrittlement of the alloy.

Void swelling has been studied extensively in other alloys such as austenitic stainless steels, but very little systematic experimental work has been performed on void swelling in FM alloys due, in part, to the high degree of swelling resistance. Very few voids form at the relatively low damage levels examined. However, some limited results for neutron (up to 200 dpa) and ion irradiation (up to $600 \mathrm{dpa}$ ) do exist. Neutron irradiations performed in FFTF up to 200 dpa indicates that swelling remains below $3.2 \%$ in the temperature range $400-460^{\circ} \mathrm{C}$ [4-10]. However, the scarcity of data makes it difficult to determine whether or not swelling is in the so-called steady state swelling regime, which is characterized by void growth-dominated swelling and a linear or near linear swelling rate. If the highest neutron damage (165-200 dpa) irradiations are in the growth-dominated steady state swelling regime, then the linear swelling rate is expected to be $\sim 0.01-0.02 \% / \mathrm{dpa}[5,7,10,11]$.

More recently, ion irradiation has proven effective as a surrogate for neutron irradiations $[2,12,13]$ resulting in more systematic studies of temperature and damage, but has yielded conflicting results. Smidt et al. [14] found swelling of up to $4.7 \%$ at $450^{\circ} \mathrm{C}$ and 250 dpa in HT9. Assuming swelling is linear by $250 \mathrm{dpa}$, the swelling rate is $\sim 0.02 \% / \mathrm{dpa}$. In contrast, Toloczko et 
al. [15] in irradiations performed with $1.8 \mathrm{MeV} \mathrm{Cr}^{3+}$ at $450^{\circ} \mathrm{C}$ determined a final swelling rate of $0.2 \% / \mathrm{dpa}$ at damage levels of $400 \mathrm{dpa}$ and above.

In addition to uncertainty of the high damage void swelling behavior, secondary phases have not been examined at commensurately high damage levels. The main precipitates observed in reactor or under ion irradiation in this temperature and damage regime are $\mathrm{G}$ phase, $\mathrm{M}_{2} \mathrm{X}$ and $\alpha^{\prime}$ [3]. Finally, there exists no systematic study of dislocation formation without stress beyond 10 dpa, but recent results from Gupta et al. [16] and Jiao et al. [17] suggest that dislocation loop diameter saturates by 10 to $20 \mathrm{dpa}$, well below the void growth-dominated swelling regime.

The limited and somewhat contradictory experimental results from both neutron and ion irradiations highlight the need for a systematic study of the evolution of void swelling and other microstructure features to a very high damage level in FM alloys. In particular, and especially relevant to this thesis, is that the so called terminal swelling rate of FM alloys is a subject of some debate. Consequently, there are no studies on the effect of other irradiation-induced features such as secondary phases and dislocations on voids at this high damage and high swelling rate.

The objective of this thesis is to understand the co-evolution of microstructure features at very high damage in self-ion irradiated HT9. This will be accomplished with a combination of ion irradiation experiments followed by post irradiation microstructure characterization and computational modeling. Chapter 2 will provide extended background from the literature relevant to this thesis. Chapter 3 will present the detailed objective and associated approach. Chapter 4 presents the experimental details and methodology utilized in ion experiments. Results from ion irradiation experiments and post-irradiation characterization will be presented in Chapter 5. Chapter 6 will present the modeling portion of the thesis, including additional background relevant to the computational model. Chapter 7 will present a discussion and analysis of the combined 
experimental and modeling results. Chapter 8 will present final conclusions and finally, Chapter 9 will suggest possible future work. 


\section{CHAPTER 2}

\section{BACKGROUND}

Cladding and structural components will be subject to a high fluence of fast neutrons in Generation IV reactors, especially in TerraPower's traveling wave reactor concept. Although there is a large database of knowledge of radiation effects on materials under light water reactor conditions, a limited amount of these results can be applied to next generation reactors since they are at relatively low damage ( $<100 \mathrm{dpa}$ ) levels. Nevertheless, significant materials degradation has occurred at these damage levels via radiation-induced segregation (RIS), nucleation and growth of dislocation loops, secondary phase formation causing hardening and embrittlement as well as dimensional changes such as creep and void swelling.

FM alloys are being considered as alternatives to stainless steels used in previous fast reactor designs because of improved radiation tolerance. However, the existing dataset is small and little to nothing is known about void swelling in the very high damage regime in these alloys, due to their high degree of swelling resistance and the low damage levels achievable in test reactors. Additionally, the coupling of temperature and flux makes systematic damage or temperature studies challenging.

This chapter will provide background necessary to place results of this thesis in context within the existing literature. First, void swelling will be introduced and mechanisms described in 
a theoretical framework. Then, the existing experimental measurements of void swelling as well as other irradiation-induced phenomena that may influence void swelling will be presented.

\subsection{Mechanisms of Void Swelling}

Swelling is caused by the formation and growth of cavities under irradiation. The supersaturation of vacancies due to irradiation and biased sinks drives void nucleation and growth by vacancy agglomeration, which in turn causes bulk dimensional change. Although it is well understood in austenitic alloys that voids undergo a nucleation period followed by linear or "steady state" swelling rate of $\sim 1 \% / \mathrm{dpa}[8,18]$, there is little evidence that ferritic-martensitic alloys exhibit linear swelling behavior at any levels approaching 1\%/dpa. Furthermore, the influence of other irradiation features on void swelling has not been studied systematically. An introduction to void swelling followed by a discussion of ferritic-martensitic alloys will be presented. The current void swelling results under neutron and ion irradiation will be reviewed.

The term cavity is inclusive of both bubbles, which by definition require helium to stabilize, whereas voids do not require helium to be stabilized. In other words, all voids are cavities but all cavities may not be voids. At large sizes $(>20 \mathrm{~nm})$, it is a safe assumption that all cavities are voids. Thus, for the swelling range examined in this study, it is safe to assume all cavities are in fact voids and for preference will be given to this nomenclature from here on.

\subsubsection{Introduction to Void Swelling}

Irradiation-induced void swelling in susceptible metals and alloys evolves through three different void behavior regions shown in Figure 2.1 [19]. In the nucleation regime, cavity embryos are nucleated then stabilized to form bubbles or small voids, which are resolvable by transmission

electron miscopy (TEM) or scanning transmission electron microscopy (STEM) at $\sim 1$ to $2 \mathrm{~nm}$. 
Swelling increase in this regime is dominated by the nucleation of new stable bubbles or voids. There are two nucleation pathways for void formation under irradiation [18,20,21]. The first pathway is agglomeration of small vacancies or vacancy clusters that then reach a critical size; which is a mechanism that is dependent on stochastic fluctuations in the damaged microstructure. The second pathway is accumulation of small gas-stabilized bubbles (typically helium) until they reach a critical size beyond which gas accumulation is not needed for growth and they convert from bubbles to voids. Helium decreases the critical size necessary for a stable cavity by reducing the free energy barrier.

As these void embryos nucleate and stabilize, they start to accumulate vacancies and grow in size. At this point, the void distribution is centered at low diameters $(<10 \mathrm{~nm})$ and has a small full width half maximum, as growth is not yet the dominant process. In the transition region, there is simultaneous nucleation of new stable embryos while existing voids grow by vacancy agglomeration. Thus the void size distribution expands and the mean void diameter increases as stable voids grow. The increase in swelling is due to both nucleation of new voids as well as growth of already nucleated voids. After a period of time depending on the swelling resistance of the alloy in question, growth dominates and the swelling reaches the growth-dominated regime, which continues to very high damage levels and is characterized by a near constant swelling rate [19]. This has also been referred to as steady state swelling due to linear final swelling value observed in reactor for austenitic and pure alloys [8,22-24]. An alternative theory suggests that swelling may even saturate at sufficiently high swelling values, but this has only been shown to happen in AISI 316 at $260 \%$ swelling at 600 dpa [25]. Although void swelling is classified into these three regimes, the transition is not distinct as the regimes overlap in time. 
Void evolution is well documented in both FCC austenitic alloys $[8,22,25]$ and pure metals $[3,23,26]$, which are relatively susceptible to void swelling. The final linear swelling rate appears to be independent of heat treatment and temperature for austenitics $[8,27]$ within the temperature region where metals/alloys are susceptible to void swelling $\left(T \approx 0.3 T_{m}-0.5 T_{m}\right)$ [20]. Although void swelling is well understood in austenitic alloy, there is limited data, particularly at high damage level, for ferritic-martensitic (FM) alloys, which are candidate materials for Gen IV fast reactors. The lack of swelling data in FM alloys is due to a combination of the swelling resistance of FM alloys, as well as their relatively limited use in reactors to date $[4,6-8]$.

\subsection{Ferritic-Martensitic Alloys}

High chromium steels refer to those that contain $7-12 \%$ chromium. The first high chromium steel was developed in 1912 for steam turbine blades in Germany. British scientists then discovered that martensitic steels were rust resistant during unrelated experiments for gun barrel applications. Initial commercial applications included knives, tableware, razors and heat resistant bearings and tools [3]. High chromium steels continued to be developed for applications in chemical plants, aerospace technologies, electrical plants and gas turbine engines throughout the rest of the $20^{\text {th }}$ century. There was renewed interest in high chromium FM steels during the fifties and sixties due to a large push to improve efficiencies of coal power plants [28]. Increased efficiency was accomplished by increasing the temperature and pressure of the steam, leading to a need for supercritical boilers, designed to operate to ever increasing temperatures and pressures. As a result, steels needed to be developed that operated with low thermal fatigue, high thermal conductivity and low thermal expansion. Austenitic steels, previously used in coal plants, did not possess sufficient thermal properties to operate safely and effectively under the new required operating conditions [28]. Interest continued to grow throughout the seventies and early eighties, 
due to the energy crisis of the seventies and interest in first fast breeder then fusion applications [3].

It became apparent that austenitic steels were susceptible to both irradiation-induced creep and void swelling so scientists turned to the high chromium steels that were being utilized in fossil fuel applications. In 1974, scientists worldwide began an effort to optimize breeder reactors with a new type of steel, instead of the previously utilized AISI 316. Two FM alloys that were under consideration were HT9 and T91. HT9 was used in many fuel tests as primary core materials including duct and cladding as well as pressurized tubes used to study creep at the Fast Flux Test Facility (FFTF) in Hanford, Washington [4-7,11,29].

FM steels, in general, possess a number of useful properties [3]. These properties include high thermal conductivity, low thermal expansion and resistance to thermal fatigue; all of which are necessary for usage in high temperature fossil fuel plants as well as in high or very high temperature reactors. They also exhibit sufficient strength to be of practical use in power plants. $12 \mathrm{Cr}$ steel, in particular, exhibits excellent oxidation resistance. They show particular resistance to irradiation-induced creep and void swelling.

There are several limitations with FM steels. Body-centered-cubic (BCC) structures exhibit a ductile-brittle transition temperature (DBTT) that increases significantly with radiation [3]. Furthermore, radiation embrittlement and irradiation-induced creep will still be a consideration for FM steels. Finally, welding is much more difficult than for conventional austenitic stainless steels.

\subsubsection{Physical Metallurgy}

Processing of FM alloys involves three heat treatments: austenization, transformation to martensitic phase and tempering. The phase diagram relevant for FM alloy formation is given in 
Figure 2.2 [3]. Typically, austenization occurs when the alloy is heated up to $\sim 800-1450^{\circ} \mathrm{C}$ for 9 $12 \mathrm{wt} \% \mathrm{Cr}$ alloys. The austenizing temperature can be affected by the addition of austenite $(\gamma)$ stabilizers, which typically lower the temperature required for complete austenization of the steel, but at the risk of the formation of $\mathrm{MC}$ carbides if $\mathrm{C}$, the most commonly used and inexpensive austenite stabilizer is used. Thus, a higher temperature is desirable as it has the added effect of dissolving $\mathrm{MC}$ carbides resulting in coarser prior austenite grain (PAG) sizes and complete transformation to austenite before cooling. Austenization temperature for HT9 is typically around $1050-1100^{\circ} \mathrm{C}$. Following cooling, all or most of the phase transforms to martensite, unless austenite or ferrite stabilizers such as nickel or chromium are added. In general, HT9 may consist of all three phases: austenite, martensite and $\delta$ ferrite, but an effort is made to minimize both retained austenite and ferrite. Initially, the austenite to martensite transformation yields a very brittle microstructure prone to brittle fracture due to the high dislocation density.

Martensite is formed in thick sections known as laths, due to inhibition of pearlite transformation and absence of bainite. A continuous cooling transformation (CCT) diagram is shown in Figure 2.3. Lathes tend to be low in carbon and are usually around $0.5-1 \mu \mathrm{m}$ by $5 \mu \mathrm{m}$ in length [30]. Upon cooling, the austenite phase normally transforms to martensite, but when the martensite start temperature is close to room temperature austenite is retained. In an ideal microstructure, PAG has packets of martensite laths that are parallel. Laths within a single pack occupy the same habit plane, have the same orientation and are often very closely aligned in terms of crystallography.

The final step, tempering, is required to improve toughness by refining the microstructure. Tempering causes formation of small precipitates such as $\mathrm{M}_{23} \mathrm{C}_{6}$, which improve toughness and reduce softening, and is performed at a temperature below austenization to avoid re-austenization. 
Typically, tempering is performed around $760-850^{\circ} \mathrm{C}$ for conventional $\mathrm{FM}$ alloys and $870-960^{\circ} \mathrm{C}$ for reduced activation steels [3]. The effect of tempering temperature is as follows:

- $\quad<350^{\circ} \mathrm{C}: \mathrm{M}_{3} \mathrm{C}$ precipitates form and grow into a dendrite morphology

- $450-500^{\circ} \mathrm{C}$ : Fine needles of $\mathrm{M}_{2} \mathrm{X}$ precipitate nucleate on dislocations causing retardation of softening

- $500-550^{\circ} \mathrm{C}: \mathrm{M}_{7} \mathrm{C}_{3}$ and $\mathrm{M}_{2} \mathrm{X}$ phases coarsen and decrease hardness

- $\quad>550^{\circ} \mathrm{C}$ : $\mathrm{Cr}$-rich $\mathrm{M}_{23} \mathrm{C}_{6}$ precitiates nucleate on the martensite laths and PAG boundaries

- $\quad>650^{\circ} \mathrm{C}: \mathrm{M}_{23} \mathrm{C}_{6}$ precipitates coarsen, reducing dislocation density

- $\quad>750^{\circ} \mathrm{C}$ : sub-cell within laths grow into equi-axed grains and continued growth of $\mathrm{M}_{23} \mathrm{C}_{6}$ [3]

During tempering, the dislocation networks rearrange into a lower energy configuration with the lath forming small subgrains. Dislocation motion and rearrangement is caused by thermally-activated dislocation climb and glide and results in small grain orientation angles. The martensitic phase has lathes with high dislocation density, even after tempering and formation of subgrains. The resulting dislocation network density is on the order $10^{14}-10^{15} \mathrm{~m}^{-2}$ [3,31]. A schematic and typical microstructure is presented in Figure $2.4[3,32]$.

Finally, tempering also results in precipitation of carbon out of the matrix, which then precipitate as carbides on the PAGB (prior austenite grain boundaries). They preferentially nucleate on PAGBs rather than lath or subgrain boundaries due to the increased diffusion rate along high angle boundaries [30,32].

\subsection{Void Swelling Experiments in Ferritic-Martensitic Alloys}


Although there is limited data at high (>100 dpa) or very high damage (>200 dpa) for void swelling in FM alloys, there exists a non-trivial dataset at lower damage levels that may approach the high damage/growth-dominated swelling regime. The following is a summary of both inreactor (Section 2.3.1) and ion (Section 2.3.2) irradiations. A discussion of irradiation-induced defect partioning and its relation to void swelling is also included.

\subsubsection{Void Swelling under Neutron Irradiation}

Sencer et al. performed a study using neutron-irradiation at the Fast Flux Test Facility (FFTF) on HT9 [9,10]. A duct was irradiated under a variety of conditions at a temperature of $443^{\circ} \mathrm{C}$ up to $155 \mathrm{dpa}$. Swelling was calculated via TEM measurement of the voids and was $0.3 \%$ on average. However, it is unclear whether void growth-dominated swelling was reached or if the swelling was in the transition regime since average void diameter was $\sim 30 \mathrm{~nm}$. If the swelling was transient in nature, it would be expected that the swelling could increase at a faster rate at higher damage levels. Assuming linear swelling, the swelling rate could be as low as 0.002\%/dpa. However, the results of this study do not provide enough data to support a prediction for swelling amount or rate, especially given the likelihood that the swelling is still exhibiting transient-type behavior. It is worth mentioning that this is the same heat (84425) as was examined in this thesis.

Gelles [7] performed microstructure examination of commercial alloys (HT9 and T91) irradiated at FFTF up to approximately 200 dpa. A single heat of HT9 (9607R2) with two different heat treatments was examined: RFFL (Heat $9607 \mathrm{R} 2,1000^{\circ} \mathrm{C} / 20 \mathrm{~h}+1100^{\circ} \mathrm{C} / 5 \mathrm{~min}+700^{\circ} \mathrm{C} / 2 \mathrm{~h}$ ) and RHFL (Heat $9607 \mathrm{R} 2,1050^{\circ} \mathrm{C} / 5 \mathrm{~min}+760^{\circ} \mathrm{C} / 0.5 \mathrm{~h}$ ) and compared to a third heat/heat treatment of T91 designated as PTFL (Heat $30176,1040^{\circ} \mathrm{C} / 1 \mathrm{~h}+760^{\circ} \mathrm{C} / 1 \mathrm{~h}$ ). Overall, the T91 (PTFL) swelled the most when measured via the density change method with $1.76 \%$ average swelling. Compared to the RHFL heat treatment of HT9, which swelled $1.02 \%$ and the RFFL heat treatment of HT9 
that swelled by only $0.09 \%$. This result indicates that heat treatment can be at least as important as alloy composition in terms of swelling resistance. Additional evidence is that the HT9-RHFL and T91-PTFL heat treatments, which swelled the most, also had the most similar heat treatments, with shorter tempering and austenization times. However, swelling in any of the 3 alloys/heats/heat treatments examined likely had not reached the growth-dominated swelling regime, so this was likely a measurement of the resistance to void nucleation rather than an examination of the growth regime. The observed void structures were consistent with expected void structures in martensitic steels; i.e. void arrays between lath boundaries and void denuded zones on and adjacent to boundaries. The HT9 microstructure had blockier carbide precipitation on boundaries and lower density of larger voids when compared to T91. Swelling was also found to be independent of Burgers vector distribution.

Toloczko and Garner [4,6] examined irradiated HT9 from the FFTF facility to examine creep and swelling behavior. Swelling behavior was examined at damage levels up to 165 dpa and temperatures in the range $384-427^{\circ} \mathrm{C}$. Swelling remained under $1 \%$ for all HT9 heats up to 165 dpa at $400^{\circ} \mathrm{C}$, without stress. Maximum swelling rate appeared to be $\sim 0.01 \% / \mathrm{dpa}$. A comparison of incremental swelling with swelling rate is shown in Figure 2.5. Heat treatment as well as heatto-heat variation are major factors in swelling and were found to cause orders of magnitude changes in swelling rate. Although the samples were nominally the same composition and heat treatment, the swelling variance was attributed to differences in production variables, which were not characterized by the authors.

Toloczko et al. [5] also examined HT9 at a damage level up to $208 \mathrm{dpa}$ at $\sim 400^{\circ} \mathrm{C}$ in FFTF. Swelling reached up to $\sim 1 \%$ swelling at $208 \mathrm{dpa}$ in the absence of a hoop stress. Assuming linear swelling, the swelling rate again was found to be less than $\sim 0.01 \% / d p a$. Swelling measured from 
165-208 dpa did not increase substantially. It was also found that swelling was accelerated by hoop stress. A 9 Cr-1Mo alloy was also examined and swelling was measured to be $0.8 \%$ at the same temperature and damage level.

The effect of the chromium composition was examined by Gelles [33] in neutron irradiations of up to $200 \mathrm{dpa}$ at $425^{\circ} \mathrm{C}$. Using previous irradiation data from EBR-II and results from FFTF, the void swelling and microstructures were characterized as a function of chromium composition. Swelling peaked between 6 and $9 \mathrm{Cr}$ wt $\%$. The maximum swelling of $7.4 \%$ was observed in the Fe-9Cr alloy. The Fe-12Cr alloy (similar chromium content as HT9) had swelling of $2.8 \%$. Fe-18Cr and $\mathrm{Fe}-15 \mathrm{Cr}$ had the lowest swelling levels. For all chromium compositions, there appeared to be a slower swelling rate up to just under $150 \mathrm{dpa}$ (presumed transient regime) and an accelerated swelling rate afterwards (Figure 2.6), indicating possible linear swelling behavior. However, very few damage levels were examined. The highest average swelling rate was estimated to be $0.1 \% / \mathrm{dpa}$ for Fe-9Cr. However, irradiations were performed in two different reactors with two different neutron spectra and He/dpa ratios.

Another important value examined in this study is the sink density ratio (Q). Q is the ratio of the void sink strength to that of dislocations. Q analysis was developed by Mansur [34] to predict the damage dependence of swelling in austenitic alloys and ferritic-martensitic alloys to a limited extent. Q is defined as:

$$
Q=\frac{Z_{i, v}^{d} \rho_{d}}{Z_{i, v}^{c} 4 \pi r_{c} \rho_{c}}
$$


where $z_{i, v}^{d}$ is the bias factor for either dislocations or cavities, $\rho_{d}$ is the dislocation density including both network and loops, $r_{c}$ is the cavity radius and $\rho_{c}$ is the cavity density. Assuming bias factors are similar, the expression for Q can be simplified to the following:

$$
Q=\frac{\rho_{d}}{4 \pi r_{c} \rho_{c}}
$$

The peak swelling rate is expected at values of Q 1, which was observed in this study at 200 dpa. The maximum swelling rate is likely to be synonymous with linear swelling [34]. The implications for $\mathrm{Q}$ will be further discussed in Section 2.3.3. Voids were homogeneously distributed throughout the microstructure except for the void denuded zones near grain boundaries [33]. Larger voids were imaged attached to needle-shaped precipitates in the $18 \mathrm{Cr}$ alloy only.

After the publication of Gelles' paper ([33]) on the effect of chromium content on swelling, Garner et al. [8] suggested an error had been made in the damage calculation and the dpa levels were adjusted. Garner et al. suggest that the calculated $0.06 \% / \mathrm{dpa}$ swelling rate from 0-150 dpa was mistaken. A comparison of their corrections next to the original data from EBR-II is given in Figure 2.7. Assuming that the new damage is more accurate, this increases the swelling rate by approximately an order of magnitude. Additionally, it was suggested in Garner's study that the temperature dependence of swelling arises primarily by extension of the transient regime, rather than a difference in the swelling rate observed in the high damage level growth-dominated regime, which is still consistent with the bell-shaped curve predicted by swelling theory [20]. The author also suggested that final swelling could be as high as $0.5 \% / \mathrm{dpa}$, and could approach the swelling rate of austenitic steels; however, there was no experimental evidence of such a swelling rate. 
A summary of void swelling results of neutron-irradiated HT9 is given below in Figure 2.8. Clearly, swelling varies greatly with heat treatment but in general tends to increase at a rate of $0.01 \% / \mathrm{dpa}$. In addition, there is no data beyond 208 dpa under neutron irradiation.

\subsubsection{Void Swelling under Ion Irradiation}

Kai and Kulcinski [35] investigated the effect of helium preimplantation on $14 \mathrm{MeV}$ nickel irradiated HT9 in damage levels up to $200 \mathrm{dpa}$. There was no void formation in samples without helium preimplantation at $500^{\circ} \mathrm{C}$ to $200 \mathrm{dpa}$. However, the $200 \mathrm{dpa}$ location was at the peak, where the injected interstitial has been shown to suppress void swelling [12,25,36]. At 30 dpa, voids did not form at temperatures other than $500^{\circ} \mathrm{C}$. The highest swelling value $(0.02 \%)$ reached was at 60 dpa, $500^{\circ} \mathrm{C}$ with 100 appm He preimplanted.

Comparisons between cold pre-injection of helium and simultaneous co-implantation was examined by Ayrault in a 9Cr-1Mo [37]; both damage dependence (5, 10 and 25 dpa) and temperature dependence $\left(450,500,550\right.$ and $\left.600^{\circ} \mathrm{C}\right)$ experiments were performed. Helium levels were 15 appm He/dpa and implanted using a degraded $0.83 \mathrm{MeV} \mathrm{He}^{+}$ion beam at the Argonne National Lab Dual-Ion Beam Facility. 3.0 $\mathrm{MeV} \mathrm{Ni}^{+}$ions were used for the irradiation and damage rates were $3-4 \times 10^{-3} \mathrm{dpa} / \mathrm{s}$. There was more cavity formation in the co-implanted samples then the pre-injected samples. It was also observed that there was a peak swelling temperature at $450^{\circ} \mathrm{C}$, regardless of method of helium implantation. All swelling was below $0.05 \%$, which is likely far below the onset of linear or growth-dominated swelling.

Smidt et al. [14] irradiated HT9 and EM12 steel with $2.8 \mathrm{MeV} \mathrm{Fe}^{+}$ions up to 250 dpa preimplanted with 1 appm He. The primary objective of this study was to understand the relationship between temperature and swelling. The peak swelling temperature was determined to 
be $500^{\circ} \mathrm{C}$ for $\mathrm{HT} 9$ and $550^{\circ} \mathrm{C}$ for EM12. Once peak swelling temperature was determined, a damage dependence study was performed up to $250 \mathrm{dpa}$. Temperature dependence for both steels is plotted in Figure 2.9a. Swelling was maximized at $500^{\circ} \mathrm{C}$ and $3.7 \%$ for HT9 at 150 dpa. The swelling curve as a function of damage is plotted in Figure 2.9b. The linear swelling rate was calculated to be $0.017 \% / \mathrm{dpa}$ for HT9 and $0.011 \% / \mathrm{dpa}$ for EM12. EM12 had a transient regime of approximately 10 dpa and HT9 appeared to have a higher swelling rate from 0-40 dpa, which does not agree with swelling theory. Swelling behavior was atypical and did not exhibit nucleation; the transient swelling followed what appeared to be a linear swelling rate. Swelling values were higher than those of neutron irradiated samples at similar temperatures and damage levels.

Borodin et al. [38] examined various 10-13\% chromium-containing steels with heavy ions (3 $\mathrm{MeV} \mathrm{Cr}^{+3}$ ) including $13 \mathrm{Cr}-2 \mathrm{Mo}-\mathrm{Nb}-\mathrm{V}-\mathrm{B}, 13 \mathrm{Cr}-2 \mathrm{Mo}^{-\mathrm{TiO}} 2,10 \mathrm{Cr}-6 \mathrm{Mo}-\mathrm{Nb}-\mathrm{V}$ and $\mathrm{Fe}-12 \mathrm{Cr}$ from 1 to $150 \mathrm{dpa}$. Maximum swelling of $0.5 \%$ was observed at $500^{\circ} \mathrm{C}, 150$ dpa suggesting a peak swelling temperature of $500^{\circ} \mathrm{C}$ for the $\mathrm{Fe}-12 \mathrm{Cr}$ alloy. Swelling was examined in both ferrite and martensite phases and remained below $0.5 \%$, regardless of grain type. Void behavior differed in each of the phases; for the ferrite phase, voids were distributed homogeneously and for the martensite phase, voids were highest near cell walls.

Ferritic steels irradiated with heavy ions were examined by Ward and Fisher [39]. Fe-10Cr and FV448 (10Cr with solute additions) were irradiated up to 50 dpa with helium preimplantation with $52 \mathrm{MeV} \mathrm{Cr}$ ions. Swelling in the $\mathrm{Fe}-10 \mathrm{Cr}$ was $4.6 \%$ and in the FV448 was $0.1 \%$, indicating the improved swelling resistance of commercial alloys with solute additions. The swelling rate of the Fe-10Cr was the highest observed in an FM alloy at the time. Lower damage experiments showed that a dislocation network formed very quickly in the high purity metals, and slower in the 
commercial alloys. Since a stable dislocation network is correlated to higher swelling rates, these results are consistent with expected swelling behavior.

Hide et al. [40] irradiated FM steels in a two-step process. Specimens were first injected with $42 \mathrm{keV}$ helium ions and then irradiated with either carbon $(200 \mathrm{keV})$ or nickel ions (3 MeV) up to $200 \mathrm{dpa}$ with damage rates of $1 \times 10^{-2} \mathrm{dpa} / \mathrm{s}$. Irradiations were performed between $425-625^{\circ} \mathrm{C}$. Maximum swelling was $0.3 \%$ at $575^{\circ} \mathrm{C}$ and $150 \mathrm{dpa}$. Larger cavities of around $17 \mathrm{~mm}$ were formed at $525^{\circ} \mathrm{C}$ and were homogeneously distributed. For all FM steels, swelling was very low and temperature and damage relationships are plotted in Figure 2.10. Estimated swelling rate was 0.001-0.003\%/dpa at peak temperatures, suggesting void evolution had not proceeded to any great extent.

Toloczko et al. [15] examined swelling in $\mathrm{Cr}^{3+}$-irradiated ODS ferritic alloy MA957, EP 450 and HT9 up to 600 dpa. Damage and temperature dependence experiments were performed. At $450^{\circ} \mathrm{C}$, all three alloys were examined up to $600 \mathrm{dpa}$. The authors determined that steady state/linear swelling in HT9 was not reached until approximately 400-600 dpa and the swelling rate was determined to be approximately $0.2 \% / \mathrm{dpa}$, which was consistent with previous results from Garner et al. under neutron irradiation [8]. MA957 did not reach this high of a swelling rate, which the authors attributed to an extended nucleation regime. The temperature dependence results are shown in Figure $2.11 \mathrm{~b}$. Peak swelling temperature was determined to be $450^{\circ} \mathrm{C}$ at 500 dpa.

A summary of ion irradiations, analogous to Figure 2.8, is shown below in Figure 2.12. It highlights the conflicting results between different experiments. The overall results of this literature search show that void swelling evolution to high damage is not well understood in FM alloys. Specifically, neither the length of void incubation nor the high damage swelling rate has been determined to any degree of consistency because FM alloys have been shown to be highly 
resistant to void swelling both in reactor [4-10] and by ion irradiation [15,35,37-40]. Since accessing the post-transition swelling regime requires high damage levels, there is little data in the literature at damage levels in excess of 100-200 dpa. Such damage levels have only recently become experimentally feasible due to recently improved ion source performance.

\subsubsection{Defect Partitioning}

Related to understanding void swelling, the evolution of various microstructure features with increased damage can be tracked by determining how the freely migrating defects are partitioned. In other words, at which sinks do they agglomerate, how many recombine, and how many annihilate at various other sinks. Defect absorption at sinks (i.e. microstructure features) is determined by the sink strength. Sink strength determines the affinity of a microstructure feature for defects. The simplest method to analyze defect partitioning is by a $\mathrm{Q}$ analysis, which uses void and dislocation sink strengths (i.e. defect partitioning) described in Section 2.3.1.

This model is simplistic in that it only takes into account dislocations and voids. Other features, such as coherent and incoherent precipitates, grain boundaries and lath boundaries also act as sinks, which are not addressed in this analysis. An additional simplification is the assumption that bias factors are approximately equal. Since swelling is bias driven, this makes little physical sense for large values of bias. Ferritic-martensitic alloys are expected to have low values of bias $(<10 \%)$ [8,41-43], especially when compared to austenitic stainless steels. Finally, Q analysis has only been applied at limited damage levels and correspondingly low swelling values. However, a summary of the available results is presented in Figure 2.13. A more sophisticated defect partitioning analysis via modeling of individual defect behavior at various sinks including precipitates, dislocations and voids would be both more useful and applicable to the physical behavior under irradiation. 


\subsection{Dislocation Microstructure}

Dislocation analysis in FM alloys has primarily been studied within the context of mechanical properties such as creep [30,44], fatigue [45,46] or tensile deformation [47], but limited data is available for FM alloys not under stress. The interaction between biased loops and voids is critical. The bias for interstitials enables the free vacancies necessary for void swelling to initiate and continue. The dislocation network also makes a significant contribution to irradiation hardening, which will be discussed later in this chapter, for completeness but will not be analyzed in this work.

\subsubsection{Loop Character}

Prior to irradiation, lathes in FM alloys [3] contain dislocations with a Burgers vector of $\mathbf{a} / 2<111>$ and a density typically around $10^{14} \mathrm{~m}^{-2}$. This is large when compared to typical dislocation densities in pure metals or solution annealed stainless steel alloys that have not been cold worked. Under irradiation below $0.3 T_{m}$ (where $T_{m}$ is the melting temperature of the alloy), interstitials are much more mobile than vacancies, leading to formation interstitial-type dislocation loops. Vacancy loops, nucleated by collapse of depleted zones within the cascade, are theoretically possible but have not been observed in ferrite and as such are not expected in FM alloys [3].

Dislocation loops form under irradiation with two possible Burgers vector orientations: a cube edge-loop with $\mathbf{a}<100>$ or glissile edge loops with $\mathbf{a} / 2<111>$ Burgers vectors on $\{111\}$ habit planes. Loops tend to vary in size from 10 to $40-50 \mathrm{~nm}$ in size. They can be formed by shearing from a common $\mathbf{a} / 2<110>$ nucleus. The high stacking fault energy in high-Cr FM alloys means that loops unfault very early by shear in one or two directions [47] and faulted loops are not observed. 
Interactions between dislocations form a complex dislocation network. $\mathbf{a}<100>$ loops have strong interstitial bias and it has been theorized that the $\mathbf{a} / 2<111>$ network has less of a bias $[3,48]$. Assuming this theory is correct, it means that $\mathbf{a} / 2<111>$ are essentially neutral sinks whereas $\mathbf{a}<100>$ are more biased sinks. Thus, the formation of a stable $\mathbf{a}<100>$ loop population instead of the preexisting $\mathbf{a} / 2<111>$ network has been correlated with the extended nucleation regime for void swelling as the bias increases with the growth of loops as a function of damage [41]. Under irradiation, loops have been observed to be primarily of $\mathbf{a}<100>$ character while the network still tends to consist of dislocations with Burgers vector $\mathbf{a} / 2<111>$ as in the unirradiated microstructure.

\subsubsection{Dislocation Microstructure under Irradiation}

The dislocation microstructure of commercial FM alloys and binary $\mathrm{Fe}-\mathrm{Cr}$ model alloys irradiated at $420^{\circ} \mathrm{C}$ to nominally $200 \mathrm{dpa}$ were characterized by Gelles $[7,11,33]$. HT9 exhibited a dislocation microstructure dominated by $\mathbf{a} / 2<111>$ network with a low density of $\mathbf{a}<100>$ loops. Loop sink strength $\left(0.83 \times 10^{14} \mathrm{~m}^{-2}\right)$ was approximately an order of magnitude lower than the network $\left(9.8 \times 10^{14} \mathrm{~m}^{-2}\right)$ in Fe-12Cr [33]. Similar behavior was observed in T91 irradiated under the same conditions; with the loop population dominated by $\mathbf{a}<100>$ type loops and network of $\mathbf{a} / 2<111>$ character. The dominance of the $\mathbf{a} / \mathbf{2}<111>$ network was attributed to the subgrain structure in the as-received condition.

Binary alloys were also irradiated to similar conditions $\left(420^{\circ} \mathrm{C}, 200 \mathrm{dpa}\right)$ and will be considered, as there is a shortage of dislocation data in commercial FM alloys. At 200 dpa, [11,33] model Fe-12Cr alloys exhibited small $(\sim 15 \mathrm{~nm}) \mathbf{a}<100>$ loops and both $\mathbf{a}<100>$ and $\mathbf{a}<111>$ lines in the network. In terms of morphology, there were fewer dislocations with Burgers vectors of $\mathbf{a}<100>$ located at or near subgrain boundaries, which is likely a result of the tempering step. The behavior of $\mathrm{Fe}-9 \mathrm{Cr}$ was the same in terms of the character of the loops and the network. 
FM alloys examined by Dubuisson et al. [49] showed formation of dislocation loops from $400-450^{\circ} \mathrm{C}$ as well as a large dislocation network with small unfaulted loops at high temperatures of $500^{\circ} \mathrm{C}$ and above. There was little dislocation evolution with increasing damage levels up to 100 dpa, but no quantitative results were given.

Kai and Kulcinski characterized the dislocation microstructure of $14 \mathrm{MeV}$ Ni-irradiated HT9 $[19,35]$. A high density of dislocation loops was found after irradiation in the temperature range of $300-600^{\circ} \mathrm{C}$. Loops were interstitial in nature with $\mathbf{b}=\mathbf{a}<100>$. The high network density prior to irradiation was replaced by irradiation-induced loops. Damage dependence was not examined. Dislocation loop diameter increased while number density decreased with temperature as shown in Figure 2.14 and there was virtually no difference in dislocation microstructure after irradiation at $600^{\circ} \mathrm{C}$.

The dislocation microstructure after irradiation in FFTF was also characterized by Sencer et al. $[9,10]$. At $384^{\circ} \mathrm{C}$ and $28 \mathrm{dpa}$, loops of type $\mathbf{a}<100>$ of average size of approximately $14 \mathrm{~nm}$ were observed in densities of around $9.3 \times 10^{20} \mathrm{~m}^{-3}$. At $443^{\circ} \mathrm{C}$ and $155 \mathrm{dpa}$, the total loop and line density was $\sim 3 \times 10^{15} \mathrm{~m}^{-3}$ and was dominated by a network of $\mathbf{a} / 2<111>$ with a network density of $2.2 \times 10^{15} \mathrm{~m}^{-2}$. Dislocation loops of type $\mathbf{a}<100>$ increased in size to an average of $18 \mathrm{~nm}$ while the loop number density decreased to $5 \times 10^{20} \mathrm{~m}^{-2}$.

Under low damage irradiation ( $<10 \mathrm{dpa})$, loops have been shown to nucleate and grow until saturation has been reached. Systematic studies by Gupta et al. [16] and Jiao et al. [17] on T91 suggest that loop size and density increase as a function of irradiation damage until reaching saturation by $10 \mathrm{dpa}$, regardless of temperature. However, the regime above 10 dpa was not considered and still remains unexamined. 
A summary of dislocation loop data is given below in Figure 2.15 and Table 2.1. Throughout all data examined, loop density tended to decrease with increased temperature while the average diameter increased with temperature until the loops became large enough to form a network, usually around $500^{\circ} \mathrm{C}$. In addition to the limited data for dislocations at low damage levels in FM alloys not subjected to stress under irradiation, the coupling of temperature and flux in reactor makes a systematic study of either temperature or damage dependence difficult. In addition, there is a distinct lack of characterization of the evolution of these dislocations above 10 dpa to high damage levels of 150 dpa or higher.

\subsection{Precipitation of Secondary Phases}

The formation of secondary phases, either enhanced or induced by irradiation, is considered for several reasons. First, formation of secondary phases may lead to embrittlement or hardening of the materials. Second, and more important to the objective of this thesis, the formation of secondary phases provides alternative sinks for defects and thus could affect swelling behavior.

\subsubsection{Precipitate Types in HT9}

Many types of precipitates can form under irradiation in the temperature range considered for this thesis. Below is a summary of types of precipitates that may form under ion irradiation in the relevant temperature range.

$\underline{\alpha^{\prime}}$

$\alpha^{\prime}$ is a chromium-rich ferrite radiation-enhanced precipitate. It is a $\mathrm{BCC}$ phase that is usually produced in the tempered martensite phase by spinoidal decomposition [3]. It is coherent with the iron matrix. Sizes of the precipitate vary from 2 to $20 \mathrm{~nm}$. $\alpha^{\prime}$ precipitate is highly resistant 
to coarsening. An interesting effect of $\alpha^{\prime}$ composition is that it is very difficult to directly observe because of its chemical similarity to the bulk steel as well as small size [50]. $\alpha^{\prime}$ precipitation is enhanced by irradiation and is a contributor to embrittlement. It is one of the most undesirable precipitates enhanced by irradiation because it increases hardness, yield strength and tensile strength while decreasing corrosion resistance, elongation, ductility and impact resistance [50]. Although the $\alpha^{\prime}$ phase has been observed in irradiated alloys with $12-13 \% \mathrm{Cr}$ content, it has not been observed in 9Cr-1Mo (T91). Thus, it was hypothesized that there is a critical bulk $\mathrm{Cr}$ concentration $(>12 \mathrm{wt} \%)$ for the formation of $\alpha^{\prime}$ precipitates [3].

\section{$\chi$}

$\chi$ is a $\mathrm{BCC}$ intermetallic phase. The most common composition is $\mathrm{Fe}_{36} \mathrm{Cr}_{12} \mathrm{Mo}_{10}$ [3]. It only occurs in Fe-Cr-Mo ternary systems and Fe-Cr-Ni-Mo and Fe-Cr-Ni-Ti quaternary systems. It increases susceptibility to intergranular stress corrosion cracking and may also be enriched in silicon and nickel under irradiation. A minimum molybdenum content, which is dependent on temperature, is needed for precipitation; for instance, $2 \mathrm{wt} \%$ at $600^{\circ} \mathrm{C}$ is required for precipitation $[50]$.

$\underline{M_{6}} \underline{X(\eta)}$

$\mathrm{M}_{6} \mathrm{X}(\eta)$ is a diamond cubic phase in the form of fine and coarse precipitates in martensitic steels with $>0.3 \mathrm{wt} \%$ nickel composition. $\eta$ phase is primarily observed in thermally aged highchromium steels. Under irradiation, it may become enriched in Si and Ni similar to $\chi$-phase [3].

$\underline{\mathrm{M}_{23} \mathrm{C}_{6}}$ 
$\mathrm{M}_{23} \mathrm{C}_{6}$ precipitates, also known as $\tau$-phase, are chromium rich carbides that nucleate on the martensite lath and PAG boundaries. These precipitates are a primary cause of intergranular stress corrosion cracking [3].

$\underline{\mathrm{M}_{2} \mathrm{X}(\varepsilon)}$

$\mathrm{M}_{2} \mathrm{X}$ precipitates are predominately $\mathrm{Cr}_{2} \mathrm{~N}$, but can also form as carbides. They are structured as fine needles. They generally precipitate on dislocations within martensite laths [3]. $\mathrm{M}_{2} \mathrm{X}$ is a contributor to embrittlement under irradiation [51]. Large (100 to $1000 \mathrm{~nm}$ ) needles of can be formed during thermal processing. Conversely, $\mathrm{M}_{2} \mathrm{X}$ can form under irradiation, typically as a platelike precipitate of length below $100 \mathrm{~nm}[3,38]$.

\section{$\underline{\text { Laves }}$}

Laves phase is an irradiation-enhanced phase, and also is found after prolonged thermal aging. The composition is $\mathrm{Fe}_{2} \mathrm{Mo}$. Enhancement of this phase was not observed in HT9 at irradiation temperatures between 300 to $615^{\circ} \mathrm{C}$, which include applicable temperature ranges for this research [3].

$\underline{\sigma}$

$\sigma$-phase can occur in austenitic, ferritic and duplex steels. It is an intermetallic with a tetragonal crystal structure precipitate with 30 atoms per unit cell composed of Fe-Mo-Cr. It causes loss of toughness and can cause depletion of $\mathrm{Cr}$ and Mo from the matrix. Generally, $\sigma$-phase replaces the ferrite portion of the microstructure. Higher chromium and molybdenum content tend to lead to quicker precipitation of this phase [3,50].

$\underline{\text { G phase }}$ 
G phase has been observed in irradiated HT9 steels. G phase is a complex FCC silicide of the form $\mathrm{M}_{6} \mathrm{Mn}_{16} \mathrm{Si}_{7}$ where $\mathrm{M}=\mathrm{Ni}$ typically [3]. Precipitates are around $10 \mathrm{~nm}$ in size and occur on grain boundaries, but can be somewhat bigger when occurring within subgrains. Irradiation hardening is attributed in part to this phase [51].

\subsubsection{Precipitate Behavior under Irradiation}

After irradiation, $\alpha^{\prime}$-phase [49], G phase [10], $\mathrm{M}_{6} \mathrm{C}$ and $\chi$-phases [49], $\sigma$-phase and the $\mathrm{Fe}_{2} \mathrm{Mo}$ type Laves phase have been reported in FM alloys.

The microstructure stability of the ACO3 duct was examined by Sencer et al. $[9,10]$. At 4 dpa and an irradiation temperature of $505^{\circ} \mathrm{C}$, the microstructure was essentially unchanged from the unirradiated microstructure, meaning that large blocky carbides $\left(\mathrm{M}_{23} \mathrm{C}_{6}\right)$ were observed on the martensite lath boundaries and carbides were distributed on or near the subgrain boundaries. After $28 \mathrm{dpa}$ and $384^{\circ} \mathrm{C}$, there was enhancement of the carbides on the subgrain boundaries. In addition, G phase developed within grains and on lath boundaries. No voids were observed. At 155 dpa and $443^{\circ} \mathrm{C}$, there was an enhancement of carbide size and density on and around the subgrain boundaries. Radiation enhanced precipitation was clearly observed and was dominated by G phase and $\eta$-phase at 155 dpa Smaller precipitates were obscured but were presumed to be $\alpha^{\prime}$. A comparison of these microstructures is given in Figure 2.16. It was theorized that the precipitates may act as sinks for the vacancies, which elongates the transient regime of swelling and could decrease the final swelling rate.

Gelles [7] identified G phase formation in HT9 and T91 irradiated in FFTF via neutron irradiation to $200 \mathrm{dpa}$ at $420^{\circ} \mathrm{C}$. The $700^{\circ} \mathrm{C}$ heat treated HT9 had well developed precipitate arrays decorating the subgrain lath boundaries, non-uniform void arrays within the laths and fine 
precipitates on and within the boundaries. There were no phases observed that were not visible at lower radiation damage levels. Precipitated $\mathrm{G}$ phase that had a diameter of $10 \mathrm{~nm}$ formed within laths, but larger precipitates formed on prior austenite grain boundaries (PAGBs) and on packet boundaries.

Recent results from Z. Jiao and G.S. Was [51] suggest that precipitate behavior (specifically $\mathrm{Ni} / \mathrm{Si} \mathrm{G}$ phase) continues to evolve at high damage levels (>100 dpa). Increased diameter and decreased number density of $\mathrm{Ni} / \mathrm{Si} / \mathrm{Mn}$ and copper rich precipitates was observed up to 500 dpa in $\mathrm{Fe}^{++}$-irradiated HCM12A suggested coarsening behavior up to high damage levels. However, the volume fraction of the precipitates decreased at damage levels beyond $7 \mathrm{dpa}$, an unexpected result that suggests that precipitates continue to evolve as a function of dpa.

Coarsening or dissolution of carbides [51] $\left(\mathrm{M}_{23} \mathrm{C}_{6}\right)$ was observed at low damage levels in the temperature range of $420-460^{\circ} \mathrm{C}$ in unalloyed $12 \% \mathrm{Cr}$ steels. However, this effect was only observed in high alloy grades. $\alpha$ ' precipitation has been observed in temperatures at or below $400^{\circ} \mathrm{C}$ or for high $\mathrm{Cr}(17 \%) \mathrm{FM}$ alloys. $\mathrm{G}$ phase was also shown to be closely correlated with strong radiation-induced segregation (RIS) to point defect sinks, such as carbides and dislocations. Prior to more recent results, there had been a debate of whether $\mathrm{G}$ phase actually formed, as it was only observed in samples irradiated above $20 \mathrm{dpa}$, so it may be classified as a "high damage phenomenon" and primarily nucleated on dislocation networks.

Although the predominant mode of precipitation in FM alloys is formation of $\mathrm{G}$ phase or $\alpha^{\prime}$, other modes have been observed. $\sigma$ phase (Fe-Cr intermetallic) was observed in the temperature range of $420-460^{\circ} \mathrm{C}$ [3]. It precipitated as large sheets and thin ribbons surrounding carbides, primarily in the ferritic portion of $9-13 \mathrm{Cr}$ steels. The Laves phase [3] formed prior to irradiation 
upon thermal ageing in some high $\mathrm{Cr}$ martensitic steels, but was suppressed upon irradiation in the temperature range of $300-615^{\circ} \mathrm{C}$.

$\mathrm{M}_{2} \mathrm{X}(\varepsilon)[38,52]$ were observed by Borodin et al. after ion irradiation at $500^{\circ} \mathrm{C}$ to $30 \mathrm{dpa}$ and $150 \mathrm{dpa}$. Irradiations were performed with $3 \mathrm{MeV} \mathrm{Cr}$ ions on $13 \mathrm{Cr}-2 \mathrm{Mo}-\mathrm{TiO}_{2}$. Rod-like precipitates formed by $30 \mathrm{dpa}$ and grew in size up to $150 \mathrm{dpa}$, shown in the Figure 2.17. Similar $\mathrm{M}_{2} \mathrm{X}$ precipitates were also observed by Maziasz [53] in 12Cr-1MoVW (HT9) irradiated in HFIR at $500^{\circ} \mathrm{C}$ to $38 \mathrm{dpa}$ with 87 appm He co-generated, indicating that the phase forms both in neutron and ion irradiation.

Although precipitate formation in FM alloys has been observed and reported at a number of different damage levels and in in a large temperature range, there has been no systematic study of precipitate evolution with high damage, especially in excess of 100 to $200 \mathrm{dpa}$.

\subsection{Other Irradiation Effects}

The addition of irradiation to the microstructure has been shown to also affect mechanical properties. Other irradiation effects not central to the scope of this thesis are presented below. Specifically, radiation-induced segregation and hardening behavior will be briefly summarized, but will not be examined in this study.

\subsubsection{Radiation-Induced Segregation}

Radiation-induced segregation, or RIS, can contribute to the formation of precipitates. Jiao and Was [54] irradiated FM alloys (T91, HCM12A, and HT9) with protons and heavy ions to 7 and 100 dpa, respectively. For HCM12A, the focus of the study, silicon, nitrogen and phosphorous were enriched at the grain boundaries. Chromium enrichment/depletion was highly variable and 
different at each of the grain boundaries analyzed. It was theorized that enrichment levels were affected by the competing mechanism of solute precipitation. Copper enrichment at the grain boundaries was lower than expected. It was also theorized, much like for chromium, that copperrich precipitates decrease enrichment levels by diverting $\mathrm{Cu}$ atoms. Molybdenum and manganese concentration remained constant or slightly increased at the grain boundaries. Tungsten depleted, which was expected due to its large size. Segregation to dislocations was also observed in HCM12A. Solute segregation to dislocations was similar to segregation at the grain boundaries.

Wharry and Was [55] also examined RIS in FM alloys irradiated with protons up to 10 dpa. Under irradiation in the temperature range of $300-600^{\circ} \mathrm{C}$, they observed chromium, nickel and silicon enrichment at grain boundaries. A damage dependence study in a $9 \mathrm{Cr}$ model alloy suggested that $\mathrm{Cr}$ enrichments saturates between 7-10 dpa, whereas T91 enrichment reached a peak at 7 dpa and decreased beyond that damage. A similar trend was observed in $\mathrm{Ni}$ and $\mathrm{Si}$, and it was suggested that the lattice parameter in the vicinity decreased with the addition of undersized solutes, which decreased the migration energy of $\mathrm{Cr}$, thus decreasing enrichment. Higher damage levels were not examined.

\subsubsection{Hardening}

Irradiation-induced microstructural and microchemical changes contribute to the macroscopic effect of irradiation-induced hardening. Hardening then increases the yield stress and ultimate tensile strength of the examined material, while decreasing the uniform and total elongation. This can be observed in changes in the stress-strain curve of the alloy after irradiation. Representative stress-strain curves are shown in Figure 2.18 [56]. Irradiation of a metal causes strengthening by source hardening and friction hardening. The formation of defect clusters, voids,

precipitates and dislocation loops impede the motion of dislocation lines [20]. In general, the 
magnitude of irradiation hardening decreases with temperature and disappears between 400 and $500^{\circ} \mathrm{C}$, likely due to annealing of radiation-induced defect clusters at higher temperatures [56].

Yield strength $\left(\Delta \sigma_{y}\right)$ is related to hardening $\Delta H_{V}$ via the following equation:

$$
\Delta \sigma_{y}=3.06 \Delta H_{V}
$$

for FM alloys [57].

Gelles and Schäublin [58] tensile tested irradiated F82H samples and examined the post deformation microstructures. $\mathrm{F} 82 \mathrm{H}$ samples were irradiated to $2.57 \mathrm{dpa}$ at $327^{\circ} \mathrm{C}$. Deformation only occurred $<0.5 \mathrm{~mm}$ from the failure site. Yield strength increased to an average of $619 \mathrm{MPa}$ compared to $540 \mathrm{MPa}$, prior to irradiation. Hardening was attributed to the small loops which formed near preexisting dislocation microstructure. In a similar study, de Carlan et al. [59] also surveyed a variety of types of steels and compared post-irradiation tensile behavior. Austenitic, martensitic and ODS FM steels were irradiated up to 9.5 dpa in the Osiris reactor in France. All steels exhibited irradiation hardening and increases in yield strength. Hardening and ductility for all the steels appeared to saturate by 9 dpa. Saturation of yield strength was observed in Figure 2.19 for a variety of FM alloys. HT9 exhibited brittle behavior and intergranular failure mode, especially relative to ODS alloy MA 957, which showed more ductile fracture.

Radiation damage at high damage levels is a dynamic process where the resultant defect flow is complex and there are many possible mechanisms for interactions between the various radiation-induced microstructure features. Defect clusters form and are annealed. Dislocation loops form, grow and climb and glide. Solutes segregated to and away from grain boundaries and sinks. Secondary phases form and provide additional sinks. Finally, vacancies agglomerate into 
void nuclei and grow into voids. The scope of this thesis will be limited to the interactions of voids, secondary phases and the dislocation loops and network. 
Table 2.1: Summary of observed dislocation behavior in FM alloys.

\begin{tabular}{|c|c|c|c|c|c|c|c|c|}
\hline Alloy & $\begin{array}{c}\text { Irradiation } \\
\text { Temperature } \\
\left({ }^{\circ} \mathrm{C}\right) \\
\end{array}$ & $\begin{array}{l}\text { Damage } \\
\text { (dpa) }\end{array}$ & Dislocation Character & $\begin{array}{l}\text { Loop Number } \\
\text { Density }\left(\mathrm{m}^{-2}\right)\end{array}$ & $\begin{array}{c}\text { Loop } \\
\text { Diameter } \\
(\mathrm{nm}) \\
\end{array}$ & $\begin{array}{l}\text { Loop Line } \\
\text { Density }\left(\mathrm{m}^{-2}\right)\end{array}$ & Type & Reference \\
\hline HT9 & 420 & 200 & $\mathrm{a} / 2<111>$ network and $\mathrm{a}<100>$ loops & & & \multicolumn{2}{|c|}{ Not Reported } & {$[33]$} \\
\hline \multirow[t]{5}{*}{$\begin{array}{c}\mathrm{Fe}-12 \mathrm{Cr} \\
\text { model }\end{array}$} & 420 & 15 & $\begin{array}{c}\text { small and large a[001] segments; } \mathrm{a} / 2<111> \\
\text { network }\end{array}$ & & & $4.70 \mathrm{E}+14$ & Total & {$[33]$} \\
\hline & & 21 & $\begin{array}{c}\text { small and large } a[001] \text { segments; } a / 2<111> \\
\text { network }\end{array}$ & & & $6.80 \mathrm{E}+13$ & Total & {$[33]$} \\
\hline & & 200 & $\begin{array}{c}\text { small and large } \mathrm{a}[001] \text { segments; } \mathrm{a} / 2<111> \\
\text { network }\end{array}$ & & & $8.30 \mathrm{E}+13$ & $\mathrm{a} / 2<111>$ & {$[33]$} \\
\hline & & & & & & $4.11 \mathrm{E}+14$ & $a<100>$ & [33] \\
\hline & & & & & & $4.90 \mathrm{E}+14$ & Total & {$[33]$} \\
\hline \multirow[t]{10}{*}{ HT9 } & 450 & $10-100$ & loops +network & & & \multicolumn{2}{|c|}{ Not Reported } & [49] \\
\hline & 500 & $10-100$ & network + small unfaulted loops & & & \multicolumn{2}{|c|}{ Not Reported } & [49] \\
\hline & 300 & 40 & $\mathrm{a}<100>$ & $1.00 \mathrm{E}+22$ & 8 & $2.51 \mathrm{E}+14$ & $\mathrm{a}<100>$ & {$[35]$} \\
\hline & 400 & 40 & $\mathrm{a}<100>$ & $8.00 \mathrm{E}+21$ & 25 & $6.28 \mathrm{E}+14$ & $\mathrm{a}<100>$ & {$[35]$} \\
\hline & 500 & 40 & $a<100>$ & $6.00 \mathrm{E}+19$ & 800 & $1.51 \mathrm{E}+14$ & $\begin{array}{c}\mathrm{a} / 2<111> \\
\text { network } \\
\end{array}$ & {$[35]$} \\
\hline & 384 & 28 & $\mathrm{a}<100>$ loops & $9.30 \mathrm{E}+20$ & 14 & $4.09 \mathrm{E}+13$ & $\mathrm{a}<100>$ & {$[9,10]$} \\
\hline & 443 & 155 & $\mathrm{a} / 2<111>$ network and $\mathrm{a}<100>$ loops & & & $2.20 \mathrm{E}+15$ & $\begin{array}{c}\mathrm{a} / 2<111> \\
\text { network }\end{array}$ & {$[9,10]$} \\
\hline & 443 & & & $5.00 \mathrm{E}+20$ & 18 & $8.00 \mathrm{E}+14$ & $\mathrm{a}<100>$ & {$[9,10]$} \\
\hline & & & & & & $3.00 \mathrm{E}+15$ & total & {$[9,10]$} \\
\hline & 505 & 4 & Microstructure unchanged with irradiation & & & Not $\mathrm{Re}$ & orted & {$[9,10]$} \\
\hline
\end{tabular}




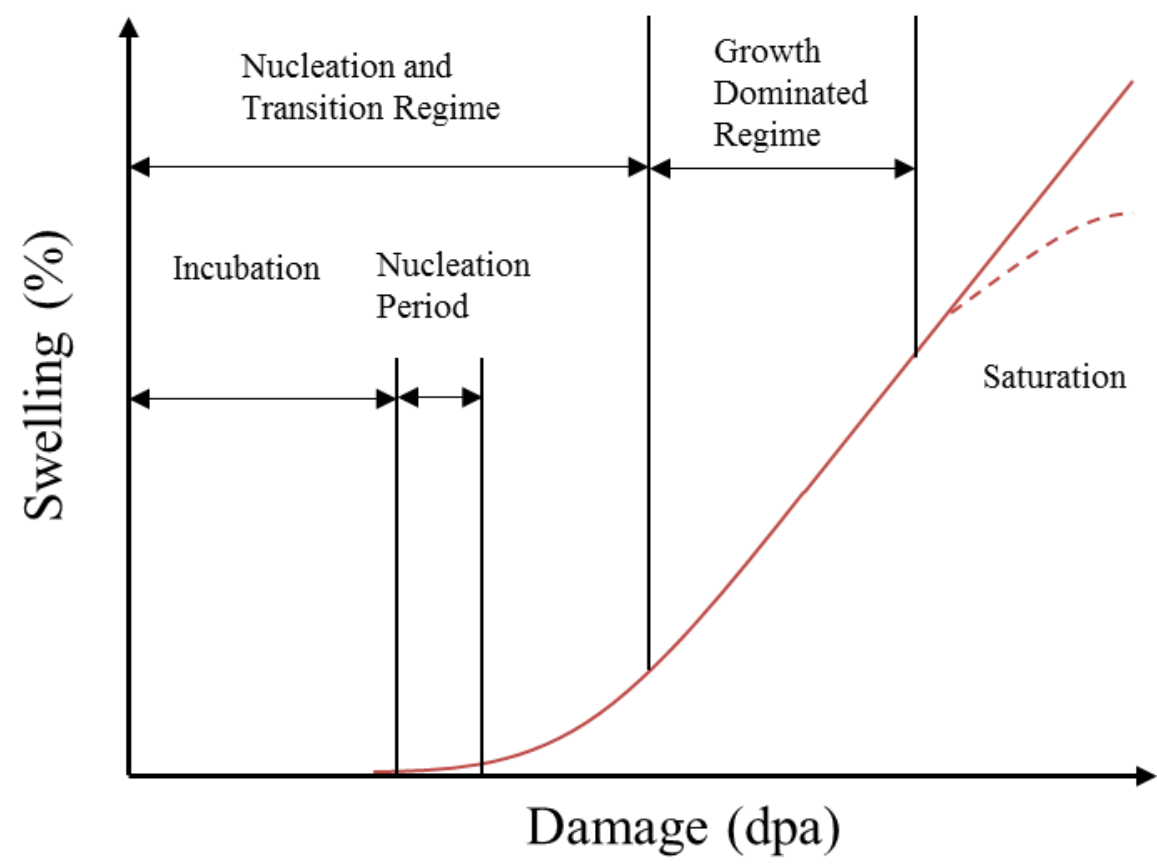

Figure 2.1: Expected swelling behavior as a function of damage. Adapted from [19]. 


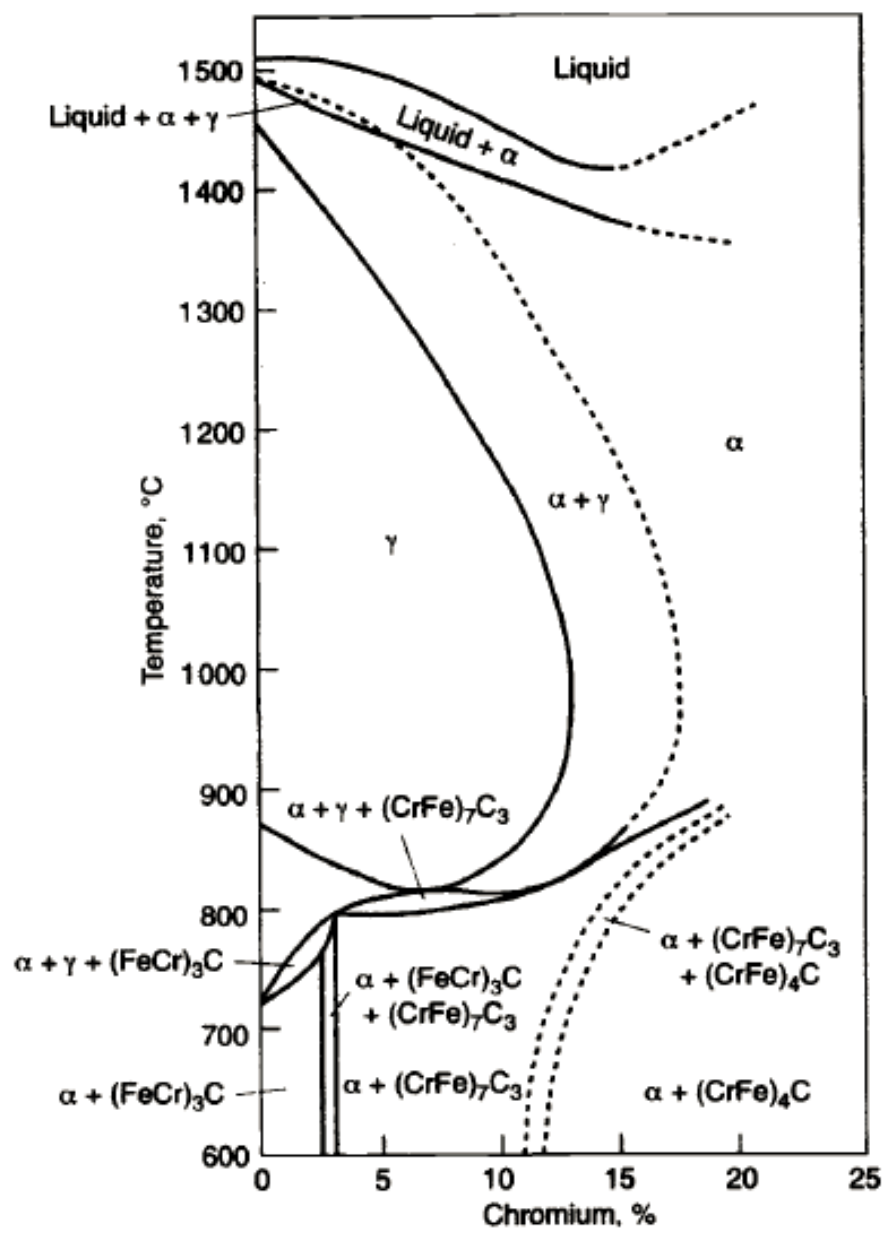

Figure 2.2: Effect of chromium on the constitution of Fe-Cr-C alloys with $0.1 \%$ carbon. $(\mathrm{CrFe})_{4} \mathrm{C}$ is the $\mathrm{M}_{23} \mathrm{C}_{6}$ carbide [3]. 


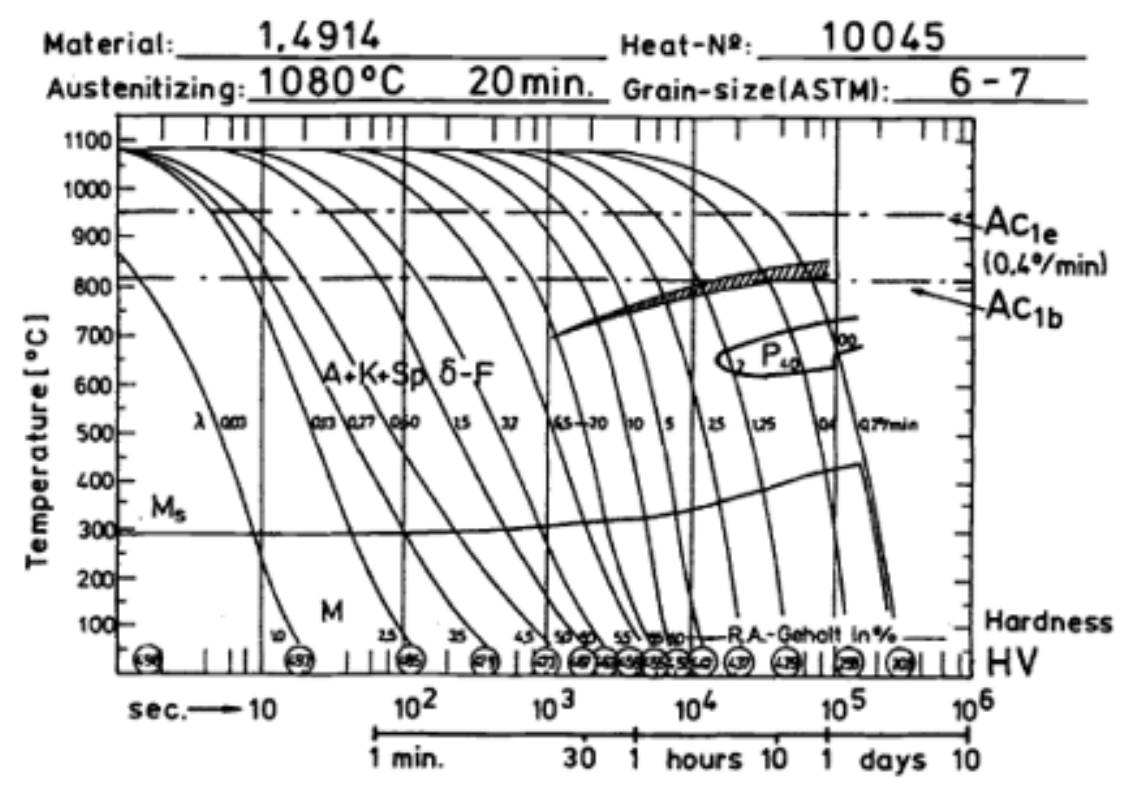

Figure 2.3: Continuous cooling transformation (CCT) diagram for $12 \mathrm{Cr}-\mathrm{MoVNb}$ martensitic steel where A refers to austenite, $\mathrm{K}$ refers to carbide, Sp- $\delta$ refers to trace ferrite, $\mathrm{M}$ refers to martensite, $\mathrm{P}=$ pearlite, $\mathrm{A}_{\mathrm{Cl}}$ is the start of austenite formation upon heating, $\mathrm{A}_{\mathrm{Cle}}$ is the completion of austenite formation upon heating and $\lambda$ is the cooling rate $\left({ }^{\circ} \mathrm{C} / \mathrm{min}\right)$ from [3]. 


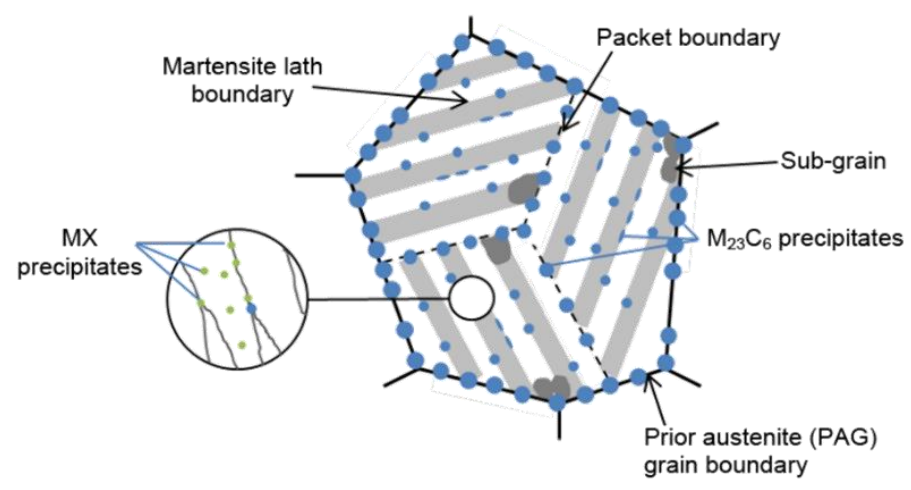

(a)

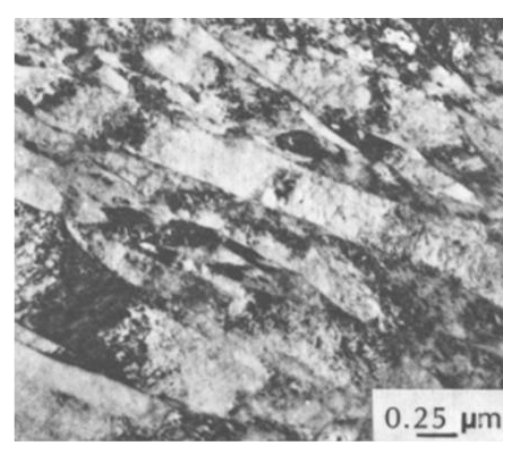

(b)

Figure 2.4: a) Schematic [32] and b) micrograph of typical HT9 microstructure prior to irradiation [3]. 


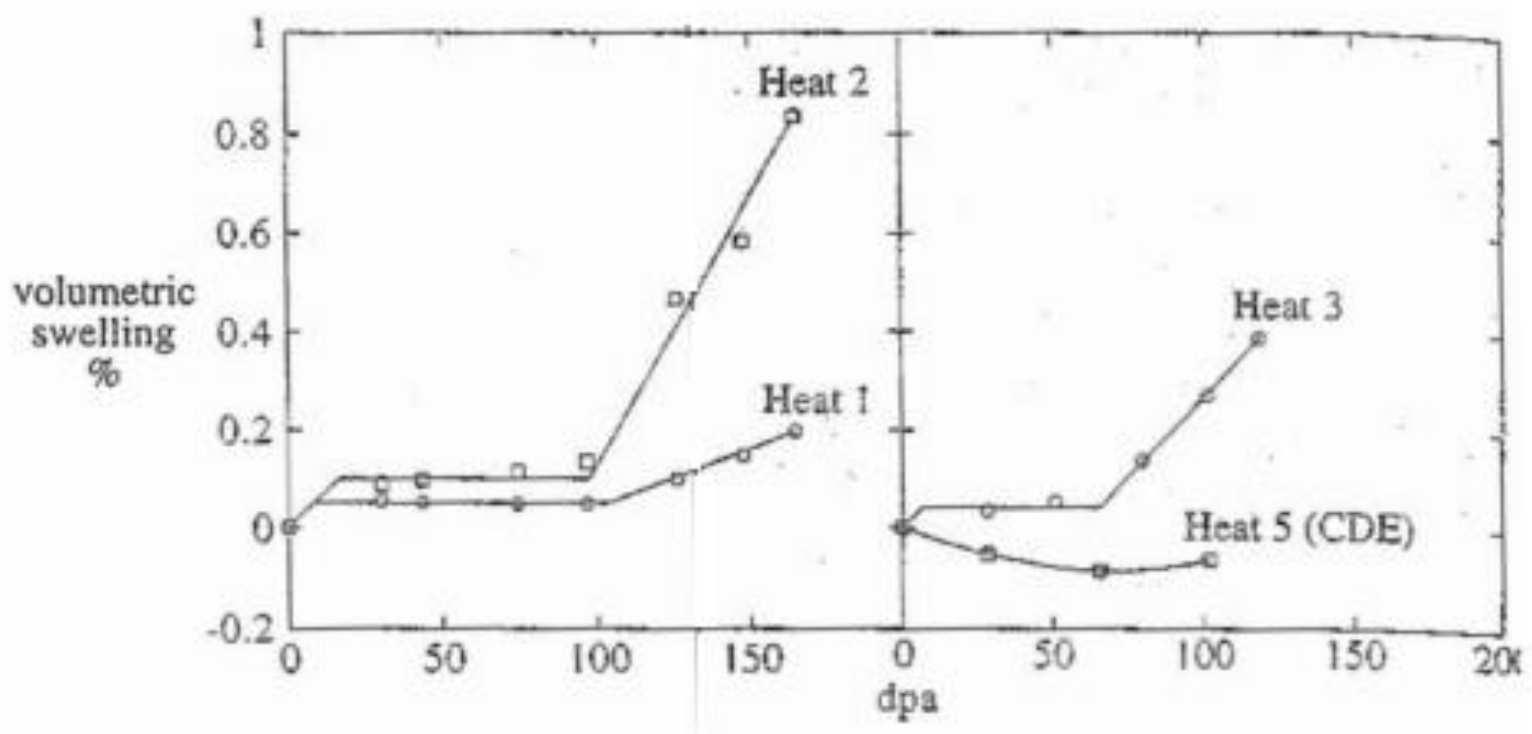

Figure 2.5: Stress-free swelling of irradiated HT9 up to 165 dpa for 4 heat treatments. Heat 1 and Heat 2 were subjected to the same heat treatment: $1038^{\circ} \mathrm{C} / 5 \mathrm{~min} /$ air followed by $760^{\circ} \mathrm{C} / 30 \mathrm{~min} /$ air cool. Heat 3 and 5 were subjected to $1100^{\circ} \mathrm{C} / 2 \mathrm{~min} /$ air cool followed by $650 \% 120 \mathrm{~min} /$ air cool [4]. 


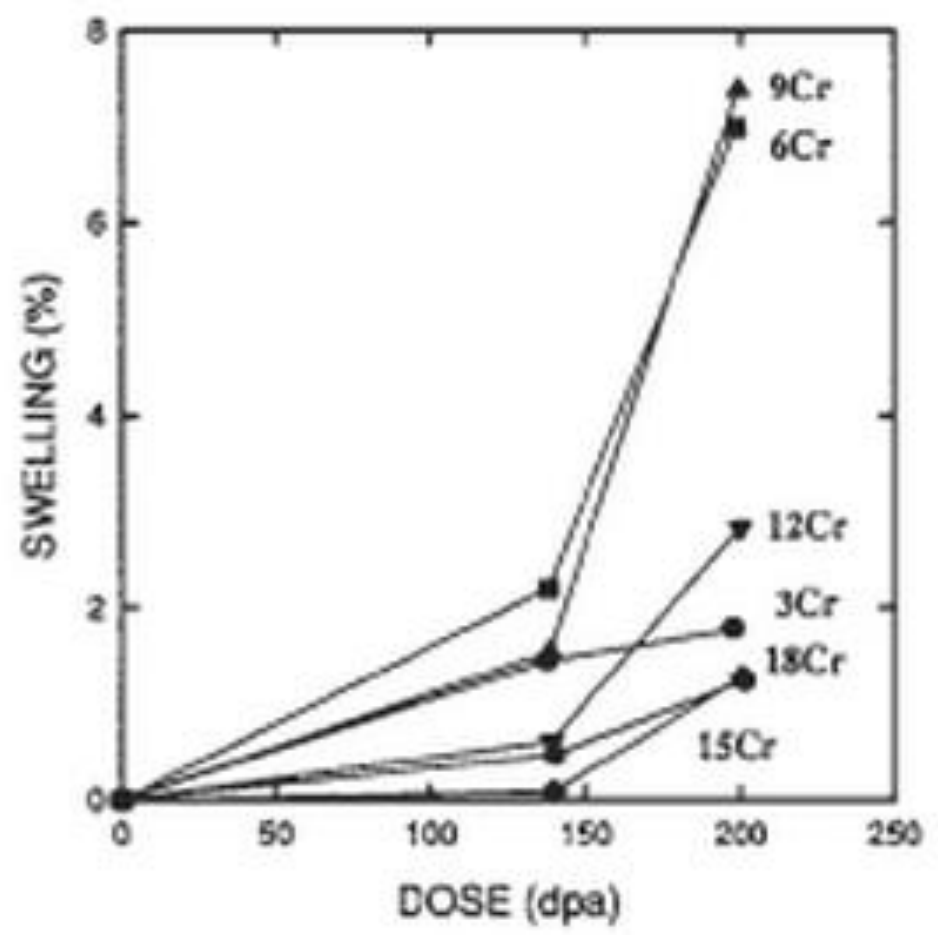

Figure 2.6: Swelling in Fe-Cr alloys as a function of damage [33]. 

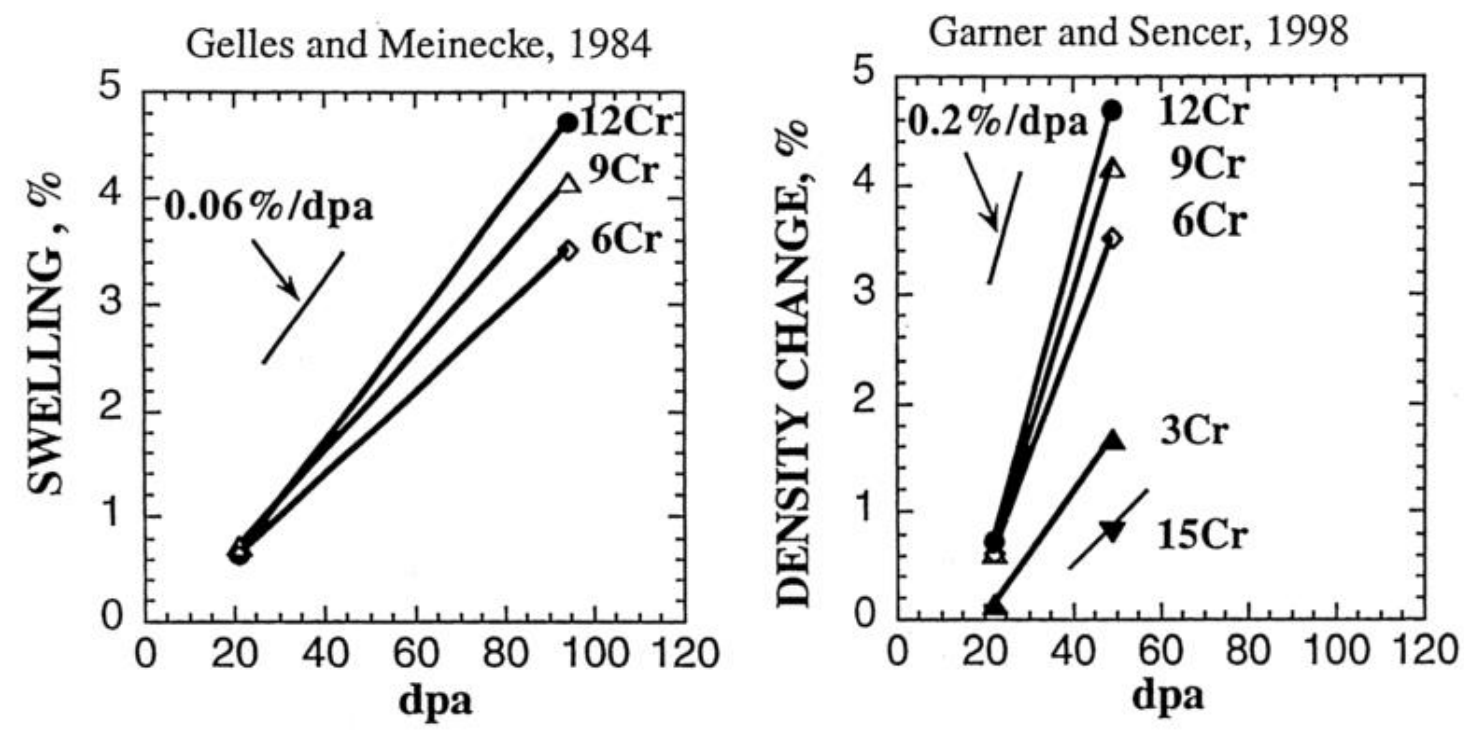

Figure 2.7: Adjustment of damage levels in EBR-II results by Garner et al. [8] to data collected by Gelles [33]. 


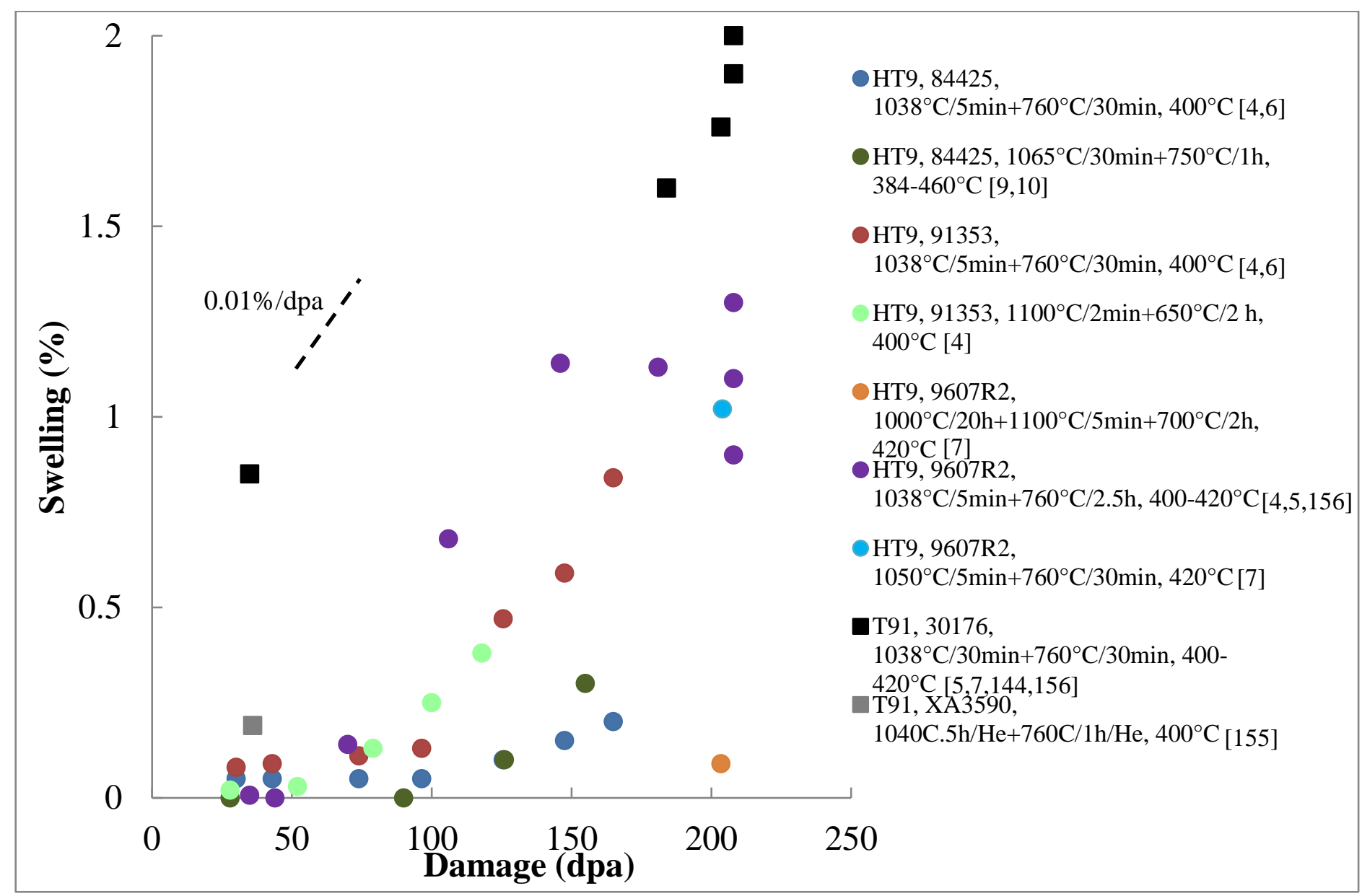

Figure 2.8: Summary of HT9 and T91 irradiated in-reactor up to 208 dpa. 


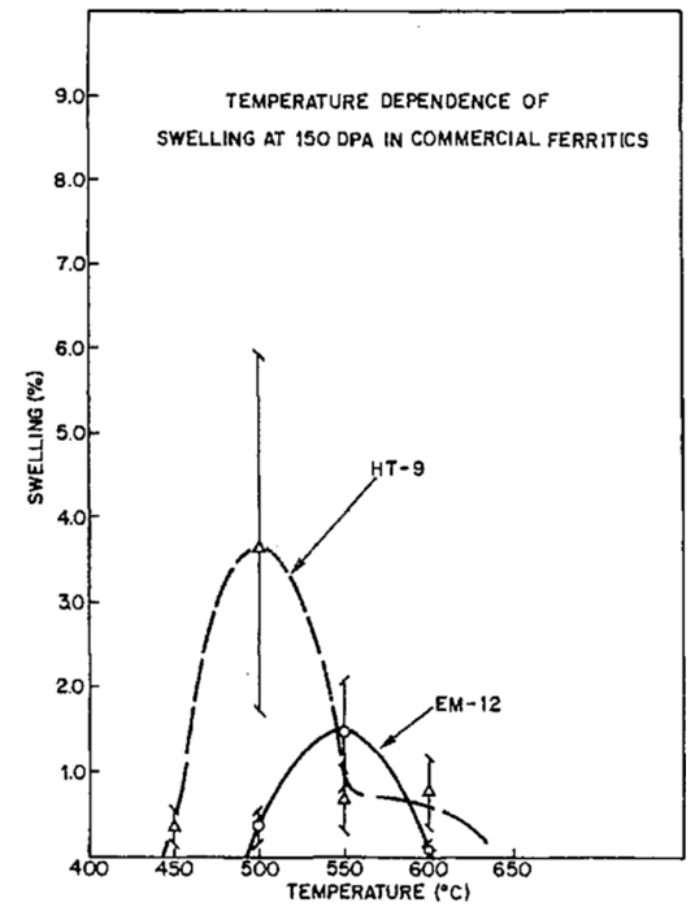

(a)

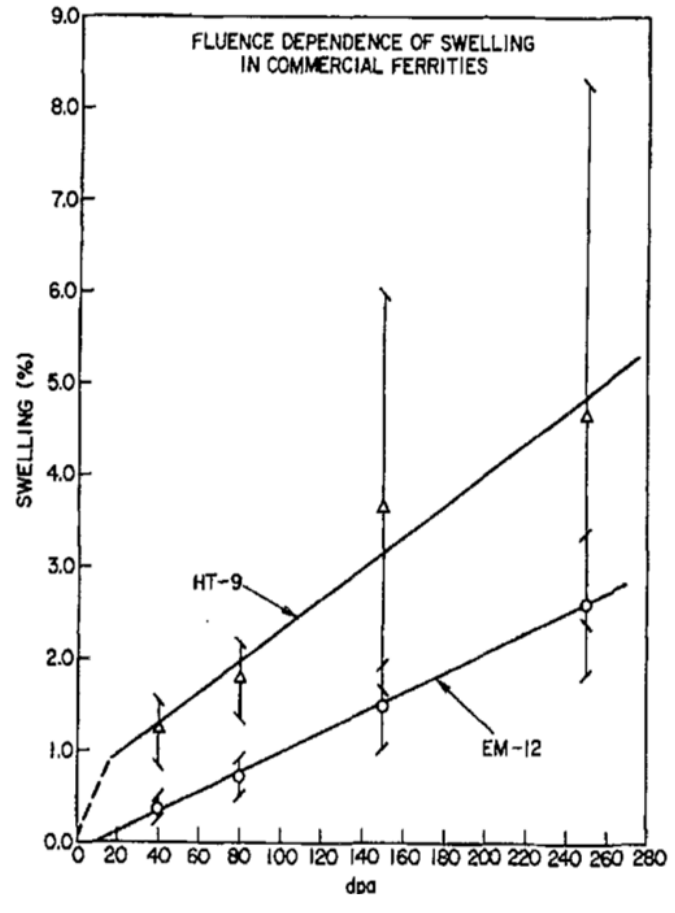

(b)

Figure 2.9: a) Temperature dependence of void swelling in EM12 and HT9 irradiated to $150 \mathrm{dpa}$ b) Damage dependence of swelling of EM12 and HT9 up to at $500^{\circ} \mathrm{C}$ [14]. 


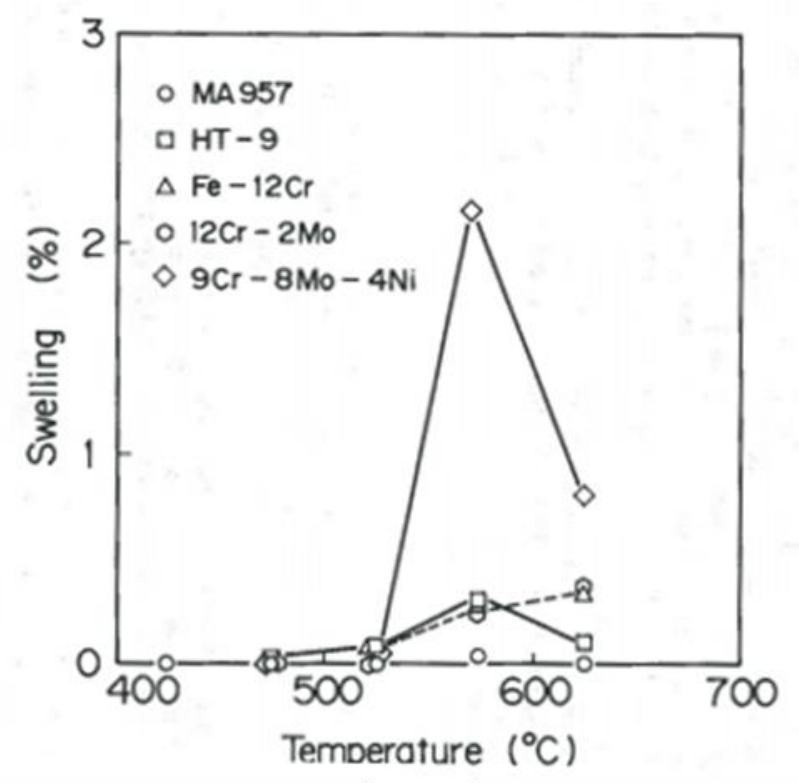

(a)

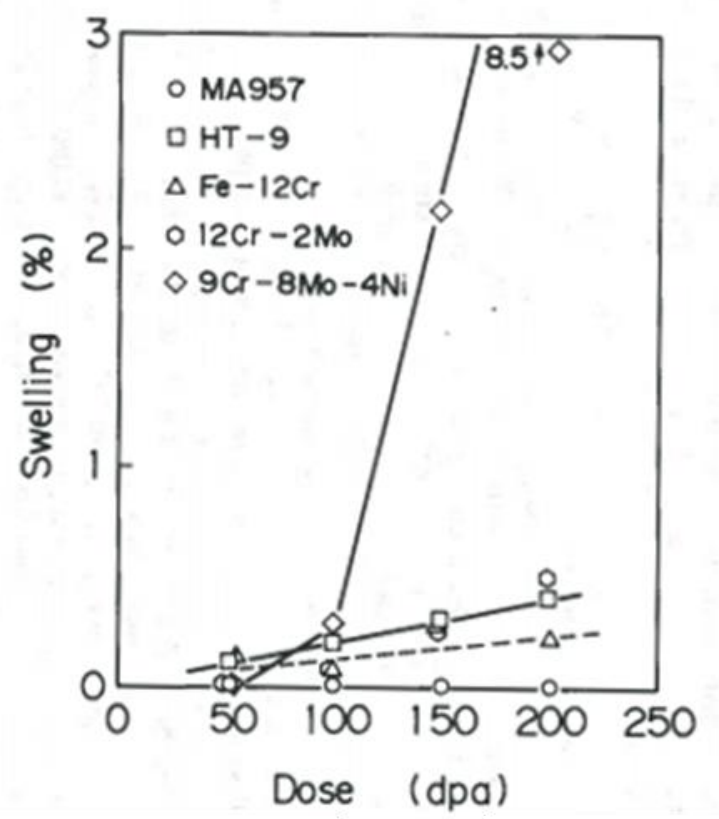

(b)

Figure 2.10: a) Temperature dependence of swelling in ferritic steel irradiated with 200 $\mathrm{keV} \mathrm{C}^{+}$ions to $150 \mathrm{dpa}$ and $\mathrm{b}$ ) damage dependence of swelling in ferritic steels irradiated with $200 \mathrm{keV} \mathrm{C}^{+}$ions at $575^{\circ} \mathrm{C}$ [40]. 


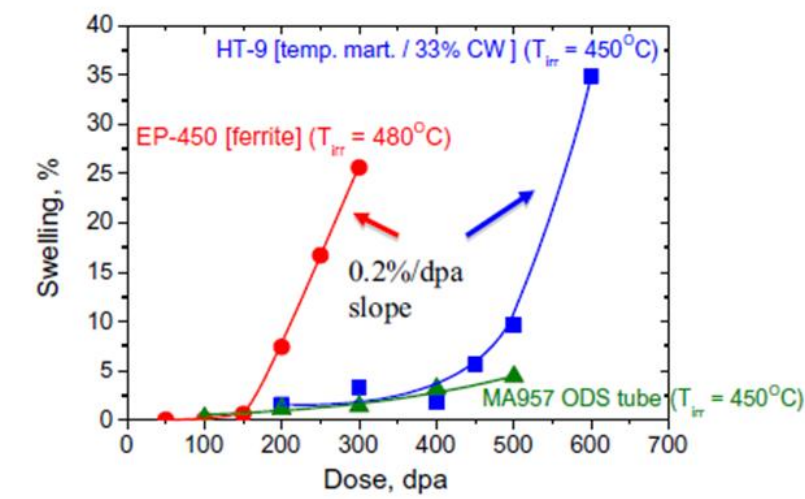

(a)

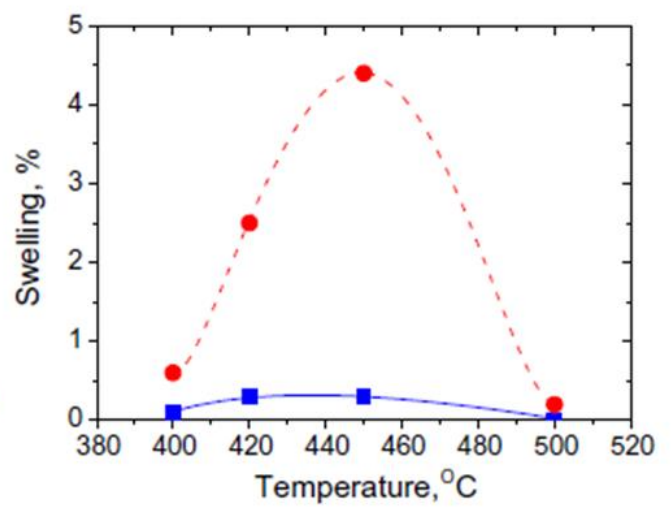

(b)

Figure 2.11: a) Damage dependence of commercial alloys up to $600 \mathrm{dpa}$ at 450 or $480^{\circ} \mathrm{C}$. b) Temperature dependence experiment at $100 \mathrm{dpa}$ and $500 \mathrm{dpa}$ on MA957 ODS ferritic steel [15]. 


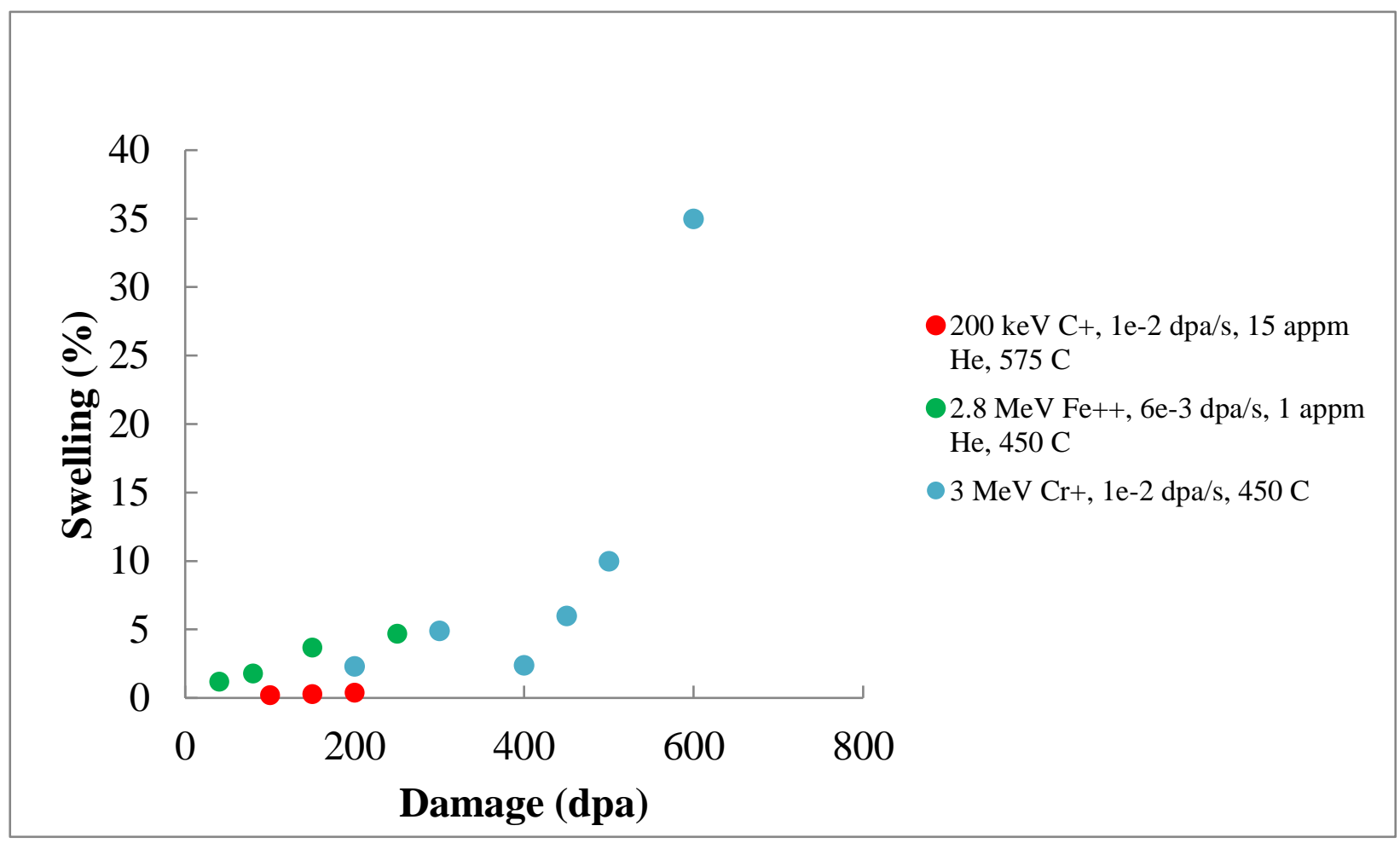

Figure 2.12: Summary of ion irradiations performed upon HT9 [14,15,40]. 


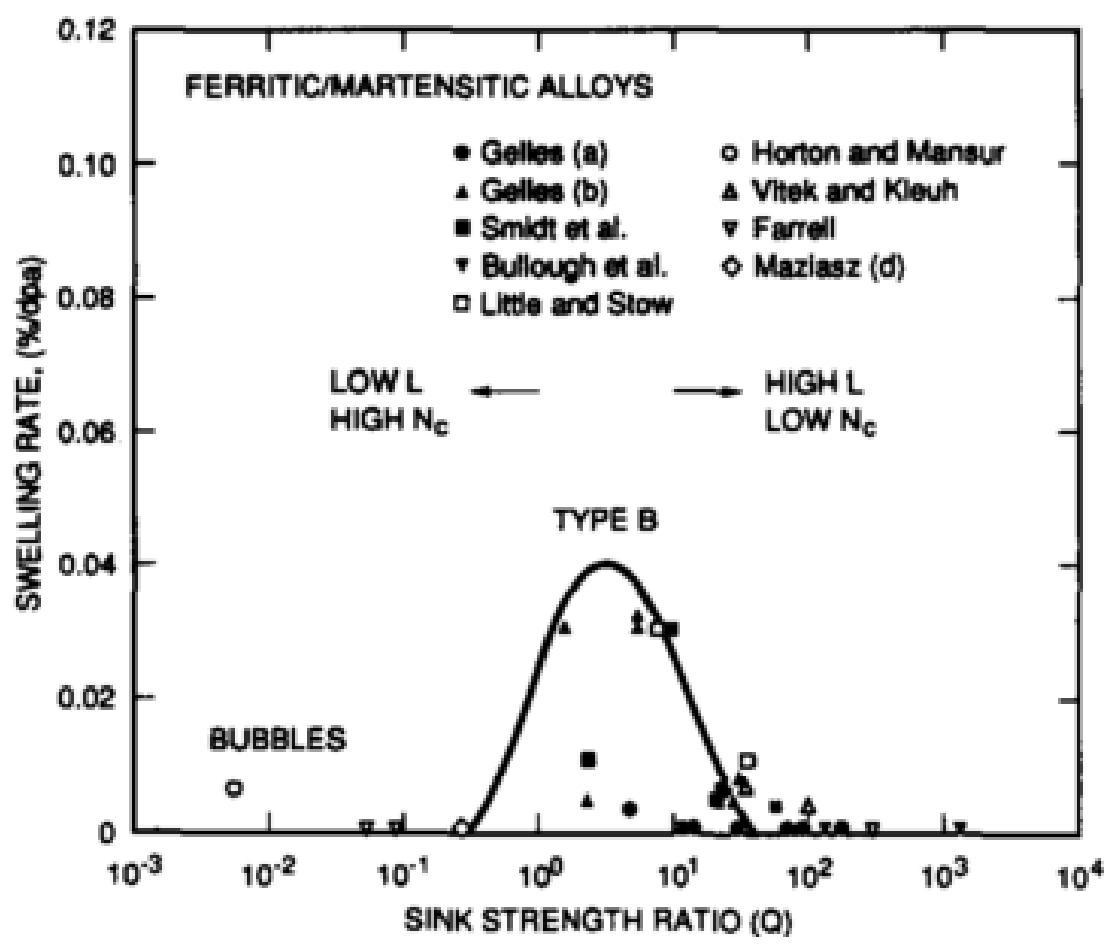

Figure 2.13: Q analysis for various neutron irradiations [34]. 


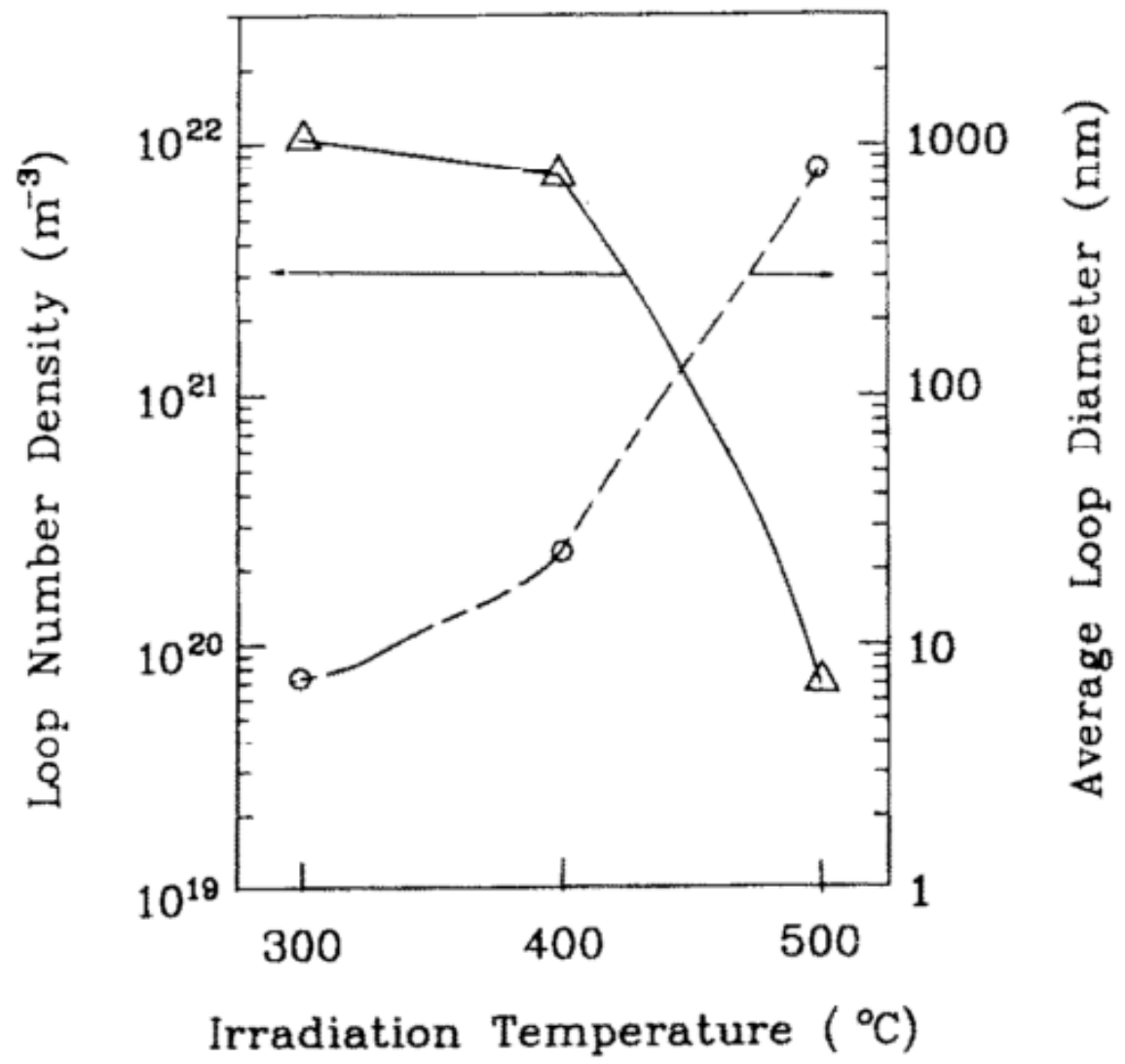

Figure 2.14 Temperature dependence of loop number density and diameter in $14 \mathrm{MeV}$ Ni-irradiated HT9 [35]. Note that loops of size of $800 \mathrm{~nm}$ would typically be classified as an unfaulted network. 


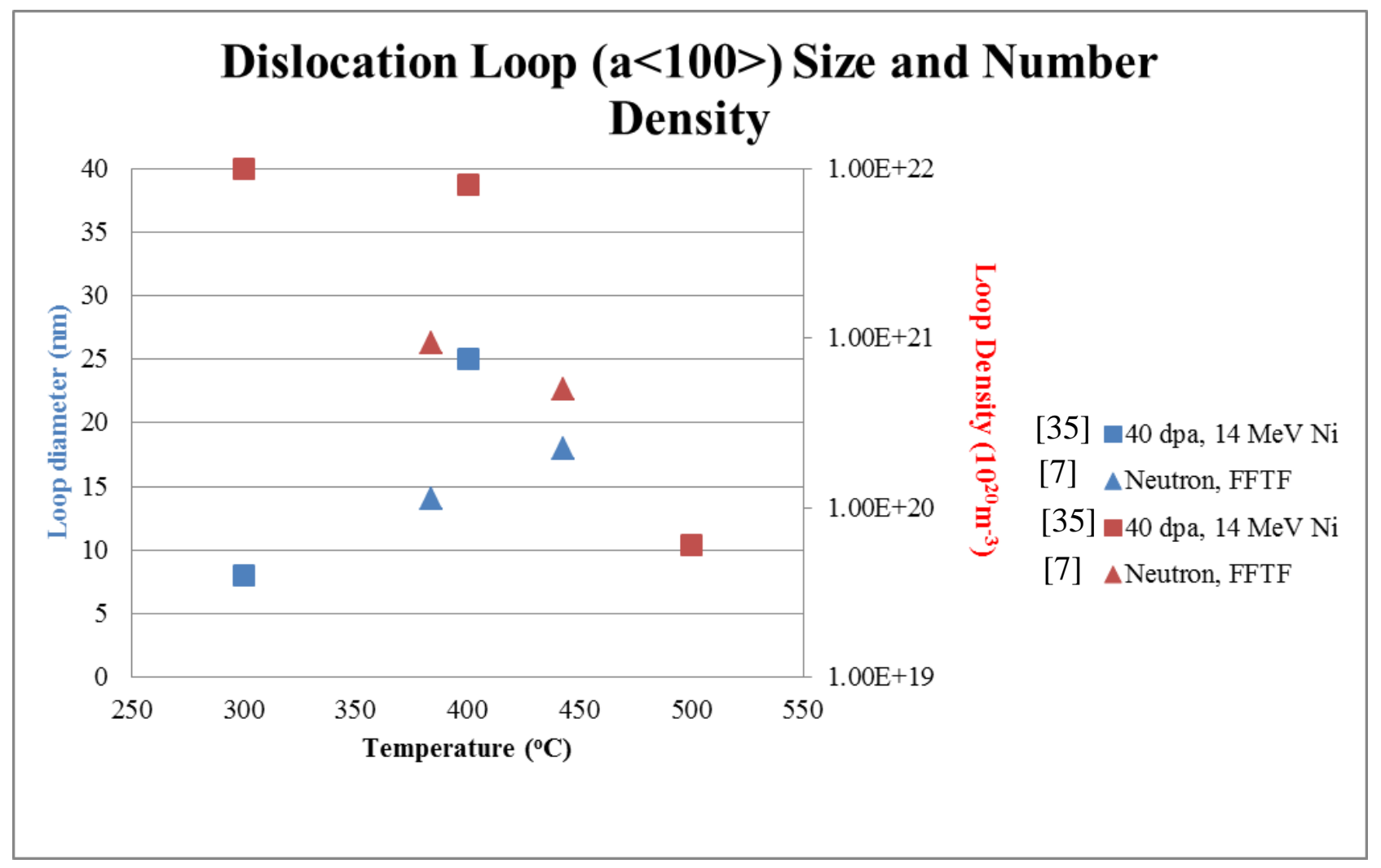

Figure 2.15: A summary of dislocation loop results as a function of temperature in neutron and ion irradiated HT9 [7,35]. 


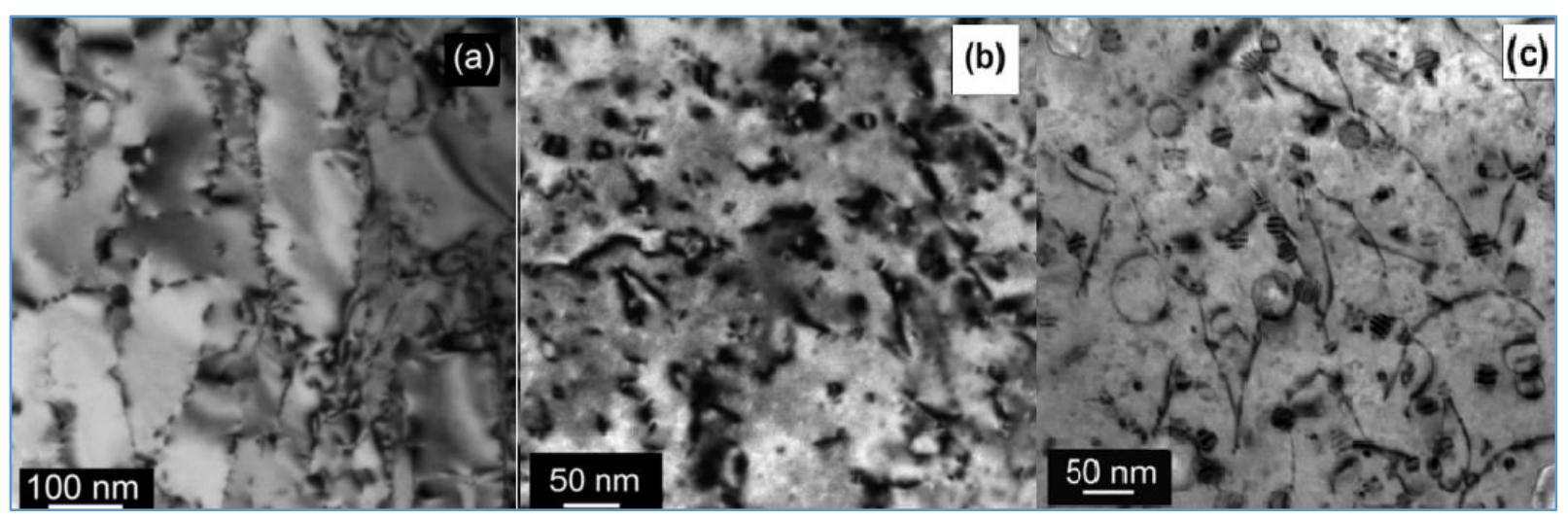

Figure 2.16: HT9 irradiated to a) $4 \mathrm{dpa}$ at $505^{\circ} \mathrm{C}$ b) $28 \mathrm{dpa}$ at $384^{\circ} \mathrm{C}$ c) $155 \mathrm{dpa}$ at $443^{\circ} \mathrm{C}$ [10]. 

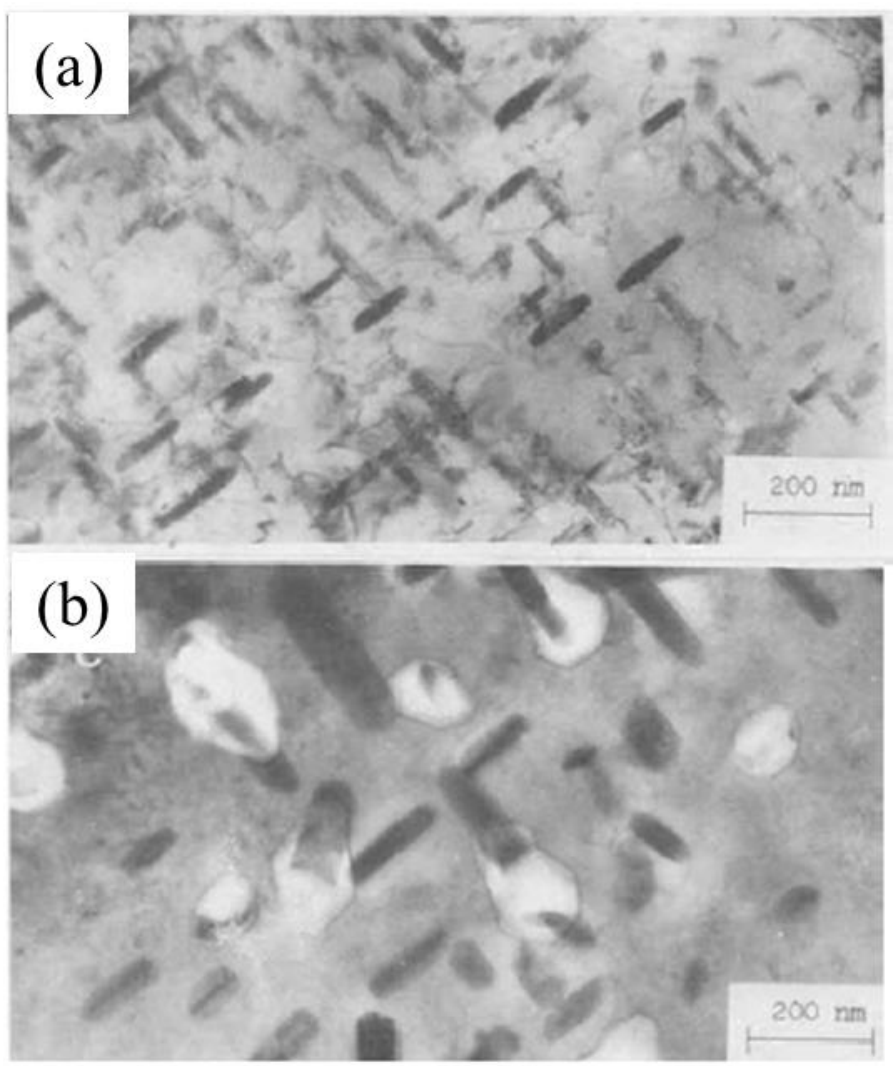

Figure 2.17: Formation of rod-like precipitates in $13 \mathrm{Cr}-2 \mathrm{Mo}-\mathrm{TiO}_{2}$ after $\mathrm{Cr}$ irradiation at $500^{\circ} \mathrm{C}$ to a) $30 \mathrm{dpa}$ and b) $150 \mathrm{dpa}[38]$. 


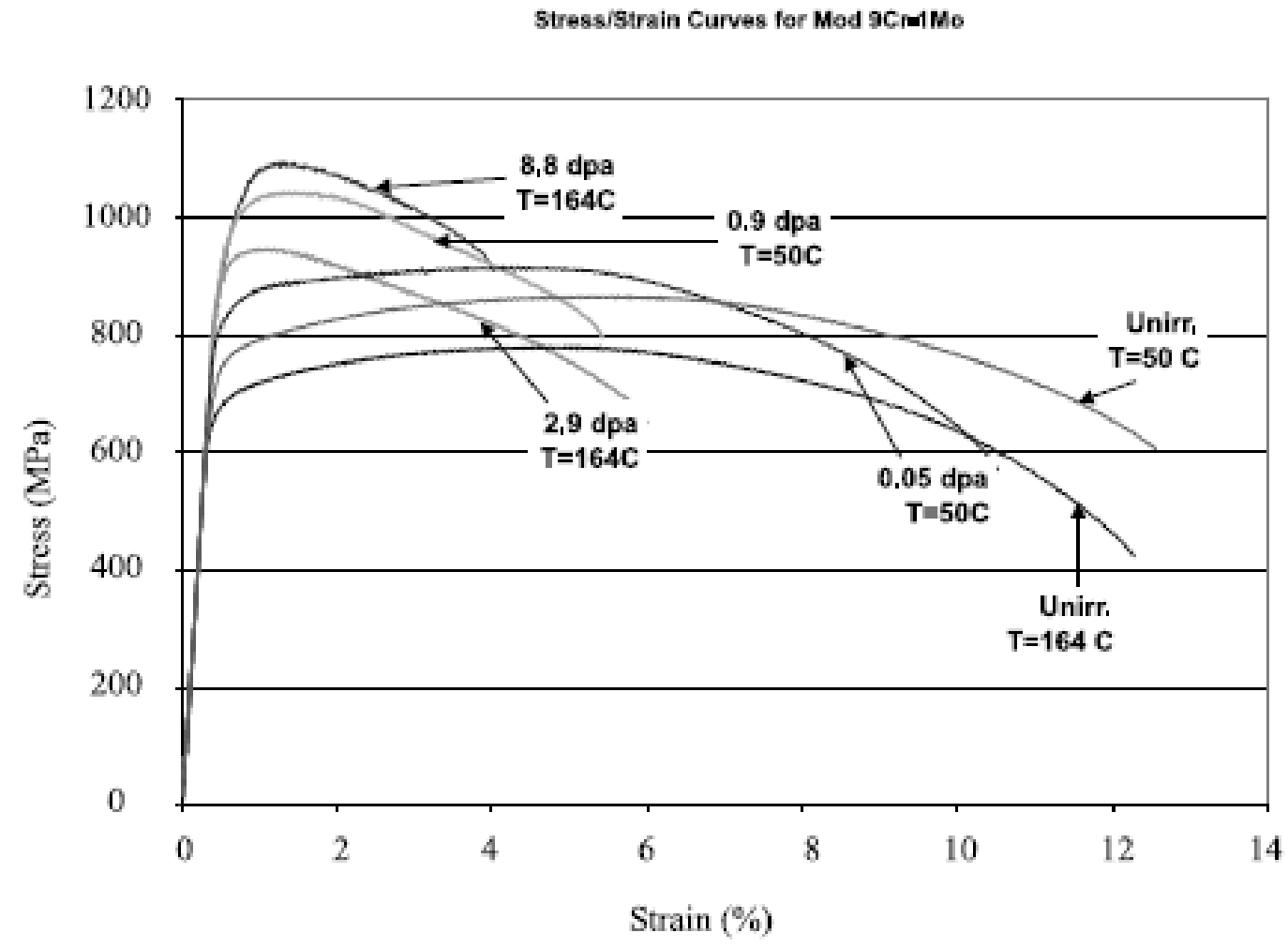

Figure 2.18: Representative stress/strain curves for Mod 9Cr-1Mo after irradiation in spallation environment [56]. 


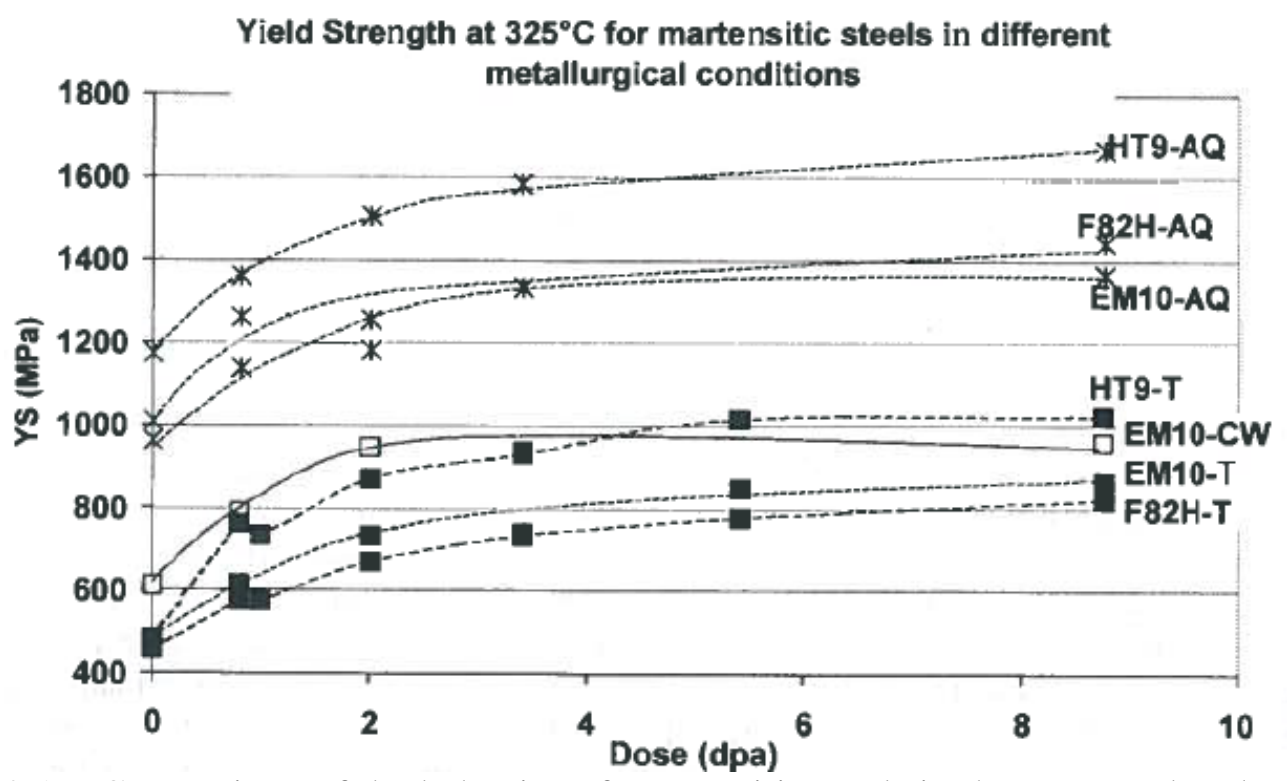

Figure 2.19: Comparison of the behavior of martensitic steels in the tempered and asquenched conditions [59]. 


\section{CHAPTER 3}

\section{OBJECTIVE}

The objective of this thesis is to understand how microstructure features co-evolve in the high damage level, growth-dominated swelling regime of self-ion irradiated HT9. The hypothesis is that continued defect absorption at precipitate and dislocations maintains the supersaturation of vacancies necessary for linear swelling to continue to high damage levels.

A combination of ion irradiation, microstructure characterization and computational modeling will be used to understand how voids evolve in the high damage regime where growth rather than nucleation of voids dominates the swelling behavior. Three sub-objectives have been identified.

First, Sub Objective 1 will determine the temperature and damage regime relevant to void swelling. Prior to this work, the majority of analysis of the microstructure has been performed in

the incubation and transition regime. Although void swelling is postulated between $0.3-0.5 \frac{T}{T_{m}}$, void formation in this high swelling resistant alloy only occurs at high damage. Thus, identifying the peak swelling temperature will be key to reaching the growth dominated swelling regime.

Second, Sub Objective 2 will determine microstructure (void, dislocation, precipitate) evolution at the peak swelling temperature after the onset of linear swelling in the growth regime using a combination of ion irradiation and post-irradiation microstructure characterization. Specifically, a damage dependence experiment at the peak swelling temperature will be performed 
then characterized via STEM imaging. The high damage level set of experiments will determine the damage evolution for all features of interest (voids, dislocations, precipitates), which will isolate the onset of growth-dominated swelling regime (in terms of damage). The characterized microstructure at the onset of growth-dominated swelling will be used as an input for a cluster dynamics/rate theory model and will provide a database of microstructure results with which to 1) use as input into the model in the linear swelling regime 2) use to verify model results are reasonable and finally 3 ) use to track the defect partitioning to various sinks at high damage levels.

The third sub-objective will be to adapt and utilize the Radiation Induced Microstructure Evolution (RIME) model to understand how precipitates, dislocations and voids co-evolve in HT9. The sub-objective is to understand the processes by which voids, dislocations and precipitates coevolve at high damage, with the focus being on the interactions between the microstructure features rather than benchmarking the code explicitly to the database. The interdependencies will be determined by examining how defect partitioning evolves with increasing damage in the growth regime. Thus, the model will be able to track the evolution of defect absorption at sinks. It is expected that the voids will continue to grow in both sink strength as well as the relative amount of defect absorption at sinks, but the focus of the modeling effort will be on analyzing how the dislocation and precipitate microstructure affect defect partioning. The combination of defect behavior in the bulk and at sinks can then be used to determine what, if any, interdependencies/interactions between voids, precipitates and dislocations occurred. Thus, the results of Sub Objective 1-3 will demonstrate both how the microstructure evolves and why it evolves in such a way. 


\section{CHAPTER 4}

\section{EXPERIMENTAL PROCEDURE}

The chapter will describe experimental techniques used in this thesis. It is organized into the following sections: sample preparation, ion irradiations and post-irradiation sample preparation and microstructure characterization.

\subsection{Sample Preparation}

The focus of this thesis is on the ion irradiation-induced microstructure evolution of HT9a FM alloy.

\subsection{1 $\underline{\text { Sample Processing }}$}

The commercial alloy chosen for this study was HT9, which is an iron based alloy with major additions of $\mathrm{Cr}$ and minor additions of $\mathrm{Mo}, \mathrm{V}$, and $\mathrm{W}$. The heat number is 84425 and has the composition shown in Table 4.1. HT9 was heat treated first with a $1065^{\circ} \mathrm{C} / 30 \mathrm{~min} /$ air cooling to room temperature (RT) austenization step followed by a $750^{\circ} \mathrm{C} / 60 \mathrm{~min} /$ air cooling to $\mathrm{RT}$ tempering step $[29,60]$. A number of publications of data from this duct reflect different heat treatment parameters $[9,10,29,31,60-62]$, but a propriety certification from Carpenter verified that this was the correct heat treatment [63]. The heat treatment results in a transformation from austenite to martensite lathes with carbides forming primarily at the grain boundaries during the tempering step, shown in Figure 4.1. There were also retained grains of $\delta$-ferrite. The heat was 
formed into ducts and irradiated in the Fast Test Flux Facility $[9,10]$ as well as under ion irradiation [2,13]. The duct removed from FFTF and analyzed was designated as ACO3.

Samples were cut from archive duct material into 1.5 x 1.4 x $18 \mathrm{~mm}$ TEM bar specimens by electrical discharge machining (EDM). EDM, also known as spark machining, is a manufacturing process where a voltage applied between an electrode wire and the block results in high frequency sparks. These sparks, across a distance of 10-100 $\mu \mathrm{m}$ cause material removal near the wire, which is placed at the desired cut area. Overall, the EDM process results in a sample cut without cold working, which is induced by traditional machining techniques. The schematic of the samples post-EDM is shown in Figure 4.2.

\subsubsection{Sample Preparation}

After samples have been machined, they were then subjected to a series of polishing steps, starting with mechanical polishing followed by a final finish of vibratory polishing and/or electropolishing. A flat, aluminum polishing block or disk was heated to a sufficiently high temperature to melt Crystalbond ${ }^{\mathrm{TM}}$ resin wax. The samples were placed on the block in the configuration that they would be mounted onto the irradiation stage. Upon cooling, the resin wax bonded the samples to the block. Samples were placed in this configuration to ensure a planar surface, which resulted in good thermal contact between the samples and the hold down bars. When resin wax is fully cooled, the samples were wet polished using Buehler® $\mathrm{SiC}$ paper, beginning with grit \#240 and working up to grit \#4000.

The back surface (opposite to irradiation surface) was polished up to grit \#4000, after which the samples were cleaned ultrasonically in acetone, flipped over, remounted with Crystalbond ${ }^{\mathrm{TM}}$ and the irradiated surface was mechanically polished with $\mathrm{SiC}$ paper up to grit \#4000. After that, 
samples were mechanically polished using a diamond solution from $9 \mu \mathrm{m}$ to $0.25 \mu \mathrm{m}$ ensuring a very smooth surface. A final mechanical polishing step using $0.02 \mu \mathrm{m}$ silica solution was performed either by hand or on a vibratory polisher.

To ensure that no plastic deformation remains after mechanical polishing, some samples were then electropolished. An electropolishing study was performed to select optimum electropolishing conditions such that the samples had a mirror finish, with no observable pitting or etching.

A $1000 \mathrm{ml}$ beaker containing a solution of $500 \mathrm{ml}$ of $90 \%$ methanol and $10 \%$ perchloric acid was immersed in a bath of methanol and dry ice. The temperature of the solution was maintained between -40 and $-50^{\circ} \mathrm{C}$. A square platinum mesh cathode with sides approximately 70 $\mathrm{mm}$ and in length and $40 \mathrm{~mm}$ in height was placed in the bottom of the beaker. During electropolishing, individual samples were held at one end with alligator clips and placed, upright, centered vertically in the solution-containing beaker. Samples were electropolished for 20 seconds with an applied potential of $35 \mathrm{~V}$ between the sample and the platinum mesh cathode, measured by a voltmeter. A magnetic stirring bead rotating with a frequency of approximately $650 \mathrm{rpm}$ created a circular flow inducing a vortex of 0.75 inches in the polishing solution. The sample face to be irradiated was immersed in the vortex so that oxygen bubbles would be agitated off from the sample surface, which reduced pitting. A schematic of this electropolishing assembly is shown in Figure 4.3. After electropolishing, the samples were rinsed in acetone, followed by methanol, followed by ethanol; after rinsing, a final ultrasonic cleaning with ethanol was performed. Samples were stored in membrane boxes until the time of irradiation.

\subsection{Helium Preimplantation}


To more accurately account for helium formed in-reactor by (n, $\alpha$ ) reactions, preimplantation of helium was performed prior to irradiation of the samples. A $400 \mathrm{keV}$ National Electrostatics Company (NEC) ion implanter was used. Helium pre-injection was performed at energies of $80,140,220,310$ and $420 \mathrm{keV}$, to yield an approximately flat distribution of helium $( \pm 10 \%)$ over a depth range of $300-1000 \mathrm{~nm}$ from the surface shown in Figure 4.4. In this thesis, 10 appm He was determined to be sufficient to enhance nucleation of voids without unduly suppressing the growth of the voids [12]. For preimplantation, samples were mounted to the implanter stage in the configuration that they would be mounted onto the irradiation stage. Preimplantation were performed at room temperature, with no heating from the He beam or cooling from the stage assembly.

\subsection{Fe $^{++}$Irradiations}

This section covers all the relevant experimental details and techniques utilized for iron irradiations. Samples were irradiated in either a 1.7 MV General Ionex Tandetron accelerator or a 3 MV National Electrostatics Company (NEC) Pelletron accelerator at the Michigan Ion Beam Laboratory. During the course of this thesis, based upon increased user experience, a number of experimental improvements were implemented. Additionally, a renovation of the laboratory that resulted in improvements to the experimental setup and the switch to a new accelerator; all experimental set-ups will be described later. To perform a successful iron irradiation, a specialized irradiation set-up including stage design, aperture systems, temperature measurement, experimental monitoring and optimal vacuum conditions were utilized.

\subsubsection{Irradiation Set-up}


Irradiations were performed under high vacuum conditions. The entire accelerator beam line was maintained at pressures ranging from approximately $10^{-7}$ torr near the accelerator to below $10^{-7}$ torr near the stage. The stage was outgassed prior to the start of each irradiation, ensuring that experiments only started after the pressure dropped below $10^{-7}$ torr after stage. To improve vacuum further, a second cryopump was added directly below the stage to minimize any oxide formation due to the high energy incident iron ions at high temperature.

Samples were mounted on an irradiation stage, which was attached to the accelerator beam line. Two stages were used in these experiments, both of identical design, but constructed using two different metals $(\mathrm{Cu}$ and $\mathrm{Ni})$. The "first generation" stage, made of copper, was used initially when irradiations were performed using liquid indium to ensure good thermal contact between the sample and stage. The prevalence of indium leaks with this configuration lead to design of an intermediate "second generation" stage configuration using a combination of indium and graphite foil to mitigate leaks. Due to concerns about vacuum quality, a final "third generation" stage configuration was implemented which utilized a nickel stage with copper foil for heat conduction. Regardless of stage or stage configuration used, a combination of resistive heating and air cooling monitored by an in-situ 2D infrared thermal imager was used to ensure excellent temperature control, further explained in Section 4.3.5.2. Figure 4.5 shows a side view of the beam line at the stage showing the relative position of the $2 \mathrm{D}$ infrared thermal imager. The following are descriptions of each of the three stage configurations used for this thesis.

\subsubsection{Copper Stage with Indium}

First, a stainless steel shim was placed on the copper stage. In the center of the shim, an opening of 16 or $10 \mathrm{~mm}$ (length) by $10 \mathrm{~mm}$ (width) which was filled with an indium foil. The shim 
was $0.65 \mathrm{~mm}$ in height and was filled with an approximately $0.60 \mathrm{~mm}$ of indium foil(s), which was sufficient to fill the reservoir upon melting. Samples were then placed on top of the stage and centered over the indium reservoir. Indium was used to ensure good thermal conductivity between the copper stage and steel samples. TEM bar samples were placed on top of the stage between two guide bars. Guide bars were included to 1) ensure uniform irradiation across the width of the stage and to 2) provide a surface to attach thermocouples for calibration of the $2 \mathrm{D}$ thermal imager.

Samples and guide bars were held in place with hold-down bars, shown in Figure 4.6. The hold down bars fit tightly over the samples to ensure good, uniform thermal contact and the "halfmoon" shape minimizes thermal reflections to the thermal imager (Figure 4.6a). They were secured with 4 set screws which were tightened slowly to maintain even pressure on the samples (Figure 4.6b). This stage configuration was designated as "first generation" and a schematic is given in Figure 4.7a. J-type thermocouples were spot-welded to guide bars for use in calibration of the 2D thermal imager and will be further described in Section 4.3.5. Lastly, a tantalum aperture assembly for beam alignment was mounted onto the stage using isolating ceramic standoffs immediately adjacent to the stage. A schematic view from the top is shown in Figure 4.8 and Figure 4.9 shows a completed stage. Stage and slit aperture systems will be further described in detail in Section 4.3.4.

Indium melts at $156^{\circ} \mathrm{C}$, and was originally used to maintain thermal contact, which is especially necessary for higher flux proton irradiations that result in high beam heating. This stage design was only used with stage apertures. However, temperature cycling due to frequent iron source failures led to indium leaks, which limited damage levels that could be reached in each irradiation. Thus, the stage configuration was improved to mitigate the possibility of indium leaks. 


\subsubsection{Copper Stage with Indifoil}

To limit indium leaks and the subsequent detrimental effect on sample surface, a graphite foil was placed in between the indium and the samples on the stage (Figure 4.7b), colloquially known as "indifoil." The effect of the graphite foil was twofold. First, it maintained the necessary thermal contact to ensure uniform temperature across the samples. Second, it eliminated leaks entirely, even with thermal cycling due to interruptions during irradiation to rebuild the iron source or replace the iron target. The graphite foil used was a $0.1 \mathrm{~mm}$ thick, high thermal conductivity pyrolytic graphite sheet. The stage design was used for a limited period (two experiments presented: $480^{\circ} \mathrm{C}, 188 \mathrm{dpa}$ and $400^{\circ} \mathrm{C}, 250 \mathrm{dpa}$ ) before it was replaced with the final stage configuration that was used for the majority of the experiments in this thesis. Again, this stage design was only used with stage apertures.

\subsubsection{Nickel Stage with Copper Foil}

Concerns about the formation of a surface oxide prompted a final stage configuration change. Despite the low $\left(<10^{-7}\right.$ torr $)$ vacuum near the stage, a surface oxide formed under irradiation. To minimize this oxide, the graphite foil was removed because water vapor and other light molecules can adsorb to the graphite foil and decrease vacuum quality. Thus, two changes were made. First, the nickel stage was machined flat. Second, the indium and graphite foil were removed entirely from the stage and replaced with a $0.25 \mathrm{~mm}$ copper foil (Figure $4.11 \mathrm{a}$ ). Given the low current of $\mathrm{Fe}^{++}$, very little beam heating $\left(<10^{\circ} \mathrm{C}\right)$ was observed and a copper foil maintained good enough thermal contact ensure uniform heating across samples.

Prior to irradiation, the temperature uniformity was assessed. Four FM samples (T91) were polished and loaded onto the stage. Eight thermocouples were welded to the stage (Figure 4.10a- 
b). On the benchtop, the stage was heated to $200^{\circ} \mathrm{C}$ and 6 of the $8 \mathrm{TCs}$ were within $200 \pm 3^{\circ} \mathrm{C}$. Upon cooling, it was found that the welds of the two thermocouples that were not within $3^{\circ} \mathrm{C}$ of $200^{\circ} \mathrm{C}$ were loose. The loose thermocouples were re-welded and the stage was loaded onto the beamline and heated up to $500^{\circ} \mathrm{C}$. It was found that 6 of the 8 thermocouples were within $4^{\circ} \mathrm{C}$ for a period of 20 hours (Figure 4.10c), with a brief interruption due to a power outage. Upon unloading, the two thermocouples that had readings outside of the $4^{\circ} \mathrm{C}$ window were again loose. These results were deemed sufficient to demonstrate temperature uniformity. However, this experiment also highlighted limitations in thermocouple welding and the procedure for spot welding thermocouples was improved as a result, to minimize loosening of thermocouples.

The copper foil stage design was used with both stage (Figure 4.11a) and slit apertures (Figure 4.11b). After the upgrade to slit apertures, the stage was fitted with 2 thermocouple feedthroughs, which allowed up to 8 thermocouples to be used per stage. (Figure 4.12)

\subsubsection{Displacement Damage}

Displacement damage of the incident $5 \mathrm{MeV} \mathrm{Fe}^{++}$is calculated with the Stopping and Range of Ions in Matter (SRIM) 2010 ${ }^{\mathrm{TM}}$ program [64]. The SRIM program outputs a number of displacements produced per unit length per ion (displacements/Å-ion), which is depth dependent. For this thesis, the SRIM calculations were calculated using the "quick" Kinchin-Pease approximation, which has been shown to be more appropriate for calculating damage levels of ion experiments [65], when they are compared to damage calculated in neutron irradiations by the Norgett, Robinson and Torrens (NRT) method [66]. 100,000 incident ions were used to improve counting statistics, which provides an accurate and smooth damage curve. 
Displacement energy for each element was defined in the SRIM calculation. For this work, the input composition was $88 \% \mathrm{Fe}$ and $12 \% \mathrm{Cr}$. For both $\mathrm{Fe}$ and $\mathrm{Cr}, 40 \mathrm{eV}$ was used as the displacement energy for both elements, per the recommendation offered by ASTM E 521-89 [67]. Figure 4.4 shows both the damage curve (red) as well as the implanted ion fraction (green) overlaid on the preimplanted helium concentration (blue). As shown, the damage curve is highly peaked so a specific depth was chosen to avoid both surface and damage peak effects. Peak damage due to $\mathrm{Fe}^{++}$was at $1.3 \mu \mathrm{m}$, but nominal damage was measured and recorded at $0.6 \mu \mathrm{m}$. The damage rate was calculated to be 0.355 displacements/Å-ion.

\subsubsection{Damage Calculation}

The irradiation damage is related to the current of iron ions on the stage, so accurate measurement of the current throughout the irradiation is paramount to reaching the appropriate damage level. $5 \mathrm{MeV} \mathrm{Fe}^{++}$ions incident on a metal surface cause electron showers, [68] which means that the current cannot be read accurately unless the measurement was actively electronically suppressed. Thus, in situ monitoring of the current on the stage was not possible, as with proton irradiations $[32,69,70]$. Current was measured with a suppressed Faraday cup either before or after the apertures every 45-60 minutes, depending on source stability. Installation of slit aperture system allowed the current to be measured immediately before the samples. The damage level (dpa) is calculated using the following equation:

$$
\boldsymbol{d p a}=\frac{\left(\sum t_{i}(\text { minutes }) * f_{1}\left(\frac{s}{\text { minute }}\right) * i_{\text {stage }}(\mu A) * R_{D}\left(\frac{\text { displacement }}{\AA}\right) * f_{2}\left(\frac{A}{c m}\right)\right)}{N\left(\frac{a t}{c^{3}}\right) * Q\left(\frac{C}{i o n}\right) * f_{3}\left(\frac{\mu C}{C}\right) * A\left(c^{2}\right)}
$$

where $\sum t_{i}$ is the total time under irradiation, $f_{1}$ is the conversion factor from seconds to minutes, $i_{\text {stage }}$ is the time-averaged stage current, $R_{D}$ is the displacement rate from SRIM, $f_{2}$ is the 
conversion factor from angstroms to centimeters, $N$ is the atomic density, $Q$ is the charge per $\mathrm{Fe}^{++}, f_{3}$ is conversion factor from micro-coulombs to coulombs and $A$ is the area receiving full irradiation (i.e. aperture opening). A summary of these terms and their typical values is given in Table 4.2.

\subsubsection{Apertures and Beam Scanning}

The aperture system provided a method for both aligning and sizing the beam. Two different methods of beam scanning were used throughout this thesis. First, apertures attached to the stage were used. Although stage apertures have been effective in previous proton and iron irradiations, the apparatus could shift upon loading to the beamline, which changes the area of the samples that is irradiated. Shifting of the apertures and consequently the irradiated area was mitigated by an upgrade to an in situ slit aperture system.

\subsubsection{1 $\quad$ Stage Aperture System}

The stage aperture system consisted of four tantalum plates, which were electrically isolated from each other. They were mounted to the stage using ceramic standoffs to ensure that they were electrically isolated from the stage as well. The stage aperture system is shown in Figure 4.13. Aperture sizes were adjusted using the screws to reach the desired irradiated area. Typically, the aperture size was $12 \mathrm{~mm}$ in $\mathrm{x}$ by $5 \mathrm{~mm}$ in $\mathrm{y}$, though it varied depending on the number of samples; typically, 4 to 6 samples plus 2 guide bars per stage. Each aperture piece was individually connected with a wire to a feedthrough that output current to a monitoring computer. The relative current on each aperture was then used to balance the beam on desired irradiated area. 
Aperture alignment was performed ex situ. The alignment was performed on the benchtop with the stage oriented on its side as it would be on the beamline. A laser pen was positioned $\sim 20$ feet away from the irradiation stage. The laser is aligned to the center of the aperture then diffused with a thin plastic sheet to mimic a raster-scanned beam. The aperture was adjusted with screws so that the irradiated area was clearly illuminated by the laser. The alignment assembly is shown in Figure 4.14.

Before starting an irradiation, the focused iron beam was measured for size in a beam profile monitor. The beam size was verified to be at most $3 \mathrm{~mm}$. The beam was then rasterscanned across the samples at a frequency of $255 \mathrm{~Hz}$ in $\mathrm{x}$ and $2061 \mathrm{~Hz}$ in $\mathrm{y}$, corresponding to 3.92 and $0.48 \mathrm{~ms}$ cycle time in the $\mathrm{x}$ and $\mathrm{y}$ directions, respectively. The ratio of these cycles was not an integer, which results in an offset of the beam between scan cycles, ensuring uniform irradiation. A schematic is shown in Figure 4.15.

The raster-scanned beam must overlap onto all the aperture pieces to ensure full, uniform irradiation for all samples. One full beam diameter, or $3 \mathrm{~mm}$ must overlap onto each aperture piece during raster-scanning. The size of the aperture opening sets the size of the beam. For a 12 $\mathrm{mm} \times 5 \mathrm{~mm}$ aperture with a $3 \mathrm{~mm}$ beam diameter, the beam must be scanned $18 \mathrm{~mm}$ x $11 \mathrm{~mm}$. The desired irradiation area was drawn on a ceramic, which was the loaded in the target chamber before the stage. When the $\mathrm{Fe}^{++}$beam hits the ceramic, it fluoresced so the beam was able to be scanned to an appropriate size. After sizing, the ceramic was moved out of the incident beam so the beam is allowed to reach the aperture and irradiated area. A schematic of the appropriately sized beam is shown in Figure 4.16a. 
To measure $i_{\text {Stage }}$, the Faraday cup was inserted periodically ( 45-60 minutes) into the beam. The value of $i_{\text {Stage }}$ was recorded and time averaged. However, since the current was being monitored before the slits, an additional factor was required to calculate the current at the stage. That factor was calculated by following the center of the beam as it rastered, even though the scanned area is $3 \mathrm{~mm}$ in each direction, the center of the beam only travels $1.5 \mathrm{~mm}$ off each direction. The result of this beam path was demonstrated by the beam intensity curve plotted in Figure 4.16. Thus, the percentage of current on the stage using Equation 4.2 below:

$$
\frac{i_{\text {stage }}}{i_{\text {total }}}=\frac{x * y}{\left(x+\frac{d_{\text {beam }}}{2}\right)\left(y+\frac{d_{\text {beam }}}{2}\right)}
$$

where $i_{\text {stage }}$ is the current on stage, $i_{\text {total }}$ is the current before the slits, $x$ is the aperture width, $y$ is the aperture height and $\mathrm{d}_{\text {beam }}$ is the beam diameter. Considering the nominal case of $x=5 \mathrm{~mm}, y=$ $12 \mathrm{~mm}$ and $d_{\text {beam }}=3 \mathrm{~mm}$, the percentage of current on the stage is $50 \%$. Thus, this factor can be multiplied by the total current before slits to calculate $i_{\text {stage }}$, which is used to calculate damage.

\subsubsection{Slit Aperture System}

The stage aperture system had several drawbacks. First, the slit size was manually adjusted and measured with calipers. Second, the alignment took place ex situ, which had the disadvantage of not being adjustable once the stage was loaded. In addition, the assembly was prone to shifting or translation during the loading process, which was only discovered upon completion of the irradiation. For the purposes of re-irradiating samples, this was a major concern. Thus, with the renovation of the ion irradiation beam line, slit apertures were included so alignment could be performed in situ. 
The slit aperture system contains 4 tantalum plates attached to independent motors. The plates were electrically isolated so current can be read off of each plate (X+, $\mathrm{X}-, \mathrm{Y}+$, and $\mathrm{Y}-)$. The slit system was 24 inches in front of the stage. An alignment laser had been installed and was aligned from the magnet to the stage. It was a green laser diffuse enough so that entire irradiated region of interest (maximum of $20 \mathrm{~mm}$ x $20 \mathrm{~mm}$ ) can be observed. After the stage had been loaded, the alignment laser before the magnet is turned on. Slits were opened to the desired irradiation size (for most cases, $12 \mathrm{~mm}$ x $5 \mathrm{~mm}$ ). The laser was turned on and the stage was adjusted, if necessary, by physically adjusting the stage holder. An image of the aligned stage is presented in Figure 4.17.

The beam sizing procedure described previously had the disadvantage of being dependent on 1) the florescence of the beam on the ceramic, and 2) visual inspection of the size of the beam scanned area from a distance of $\sim 18$ inches. With the upgrade to the slit aperture system, and the installation of Faraday cups before and after the slits, a more precise beam sizing procedure was developed. First, the slits were closed to $3 \mathrm{~mm}$ x $3 \mathrm{~mm}$ and the beam was maximized in the stage Faraday cup using the magnet and quadruples, ensuring the beam was approaching the stage head on, and also minimized the size of the beam. After this focusing step, the beam was typically $\leq 2 \mathrm{~mm}$, measured by the beam profile monitor. The slits were then opened to the desired irradiated area (usually $12 \mathrm{~mm}$ x $5 \mathrm{~mm}$ ). The maximum ratio of beam to be transmitted through the slits to the stage was calculated using Equation 4.2 mentioned previously. Similarly, for a $12 \mathrm{~mm}$ by $5 \mathrm{~mm}$ aperture, $50 \%$ of the beam is on the stage and $12.5 \%$ is on each aperture. It should be noted that this ratio was identical to what was used to calculate the damage on the stage in the stage aperture method. Beam raster-scanning was adjusted in $\mathrm{x}$ and $\mathrm{y}$ directions such that these parameters were measured when the irradiation was ready to commence. 


\subsubsection{Temperature Control}

Temperature control of the irradiation status was accomplished by a combination of heating and cooling. A Watlow FIREROD ${ }^{\circledR}$ resistance cartridge was inserted to the stage base. The heater cartridge was $4 \mathrm{~cm}$ in length with a $1 \mathrm{~cm}$ diameter and was rated up to $760^{\circ} \mathrm{C}$, which was well above desired irradiation temperature, which varied from $400-480^{\circ} \mathrm{C}$. Cooling lines ran through the stage and air cooling removed heat during or after completion of irradiations. The combination of heating and cooling was ideal for minimizing temperature variations.

\subsubsection{Thermocouples}

Four J-type thermocouples were spot welded to the guide bars or portions of the sample not under irradiation on the stage. These were used upon start up to calibrate the $2 \mathrm{D}$ infrared thermal imager, which was used for monitoring the temperature during irradiations. The thermal infrared imager will be discussed in Section 4.3.5.2.

J-type thermocouples have an operating temperature of up to $750^{\circ} \mathrm{C}$, well within what was necessary for these experiments. They were made from $0.0013 \mathrm{~mm}$ iron and constantan wires, which were insulated with ceramic beads to ensure that there were no shorts between the wires, other thermocouples or with the stage. Four thermocouples were used as a redundant check on temperature and to verify that there were no non-uniformities across the stage between the unique samples.

Thermocouples were attached to the samples using a spot weld at the "cross" of the two wires. The thermocouple beads and wire cross on the sample are shown in Figure 4.9 on the irradiation stage. Since repeated thermal cycling and irradiation can embrittle the wire, they were made separately for each irradiation. A fifth coated probe J-type thermocouple from Omega® 
was inserted into a port on the back of the stage, which was used to monitor the temperature of the back of the stage throughout the irradiation.

\subsubsection{Thermal Imager}

Prior to sending the iron beam onto the samples, the samples were heated to irradiation temperature using the resistance heater. They were allowed to stabilize at the desired irradiation temperature, measured by the thermocouples. At this point, the $2 \mathrm{D}$ infrared thermal imager was calibrated to the thermocouples readings. Shown in Figure 4.5, an IRCON thermal imaging system was positioned outside the irradiation chamber. Areas of interest (AOIs) were created on each TEM bar sample; each corresponded to 5 pixels or $1 \mathrm{~mm}$ in diameter. At least 2 AOIs per sample were monitored and the resulting image is shown in Figure 4.18. Each AOI was calibrated to the thermocouples by adjusting the emissivity, which was dependent on the angle of imaging as well as surface condition and material. Beam heating was measured by the thermal imager and was typically between $2-8^{\circ} \mathrm{C}$, depending on current density from the source.

Some experiments were run entirely, or partially, with previously irradiated samples. Since annealing of irradiation damage was a major concern, the sample temperature, measured in the AOIs from the 2D thermal image, was not calibrated prior to starting the irradiation. The samples were out-gassed, typically overnight at $150^{\circ} \mathrm{C}$. When the beam was ready, the samples were heated up (with the beam on the stage from $350^{\circ} \mathrm{C}$ to the irradiation temperature). The AOI temperatures were then calibrated to the thermocouples with the irradiation already started. Calibration of the AOIs typically took less than 5 minutes to complete, once the temperature was stabilized.

\subsubsection{Irradiation Setup and Control}


Once the samples were at temperature and the AOIs calibrated, the beam was allowed to reach the samples. The temperature was maintained with a combination of resistive heating and air cooling, which maintained the temperature within $\pm 10^{\circ} \mathrm{C}$. With iron irradiations, this was typically much lower and closer to within $\pm 5^{\circ} \mathrm{C}$ on average. With the beam sent through, the current was balanced using the beam steerer, which set the horizontal and vertical positions. If the beam scanning has not already been set, it was then set to the appropriate ratio between stage and apertures calculated in Section 4.3.4.

Temperature (from both the AOI output from the thermal imager as well as from the thermocouples) and current were monitored throughout the duration of the irradiation. Each AOI temperature was collected once per second then averaged over a 15 second period and that value was recorded in LabVIEW ${ }^{\mathrm{TM}}$. The thermocouple temperature, including the back thermocouple, was recorded once every 30 seconds. The current was recorded manually by insertion of the Faraday cup into beam line every 45-60 minutes. The aperture current and pressure were also recorded once per 15 seconds. Data acquisition was performed using LabVIEW ${ }^{\mathrm{TM}}$ program.

The monitoring frequencies were set to high frequency (every 15 and 30 seconds for AOI and thermocouple temperature, respectively) to ensure the highest quality irradiations in terms of temperature control and current monitoring, without being unduly disruptive to the experiment. If any of the AOI temperatures drifted outside of the acceptable $\pm 10^{\circ} \mathrm{C}$ an alarm sounded, alerting the experimenter. Similarly, the aperture currents were monitored so if the beam drifted an alarm sounded alerting the experimenter that the steerer needed to be adjusted.

\section{4 $5 \mathrm{MeV} \mathrm{Fe}^{++}$Irradiation Results}


Sample designations have been developed to ensure easy identification. Each irradiated bar was designated as 'heat name-temperature-damage-helium-date.' For instance, 'ACO3-460C350dpa-10He-011215' refers to a bar of the ACO3 heat of HT9 preimplanted with 10 appm He irradiated on $01 / 12 / 15$ at $460^{\circ} \mathrm{C}$ up to a final damage of $350 \mathrm{dpa}$. If a specific liftout is referenced, then the designation also includes the grid post and date of liftout taken as well ('alloytemperature-damage-helium-irradiation date-grid post-liftout date'). For instance, a liftout on grid post B from the previously mentioned 350 dpa irradiation would be referred to as ACO3-460C350dpa-10He-011215-Grid B-011715. A list of bar samples used in this thesis is given in Table 4.3. A total of 15 irradiations were performed for this thesis to fulfill the requirements of Sub Objectives 1 and 2.

This section will report on irradiations performed using consistent and appropriate experimental techniques. For any type of ion irradiation, there were three key parameters that were defined: temperature, damage and damage rate. Temperature and final damage level were of primary concern. Regarding damage rate, an effort was made to ensure the highest possible damage rate, while still maintaining stable irradiation parameters. Practically speaking, this varied between $0.5-1 \times 10^{-3} \mathrm{dpa} / \mathrm{s}$ for the majority of irradiations. Within an irradiation, the ion current and as a consequence, the instantaneous damage rate, was maintained within a factor of 2 . An attempt was also made to ensure all damage rates were within a factor of 2-3 between irradiations.

A summary of all the irradiations conducted is provided in Table 4.4. All irradiations reached $\pm 0.5 \mathrm{dpa}$ of the target damage level. The damage rate varied between $0.27-1.20 \times 10^{-3} \mathrm{dpa} / \mathrm{s}$. The lower damage rates were prior to replacement of the $1.7 \mathrm{MV}$ Tandem accelerator with a $3 \mathrm{MV}$ Pelletron accelerator, which had increased beam transmission by approximately a factor of two. After this upgrade, the damage rates were within a factor of two for the irradiation campaign from 
0-650 dpa referred to as part of Sub Objective 2 described in Chapter 3. Irradiation temperature was calibrated with $\mathrm{J}$ type thermocouples; the average temperature is shown in Table 4.4. Prior to irradiation, at least 3 thermocouples varied less than $<5^{\circ} \mathrm{C}$ from the target irradiation temperature. Irradiations only commenced upon the pressure reaching $9.9 \times 10^{-8}$ torr, ensuring a high vacuum environment.

The irradiation temperature, measured by at least two AOIs on each sample, is also shown in Table 4.4. All samples have been irradiated within $<4^{\circ} \mathrm{C}$ of the target irradiation temperature. The frequency of temperature measurements has been plotted as a series of histograms and the temperature distribution for each irradiation was normally distributed with a target $2 \sigma< \pm 10^{\circ} \mathrm{C}$. Presented in Table 4.4 , most $2 \sigma$ values were considerably lower and averaged around $\pm 5^{\circ} \mathrm{C}$. Temperature histograms for AOIs on all samples included in this thesis are available in "Appendix A: Temperature Histograms", but an example is also shown in Figure 4.19.

\subsection{Post-Irradiation Sample Preparation and Microstructure Characterization}

Following iron irradiation, the samples were removed from the stage for preparation of TEM specimans using the focused ion beam (FIB) in-situ liftout method. The procedures for this technique are discussed in this section.

\subsubsection{TEM Specimen Preparation}

The shallow penetration depth of $\mathrm{Fe}^{++}$ions into the irradiated sample presented a unique challenge. In addition, magnetic materials such as HT9 distort the TEM electron beam. A practical solution to both of these challenges is to limit the volume of examined material to the near surface, which was accomplished using the FIB in-situ lift-out technique to create crosssectional TEM specimens. 
The dual beam FIB system used consists of a low energy $(\leq 30 \mathrm{keV})$ electron beam and a gallium beam oriented $52^{\circ}$ from the e-beam. It also included a tilting stage so that the stage can be tilted so it was orthogonal to the e-beam $\left(0^{\circ}\right.$ tilt $)$ or orthogonal $\left(52^{\circ}\right.$ tilt $)$ to the gallium beam. Lift-outs prepared for this thesis were made using the FEI Company's Helios NanoLab ${ }^{\mathrm{TM}}$ DualBeam $^{\mathrm{TM}}$, Nova NanoLab ${ }^{\mathrm{TM}}$ DualBeam $^{\mathrm{TM}}$ or Quanta $^{\mathrm{TM}} 200$ 3D Focused Ion Beam Workstation. TEM specimens were prepared in the following way.

First, the stage was tilted normal to the ion beam $\left(52^{\circ}\right)$. A gas injector was inserted and a low current ion beam $(\sim 0.1-0.23 \mathrm{nA})$ was used to deposit platinum (Figure 4.20a) onto the sample surface. The gallium beam, at a higher current ( 7-9 nA), was then used to mill away trenches surrounding the area of interest, known as a lamella (Figure 4.20b). The sample was then tilted back normal to the e-beam $\left(0^{\circ}\right.$ tilt $)$. The gallium beam, still at a high current, was used to cut the bottom of the lamella, so it was held only on one side (Figure 4.20c). At this point, a micromanipulator (Ominoprobe) was inserted and positioned on the side of the lamella and was then jointed to the lamella with platinum (Figure 4.20d). The lamella was then totally free from the surrounding materials by further cutting and was lifted out of the trench by moving up the Ominoprobe. The lamella was then transferred on the micromanipulator to a copper half grid (Figure 4.20e). Once it was jointed to the grid, the micromanipulator was cut away using the gallium beam. The grid was then tilted such that the sample surface was orthogonal to the ion beam. Thinning was performed using low current ion beam in stages until the TEM specimen was electron transparent (Figure 4.20f). A final cleaning was performed with $5 \mathrm{keV}$ ion energy. Liftouts were usually $100-150 \mathrm{~nm}$ in thickness. A schematic of the cross sectional liftout is shown in Figure 4.21.

\subsubsection{Void Swelling Measurement and Characterization}


The objective of this thesis is to determine how microstructure features co-evolve. Thus, a systematic and careful characterization of microstructure features was essential. There are a number of challenges in imaging voids in particular. First, the irradiation area is limited to the first $1.6 \mu \mathrm{m}$ and there were many gradients in terms of both helium and damage. Second, HT9 is swelling resistant and void nucleation is highly heterogeneous. Thus, it was expected that there would be a low density of voids nucleated over large areas making statistical significance a challenge. Third, FM alloys such as HT9 are very complex and include many features including dislocation loops, lines, as well as small grain and lath sizes. These features have the potential to obscure voids, particularly those that are small $(<5-10 \mathrm{~nm})$.

\subsubsection{Imaging Conditions}

Void imaging was performed using either a JEOL 2100F Cs Corrected Analytic Electron Microscope (AEM) located at the Michigan Center for Materials Characterization $\left(\mathrm{MC}^{2}\right)$, formerly the Electron Microbeam Analysis Laboratory (EMAL) at the University of Michigan, or a JEOL 2010F AEM. The JEOL 2100F operates only in scanning transmission electron microscopy (STEM) mode. The JEOL 2010F operates in either CTEM or STEM mode. Both microscopes were operated using an accelerating voltage of $200 \mathrm{kV}$. Specimens were loaded on either a single-JEOL or double-tilt Gatan holder, operated at room temperature. The Digital Micrograph® software package was used for STEM and CTEM image acquisition. It was also used for energy-dispersive X-ray spectroscopy (XEDS), a technique utilized to obtain microchemical data collection and analysis when imaging secondary phases.

Void imaging was performed using STEM mode. The entire liftout was imaged in both high angle annular dark field (HAADF) and in bright field (BF) modes. Traditionally, void 
imaging has been performed using CTEM with the through focus technique, meaning that voids were over and under focused in a series of imaging conditions. There are several issues with this technique. First, although conventional BF has better resolution than BF STEM, the voids varied in size with depth, since not all voids were in focus at a single objective focus value. Second, the BF imaging suffers diffraction contrast effect causing voids to be obscured by BF imaging under strong Bragg scattering conditions. Since HAADF imaging in STEM has $\mathrm{Z}$ contrast ( $\mathrm{Z}$ is atomic number here) in nature, the void size can be accurately measured irrespective of the position of the void in the lamella and most voids were in focus as long as the a smaller beam convergent angle used and the specimen is not too thick, shown in Figure 4.22. So, HAADF is optimal for imaging voids, as there is less diffraction contrast in HAADF relative to that observed in BF; thus, voids that were obscured by diffraction contrast in BF (Figure 4.23b) were clearly visible in HAADF (Figure 4.23a).

Void images in STEM HAADF and STEM BF modes were taken across the entire liftout at a magnification of 50,000x and were then "stitched" together to show the whole area. An example of this is shown in Figure 4.24, where both HAADF and BF images clearly show the void distribution within the sample. In addition, as it is hard to reach a "pure Z-contrast" imaging condition experimentally, if diffraction contrast was still visible in HADDF, the stage was then tilted so that all grains can be clearly illuminated without much diffraction contrast.

Specimen thicknesses was measured using Electron Energy Loss Spectroscopy (EELS) low loss method. The EELS spectrum, which was performed in conjunction with STEM imaging, measures the loss in energy of electrons penetrating the samples using an energy filter here a Gatan Imaging Filter (GIF). The lenses for STEM imaging were set to give a probe size of $\sim 1 \mathrm{~nm}$ with an EELS collection angle about $38 \mathrm{mrad}$. The EELS low loss method used to 
determine specimen thickness has an error of $10 \%$ [71], which was determined by calculating the error in logarithmic fit of the inelastic mean free path of electrons through the sample. The specimen thickness was measured at a depth of 500-700 nm, corresponding to the depth of interest for void data, in at least 2 locations per liftout.

\subsubsection{Void Counting and Determination of Region of Interest}

After imaging of the liftout was complete, the voids were counted from HAADF images. Features that appeared to be voids were confirmed by examining the same image in BF to ensure that the feature was in fact a void and not a precipitate or defect cluster. Counting was performed using ImageJ software. Micrographs were opened in the program. Using the length measurement tool, the experimenter drew straight lines across the voids to measure the diameter. Voids were counted in $100 \mathrm{~nm}$ depth increments, or "bins," starting from the surface and proceeding to a depth of at least $1000 \mathrm{~nm}$ up to $1300 \mathrm{~nm}$, depending on if there were any voids at greater depths, which occurred at high damage/swelling. After an image was counted, the experimenter repeated the process with the next image, taking care not to double count voids that may overlap in both of the images. Swelling was calculated using the following equation:

$$
\frac{\Delta V}{V}=\frac{\frac{\pi}{6} \sum_{i=1}^{N} d_{i}^{3}}{A * \delta-\frac{\pi}{6} \sum_{i=1}^{N} d_{i}^{3}} * 100
$$

where $A$ is the image area, $\delta$ is the sample thickness, $d_{i}$ is the void diameter and $N$ is the number of voids. Sink strength for voids is calculated below:

$$
k_{v}^{2}=4 \pi \frac{d_{a v g}}{2} \rho_{V}
$$

where $\rho_{V}$ is the void number density. 
Swelling rate $\left(\frac{\dot{\Delta V}}{V}\right)$ was calculated from a linear regression using either linear or parabolic least squares regression line from the swelling vs. dpa curve where

$$
\frac{\Delta V}{V}=\frac{\sum\left(\Phi_{i}-\bar{\Phi}\right)\left(\frac{\Delta V}{V}-\frac{\overline{\Delta V}}{V}\right)}{\sum\left(\Phi_{i}-\bar{\Phi}\right)^{2}}
$$

where $\Phi$ is the damage level in dpa for a linear least squares regression. Swelling rate for the linear swelling regime is calculated with all values from 188 to 650 dpa unless otherwise marked. Least squares regression can also be applied to polynomial fits. In this case, the instantaneous swelling rate is taken as the tangent of least squares polynomial fit of $n=2$ :

$$
\frac{\Delta \dot{V}}{V}=\frac{d}{d \Phi}\left(a_{0}+a_{1} \Phi+a_{2} \Phi^{2}\right)=a_{1}+2 a_{2} \Phi
$$

where $a_{0}, a_{1}$ and $a_{2}$ are the polynomial coefficients calculated in Excel.

A typical result of depth profiling is shown in Figure 4.25. Several effects can be observed. Figure 4.25a-b shows that there was a high density of small voids near the surface, which was observed in the majority of conditions, regardless of temperature or presence of helium, which resulted in relatively high values of swelling in the near surface region. A similar result was observed by Shao et al. [72] Enhanced nucleation of voids within $300 \mathrm{~nm}$ of the surface where the dpa increases from 0 to $68 \%$ and the He concentration is below the nominal level of the nominal value (Figure 4.4) indicates that some mechanism is increasing the damage efficiency in this region. Furthermore, this effect was found to be independent of nominal helium concentration. Thus, it is likely caused by the close proximity of the surface. In addition, at depths greater than 
$700 \mathrm{~nm}$ a decrease in number density (Figure 4.25b) was observed and found to be coincident with the increase of the injected interstitial [73].

A full discussion of these atypical effects is in Section 5.2.1, and the valid region for void analysis was determined to lie between 300 and $700 \mathrm{~nm}$. While the He concentration was constant in this region, the damage varied substantially, increasing from 68 to $117 \%$ of the nominal damage across this $400 \mathrm{~nm}$ region. Swelling was analyzed in either the region from 300 to $500 \mathrm{~nm}$ or from 500 to $700 \mathrm{~nm}$, which limited the damage variation over the analyzed area while still maintaining a larger enough area to capture the microstructural variations and to ensure statistically significant numbers of voids. The creation of two regions of interest effectively doubled the damage levels that could be analyzed in each sample, assuming there were statistically significant numbers of voids in both regions. However, it should be noted that there will be a different damage rate in each region.

\subsubsection{Error Analysis}

A variety of measurement errors and inherent variations in void formation and measurement contributed to uncertainty regarding the void diameter, number density and overall swelling. Measurement errors were treated separately from variations. For the purposes of this analysis, fractional error $\left(\mu_{X}\right)$ is denoted as the following:

$$
\mu_{X}=\frac{\sigma_{X}}{X}
$$

where $\sigma$ is the measurement error and $\mathrm{X}$ is the measured quantity. Fractional error is typically presented as a percentage. There are two types of measurement error to be considered: TEM Resolution and EELS Measurement Error. 
The resolution of the image is $0.7 \mathrm{~nm} / \mathrm{pixel}$ and using line measurement, the error would involve at least one pixel on each end. So the error in resolution $\left(\sigma_{\mathrm{Res}}\right)$ is $\sim 1 \mathrm{~nm}$, regardless of void size. This error should be void diameter dependent according to the following equation:

$$
\mu_{\operatorname{Res}}=\frac{1 n m}{D}
$$

where $\mu_{\text {Res }}$ is the fractional error from TEM Resolution and D is the average diameter. Measurement error contributes to the diameter and swelling, since it affects the diameter of the voids, used in calculating average diameter as well as swelling.

The EELS zero loss method has inherent measurement error of $10 \%$ or fractional error of 0.1 , due to the error in the logarithmic fit of inelastic mean free path of electrons through the sample. Fractional error in thickness ( $\left.\mu_{\text {EELS }}\right)$ contributed to error in number density and swelling, only.

$$
\mu_{E E L S}=0.1=10 \%
$$

For diameter, the only measurement in error under consideration is that from TEM resolution. Applying Equation 4.8 to voids from size of $2 \mathrm{~nm}$ up to $50 \mathrm{~nm}$ results in a void size dependent error shown in Figure 4.26. Thus, the appropriate for diameter can be selected from this graph. For void density, the error is constant regardless of void diameter and is $10 \%$, which is also plotted on Figure 4.26.

Since swelling includes error contributions from TEM resolution as well as EELS measurement, it is necessary to apply linear propagation to determine the swelling error. Linear propagation for multiplication of quantities is shown below in Equation 4.10. 


$$
\mu=\frac{\sigma_{u}}{u}=\sqrt{\left(\frac{\sigma_{x}}{x}\right)^{2}+\left(\frac{\sigma_{y}}{y}\right)^{2}}
$$

For the case of void swelling, swelling is proportional to the number density and diameter (D) via the following formula:

$$
\frac{\Delta V}{V} \propto N_{D} *\left(\frac{D}{2}\right)^{3}
$$

For the case of error propagation with quantities raised to power (diameter in this case), $\mu_{\mathrm{D}}$ can be calculated as:

$$
\mu=n * \frac{\sigma_{D}}{D}
$$

where $\mathrm{n}$ is the exponent. For swelling, combining Equations 4.10 and Equation 4.12 results in the following error for swelling:

$$
\begin{aligned}
\mu_{\frac{\Delta V}{V}} & =\sqrt{\left(\frac{\sigma_{N_{D}}}{N_{D}}\right)^{2}+\left(n * \frac{\sigma_{D}}{D}\right)^{2}} \\
& =\sqrt{\mu_{N_{D}}^{2}+\left(3 * \mu_{D}\right)^{2}}
\end{aligned}
$$

Thus, swelling error is dependent on void diameter and is also plotted in Figure 4.26.

An additional consideration is the high degree of spatial heterogeneity of the void distribution, common to FM alloys. Variation in void nucleation behavior adds an additional uncertainty into the volume averaged void swelling, which is dependent on the volume of 
material characterized. To report for such variability, the number of voids counted and the area counted is provided for all void results presented in this thesis. An effort was made to examine at least 100 voids per irradiation condition in at least 2 liftouts, or nominally $2 \mu \mathrm{m}^{2}$ of $120 \mathrm{~nm}$ thickness to minimize the effect of the heterogeneity.

\subsubsection{Precipitate Measurement, Characterization and Imaging}

Two major types of irradiation-induced precipitates were observed in this study. G phase precipitates were imaged, either in CTEM or STEM mode. G phase were imaged at magnifications of up to 50,000x. Thickness of the sample was again measured by the EELS low loss method described in Section 4.5.2.1. Either XEDS maps or diffraction patterns were used to confirm the formation of the G phase. XEDS was used to verify that precipitates too small for a usable diffraction pattern were in fact $\mathrm{Ni} / \mathrm{Si}$ rich. Diffraction patterns were used on larger precipitates. $\mathrm{M}_{2} \mathrm{X}$ precipitates were imaged in low angle annular dark field (LAADF) STEM mode analog to the dark-field imaging in CTEM at magnifications of 50,000x. Compositions were confirmed using EELS and diffraction patterns were also taken in conventional mode. The calculation for sink strength for spherical, unbiased precipitates (G phase) is shown below.

$$
k_{p p t}^{2}=4 \pi \frac{d_{p p t}}{2} \rho_{p p t}
$$

where $d_{p p t}$ is the precipitate diameter and $\rho_{p p t}$ is the precipitate number density. When precipitates are planar with $l>>w$ as in the case of $\mathrm{M}_{2} \mathrm{X}$, a more rigorous calculation is required to account for the acicular shape. The calculation for non-spheroid precipitate sink strength is shown below in Equation 4.15. 


$$
k_{p p t}^{2}=4 \pi r_{p} \rho_{p p t} \frac{\kappa}{r_{p}}=4 \pi \kappa \rho_{p p t}
$$

where $r_{p}$ is the effective radius and $\kappa$ is the capacitance for a precipitate such that $w \approx t$.

$$
\begin{gathered}
r_{p}=\sqrt[3]{l w t} \\
\kappa=\frac{\sqrt{l^{2}-t^{2}}}{\ln \left[\left(\frac{l}{t}\right)^{2}+\sqrt{\left(\frac{l}{t}\right)^{2}-1}\right]}
\end{gathered}
$$

\subsubsection{Dislocation Imaging}

Both dislocation loops and lines are present after irradiation. Dislocation loop imaging in FM alloys is challenging, especially where there is a high population of voids, which can obscure the dislocation loops. Mentioned in Chapter 3, two loop types exist in FM alloys: cube-edge loops with $\mathbf{b}<100>$ on [100] planes and glissile edge loops with $1 / 2 \mathbf{b}<111>$ on [111] planes [3]. The simplest way to image all loops is by tilting to the $<001>$ axis, so $<001>$ loops will appear circular, the $<100>$ and $<010>$ appear as ovals perpendicular to each other and $1 / 2<111>$ appear as ovals at an angle of $45^{\circ}$ to the $<100>$ loops. Imaging was performed in CTEM with weak 2 beam imaging conditions. A diagram of this is shown in Figure 4.27. Loop diameter and number density were measured and from these values, a dislocation loop line length may be calculated.

Dislocation lines were measured and counted using TEM BF imaging at a magnification of 50,000x, which resulted in the average dislocation line length. Sample thickness used in this calculated line length was measured using the EELS low loss method described in Section 4.5.2.1. 
The method for determining dislocation network was developed by Smith et al. [74] and utilized in T91 recently by $\mathrm{Xu}$ [70]. Using an equidistant circular grid, intersections between the grid and dislocation lines can be used to obtain a planar density. Consider $p$, a probability of a randomly oriented line segment that intersects a grid which is defined by line segment $L_{i}$ and grid spacing of $d_{c}$.

$$
p=\frac{2}{\pi} \frac{L_{i}}{d_{c}}
$$

If $L_{i}<<d_{c}$ and $L$ is made up of $M$ segments of $L_{i}$, the number of intersection will be $p M$ thus Equation 4.18 becomes the following:

$$
N=\frac{2}{\pi} \frac{L}{d_{c}}
$$

The 2D planar density is the total line length divided by the effective area of the grid:

$$
\rho_{2 D}=\frac{L}{A}
$$

Considering a circular grid with a number of concentric lines $n_{c}$ that were spaced $d_{c}$ apart, the effective density is thus:

$$
A=\pi\left(n_{c} d_{c}\right)^{2}
$$

Combing these equations results in a planar density with units of $\mathrm{m}^{-1}$. 


$$
\rho_{2 D}=\frac{N}{2 n_{c}^{2} d_{c}}
$$

Since TEM specimans have a finite thickness, this can be translated into a volumetric density. The length of the projection of a dislocation line in a TEM image is related to the actual length by a factor of $2 / \pi$, when added to the area density results in a dislocation line density (length) of:

$$
\rho_{3 D}=\frac{N \pi}{4 n_{c}^{2} d_{c} t}
$$

Thus, this equation was used for calculation of network dislocation line density of either the asreceived or irradiated sample.

The equation for calculation of sink strength for dislocation loops and network are given below.

$$
\begin{gathered}
k_{\text {loop }}^{2}=4 \pi \frac{d_{\text {loop }}}{2} \rho_{\text {loop }} \\
k_{\text {network }}^{2}=\rho_{\text {network }}
\end{gathered}
$$


Table 4.1 Chemical composition of HT9 in wt\% [9].

\begin{tabular}{|c|c|}
\hline Element & Chemical composition (wt\%) \\
\hline $\mathrm{Fe}$ & 85.1 \\
\hline $\mathrm{Cr}$ & 11.8 \\
\hline $\mathrm{Ni}$ & 0.51 \\
\hline $\mathrm{Mo}$ & 1.03 \\
\hline $\mathrm{Mn}$ & 0.50 \\
\hline $\mathrm{C}$ & 0.21 \\
\hline $\mathrm{Ti}$ & $<0.01$ \\
\hline $\mathrm{V}$ & 0.33 \\
\hline $\mathrm{W}$ & 0.24 \\
\hline $\mathrm{Si}$ & 0.21 \\
\hline $\mathrm{Al}$ & 0.03 \\
\hline $\mathrm{S}$ & 0.003 \\
\hline $\mathrm{P}$ & 0.008 \\
\hline $\mathrm{N}$ & 0.006 \\
\hline
\end{tabular}


Table 4.2: Variables used in calculation of damage under $5 \mathrm{MeV} \mathrm{Fe}^{++}$irradiation.

\begin{tabular}{|c|c|l|c|}
\hline Variable & Meaning & Origin & Value \\
\hline$t_{i}$ & Total irradiation time & Measured & $\begin{array}{c}\text { Irradiation } \\
\text { dependent }\end{array}$ \\
\hline $\mathrm{A}$ & $\begin{array}{c}\text { Time averaged stage } \\
\text { current }\end{array}$ & Measured & $\begin{array}{c}\text { Irradiation } \\
\text { dependent }\end{array}$ \\
\hline$f_{1}$ & $\begin{array}{c}\text { Conversion from } \\
\text { minutes to seconds }\end{array}$ & Constant & $\begin{array}{c}\text { Irradiation } \\
\text { dependent }\end{array}$ \\
\hline$f_{2}$ & $\begin{array}{c}\text { Conversion from } \\
\text { angstroms to } \\
\text { centimeters }\end{array}$ & Constant & $10^{8} \AA / \mathrm{min}$ \\
\hline$f_{3}$ & $\begin{array}{c}\text { Conversion from micro- } \\
\text { coulomb to coulomb }\end{array}$ & Constant & $10^{6} \mu \mathrm{C} / \mathrm{C}$ \\
\hline$R_{D}$ & $\begin{array}{c}\text { Damage rate calculated } \\
\text { from SRIM }\end{array}$ & Constant & $\begin{array}{c}0.355 \\
\text { displacement } / \AA- \\
\text { ion }\end{array}$ \\
\hline$N$ & Atomic density & Constant & $\begin{array}{c}8.34 \times 10^{22} \\
\text { atoms } / \mathrm{cm}^{3}\end{array}$ \\
\hline $\mathrm{Q}$ & Charge per ion & Constant & $3.204 \times 10^{19} \mathrm{C} /$ ion \\
\hline
\end{tabular}


Table 4.3: List of samples utilized in this thesis, including target irradiation temperature, damage and date.

\begin{tabular}{|c|c|c|c|c|c|c|}
\hline Sample Designation & Alloy: Heat & $\begin{array}{c}\text { Target } \\
\text { Irradiation } \\
\text { Temperature } \\
\left({ }^{\circ} \mathrm{C}\right) \\
\end{array}$ & $\begin{array}{l}\text { Target Irradiation } \\
\text { Damage (dpa) }\end{array}$ & $\begin{array}{l}\text { Final Intended } \\
\text { Damage (dpa) }\end{array}$ & $\begin{array}{l}\text { Preimplanted } \\
\text { Helium (appm) }\end{array}$ & $\begin{array}{c}\text { Date of } \\
\text { Irradiation }\end{array}$ \\
\hline ACO3-440C-140dpa-10He-032812 & HT9:84425 & 440 & 140 & 140 & 10 & $3 / 28 / 2012$ \\
\hline ACO3-440C-25dpa-10He-081412 & HT9: 84425 & 440 & 25 & 25 & 10 & $8 / 14 / 2012$ \\
\hline ACO3-440C-188dpa-10He-091312 & HT9: 84425 & 440 & 188 & 188 & 10 & $9 / 13 / 2012$ \\
\hline ACO3-480C-188dpa-10He-111312 & HT9: 84425 & 480 & 188 & 188 & 10 & $11 / 13 / 2012$ \\
\hline ACO3-400C-250dpa-10He-011913 & HT9: 84425 & 400 & 250 & 250 & 10 & $1 / 19 / 2013$ \\
\hline ACO3-460C-188dpa-10He-062413 & HT9: 84425 & 460 & 188 & 188 & 10 & $6 / 24 / 2013$ \\
\hline ACO3-440C-50dpa-10He-090413 & HT9: 84425 & 440 & 50 & 50 & 10 & $9 / 4 / 2013$ \\
\hline ACO3-460C-188dpa-10He-111313 & HT9: 84425 & 460 & 188 & 375 & 10 & $11 / 13 / 2013$ \\
\hline ACO3-460C-130dpa-10He-092214 & HT9: 84425 & 460 & 130 & 130 & 10 & $9 / 22 / 2014$ \\
\hline ACO3-460C-75dpa-10He-102914 & HT9: 84425 & 460 & 75 & 75 & 10 & $10 / 29 / 2014$ \\
\hline ACO3-460C-120dpa-10He-120814 & HT9: 84425 & 460 & 120 & 250 & 10 & $12 / 8 / 2014$ \\
\hline ACO3-460C-100dpa-10He-011215 & HT9: 84425 & 460 & 100 & 350 & 10 & $1 / 12 / 2015$ \\
\hline ACO3-460C-100dpa-10He-021615 & HT9: 84425 & 460 & 100 & 450 & 10 & $2 / 16 / 2015$ \\
\hline ACO3-460C-100dpa-10He-030515 & HT9: 84425 & 460 & 100 & 550 & 10 & $3 / 5 / 2015$ \\
\hline ACO3-460C-100dpa-10He-050715 & HT9: 84425 & 460 & 100 & 650 & 10 & $5 / 7 / 2015$ \\
\hline
\end{tabular}


Table 4.4: Summary of irradiation data from $5 \mathrm{MeV} \mathrm{Fe}^{++}$irradiations referred to in this thesis.

\begin{tabular}{|c|c|c|c|c|c|c|c|c|c|c|c|}
\hline \multirow[t]{2}{*}{$\begin{array}{c}\text { Irradiation } \\
\text { Date }\end{array}$} & \multirow[t]{2}{*}{$\begin{array}{c}\text { Temperature } \\
\left({ }^{\circ} \mathrm{C}\right)\end{array}$} & \multirow{2}{*}{$\begin{array}{c}\text { Incremental } \\
\text { Damage } \\
\text { (dpa) }\end{array}$} & \multirow{2}{*}{$\begin{array}{c}\text { Final } \\
\text { Damage } \\
\text { (dpa) }\end{array}$} & \multirow{2}{*}{$\begin{array}{c}\text { Damage } \\
\text { Rate }\left(10^{-3}\right. \\
\mathrm{dpa} / \mathrm{s})\end{array}$} & \multirow[t]{2}{*}{$\begin{array}{c}\text { Stage } \\
\text { Configuration }\end{array}$} & \multirow[t]{2}{*}{ Apertures } & \multicolumn{4}{|c|}{$\begin{array}{l}\text { Thermocouple at Calibration } \\
\left({ }^{\circ} \mathrm{C}\right)\end{array}$} & \multirow{2}{*}{$\begin{array}{c}\text { Pressure at } \\
\text { Start } \\
\left(10^{-8} \text { torr }\right)\end{array}$} \\
\hline & & & & & & & 1 & 2 & 3 & 4 & \\
\hline $3 / 28 / 2012$ & $440.5 \pm 8.8$ & 140.1 & 140.1 & 0.40 & $\mathrm{Cu}+\mathrm{In}^{1}$ & Stage & 444 & n. $\mathrm{r}^{2}$ & 440 & 441 & 9.7 \\
\hline $8 / 14 / 2012$ & $443.8 \pm 5.7$ & 25.5 & 25.5 & 0.50 & $\mathrm{Cu}+\mathrm{In}$ & Stage & 443 & 419 & n. $\mathrm{r}$ & 440 & 5.6 \\
\hline $9 / 13 / 2012$ & $440.8 \pm 7.3$ & 186.8 & 186.8 & 0.27 & $\mathrm{Cu}+\mathrm{In}$ & Stage & 444 & 438 & 440 & n. $r$ & 5.3 \\
\hline $11 / 13 / 2012$ & $480.0 \pm 5.9$ & 187.5 & 187.5 & 0.53 & $\mathrm{Cu}+\mathrm{InF}^{3}$ & Stage & n.r. & 483 & 480 & 481 & 5.3 \\
\hline $1 / 19 / 2013$ & $401.3 \pm 7.2$ & 250.1 & 250.1 & 0.40 & $\mathrm{Cu}+\mathrm{InF}$ & Stage & 404 & 395 & 402 & n. $r$ & 8.8 \\
\hline $6 / 24 / 2013$ & $460.7 \pm 7.4$ & 187.5 & 187.5 & 0.35 & $\mathrm{Ni}+\mathrm{CuF}^{4}$ & Stage & 456 & 459 & 458 & 460 & 5.6 \\
\hline $9 / 4 / 2013$ & $442.0 \pm 10.7$ & 50.0 & 50.0 & 0.40 & $\mathrm{Ni}+\mathrm{CuF}$ & Stage & 443 & 442 & 440 & 439 & 9.9 \\
\hline $11 / 13 / 2013$ & $461.4 \pm 7.5$ & 187.5 & 375 & 0.34 & $\mathrm{Ni}+\mathrm{CuF}$ & Stage & n. $\mathrm{r}$ & 460 & 458 & 463 & 8.7 \\
\hline $9 / 22 / 2014$ & $460.2 \pm 4.4$ & 127.6 & 127.6 & 0.57 & $\mathrm{Ni}+\mathrm{CuF}$ & Slit & 458 & 460 & 461 & n. $r$ & 5.7 \\
\hline $10 / 29 / 2014$ & $460.2 \pm 7.7$ & 75.0 & 75.0 & 0.37 & $\mathrm{Ni}+\mathrm{CuF}$ & Slit & n. $\mathrm{r}$ & 462 & 459 & 461 & 5.9 \\
\hline $12 / 8 / 2014$ & $462.4 \pm 3.9$ & 122.4 & 250.0 & 11.5 & $\mathrm{Ni}+\mathrm{CuF}$ & Slit & 456 & 460 & 458 & n. $r$ & 4.7 \\
\hline $1 / 12 / 2015$ & $462.2 \pm 5.2$ & 100.0 & 350.0 & 0.93 & $\mathrm{Ni}+\mathrm{CuF}$ & Slit & 456 & 460 & 458 & 459 & 5.9 \\
\hline $2 / 16 / 2015$ & $461.1 \pm 5.8$ & 100.0 & 450.0 & 11.9 & $\mathrm{Ni}+\mathrm{CuF}$ & Slit & 457 & 460 & n. $r$ & 459 & 4.9 \\
\hline $3 / 5 / 2015$ & $461.6 \pm 3.9$ & 100.0 & 550.0 & 0.63 & $\mathrm{Ni}+\mathrm{CuF}$ & Slit & 457 & 459 & n. $r$ & 460 & 4.8 \\
\hline $5 / 7 / 2015$ & $461.9 \pm 3.1$ & 100.0 & 650.0 & 085 & $\mathrm{Ni}+\mathrm{CuF}$ & Slit & n. $r$ & 463 & 459 & 460 & 2.9 \\
\hline
\end{tabular}

${ }^{1} \mathrm{Cu}+\mathrm{In}$ : Copper Stage with Indium

${ }^{2}$ N.R.: Not recorded

${ }^{3} \mathrm{Cu}+\mathrm{InF}$ : Copper Stage with Indiphoil

${ }^{4} \mathrm{Ni}+\mathrm{CuF}$ : Nickel Stage with Cooper foil 


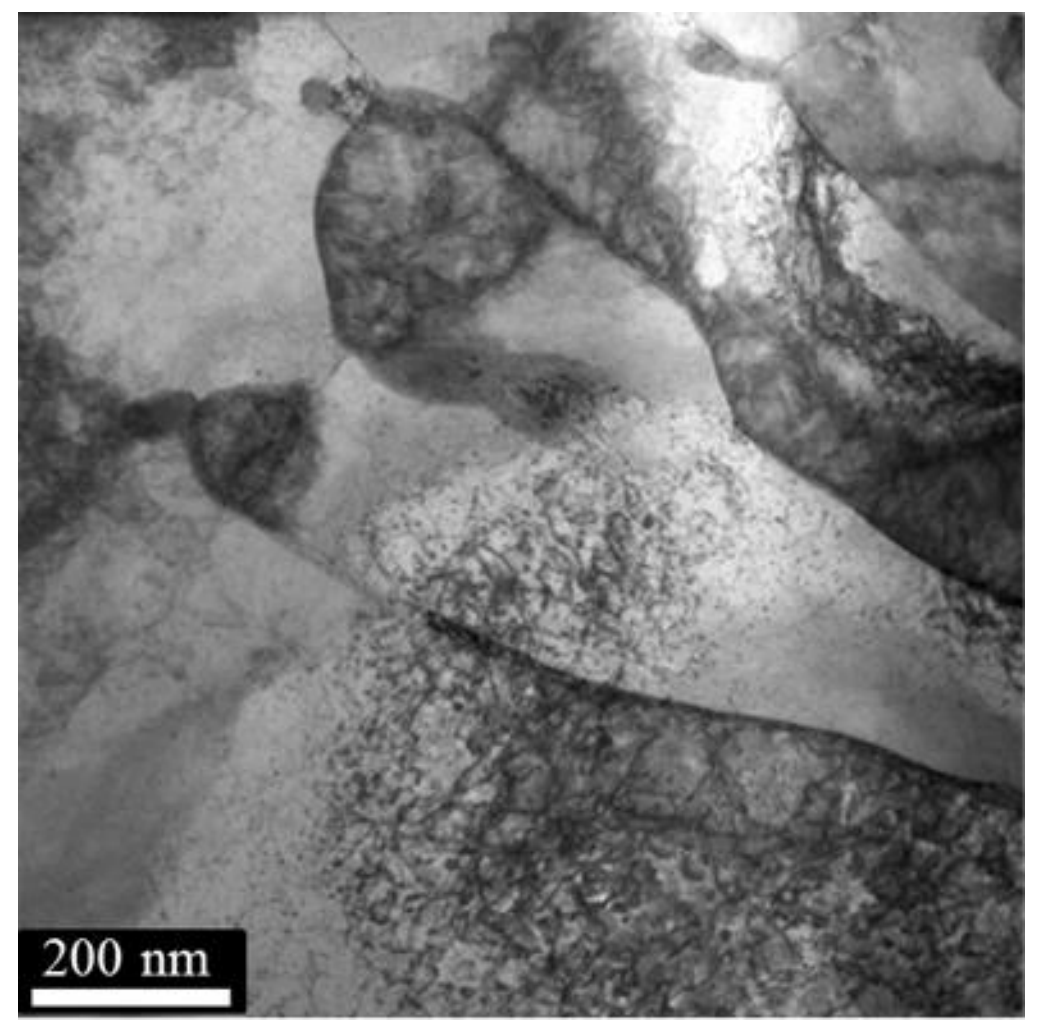

Figure 4.1: Unirradiated HT9 (Heat 84425) imaged in STEM-BF. Sample made via FIB liftout method 


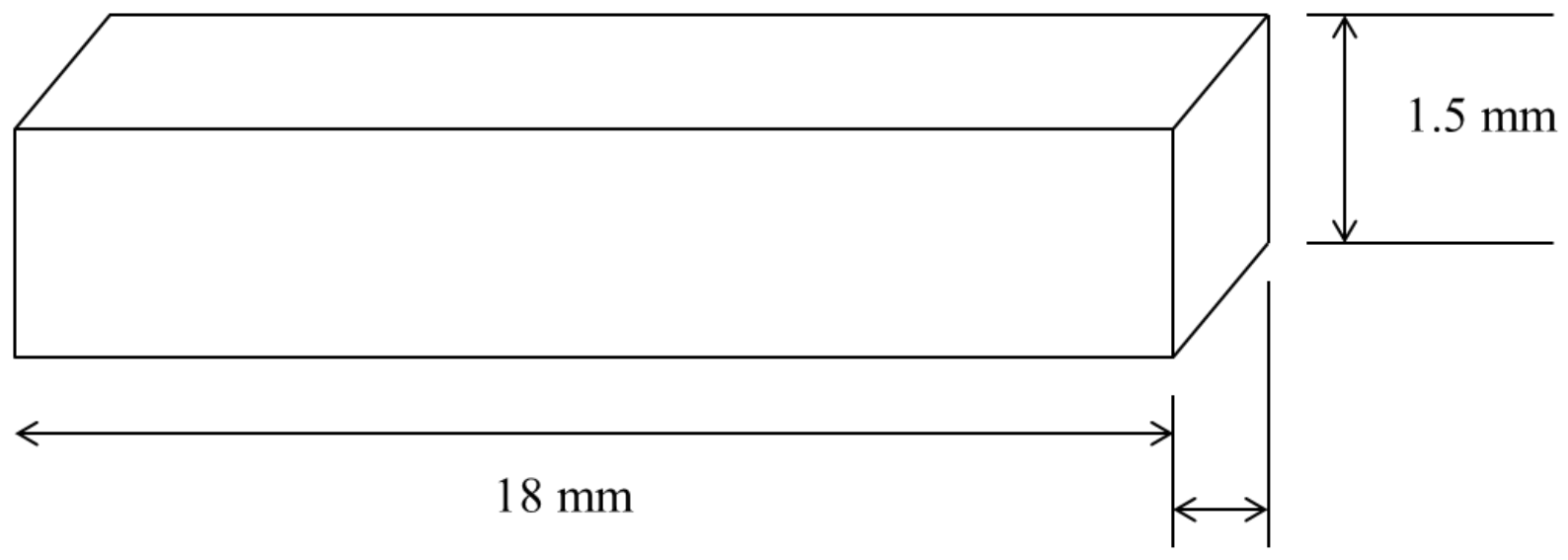

$1.4 \mathrm{~mm}$

Figure 4.2 Sample geometry following EDM. Only one face will be irradiated. 


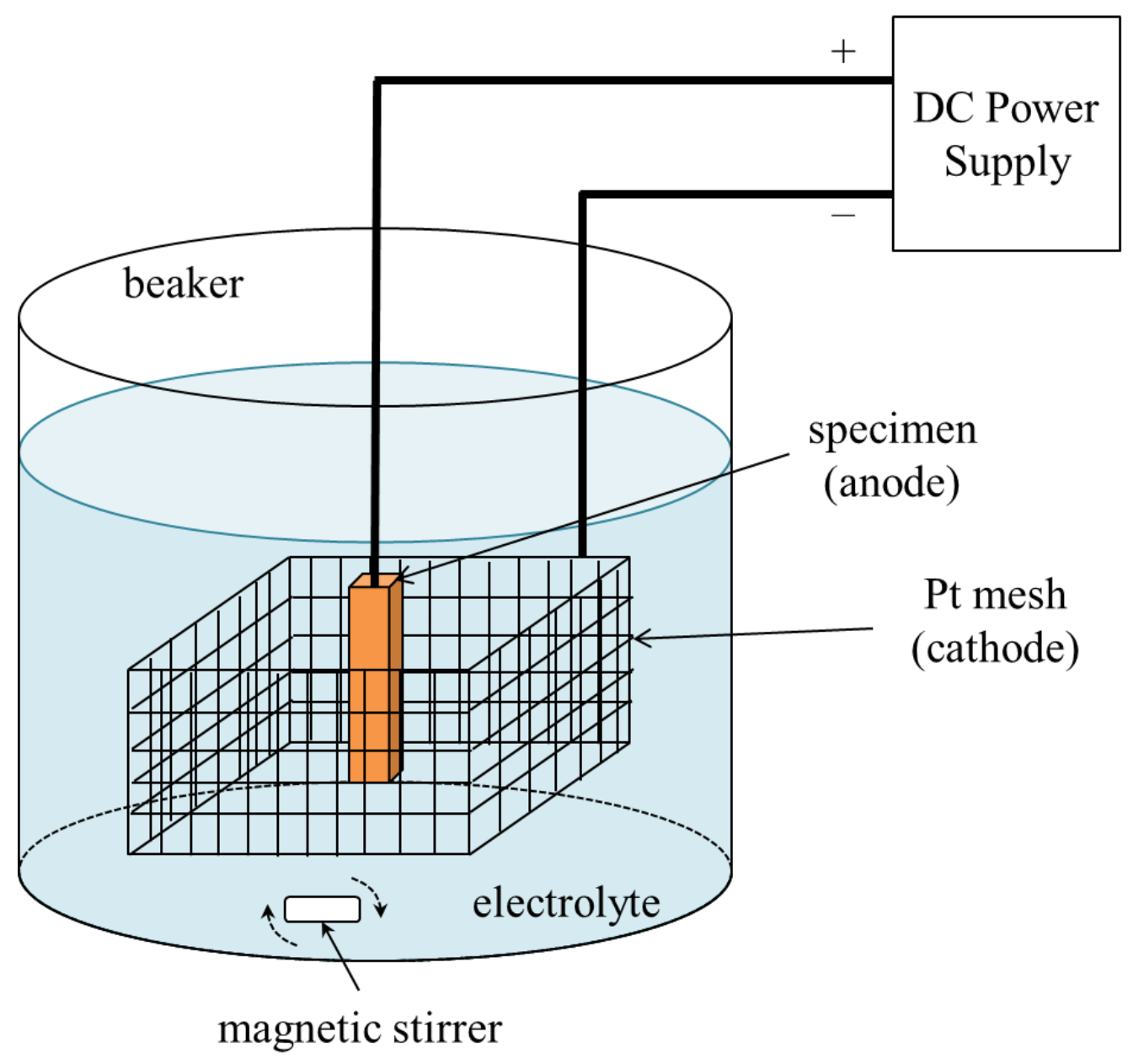

Figure 4.3: Assembly used for electropolishing of samples. Samples polished in $90 \%$ methanol/10\% perchloric acid solution. 


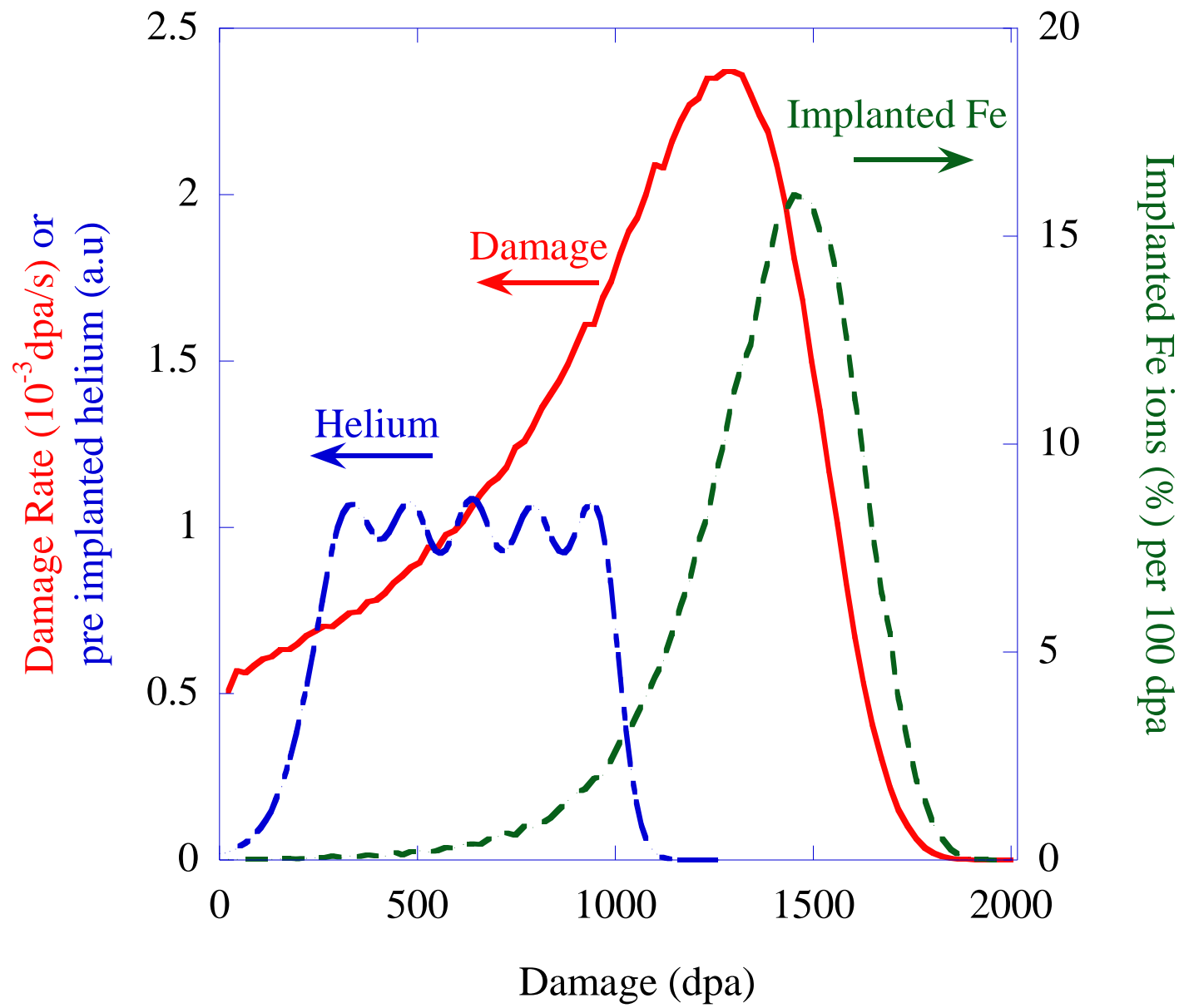

Figure 4.4: The helium profile (blue) is overlaid on the damage rate (red) and implanted ion concentration (green) for $5 \mathrm{MeV} \mathrm{Fe}^{++}$in $\mathrm{Fe}-12 \mathrm{Cr}$ calculated from SRIM [64]. 


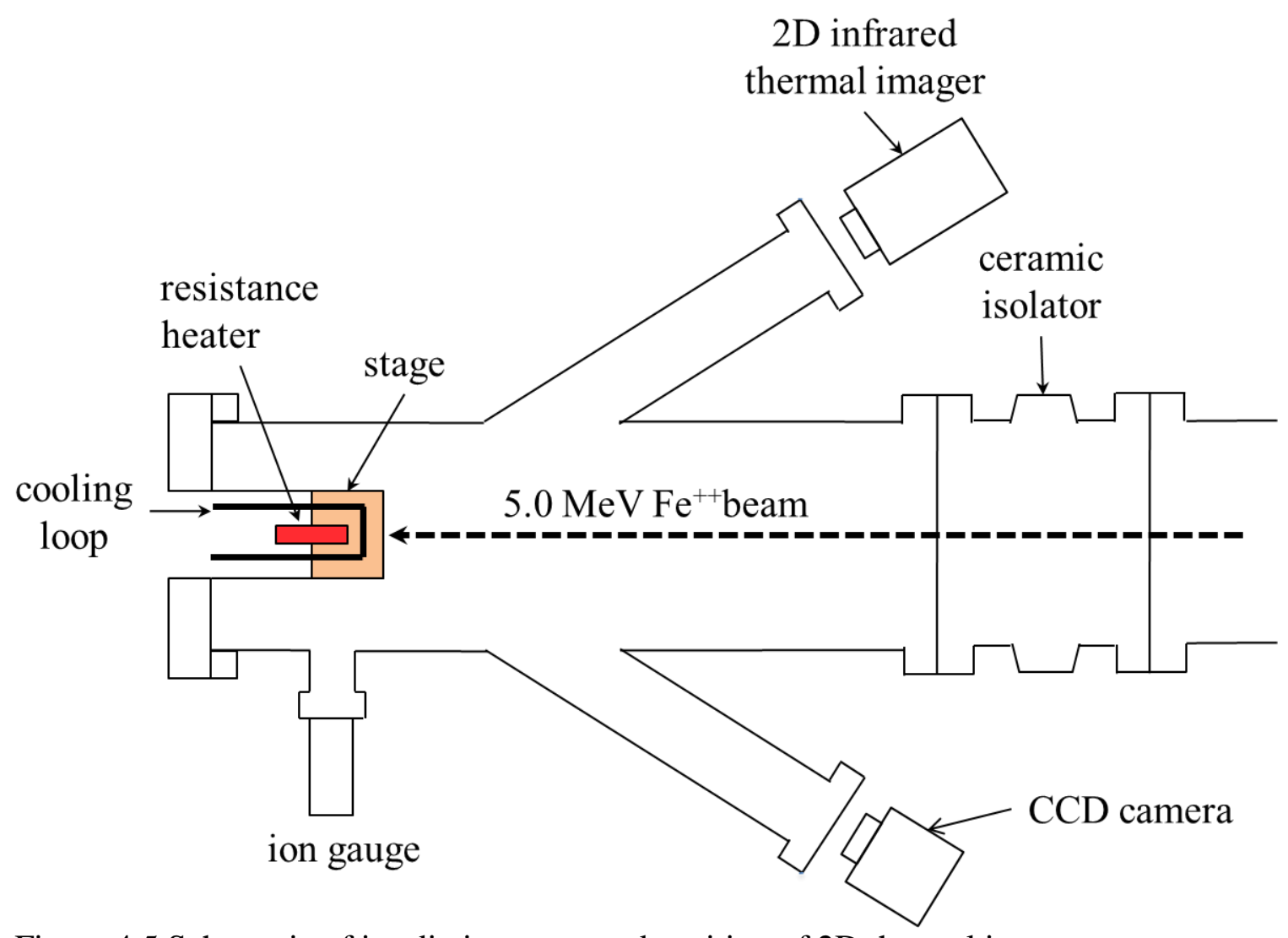

Figure 4.5 Schematic of irradiation stage and position of 2D thermal imager on beamline. 
(a)

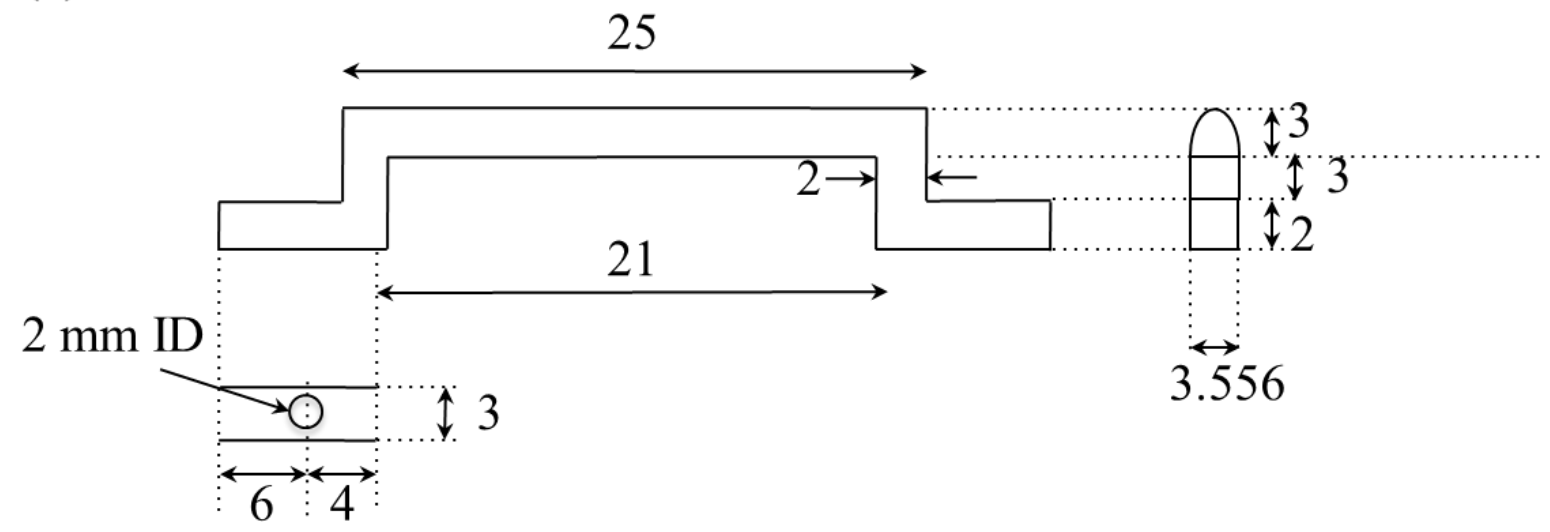

(b) set screws hold-down bar

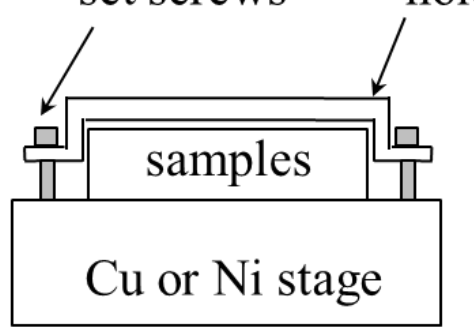

Figure 4.6: a) Schematic of hold-down bars and b) view of hold-down bars on the stage [32]. 


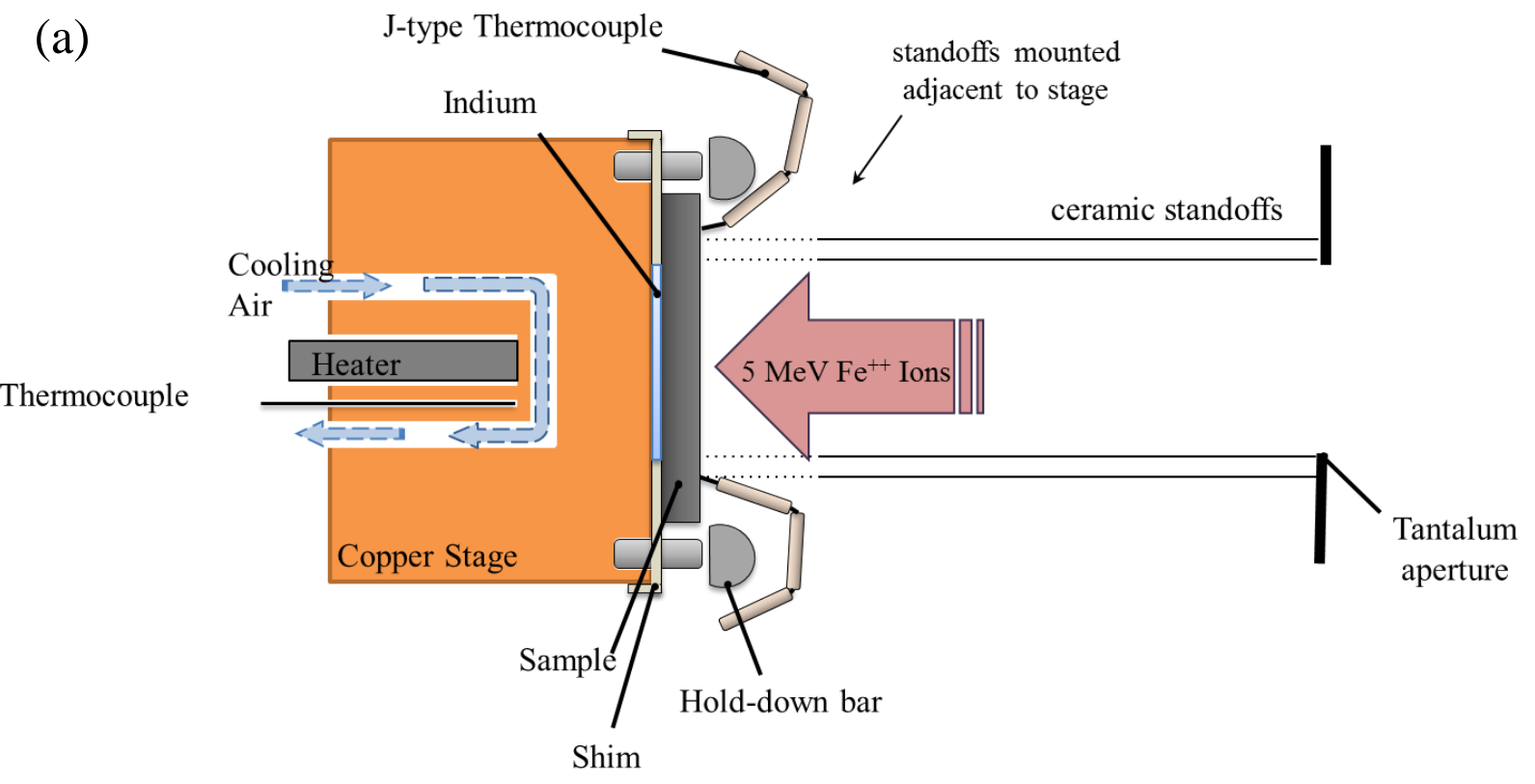

(b)

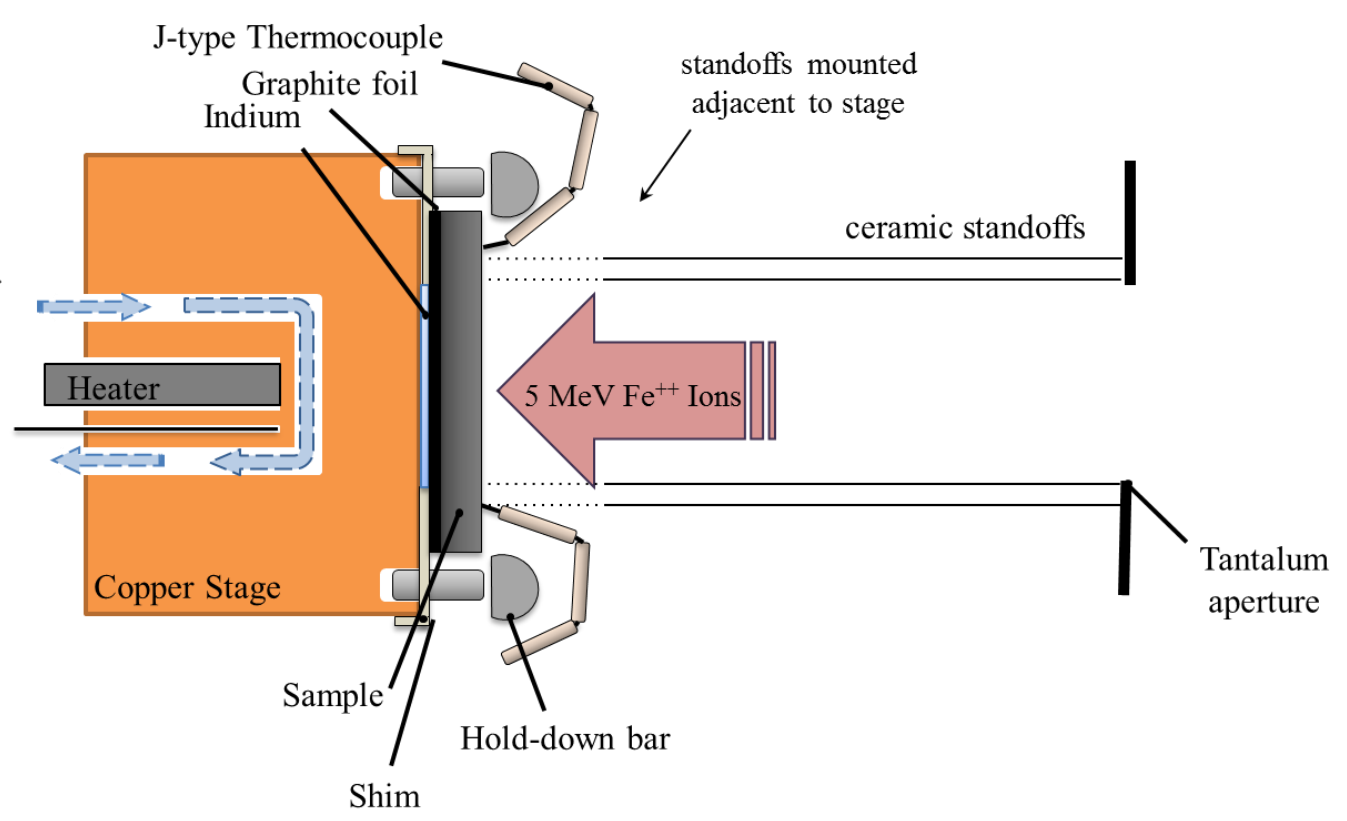

Figure 4.7: a) "First generation stage" schematic where indium is used to ensure good thermal contact and apertures were attached to the stage. b) "Second generation stage" schematic where a combination of indium and graphite foil were utilized to ensure good thermal conductivity and leak protection. 


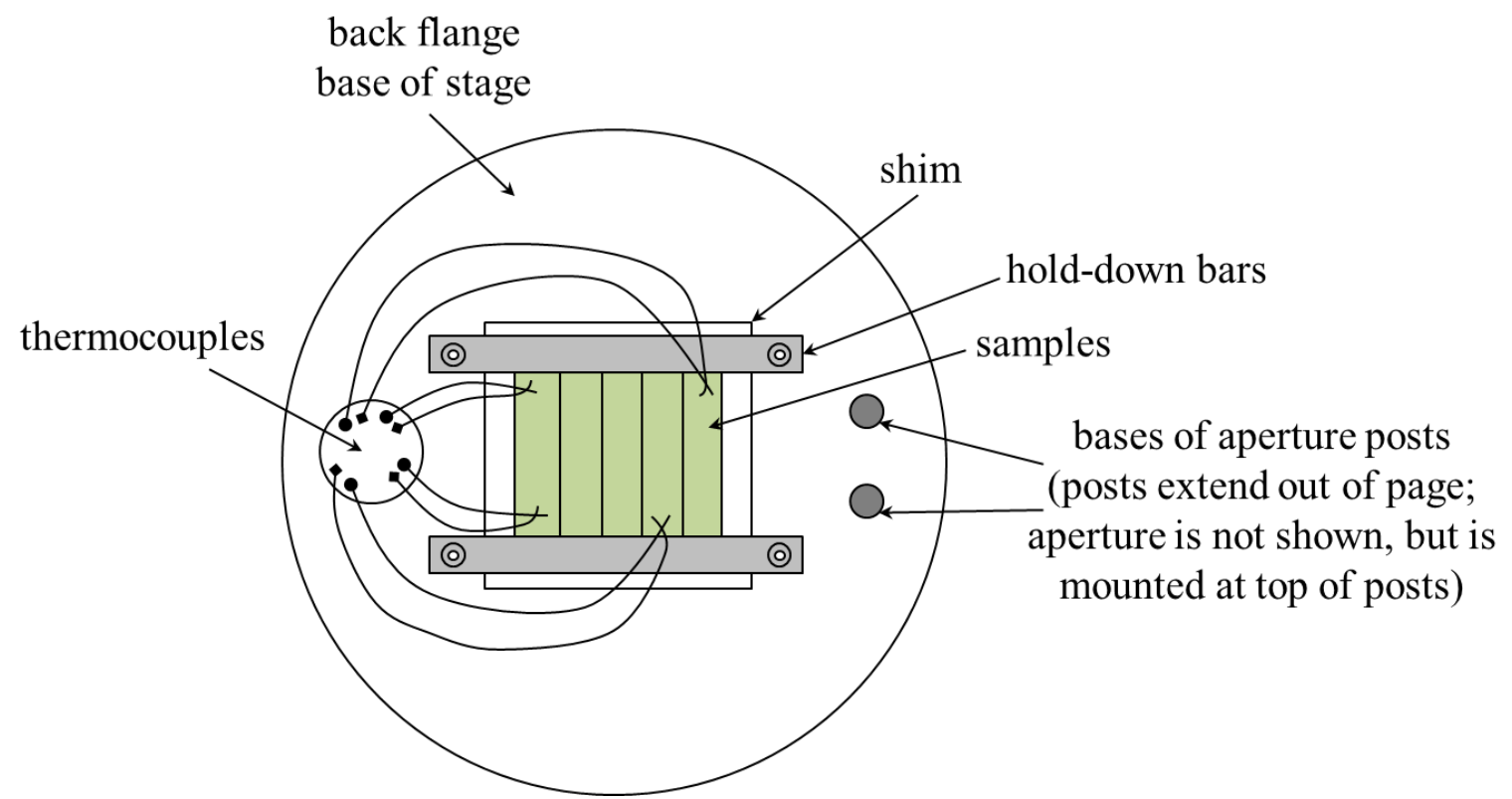

Figure 4.8: Stage design as viewed from the top [32]. 


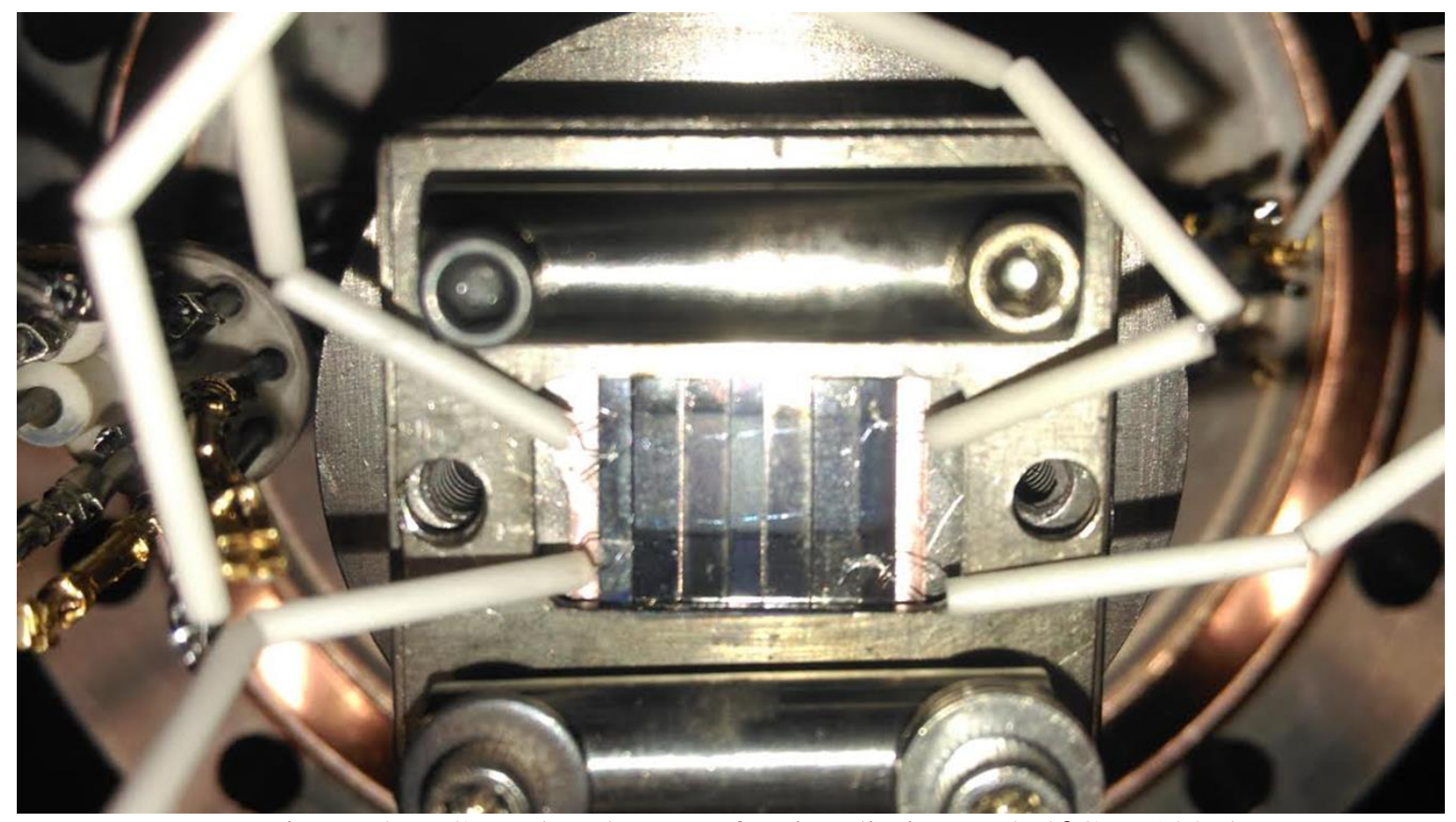

Figure 4.9: Completed stage after irradiation at $460^{\circ} \mathrm{C}$ to $130 \mathrm{dpa}$. 


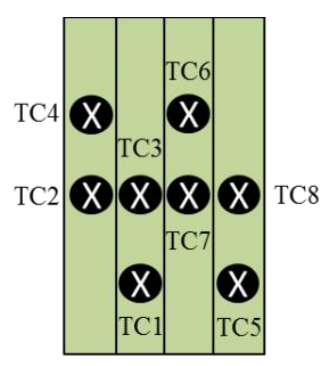

(a)

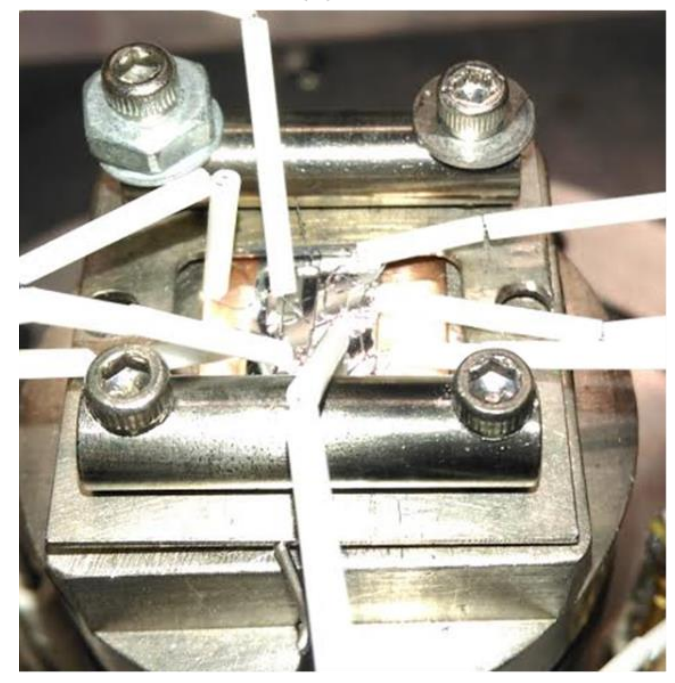

(b)

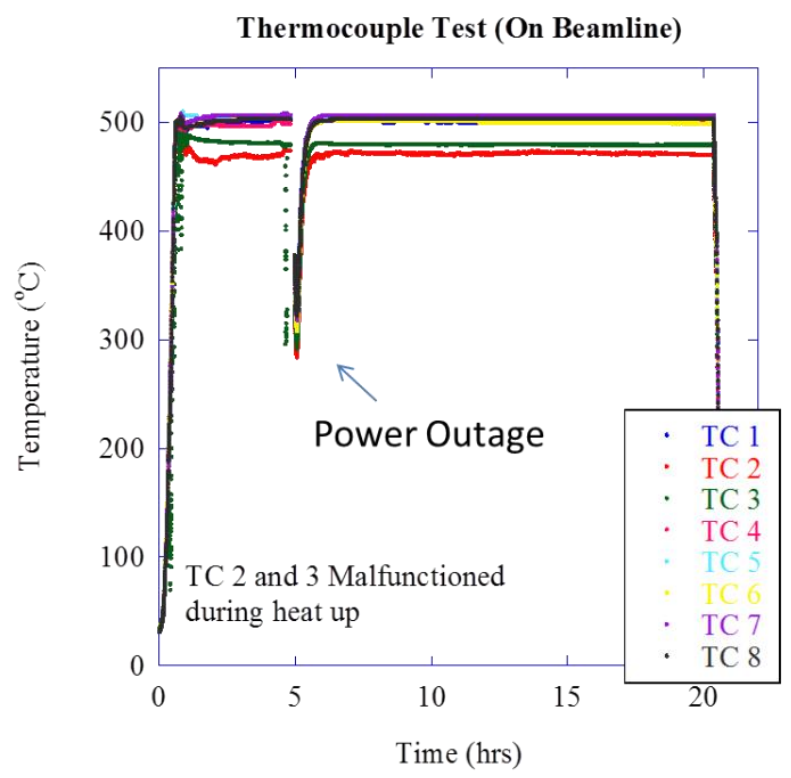

(c)

Figure 4.10: a) Schematic and b) stage image for temperature uniformity test. c) Results of the 24-hour test on the beamline. 


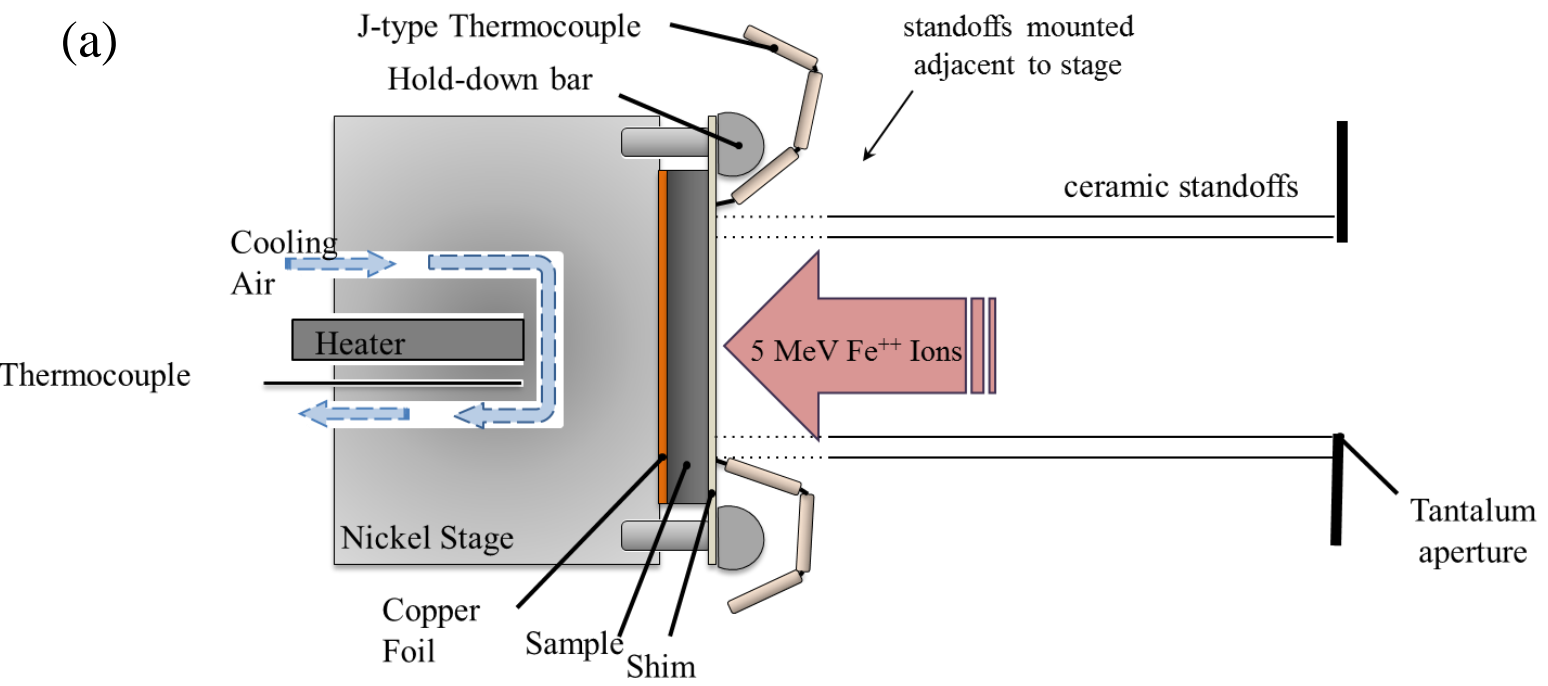

(b)

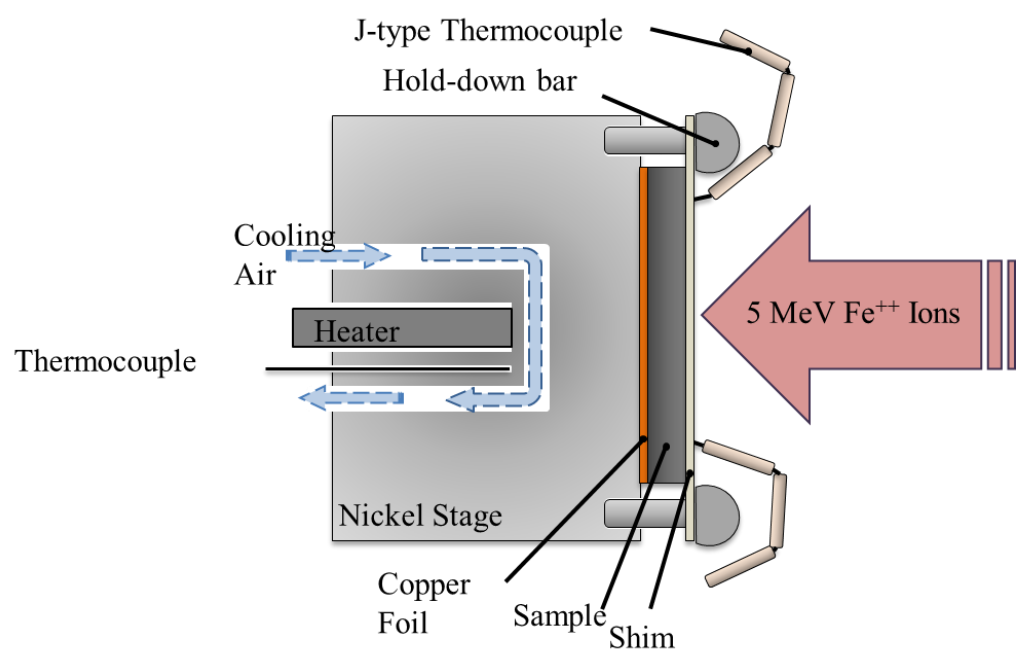

Figure 4.11: Schematic of "third generation" stage configuration with a) stage and b) slit apertures. Copper foil provided sufficient thermal contact and eliminated indium leaks. Slit apertures eliminated danger of aperture shift, which could occur during stage loading onto beamline. 


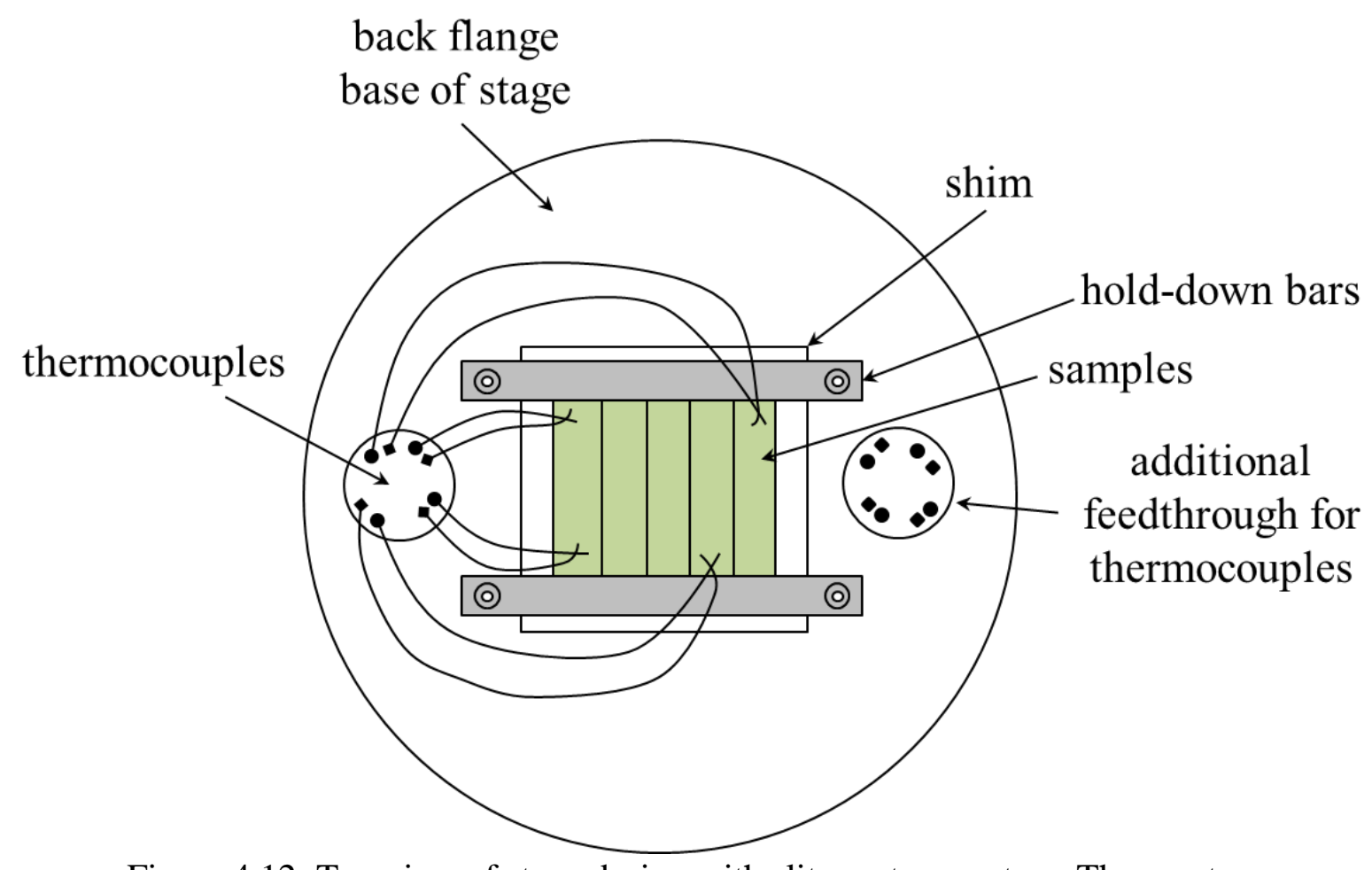

Figure 4.12: Top view of stage design with slit aperture system. The aperture feedthrough was replaced with an additional thermocouple feedthrough. 


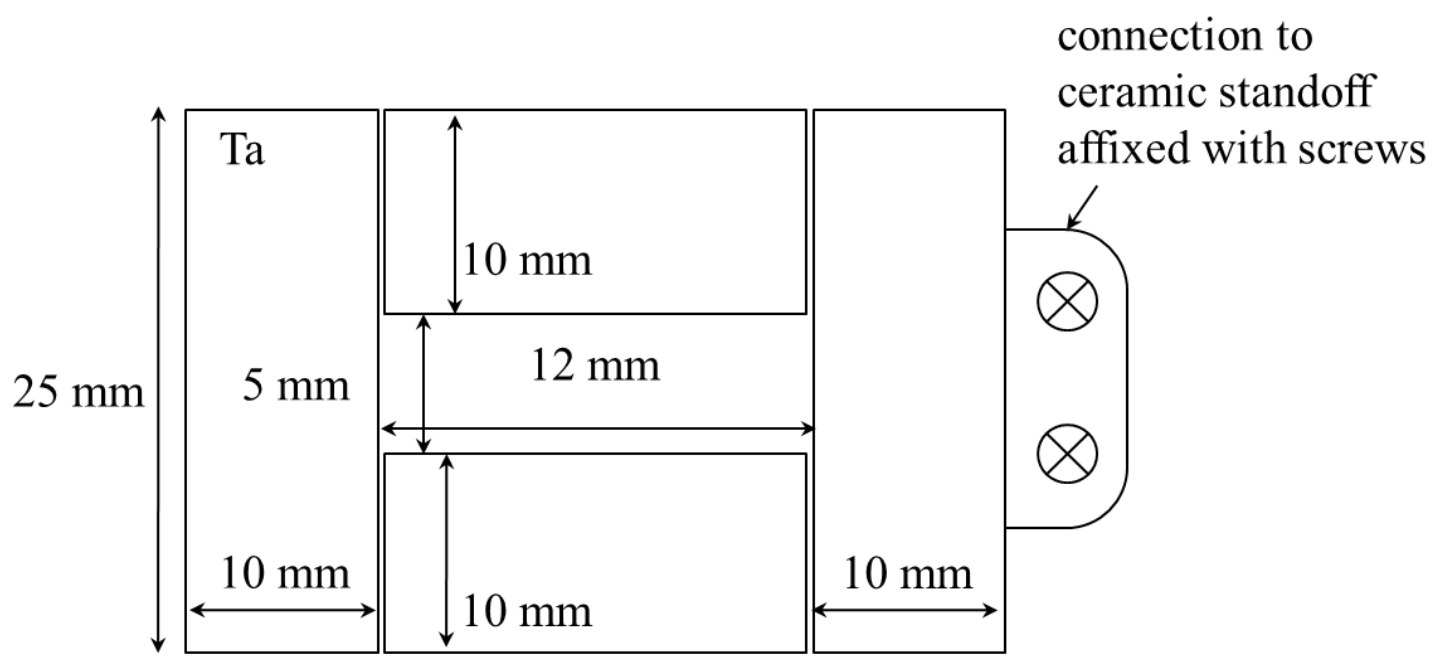

Figure 4.13: Schematic of tantalum aperture system. 


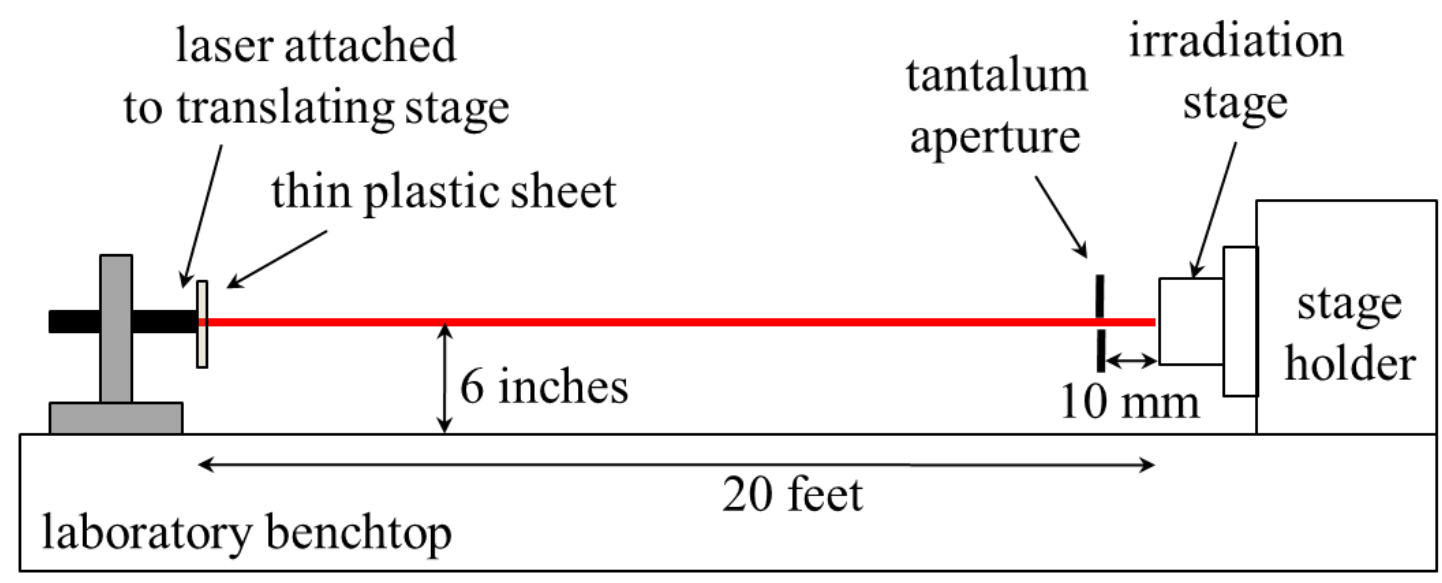

Figure 4.14: Schematic of benchtop alignment setup of stage apertures [32]. 


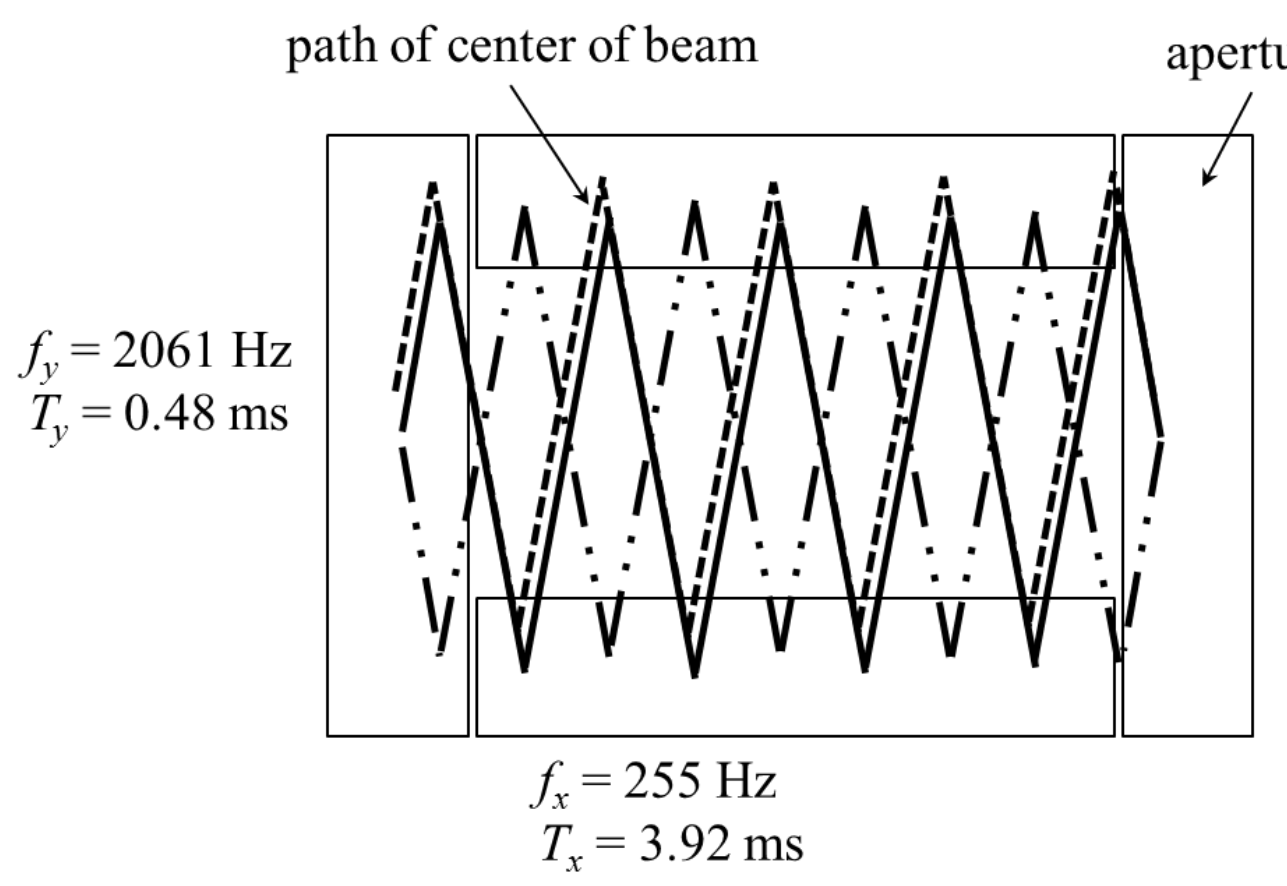

Figure 4.15: Path of raster scanned beam center across samples over irradiated area $[13,32]$. 
(a)

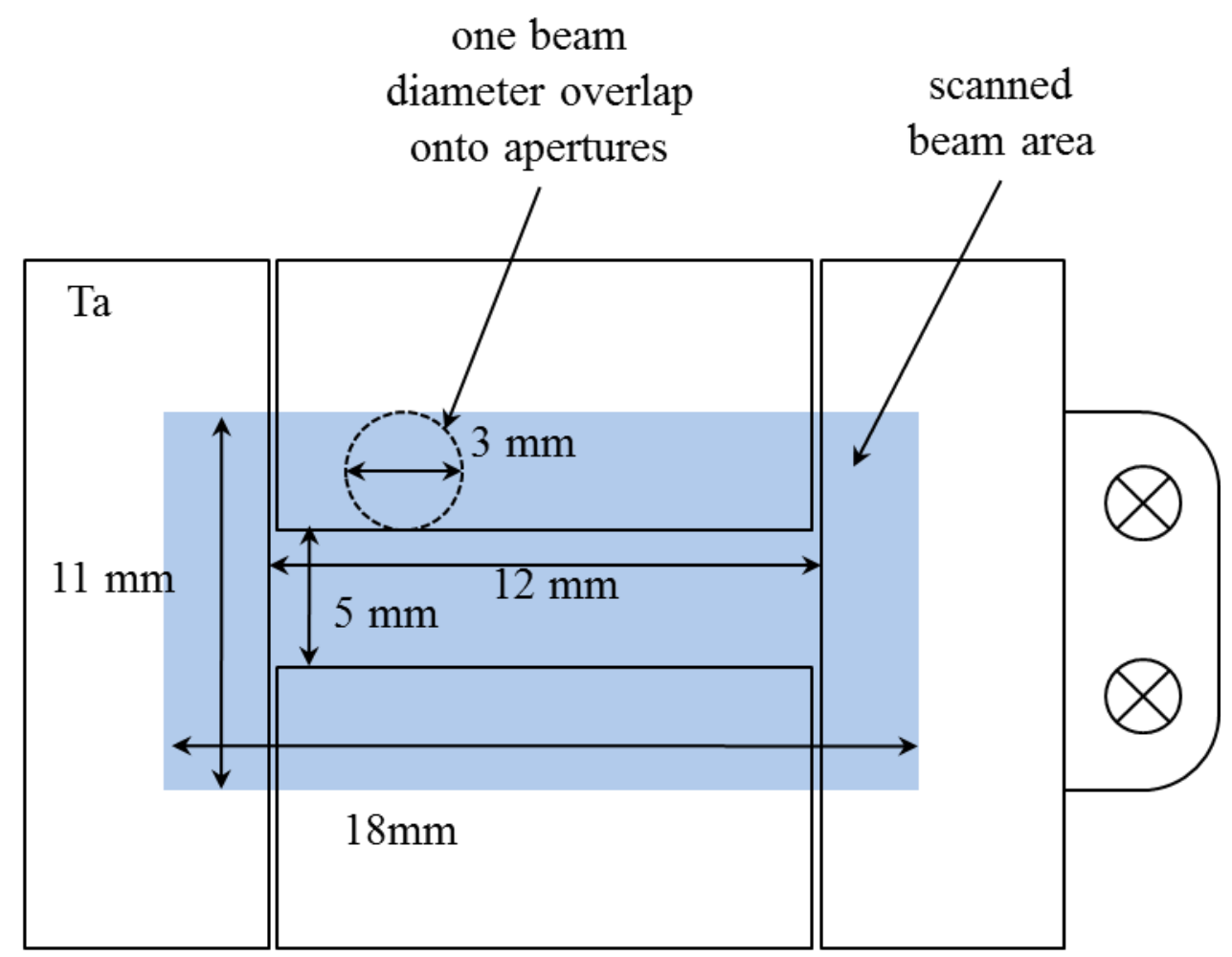

(b)

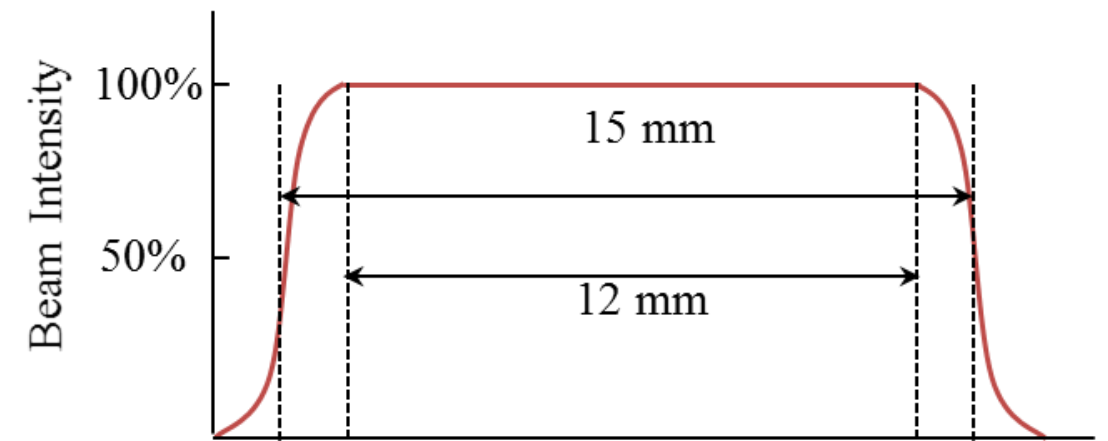

Figure 4.16: a) Schematic of raster scanned beam area after beam sizing procedure with ceramic. b) Beam intensity profile due to Gaussian beam shape 


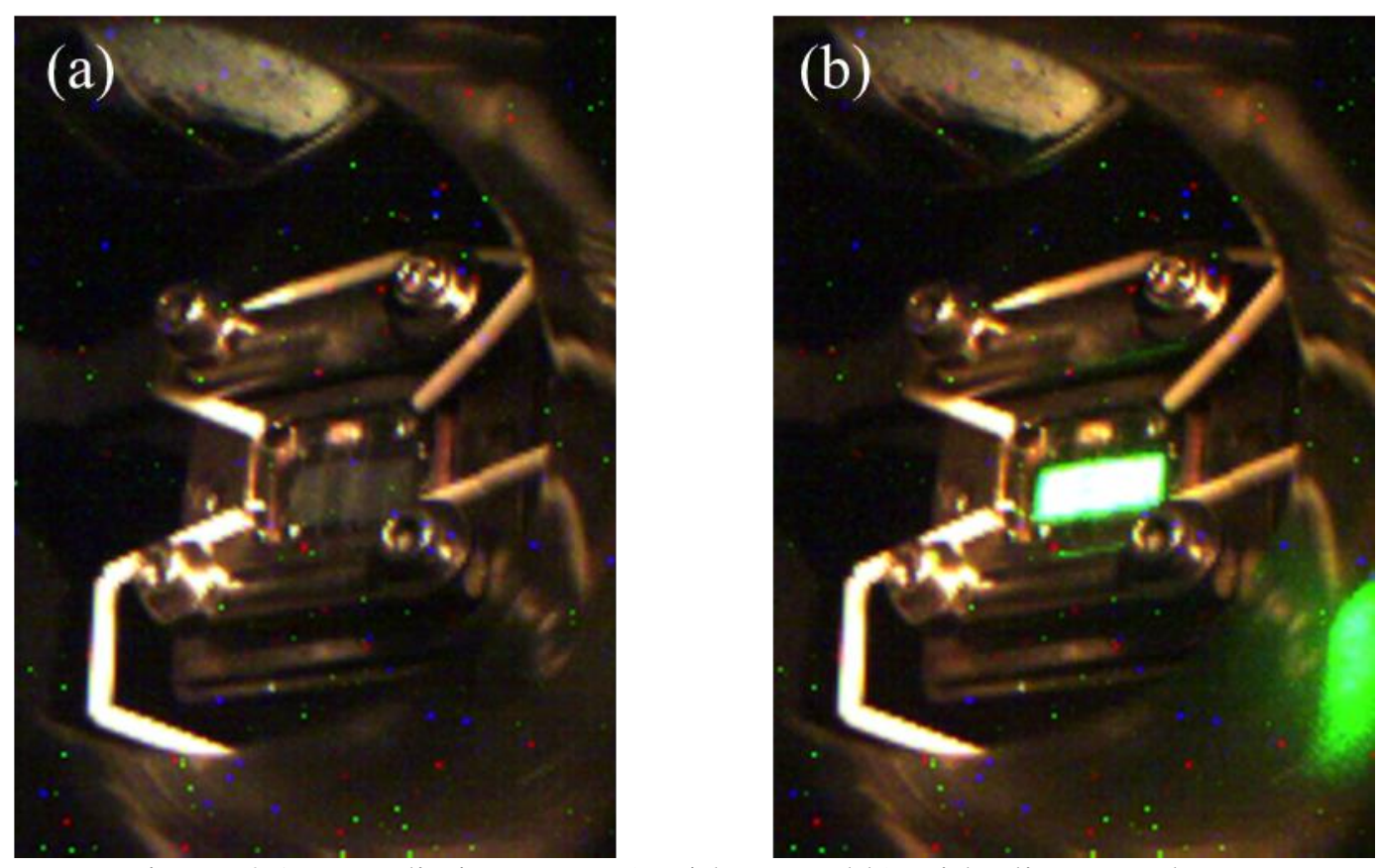

Figure 4.17: Irradiation stage a) without and b) with alignment laser. 


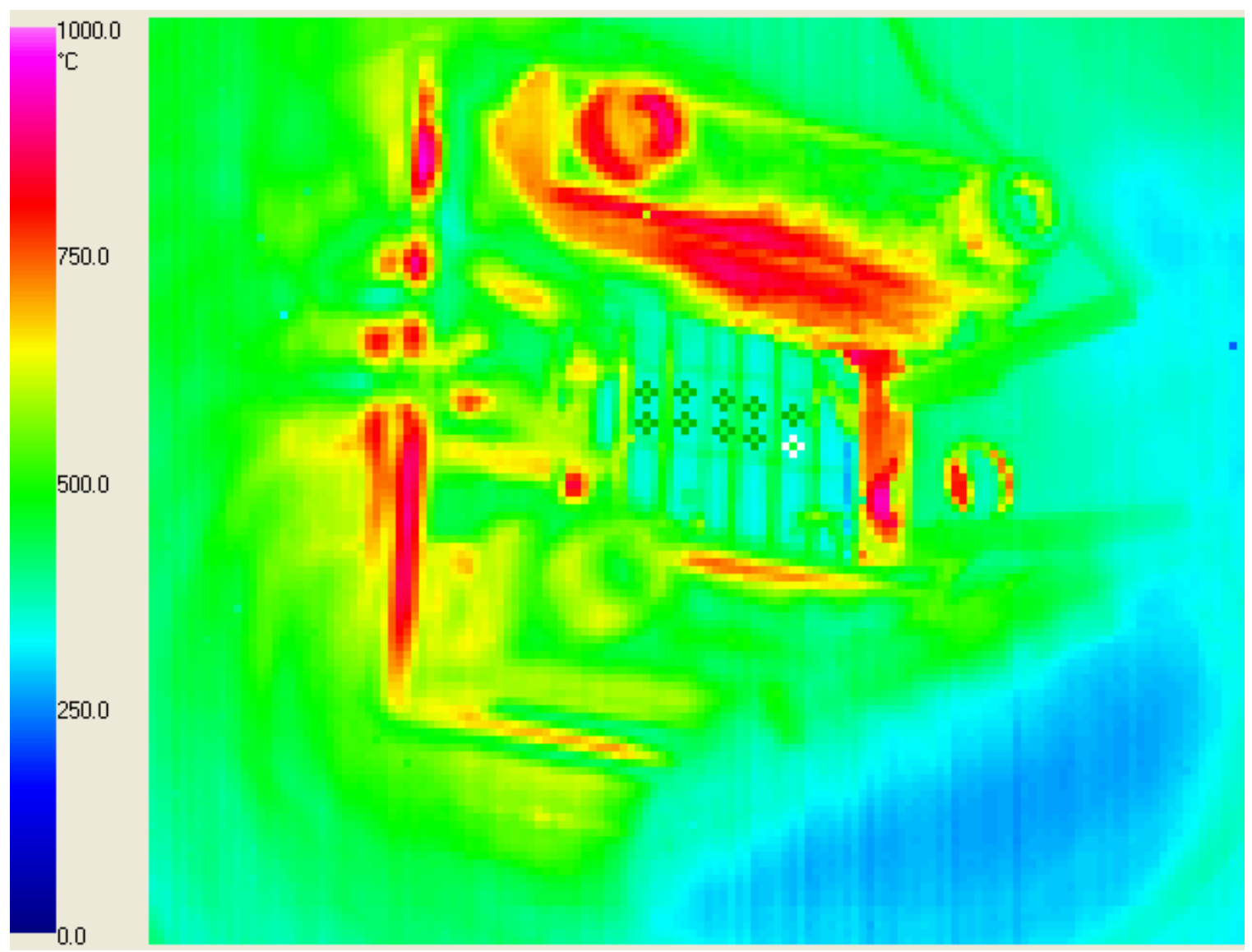

Figure 4.18: 2D thermal image from $460^{\circ} \mathrm{C}, 130 \mathrm{dpa}$ irradiation. Two AOIs per samples were placed on 5 samples with two guide bars bookending the samples. 


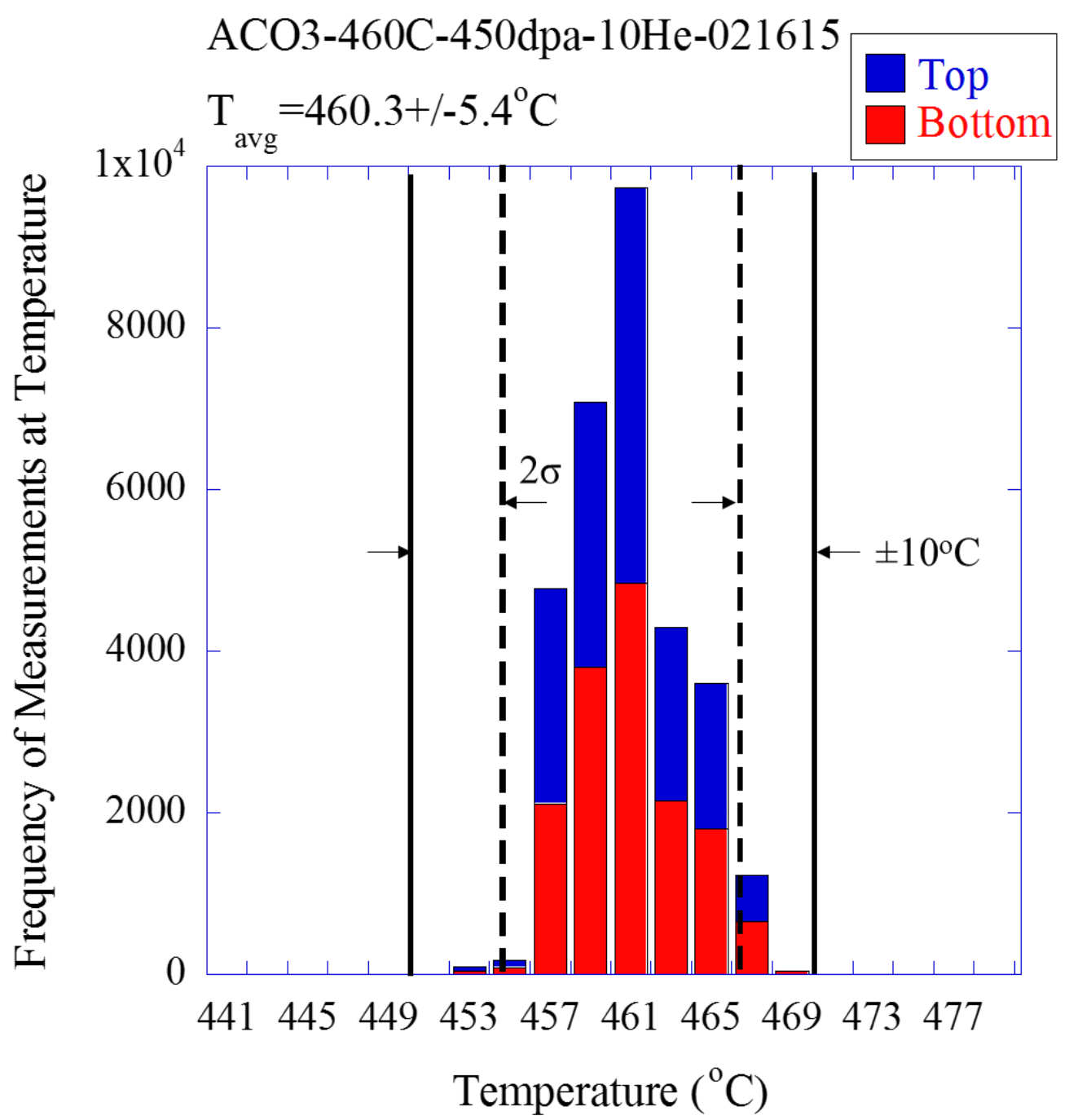

Figure 4.19: Example temperature histogram for $5 \mathrm{MeV} \mathrm{Fe}^{++}$irradiation of ACO3-460C-450dpa-10He-021615 showing approximately normal distribution in both areas of interest (top and bottom) on the sample and having $2 \sigma$ well within $\pm 10^{\circ} \mathrm{C}$. 


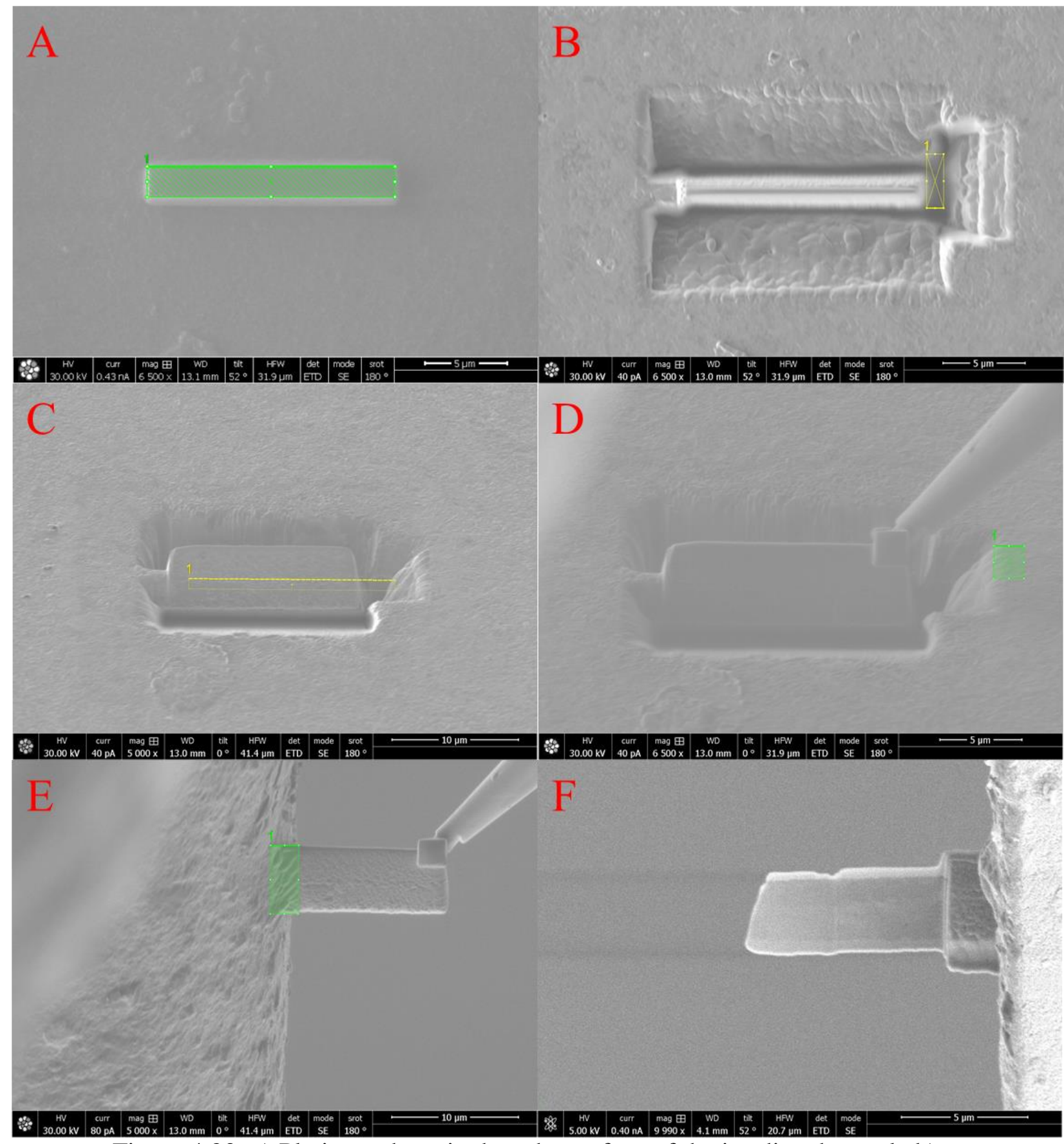

Figure 4.20: a) Platinum deposited on the surface of the irradiated sample b) trenching around the platinum protected surface c) under cut of the samples d) attaching the needle to the lamella using platinum e) attaching the lamella to the copper grid $f$ ) thinning using the Ga beam. 


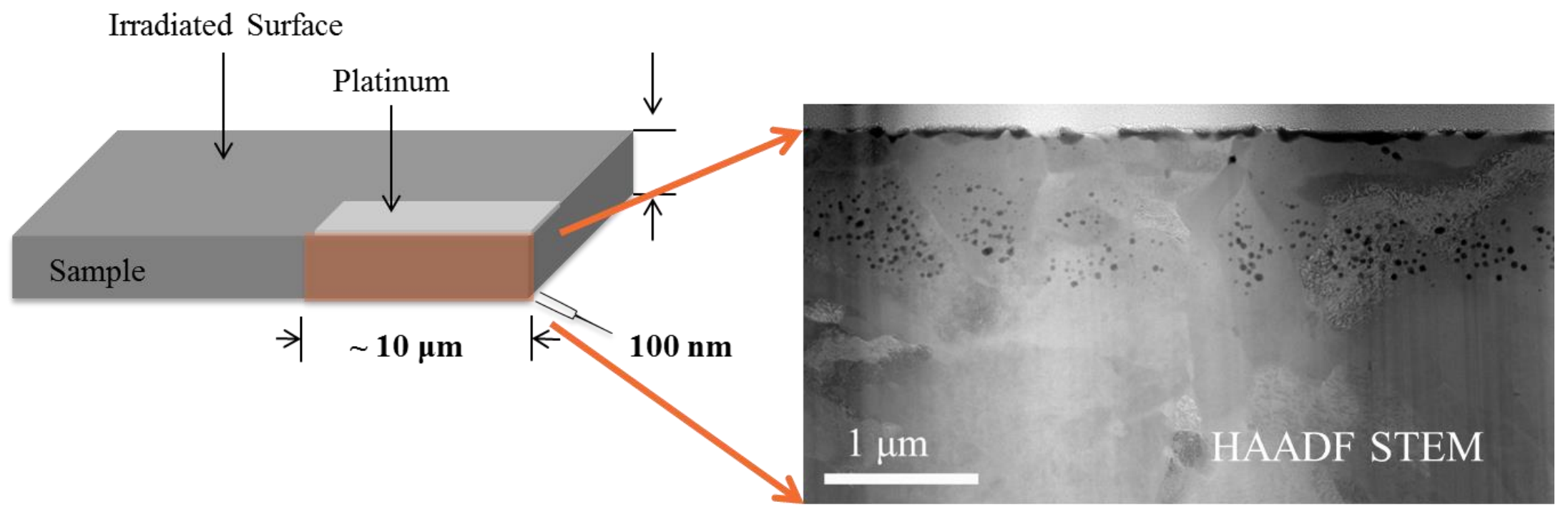

Figure 4.21: Schematic of cross-sectional liftout and resulting image in HAADF STEM. The PT layer and irradiated surface are at the top of the image. Voids are dark circles. Sample irradiated at $460^{\circ} \mathrm{C}$ to $188 \mathrm{dpa}$ with $10 \mathrm{appm} \mathrm{He}$ preimplanted. 


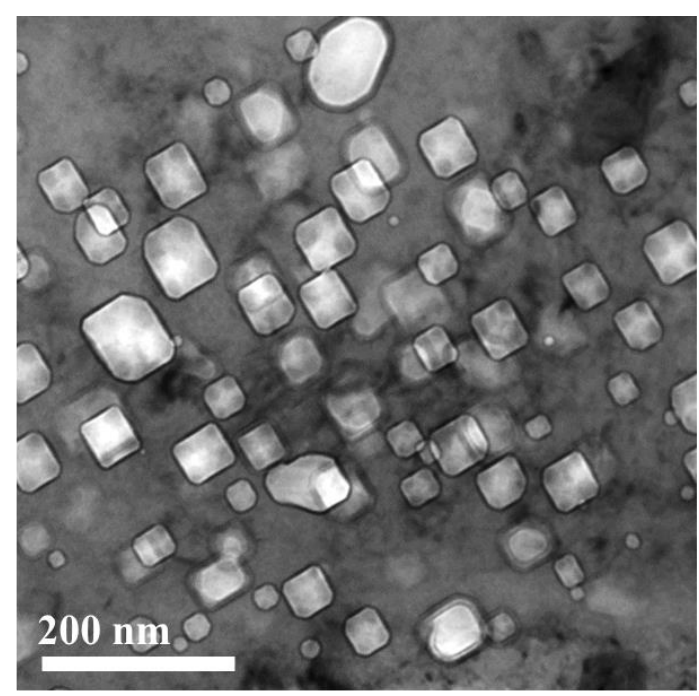

(a)

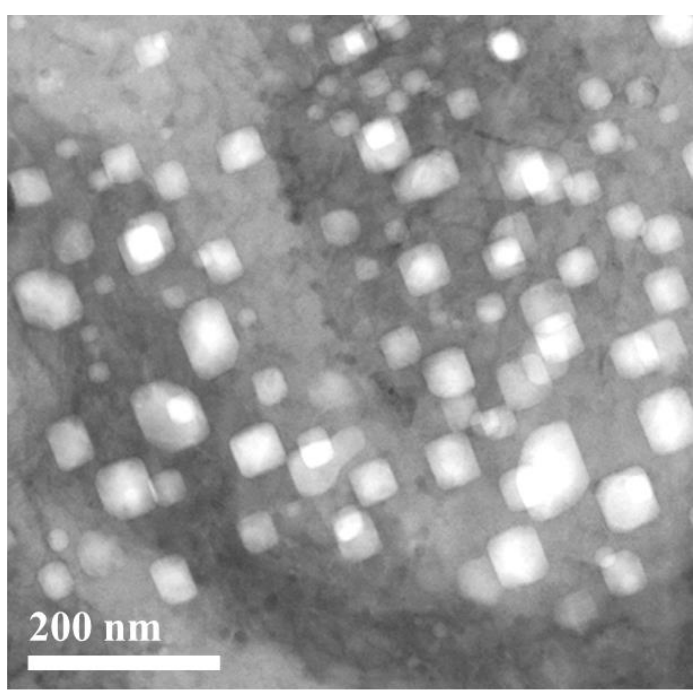

(b)

Figure 4.22: Conventional BF (a) and STEM BF (b) images of voids in HT9 irradiated with $5 \mathrm{MeV} \mathrm{Fe}^{++}$at $460^{\circ} \mathrm{C}$ to 375 dpa with 10 appm He preimplanted. Note that all voids are in focus in (b) while conventional images show some voids in over or under focus. 


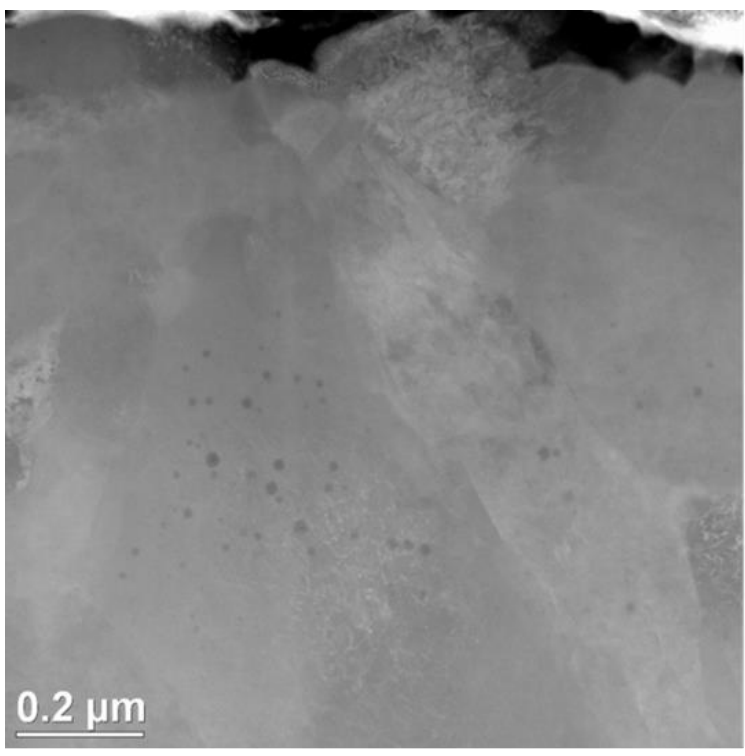

(a)

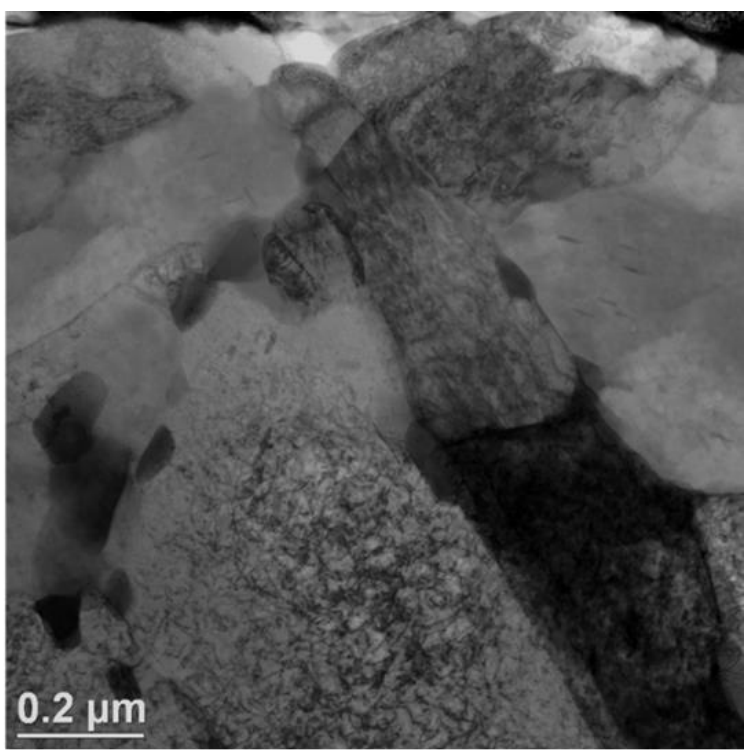

(b)

Figure 4.23: STEM ADF (a) and BF (b) images of HT9 irradiated with $5 \mathrm{MeV}$ $\mathrm{Fe}^{++}$at $440^{\circ} \mathrm{C}$ to $140 \mathrm{dpa}$ with $100 \mathrm{He}$ preimplanted. Voids obscured by diffraction contrast in BF (b) are clearly observed in ADF (a) as black circles. 


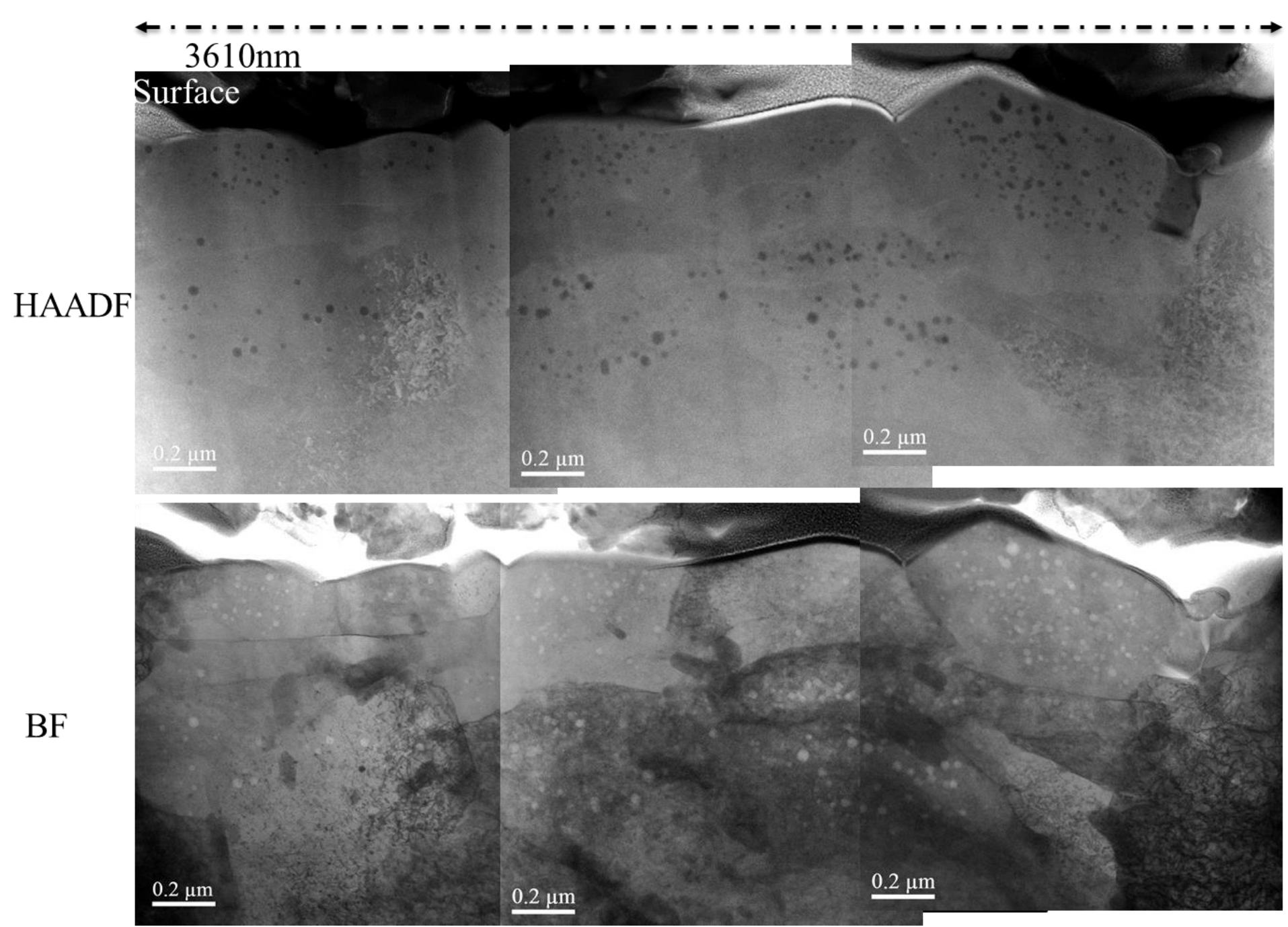

Figure 4.24: Example of STEM HAADF and BF images stitched together to show entire liftout. (HT9, $440^{\circ} \mathrm{C}, 188 \mathrm{dpa}$, 10 appm $\mathrm{He}$ ) 


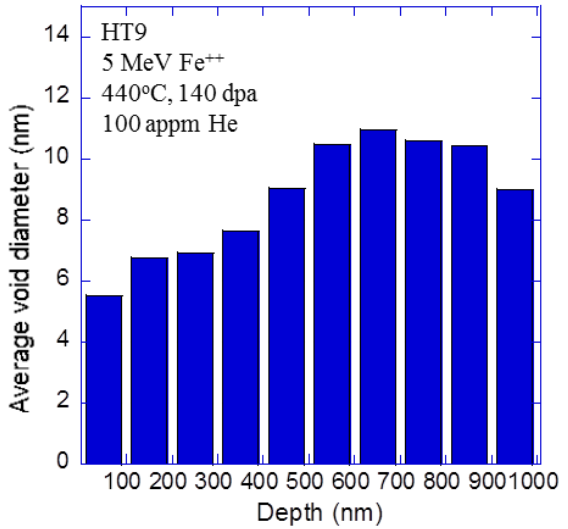

(a)

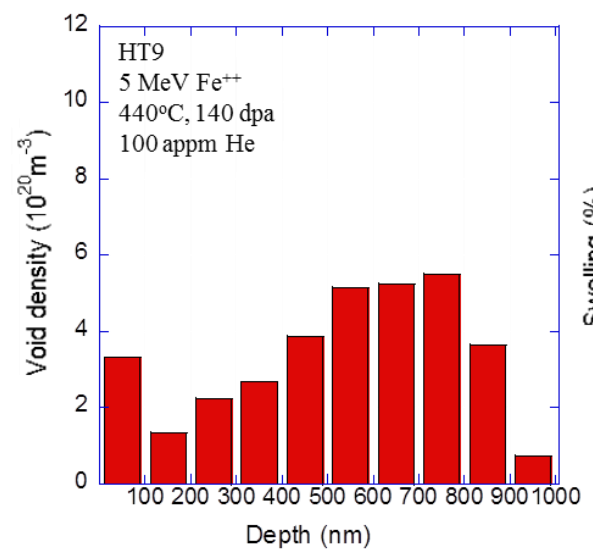

(b)

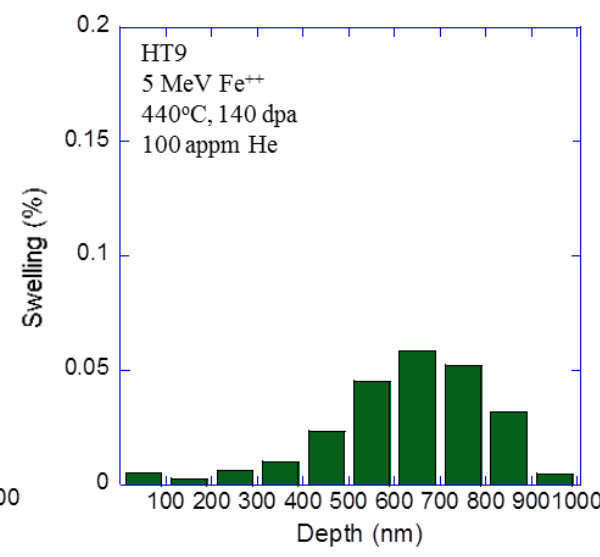

(c)

Figure 4.25: Depth profiles for void a) diameter b) number density and c) swelling in HT9 irradiated at $440^{\circ} \mathrm{C}$ to 140 dpa with 100 appm He preimplanted [12]. 
Error in Diameter, Number Density and Swelling as a Function of Average Void Diameter

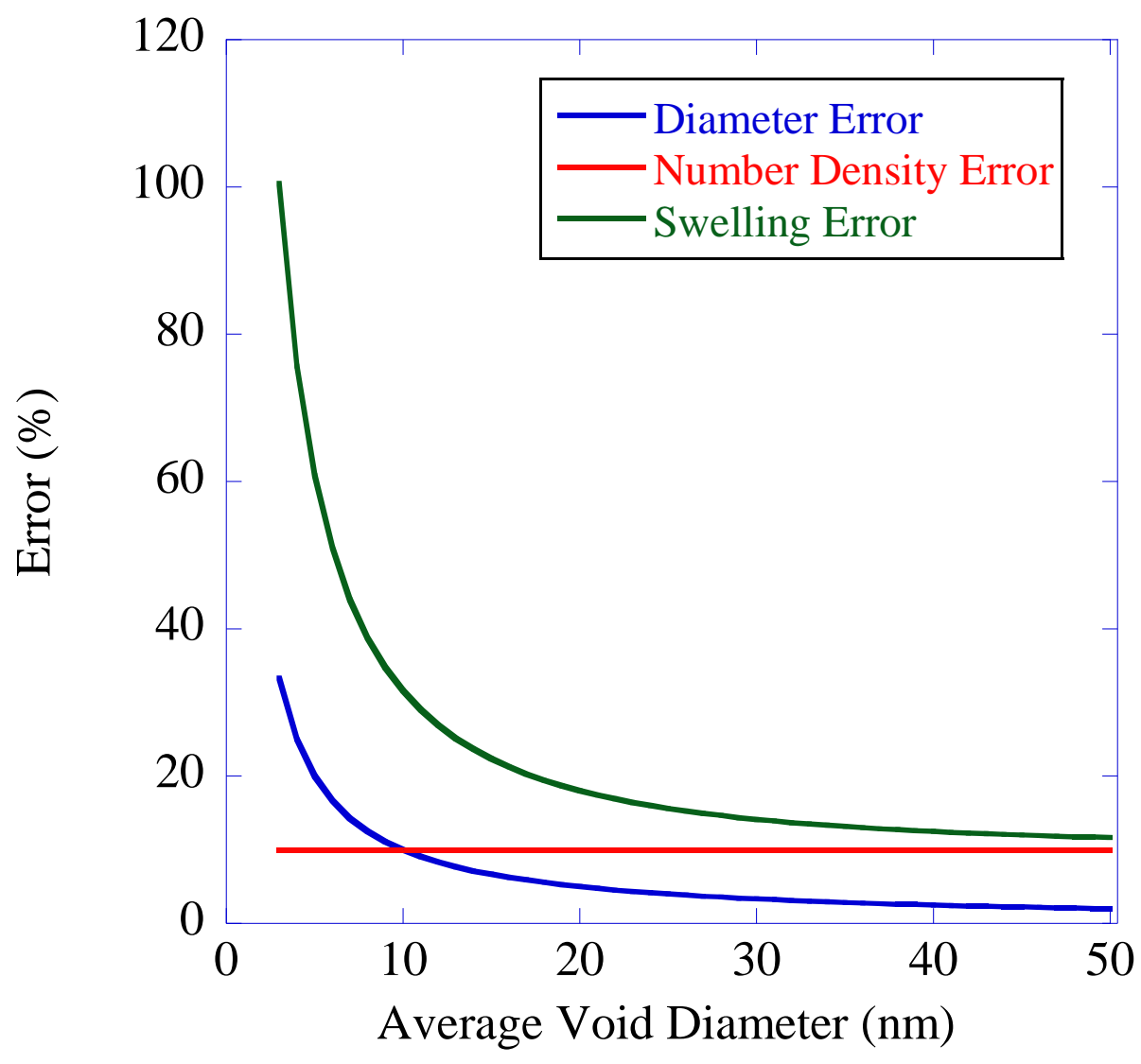

Figure 4.26: Error for diameter, number density and swelling as a function of average void diameter. 


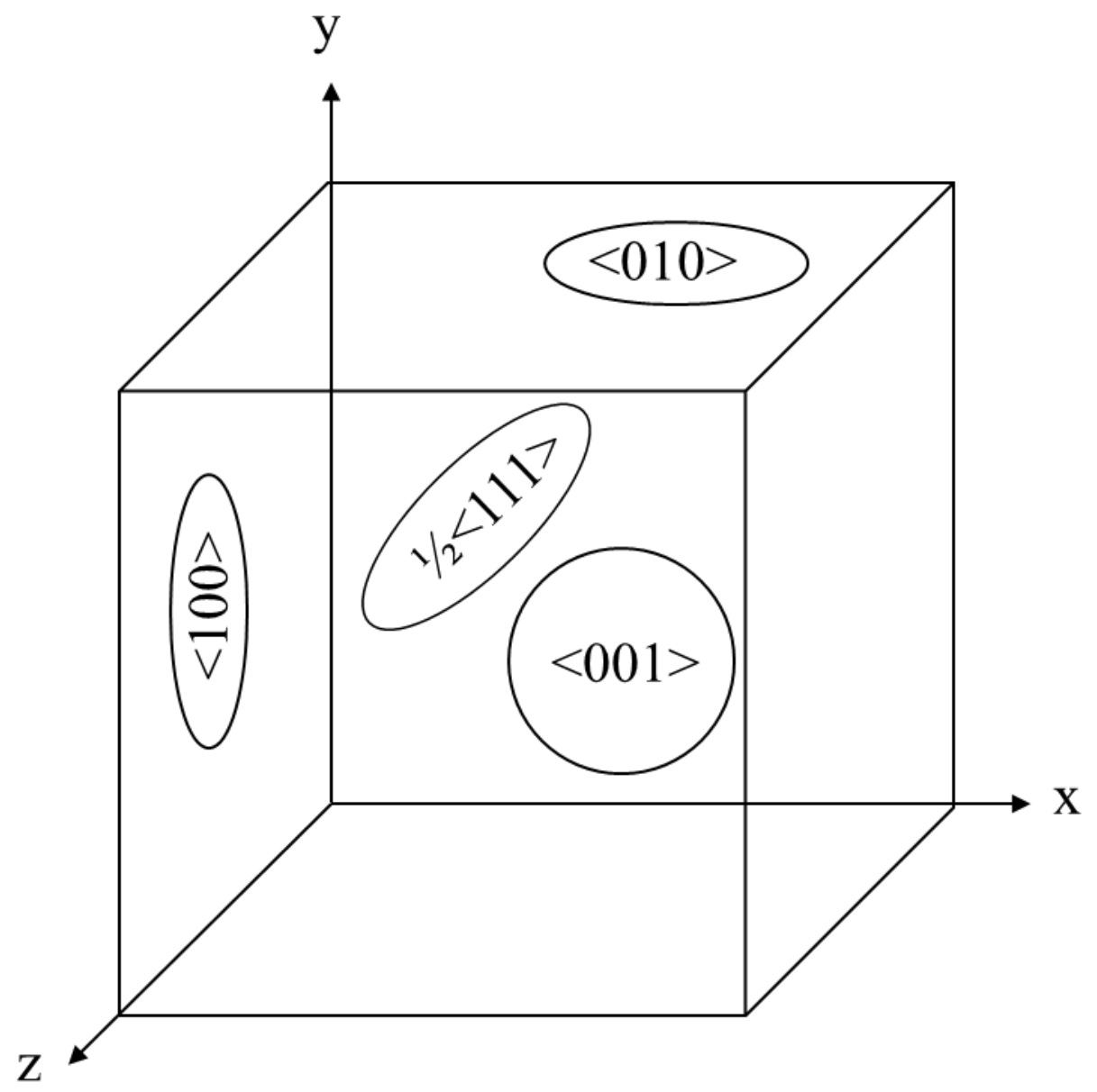

Figure 4.27: Orientation and shapes of dislocation loops in FM alloys imaged along <001> axis [32]. 


\section{CHAPTER 5}

\section{RESULTS OF MICROSTRUCTURE EVOLUTION UNDER ION IRRADIATION}

This chapter will describe results of the experimental portion of the thesis. There are two sections; the results of the $5 \mathrm{MeV} \mathrm{Fe}^{++}$irradiation experiments followed by the post-irradiation microstructure characterization. The first part of the chapter will verify that irradiations were performed at the targeted conditions in terms of temperature, damage, and pressure. The second part of the chapter will present all relevant microstructure results, which will serve as both inputs and a baseline comparison for the modeling section of the thesis in Chapters 6 and 7.

\subsection{Unirradiated Microstructure Characterization}

Prior to irradiation, FIB liftouts of the as-received alloy were prepared and characterized to establish a baseline microstructure for comparison to the irradiated microstructure. Measurements of the grain sizes, precipitate $\left(\mathrm{M}_{23} \mathrm{C}_{6}\right.$ carbides $)$ size and density and dislocation line length are presented in this section.

Grain size (measured as lath length and width), precipitate diameters and densities as well as dislocation lines were measured from STEM bright field images. The precipitate observed in the as-received microstructure was $\mathrm{M}_{23} \mathrm{C}_{6}$, which primarily decorate grain boundaries. An example as-received microstructure is shown in Figure 5.1. The as-received characterization of this alloy is quantified in Table 5.1. 
Laths in HT9 have, on average, a length of $880 \mathrm{~nm}$ and width of $317 \mathrm{~nm}$. At least 50 grains were measured. Grain boundary sink strength requires calculation of an effective grain size parameter $d . d$ is calculated by mathematically equating the rectangular lath area to an equivalent square area, shown in Equation 5.1, where $l$ is the lath length and $w$ is the lath width.

$$
d=\sqrt{l * W}
$$

The effective grain width can be used to calculate grain boundary sink strength using Equation 5.2 .

$$
k_{g b}^{2}=\left\{\begin{array}{l}
\frac{6 k}{d}, d>10^{-5} m \\
\frac{24}{d^{2}}, d<10^{-5} m
\end{array}\right\}
$$

where $k$ is the sum of all other sink strengths, which in this case was approximately $\mathrm{k}=2 \times 10^{14} \mathrm{~m}^{-2}$ [20]. Using $d$, which was calculated as $528 \mathrm{~nm}$, the grain boundary sink strength was calculated to be $8.6 \times 10^{13} \mathrm{~m}^{-2}$.

Finally, retained $\delta$-ferrite due to incomplete tempering was observed. Metallography of the as-received HT9 was performed and a representative micrograph is shown in Figure 5.2. Several $\delta$-ferrite are marked with yellow arrows. $\delta$-ferrite is marked by a characteristically "clean" matrix, free of $\mathrm{M}_{23} \mathrm{C}_{6}$. The fraction of retained $\delta$-ferrite was measured to be approximately $8 \%$ by area. A comparison of the void behavior in each grain type is further discussed in Section 5.2.4.1.

A copy of the indexed diffraction pattern is given in Figure 5.3 using selected area electron diffraction (SAED) along the [001] zone axis, which was consistent with body centered structure. No asymmetry was noted between the $a$ and $c$, which indicates that HT9 is body centered cubic 
(BCC) rather than body centered tetragonal (BCT) with $a=0.282 \mathrm{~nm}$. (The calculated c/a ratio was 1.002, which was within TEM error). The classification of HT9 as BCC is consistent with a well establish observation in the literature that martensite has BCC structure when carbon levels are below $0.6 \mathrm{wt} \%$ [75-78]. (The concentration of carbon in HT9 heat 84425 is $0.2 \mathrm{wt} \%$ ). These results are also consistent with recent X-ray diffraction (XRD) results of the c/a ratio of 1.0003 in T91, a similar alloy [79].

$\mathrm{M}_{23} \mathrm{C}_{6}$ carbides were observed primarily decorating the grain boundaries and were determined to be on average, $47.7 \mathrm{~nm}$ in diameter with a density of $1.66 \times 10^{19} \mathrm{~m}^{-3}$ yielding a sink strength of $4.96 \times 10^{12} \mathrm{~m}^{-3}$, an order of magnitude lower than the sink strength of the grain boundaries.

The dislocation network was also measured. The as-received alloy had a network dislocation line length of $2 \times 10^{14} \mathrm{~m}^{-2}$, which precisely corresponds to its sink strength. From the unirradiated characterization, it was clear that the dislocation network was the strongest sink contributor by an order of magnitude and dominated the calculated total sink strength of $2.50 \times 10^{14}$ $\mathrm{m}^{-2}$ of the as-received alloy.

\subsection{Void Swelling Results}

The focus of this thesis is on the co-evolution of the microstructure with particular focus on the behavior of voids under self-ion irradiation. Before analytic void results in terms of void diameter, number density and swelling can be determined, several unique effects due to the ion irradiation must be considered to determine a "valid" depth region of interest. These atypical effects include the shallow depth penetration (less than $1.6 \mu \mathrm{m}$ in the sample surface), proximity to the surface as well as the implantation of iron ions, referred to as the "injected interstitials." 


\subsubsection{Determination of Valid Region of Interest}

A meaningful characterization of void swelling requires that the extrinsic factors previously mentioned be properly characterized. A complete analysis was performed and presented in [12]; for completeness, a summary is included here. First, a representative void image is shown in Figure 5.4a from a sample irradiated at $460^{\circ} \mathrm{C}$ up to 188 dpa with 10 appm $\mathrm{He}$ preimplanted. By visual inspection, a high density of mostly small voids near the irradiated surface was observed (Figure 5.4b). With increasing depth, it was also observed that there was a second band of larger voids from approximately 400 to 1000 nanometers from the surface. Finally, the diameter increased with depth into the bulk. The void distribution with depth was characterized using the method described in Chapter 4 and the results for void diameter, number density and swelling as a function of depth are presented in Figure 5.5a-c. Quantitative comparisons verify the qualitatively observed trends regarding void density; a peak near the surface and a decrease in void density beyond $700 \mathrm{~nm}$.

Small voids near the surface were observed in a majority of irradiated conditions, regardless of temperature or presence of helium, which resulted in relatively high values of swelling in the near surface region, especially at low damage levels. The enhanced nucleation cannot be attributed to radiation damage; within the first $300 \mathrm{~nm}$, the damage increased from up to $68 \%$ of the nominal calculated damage and the helium concentration was below the nominal level, shown in Figure 4.4. Furthermore, this effect was found to be independent of nominal helium concentration. (The effect of helium on void behavior is outside the scope of this thesis, but relevant to the discussion at hand and was addressed in [12].) Thus, it was assumed that this is a surface effect. 
To quantify the surface effect, swelling depth profiles were examined for fourteen conditions of varying helium, damage and temperature. The depth at which the swelling began to increase is plotted in Figure 5.6, which shows that the onset of the swelling increase was most commonly at a depth of $400 \mathrm{~nm}$, demonstrating that the surface effect was confined to the first 300 $\mathrm{nm}$. The surface effect was also observed by Shao et al. [72], who attributed this effect to a defect imbalance near the surface. A vacancy-rich region forms near the surface because of the preferential loss of interstitials due to their higher mobility. The surface effect was modeled by Xu et al. [80] in molybdenum irradiated in the TEM with $1 \mathrm{MeV} \mathrm{Kr}^{+}$. The ratio of mono-vacancies to mono-interstitials was always larger near the surface of the examined foil rather than near the middle of the foil. This ratio varied from $1.81 \times 10^{11}$ at the surface to $3.89 \times 10^{10}$ at $30 \mathrm{~nm}$ from the surface in a TEM foil with thickness of $60 \mathrm{~nm}$ under $1 \mathrm{MeV} \mathrm{Kr}^{+}$irradiation at fluence of $4.8 \times 10^{13} / \mathrm{cm}^{2}$ at $80^{\circ} \mathrm{C}$. In addition, MD simulation of $100 \mathrm{keV} \mathrm{Fe}$ irradiated iron performed by Stoller [81] compared surviving defects after cascades near the surface and the bulk. It was found that 0.48 vacancies per NRT survived cascades versus 0.33 for interstitials near the surface. In the bulk, there was no difference in survival efficiency between vacancies and interstitials. There was also an increase in vacancy clustering near the surface relative to the bulk. The vacancy/interstitial imbalance promotes void nucleation by stabilization of vacancy clusters and void embryos. Regardless of the mechanism, the surface affected void formation within the first $300 \mathrm{~nm}$.

Figure 5.5c also showed a decrease in void swelling at depths greater than 700 to $900 \mathrm{~nm}$ due primarily to a decrease in number density, with little to no effect on diameter. The damage in this region was $80 \%$ above the nominal damage level measured at $600 \mathrm{~nm}$, which should result in increased void swelling. The decrease was likely due to the presence of injected interstitials, which have been shown throughout the literature $[36,73,82]$ to suppress swelling. A similar decrease in 
number density at increasing depth/damage was also observed by Yamamoto et al. [83] in irradiations performed with $6.4 \mathrm{MeV} \mathrm{Fe}^{3+}$ ions. They performed a similar depth profiling study up to $1.6 \mu \mathrm{m}$ in dual ion-irradiated MA957 and PM2.

For the results considered in this analysis, the depth at which the apparent decrease in void swelling occurred is plotted in Figure 5.7. Fifteen different cases varying in temperature, damage and helium implantation level were examined. Irrespective of temperature, helium or damage, the drop in swelling occurred between 700 and $900 \mathrm{~nm}$. A rate theory calculation of void swelling by Brailsford and Mansur determined that excess interstitials decrease void growth by less than $10 \%$ when the ratio of interstitials to dpa was $\leq 2.5 \times 10^{-4}$ when modeling $4 \mathrm{MeV} \mathrm{Ni}{ }^{+}$irradiation of pure $\mathrm{Ni}$ at $450^{\circ} \mathrm{C}$ [82]. Between the depths of 700 to $900 \mathrm{~nm}$, the ratio of implanted ions to dpa in this experiment varied between $5.26 \times 10^{-6}$ and $5.45 \times 10^{-5}$. The rate theory calculation pertains to the growth-dominated swelling regime. Since most of the data in this analysis was in the nucleation or transition regimes, the primary effect of the injected interstitial was on void nucleation. The decrease in swelling was due to a decrease in the void number density over a variety of damage, temperature and helium conditions. Fifteen temperature conditions that were examined in Figure 5.7 for the depths at which there was an apparent swelling decrease were then compared to the locations where diameter and number density decreased. It was found that the decrease in swelling was due to a decrease in void number density in 7 cases, a decrease in void diameter in 2 cases and a decrease in both number density and diameter in 3 conditions. There was no correlation in the remaining 3 cases.

After accounting for the effects of the surface and the implanted interstitial, the valid region for void analysis was determined to lie between 300 and $700 \mathrm{~nm}$. While the He concentration was constant in this region, the damage varied substantially, increasing from 68 to $117 \%$ of the nominal 
damage across this $400 \mathrm{~nm}$ region. Swelling and void characteristics for the rest of this thesis were analyzed in either the region from 300 to $500 \mathrm{~nm}$ or from 500 to $700 \mathrm{~nm}$, which limited the damage variation over the analyzed area while still maintaining a large enough area to capture microstructural variations and to ensure statistically significant numbers of voids; effectively doubling the number of damage levels that can be analyzed in each sample, assuming there were statistically significant numbers of voids in both regions. However, it should be noted that there was a different damage rate and He/dpa ratio in each region and preference was given to analyzing voids in the 500 to $700 \mathrm{~nm}$ region.

\subsubsection{Damage Dependence at $440^{\circ} \mathrm{C}$}

Using the characterization method outlined in Chapter 4 combined with the region of interest selected in Section 5.2.1, void results were analyzed for each of the damage and temperature combinations outlined in the approach in Chapter 3. Representative images for each examined condition are included in this chapter, and Appendix B: Void images, presents images of an entire liftout from each examined condition. Void depth profiles for each irradiation condition are included in Appendix C: Void Depth Profiles.

The first damage dependence study was performed at $440^{\circ} \mathrm{C}$ from $25-188$ dpa with 10 appm He preimplanted. The primary motivation of this damage series was to determine 1) where void swelling occurred in terms of damage level and 2) what damage level was sufficiently high enough to provide a large enough void population to perform a statistically significant temperature dependence experiment. The temperature, $440^{\circ} \mathrm{C}$, was chosen because $440^{\circ} \mathrm{C}$ was believed to be the approximate peak swelling temperature in reactor irradiations of the same heat of HT9 $[9,10]$. Due to the high sink density of HT9, temperature shift theory from Mansur [34] suggested that there would not likely be a large temperature shift with accelerated damage rates reached by ion 
irradiations. Thus, $440^{\circ} \mathrm{C}$ was chosen on the assumption that voids may be observed at similar damage levels as those reached in reactor at $443^{\circ} \mathrm{C}, 155 \mathrm{dpa}$ [9].

Representative images from each damage level at $440^{\circ} \mathrm{C}$ are presented in Figure 5.8. No voids were observed at 25 or $50 \mathrm{dpa}$ (Figure 5.8a-b). By $140 \mathrm{dpa}$ (Figure 5.8c), a few isolated voids were observed. By 188 dpa (Figure 5.8d), a significant population of voids were observed from 0 to $1000 \mathrm{~nm}$.

Using both the 300 to 500 and 500 to $700 \mathrm{~nm}$ depth regions described previously, the diameter and number density as a function of damage are plotted in Figure 5.9 and tabulated in Table 5.2. Figure 5.9a showed a modest increase in diameter with damage from 92-188 dpa. A larger relative increase was observed in number density (Figure $5.9 \mathrm{~b}$ ) from $0.214 \times 10^{20} \mathrm{~m}^{-3}$ to $5.60 \times 10^{20} \mathrm{~m}^{-3}$ at damage levels from 140 to $188 \mathrm{dpa}$. The resulting effect of increased diameter and number density is plotted in Figure 5.10. A clear increase in overall swelling was observed. For a simple comparison, the final swelling rate was calculated using linear regression Equation 4.5 from a damage of 140 (with $0.03 \%$ swelling) and 188 dpa (with $0.22 \%$ swelling) and was determined to be $0.004 \% / \mathrm{dpa}$. This swelling rate was low compared to the swelling rates observed in reactor at similar damage levels, $(\sim 0.01 \% / \mathrm{dpa})[7,9,10]$ indicating that linear, growth-dominated swelling may not have yet been reached. However, given that there was significant void swelling by $188 \mathrm{dpa}(0.22 \%$ measured from 179 voids counted in the region of interest), 188 dpa was deemed a sufficiently high enough damage level at which to perform a temperature dependence experiment.

\subsubsection{Temperature Dependence at $188 \mathrm{dpa}$}


Following the $440^{\circ} \mathrm{C}$ damage dependence experiment, additional temperatures (460 and $480^{\circ} \mathrm{C}$ at $188 \mathrm{dpa}$ ) were examined to verify that $440^{\circ} \mathrm{C}$ was the peak swelling temperature or alternatively, to determine where swelling peaks as a function of temperature. Representative images from the temperature dependence experiment are included in Figure 5.11. Qualitatively, the results were striking. At $460^{\circ} \mathrm{C}$, there was a higher number density of voids with a larger diameter than those at $440^{\circ} \mathrm{C}$, which was strong evidence suggesting that $460^{\circ} \mathrm{C}$ was the peak swelling temperature. At $480^{\circ} \mathrm{C}$, the voids were larger than those at $440^{\circ} \mathrm{C}$, but not at $460^{\circ} \mathrm{C}$. They also appeared to have a smaller number density than any other temperature.

These results are quantified in Figure 5.12 and Figure 5.13. The void diameter (Figure 5.12a) variation with temperature was highly peaked, which did not agree with previous observations from the literature. The literature [14,15] suggests that diameter increases with temperature until it becomes thermodynamically unfavorable to maintain void stability due to thermal emission from vacancy clusters [20]. The number density (Figure 5.12b) also peaked at $460^{\circ} \mathrm{C}$, and sharply dropped off by $480^{\circ} \mathrm{C}$ by two orders of magnitude. Previous results from neutron and ion irradiation [10,14,15] suggested that number density should decrease with temperature. The results from this study did not reflect the expected diameter and number density behavior. The reason for this is that at $188 \mathrm{dpa}$, the different temperatures examined may not be in the same swelling regime i.e. transition versus void growth-dominated. To demonstrate this, the damage evolution of all three temperatures is plotted in terms of diameter and number density in Figure 5.14. Dashed lines were added to extrapolate the expected damage evolution at $440^{\circ} \mathrm{C}$ and $480^{\circ} \mathrm{C}$ to higher damage levels. At 188 dpa (marked by a black line), $460^{\circ} \mathrm{C}$ is at the beginning of the void growth dominated regime whereas new voids are likely being nucleated in 440 and $480^{\circ} \mathrm{C}$. At higher dpa, such as the 300 dpa marked by a black line, all three temperatures will likely be in 
the void growth-dominated regime with the diameter directly proportional to temperature and number density inversely proportional temperature. In other words, the period of sudden growth at $460^{\circ} \mathrm{C}$ from 130 to 188 dpa was not yet observed in either 440 or $480^{\circ} \mathrm{C}$. Thus, a better comparison of temperature dependence is if all three were in the void growth-dominated regime.

Regardless of the observed diameter and number density behavior, swelling exhibited the expected bell-shaped curve, which was consistent with theory [20] and literature $[10,14,15]$, despite the inconsistency in the diameter and number density trends. Smidt et al. [14] observed a similar swelling peak in both $\mathrm{HT} 9$ and EM12, which was centered at $500^{\circ} \mathrm{C}$ rather than $460^{\circ} \mathrm{C}$ as in this study. These irradiations were performed with $2.8 \mathrm{MeV} \mathrm{Fe}^{+}$with a damage rate of $6 \times 10^{-2}$ dpa/s up to 150 dpa with 1 appm He preimplanted. The full width at tenth maximum (FWTM) for alloy HT9 in Smidt's experiment was approximately $100^{\circ} \mathrm{C}$. Toloczko [15] recently reported on the temperature dependence on swelling of MA957, in which the FWTM was $\sim 90^{\circ} \mathrm{C}$. Toloczko's irradiations were performed on MA957 with $1.8 \mathrm{MeV} \mathrm{Cr}^{+}$at $1 \times 10^{-2} \mathrm{dpa} / \mathrm{s}$ up at 100 and $500 \mathrm{dpa}$. However, these results differed in that the swelling peak was narrower than that reported in the literature $[14,15]$. A FWTM value of $\sim 30^{\circ} \mathrm{C}$ was observed in this study. The cause of this difference was unclear but may be in part due to improvements in temperature control under ion irradiation. Regardless, the peak swelling temperature was quite clearly demonstrated to be $460^{\circ} \mathrm{C}$.

\subsubsection{Damage Dependence at $460^{\circ} \mathrm{C}$}

A damage dependence experiment was then performed at $460^{\circ} \mathrm{C}$; the motivation was previously described in Chapter 3, but is repeated here. The peak swelling temperature was chosen for several reasons. First, the focus of this thesis is on the high damage, void growth-dominated regime. To this end, it was desirable to reach the growth-dominated swelling regime as quickly as possible in terms of damage/time. Damage levels examined were from 75-650 dpa for the purpose 
of mapping out void evolution from nucleation, through transition and into growth-dominated regime. A summary of all void results following irradiation at $460^{\circ} \mathrm{C}$ is presented in Table 5.3. Due to the large amount of swelling and small amount of helium, "cavities" are hereafter referred to as "voids" for the rest of these results.

No voids were observed at $75 \mathrm{dpa}$ in the 500 to $700 \mathrm{~nm}$ region. Figure 5.15 shows micrographs of the self-ion irradiated HT9 from 130 to $650 \mathrm{dpa}$. The lowest damage at which voids were observed was $130 \mathrm{dpa}$, where there was a large population of small voids (Figure 5.15a). As the damage increased, the void diameter also increased (Figure 5.15b-f). In general, the void diameter increased with increasing depth into the sample, but decreased in number density at depths greater than 700 to $1000 \mathrm{~nm}$.

Voids were characterized as a function of depth for each irradiation damage level, previously described in Chapter 4 and also in Appendix C: Void Depth Profiles. Void diameter from the 500 to $700 \mathrm{~nm}$ region of interest is shown in Figure 5.16. Diameter increased as a function of damage up to and including $650 \mathrm{dpa}$. The void diameter growth rate was determined to be nearly $0.1 \mathrm{~nm} / \mathrm{dpa}(0.063 \mathrm{~nm} / \mathrm{dpa}$ from 188 to $650 \mathrm{dpa})$ from $188 \mathrm{dpa}$ and above. A constant or near constant void diameter growth rate was a strong indication that linear swelling had been achieved. Figure 5.17 presents the number density as a function of damage. Number density was approximately constant throughout the damage levels examined until $450 \mathrm{dpa}$. The decrease was attributed to void coalescence as voids grew large enough for agglomeration to occur. Atypical void shapes, meaning those not circular or faceted, provided additional evidence of void coalescence.

Figure 5.18 shows the swelling as a function of damage. Consistent with the damage behavior demonstrated in Figure 5.16, there was linear swelling from approximately 188 dpa up 
to $650 \mathrm{dpa}$. A swelling rate was determined to be $\sim 0.033 \% / \mathrm{dpa}$, measured from 188 to $650 \mathrm{dpa}$ using Equation 4.5, and this will be compared to the literature as it serves as a useful metric. Smidt et al. [14] determined a linear swelling rate of $\sim 0.02 \% / \mathrm{dpa}$ up to $250 \mathrm{dpa}$ for HT9 under 2.8 $\mathrm{MeV} \mathrm{Fe}^{++}$-irradiated at $500^{\circ} \mathrm{C}$ with 1 appm He preimplanted. Toloczko et al. [15] determined a final swelling rate of $0.2 \% / \mathrm{dpa}$ from $1.8 \mathrm{MeV} \mathrm{Cr}^{3+}$ at $450^{\circ} \mathrm{C}$ from 400 to $600 \mathrm{dpa}$. For this study, recall that 10 appm He was purposefully chosen to accelerate the void swelling evolution. In spite of this, the approximate swelling rate remained an order of magnitude lower at a higher damage when compared to Toloczko's results. The swelling rates at $460^{\circ} \mathrm{C}$ were more consistent with Smidt's results and those of the HT9/T91 in-reactor database showing swelling rates of 0.01\%/dpa up to 208 dpa shown in Figure 2.8.

The inconsistency in void response between these results and [15] at high damage levels need to be addressed. Toloczko's irradiation were performed using a lower ion energy resulting in void data that was taken much closer to the surface (from 100 to $200 \mathrm{~nm}$ compared to 500 to $700 \mathrm{~nm}$ in this study); the surface has previously been shown to promote an atypical void response $[12,72,83]$. Furthermore, the differences in sample preparation and characterization methods (conventional BF vs. STEM ADF) could account for some of the variability. Samples in Toloczko's study made using electropolishing have more variability in terms of isolating the exact depth of the sample. Finally, electropolishing could also have artificially increased the size of the voids in the examined samples, especially at higher swelling values.

Linear regression analysis, calculated from Equation 4.5, has classically been used to determine the so-called "steady state" swelling rate. An alternative interpretation is that of a strictly monotonically increasing swelling rate i.e. swelling modeled by a parabolic curve with a slowly increasing instantaneous swelling rate, rather than as linear. The comparison of regression 
analyses is shown in Figure 5.19. Both regression types are within error bars, which indicates that either was appropriate for describing the overall swelling curve shape, though the parabolic fit has a slightly better fit measured by the $\mathrm{R}^{2}(0.99$ vs 0.97$)$. Despite the fact that both regression methods are within error bars, a parabolic fit indicates that the "linear" swelling observed may actually indicate a very slowly increasing swelling rate cannot be discounted and will be considered in Chapter 7.

\subsubsection{Grain to Grain Void Variation}

The void results reported here are representative to the average behavior across a number of grains. Typically, at least 2 or 3 liftouts with lengths of 8 to $12 \mu \mathrm{m}$ were examined with approximately 15-20 grains per samples. Examining the void behavior of 30 to 50 represents an appropriate average microstructure, which will then be applicable to the modeling mean field approach that will be discussed in Chapters 6 and 7.

However, it was observed in Figure 5.2 that there was measureable retained $\delta$-ferrite (approximately $8 \%$ by area measured by metallography). $\delta$-ferrite has been correlated with high levels of local swelling $[15,49,84]$. For comparison, a $\delta$-ferrite grain is compared to a typical martensite grain in Figure 5.20 at both 130 and 450 dpa. At 130 dpa, voids appear to be more homogeneous in the image of the ferrite grain (Figure 5.20a) relative to the image of the martensite grains (Figure 5.20b). At $450 \mathrm{dpa}$, the voids are also much more homogenously distributed in the ferrite (Figure 5.20c) relative to the martensite (Figure 5.20d). These results are quantified in Figure 5.21. At both damage levels, nucleation was higher in the ferrite grain. The void diameter between grain types was within error bars and the swelling was higher in ferrite rather than martensite as a result. The effect of ferrite was stronger at $130 \mathrm{dpa}$, which is likely due to the fact that HT9 at $130 \mathrm{dpa}$ is within the nucleation dominated regime rather than void 
growth-dominated regime, as for $450 \mathrm{dpa}$. However, it is difficult to determine if grain boundaries in the martensite are influencing the observed void inhomogeneity. It cannot be discounted that the decrease in nucleation was due to the larger proportion of void denuded zones in martensite as in ferrite. However, qualitatively it was observed at all damage levels there are always voids in a ferrite grain but there are not always voids in a martensite grain. Thus, the results from this study are still consistent with results in the literature that suggest that $\delta$-ferrite is more susceptible to void swelling than martensite $[15,49,84]$; in this case, by enhanced void nucleation.

\subsubsection{High Swelling Correction}

To verify that there were no atypical effects from ion irradiation, an additional analysis was performed to determine whether there was a significant effect of increased porosity due to void swelling in terms of the ion damage curve. Void swelling decreased the effective density of the material. Thus, ions are able to penetrate further into the bulk altering the dpa versus depth curve. A full analysis is available in [85], but a summary is presented here.

After void depth profiling, SRIM was used to calculate the damage in HT9 specimens. Figure 4.4 shows the damage vs. depth profile and the implanted ion distribution for $5 \mathrm{MeV} \mathrm{Fe}^{++}$ in HT9. After voids were profiled in depth, the resulting swelling profile was used to recalculate the damage profile at each of the 6 damage levels at which voids were observed. Using the swelling profiles, the SRIM calculation was adapted for the change in material density as a result of void swelling at each nominal damage level. The updated densities were input into SRIM as discrete layers of $100 \mathrm{~nm}$ thickness. The effective density is then given by: 


$$
\rho_{\text {effective }}=\left(1-\frac{\Delta V}{V}\right) * \rho_{H T 9}
$$

where $\rho_{\text {effective }}$ is the effective density, $\frac{\Delta V}{V}$ is the fractional change in volume (measured swelling) and $\rho_{H T 9}$ is the density of HT9. The results of this calculation, i.e. the effective damage rate under ion irradiation with voids were used to determine an appropriate method for correction of the damage curve.

Using the effective densities calculated from the depth profiles in Appendix $\mathrm{C}$ as input into the SRIM calculation described in Chapter 4, the damage rate vs. depth are directly compared at each nominal damage level in Figure 5.22. The beam current was assumed to be constant regardless of irradiation to directly compare damage rates measured in dpa/s. With increasing dpa and hence, swelling, the range of the ions increased due to increased porosity from void swelling. The effect of porosity became significant at nominal damage levels of 450 dpa. Between 550 and $650 \mathrm{dpa}$, peak swelling remained approximately the same at $\sim 23 \%$ at $900 \mathrm{~nm}$; however, at 650 dpa, the swelling prior to the peak (300 to $700 \mathrm{~nm}$ ) continued to increase. Related to this, at any given depth (before the damage peak) the damage rate decreased with increasing nominal damage level. Finally, all of these effects were exacerbated at high swelling (>10\%) of $550 \mathrm{dpa}$ and above.

Figure 5.23a shows the results of the SRIM calculation with effective density of the irradiated HT9. The decrease in damage rate at $600 \mathrm{~nm}$ with damage was marked by a black arrow. Since this correction method maintains the same 500 to $700 \mathrm{~nm}$ area of interest, the damage must then be corrected with the results of the SRIM calculation. For instance, at 450 dpa, the effective damage rate was $0.91 \times 10^{-3} \mathrm{dpa} / \mathrm{s}$ instead of $1.00 \times 10^{-3} \mathrm{dpa} / \mathrm{s}$, for a decrease of 
9\%. The 100 dpa increment from 350 to 450 dpa was actually a 91 dpa increment, if the worst case swelling profile was used. A more realistic application uses the average damage rate from $350 \mathrm{dpa}\left(0.94 \times 10^{-3} \mathrm{dpa} / \mathrm{s}\right)$ and $450 \mathrm{dpa}\left(0.91 \times 10^{-3} \mathrm{dpa} / \mathrm{s}\right)$, which was calculated as $0.92 \times 10^{-3}$ $\mathrm{dpa} / \mathrm{s}$, for a decrease in $8 \%$ of the total damage. The resulting swelling curves are plotted in Figure 5.23b, which includes both the average and the "worst case scenario" corrections. Up to 450 dpa, there was almost no difference between the nominal swelling and the damage corrected swelling, but the effect became non-negligible from 450 to 650 dpa. For the fixed depth method, one downside was that the actual damage rate changed from irradiation to irradiation. However, the change in damage rate was $\sim 20 \%$ at 650 dpa when compared to the unirradiated damage rate. The effect of damage rate on swelling was therefore minimal.

Another interesting result was that the difference between the "average" and "worst case" swelling profile was almost negligible, even up to $650 \mathrm{dpa}$. The relative consistency was likely because the largest amounts of swelling were at $600 \mathrm{~nm}$ or greater as shown in Figure 5.24 and Figure 5.25, meaning that the largest effect of the increased swelling was at greater depth where there was a greater cumulative effect of the porosity. The cumulative effect of increased swelling also increases with each subsequent irradiation. The resultant swelling rates from the two cases were very close for damage levels up to $650 \mathrm{dpa}$. The swelling rate was also within the error bars when compared to the nominal swelling rate.

This "fixed depth" method has the benefit of maintaining a usable void distribution, which was useful for examining void growth behavior. The correction was based upon a relatively simple SRIM calculation only. It was also the most similar to the previous correction methods used by Odette et al. [86] and Johnston et al. [87]. Finally, and perhaps most 
importantly, maintaining the same region of interest ensured that there are no complicating effects from the injected interstitial deeper in the sample.

A key observation was that there was little variation in the ion damage profile in irradiations below 450 dpa or $\sim 10 \%$ swelling. Thus, the method was most useful in the high swelling regime. It was expected that the correction methodology will be even more important at even higher swelling values of $20 \%$ or more, assuming a reactor design can accommodate that level of void swelling. The depth correction method would be especially relevant for higher swelling materials such as austenitic stainless steels. Nevertheless, even at lower swelling amounts, the value of the method was that it provides more accurate data to swelling model development to high damage levels. Thus, these results were convincing evidence that even with the most liberal correction, the calculated linear swelling rate remained more or less constant and the reported swelling rate remains approximately $0.033 \% / \mathrm{dpa}$ from 188 to $650 \mathrm{dpa}$.

\subsection{Secondary Phase Results}

In this section, the formation of secondary phases (precipitates) with irradiation will be discussed and characterized as a function of damage.

\subsection{1 $\underline{\mathrm{G} \text { Phase }}$}

Previously mentioned in Chapter 2, G phase is a complex FCC silicide of the form $\mathrm{M}_{6} \mathrm{Mn}_{16} \mathrm{Si}_{7}$ where $\mathrm{M}=\mathrm{Ni}$ typically. Images of $\mathrm{G}$ phase observed from 130 to 650 dpa are shown in Figure 5.26. Atom probe analysis was used to confirm the composition of G phase in samples irradiated at $440^{\circ} \mathrm{C}$ to $140 \mathrm{dpa}$ (Figure 5.27). For the $\mathrm{M}=\mathrm{Ni}$ type precipitates observed, the expected concentration (at $\%$ ) of elements is $55 \% \mathrm{Ni}-24 \% \mathrm{Si}-21 \% \mathrm{Mn}$. This was consistent with Figure $5.27 \mathrm{~b}$ and Figure 5.27c, which show two precipitates that have concentrations of nearly $55 \% \mathrm{Ni}, 24 \% \mathrm{Si}$ 
and $21 \% \mathrm{Mn}$, within error bars. There was some uncertainty in exact composition due to how atom probe analyses are performed, but these compositions are close enough to be appropriate in identifying these precipitates as $\mathrm{G}$ phase. These results are also consistent with representative a XEDS image from HT9 irradiated to $130 \mathrm{dpa}$ at $460^{\circ} \mathrm{C}$ that is shown in Figure 5.28, confirming that these are Ni/Si rich since APT analysis of all irradiation results was not feasible. $\gamma^{\prime}$, also a Ni Si rich precipitate with no Mn was not observed in this study nor in any other irradiations of FM alloys [3].

A representative HRTEM image of $\mathrm{G}$ phase at $460^{\circ} \mathrm{C}, 650$ dpa is presented in Figure 5.29. Analysis of the image verifies that $G$ phase has an fcc cubic structure and a lattice parameter of $\mathrm{a}=1.148 \mathrm{~nm}$. Furthermore, the cubic-on-cubic growth relationship to the surrounding matrix was verified to be $(001)_{\mathrm{G}} / /(001)_{\alpha},(110)_{\mathrm{G}} / /(110)_{\alpha}$, and $(111)_{\mathrm{G}} / /(111)_{\alpha}$, which was consistent with results from Mateo et al. [88] and Vitek et al. [89].

G phase was observed in self-ion irradiated HT9 by 75 dpa and G phase diameter, number density and volume fraction are plotted as a function of damage in Figure 5.30 and are included in Table 5.4. Initially, $\mathrm{G}$ phase formed at a high number density of $2.38 \times 10^{21} \mathrm{~m}^{-3}$ and a small average diameter of $7.2 \mathrm{~nm}$. The number density was approximately constant from 75-130 dpa, and then decreased to $\sim 8.5 \times 10^{20} \mathrm{~m}^{-3}$ between 188 and $650 \mathrm{dpa}$ as the diameter increased approximately linearly. Since the number density was more or less constant, the increasing growth in diameter led to an overall linear increase in volume fraction beyond 188 to $650 \mathrm{dpa}$. Two key observations are emphasized: first, the continuing evolution of the $G$ phase precipitates up to very high damage levels of 650 dpa was consistent with recent results from Jiao and Was that suggest that the several types of precipitates continue to evolve up to very high damage levels [51]. Second, the constant number density and linear diameter growth rate resulted in an evolution similar to that of the voids. 
$\mathrm{G}$ phase is an incoherent precipitate, meaning that it acts as an unbiased sink in terms of defect absorption behavior similar to voids [20].

\subsection{2 $\underline{\mathrm{M}}_{2} \underline{\mathrm{X}}$}

In addition to the formation of $\mathrm{G}$ phase under irradiation, another irradiation-induced secondary phase was first observed at $250 \mathrm{dpa}$. Using low angle annular dark field (LAADF), oriented precipitates were observed in low number densities at 250 dpa up to 650 dpa. Figure 5.31 shows both the HAADF and LAADF images of the precipitates formed from 250 up to 650 dpa. $\mathrm{M}_{2} \mathrm{X}$ are planar type precipitates. With damage, there was a clear increase in number density and with size up to 450 dpa.

Intragranular precipitation was observed and was consistent with previous observations of $\mathrm{M}_{2} \mathrm{X}$ formation from Borodin et al., Wang et al. and Maziasz [3,38,53,90]. $\mathrm{M}_{2} \mathrm{X}$-denuded zones were observed near grain boundaries, which is shown in Figure 5.33. Several authors have suggested that $\mathrm{M}_{2} \mathrm{X}$ forms on $\mathbf{a}<100>$ dislocation loops, which is further supported by evidence that the habit plane is the same $[3,38] . \mathrm{M}_{2} \mathrm{X}$ was not observed to precipitate on grain boundaries. In ferritic-martensitic alloys, $\mathrm{Cr}$ enriches at the grain boundary via radiation-induced segregation [54,55] and some limited $\mathrm{C}$ segregation to grain boundaries has been observed [54], yet the enrichment does not result in precipitation. This observation is not surprising since in austenitic alloys, Ni and Si have been observed to strongly enrich at grain boundaries, but rarely results in precipitation $[54,69,91,92]$. In Figure 5.26, most Ni/Si precipitated within the grains as well.

The orientation relationship with respect to the miller indices is shown in a schematic in Figure 5.32. As it was determined that these are oriented on the $\{001\}$ family of planes along the 
$<001>$ direction. The schematic shows the top view that corresponds with Figure 5.31a-e, and also defines the dimensions of $\mathrm{M}_{2} \mathrm{X}$ with respect to the miller indices.

Additional characterization of the irradiation-induced $\mathrm{M}_{2} \mathrm{X}$ was performed to verify the composition, structure, and coherency. EFTEM imaging (Figure 5.34) was used to verify that they are rich in chromium and carbon, and that the stoichiometric composition was $\mathrm{Cr}_{2} \mathrm{C}$. This was further confirmed with an EDX spectrum taken on $\mathrm{M}_{2} \mathrm{X}$ that had been polished to $<50 \mathrm{~nm}$ in thickness to perform HRTEM, which is shown in Figure 5.35.

Selected area electron diffraction (SAED) was performed at three different tilt angles to deterime the lattice parameter of M2X, shown in Figure 5.36. The diffraction patter was consistent with hexagonal crystal structure (HCP). This was later confirmed with HRTEM in HT9 irradiated to 650 dpa. Figure 5.37a shows the bright field HRTEM image. Figure 5.37b shows the fast Fourier transform (FFT) from the HRTEM images. This pattern was identical with that in Figure 5.36. Lattice parameters for $\mathrm{M}_{2} \mathrm{X}$ are $a=0.271 \mathrm{~nm}$ and $c=0.452 \mathrm{~nm}$, consistent with [90].

$\mathrm{M}_{2} \mathrm{X}$ was determined to be semi-coherent with the matrix. To prepare specimens for HRTEM imaging of the $\mathrm{M}_{2} \mathrm{X}$ phase free from the interference of the matrix, a FIB liftout was flash electropolished yielding specimen thicknesses less than $50 \mathrm{~nm}$ (Figure 5.38). The orientation relationship between the precipitate and the matrix is $[100]_{M_{2} X} / /[100]_{\alpha}$ or $[11 \overline{2} 0]_{M_{2} X} / /[100]_{\alpha}$; $(\overline{1} 20)_{M_{2} X} / /(0 \overline{1} 0)_{\alpha}(\overline{1} \overline{1} 20)_{M_{2} X} / /(0 \overline{1} 0)_{\alpha}$, shown in Figure 5.39. The semi-coherency and growth directions of $\mathrm{M}_{2} \mathrm{X}$ were consistent with recent results from Wang et al. in FM alloy CNS II, a Fe$12 \mathrm{Cr}$ analogue to $\mathrm{HT} 9$ that was irradiated at $460^{\circ} \mathrm{C}$ up to 450 dpa with $5 \mathrm{MeV} \mathrm{Fe}^{++}$[93].

To further explore coherency in the [011] direction, FFTs from the HRTEM image in Figure 5.40a the simulated SAED patterns (Figure 5.40b) were overlaid on the FFT shown in 
Figure 5.40c. From the simulated SAED pattern, it can be shown that $[0 \overline{2} 0]$ spot from the Fe matrix had $\mathrm{d}=0.141 \mathrm{~nm}$ and coincident with the $[\overline{1} 20]_{M_{2} X}$ spot from $\mathrm{M}_{2} \mathrm{X}$, which had $\mathrm{d}=0.136 \mathrm{~nm}$.

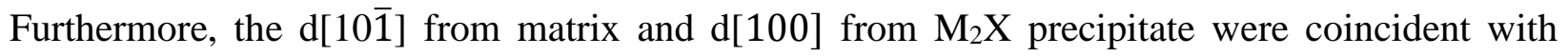
$\mathrm{d}=0.202 \mathrm{~nm}$ and $\mathrm{d}=0.235 \mathrm{~nm}$, respectively, indicating a $16 \%$ mismatch, which is consistent with semi-coherency. The orientation relationship between the precipitate and the matrix along the [011] direction is thus $[001]_{M_{2} X} / /[10 \overline{1}]_{\alpha}$ or $[0001]_{M_{2} X} / /[10 \overline{1}]_{\alpha} ;(100)_{M_{2} X} / /(10 \overline{1})_{\alpha}$ or $(11 \overline{2} 0) / /(10 \overline{1})_{\alpha}$. The orientation relationship is also consistent with results from [94,95].

There are differences in both the $a$ lattice parameter $(a=0.28 \mathrm{~nm}$ for matrix, $a=0.271 \mathrm{~nm}$ for $\mathrm{M}_{2} \mathrm{X}$ ) and the $c$ lattice parameter, which is $0.452 \mathrm{~nm}$ in $\mathrm{M}_{2} \mathrm{X}$. Since, by definition, if any direction is semi-coherent the precipitate must be classified as semi-coherent, therefore $\mathrm{M}_{2} \mathrm{X}$ is semi-coherent. Furthermore, semi-coherency is much more plausible than coherency because of the differing crystal structure (hcp versus bcc) and the much larger $c$ lattice parameter of $\mathrm{M}_{2} \mathrm{X}$. Furthermore, the growth directions of [010] and [10 $\overline{1}]$ determines in Figure 5.39 and Figure 5.40 explains the platelike shape on the $\{100\}$ family of planes as $\mathrm{M}_{2} \mathrm{X}$ forms from growth inside the

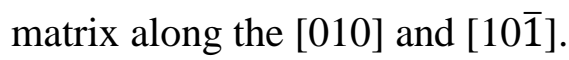

To further quantify the precipitate response to increasing damage, Figure 5.41 and Table 5.4 present the length, width, thickness, number density and volume fraction as a function of dpa. Similar to what was observed by visual inspection in Figure 5.31, the length, width and thickness saturated with damage by $\sim 450 \mathrm{dpa}$. The number density peaked at $450 \mathrm{dpa}$, then decreased leading to a corresponding decrease in the volume fraction.

\subsection{Dislocation Microstructure}


A systematic characterization of the dislocation network and loop microstructure is essential since swelling and dislocation microstructure are linked through the interstitial bias of the dislocations, which provides vacancy supersaturation and drives defect partitioning. The following is a discussion of the dislocation lines and loops as a function of damage level.

\subsubsection{Dislocation Network}

Dislocation lines were characterized at each damage level in the manner described in Chapter 4 using representative bright field STEM images shown in Figure 5.42 and tabulated in Table 5.5. A representative area was chosen for each damage level. Several higher magnification images are also presented in Figure 5.43 so that the network is more clearly visible. The Burgers vector was $\mathbf{a} / 2<111>$ type network. Very little evolution of the line network was observed, evidenced by Figure 5.44. The initial network density was $2 \times 10^{14} \mathrm{~m}^{-2}$. By $130 \mathrm{dpa}$, the network line length was $\sim 3 \times 10^{14} \mathrm{~m}^{-2}$, which was consistent (same order of magnitude) with results from Gelles [7,11] and Kai [19], but was an order of magnitude lower than that of Sencer observed in the same heat of HT9 [9]. Variations in results may be due to different imaging conditions and counting methods.

\subsubsection{Dislocation Loops}

Dislocation loops were characterized in the weak 2 beam condition described in Chapter 4 using BF conventional TEM or STEM imaging. An effort was made to examine loops only in the approximate depth range of 500 to 700 nanometers, reflecting the nominal damage. No loops were

observed in the unirradiated condition, which was expected [20]. A summary table is available in Table 5.5 and micrographs are presented in Figure 5.45. 
Loops were first characterized at 130 dpa. Loops formed primarily were of $\mathbf{a}<100>$ character with very few observed $\mathbf{a} / 2<111\rangle$, which was consistent with literature $[3,10]$. Since so few $\mathbf{a} / 2<111>$ loops were observed, all results reported are from $\mathbf{a}<100>$ loops only. A high density of relatively small loops formed at the lowest damage imaged. Loops had an average diameter of $\sim 22 \mathrm{~nm}$. With damage, the loop diameter increased up to $30 \mathrm{~nm}$ and remained relatively constant up to 375 dpa varying between $23-32 \mathrm{~nm}$, which is shown in Figure 5.46. Between 375 to $450 \mathrm{dpa}$, there was a sudden increase in dislocation loop diameter growth rate resulting in loops of the unusual size of $78 \mathrm{~nm}$ at $450 \mathrm{dpa}$ up to a final loop diameter of $92 \mathrm{~nm}$ at 650 dpa.

Figure 5.47 presents the loop number density from 130 to $650 \mathrm{dpa}$. At the lowest damage, the highest number density of loops $\left(9.7 \times 10^{20} \mathrm{~m}^{-3}\right)$ was observed. As damage increased, the number density dropped off precipitously from 130 to $188 \mathrm{dpa}$ and more slowly to $450 \mathrm{dpa}$. Given the typical uncertainty inherent in loop number density due to the difficulty in imaging loops in ferritic-martensitic alloys, it was appropriate to conclude that the loop number density remains approximately constant from 450 to $650 \mathrm{dpa}$.

The combined effect of the increasing diameter and decreasing number density, in general, resulted in a dynamic evolution of the loop line length. Figure 5.48 presents the loop line length as a function of damage. Due to the high density of loops, the loop line length, analogous to sink strength, was the highest at $130 \mathrm{dpa}$. With the drop off in number density, line length decreased before it recovered to the previously maximum value via increased diameter growth. The loop line length at 250 dpa did not quite follow this trend due to its higher observed number density, but in general the trend was still consistent. Another key observation was that even though the loop line 
length did not vary significantly with damage, the character of the loops (i.e. diameter and number density) evolved consistently up to high damage.

Calculation of the loop line length can be directly compared with the network line length shown in Figure 5.49. The striking result of this comparison was that the dislocation behavior was dominated by the network rather than the loops by approximately an order of magnitude. Regardless of the dynamic nature of loop evolution, defect accumulation was likely dominated by network behavior, rather than the loops.

The increase in network line length from 450 to 550 dpa could be explained by the observed decrease in loop number density in which the large loops join the network. Since no loops were observed experimentally above $100 \mathrm{~nm}$, it is a good assumption that loops are joining the network at approximately $100 \mathrm{~nm}$. A measurable decrease $\left(2 \times 10^{20} \mathrm{~m}^{-3}\right)$ in loop number density from approximately 4 to $2 \times 10^{20} \mathrm{~m}^{-3}$ (reflecting the decrease in number density from approximately 250 to $550 \mathrm{dpa}$ ) was observed. Assuming $100 \mathrm{~nm}$ loops have left the network at $2 \times 10^{20} \mathrm{~m}^{-3}$, this corresponds to an increase in sink strength of $7 \times 10^{13} \mathrm{~m}^{-2}$. The measured sink strength at $550 \mathrm{dpa}$ was $1 \times 10^{14} \mathrm{~m}^{-2}$ higher than at $450 \mathrm{dpa}$. Given the large error inherent in measuring loop number density and network line length, this difference is well within error.

Recall that loops observed are $\mathbf{a}<100>$ whereas the network is $\mathbf{a} / 2<111>$. Some authors have theorized that a secondary population of $\mathbf{a} / 2<111>$ loops may form and rapidly climb to grow the network [94], which could account for the increase in the dislocation network from the asreceived case $\left(2 \times 10^{14} \mathrm{~m}^{-2}\right)$ to $130 \mathrm{dpa}\left(3 \times 10^{14} \mathrm{~m}^{-2}\right)$, but likely would not account for the increase from 450 to 550 dpa from 3 to $4 \times 10^{14} \mathrm{~m}^{-2}$. 
Interactions between $\mathbf{a}<100>$ loops and the $\mathbf{a} / 2<111>$ network have been proposed in the literature. Eyre and Bullough proposed a mechanism where dislocation loops of both $\mathbf{a}<100>$ and a $/ 2<111>$ type could form from an aggregate of self-interstitials through shear reactions, resulting in the formation of a network [95]. More recently, possible interaction mechanisms between $\mathbf{a}<100>$ type loops and $\mathbf{a} / 2<111>$ network were proposed by Terentyev et al. on the basis of MD simulations and offer an explanation of how the loops observed may grow the network from 450 to 550 dpa by absorption of $\mathbf{a}<100>$ loops into the $\mathbf{a} / 2<111>$ network. Interaction of any type of $\mathbf{a}<100>$ loop with $\mathbf{a} / 2<111>$ resulted with absorption into the network by the formation of "superjogs" or segments that are pinned then absorbed in the network with a resulting change in burgers vector. Thus, it is reasonable to assume that the loops are growing the network despite the difference in loop character.

One of the most significant results from this study was the continuing evolution and growth of loops at damage levels beyond $200 \mathrm{dpa}$. The most comparable study of dislocation evolution with damage for comparison was performed on binary $\mathrm{Fe}-9 \mathrm{Cr}$ and $\mathrm{Fe}-12 \mathrm{Cr}$ alloys irradiated in FFTF by Gelles from 15 to 200 dpa [33]. Gelles observed that total dislocation density increased from 2 to $10.8 \times 10^{14} \mathrm{~m}^{-2}$ in Fe-9Cr and from 4.7 to $4.9 \times 10^{14} \mathrm{~m}^{-2}$ in Fe-12Cr. The relatively stable network in $\mathrm{Fe}-12 \mathrm{Cr}$ was consistent with these results, as total dislocation density increased from $3.6 \times 10^{14} \mathrm{~m}^{-2}$ at $130 \mathrm{dpa}$ up to $4.5 \times 10^{14} \mathrm{~m}^{-2}$ at $650 \mathrm{dpa}$.

A second finding was that the dislocation line length of the loops was an order of magnitude lower than that in the network $\left(0.4 \times 10^{14} \mathrm{~m}^{-2}\right.$ versus $3 \times 10^{14} \mathrm{~m}^{-2}$, respectively at $\left.250 \mathrm{dpa}\right)$. In Fe-Cr alloys irradiated in FFTF at $420^{\circ} \mathrm{C}$ to 130 dpa, Katoh [96] observed dislocation line length of loops and networks to be of similar value in Fe-12Cr $\left(\sim 1 \times 10^{14} \mathrm{~m}^{-2}\right.$ and $0.8 \times 10^{14} \mathrm{~m}^{-2}$ for loops and lines, 
respectively), but a much smaller loop contribution to overall dislocation density in Fe-9Cr binary alloys $\left(\sim 0.2 \times 10^{14} \mathrm{~m}^{-2}\right.$ and $0.8 \times 10^{14} \mathrm{~m}^{-2}$ for loops and lines, respectively).

Figure 5.50 presents a summary of the co-evolution of the microstructure at $460^{\circ} \mathrm{C}$. As damage increased beyond $188 \mathrm{dpa}$, which is referred to as the nucleation regime, both void swelling and $\mathrm{G}$ phase volume fraction increased linearly up to $650 \mathrm{dpa} . \mathrm{M}_{2} \mathrm{X}$ formed at $250 \mathrm{dpa}$ and increased up to $450 \mathrm{dpa}$ then decreased in terms of volume fraction. Dislocation microstructure evolution was dominated by the network, while loops continued to grow up to $650 \mathrm{dpa}$. This complete data set thus provides a database for modeling of this microstructure evolution, which will be discussed in Chapter 6. The results also provide insights into the interaction mechanisms between the voids, dislocations and precipitates, which will be further explained using the RIME model and discussed in Chapter 7. 
Table 5.1: As-received microstructure characterization of HT9 (84425).

\begin{tabular}{|c|c|}
\hline Alloy & HT9 (84425) \\
\hline Damage (dpa) & 0 \\
\hline Sample Preparation Method & FIB liftout \\
\hline Lath Length $(\mathrm{nm})$ & 880 \\
\hline Lath Width $(\mathrm{nm})$ & 317 \\
\hline Lath size, $d(\mathrm{~nm})$ & 528 \\
\hline Sink Strength of Grain Boundaries $\left(\mathrm{m}^{-2}\right)$ & $8.6 \times 10^{13}$ \\
\hline $\mathrm{M}_{23} \mathrm{C}_{6}$ Diameter $(\mathrm{nm})$ & 47.7 \\
\hline $\mathrm{M}_{23} \mathrm{C}_{6}$ Density $\left(\mathrm{m}^{-3}\right)$ & $1.7 \times 10^{19}$ \\
\hline $\mathrm{M}_{23} \mathrm{C}_{6}$ Sink Strength $\left(\mathrm{m}^{-2}\right)$ & $5.0 \times 10^{12}$ \\
\hline Dislocation Network Line Density $\left(\mathrm{m}^{-2}\right)$ & $2.0 \times 10^{14}$ \\
\hline Dislocation Network Sink Strength $\left(\mathrm{m}^{-2}\right)$ & $2.0 \times 10^{14}$ \\
\hline Total Sink Strength $\left(\mathrm{m}^{-2}\right)$ & $2.5 \times 10^{14}$ \\
\hline
\end{tabular}


Table 5.2: Void results from damage dependence study at $440{ }^{\circ} \mathrm{C}$ and temperature dependence study at $188 \mathrm{dpa}$.

\begin{tabular}{|c|c|c|c|c|c|c|c|c|}
\hline $\begin{array}{l}\text { Temperature } \\
\left({ }^{\circ} \mathrm{C}\right)\end{array}$ & $\begin{array}{c}\text { Damage } \\
\text { (dpa) }\end{array}$ & $\begin{array}{c}\mathrm{He} \\
(\mathrm{appm})\end{array}$ & $\begin{array}{c}\text { Number of } \\
\text { TEM } \\
\text { Samples } \\
\text { Analyzed } \\
\text { Per } \\
\text { Condition }\end{array}$ & $\begin{array}{l}\text { Number } \\
\text { of Voids }\end{array}$ & $\begin{array}{c}\text { Area } \\
\text { Examined } \\
(\mu \mathrm{m})\end{array}$ & $\begin{array}{c}\text { Void } \\
\text { Diameter } \\
(\mathrm{nm})\end{array}$ & $\begin{array}{c}\text { Void } \\
\text { Density } \\
\left(10^{20} \mathrm{~m}^{-3}\right)\end{array}$ & Swelling (\%) \\
\hline 440 & 25 & 10 & 2 & 0 & 2.0 & 0 & 0 & 0 \\
\hline 440 & 50 & 10 & 2 & 0 & 2.0 & 0 & 0 & 0 \\
\hline 440 & 140 & 10 & 2 & 11 & 3.8 & $12.9 \pm 1.0$ & $0.214 \pm 0.021$ & $0.0029 \pm 0.0005$ \\
\hline 440 & 188 & 10 & 2 & 179 & 1.4 & $17.7 \pm 1.0$ & $5.60 \pm 0.56$ & $0.22 \pm 0.04$ \\
\hline 480 & 188 & 10 & 2 & 17 & 3.4 & $21.3 \pm 1.0$ & $0.393 \pm 0.039$ & $0.033 \pm 0.005$ \\
\hline 460 & 188 & 10 & 2 & 343 & 2.1 & $29.8 \pm 1.0$ & $12.00 \pm 1.2$ & $2.9 \pm 0.4$ \\
\hline
\end{tabular}


Table 5.3: Summary of void data from damage dependence experiment performed at $460^{\circ} \mathrm{C}$.

\begin{tabular}{|c|c|c|c|c|c|c|c|c|}
\hline $\begin{array}{c}\text { Temperature } \\
\left({ }^{\circ} \mathrm{C}\right)\end{array}$ & $\begin{array}{c}\text { Damage } \\
(\mathrm{dpa})\end{array}$ & He (appm) & $\begin{array}{c}\text { Number of } \\
\text { TEM Samples } \\
\text { Analyzed Per } \\
\text { Condition } \\
\text { Voids }\end{array}$ & $\begin{array}{c}\text { Number of } \\
\left(\mu \mathrm{m}^{2}\right)\end{array}$ & $\begin{array}{c}\text { Area Examined } \\
(\mathrm{nm})\end{array}$ \\
\hline 460 & 75 & 10 & 2 & 0 & 2.3 & N.O. ${ }^{5}$ & N.O. \\
\hline & 130 & 10 & 2 & 829 & 3.6 & $15.0 \pm 1.0$ & $13.7 \pm 1.4$ & $0.38 \pm 0.05$ \\
\hline & 188 & 10 & 2 & 343 & 2.1 & $29.8 \pm 1.0$ & $12.0 \pm 1.2$ & $2.90 \pm 0.5$ \\
\hline & 250 & 10 & 3 & 733 & 5.3 & $33.0 \pm 1.0$ & $11.8 \pm 1.2$ & $3.35 \pm 0.4$ \\
\hline & 350 & 10 & 2 & 437 & 3.9 & $40.8 \pm 1.0$ & $10.4 \pm 1.0$ & $6.00 \pm 0.7$ \\
\hline & 375 & 10 & 2 & 549 & 2.8 & $39.9 \pm 1.0$ & $14.1 \pm 1.4$ & $8.36 \pm 1.0$ \\
\hline & 450 & 10 & 3 & 742 & 5.5 & $45.1 \pm 1.0$ & $11.9 \pm 1.2$ & $8.81 \pm 1.1$ \\
\hline & 550 & 10 & 3 & 396 & 4.1 & $55.2 \pm 1.0$ & $8.4 \pm 0.8$ & $13.3 \pm 1.6$ \\
\hline & 650 & 10 & 3 & 502 & 5.3 & $59.8 \pm 1.0$ & $7.1 \pm 0.7$ & $16.0 \pm 2.0$ \\
\hline
\end{tabular}

${ }^{5}$ N.O.: Not observed 
Table 5.4: Summary of $\mathrm{G}$ phase and $\mathrm{M}_{2} \mathrm{X}$ behavior from damage dependence experiment performed at $460^{\circ} \mathrm{C}$.

\begin{tabular}{|c|c|c|c|c|c|c|c|c|c|c|}
\hline \multicolumn{2}{|c|}{$\begin{array}{c}\text { Experimental } \\
\text { Conditions } \\
\end{array}$} & \multicolumn{3}{|c|}{ G Phase } & \multicolumn{6}{|c|}{$\mathrm{M}_{2} \mathrm{X}$} \\
\hline $\begin{array}{l}\text { Temperature } \\
\left({ }^{\circ} \mathrm{C}\right)\end{array}$ & $\begin{array}{c}\text { Damage } \\
\text { (dpa) }\end{array}$ & $\begin{array}{l}\text { Diameter } \\
(\mathrm{nm})\end{array}$ & $\begin{array}{l}\text { Number } \\
\text { Density } \\
\left(10^{20} \mathrm{~m}^{-3}\right)\end{array}$ & $\begin{array}{l}\text { Volume } \\
\text { Fraction } \\
\quad(\%)\end{array}$ & \# of ppts & $\begin{array}{l}\text { Length } \\
(\mathrm{nm})\end{array}$ & $\begin{array}{l}\text { Width } \\
(\mathrm{nm})\end{array}$ & $\begin{array}{c}\text { Thickness } \\
\text { (nm) }\end{array}$ & $\begin{array}{l}\text { Number } \\
\text { Density } \\
\left(10^{20} \mathrm{~m}^{-3}\right)\end{array}$ & $\begin{array}{c}\text { Volume } \\
\text { Fraction } \\
\quad(\%)\end{array}$ \\
\hline \multirow[t]{9}{*}{460} & 75 & $7.9 \pm 0.8$ & $2.4 \pm 0.2$ & $0.060 \pm 0.01$ & N.O. & N.O. & N.O. & N.O. & N.O. & N.O. \\
\hline & 130 & $7.2 \pm 0.7$ & $2.5 \pm 0.3$ & $0.05 \pm 0.008$ & N.O. & N.O. & N.O. & N.O. & N.O. & N.O. \\
\hline & 188 & $11.0 \pm 1.1$ & $7.9 \pm 0.8$ & $0.052 \pm 0.01$ & N.O. & N.O. & N.O. & N.O. & N.O. & N.O. \\
\hline & 250 & $13.2 \pm 1.3$ & $10.5 \pm 1.0$ & $0.132 \pm 0.02$ & 26 & $50.3 \pm 5.0$ & $12.2 \pm 1.2$ & $10.0 \pm 1.0$ & $8.0 \pm 0.8$ & $0.5 \pm 0.07$ \\
\hline & 350 & $12.3 \pm 1.2$ & $7.4 \pm 0.7$ & $0.072 \pm 0.02$ & 47 & $65.2 \pm 6.5$ & $19.6 \pm 2.0$ & $10.1 \pm 1.0$ & $11.9 \pm 1.2$ & $1.6 \pm 0.3$ \\
\hline & 375 & $12.0 \pm 1.2$ & $9.4 \pm 0.9$ & $0.077 \pm 0.02$ & O. ${ }^{6}$ & O. & $\mathrm{O}$ & O. & $\mathrm{O}$. & $\mathrm{O}$ \\
\hline & 450 & $12.0 \pm .12$ & $8.2 \pm 0.8$ & $0.19 \pm 0.03$ & 65 & $93.6 \pm 9.4$ & $20.5 \pm 2.1$ & $15.0 \pm 1.5$ & $15.9 \pm 1.6$ & $4.8 \pm 0.7$ \\
\hline & 550 & $15.3 \pm 1.5$ & $9.9 \pm 1.0$ & $0.23 \pm 0.03$ & 51 & $89.1 \pm 8.9$ & $21.0 \pm 2.1$ & $17.3 \pm 1.7$ & $8.7 \pm 0.87$ & $2.8 \pm 0.4$ \\
\hline & 650 & $19.4 \pm 2.0$ & $6.6 \pm 0.7$ & $0.29 \pm 0.04$ & 46 & $99.4 \pm 10.0$ & $22.4 \pm 2.2$ & $19.1 \pm 1.9$ & $7.5 \pm 0.75$ & $3.2 \pm 0.5$ \\
\hline
\end{tabular}

\footnotetext{
${ }^{6}$ O. Observed-not quantified
} 
Table 5.5: Summary of dislocation microstructure data from damage dependence study performed at $460^{\circ} \mathrm{C}$.

\begin{tabular}{|c|c|c|c|c|c|c|c|}
\hline $\begin{array}{c}\text { Temperature } \\
\left({ }^{\circ} \mathrm{C}\right)\end{array}$ & $\begin{array}{c}\text { Damage } \\
(\mathrm{dpa})\end{array}$ & $\begin{array}{c}\text { Number of } \\
\text { Loops Counted }\end{array}$ & $\begin{array}{c}\text { Loop } \\
\text { Diameter }(\mathrm{nm})\end{array}$ & $\begin{array}{c}\text { Dislocation } \\
\text { Loop Density } \\
\left(10^{20} \mathrm{~m}^{-3}\right)\end{array}$ & $\begin{array}{c}\text { Dislocation Loop } \\
\text { Line Length } \\
\left(10^{13} \mathrm{~m}^{-2}\right)\end{array}$ & $\begin{array}{c}\text { Dislocation } \\
\text { Network Line } \\
\text { Length }\left(10^{14} \mathrm{~m}^{-2}\right)\end{array}$ & $\begin{array}{c}\text { Total } \\
\text { Dislocation } \\
\text { Line Length } \\
\left(10^{14} \mathrm{~m}^{-2}\right)\end{array}$ \\
\hline 460 & 130 & 76 & $22 \pm 2$ & $9.7 \pm 2.0$ & $6.7 \pm 1.4$ & $3.0 \pm 0.8$ & $3.7 \pm 0.9$ \\
\hline & 188 & 39 & $30 \pm 3$ & $1.7 \pm 0.3$ & $1.6 \pm 0.3$ & $3.0 \pm 0.8$ & $3.2 \pm 0.9$ \\
\hline & 250 & 51 & $32 \pm 3$ & $4.9 \pm 1.0$ & $5.0 \pm 1.0$ & $3.0 \pm 0.8$ & $3.5 \pm 0.9$ \\
\hline & 350 & 19 & $29 \pm 3$ & $1.8 \pm 0.4$ & $1.7 \pm 0.3$ & $3.0 \pm 0.8$ & $3.2 \pm 0.9$ \\
\hline & 375 & 42 & $23 \pm 2$ & $3.8 \pm 0.8$ & $2.8 \pm 0.4$ & $3.0 \pm 0.8$ & $3.3 \pm 0.9$ \\
\hline & 450 & 22 & $78 \pm 8$ & $2.1 \pm 0.4$ & $5.2 \pm 1.1$ & $3.0 \pm 0.8$ & $3.5 \pm 0.9$ \\
\hline & 550 & 26 & $78 \pm 8$ & $2.4 \pm 0.5$ & $5.8 \pm 1.2$ & $4.0 \pm 0.8$ & $4.6 \pm 1.0$ \\
\hline & 650 & 19 & $92 \pm 9$ & $1.8 \pm 0.4$ & $5.3 \pm 1.1$ & $4.0 \pm 0.9$ & $4.5 \pm 1.0$ \\
\hline
\end{tabular}




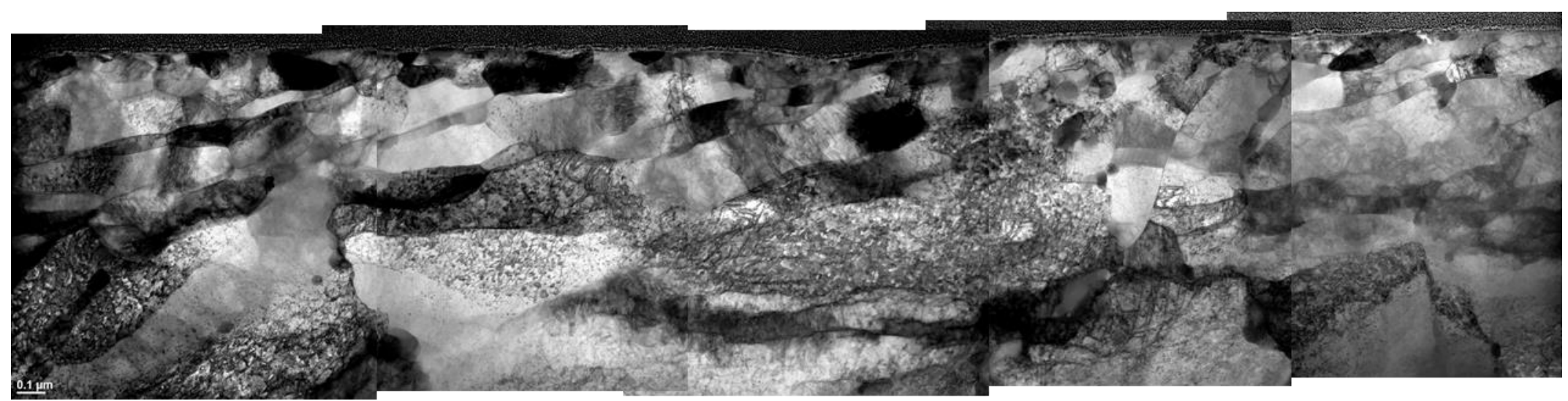

Figure 5.1: As-received HT9 sample prepared via FIB liftout method showing representative microstructure. 


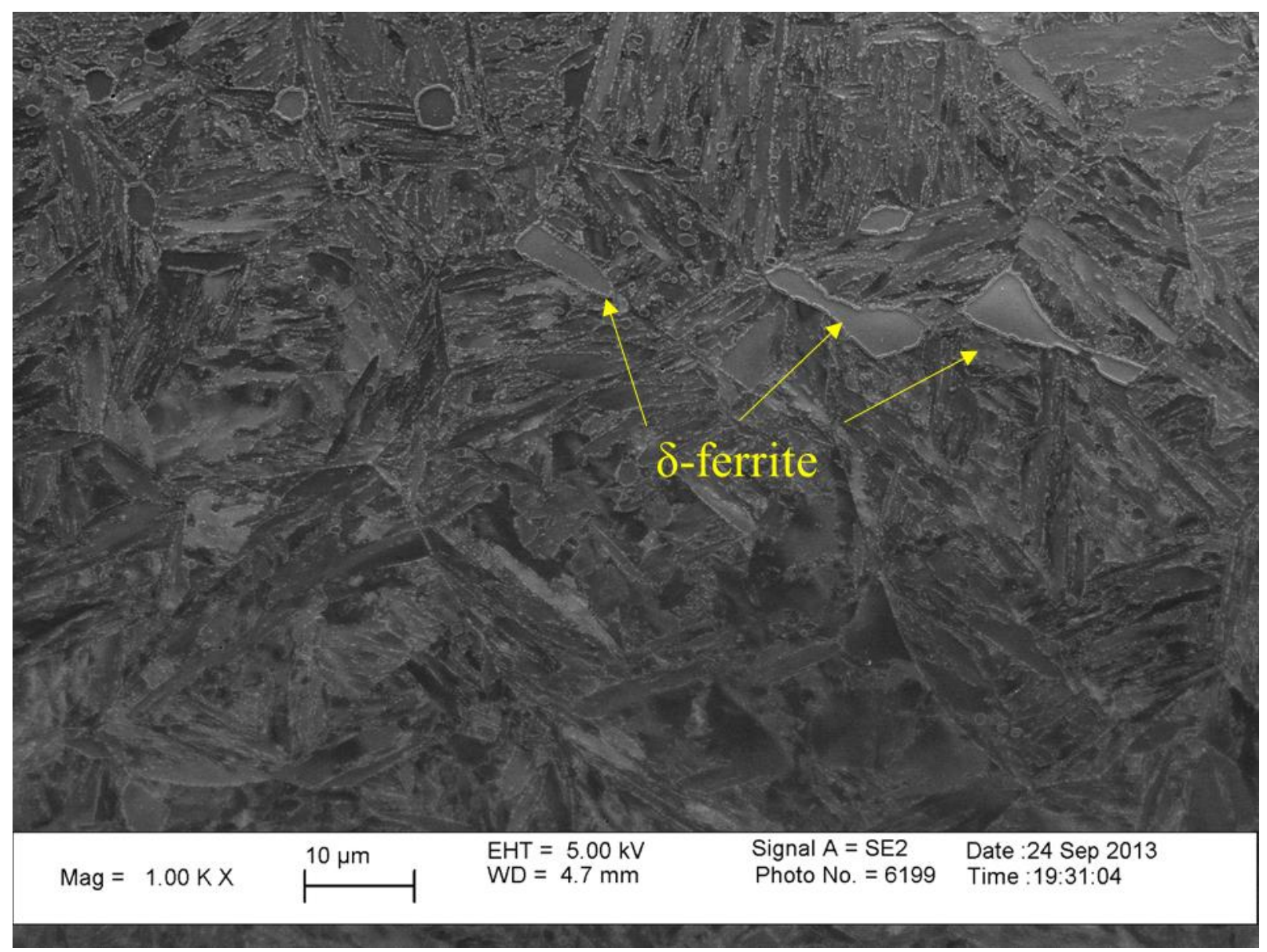

Figure 5.2: Optical micrograph of as-received HT9 after etching. The etchant used was Vilellas's reagent which includes 1 g picric acid with $5 \mathrm{~mL}$ of hydrochloric acid in a solution of $100 \mathrm{~mL}$ [63]. 


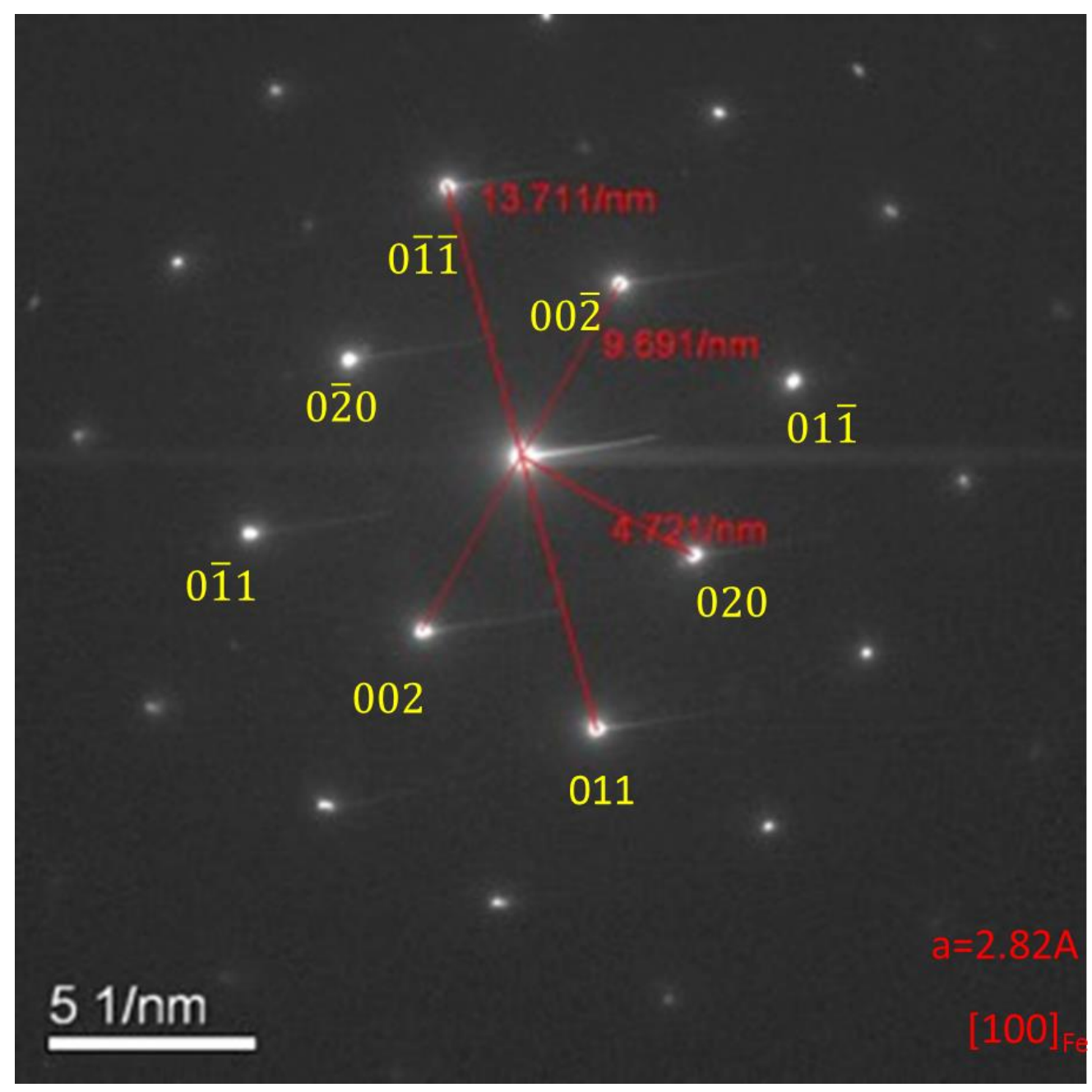

Figure 5.3: Indexed diffraction of bec matrix of HT9. 


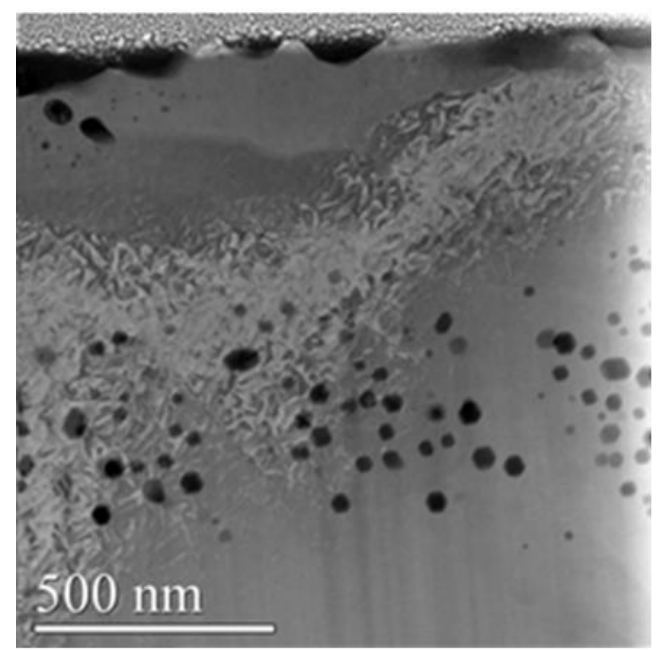

(a)

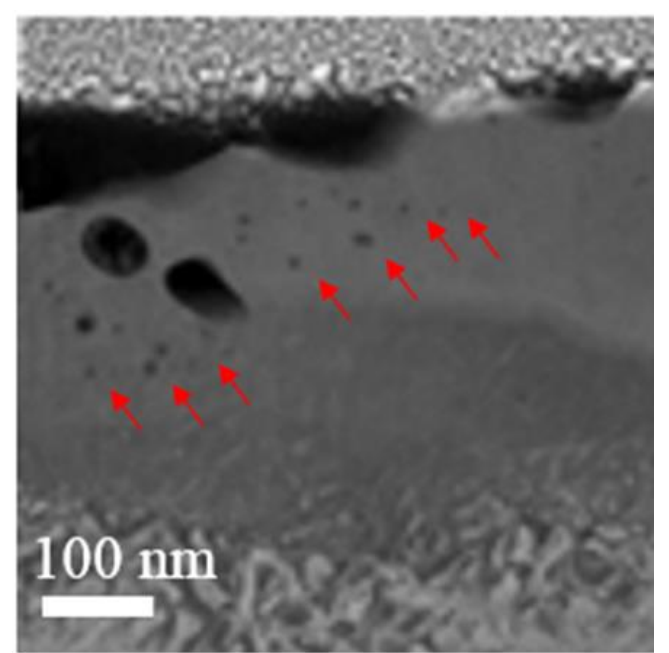

(b)

Figure 5.4: Void formation in the a) irradiated area and b) near surface in HT9 preimplanted with 10 appm $\mathrm{He}$ then irradiated with $5 \mathrm{MeV} \mathrm{Fe}^{++}$to 188 dpa at $440^{\circ} \mathrm{C}$. 


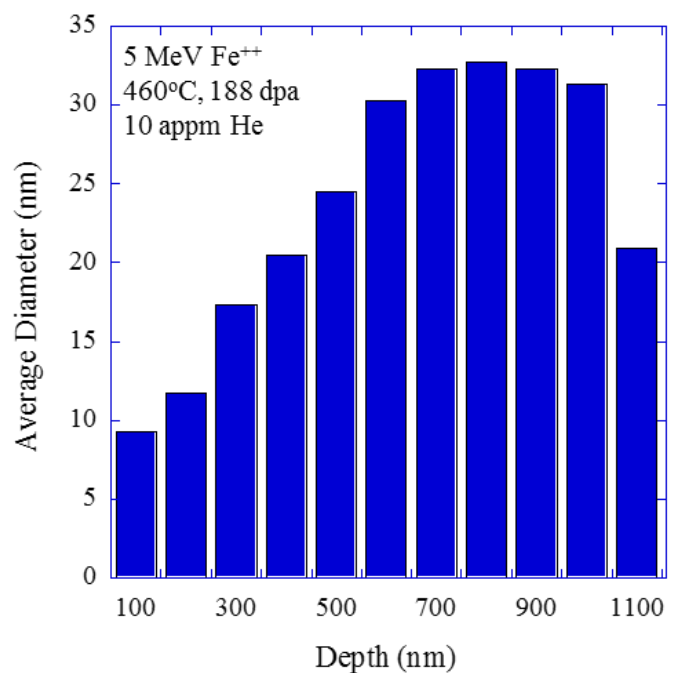

(a)

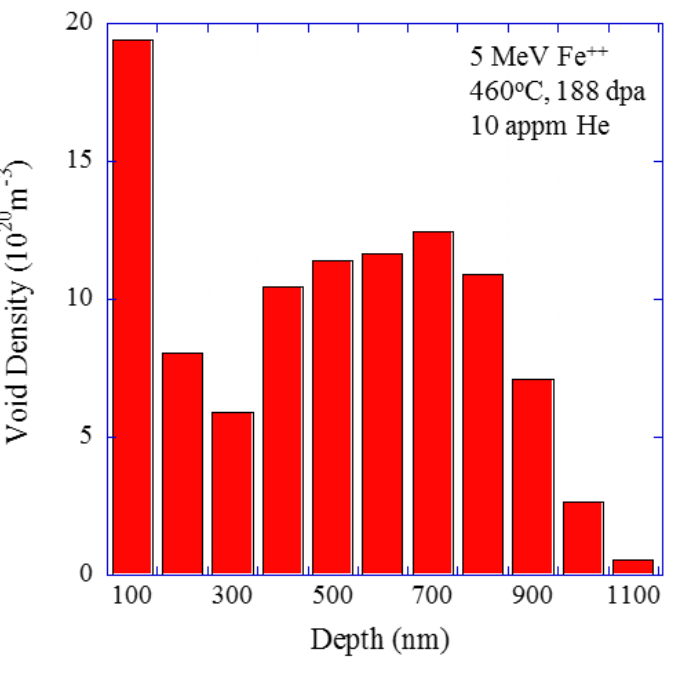

(b)

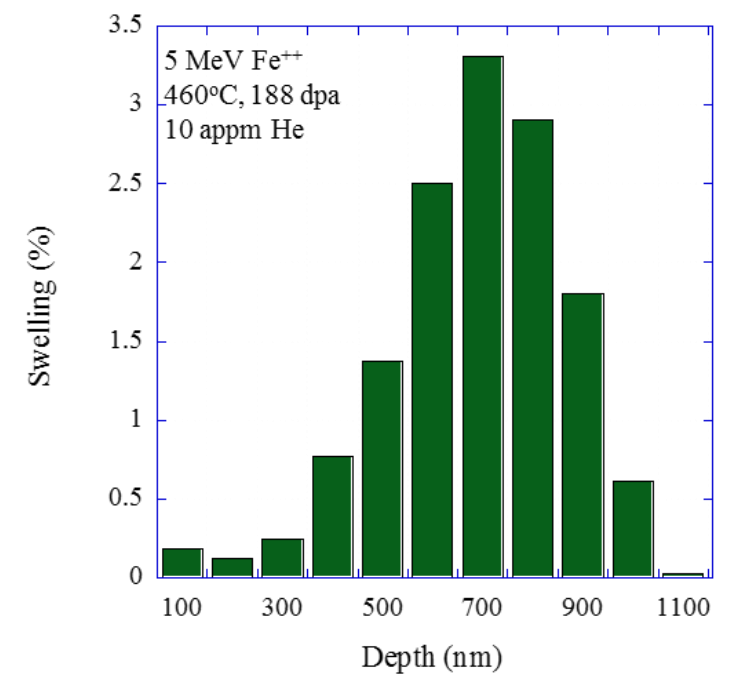

(c)

Figure 5.5: Void a) diameter b) number density c) and swelling profiles with depth in HT9 preimplanted with 10 appm He then irradiated with $5 \mathrm{MeV} \mathrm{Fe}^{++}$to 188 dpa at $460^{\circ} \mathrm{C}$. 


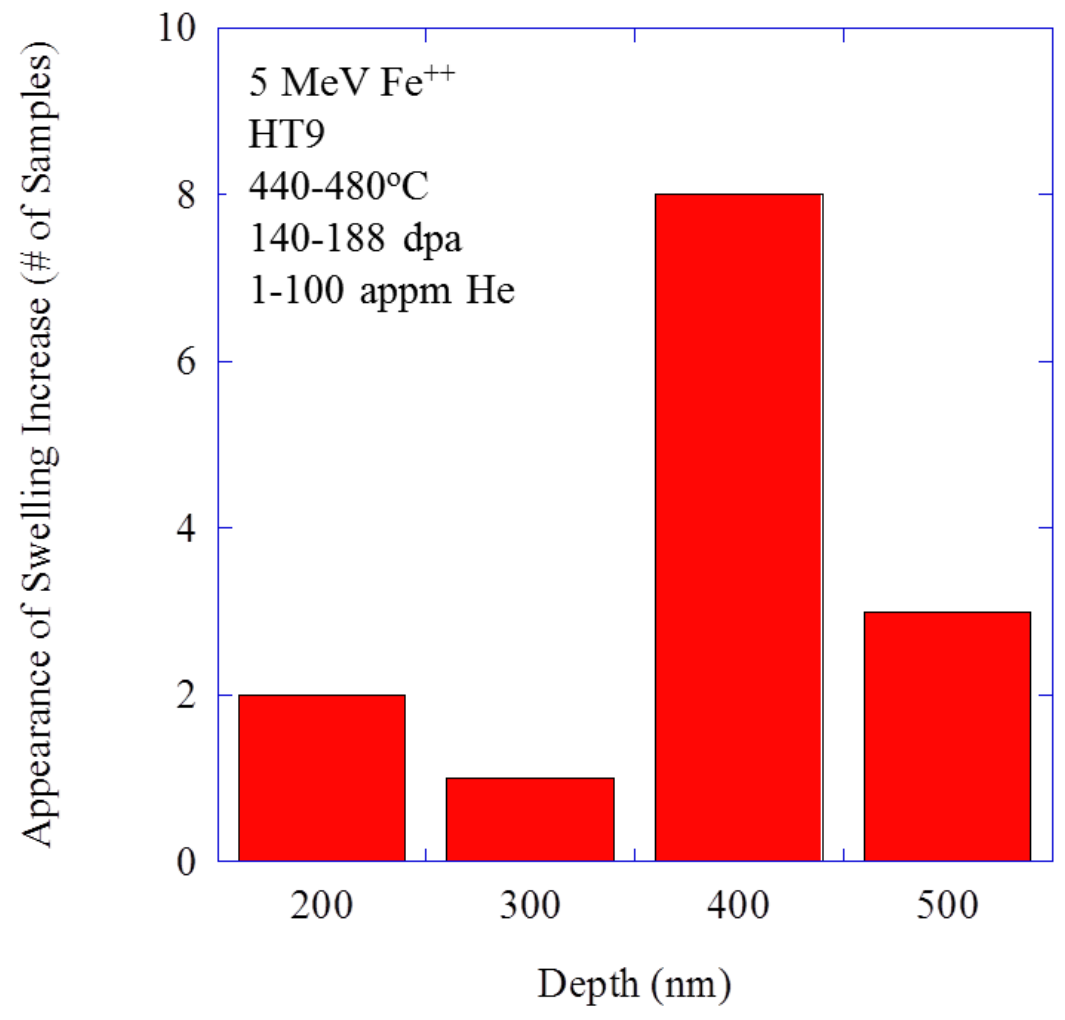

Figure 5.6: Determination of location of onset of swelling increase after swelling peak near the surface (from [12]). 


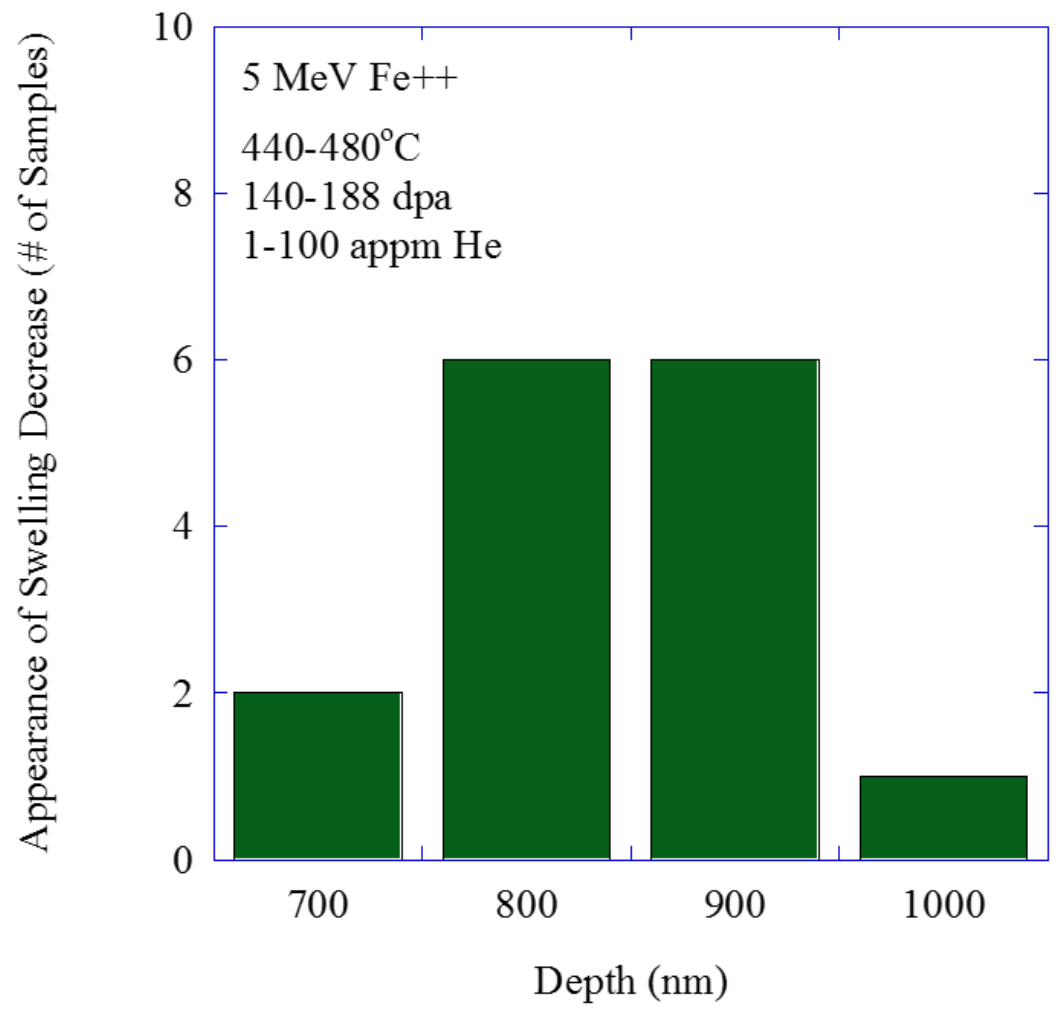

Figure 5.7: Determination of location of onset of in swelling decrease at depths of $700 \mathrm{~nm}$ or greater (from [12]). 


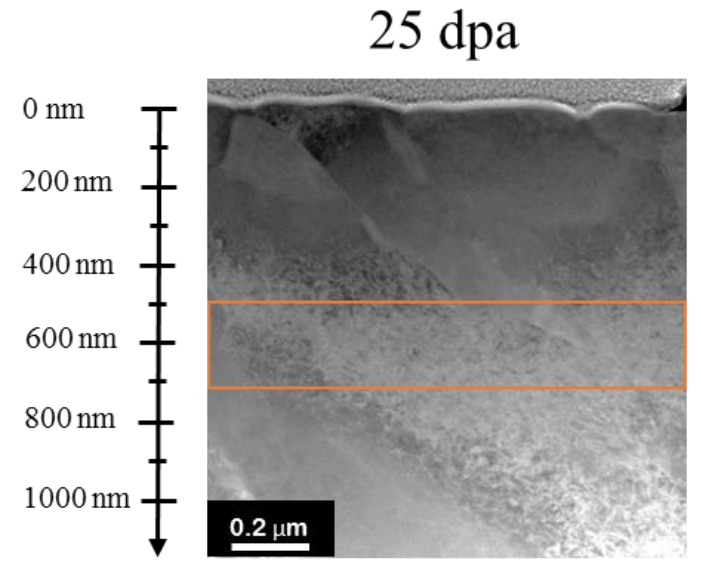

(a)

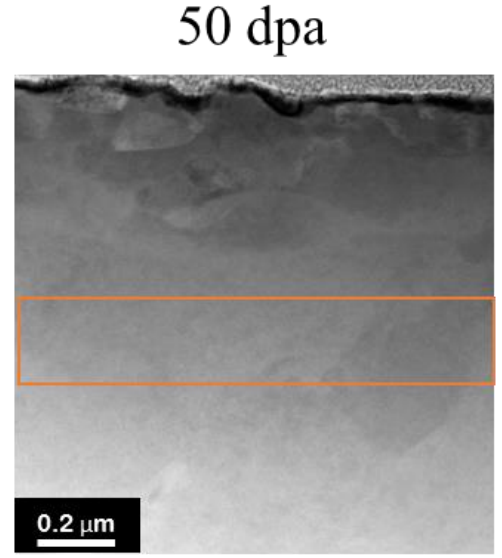

(b)

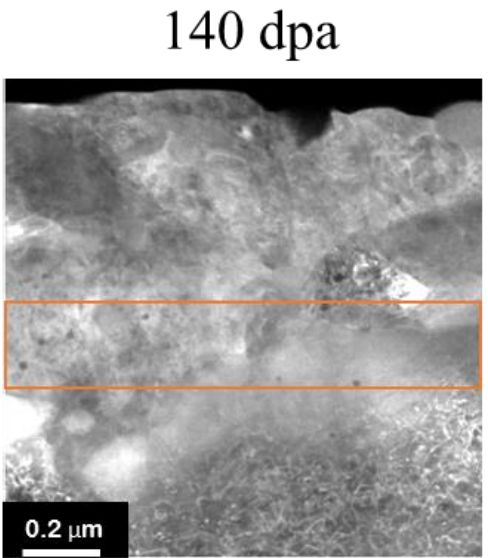

(c)

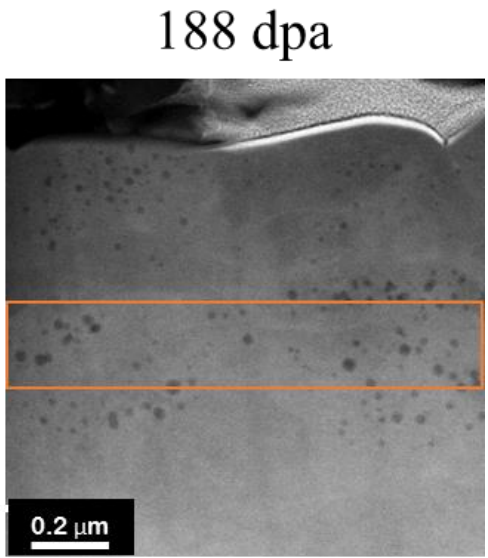

(d)

Figure 5.8: HAADF images of HT9 preimplanted with 10 appm He then irradiated with $5 \mathrm{MeV} \mathrm{Fe}^{++}$at $440^{\circ} \mathrm{C}$ to a) 25 dpa b) $50 \mathrm{dpa} \mathrm{c)} 140 \mathrm{dpa}$ and d) $188 \mathrm{dpa}$ 


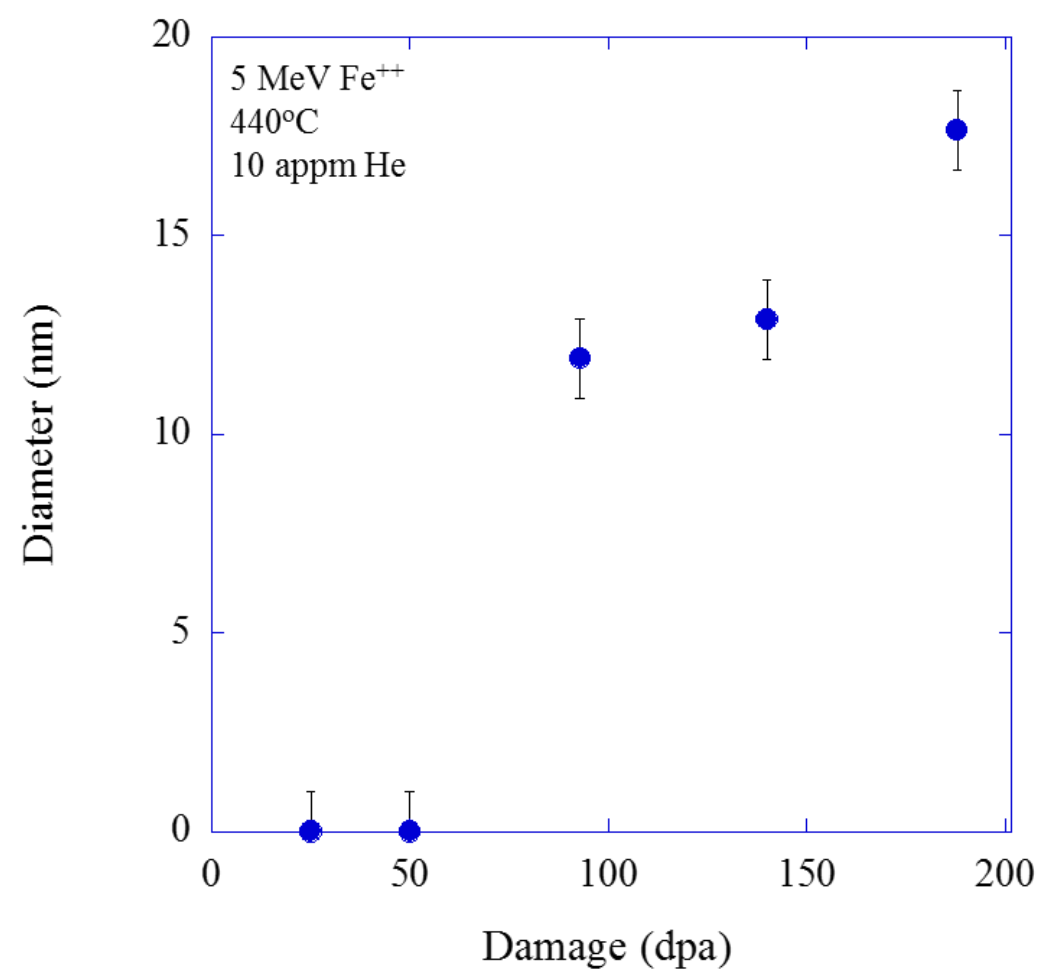

(a)

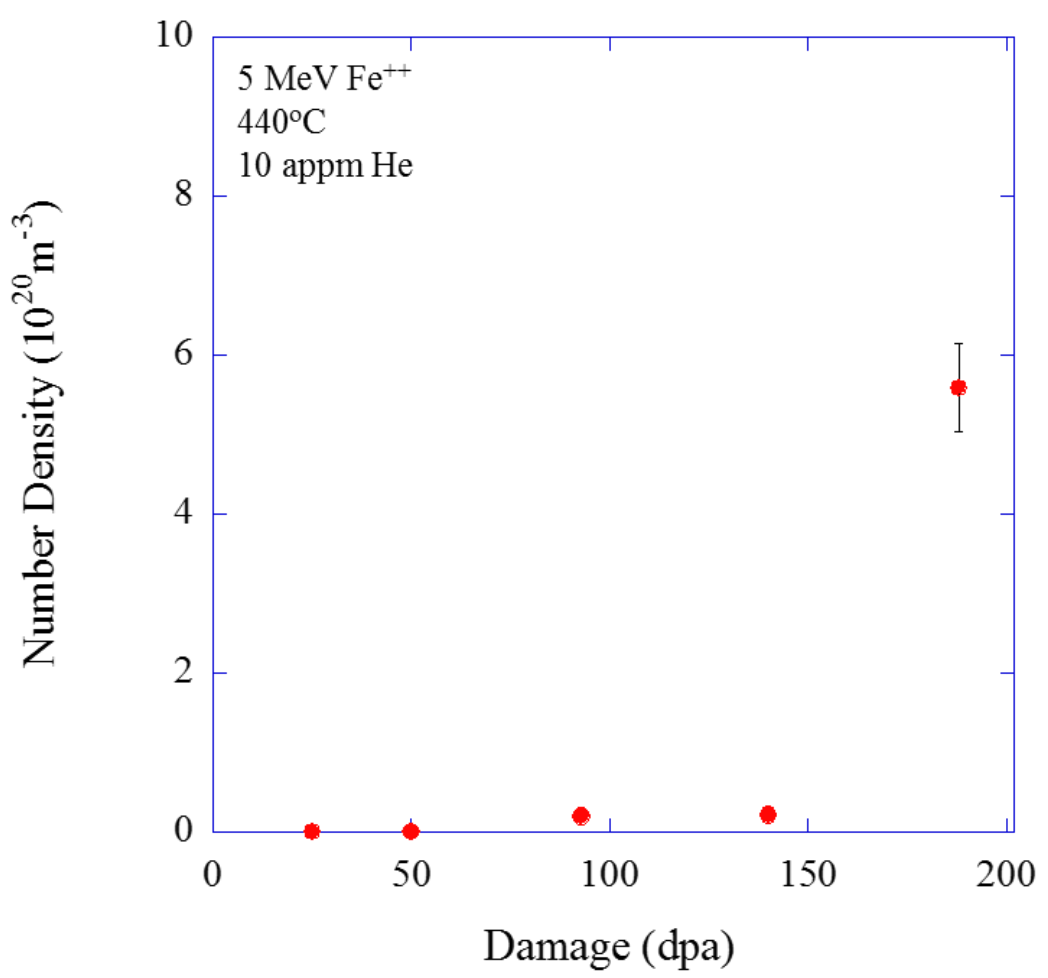

(b)

Figure 5.9: a) Diameter and b) number density of voids in HT9 preimplanted with 10 appm He then irradiated with 5 $\mathrm{MeV} \mathrm{Fe}{ }^{++}$at $440^{\circ} \mathrm{C}$ up to $188 \mathrm{dpa}$. 


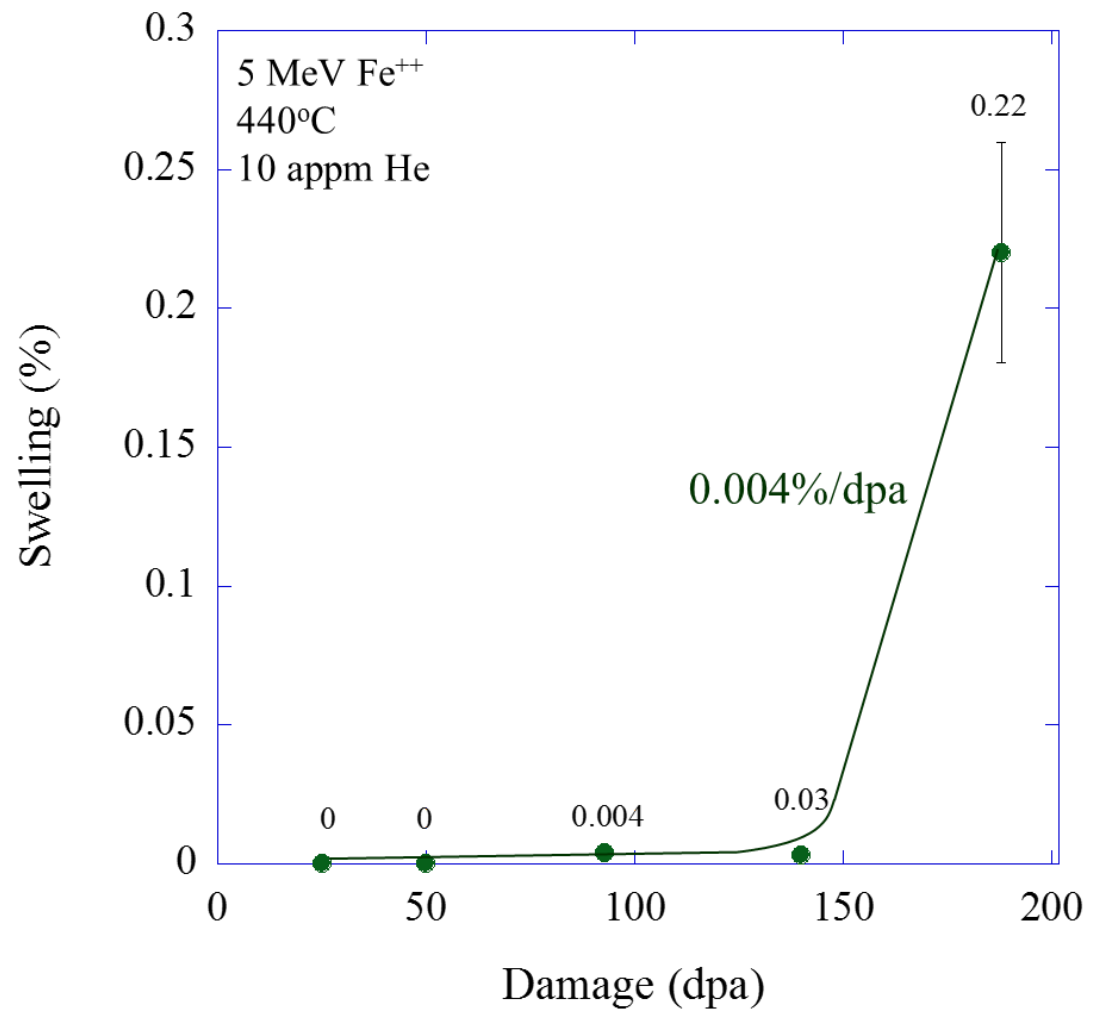

Figure 5.10: Void swelling in HT9 preimplanted with 10 appm He then irradiated with $5 \mathrm{MeV} \mathrm{Fe}^{++}$at $440^{\circ} \mathrm{C}$ up to $188 \mathrm{dpa}$. Swelling values are indicated above the data points. 


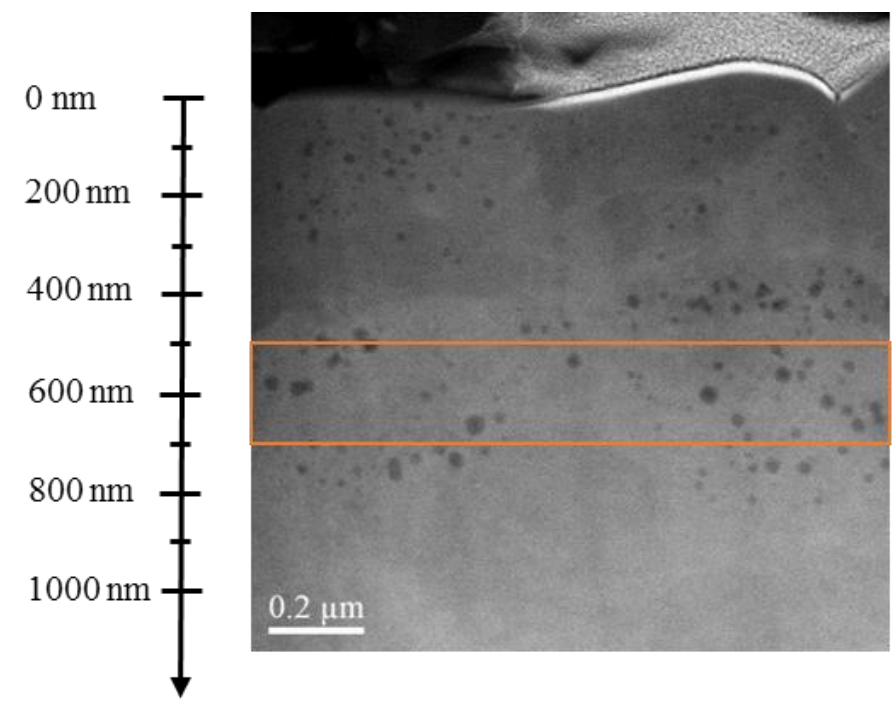

(a)

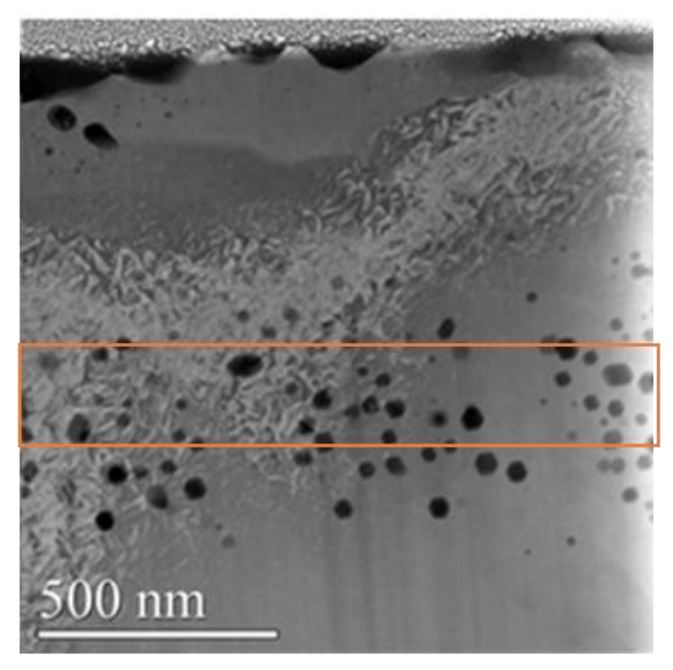

(b)

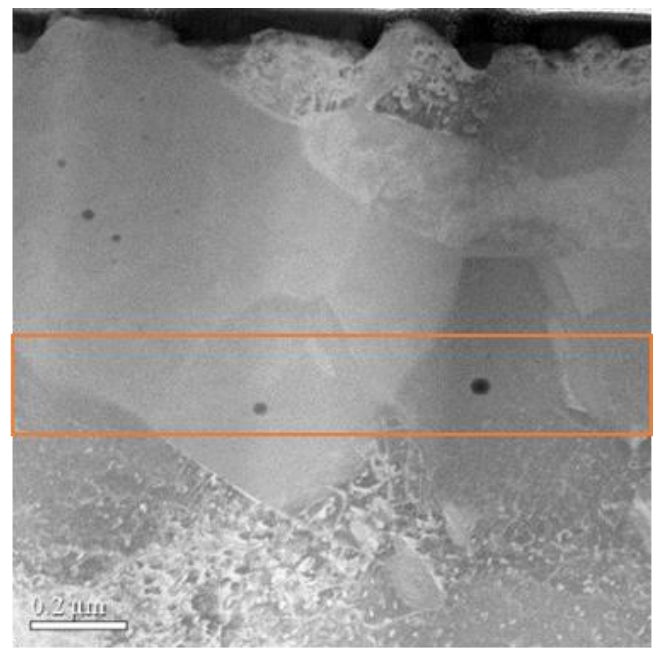

(c)

Figure 5.11: HAADF images of HT9 preimplanted with 10 appm He then irradiated with $5 \mathrm{MeV} \mathrm{Fe}^{++}$at a) $440{ }^{\circ} \mathrm{C}$ b) $460{ }^{\circ} \mathrm{C}$ and c) $480^{\circ} \mathrm{C}$ to $188 \mathrm{dpa}$. 


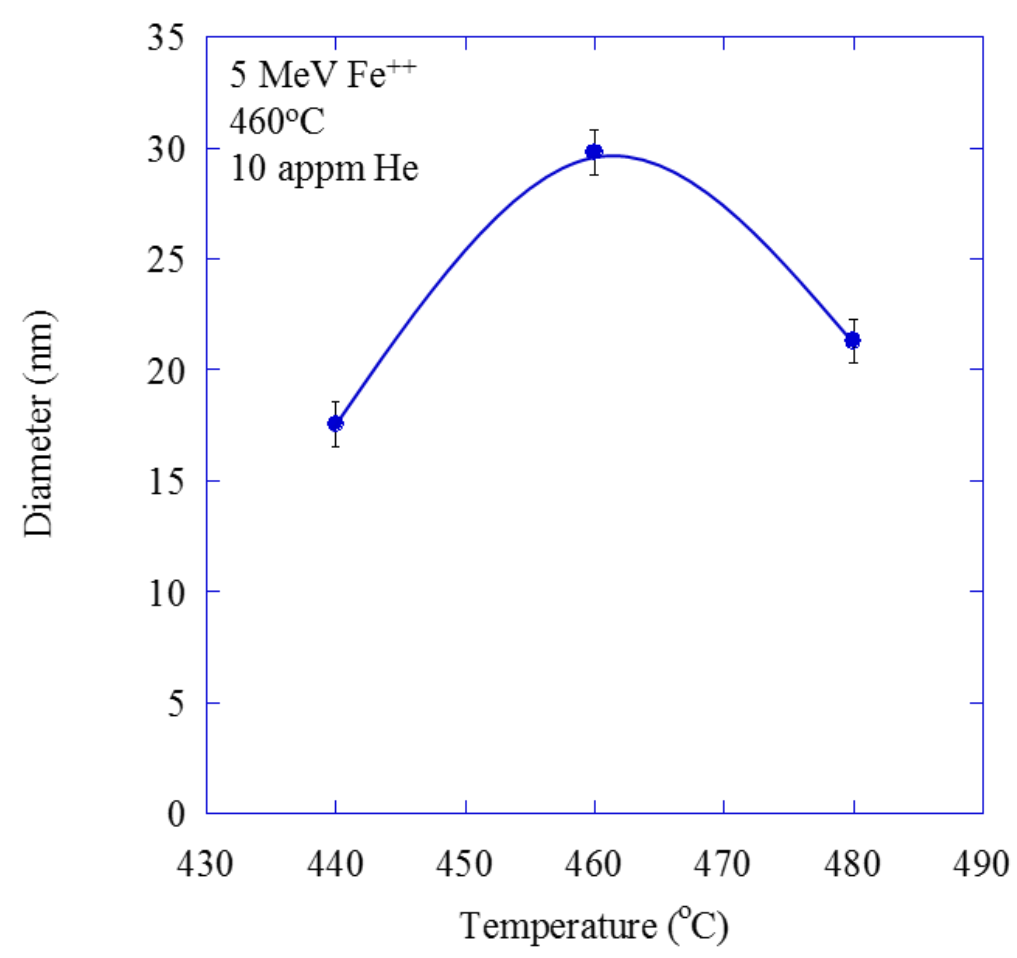

(a)

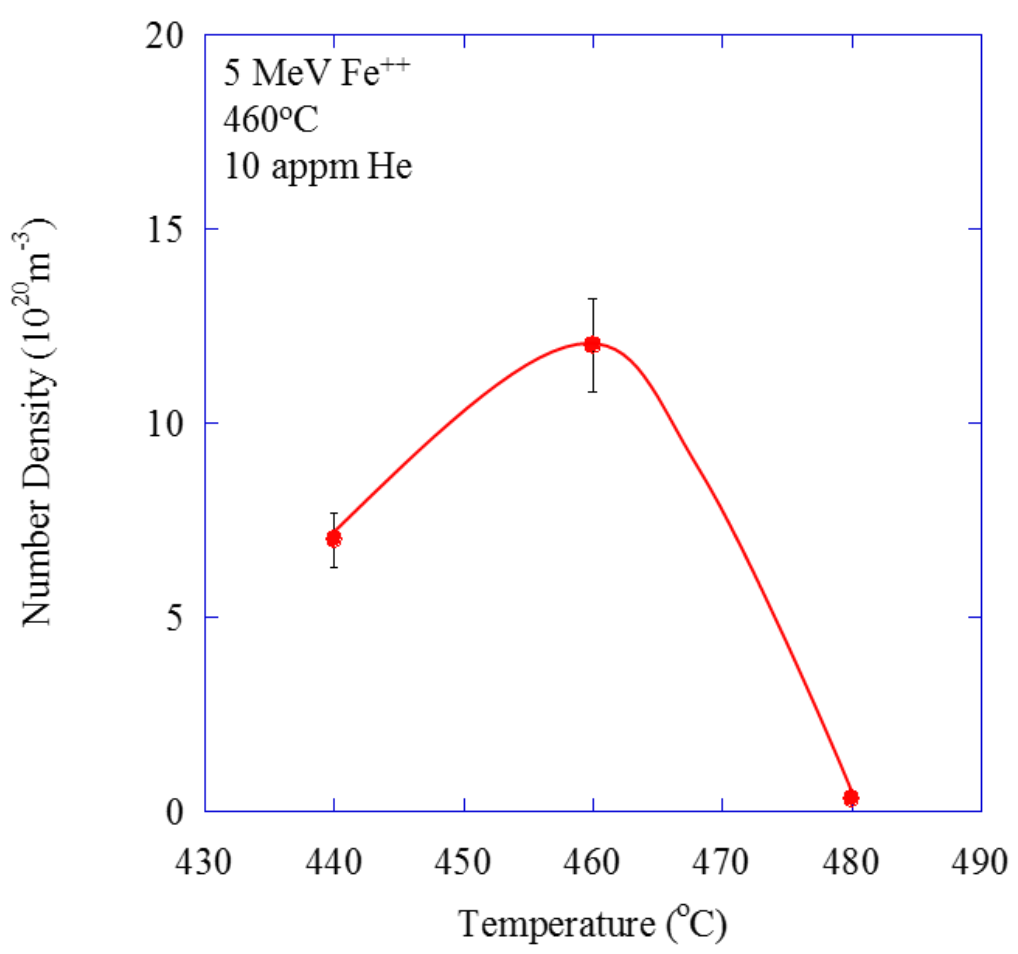

(b)

Figure 5.12: Void a) diameter and b) number density (red) in HT9 preimplanted with 10 appm He then irradiated with 5 $\mathrm{MeV} \mathrm{Fe}{ }^{++}$to 188 dpa between 440 to $480^{\circ} \mathrm{C}$. 


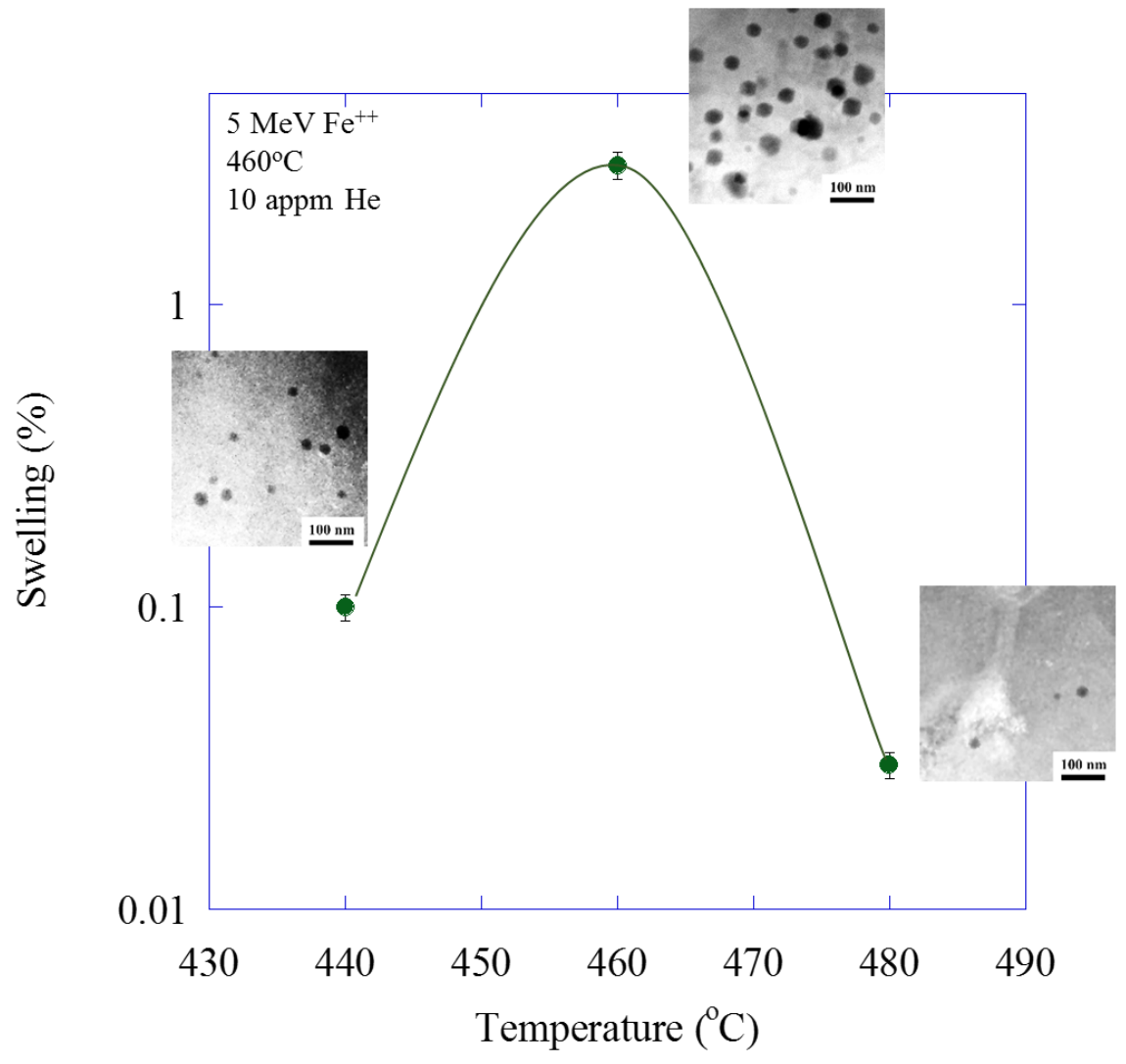

Figure 5.13: Swelling in HT9 preimplanted with 10 appm He then irradiated with $5 \mathrm{MeV} \mathrm{Fe}^{++}$to 188 dpa between 440 and $480^{\circ} \mathrm{C}$. Micrographs of typical voids are included for each temperature. 


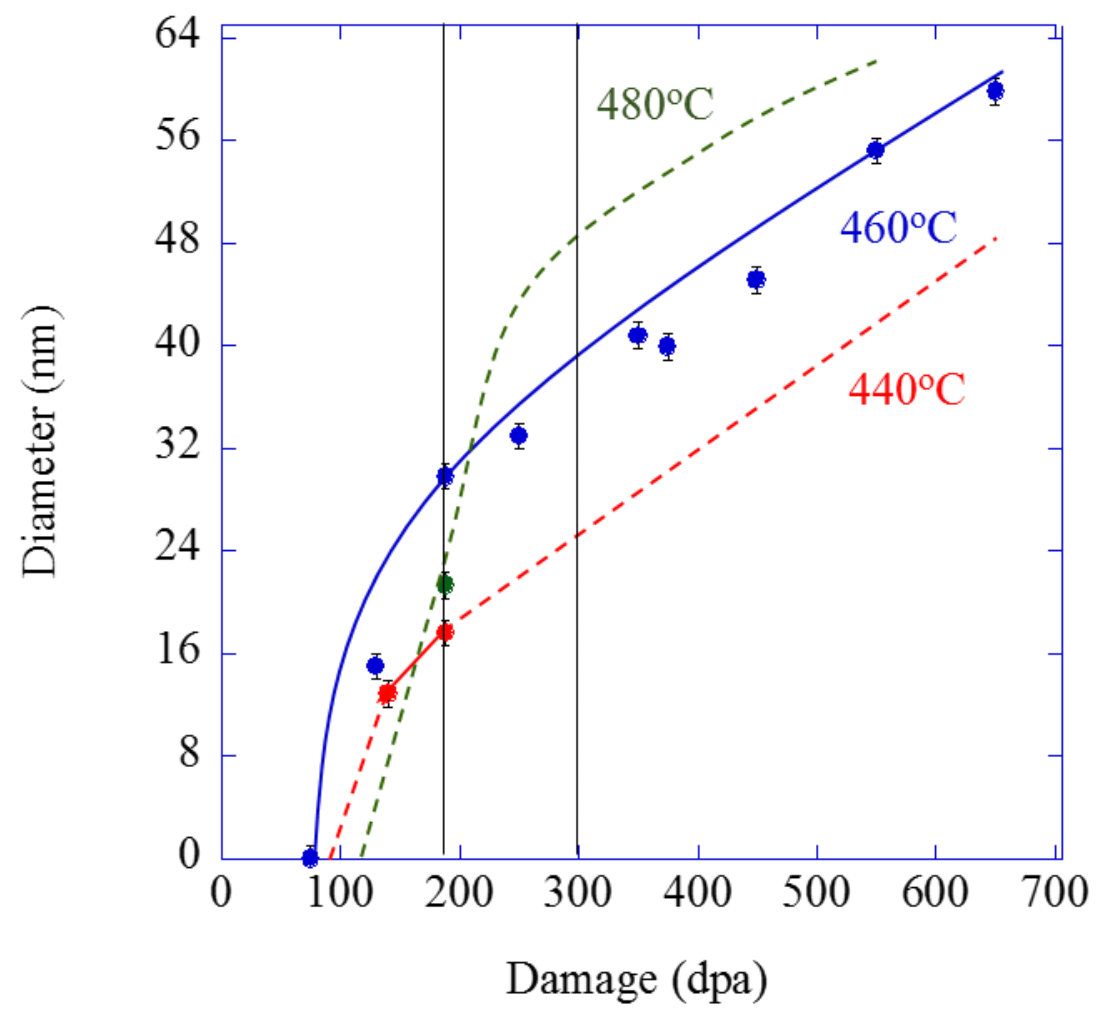

(a)

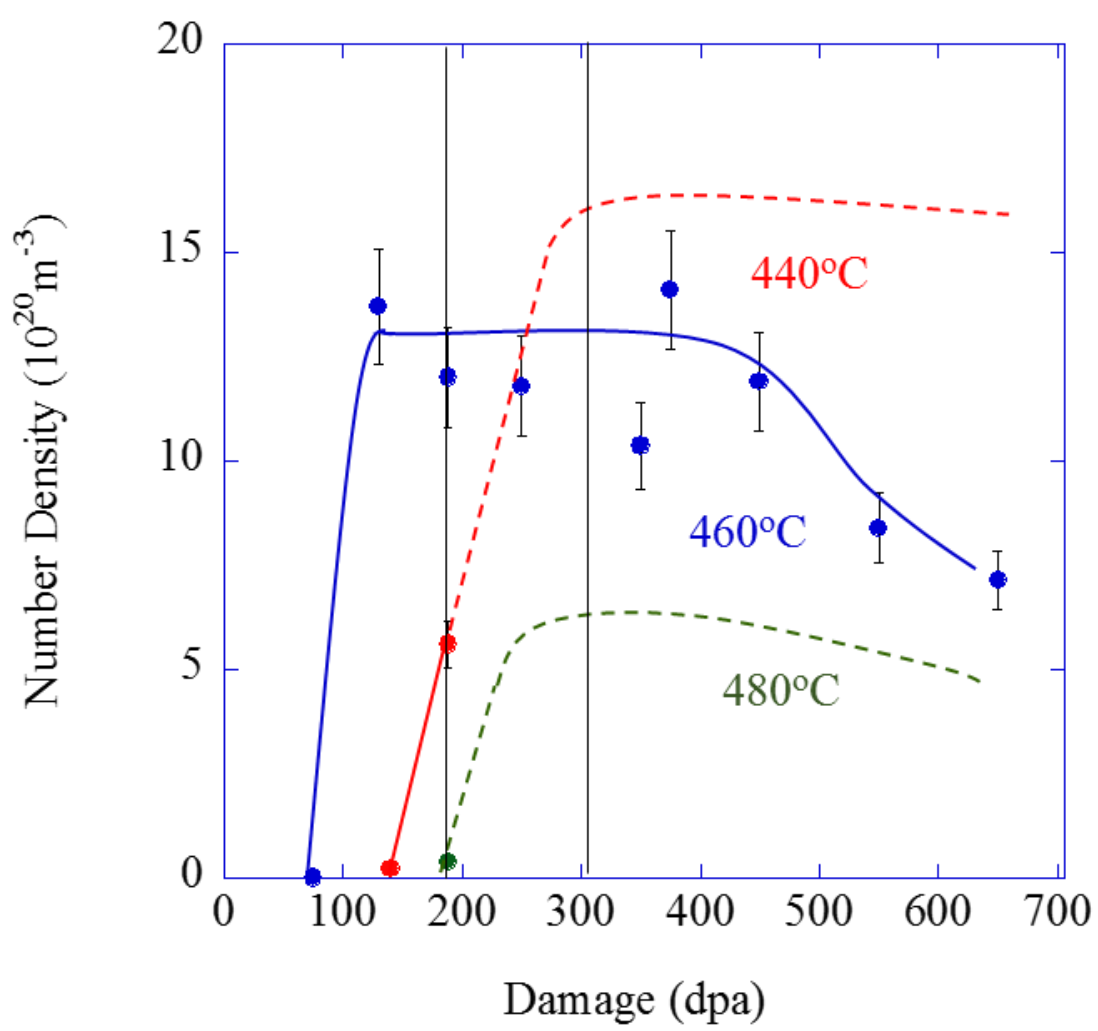

(b)

Figure 5.14: a) Diameter and b) number density as a function of damage for $440^{\circ} \mathrm{C}$ (red), $460^{\circ} \mathrm{C}$ (blue) and $480^{\circ} \mathrm{C}$ (green). Dashed lines extrapolated expected damage evolution at $440^{\circ} \mathrm{C}$ and $480^{\circ} \mathrm{C}$ resolving peak diameter and number density observed at $460^{\circ} \mathrm{C}, 188 \mathrm{dpa}$. At $300 \mathrm{dpa}$, it is expected that diameter will increase with temperature and number density will decrease as is predicted by theory since all three will be in void growth-dominated regime. 


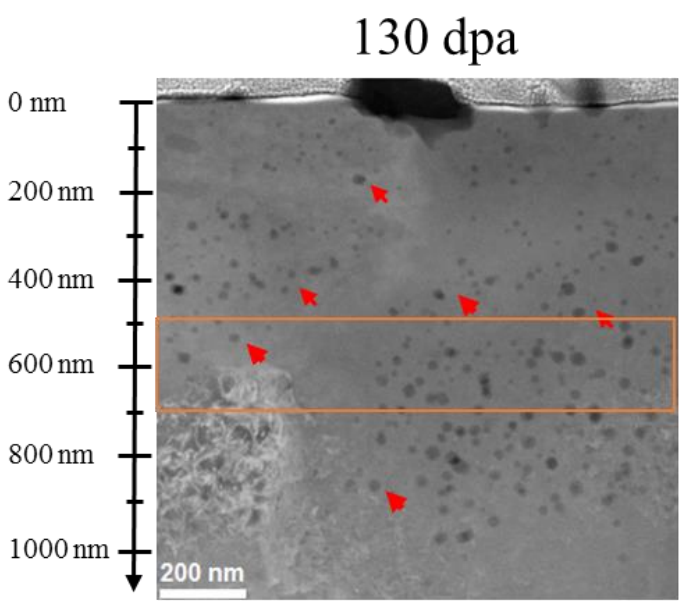

(a)

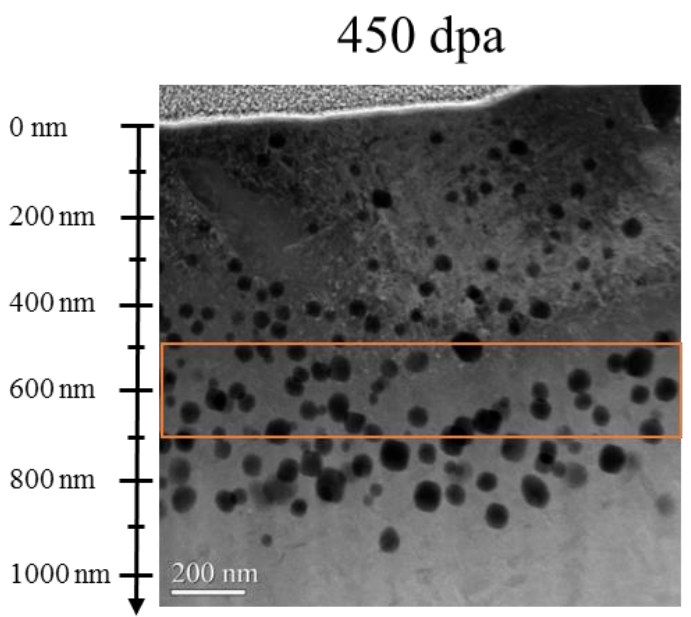

(d)

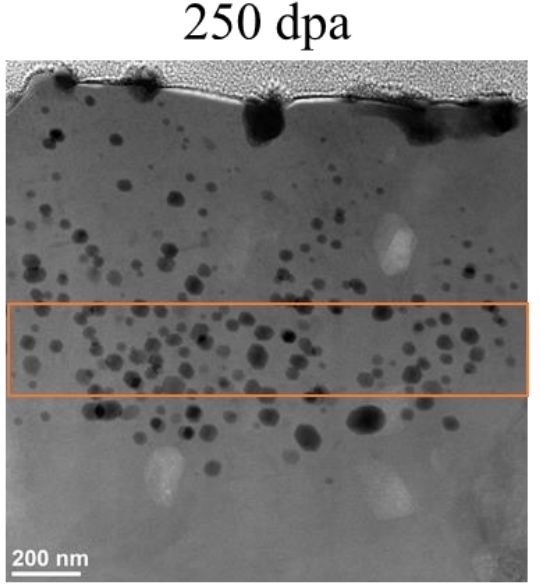

(b)

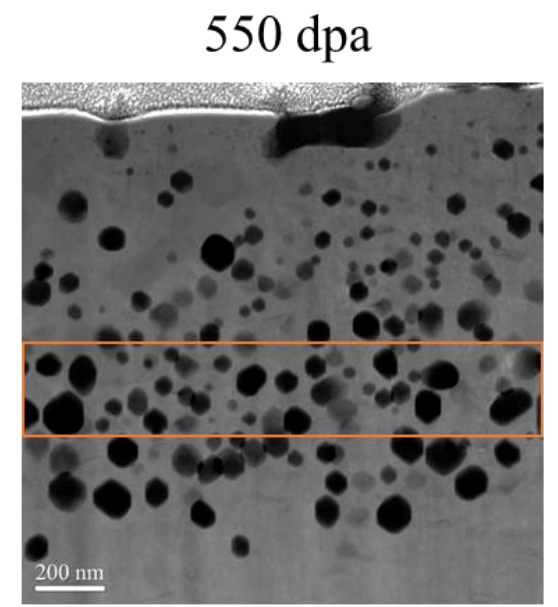

(e)

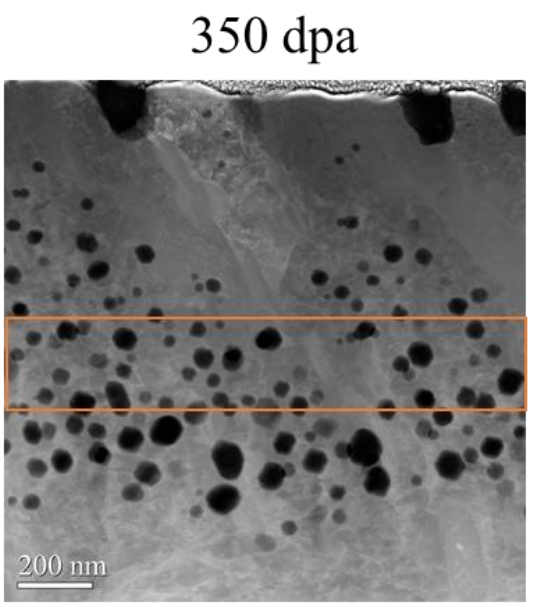

(c)

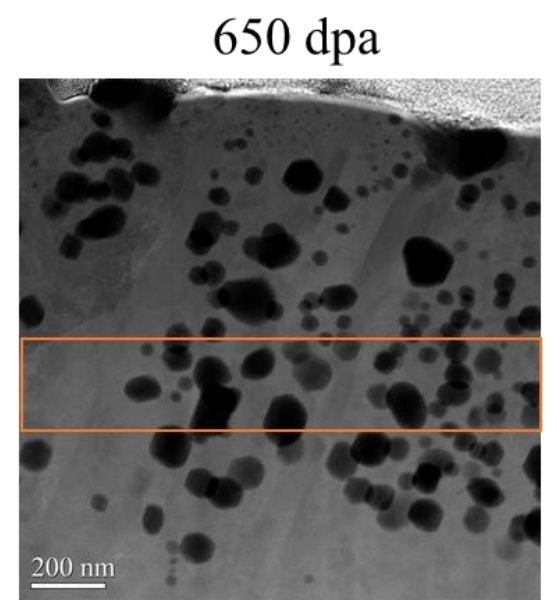

(f)

Figure 5.15: Void evolution as a function of damage in HT9 irradiated with $5 \mathrm{MeV} \mathrm{Fe}^{++}$at $460^{\circ} \mathrm{C}$ after preimplantation with 10 appm He. Voids are the dark circles and marked with red arrows. Orange rectangles denote the 500 to $700 \mathrm{~nm}$ region. 


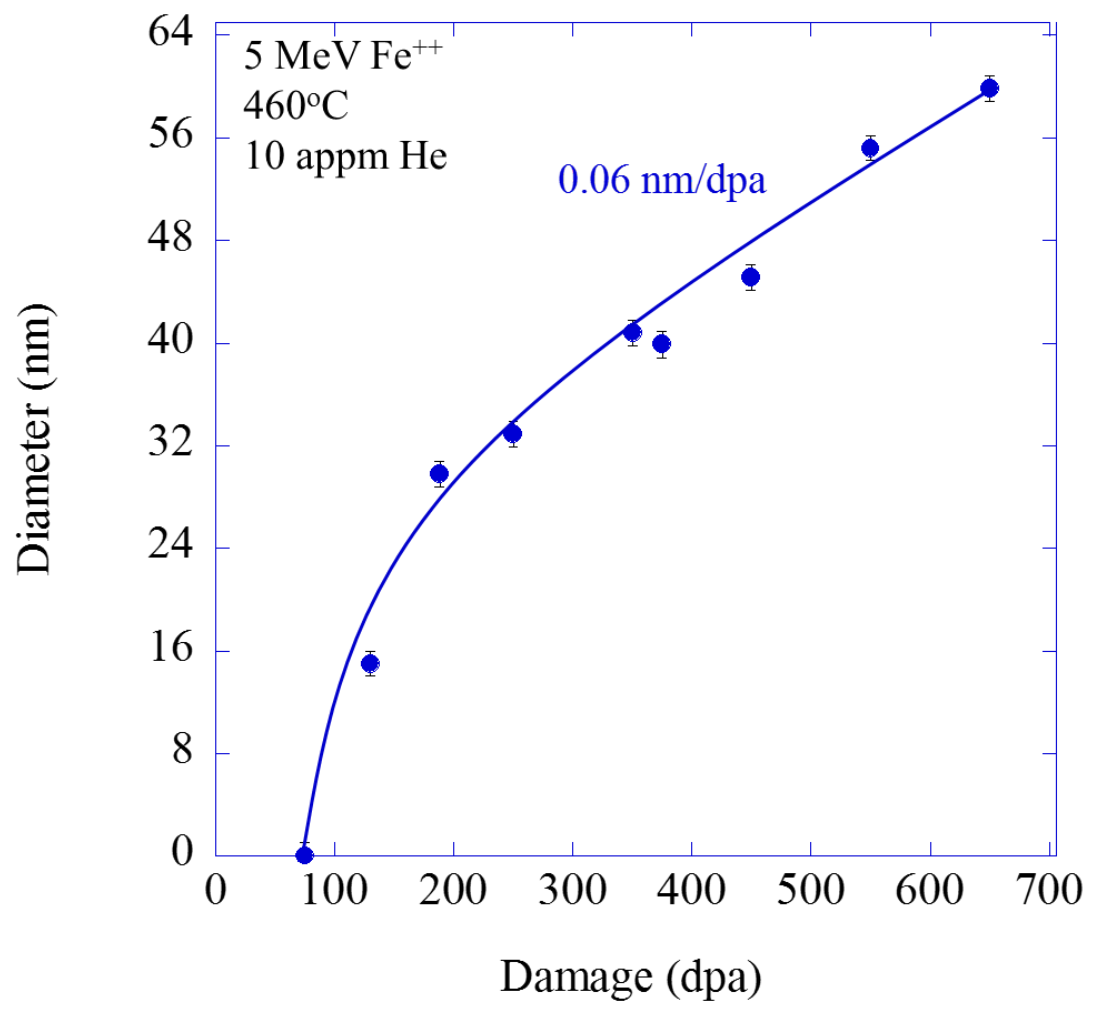

Figure 5.16: Void diameter as a function of damage in HT9 irradiated with $\mathrm{Fe}^{++}$at $460^{\circ} \mathrm{C}$ with 10 appm He preimplanted. 


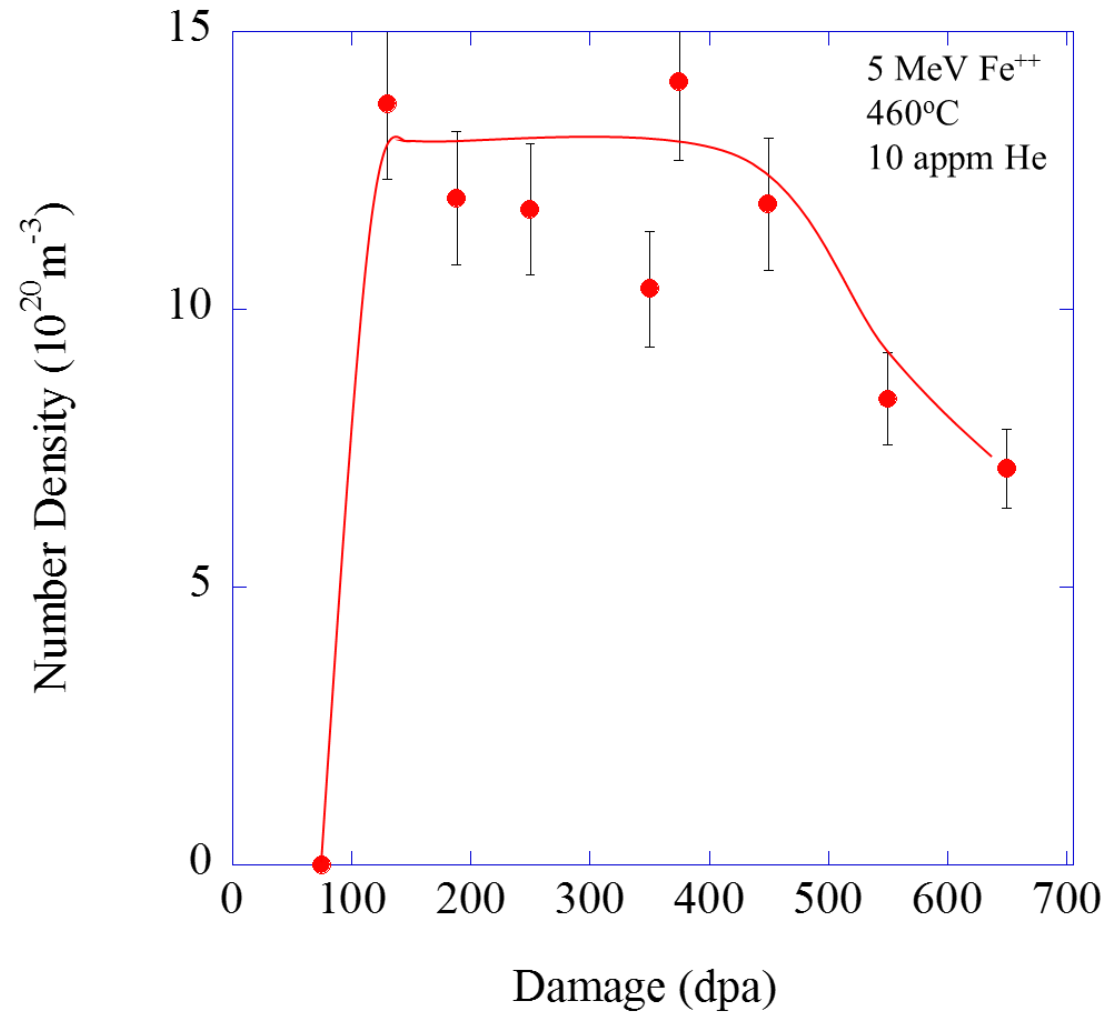

Figure 5.17: Void number density as a function of damage in HT9 irradiated with $\mathrm{Fe}^{++}$at $460^{\circ} \mathrm{C}$ with 10 appm He preimplanted. 


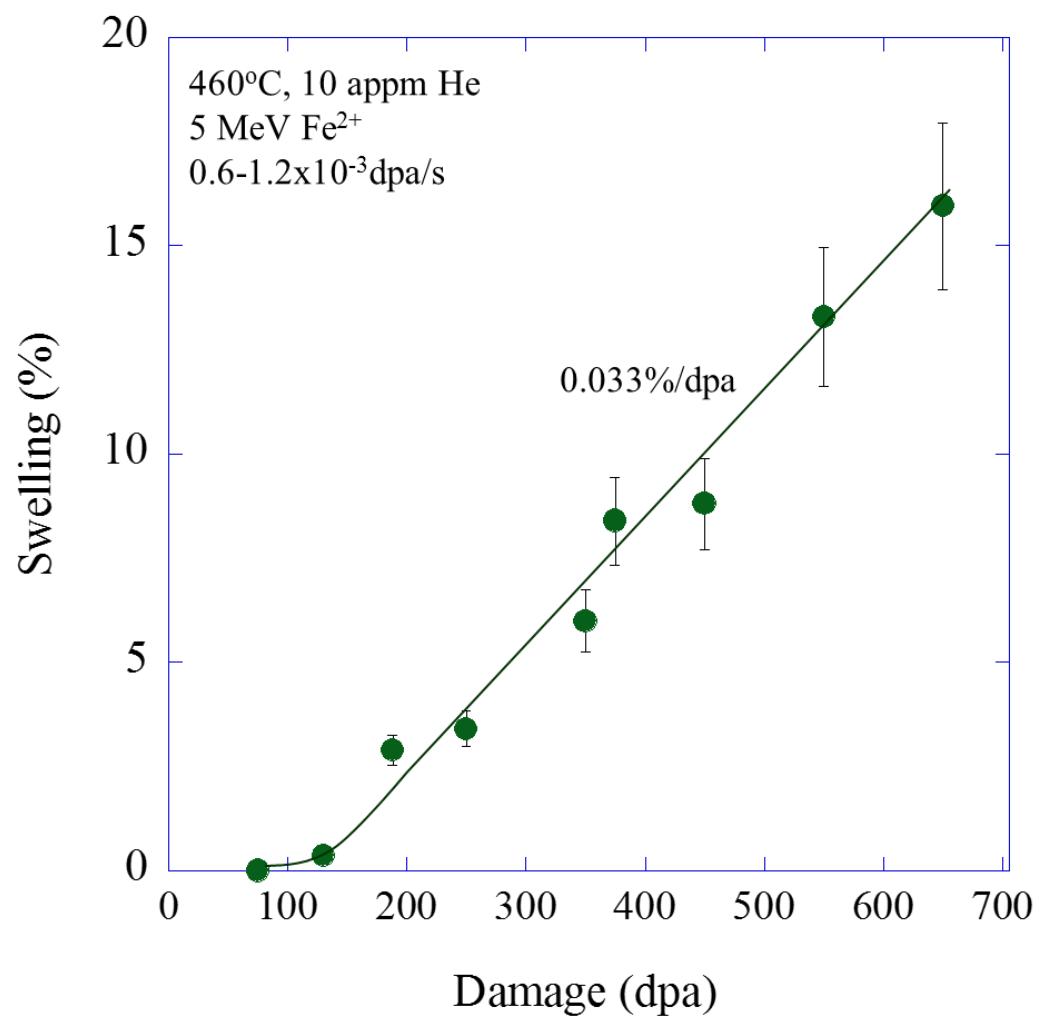

Figure 5.18: Void swelling as a function of damage in HT9 irradiated with $\mathrm{Fe}^{++}$at $460^{\circ} \mathrm{C}$ with 10 appm He preimplanted. 


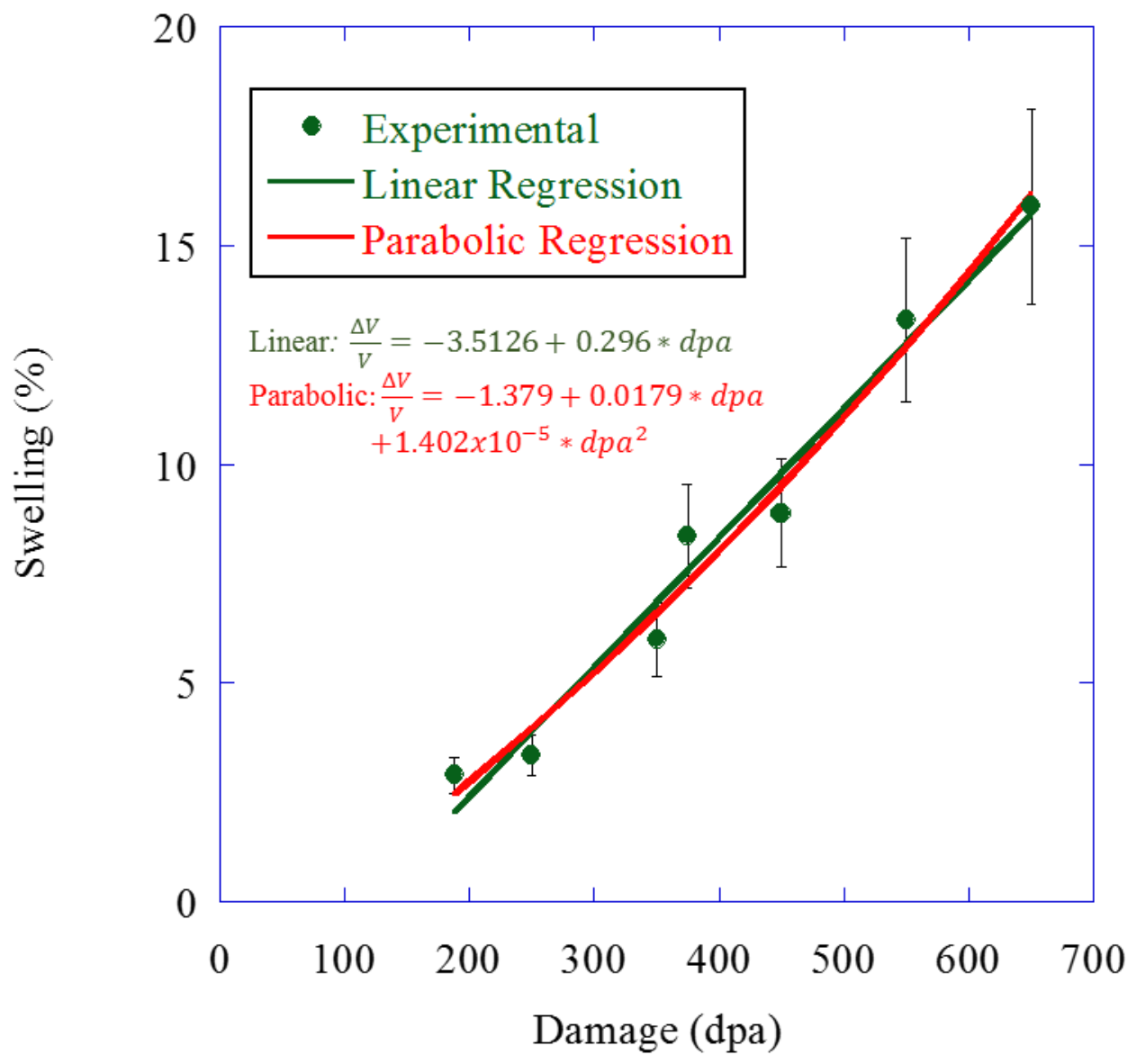

Figure 5.19: Comparison of linear or parabolic regression analysis applied to void swelling resulting from $\mathrm{HT} 9$ irradiated with $\mathrm{Fe}^{++}$at $460^{\circ} \mathrm{C}$ with 10 appm $\mathrm{He}$ preimplanted. 


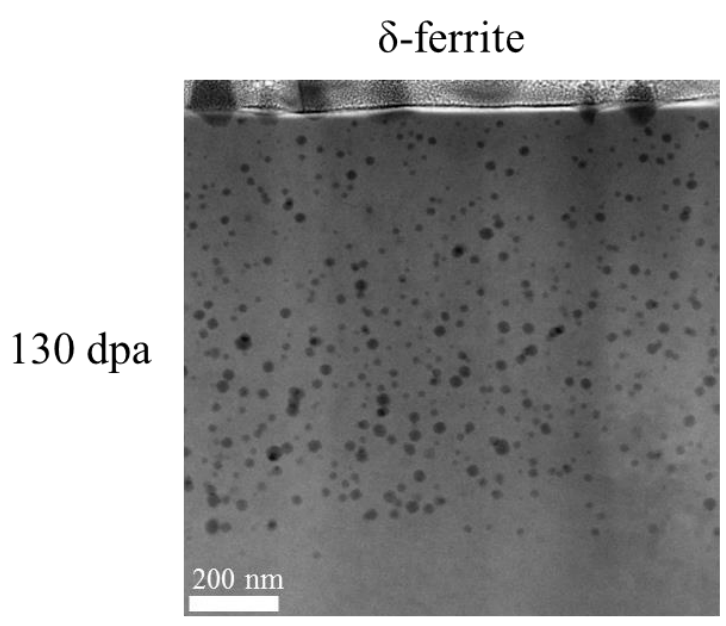

(a)

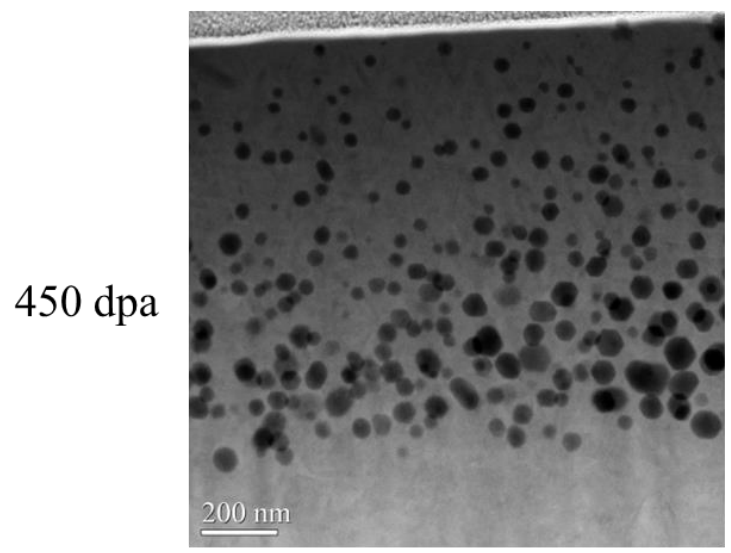

(c)

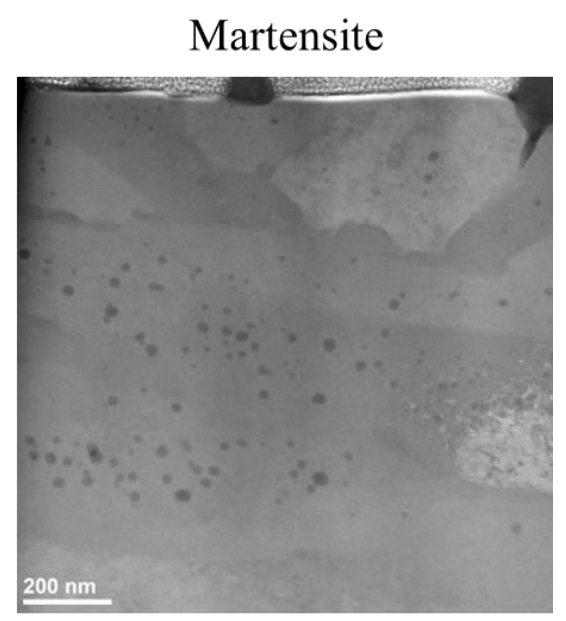

(b)

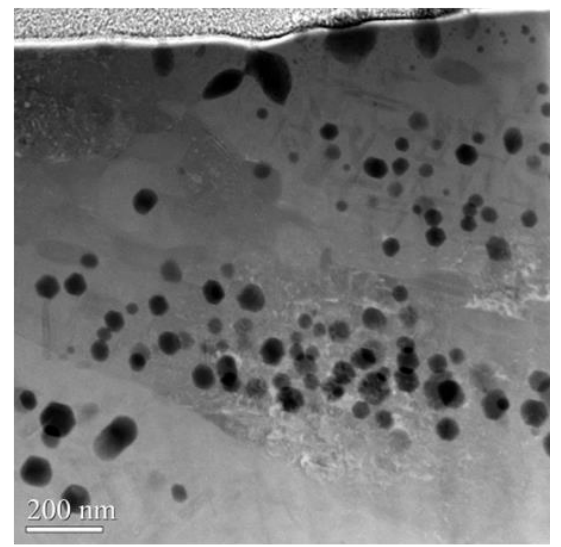

(d)

Figure 5.20: Representative images of $\delta$-ferrite (a, c) and martensite (b, d) at 130 and $450 \mathrm{dpa}$. 


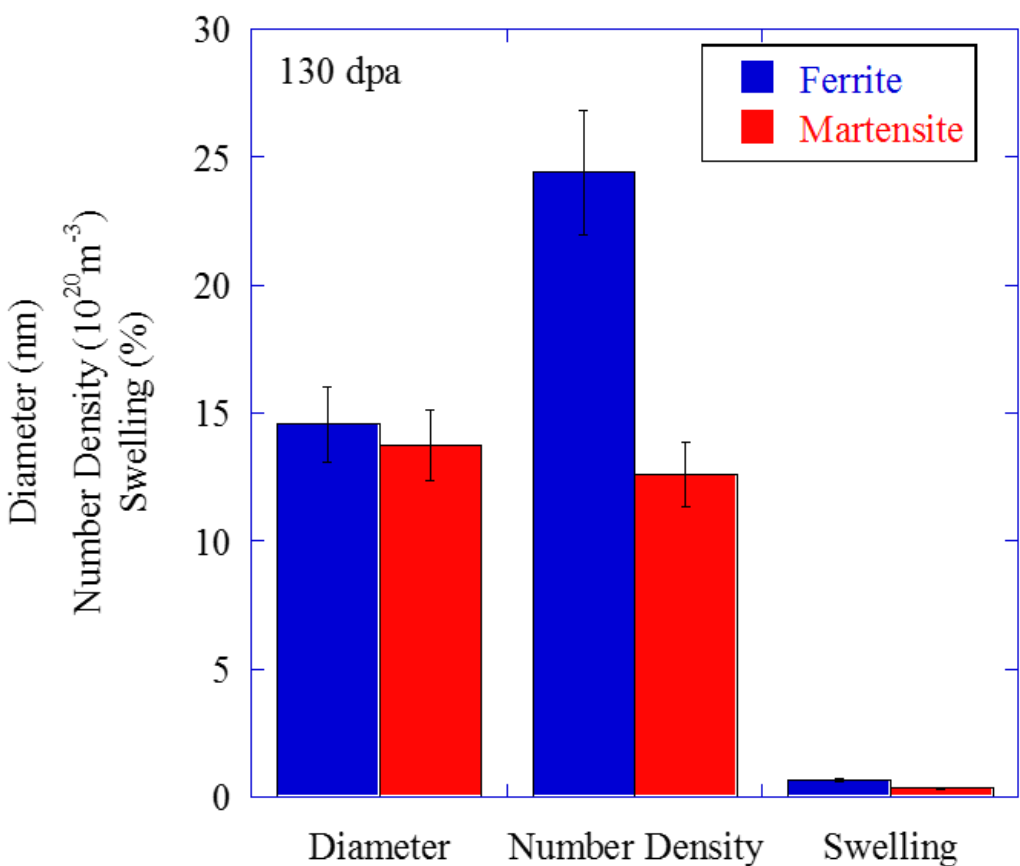

(a)

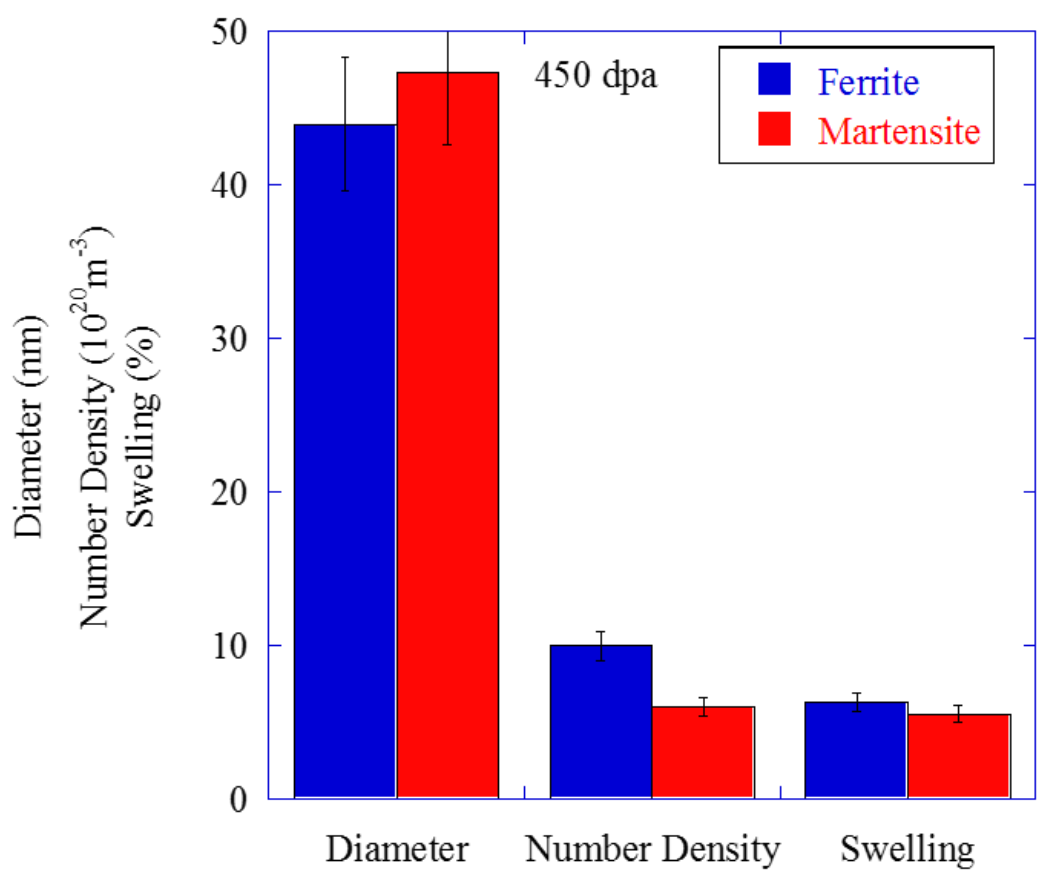

(b)

Figure 5.21: Comparison of diameter, number density and swelling in ferrite and martensite grains compared at a) 130 dpa and b) $450 \mathrm{dpa}$. 


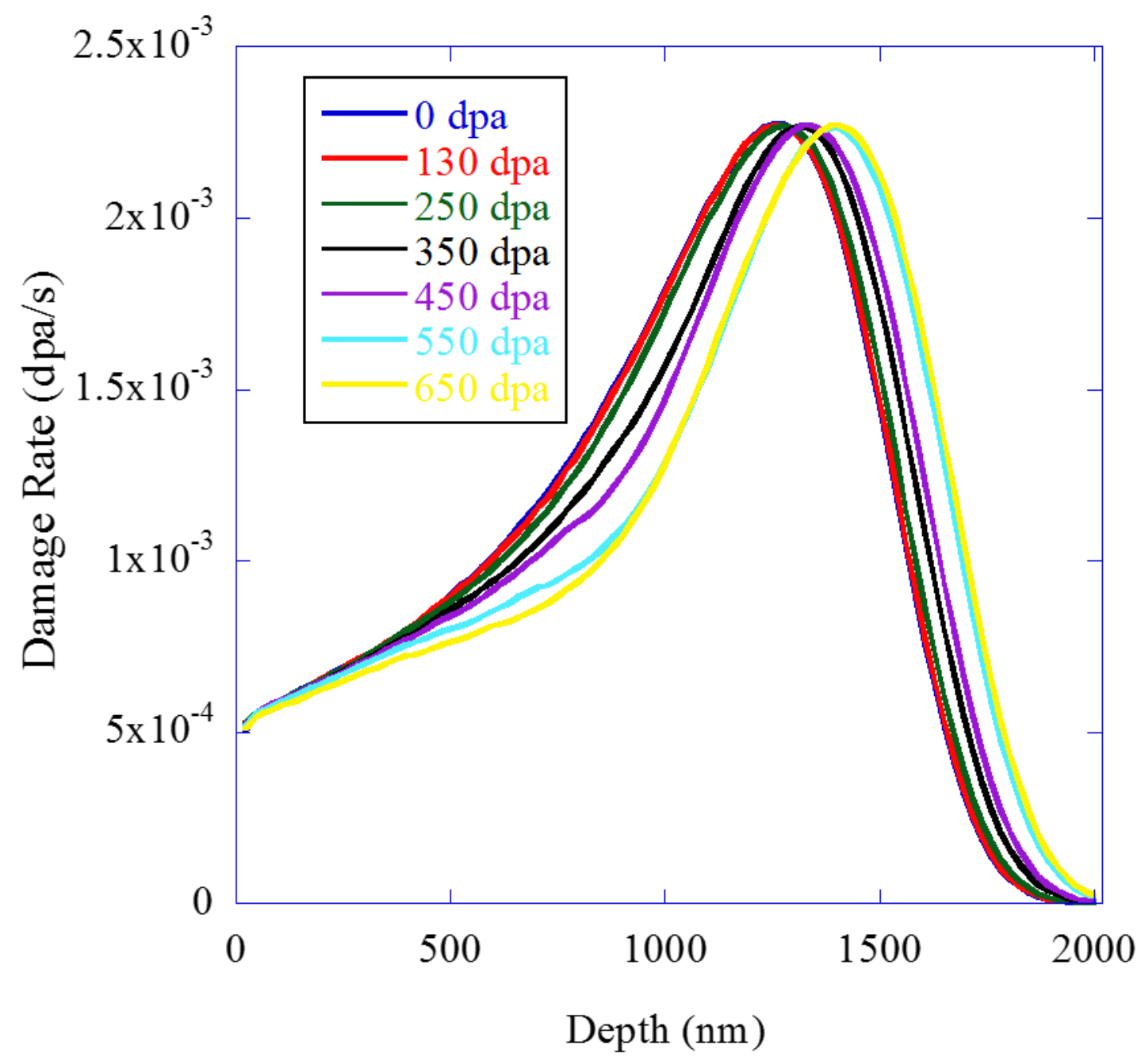

Figure 5.22: Damage rate $(\mathrm{dpa} / \mathrm{s})$ curve as a function of increasing damage due to void swelling. Beam current is assumed to be constant for all irradiations so damage rate can be directly compared. 

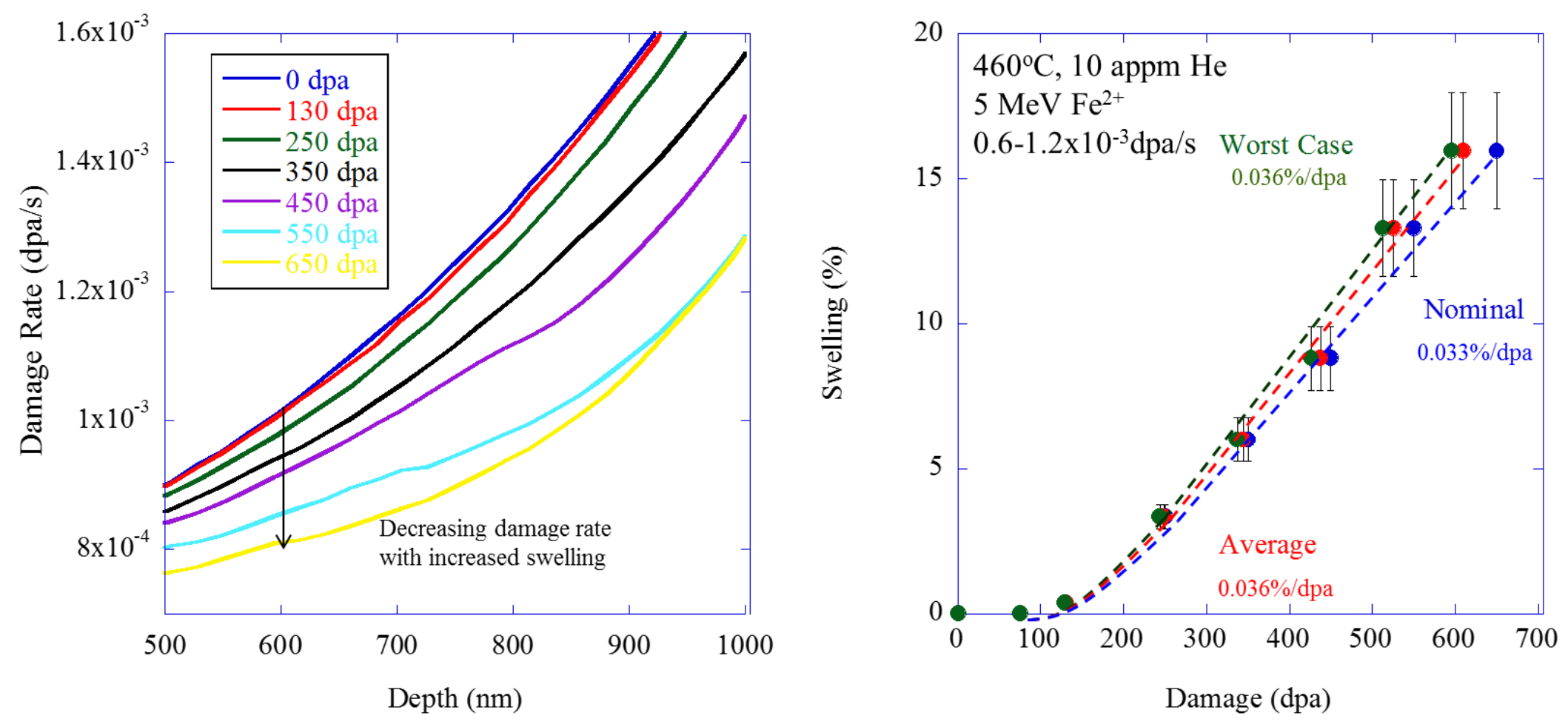

(a)

Figure 5.23: a) Effective damage rate calculated from SRIM. b) Swelling vs. damage with fixed depth correction. 

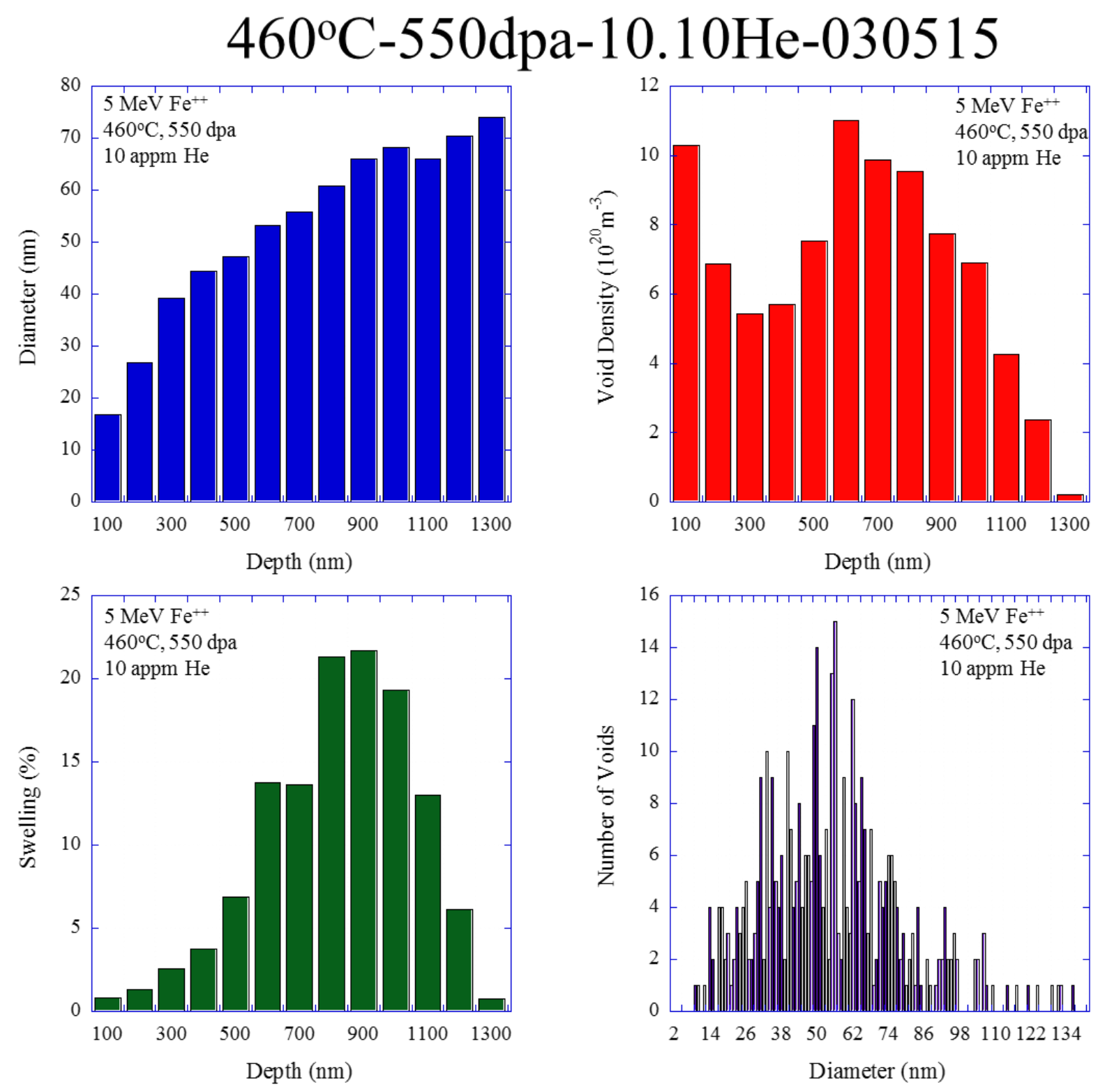

Figure 5.24: Void diameter, number density and swelling depth profiles as well as void distribution in the 500 to $700 \mathrm{~nm}$ depth region of HT9 irradiated with $5 \mathrm{MeV}$ $\mathrm{Fe}^{++}(\mathrm{ACO} 3)$ at $460^{\circ} \mathrm{C}$, to 550 dpa with 10 appm He. 

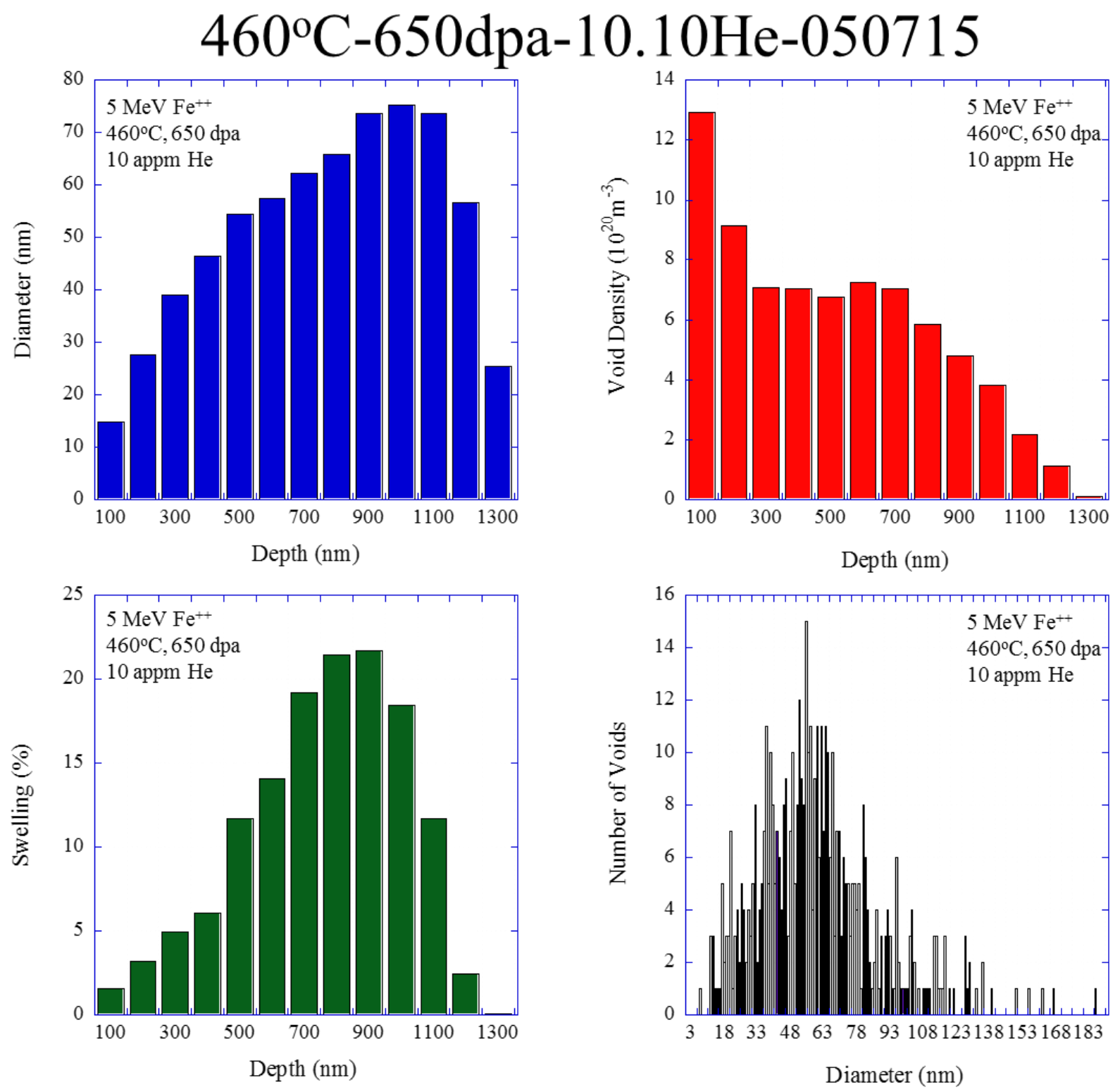

Figure 5.25: Void diameter, number density and swelling depth profiles as well as void distribution in the 500 to $700 \mathrm{~nm}$ depth region of HT9 irradiated with $5 \mathrm{MeV}$ $\mathrm{Fe}^{++}(\mathrm{ACO} 3)$ at $460^{\circ} \mathrm{C}$, to $650 \mathrm{dpa}$ with $10 \mathrm{appm} \mathrm{He}$. 


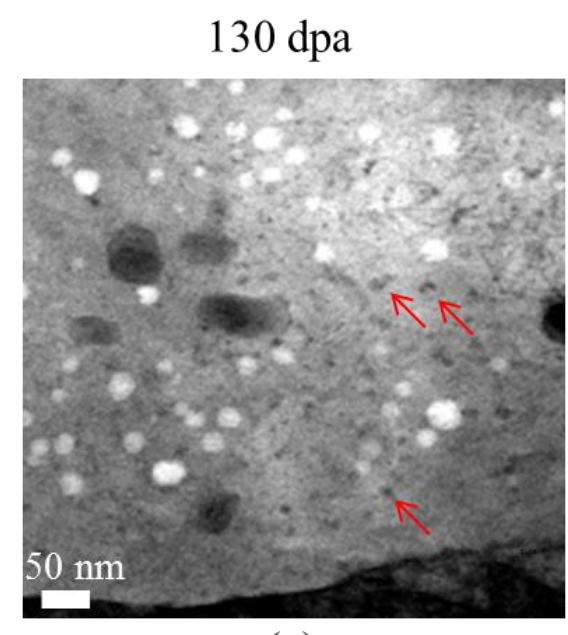

(a)

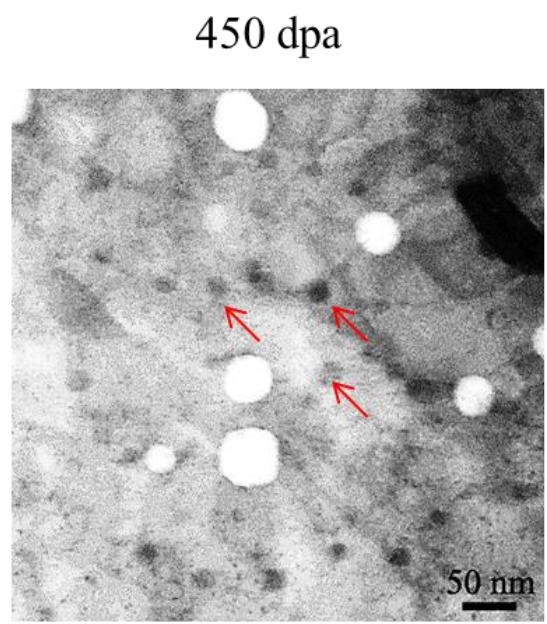

(d)

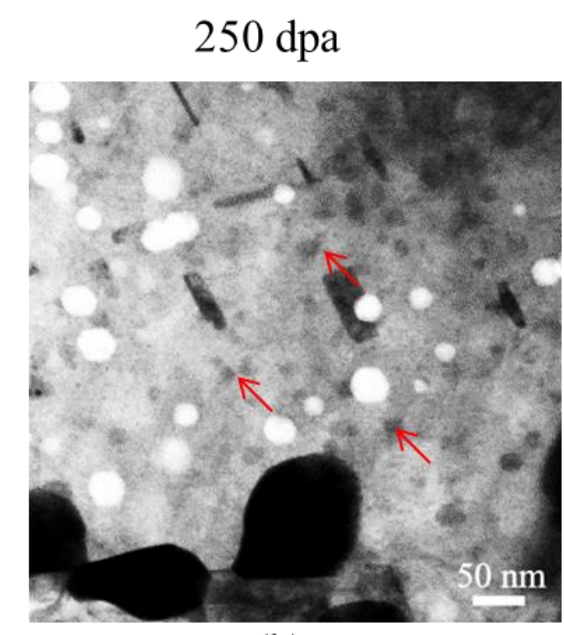

(b)

$550 \mathrm{dpa}$

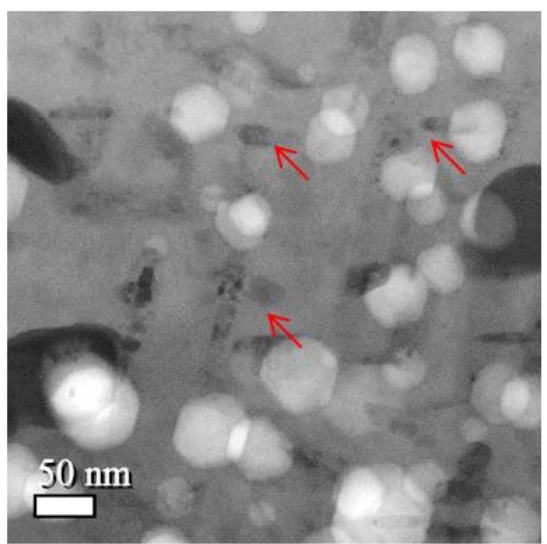

(e)

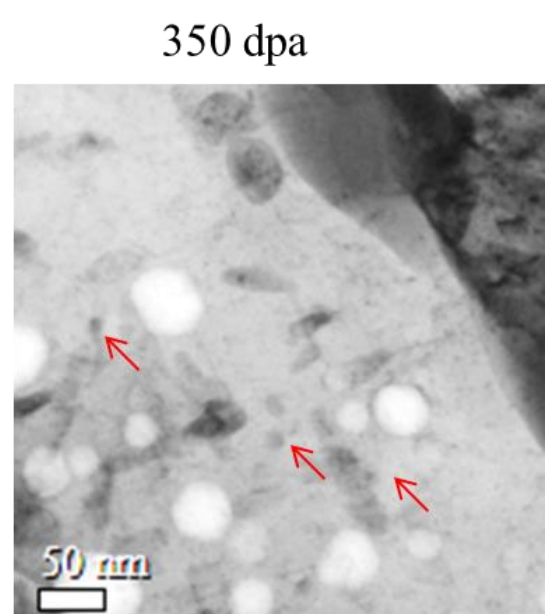

(c)

$650 \mathrm{dpa}$

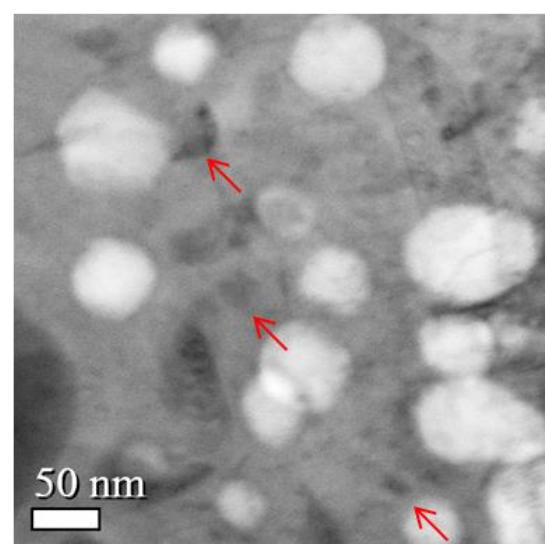

(f)

Figure 5.26: $\mathrm{G}$ phase evolution with increasing damage irradiated at $460^{\circ} \mathrm{C}$ with $5 \mathrm{MeV} \mathrm{Fe}^{++}$with 10 appm $\mathrm{He}$ preimplanted. Some $\mathrm{G}$ phase precipitates are marked by red arrows. 


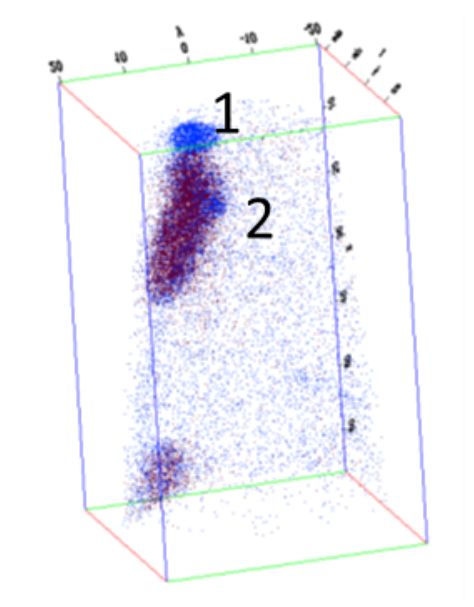

(a)

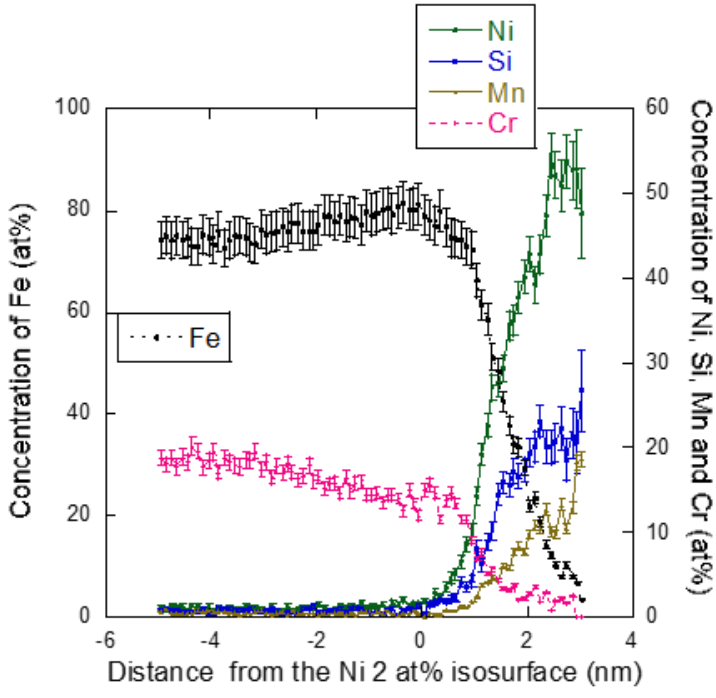

(b)

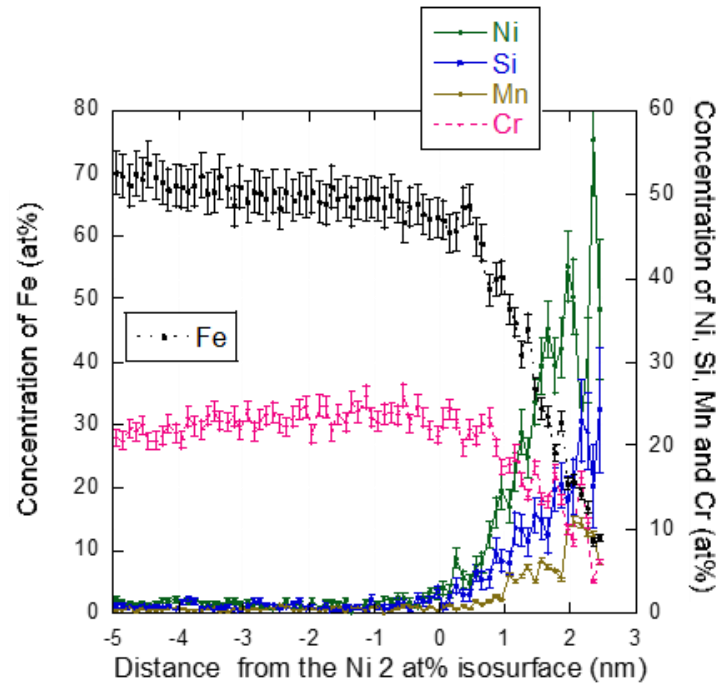

(c)

Figure 5.27: APT analysis of two $\mathrm{G}$ phase precipitates observed at $\mathrm{HT} 9$ irradiated with $5 \mathrm{MeV} \mathrm{Fe}^{++}$at $440^{\circ} \mathrm{C}$ to 140 dpa with 10 appm preimplanted. APT confirms composition of $\mathrm{Mn}_{6} \mathrm{Ni}_{16} \mathrm{Si}_{7}$ by observation of expected concentrations in at $\%(55 \% \mathrm{Ni}-24 \% \mathrm{Si}-21 \% \mathrm{Mn})$ in Precipitates b) 1 and c) 2. 


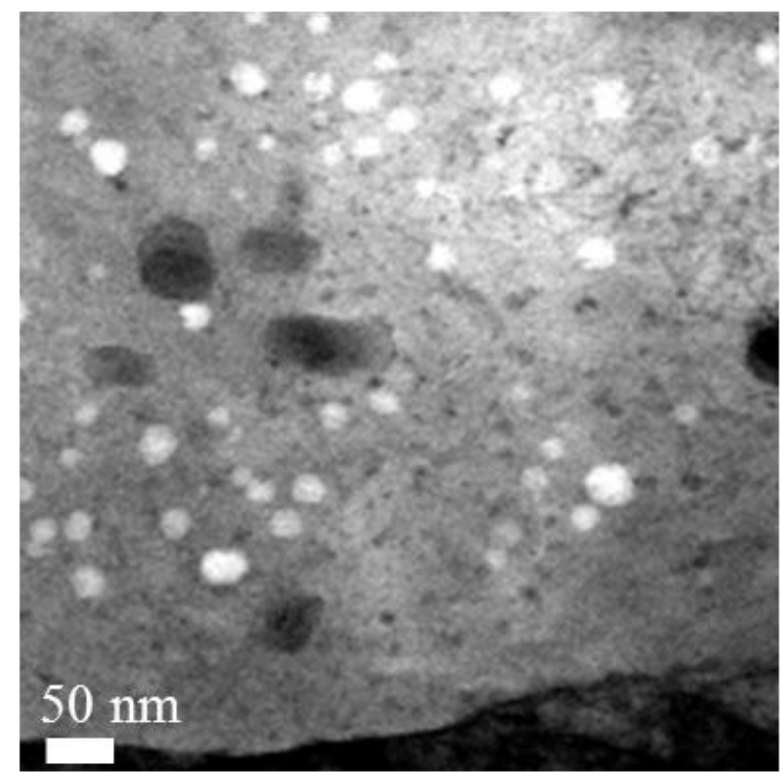

(a)

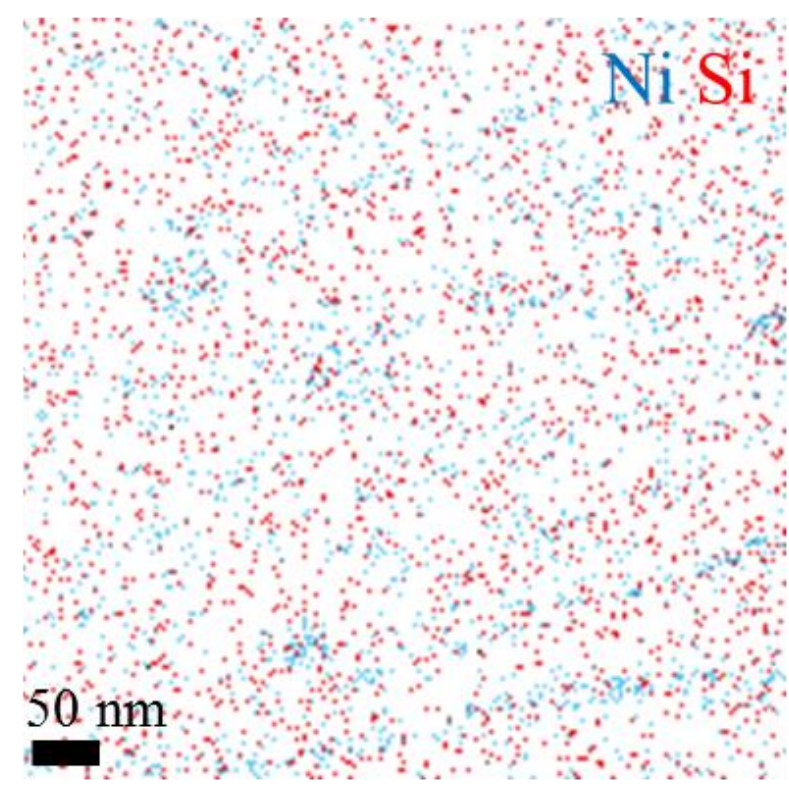

(b)

Figure 5.28: STEM BF image of G phase with corresponding XEDS map. Ni is in red and Si in blue. 


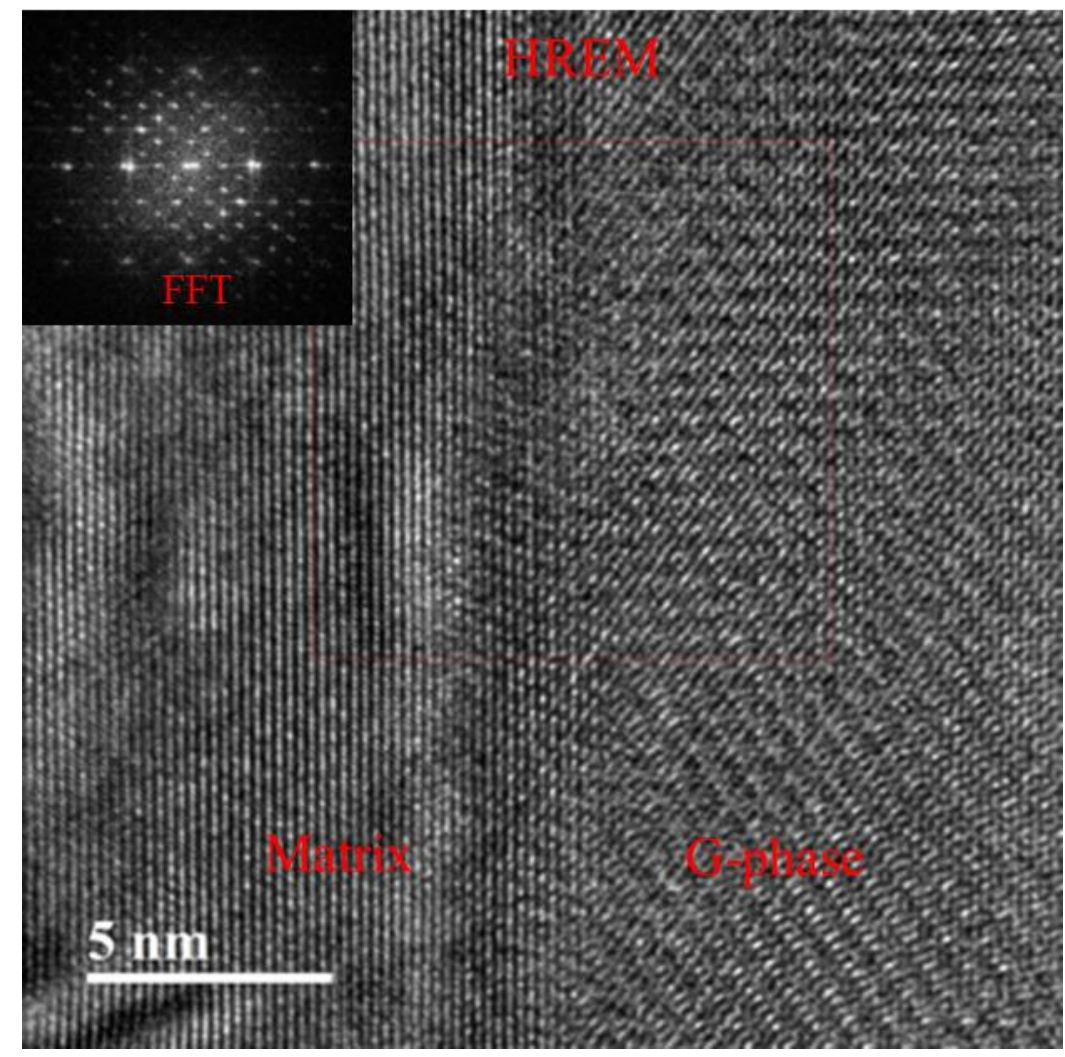

Figure 5.29: HRTEM BF image of $G$ phase in the matrix. The much larger lattice parameter of $G$ phase $(a=1.148 \mathrm{~nm})$ relative the matrix $a=0.286 \mathrm{~nm}$. The incoherent interface is clearly visible. The FFT pattern of the outlined region in the image (red frame) is inset in the top left corner and demonstrates fcc crystallographic structure. 

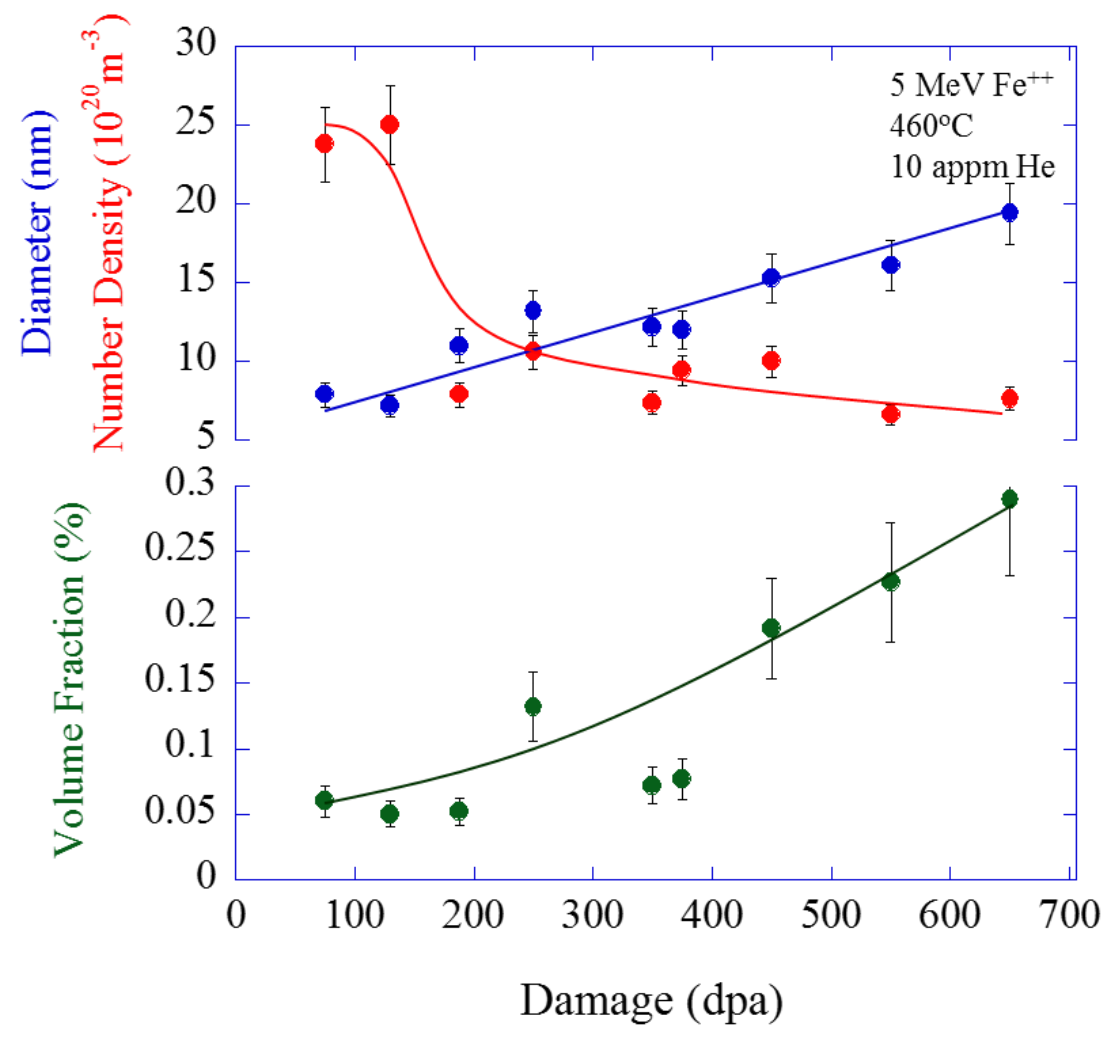

Figure 5.30: Evolution of $\mathrm{G}$ phase precipitates in terms of diameter (blue), number density (red) and volume fraction (green) at $460^{\circ} \mathrm{C}$ in $\mathrm{HT} 9$ irradiated with $5 \mathrm{MeV} \mathrm{Fe}^{++}$after preimplantation with 10 appm He. 


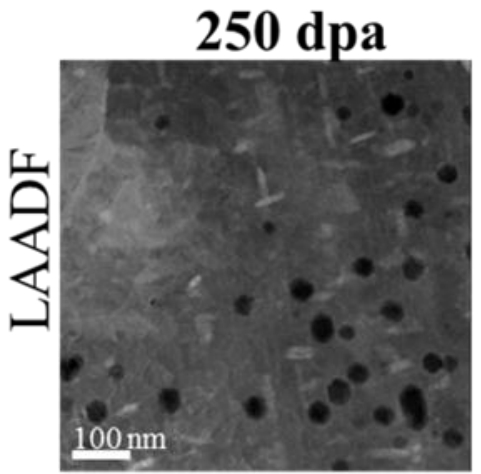

(a)

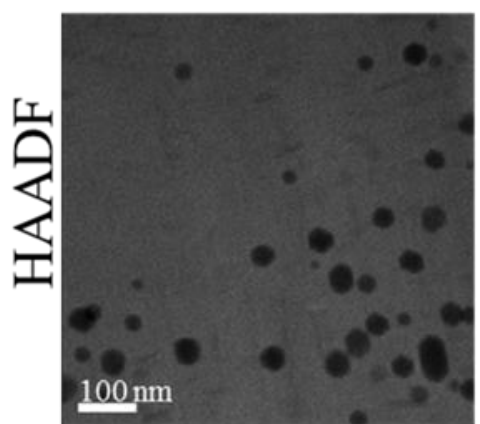

(f)

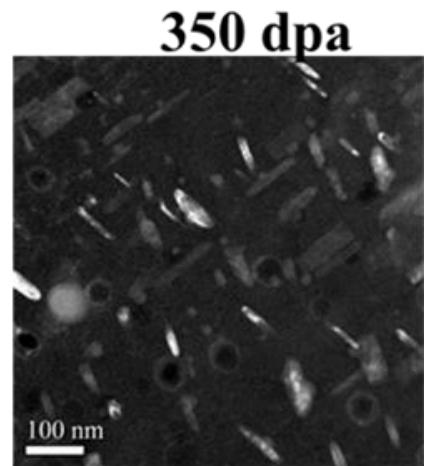

(b)

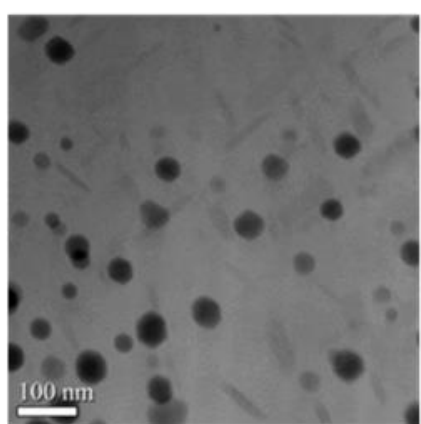

(g)

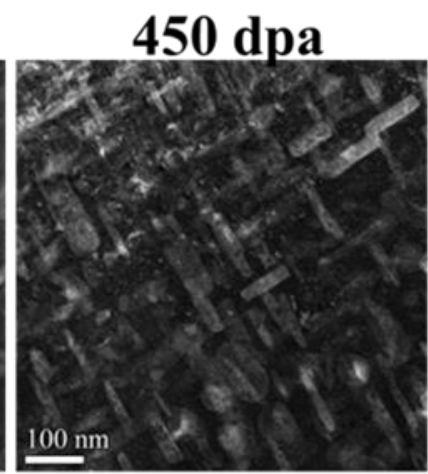

(c)

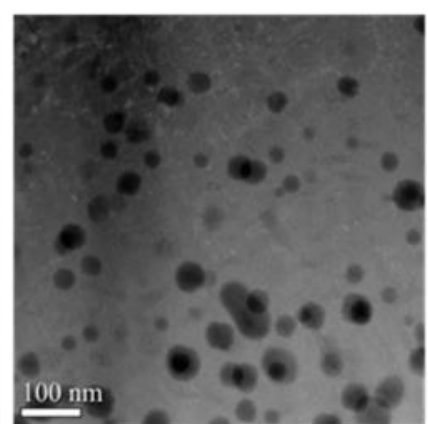

(h)

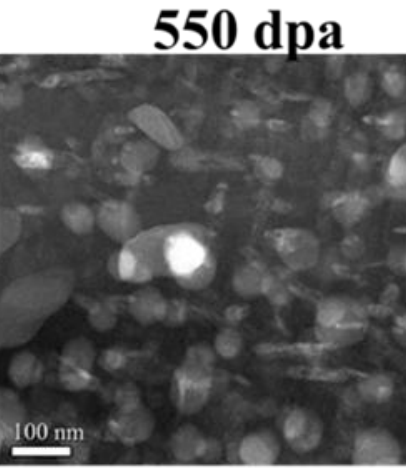

(d)

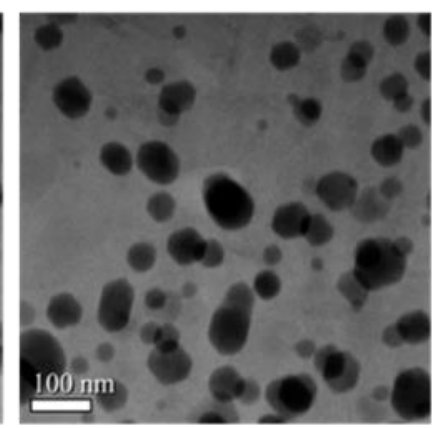

(i)

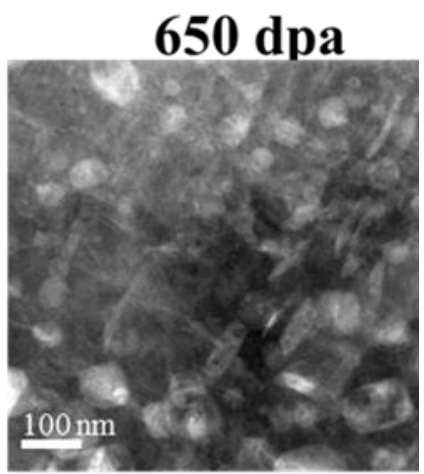

(e)

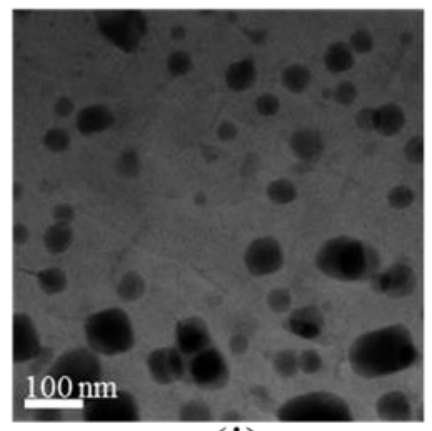

(j)

Figure 5.31: Formation of $\mathrm{M}_{2} \mathrm{X}$ precipitates in irradiated HT9 irradiated with $5 \mathrm{MeV} \mathrm{Fe}^{++}$. (a)-(e) are the low angle annular dark field images of $\mathrm{M}_{2} \mathrm{X}$, shown as light grey rods. (f)-(j) present the corresponding high angle annular dark field images with voids as dark circles and $\mathrm{M}_{2} \mathrm{X}$ can faintly be observed as light grey plates. 


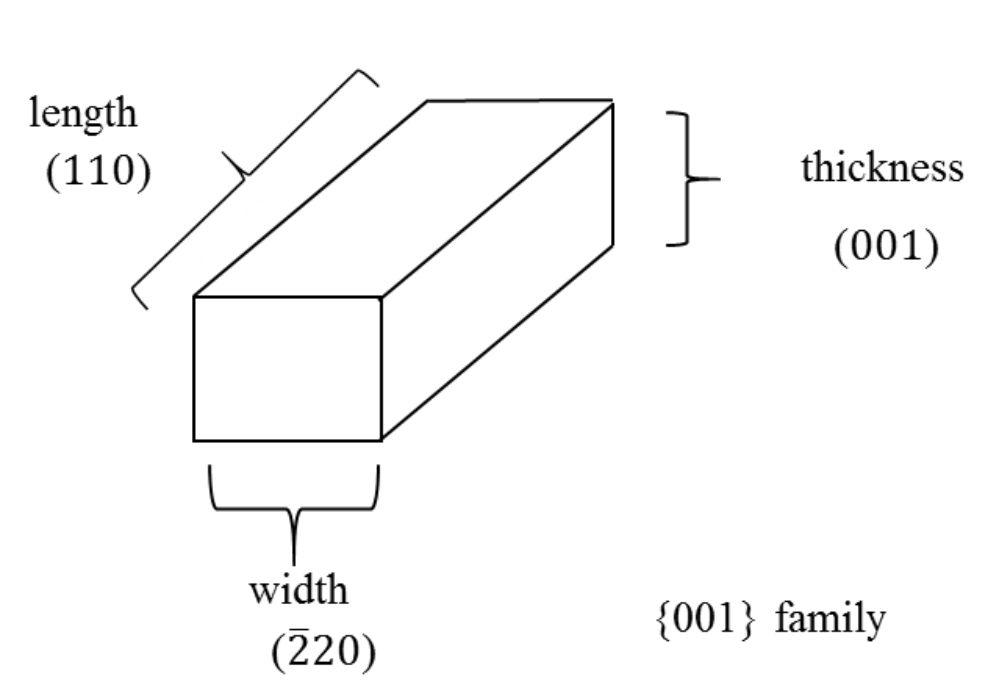

width

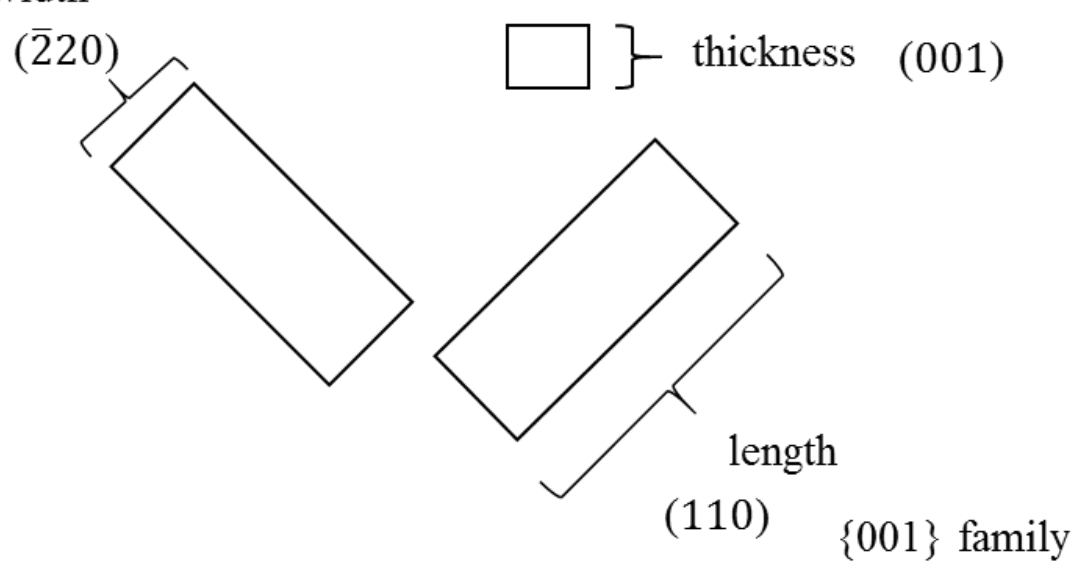

\section{End View}

$$
\begin{aligned}
& \text { Top View } \\
& (\vec{b}=001)
\end{aligned}
$$

Figure 5.32: Orientation of $\mathrm{M}_{2} \mathrm{X}$ within the matrix. The top view corresponds to what is imaged in Figure 5.31. 


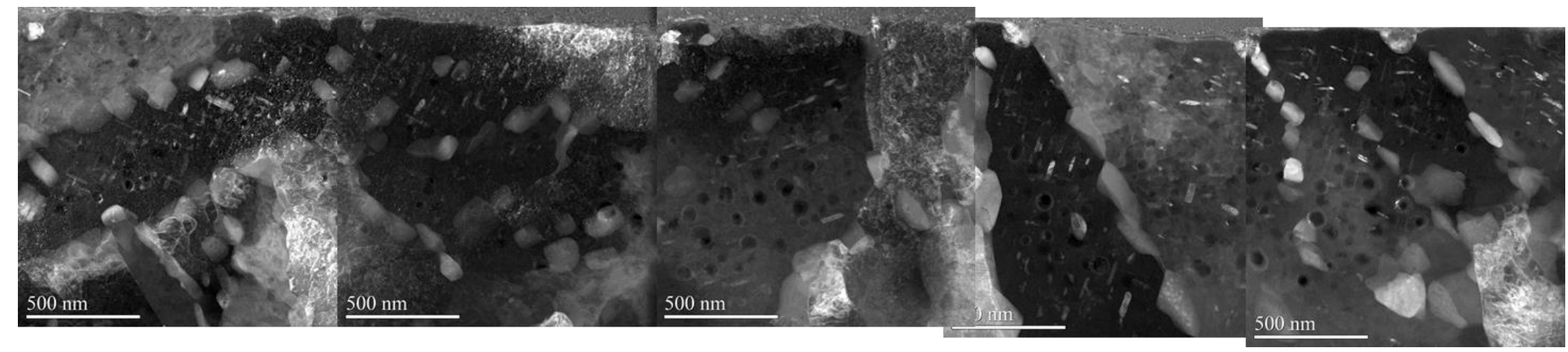

Figure 5.33: LAADF image of formation of $\mathrm{M}_{2} \mathrm{X}$ precipitates in irradiated HT9 irradiated with $5 \mathrm{MeV} \mathrm{Fe}^{++}$. Note precipitates form intragranularly. 


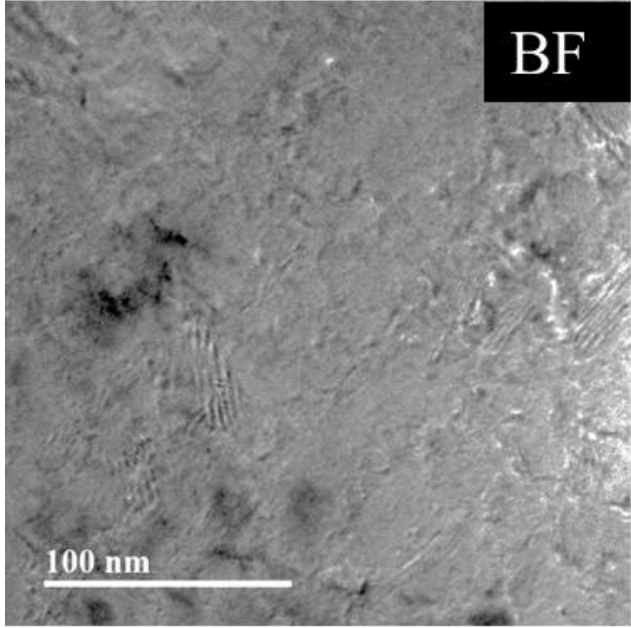

(a)

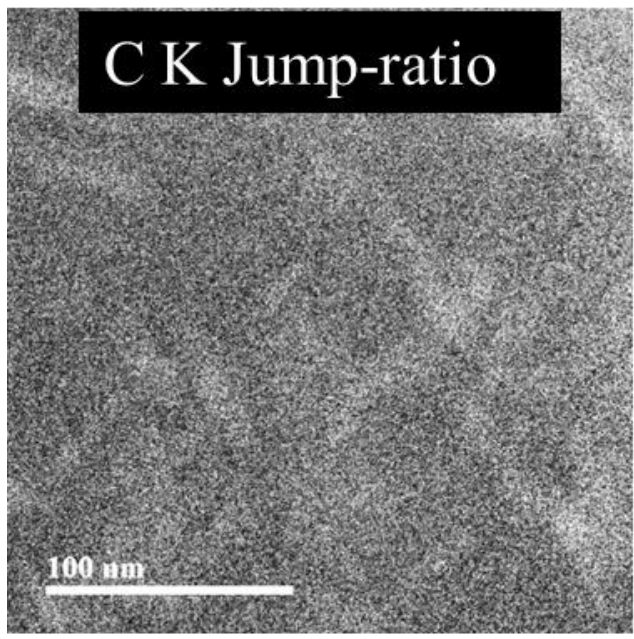

(c)

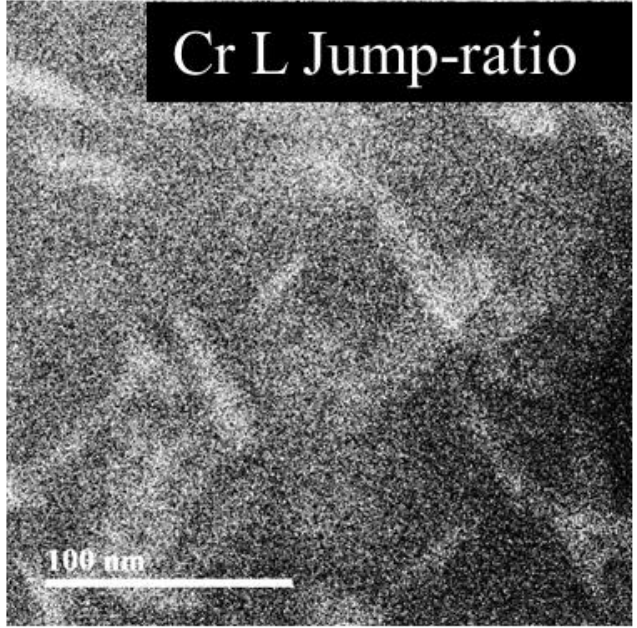

(b)

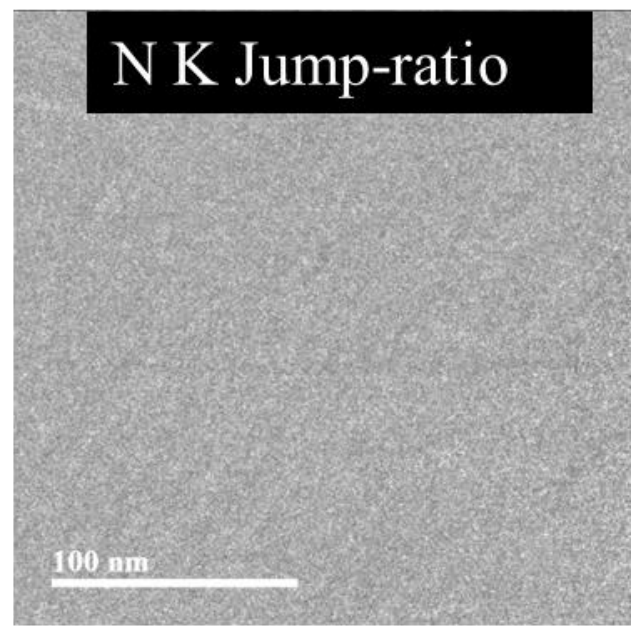

(d)

Figure 5.34: Bright field image (a) and corresponding jump ratios for $\mathrm{Cr}$ (b), $\mathrm{C}$ (c) and $\mathrm{N}(\mathrm{d})$ in $\mathrm{HT} 9$ irradiated at $460^{\circ} \mathrm{C}$ to $550 \mathrm{dpa}$ with 10 appm He preimplanted. 


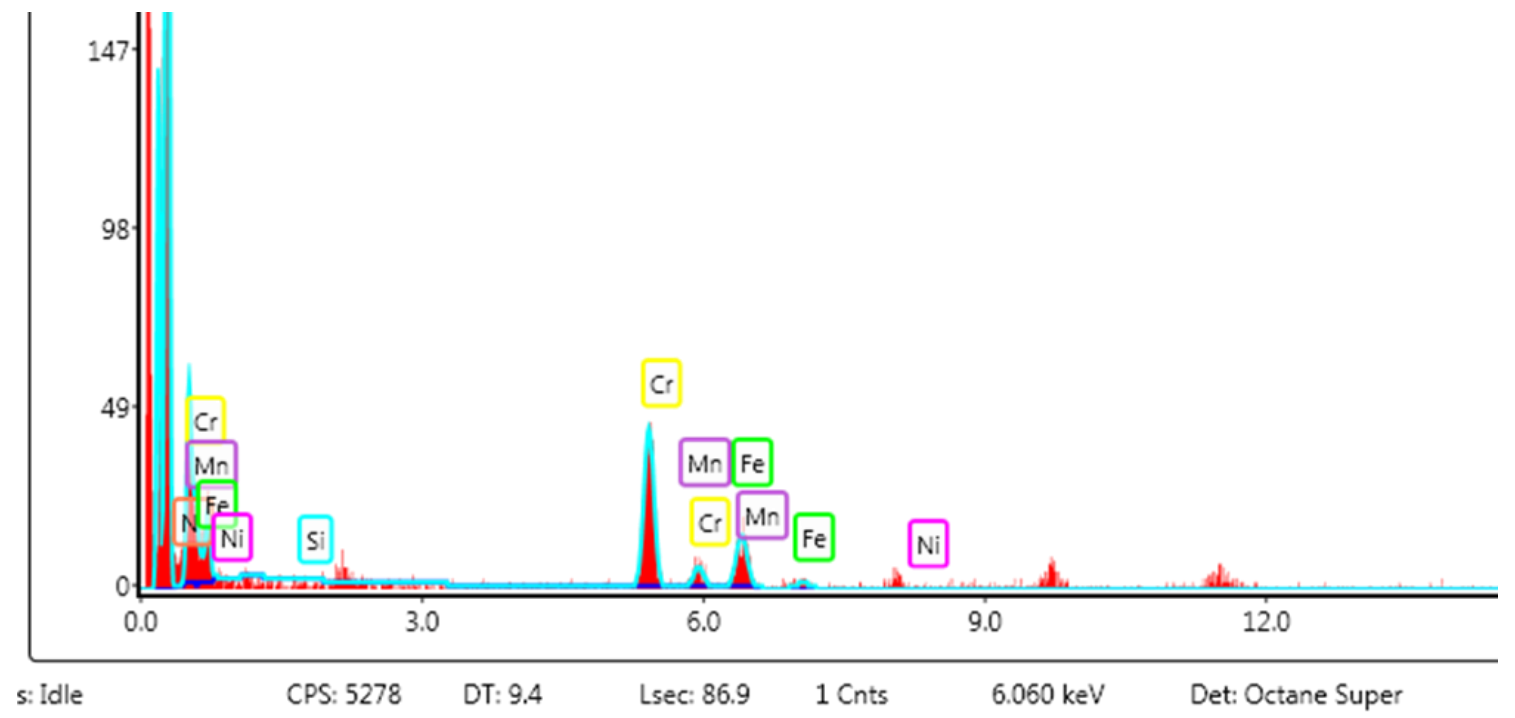

Figure 5.35: EDX spectrum of $\mathrm{M}_{2} \mathrm{X}$ confirming $\mathrm{Cr}_{2} \mathrm{C}$ in $\mathrm{HT} 9$ irradiated at $460^{\circ} \mathrm{C}$ to 550 dpa with 10 appm He preimplanted. 


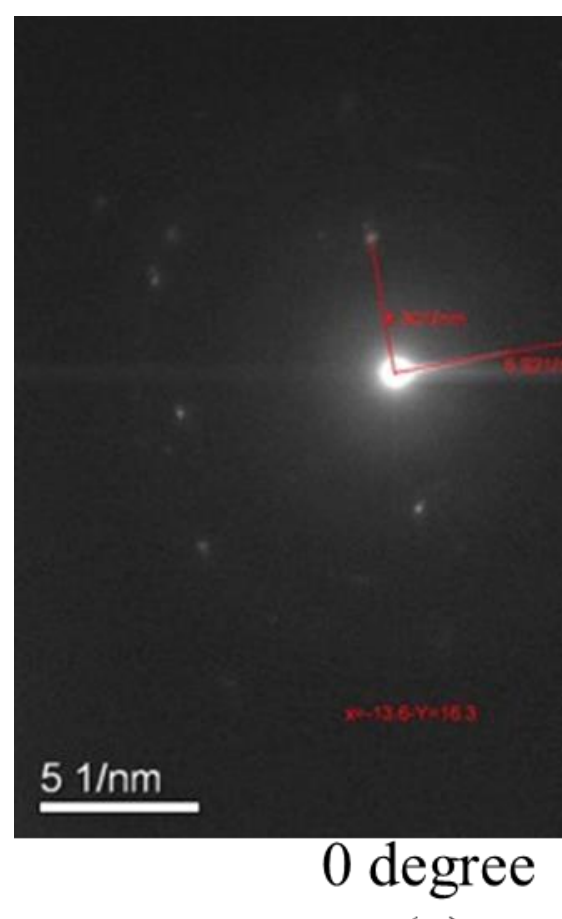

(a)

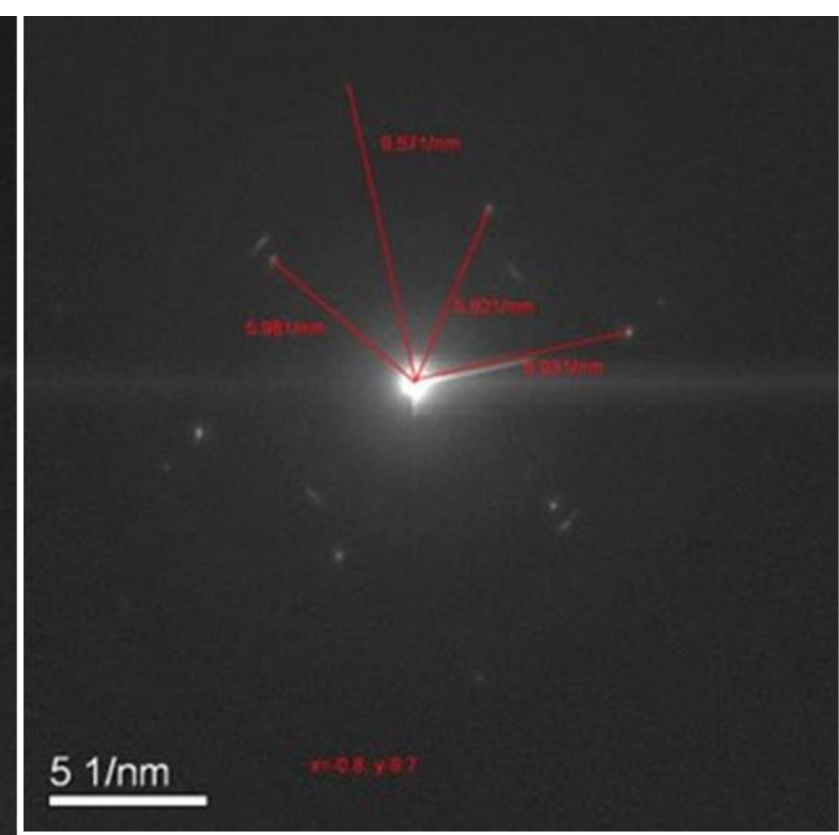

27 degree

(b)

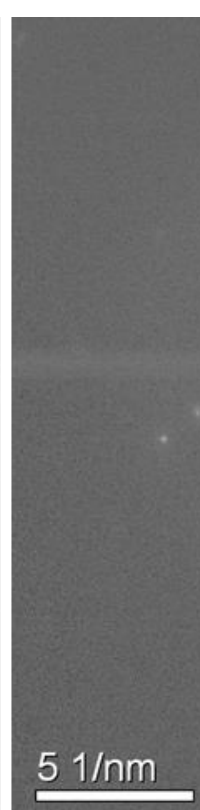

$51 / \mathrm{nm}$

\section{3 degree}

(b)

Figure 5.36: SAED patterns of $\mathrm{M}_{2} \mathrm{X}$ in $\mathrm{HT} 9$ irradiated at $460^{\circ} \mathrm{C}$ to 450 dpa with 10 appm He preimplanted with axis tilted to a) $0^{\circ}$ b) $27^{\circ}$ and c) $43^{\circ}$. 


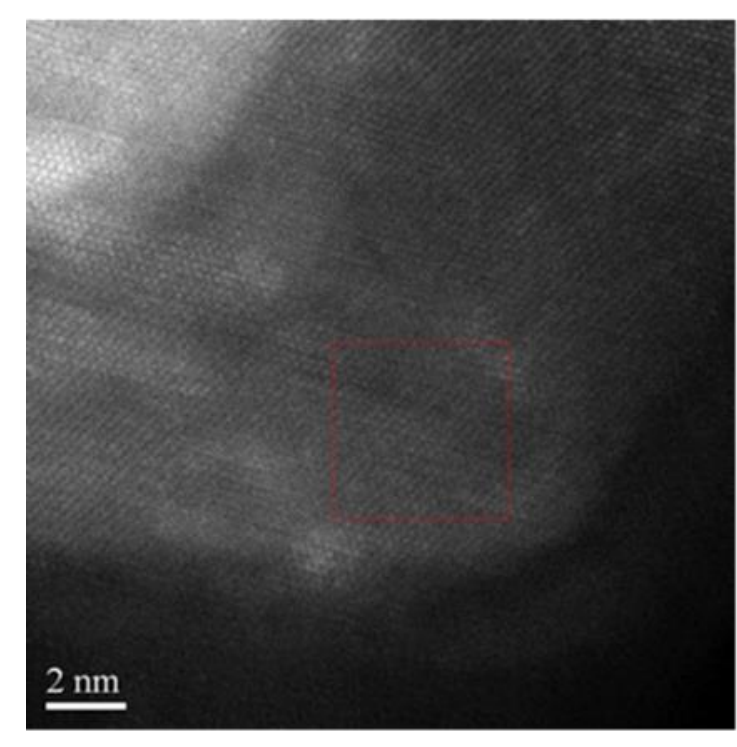

(a)

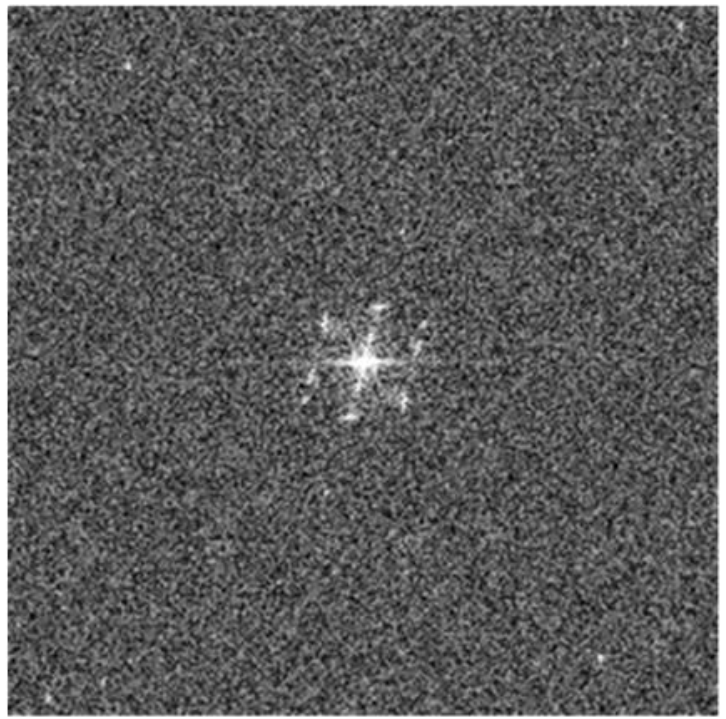

(b)

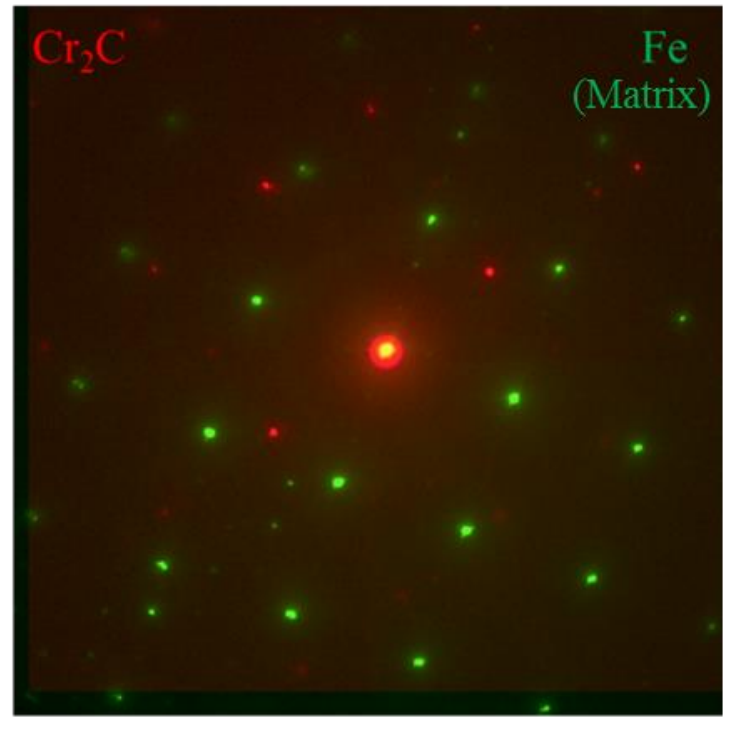

(c)

Figure 5.37: a) HRTEM of $\mathrm{M}_{2} \mathrm{X}$ b) FFT of precipitate from red box in (a), c) nanodiffraction revealing $\mathrm{M}_{2} \mathrm{X}$ (red) and $\mathrm{Fe}$ (green) matrix in HT9 irradiated at $460^{\circ} \mathrm{C}$ to 650 dpa with 10 appm He preimplanted. 


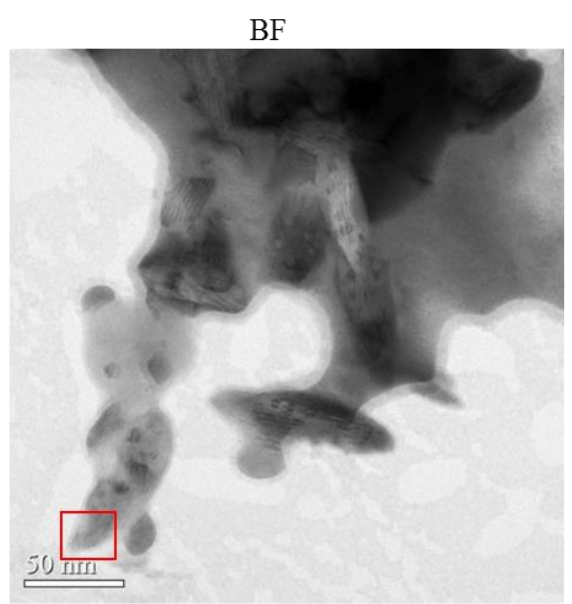

(a)

HR HAADF

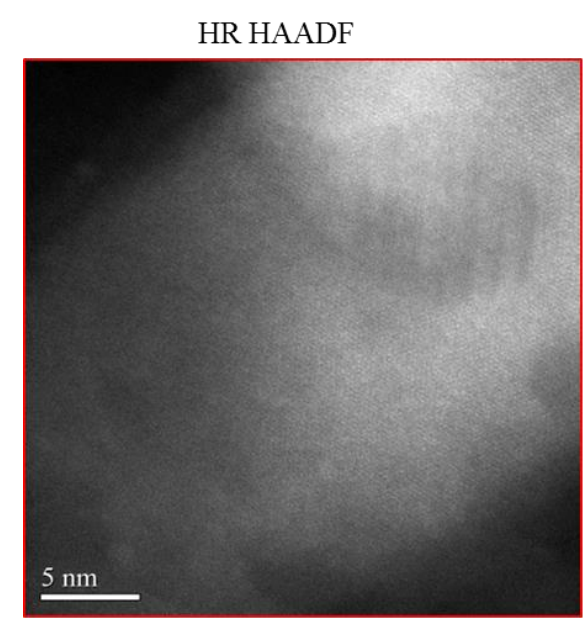

(c)

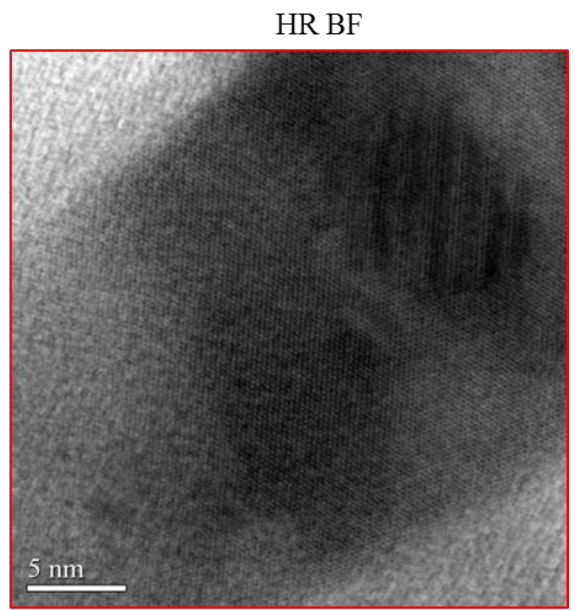

(b)

(c)

Figure 5.38: a) BF b) HR BF and c) HR HAADF images of $\mathrm{M}_{2} \mathrm{X}$ irradiated to 650 dpa at $460^{\circ} \mathrm{C}$. Moiré fringes clearly visible and indicated misfit strain due to slight lattice mismatch, consistent with coherency. 


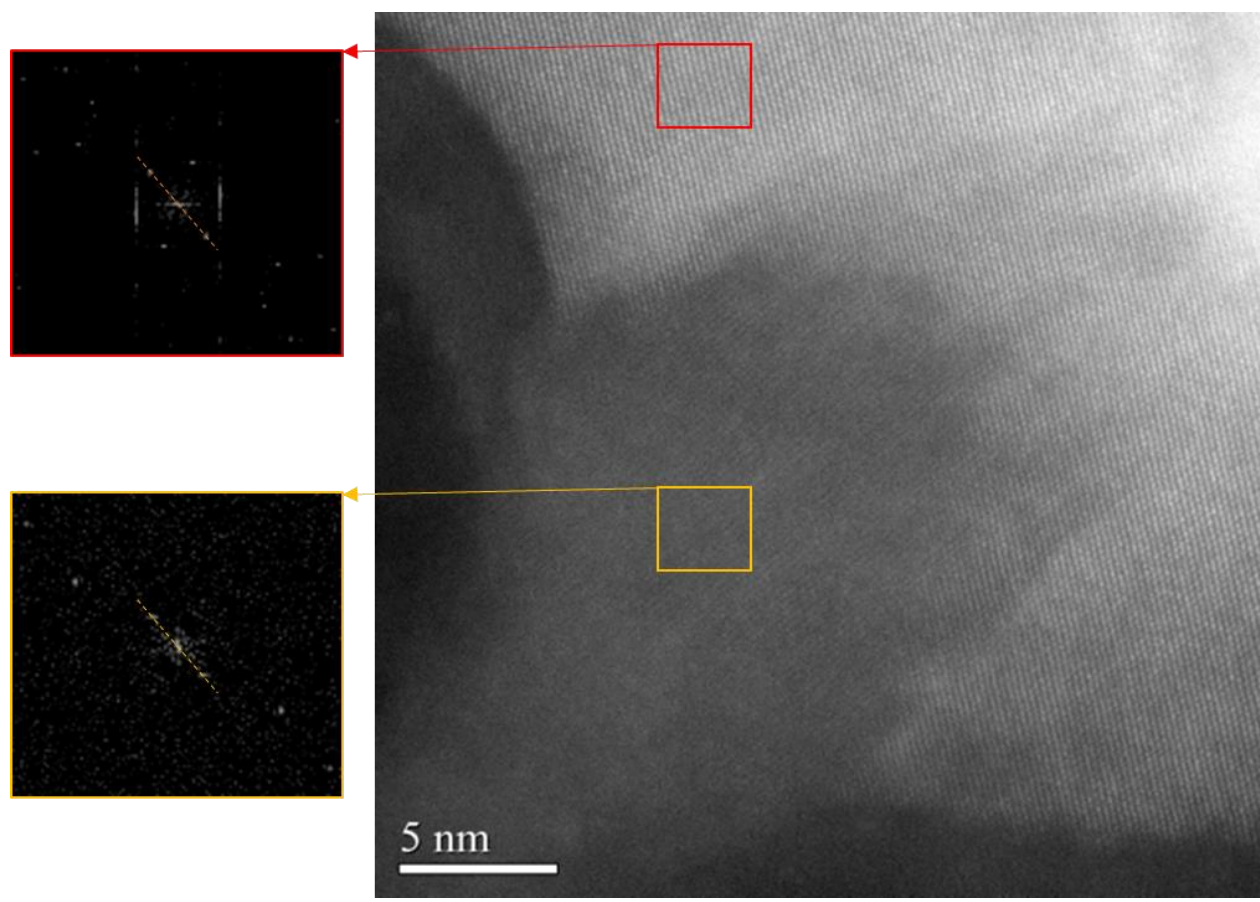

Figure 5.39: HR HAADF image of $\mathrm{M}_{2} \mathrm{X}$ (yellow) and matrix (red). FFT show same growth relationship $\left\langle 12 \overline{2} 0>_{M_{2} X} / /<100\right\rangle_{\alpha}$, demonstrated by parallel lines. 


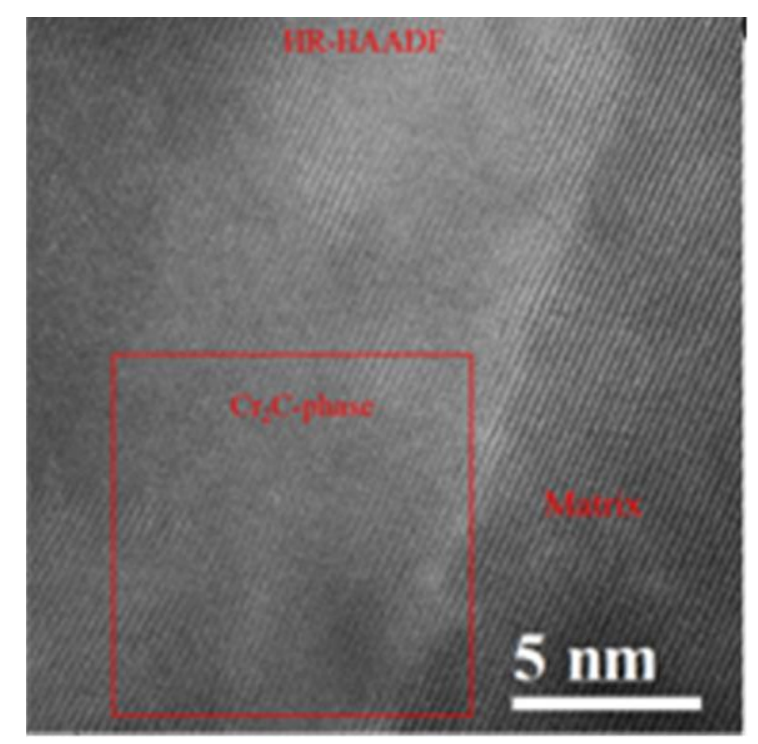

(a)

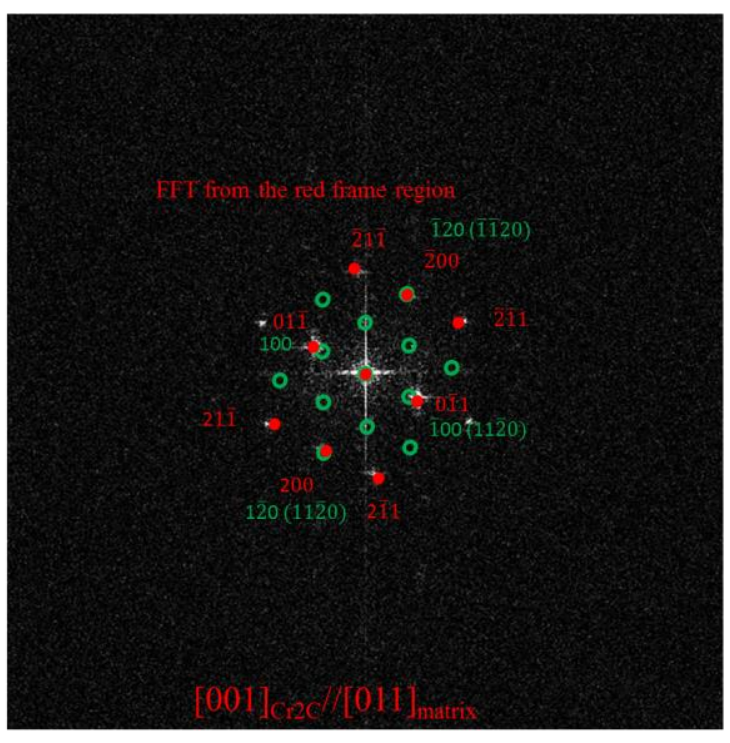

(b)

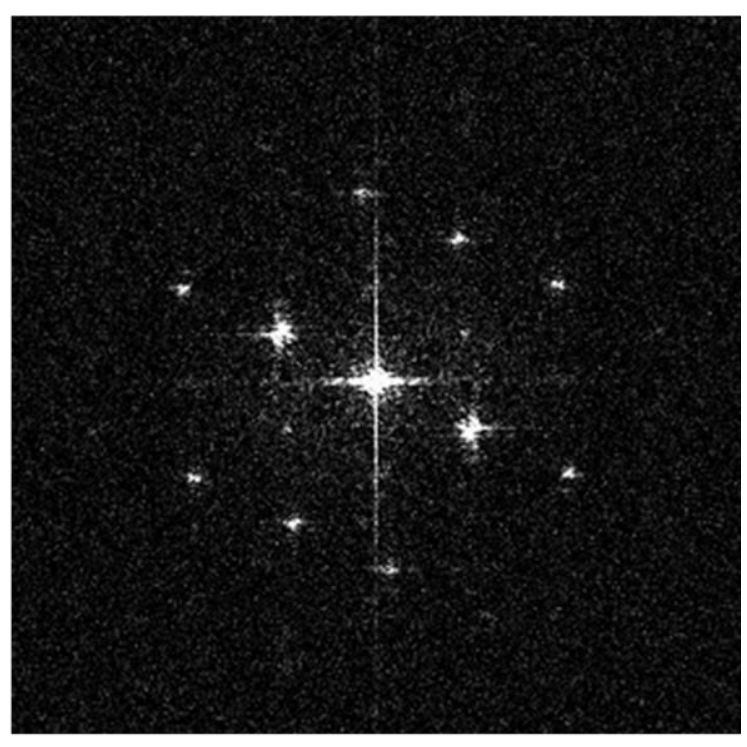

(c)

Figure 5.40: a) HR-HAADF image of HT9 irradiated at $460^{\circ} \mathrm{C}$ to $650 \mathrm{dpa}$. b) FFT with simulated SAED pattern overlaid indicating consistency of observed pattern with simulation along [011] direction of matrix, c) FFT with no simulated diffraction pattern overlaid. 


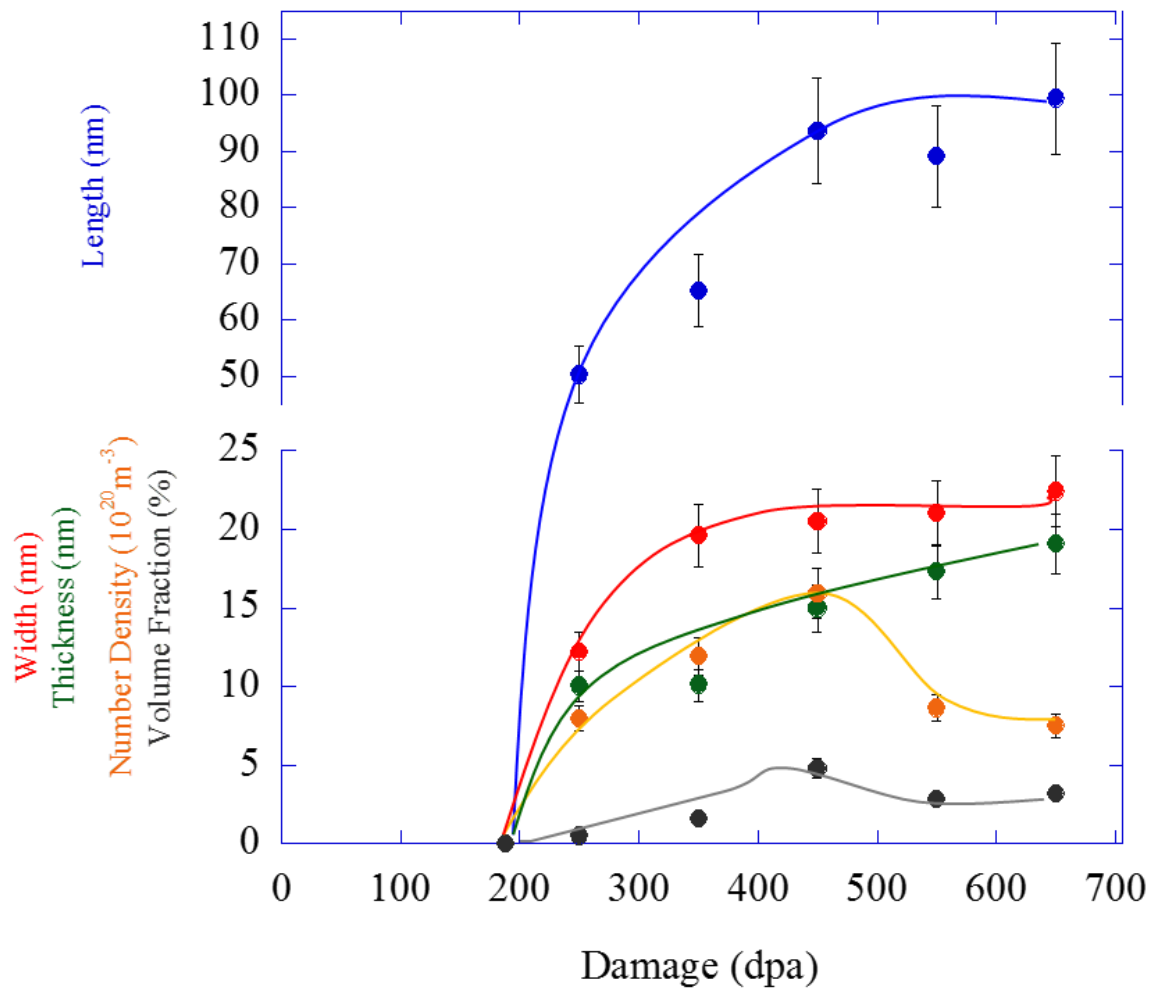

Figure 5.41: Evolution of $\mathrm{M}_{2} \mathrm{X}$ precipitates in terms of length (blue), width (red), thickness (green), number density (yellow) and volume fraction (grey) in HT9 irradiated with $5 \mathrm{MeV} \mathrm{Fe}^{++}$at $460^{\circ} \mathrm{C}$ after preimplantation of $10 \mathrm{appm} \mathrm{He}$. 
130 dpa

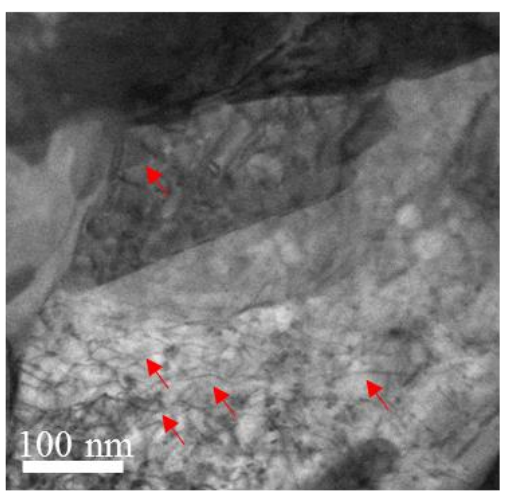

(a)

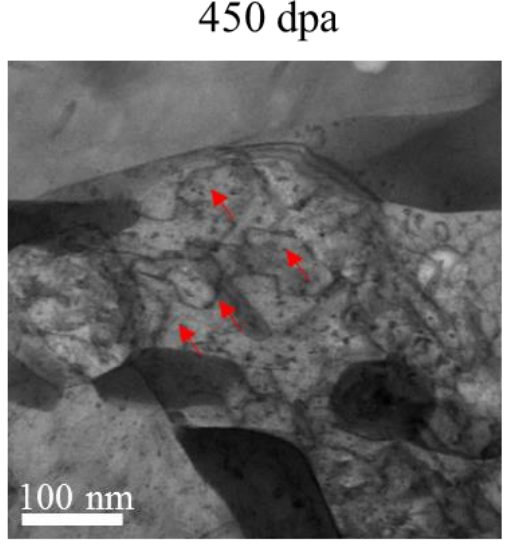

(d)

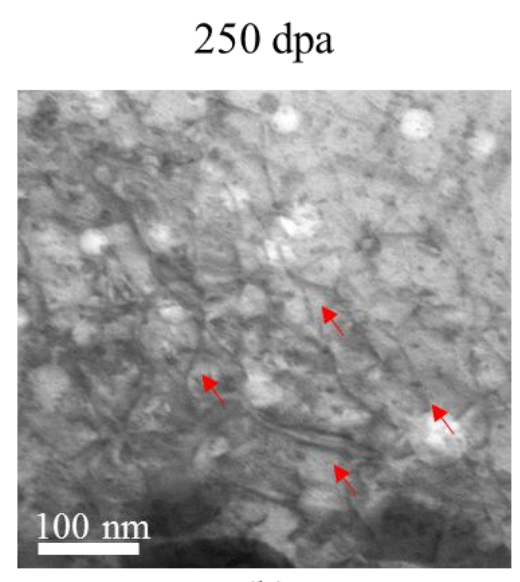

(b)

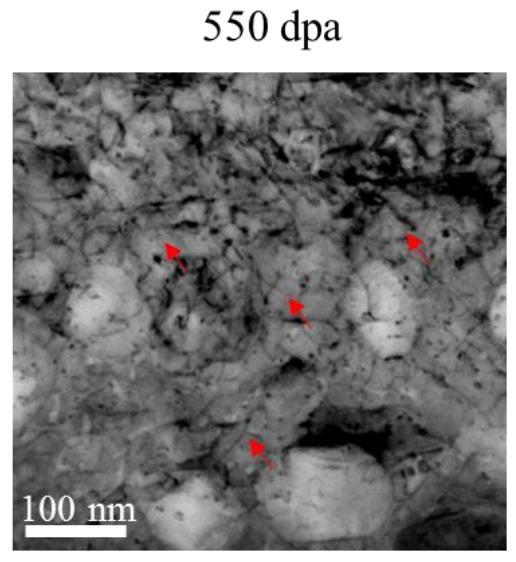

(e)

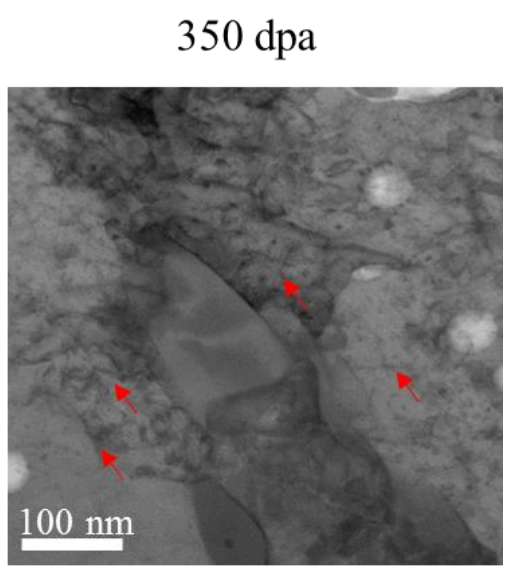

(c)

$650 \mathrm{dpa}$

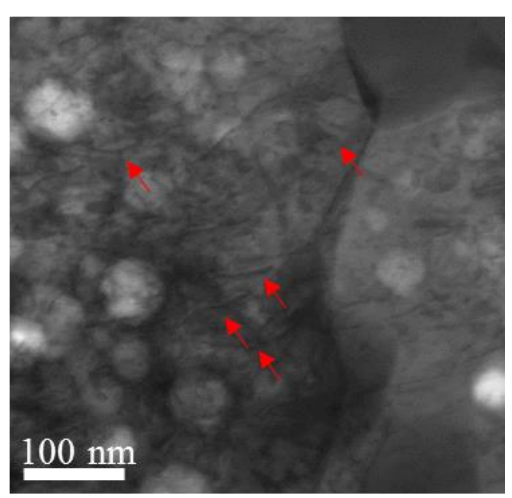

(f)

Figure 5.42: Images of representative dislocation network used for characterization of line density in HT9 irradiated with $5 \mathrm{MeV} \mathrm{Fe}^{++}$at $460^{\circ} \mathrm{C}$ with 10 appm He preimplanted. Some dislocations pointed out with red arrows. 


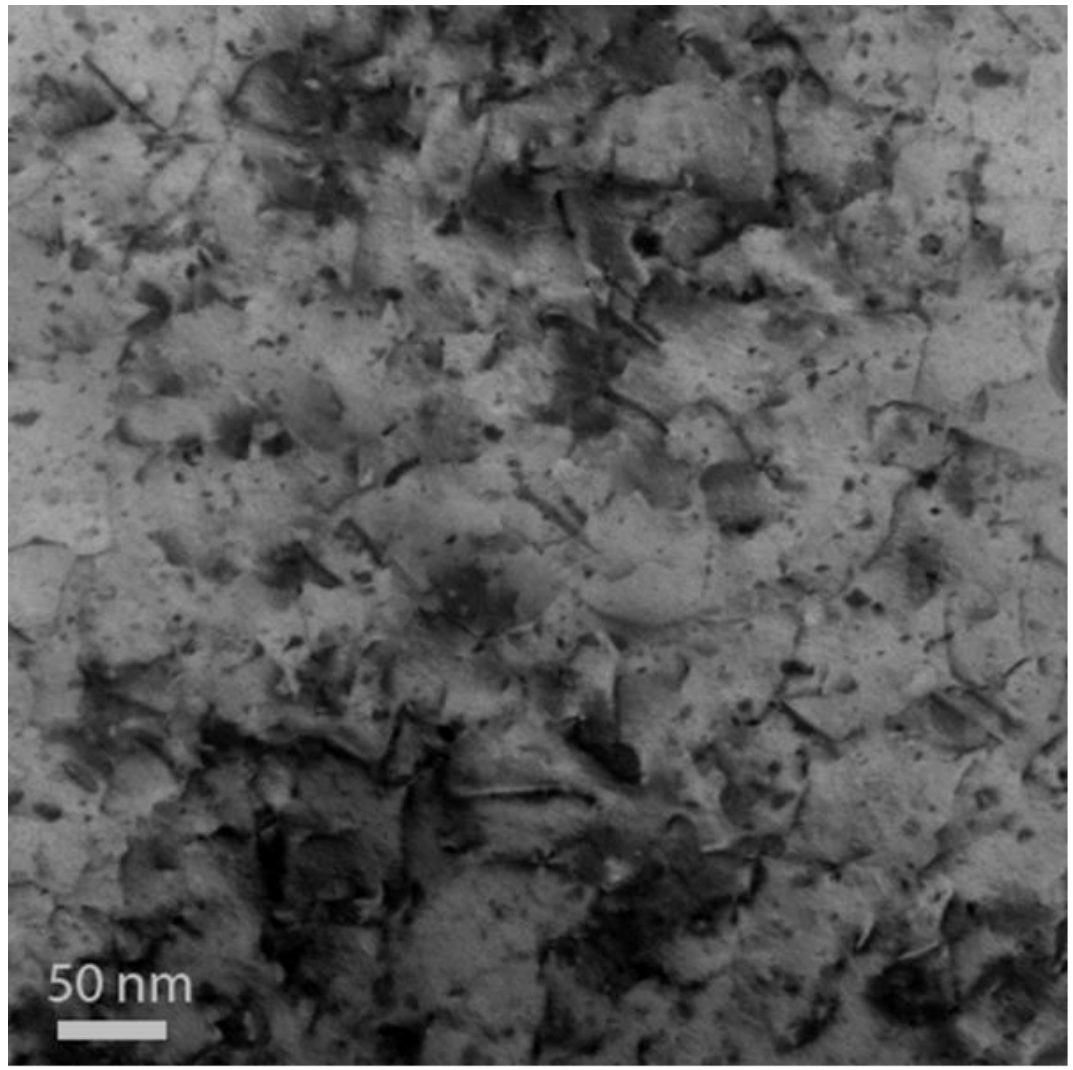

(a)

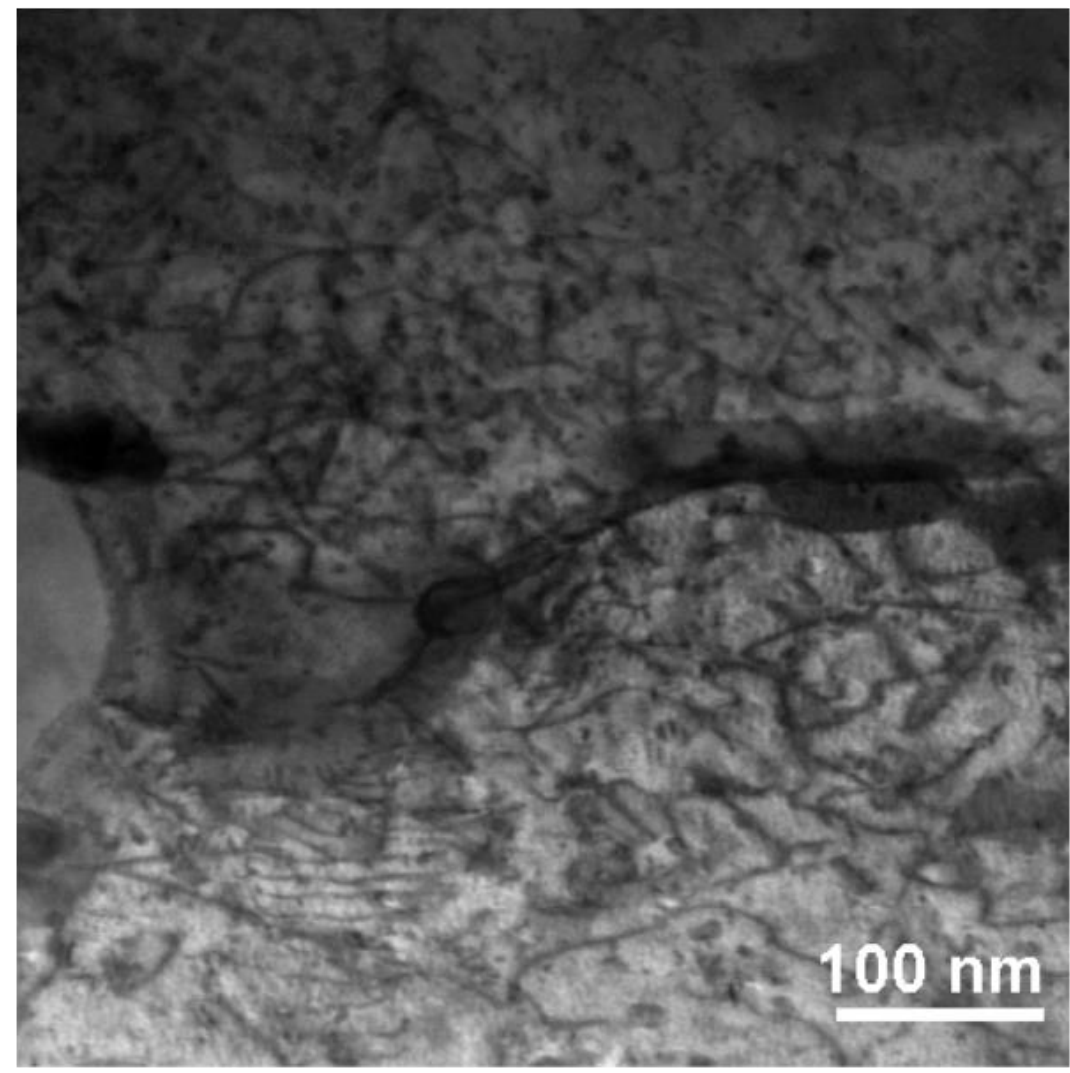

(b)

Figure 5.43: High magnification images of dislocation lines/network in HT9 irradiated with $5 \mathrm{MeV} \mathrm{Fe}^{++}$at $440^{\circ} \mathrm{C}$ with 10 appm He preimplanted to a) 140 and b) 188 dpa. 


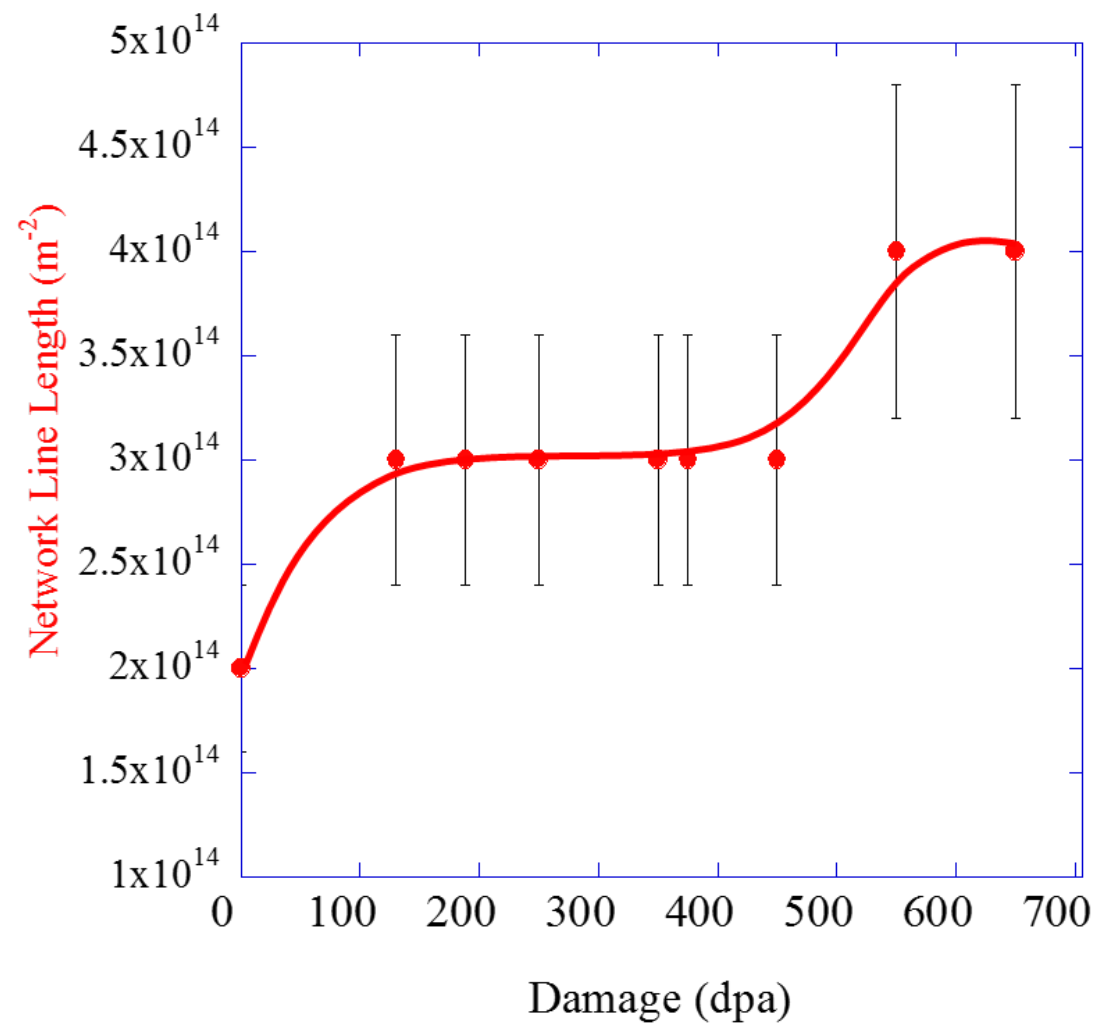

Figure 5.44: Dislocation network evolution under $5 \mathrm{MeV} \mathrm{Fe}^{++}$irradiation at $460^{\circ} \mathrm{C}$ in HT9. 


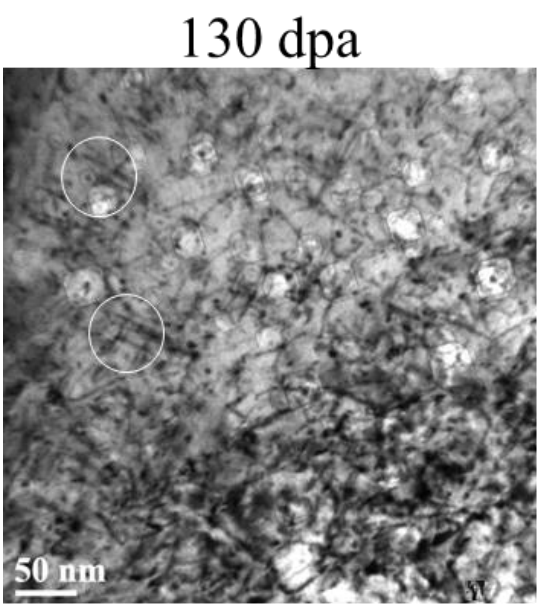

(a)

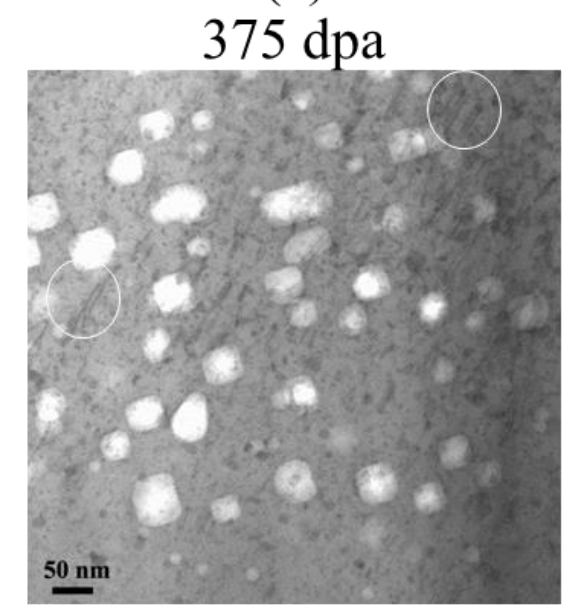

(e)

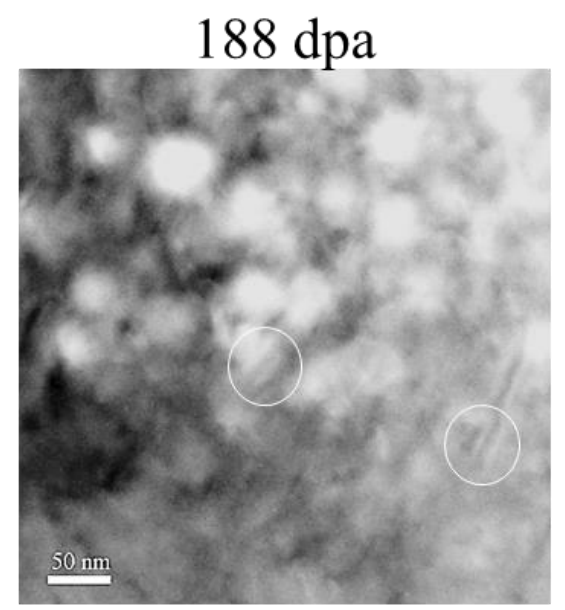

(b)

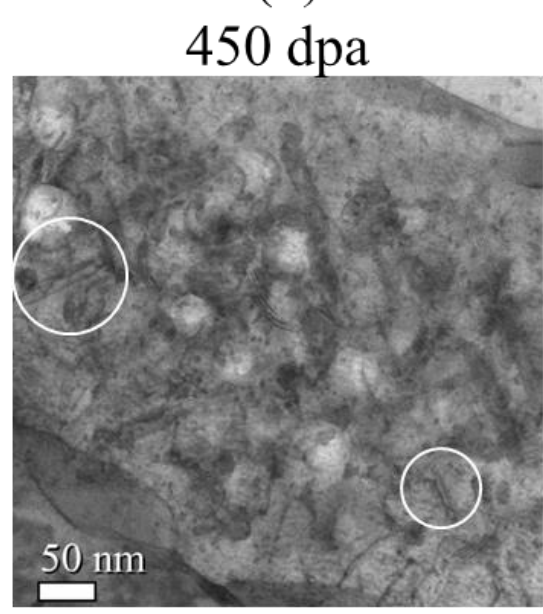

(f)

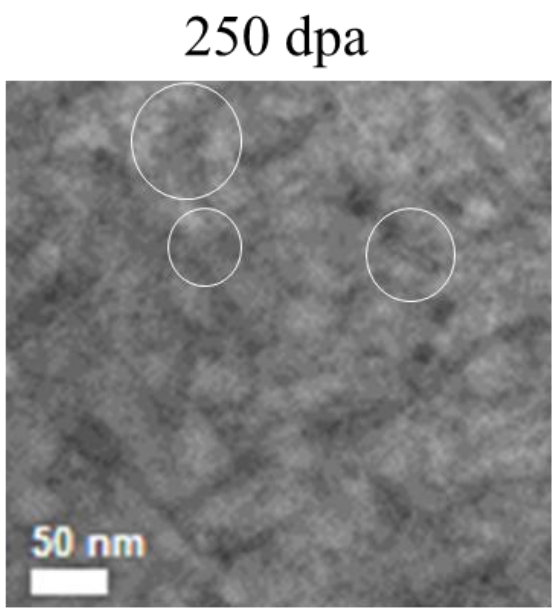

(c)

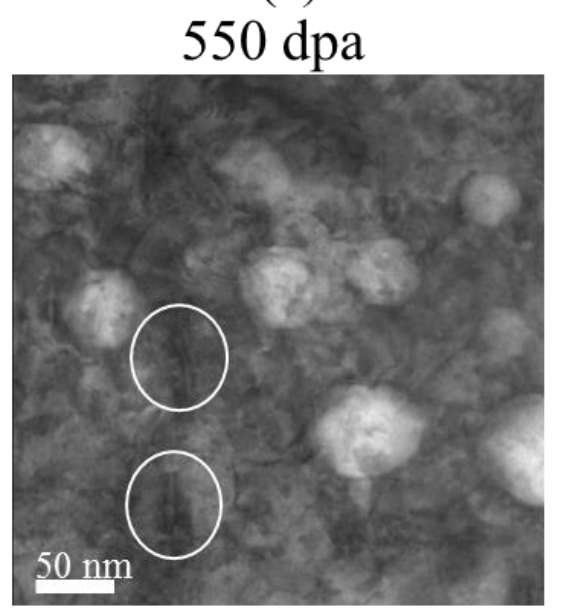

(g)

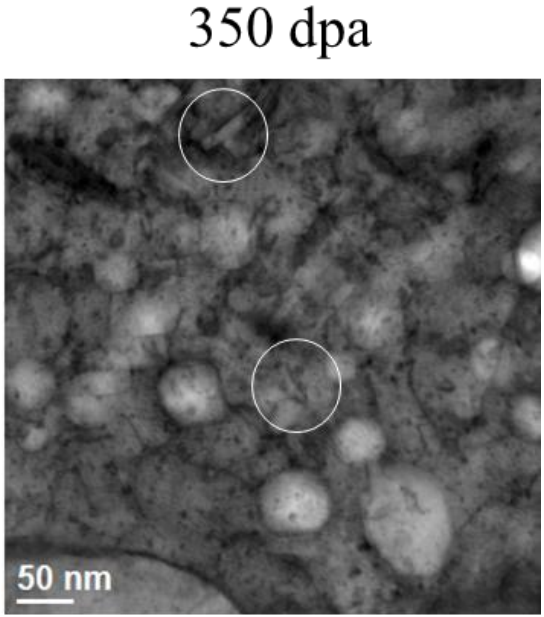

(d)

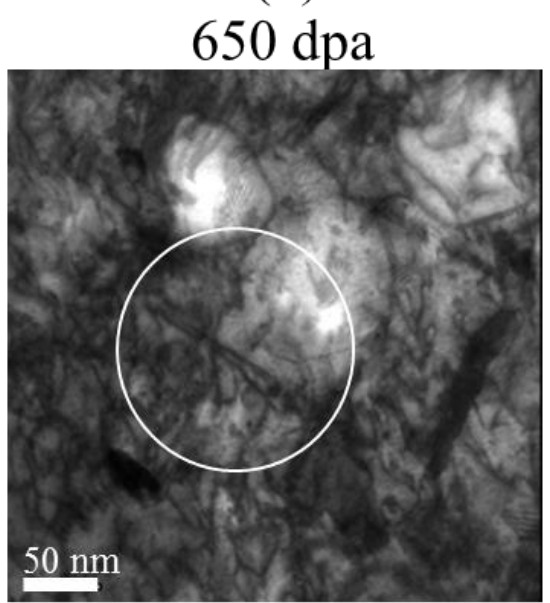

(h)

Figure 5.45: Dislocation loop evolution with increasing damage irradiated at $460^{\circ} \mathrm{C}$ with $5 \mathrm{MeV} \mathrm{Fe}^{++}$. Some loops are marked by white circles. 


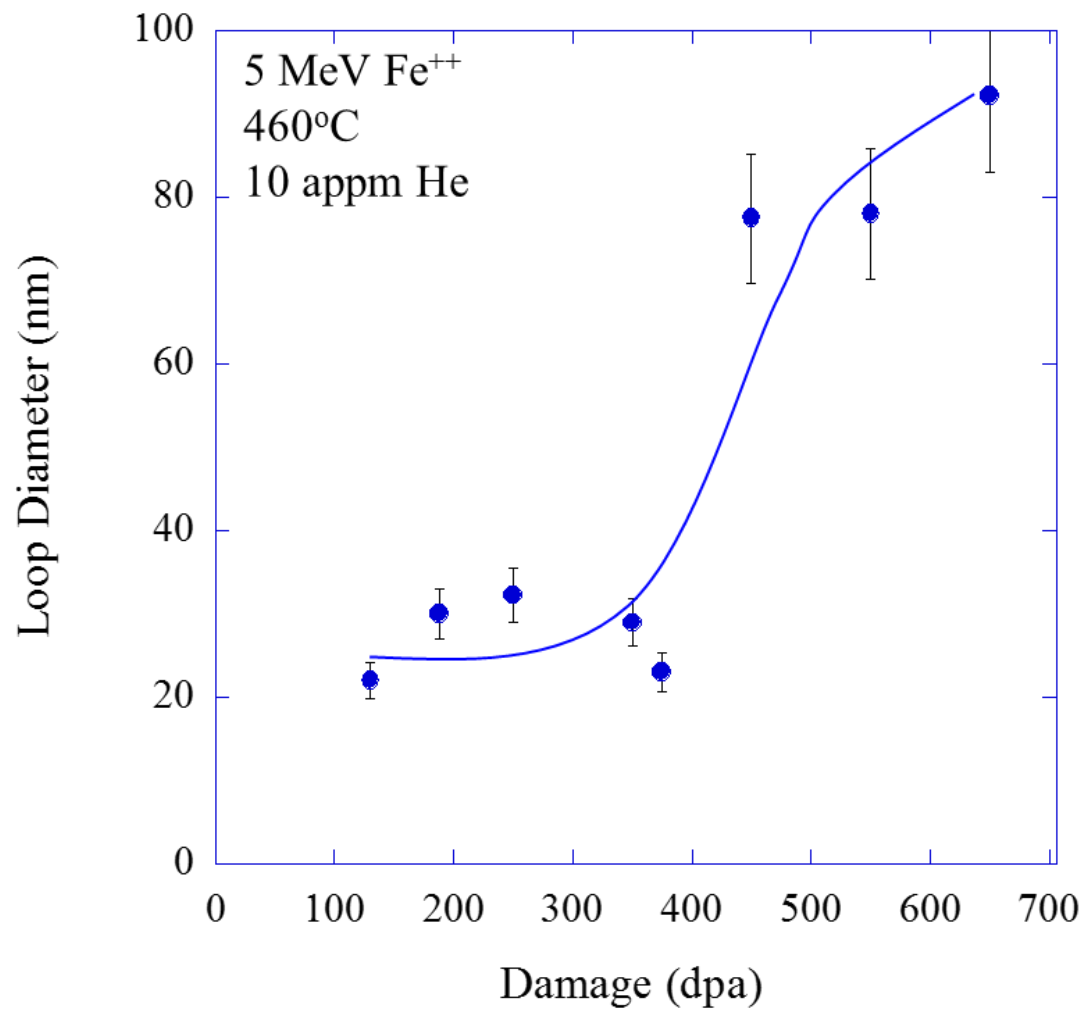

Figure 5.46: Quantification of dislocation loop diameter from 130 to $650 \mathrm{dpa}$ at $460^{\circ} \mathrm{C}$ irradiated with $5 \mathrm{MeV} \mathrm{Fe}^{++}$. 


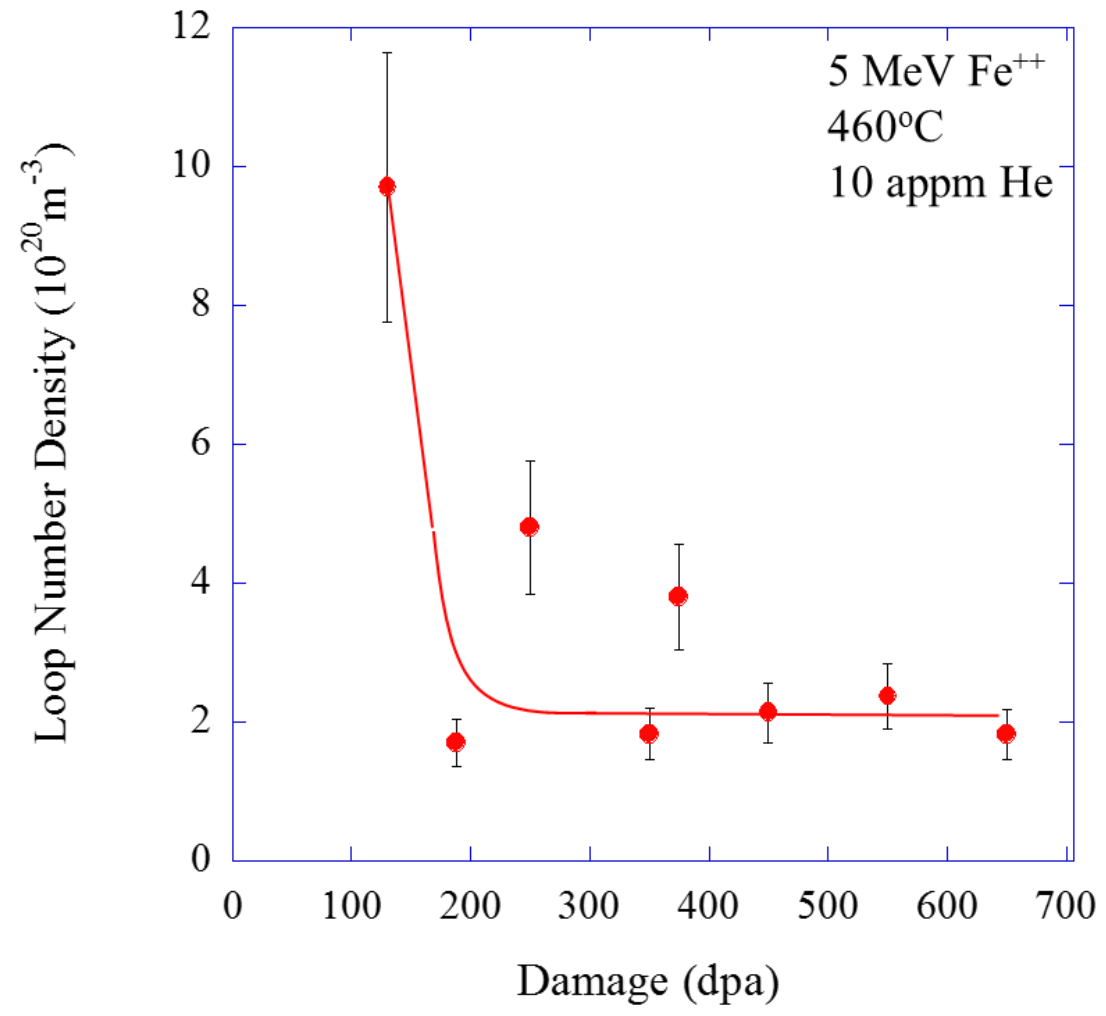

Figure 5.47: Quantification of dislocation loop number density from 130 to 650 dpa at $460^{\circ} \mathrm{C}$ irradiated with $5 \mathrm{MeV} \mathrm{Fe}^{++}$. 


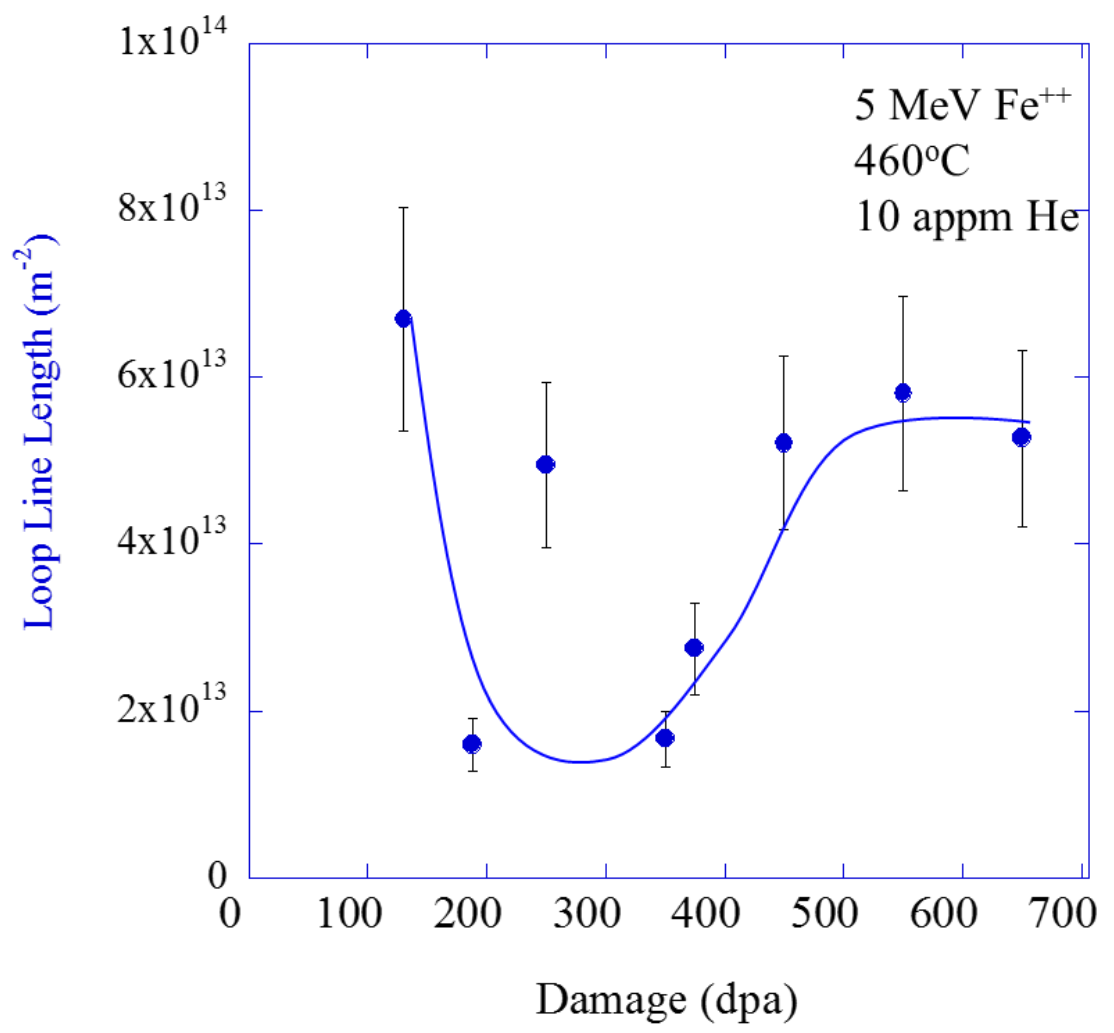

Figure 5.48: Dislocation loop line length in $\mathrm{HT} 9$ irradiated with $5 \mathrm{MeV} \mathrm{Fe}^{++}$at $460^{\circ} \mathrm{C}$ from 130 to $650 \mathrm{dpa}$. 


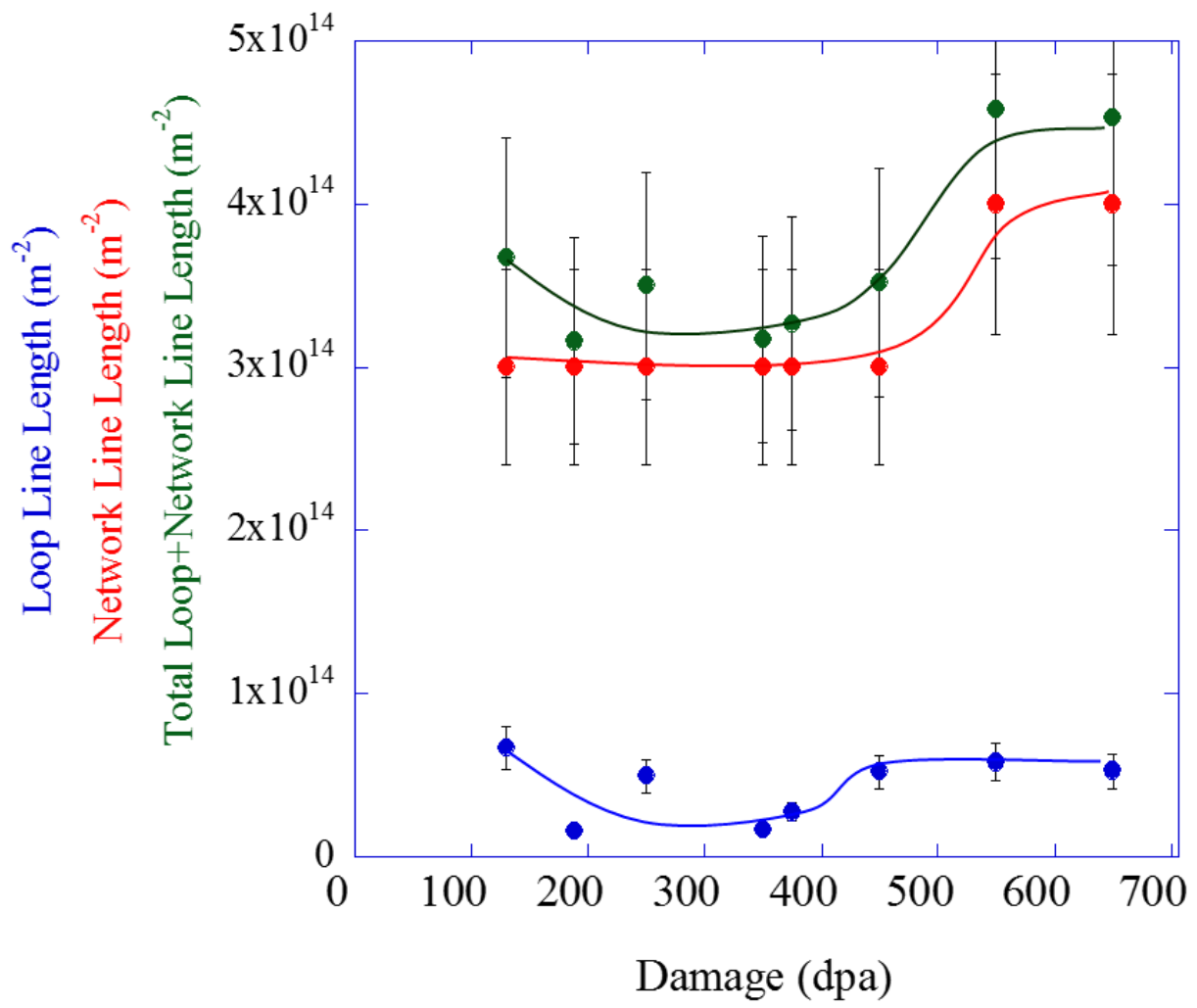

Figure 5.49: Loop (blue), network (red) dislocation line length and total line (green) length in HT9 irradiated with $5 \mathrm{MeV} \mathrm{Fe}^{++}$at $460^{\circ} \mathrm{C}$ up to $650 \mathrm{dpa}$. 


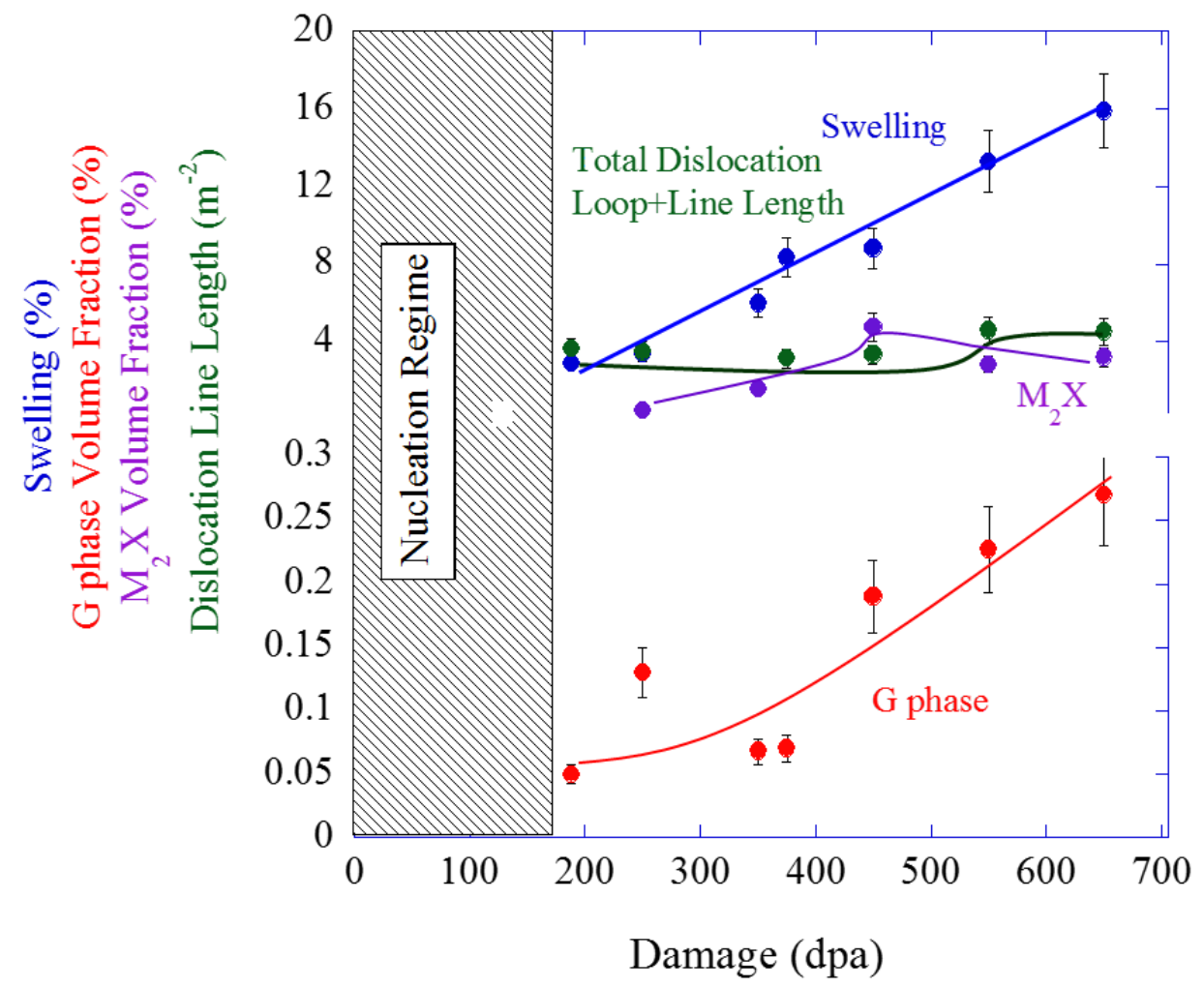

Figure 5.50: Summary of evolving microstructure with damage in HT9 irradiated with $5 \mathrm{MeV} \mathrm{Fe}^{++}$at $460^{\circ} \mathrm{C}$. 


\section{CHAPTER 6}

\section{MODELING}

The chapter will describe the Radiation Induced Microstructure Evolution (RIME) model. A summary of modifications made to the model and reference case definitions will also be included. The modeling efforts in this Chapter will demonstrate that reference cases capture the majority of microstructure evolution, which will be further discussed in Chapter 7. Thus, the behavior of the microstructure in the RIME model in response to mechanistic changes will be correlated to and used to explain the experimental results.

\subsection{Modeling Methodology}

The modeling effort is based upon a mean field approach to a cluster dynamics model. The primary purpose of this section is to describe the modeling approach and perform a sensitivity analysis to verify that the underlying physics is captured as expected. Second, the input parameter selection process will be described including the selection of appropriate values relevant to BCC ferritic-martensitic steels. Third, modifications to the RIME model relevant to discussing significant findings from the experimental results will be described (Section 6.2) and finally, the reference cases for analysis will be presented. (Section 6.3)

\subsubsection{Introduction to RIME Model}


The RIME model utilizes a mean field approach, where cluster evolution is treated using the master equation (ME) that summarizes a large number of differential equations [97,98]. To achieve realistic treatment of cluster evolution up to high damage and high temperature, the large number of equations makes a purely numerical solution computationally difficult or impossible. As such, a grouping scheme was developed to reduce the number of equations to a solvable level. An early grouping scheme was proposed by Kiritani [99]. This and other schemes were later shown to be inadequate [97] because they created an artificial dependence on the group width, leading to unphysical results. An alternative grouping scheme developed by Golubov et al. [97] demonstrated much less sensitivity to the group widths and better agreement with analytical results. This scheme approximated the size distribution within a group using a linear function, rather than a single average value as in most other methods. This linear approximation enables the simultaneous conservation of both the total number of clusters and total number of point defects in clusters. This framework was also extended to permit integration of 2-D MEs, which account for both vacancies and helium gas atoms so that bubbles could be accurately modeled.

For the purpose of this thesis, helium treatment is neglected because helium is pre-injected in the experiment while the regime of modeling interest is at high damage levels (188 dpa and beyond), where there was no simultaneous co-injection of helium, and the major effect of helium was likely on void nucleation [12]. Furthermore, there is no way to experimentally track where He migrates or coalesces in the first 188 dpa.

After adapting the RIME model for bubble kinetics under aging or irradiation in model austenitic alloys [97], the RIME method was further refined by integration of a model for dislocation evolution originally developed by Stoller and Odette $[18,100]$. A brief overview of the equations follows and a description of key subroutines is at the end of this section. 
The generalized model accounts for helium effects and is performed in the $(x, m)$ phase space where $x$ is number of vacancies and $m$ is the number of gas atoms in a cavity, respectively. Since helium is not related in this thesis, equations will only represent calculation/integration in $x$ space, which treats only the vacancies in a given void. For the purpose of this section, the superscript $i$ refers to interstitials and the superscript $v$ refers to vacancies, not voids. Superscript $V$ refers to voids and superscript $L$ refers to voids and loops.

\subsubsection{Description of Model Physics}

The following section will detail the relevant equations used in calculation of swelling and dislocation results. Since He is not included, all cavities are referred to as voids in keeping with the preferred nomenclature.

\subsubsection{1 $\quad \underline{\text { Master Equations }}$}

The size distribution of voids, $f_{x}$, accounts for reactions of voids with vacancies and interstitials in the following equation.

$$
\frac{d f_{x}}{d t}=G_{x}-P_{x}^{i} f_{x}+P_{x+1}^{i} f_{x+1}-P_{x}^{v} f_{x}+P_{x-1}^{v} f_{x-1}-Q_{x}^{v} f_{x}+Q_{x+1}^{v} f_{x+1}
$$

where $G_{x}$ is the generation rate of $x$ clusters in cascades from dpa. Association, $P$, and dissociation, $Q$, for vacancies and interstitials with voids containing $x$ vacancies are defined below: 


$$
\begin{gathered}
P_{x}^{j}=w_{x} D_{j} C_{j} \\
Q_{x}^{v}=w_{x} D_{v} \exp \left(-\frac{E_{x}^{v}}{k_{B T}}\right)
\end{gathered}
$$

where

$$
w_{x}=\frac{4 \pi r_{x}}{\Omega}
$$

where $\Omega$ is atomic volume and the void radius $r_{x}$ is can thus be calculated as:

$$
r_{x}=\sqrt[3]{\frac{3 \Omega x}{4 \pi}}
$$

Additionally, $D_{j}$ and $C_{j}$ are the diffusion coefficients $\left(\mathrm{m}^{2} / \mathrm{s}\right)$ and concentrations (site fractions) of $j$-type defects, $E_{x}^{v}$ is the binding energies of vacancy with a cavity of $x$ vacancies, $T$ is the absolute temperature, $k_{B}$ is the Boltzmann constant. Diffusion coefficients are calculated using migration energy $E_{m}^{j}$, and pre-exponential $D_{j 0}$ :

$$
D_{j}=D_{j 0} \exp \left(-\frac{E_{m}^{j}}{k_{B} T}\right)
$$


A second similar ME is used for calculation of size distribution of interstitial loops. For clarity, the superscript $L$ is used to differentiate this ME from that presented in Equation 6.1.

$$
\frac{d f_{x}^{i L}}{d t}=G_{x}^{i L}-P_{x}^{i} f_{x}^{i L}+P_{x+1}^{v} f_{x+1}^{i L}-P_{x}^{v} f_{x}^{i L}+P_{x-1}^{i} f_{x-1}^{i L}
$$

where

$$
P_{x}^{j}=w_{x} D_{j} C_{j}
$$

$w_{x}=\frac{2 \pi r}{\Omega}$ is calculated for loops. The loop radius is calculated below in Equation 6.9:

$$
r_{x}=\sqrt{\frac{\Omega x}{\pi b}}
$$

$b$ is the burgers vector. Vacancy loops are included as an option in the code and have a similar formalism, but not treated in this thesis. The conversion of loops to the network is discussed in Section 6.1.2.4.

Concentrations for each defect species are shown below in Equation 6.10 and Equation 6.11. 


$$
\begin{gathered}
\frac{d C_{i}}{d t}=G_{i}-\mu D_{i} C_{i} C_{v}-\left(z_{i}^{d} \rho_{d}+z_{i}^{L} k_{L}^{2}+z_{i}^{G B} k_{G B}^{2}+k_{b}^{2}\right) D_{i} C_{i} \\
-2 w_{l} D_{i} C_{i}^{2}+\left(P^{v} f_{L}\right)_{x=2}+P_{F P} \\
\frac{d C_{v}}{d t}=G_{v}-\mu D_{i} C_{i} C_{v}-\left(z_{i}^{d} \rho_{d}+z_{i}^{L} k_{L}^{2}+z_{i}^{G B} k_{G B}^{2}+k_{b}^{2}\right) D_{v}\left(C_{v}-C_{v}^{e}\right) \\
-2 w_{l} D_{v} C_{v}^{2}+\left(Q^{v} f\right)_{x=2}
\end{gathered}
$$

where $\mu$ is the recombination constant, $\rho_{d}$ is the dislocation density, $k_{L}^{2}$ is the sink strength for interstitial loops, $k_{G b}^{2}$ is the sink strength for grain boundaries and $k_{b}^{2}$ is the sink strength for the bubbles. $z_{j}^{G B}$ are capture efficiencies from grain boundary, $z_{j}^{d}$ is the bias for each defect species, $G_{j}$ are the defect generation rates and $C_{v}^{e}$ is the thermal-equilibrium vacancy concentration calculated as

$$
C_{v}^{e}=C_{v 0}^{e} \exp \left(-\frac{E_{f}^{v}}{k_{B} T}\right)
$$

where $C_{v 0}^{e}$ is the pre-exponential.

\subsubsection{Sink Strength Calculations}

The sink strength of voids is calculated as the integral of the overall total number of vacancy clusters in the grouping scheme: 


$$
k_{b}^{2}=\sum_{x} \frac{4 \pi r_{x}}{\Omega} f_{x}(\mathrm{x}) \Delta_{x}^{V}
$$

where $\Delta_{x}^{V}$ is the width of the groups. For planar loops, sink strength is approximated by

$$
k_{i L}^{2}=\sum_{x} \frac{2 \pi r_{x}}{\Omega} f_{x}^{i L}(x) \Delta_{x}^{i L}
$$

Again, $\Delta_{x}^{i L}$ is the group width.

\subsubsection{Defect Production Rates}

The point defect production rates are equivalent to those in the NRT standard damage rate [66], with a correction for fractions of defects recombined in cascades $\varepsilon_{r}$ and the fraction of defects clustered in cascades, $\varepsilon_{v}$ and $\varepsilon_{i}$ are shown in Equation 6.15 and Equation 6.16.

$$
\begin{aligned}
& G_{i}=G^{N R T}\left(1-\varepsilon_{r}\right)\left(1-\varepsilon_{i}\right) \\
& G_{i}=G^{N R T}\left(1-\varepsilon_{r}\right)\left(1-\varepsilon_{v}\right)
\end{aligned}
$$

The generation rate of vacancy and interstitial clusters is given below in Equation 6.17.

$$
G_{x}^{j}=A^{j} x^{-k_{j}}
$$

\subsubsection{Network Dislocation Evolution}


Network dislocations were allowed to evolve or remain static based upon user choice. For the dynamic network treatment, the network decreases due to annihilation reactions and increases due to large loops reaching a specified radius and join the network.

$$
\frac{d \rho_{d}}{d t}=G_{d}-\rho_{d}\left(\tau_{i r r}^{-1}+\tau_{t h}^{-1}\right)
$$

$\tau_{i r r}$ is the average lifetime of a dislocation for annihilation, from dislocation climb, calculated as

$$
\tau_{i r r}=\frac{b_{d}}{\sqrt{\pi \rho_{d}}}\left[z_{i}^{d} D_{i} C_{i}-z_{v}^{d} D_{v}\left(C_{v}-C_{v}^{e}\right)\right]^{-1}
$$

The thermal contribution is described by:

$$
\tau_{t h}=\left[\frac{A^{\prime} 2 \pi^{\frac{2}{3}}}{\ln \left[\frac{\left(\pi \rho_{d}\right)^{-\frac{1}{2}}}{2 b_{d}}\right]} \frac{\Omega G D_{v} C_{v}^{e}}{k_{b} T} \rho_{D}\right]^{-1}
$$

where $A^{\prime}$ is the modified back stress term and the generation term. The generation term has two contributions: first, the Bardeen Herring dislocation source and second, growth of loops into the network with radius greater than $r_{\max }$. The loop generation term is described by Equation 6.21 and $r_{\max }$ is a code input calculated from the number of interstitial groups mil and the group spacing $d r i$. 


$$
G_{d}=\left(\frac{2 \pi r_{\max }}{\Omega}\right)^{2} z_{i}^{i L} D_{i} C_{i}
$$

$z_{i}^{i L}$ is the bias of loop for interstitials.

\subsubsection{Calculation of Radii/Swelling}

Size distributions must be converted from $x$ space, where cluster size, $x$, is defined as the number of vacancies in a given cluster, to $R$ space, where the cluster size is defined as the radius $R$ of the cluster. This is performed using the following relationship:

$$
f_{x} d x=f_{R} d R
$$

For voids, this becomes

$$
f_{R}=\frac{4 \pi R^{2}}{\Omega} f_{x}
$$

For dislocation loops, this becomes

$$
f_{R}=\frac{2 \pi R b}{\Omega} f_{x}
$$

where $b$ is the loop Burgers vector. Swelling (S) is calculated by integrating the size distribution of voids similar to sink strength calculation: 


$$
S=\sum_{x} x f_{x} \Delta_{x}^{v}
$$

\subsubsection{Grouping Scheme}

Previously mentioned, a grouping scheme was developed to conserve number of defects and clusters. Size distributions are represented by a linear function within each group

$$
f_{x}=a+b(x-<x>)
$$

where $\langle x\rangle$ is the group mean values and $a$ and $b$ are constant unique to each group. The coefficient

$a$ is the mean cluster concentration in each group $\left\langle f_{x}\right\rangle$ while the mean value of vacancy in clusters of the group are calculated from

$$
<f_{x} x>=a<x>+b D_{x}
$$

where

$$
D_{j}=<j^{2}>-<j>^{2}
$$

$D_{j}$ are the dispersions for each group, not diffusivity, in this case.

Time integration is performed using the FORTRAN subroutine Livermore Solver for Ordinary Differential Equations (DLSODE) from Hindmarsh [101] and is coded in FORTRAN 90. 


\subsubsection{Consideration of Production Bias Model}

The production bias model (PBM) has been used to effectively explain behavior or pure metals with low dislocation densities [98]. The PBM includes the production of point defect clusters as well as one dimensional (1D) diffusion, which has been suggested as a better treatment of in-cascade clustering [98,102-105]. However, this model is not considered here because of several limitations of the model. The PBM predicts swelling saturation at a certain void radius, which is inconsistent with the void results from Figure 5.16 and Figure 5.18, which do not show diameter or swelling saturation. This swelling saturation is due to the change in reaction cross section being proportional to $\mathrm{R}$ for $3 \mathrm{D}$ diffusion and $\mathrm{R}^{2}$ for $1 \mathrm{D}$ diffusion, which can cause negative vacancy flux. Furthermore, if the PBM model is applied without 1D diffusion, the system is at risk for the so-called Singh-Foreman catastrophe which is the result of a high density of sessile interstitial clusters completely dominating the microstructure [102]. For these reasons, the PBM is not considered as an appropriate modeling methodology for this application.

\subsubsection{Description of Main Sub Routines}

The overall structure of the code is quite complex, with 9 categories of 38 sub routines. Categories refer to groups of sub routines, which have the same function. For instance, the s00 category includes all sub routines providing inputs and definitions required at the start of each case run. A full description of all sub routines and adaptations made in this thesis is given in Table 6.1. The main 11 sub routines relative to this work will be described in this section.

s00_rime.main.f: This is the main do loop, which calls functions for each time step. In addition, it calls the solver for each time step and calls intermediate results to both the screen and files. 
s00_preexistm.f: This sub routine is a new addition and reads in microstructure data with which to start an irradiation. Meaning, the void and dislocation distribution can be read in at $t_{0}$, to mimic an already irradiated microstructure. For the purposes of this study, this was the microstructure observed at $460^{\circ} \mathrm{C}, 188 \mathrm{dpa}$. More information on the preexisting microstructure sub routine is in Section 6.2.1.

sO2_a_void.f, s02_a_iloop.f: These sub routines define the vacancy and interstitial grid for voids and interstitial type loops, respectively, for cases not including the effect of helium. Reaction rate constants and binding energies are calculated in this section. Generation rates of vacancy and SIA clusters in cascade are also calculated.

s03_sd_void.f, s03_sd_iloop.f: Calculates the size distribution, in terms of number of voids for either voids or interstitial loops.

s04_treatment: This sub routine treats the overall results. It calls upon sub routines to determine size distributions with which to calculate results relevant to experimental data such as radii and number density. It also tallies defect absorption at various sinks.

s04_rt_void.f, s04_trt_iloop.f: This routine takes the cluster distribution calculated from s03 sub routines and translates the outputs into cavity or loop radii, number density and swelling.

s05_read_continue.f: This routine reads in data for continuation of irradiation, which allows parameters to be changed at a user determined damage level. 
s06_write_screen.f: This routine prepares intermediate results from each time step to be written to either the screen or files at each examined dpa.

s07_mobile_defects.f: This routine provides derivatives of the concentration of freely migrating defects including SIAs and vacancies to and from solver.

s08_dlsode.f: This is the sub routine containing the ordinary differential equation solver used for this model.

s09_fexjex.f: This routine calculates time derivatives output from the solver; it also calls calculations for size distributions and includes treatment of the precipitates.

A flow chart with the main structure and sub routines is provided in Figure 6.1.

For each case run, desired experimental conditions must be included in an input file. A table summarizing a generic input file is shown in Table 6.2. Parameters that are not used for this thesis are highlighted in red. Parameters that have been modified or added to the code are highlighted in yellow. A parameterization was performed based upon the results from [2,12] to verify that void and loop behavior was behaving in line with experimental results. This determination of key migration and formation energies for BCC FM alloys will be further discussed in Section 6.1.5. 
Given the complexity of this code and the varied applications, there are many data outputs that fully describe the microstructure evolution. A full list of outputs is available in Table 6.3 with the main outputs described below. The relevant output files will be described below.

$z m \_s w s s . d a t:$ Main output file used in for this analysis. This output file contains a summary of key swelling, loop, network, precipitate results and includes the sink strengths as well.

zm_liz.dat: Summary of void and loop diameter, number density. Swelling, sink strengths for network, loops, void and precipitates.

$z_{\_} s d w 3 . d a t$ : This file contains the size distribution of voids as a function of damage level.

z_sdil.dat: This file contains all relevant results regarding interstitial loop evolution.

$z m \_d e n s . d a t$ : This file includes number densities for voids and loops as a function of damage level.

$z m \_$radi.dat: This file includes radii for voids and loops as a function of damage level.

$z m \_c o n c . d a t:$ This file includes concentrations of mobile defects as a function of damage level.

This section has provided a very brief introduction to the development and utilization of the RIME model. Prior to implementation, a sensitivity analysis was performed 1) to understand the significance of several key experimental parameters and 2) to verify that the model was responding to perturbations of input in a physical way. 


\subsection{4 $\underline{\text { Sensitivity Analysis }}$}

The RIME model requires input of a large number of parameters, which control defect behavior in the system, in turn controlling the swelling behavior of the system. In this section, a sensitivity analysis was performed to identify input parameters that have the greatest effect on the model output. Output in this case will be measured by swelling/swelling rate. Furthermore, changing various parameters will verify that the essential physics are captured by the RIME model and that no unphysical responses are observed.

Model sensitivity is defined as the derivative of the swelling or swelling rate as a function of a given input parameter. The sensitivity was approximated as the ratio of the change in swelling or swelling rate relative to a change in the input parameter value. This is calculated as below:

$$
\frac{\frac{\partial \Delta V}{V}}{\partial P} \approx \frac{\frac{\delta \Delta V}{V}}{\delta P}=\frac{\frac{\Delta V^{\prime}}{V}-\frac{\Delta V}{V_{\text {reference }}}}{P^{\prime}-P_{\text {reference }}}
$$

where $\frac{\Delta V}{V}$ refers to the swelling and $\frac{\Delta \dot{V}}{V}$ refers to the swelling rate, both of which will be a calculated as part of this study. $P_{\text {reference }}$ is the reference input parameter, $P^{\prime}$ is the varied parameter and $\frac{\Delta V^{\prime}}{V}$ is the resulting swelling from $P^{\prime}$.

Sensitivity is best expressed as significance, which is the fractional change in swelling/swelling rate relative to the fractional change in input parameter. Significance is calculated as: 


$$
S_{P}^{\frac{\Delta V}{V}}=\frac{\frac{\Delta V^{\prime}}{V}-\frac{\Delta V}{V_{\text {reference }}}}{P^{\prime}-P_{\text {reference }}} * \frac{P_{\text {Reference }}}{\frac{\Delta V}{V_{\text {reference }}}}
$$

The parameters with the highest significance values most strongly impact resulting swelling.

To isolate changes in each of the parameters, a reference case (Ref.2) was run to $20 \mathrm{dpa}$. (Ref.2 parameters will be further described in Section 6.3.1.) At 20 dpa, the parameter of interest was changed (for instance, bias was perturbed) and the resulting swelling and swelling rate were measured at 21 and $100 \mathrm{dpa}$, to calculate significance at each bias. The change in parameter was performed at 20 dpa to mitigate instability in dislocation microstructure at the beginning of the irradiation. The main input parameters used in this study are given in Table 6.4 and the irradiated microstructure at $188 \mathrm{dpa}$ was used. This will be further discussed in Section 6.2.1. A sample significance graph for bias is shown in Figure 6.2 and for grain size is shown in Figure 6.3. The significance results for both swelling and swelling rate are shown in Table 6.5 and Figure 6.4.

First, the overall trends matched what was expected. With increasing bias, the swelling rate increased, indicating a higher preference of loops/network for interstitials in turn promoting excess vacancy population. Second, increased grain size also increased swelling due to the lower grain boundary sink strength, which acted as an alternate neutral sink for defects. Third, with increased temperature, the swelling slightly increased. It was not expected to have a large effect, since the pre-voided microstructure was used in this study. Examining the void characteristics revealed an increased void size with decreased number density with temperature, exactly as expected. Finally, 
with increasing damage rate the swelling rate decreased slightly. This was expected as lower damage rates are expected to promote void swelling [8].

Figure 6.4 demonstrates the magnitude of each significance. A few key observations were noted. Parameter change affected swelling rate more so than swelling. Clearly, bias was the controlling feature in terms of void swelling. The next most significant value was temperature and grain size followed by damage rate. Bias as the controlling parameter is reasonable as bias directly affects defect flow as opposed to the other parameter changes, with have more indirect effects. Therefore, choosing an appropriate bias was key in modeling this system. A final observation is that there is little change in significance with the addition of unbiased precipitates into the system, indicating that key physics was still captured with precipitation treatment.

A second significance study was performed to address other input parameters including cascade efficiency, recombination coefficient, vacancy migration and formation energies and interstitial migration energy. The methodology was identical to that used for the results presented in Table 6.5. The results are included in Table 6.6 and Figure 6.5. Of these features considered, the next most significant was the cascade efficiency, which has a significance of -2.0 . The next more significant was the interstitial migration energy followed by vacancy migration and formation energies and recombination coefficient was the least significant. Bias was also included for comparison and was again shown to be the most significant feature. The low significance of these other input values gives confidence that input chosen from literature, described in the next section, will be appropriate.

\subsubsection{Input Parameter Selection}


The previous section showed that variation of some parameters such as bias can highly influence void behavior within the RIME model. The major challenge with any rate theory model is determining an appropriate parameter set. Many of these cannot be directly measured and may only be inferred in post hoc experimental analyses. In addition, for more complex alloys such as HT9, there are many microchemical or microstructural variations which cannot be accounted for just by varying binding energies and assorted inputs. In this section, the reference input values will be discussed in modeling HT9 as Fe-Cr binary values. Suitable input values were taken from a variety of sources. In Table 6.2, values highlighted in red are those that are not relevant for this study and were thus not utilized. Parameters in yellow are those that were changed as a result of the transition from modeling FCC to BCC, were added as part of the additional capabilities of the RIME model or were used as a flag to include a more complex microstructure treatment (such as the treatment of precipitates). The non-highlighted features are those that were not changed as part of this work. A summary of the main changes is given in Table 6.7 showing the changes from FCC [97] to BCC. The main change was an increase in dislocation density. The unirradiated dislocation density was measured to be $2 \times 10^{14} \mathrm{~m}^{-2}$ with only a modest increase up to $3 \times 10^{14} \mathrm{~m}^{-2}$ by $188 \mathrm{dpa}$. Grain diameter was characterized prior to irradiation with lathes being 800 to $1000 \mathrm{~nm}$ on average and a lath width of $\sim 350 \mathrm{~nm}$. Since lathes are the smallest dimension, and as a consequence the strongest grain sink strength, the lath dimension is used in lieu of the prior austenite grain diameter. For simplicity, a $1 \mu \mathrm{m}$ lath was assumed. Vacancy and interstitial formation and migration energies are key in capturing appropriate defect kinetics. These formation, migrations and diffusion preexponentials were taken from [106-109]. The chosen cascade efficiency $(\eta)$, also referred to as point defect survival fraction, was 0.33 based upon molecular dynamics results [110,111]. (In RIME, this input value is reported as $\varepsilon_{R}$ which is the fraction of point defects recombined rather 
than survived where $\eta=1-\varepsilon_{R}$, which is the more accepted nomenclature in the literature.) In general, for mean theory applications, any value between 0.1 to 0.3 is appropriate, with object kinetic Monte Carlo (OKMC) typically reporting higher efficiencies (0.2) than atomistic kinetic Monte Carlo (AKMC) cascade efficiency (0.1), which was attributed to lack of inclusion of atomistic interactions in OKMC [112,113]. Finally, bias was found to be a key parameter in determining is swelling behavior in Section 6.1.4. As a result, a range of values from 1.01-1.3 was found in the literature for BCC iron/iron alloys [8,41-43,114,115], with a majority varying from 1.01-1.05. As such, bias variations were limited between these values. A more in depth discussion of bias is found in Section 7.2.

The generally accepted range for cascade efficiency $(\eta)$ for mean field rate theory applications is from 0.1 to 0.33 and the range for dislocation biases for interstitials $\left(z_{L}^{i}\right)$ is from 1 to $3 \%$. A comparison of the effect of cascade bias and dislocation bias in these ranges is presented in Figure 6.6. Low cascade efficiency combined with low bias decreases swelling while swelling increases in proportion to increased cascade efficiency and dislocation bias, in general. The majority of these values ranges from final swelling values at 650 dpa of 23-33\% with the lowest swelling from the $\varepsilon^{=} 0.1, z_{i}^{\text {loop }}=z_{i}^{\text {net }}=1 \%$ case and the highest swelling from the $\varepsilon=0.33, z_{i}^{\text {loop }}=$ $z_{i}^{n e t}=3 \%$ case, resulting in final swelling of $9 \%$ and $80 \%$, respectively. Thus, the chosen reference values of $\varepsilon=0.33, z_{i}^{\text {loop }}=z_{i}^{\text {net }}=1 \%$ (in blue) are reasonable within the range of swelling behavior presented in Figure 6.6.

\subsection{Adaptations to RIME Model for Void Growth-Dominated Microstructure}


To aid in more complete analysis of the irradiated microstructure, a number of microstructure options were added into the existing RIME model. These are isolated into four categories. First, since the focus of this thesis is on the void growth-dominated microstructure rather than any treatment of nucleation, an option for the existing void and dislocation loop distribution were included. Second, an explicit dislocation loop treatment was developed. Third, a treatment of carbon as a trapping site was included. Finally, treatment for precipitates as unbiased, biased or variable biased sinks or as recombination centers was also included.

\subsubsection{Pre-Existing Microstructure}

The study of void nucleation is an open question in the study of radiation effects. As such, this thesis is confined to examining the effect of microstructure features on voids in the growthdominated swelling regime. In Chapter 5, the onset of linear swelling was determined to be 188 dpa at $460^{\circ} \mathrm{C}$. At this damage level, there is a well characterized void (Figure 6.7a) and loop (Figure 6.7b) distribution in addition to a characterized network dislocation density. Thus, a model option to start irradiations at $188 \mathrm{dpa}$ is needed and a formalism for starting at a "pre-irradiated microstructure" was developed. Prior to development of the sub routine, an approximate distribution was calculated as input for the code. (Figure 6.8) The actual input used was idealized relative to the experimental distribution, to limit statistical variation.

The basic method was as follows: 1) Determine an appropriate grouping vector to reflect the observed void and loop distribution in s02a_void.f. 2) Read in an approximate void and loop dislocation distribution (Figure 6.8) 3) Use this distribution as the arbitrary $t_{0}$. The void distribution was idealized, but based upon the actual experimentally observed distribution. 


\section{$\underline{\text { SO2a_void.f }}$}

This sub routine determines the vacancy grid. The vacancy grid was determined in terms of $x$ space, meaning each number of vacancies corresponds to a radius of voids for each cluster of size $x$. For instance, a cluster of size 1 has a radiations of $0.1408 \mathrm{~nm}$. Previously, the grouping size was determined based upon increasing $x$ space, which did not yield a systematic increase in radius. This was adapted such that

$$
\begin{aligned}
& d x v \\
& =\left\{\begin{array}{cc}
1 & x<30 \\
\operatorname{dint}(x w(i-1) * 3.0 * d r v v)+1.0 & x<363, d r v v=0.12 \\
\frac{4 \pi}{3 \Omega * 10^{27}} *\left(r w h^{3}-r w h(i-1)^{3}\right) & x>363, d r v v=1
\end{array}\right\}
\end{aligned}
$$

where $d x v$ is the change in group size, dint is a FORTRAN function that converts a double to integer, $x w$ is a vector of the $x$ grid sizes, $d r v v$ is the change in radius size and $\Omega$ is the atomic volume. Essentially, this converts the vacancy grid from being controlled by $x$ to controlled by $r$ at sizes greater than $1 \mathrm{~nm}$ so that the void distribution can be more accurately input. The practical results of this alternate grouping scheme is that the change in each radius group above $1 \mathrm{~nm}$ is 1 $\mathrm{nm}$. Dislocation loop grouping scheme is adapted similarly.

\section{SO0_preexistm.f}

With the grid properly defined, an additional subroutine was developed to read in an input file (zz_microst.dat) for loop and void distributions. A flag "iliz" was added into the input file as 
well. When iliz=1, the s00_preexistm.f subroutine was called so that the radius and void distribution fraction $(f)$ at a given radius. $f$ was calculated from

$$
f=b+a(x-\bar{x})
$$

where $b$ is the group vacancy fraction, $a$ is the slope from the group fraction of the previous group to the next and $\bar{x}$ is the average number of vacancies. For the purpose of this calculation $a \approx 0 . b$ is calculated as:

$$
b=\frac{N \Omega}{x_{\max }-x_{\min }}
$$

where $N$ is the number density of voids in each group, taken from the experimental void distribution. This process is repeated similarly for interstitials as part of the interstitial loop treatment.

Finally, if the iliz flag is chosen, the void and loop distribution will not only be read in, but then sent to the subroutine initfors in s01_exchange.f which determines the initial y(i) that is sent to the DLSODE solver, effectively starting the irradiation at $188 \mathrm{dpa}$.

\subsection{2 $\underline{\text { Dislocation Treatment }}$}

The original RIME model included two dislocation treatments. The first was assuming a constant network density that was set at the beginning of the case run. The user inputs the desired 
network sink strength, which can be changed manually at any time during the irradiation by using the continue option in subroutine s05_write_continue.f.

The second dislocation treatment already incorporated into RIME, hereafter referred to as dynamic dislocation (DD) treatment, allows the dislocation loops and network to evolve based upon the initial experimental input. The existing dislocation treatment was previously described in Section 6.1.2 based upon modeling results from $[18,100]$.

To more accurately represent the overall experimental dislocation microstructure evolution, a formalism for explicit treatment of loops was added. Functions defining the dislocation loop radius and number density evolution with damage were determined and are shown in Figure 6.9. These equations were read in from the input file.

After the number density and radius of loops are read in, the sub routine s09_fexjex.f was updated to provide an additional biased sink strength, with the bias defined by the user input $z_{i}^{l p}$. In s09_fexjex.f, the variables $s s \_l p i$ and $s s \_l p v$ were added to track the sink strength of interstitials and vacancies, respectively, of the loops. Values for vacancy and interstitial cluster sizes are called from the DLSODE solver. Then, each radius and number density was updated for each time step. That sink strength is then used in s07_mobile_defects.f to calculate the reaction rate coefficients for loops, which were used in determining the number of defects that are absorbed at the loops. This is identical to how the dislocation network was treated, when network density is assumed to remain constant. One limitation is that the sink strength evolution of the loops is prescribed, so the evolution is a function of the input and does not react to the absorbed defects.

\subsubsection{Carbon-Vacancy Interactions}


Carbon is an undersized interstitial solute in steel. A number of studies have posited that carbon may act as a point defect trap for either interstitials or vacancies [116-122]. Free carbon interstitials can trap either vacancies or interstitials by raising the migration energy leaving them less mobile. This also serves the purpose of having trapping sites that form recombination centers, leave the freely migrating defect population smaller. In this case, carbon is treated as traps for vacancies with a binding energy of $\sim 0.4-0.8 \mathrm{eV}$ [123-125] will be considered. To model this mechanism, an effective vacancy diffusion coefficient due to formation of the C-v complexes is calculated via the following equation:

$$
D_{v}^{e f f}=\frac{D_{v}}{1+\alpha C_{s} \exp \left(\beta E_{v s}^{b}\right)} \approx \frac{D_{v 0}}{\alpha C_{s}} \exp \left[-\beta\left(E_{v}^{m}+E_{v s}^{b}\right)\right]
$$

where $\alpha=$ coordination number for BCC interstitials, $C_{s}$ is the concentration of vacancy traps in appm, $\beta=1 / k_{b} T$. Thus, both an appropriate binding energy and concentration of solute trap sites can be used as an input.

Carbon is considered as the primary solute available to trap dislocations and also to trap vacancies. Theoretically, other solutes could operate in a similar way. A table including all minor alloying elements with concentrations and approximate diffusion coefficients is provided in Table 6.8. Although some data suggests that other small interstitial solutes like $\mathrm{N}$ may provide similar trapping, the concentration is too low for consideration. Larger solutes such as $\mathrm{Cr}, \mathrm{Ni}$, $\mathrm{Mn}, \mathrm{Si}, \mathrm{P}$ and $\mathrm{S}$ have limited diffusivity in iron. Therefore, due to its small size, relatively large concentration and demonstrated precipitation out of solution $\mathrm{C}$ is likely as the major solute effect in the model. 


\subsubsection{Precipitation}

The original RIME model did not include precipitate behavior. A full model of precipitation behavior would include microchemical effects and is outside the scope of this thesis. However, an explicit treatment of precipitation behavior can be used to determine how the introduction of precipitates as sinks may affect void or dislocation behavior as defect partitioning is altered. Further, the precipitate dataset presented in Chapter 5 was systematic enough that equations describing the behavior of both $\mathrm{G}$ phase and $\mathrm{M}_{2} \mathrm{X}$ could be determined and input into the RIME model.

First, $\mathrm{G}$ phase is considered. $\mathrm{G}$ phase was determined to be an incoherent, unbiased sink in Section 5.3.1. The evolution as a function of dpa was determined for the radius and number density in Figure 6.10. The radius increases linearly at a slope of $0.008 \mathrm{~nm} / \mathrm{dpa}$ (Figure 6.10a). The number density remains roughly constant throughout this dpa range and so was assumed to be constant at $8.5 \times 10^{20} \mathrm{~m}^{-3}$. (Figure $6.10 \mathrm{~b}$ )

Second, $\mathrm{M}_{2} \mathrm{X}$ is considered. $\mathrm{M}_{2} \mathrm{X}$ was determined to be coherent in Section 5.3.2, and likely acts as a coherent sink in terms of defect absorption. Regardless of its sink characteristic, the effective radius and number density need to be determined as a function of dpa. Since $\mathrm{M}_{2} \mathrm{X}$ is a rectangular plate, the effective radius needs to be calculated according to the equations developed by Brailsford and Mansur [126].

$$
r_{P}=(L M N)^{\frac{1}{3}}
$$


where $r_{p}$ is the effective radius and $\mathrm{L}, \mathrm{M}, \mathrm{N}$ refer to the length, width and thickness respectively. The results of this calculation are shown in Figure 6.11. These equations can then be used to calculate the sink strength as a function of dpa in the code.

The radii and number densities for each precipitate type are updated according to the predetermined equations (Figure 6.10 and Figure 6.11) in the fex sub routine similar to the experimentally input dislocation loop treatment. Values for vacancy and interstitial cluster sizes are called from the DLSODE solver. Then, each radius and number density was updated as a function of dpa. From here, the sink strengths are also calculated according to sink type and the total vacancy and interstitial populations absorbed at the precipitates are calculated. Defects absorbed are calculated via the following equation:

$$
i_{U}=D_{i} C_{i} * S S_{U} * d t
$$

where $i_{U}$ refers to the interstitials (or vacancies) captured by unbiased precipitates, $D_{i}$ is interstitial diffusion constant and $C_{i}$ is interstitial concentration, $S S_{U}$ is the unbiased sink strength and $d t$ is the time step. For biased precipitates, a factor is included in calculation of sink strength but otherwise remains the same.

The RIME model was updated to treat $\mathrm{M}_{2} \mathrm{X}$ precipitates as either unbiased, biased, variable biased or as recombination centers. These different treatments will be compared in Chapter 7, but are described here. A variable in the input file (zipptB) is read in and used as a flag to determine whether the precipitates are treated as unbiased (zipptB $B=1)$, biased (zipptB $B=1.005$, for instance) or variable biased $(z i p p t B=10)$. Note that for coherent precipitates, the bias is not 10 , but this is used 
as a convenient flag to start the variable biased treatment described below. An alternate flag itrap can flag use of the recombination treatment.

For unbiased sinks, there is no preference for vacancies or interstitials and the sink strength remains as calculated in s09_fexjex.f. In the biased treatment, sink strength is multiplied by the bias for interstitials (zipptB). Finally, variable biased sinks are treated according to the Brailsford and Bullough formalism [127] shown below. The sink strength for interstitials $i$ and vacancies $v$ for a coherent precipitate is calculated below using Equation 6.37 and Equation 6.38, respectively.

$$
\begin{aligned}
& k_{v C P}^{2}=4 \pi R_{C P} \rho_{C P} Y_{v} \\
& k_{i C P}^{2}=4 \pi R_{C P} \rho_{C P} Y_{i}
\end{aligned}
$$

$Y_{i}$ is a factor which measures the bias for interstitials and vacancies respectively, that changes as a function of dpa. $Y_{i}$ is calculated below:

$$
Y_{i}=\frac{D_{v} C_{v}}{D_{i} C_{i}} Y_{V}
$$

and $Y_{v}$ is calculated as:

$$
Y_{v}=1+(z-1) f_{0}+(z-2) \delta f
$$

where $z$ is the coordination number and 


$$
\delta f=\frac{1}{z+1}\left[1-\frac{k_{v}^{2}}{k_{i}^{2}}\right]
$$

which represents the change in capacity for either vacancies or interstitials based upon the sink strength and

$$
f_{v}=\left(\frac{\bar{K}_{v} c_{v}}{\alpha_{s}}\right)^{\frac{1}{2}}
$$

$f_{v}$ is a recombination term at the coherent precipitates, $\bar{K}_{v}$ is the reaction rate constant, $c_{v}$ is the vacancy concentration, $\alpha_{s}$ is the bulk recombination coefficient.

The final possible treatment for coherent precipitates is as recombination centers. $\mathrm{M}_{2} \mathrm{X}$ is in a state of compressive stress relative to the matrix, making it preferential to trapping vacancies, due to its slightly smaller lattice parameter. Once vacancies are trapped at the precipitate-matrix interface, they are "held" until an anti-defect (i.e. an interstitial) diffuses to the trap site. When the flag itrap is used (itrap $=1)$, a recombination term for interstitials at the trapped vacancies (recomt) is calculated from the sink strength, analogous to surface area, and a trap efficiency (rotrp), set by the user. (Previous literature suggests that coherent precipitates may not act as $100 \%$ efficient sinks, so this was included as an option [128].)

$$
\text { recomt }=4.0 * \pi * R_{p p t} * \rho_{p p t} * D_{i} C_{i} * \operatorname{rotrp}
$$

where $R_{p p t}$ and $\rho_{p p t}$ are the radius and number density, respectively. 
Trapping and de-trapping reaction rate coefficients are calculated using Equation 6.44 and Equation 6.45 below. These reaction rates are then used to update dcv (the overall derivative of vacancy concentration, which is sent to the solver. Recomt also updates the derivative of interstitials in a similar way.

$$
\begin{gathered}
\text { rtrap }=4.0 * \pi * R_{p p t} * \rho_{p p t} * D_{v} C_{v} * \operatorname{rotrp} \\
\text { rdtrap }=4.0 * \pi * R_{p p t} * \rho_{p p t} * \exp \left(-\frac{E_{b}^{\text {trap }, v}}{k T}\right) * D_{v} C_{v t} * \operatorname{rotrp}
\end{gathered}
$$

where $E_{b}^{t r a p, v}$ is the binding energy at the precipitate interface.

\subsection{Development of Reference Cases}

Having updated the RIME model with the required formalisms to treat the experimental results from this thesis, reference cases were developed to track the effects of introducing experimental values of precipitates, carbon traps and preexisting microstructure into the model. Since there are a number of perturbations that must be analyzed, three reference cases are presented. Since the preexisting microstructure treatment is used, the damage reported in RIME ( $\left.\Phi_{\mathrm{RIME}}\right)$ starts at " 0 dpa" even though the input microstructure is irradiated, but is related to the total, or true, damage $\left(\Phi_{\mathrm{T}}\right)$.

$$
\Phi_{R I M E}=\Phi_{T}-188 d p a
$$


For clarity and ease of interpretation, all graphs will be plotted as a function of true dpa so that the RIME results and experimental results reflect the same damage level. For the purposes of benchmarking, only void and dislocation behavior is considered, as it represents the most fundamental interaction relevant to the study of void swelling.

Three reference cases will be compared to experimental data. Ref.0 is the simplest case with constant network density and no loops. Ref.1 includes the experimentally input network and loop evolution. Ref.2 includes the dynamic dislocation (DD) treatment. The input files for each reference case are presented in Table 6.9. Changes in input between reference cases are highlighted in yellow. Unless explicitly mentioned, the loop and network bias for all model cases was 1.01. A parametric analysis revealed that bias was the dominant parameter in determining void swelling behavior. A literature search shows expected bias in a range from 1.01-1.3 for BCC iron/iron alloys $[8,41-43,114,115]$, with a majority varying from 1.01-1.05. As such, bias of 1.01 was deemed to be reasonable.

\subsubsection{Comparison of Reference Cases to Experimental Data}

Three reference cases will be discussed below and compared to the experimental data below to provide a baseline before incorporation of the more complex microstructure interactions including precipitates and solute effects.

\subsubsection{Ref.0: Constant Network Density}

The most fundamental interaction is simply the interaction between dislocations and voids. The simplest treatment in RIME was to assume a constant dislocation network density. This will be designated as Ref.0 (input in Table 6.9). Ref.0 will be used 1) to verify that the swelling 
behavior was exhibiting reasonable behavior and 2) to provide a baseline for further comparisons of more complex dislocation and precipitate treatments. Figure 6.12a compares the constant network density with the experimentally observed network density. The assumption of constant network density was quite reasonable, as the only variation between Ref.0 and the experiment was from 550 to $650 \mathrm{dpa}$, where a small increase to $4 \times 10^{-14} \mathrm{~m}^{-2}$ was observed.

The results of Ref.0 compared to experimental results are shown in Figure 6.12b. The overall diameter was higher than that observed in the experiment, but was fairly reasonable within $10 \mathrm{~nm}$. (Figure 6.12b) The number density matched quite well for all damage levels. Finally, the swelling was overestimated as a result of the enhanced diameter. The final swelling value at 650 dpa was $21 \%$ relative to the experimental value of $16 \%$.

\subsubsection{Ref.1: Experimentally Input Dislocation Evolution}

The second reference case matches the experimentally observed dislocation evolution. The explicit loop treatment, detailed in Section 6.2.2, was used in conjunction with manual adjustment of the network density from 3 to $4 \times 10^{14} \mathrm{~m}^{-2}$ at $550 \mathrm{dpa}$, performed using the irradiation continuation option of RIME also described in Section 6.1.2.7. This case will be designated as Ref.1 (input in Table 6.9). Ref.1 will be used to provide an exact comparison to the experimentally observed dislocation evolution. The results of Ref.1 compared to experimental results are shown in Figure 6.13. Figure 6.12a compares the Ref.1 with the experimentally observed network density and loop line length. The explicit treatment is quite accurate in capturing the overall loop and network behavior, as was expected.

The overall void diameter was higher than that observed in the experiment, but was fairly reasonable within $15 \mathrm{~nm}$. (Figure 6.13b) The number density matched quite well for all damage 
levels. Finally, the swelling was overestimated as a result of the enhanced diameter. The final swelling value at 650 dpa was $26 \%$ relative to the experimental value of $16 \%$ and Ref.0 values of $21 \%$.

\subsubsection{Ref.2: Dynamic Dislocation (DD) Evolution}

The final reference case uses the dynamic dislocation treatment, which was based upon the physics from $[18,100]$ and detailed in Section 6.1.2. Ref.2 is also compared to experimental data in Figure 6.14. The network density (Figure 6.14a) was representative of what was observed experimentally, but loop sink strength was higher than expected by an order of magnitude. Figure 6.14b shows the dislocation loop behavior, which explains the higher than expected loop sink strength observed in Figure 6.14a. The number density of loops was an order of magnitude higher and diameter was approximately the average of overall loop diameter from experiment. Loop sink strength was overestimated.

Figure $6.14 \mathrm{c}$ shows the resulting void behavior. Again, the number density was accurately captured but the diameter/diameter growth rate was too high. Again, the void swelling was higher than expected, indicating that some other mechanism or microstructure features was not taken into account.

Given the similarity of the DD treatment to the experimentally observed behavior as well as Ref.1, DD is especially appropriate as a reference case. It can then be used for model cases where the dislocations must be allowed to respond to changes in microstructure; for instance, to determine the effect of precipitates on dislocations.

A comparison between all three of these reference case will be detailed in Chapter 7. The reference cases are consistent in that they overpredicted the void swelling relative to that observed 
experimentally. As such, Chapter 7 will focus on understanding where this behavior comes from and what mechanism results in the observed microstructure co-evolution. 
Table 6.1: Summary of sub routines in RIME model. Updates, changes and notes are included in the last column.

\begin{tabular}{|c|c|c|c|}
\hline Category & File & Description & Changes/Notes \\
\hline s00 & s00_rime_main.f90 & $\begin{array}{l}\text { Main do loop used to call functions for appropriate time steps. Calls } \\
\text { solver for each time step. Writes intermediate results for each time } \\
\text { step in files and to screen. }\end{array}$ & $\begin{array}{l}\text { Changed output files to reflect new } \\
\text { outputs including precipitates and } \\
\text { impurity traps. }\end{array}$ \\
\hline s00 & s00_modes.f90 & Initialize variables and prior to reading input data. & $\begin{array}{l}\text { Added in additional inputs and } \\
\text { variable definitions to reflect } \\
\text { explicit dislocation loops, } \\
\text { precipitates and impurity traps. }\end{array}$ \\
\hline s00 & s00_initialize.f90 & $\begin{array}{l}\text { Defines ODE system for new, continued or expanded problem. Calls } \\
\text { preexisting microstructure. }\end{array}$ & Calls preexisting microstructure \\
\hline s00 & s00_preexistm.f & $\begin{array}{l}\text { Reads in input file describing starting microstructure in terms of } \\
\text { dislocation loop and void distribution. }\end{array}$ & $\begin{array}{l}\text { Sub routine to call inputs for } \\
\text { preexisting microstructure. }\end{array}$ \\
\hline s01 & s01_constants.f & $\begin{array}{l}\text { Calculates constants based upon input data including Berger's vector, } \\
\text { diffusion coefficients. Initializes counters for calculation of point } \\
\text { defect flows. }\end{array}$ & \\
\hline s01 & s01_exchange.f & $\begin{array}{l}\text { Initializes values for solver. Passes derivative calculations to and from } \\
\text { solver. }\end{array}$ & \\
\hline s01 & s01_tolerance_change.f & Increases vectors/matrices sizes as cluster sizes increases. & \\
\hline s02 & s02_a_bubble.f & $\begin{array}{l}\text { Defines vacancy and helium grid for cases including effect of helium. } \\
\text { Calculates reaction rate constants. Outputs grid sizes to } \mathrm{z} \text { grid-wv.dat. }\end{array}$ & \\
\hline s02 & s02_a_void.f & $\begin{array}{l}\text { Defines vacancy grid for cases not including effect of helium. } \\
\text { Calculates reaction rate constants and binding energies. }\end{array}$ & $\begin{array}{l}\text { Updated to pre-determine group } \\
\text { sizes variation with increasing void } \\
\text { size. }\end{array}$ \\
\hline s02 & s02_a_iloop.f & $\begin{array}{l}\text { Defines interstitial grid for cases including interstitial type loops. } \\
\text { Calculates reaction rate constants and binding energies as well as } \\
\text { generation rates of sessile SIA clusters in cascades. }\end{array}$ & $\begin{array}{l}\text { Updated to pre-determine group } \\
\text { sizes variation with increasing loop } \\
\text { size. }\end{array}$ \\
\hline s02 & s02_v_iloop.f & $\begin{array}{l}\text { Defines vacancy grid for cases including interstitial type loops. } \\
\text { Calculates reaction rate constants and binding energies as well as } \\
\text { generation rates of sessile SIA clusters in cascades. }\end{array}$ & Not used for this analysis. \\
\hline $\mathrm{s} 02$ & s02_compressibility.f & $\begin{array}{l}\text { Calculation of compressibility }(\mathrm{Z}) \text { of a bubble for a given gas/vacancy } \\
\text { ratio (x) using modified Carnahan-Starling Correction. }\end{array}$ & Not used for this analysis. \\
\hline s03 & s03_sd_bubble.f & Calculates size distribution of gas bubbles. & Not used for this analysis. \\
\hline $\mathrm{s} 03$ & s03_sd_iloop.f & Calculates size distribution of sessile interstitial loops. & \\
\hline s03 & s03_sd_iloop_ik.f & $\begin{array}{l}\text { Interaction of SIA loops/clusters with 3D mobile SIA clusters. Also } \\
\text { includes interaction of voids with 3D mobile SIA clusters. }\end{array}$ & \\
\hline s03 & s03_sd_vloop.f & Calculates size distribution of vacancy loops. & Not used for this analysis. \\
\hline s03 & s03_sd_void.f & Calculates size distribution of voids. & \\
\hline s04 & s04_total_sink_strength & $\begin{array}{l}\text { Calculation of sink strength of voids, dislocation loops and lines, grain } \\
\text { boundaries. }\end{array}$ & Not used for this analysis. \\
\hline s04 & s04_treatment.f & $\begin{array}{l}\text { Treatment of results. Includes defect tallies for precipitates, gas } \\
\text { accumulation at features. Calculation of swelling rate. }\end{array}$ & Precipitate treatment added in \\
\hline
\end{tabular}


Table 6.1: Summary of sub routines in RIME model. Updates, changes and notes are included in the last column.

\begin{tabular}{|c|c|c|c|}
\hline Category & File & Description & Changes/Notes \\
\hline $\mathrm{s} 04$ & s04_trt_bubble.f & Treatment of size distribution of bubbles. & Not used \\
\hline $\mathrm{s} 04$ & s04_trt_iloop.f & Treatment of size distribution of interstitial loops. & \\
\hline s04 & s04_trt_vloop.f & Treatment of size distribution of vacancy loops. & \\
\hline s04 & s04_trt_void.f & $\begin{array}{l}\text { Treatment of size distribution of voids. Calculates void concentration } \\
\text { and radii. }\end{array}$ & \\
\hline s04 & s04_vickers.f & Calculation relating MPa to Vickers hardness values. & Not used \\
\hline $\mathrm{s} 05$ & s05_correct_input.f & $\begin{array}{l}\text { Correct input values for certain specific experiment types including } \\
\text { thermal aging, TEM foils, and thermal neutron irradiation. }\end{array}$ & Not used \\
\hline $\mathrm{s} 05$ & s05_read_continue.f & Reads data saved for continuation of irradiation or aging. & \\
\hline s05 & s05_read_input.f & Reads in input file. & $\begin{array}{l}\text { Explicit loops, precipitate data and } \\
\text { impurity traps have been added. }\end{array}$ \\
\hline s06 & s06_w_open_files.f & Opens files and formats headers for output data files. & Headers adjusted for new outputs. \\
\hline s06 & s06_write_continue.f & Saves data for continuation. & \\
\hline s06 & s06_write_generation.f & Writes generation rates of $\mathrm{He}$ and dpa with time. & \\
\hline s06 & s06_write_head.f & Writes headers for screen outputs. & \\
\hline s06 & s06_write_screen.f & $\begin{array}{l}\text { Writes intermediate results to screen including swelling, sink strength } \\
\text { and other key parameters. Also writes intermediate data to output files } \\
\text { for each dpa. }\end{array}$ & $\begin{array}{l}\text { Outputs changed to reflect new } \\
\text { precipitate data. }\end{array}$ \\
\hline s06 & s06_write_sd.f & $\begin{array}{l}\text { Writes size distributions in own size distribution files for loops, voids } \\
\text { and bubbles as a function of dpa. }\end{array}$ & \\
\hline s07 & s07_gb_sink_strength.f & Calculates grain boundary sink strengths. & \\
\hline s07 & s07_mobile_defects.f & $\begin{array}{l}\text { Derivatives of concentration of freely migrating SIAs, vacancies, } \\
\text { crowdions. }\end{array}$ & $\begin{array}{l}\text { Calculates defect annihilation at } \\
\text { precipitates. }\end{array}$ \\
\hline s08 & s08_dlsode.f & $\begin{array}{l}\text { Livermore Solver for Ordinary Differential Equations. DLSODE } \\
\text { solves the initial-value problem for stiff or nonstiff systems of first- } \\
\text { order ODE's, dy/dt }=\mathrm{f}(\mathrm{t}, \mathrm{y}), \text { or, in component form, dy(i)/dt }=\mathrm{f}(\mathrm{i})= \\
\mathrm{f}(\mathrm{i}, \mathrm{t}, \mathrm{y}(1), \mathrm{y}(2), \ldots, \mathrm{y}(\mathrm{N})), \mathrm{i}=1, \ldots, \mathrm{N} \text {. }\end{array}$ & \\
\hline s09 & s09_fexjex.f & $\begin{array}{l}\text { Calculation of time derivatives. Calculates radius and sink strength of } \\
\text { precipitates. }\end{array}$ & $\begin{array}{l}\text { Explicit loops, Precipitate treatment } \\
\text { is calculated here. }\end{array}$ \\
\hline $\mathrm{s} 10$ & s10_euler.f & Euler solution method & Not used \\
\hline
\end{tabular}


Table 6.2: Parameters used in a complete input file. Highlighted boxes in red are not used for the purpose of this work. Highlighted boxes in yellow have been adapted or changed.

\begin{tabular}{|c|c|c|c|c|}
\hline $\begin{array}{l}\text { Reference } \\
\text { Value }\end{array}$ & $\begin{array}{l}\text { Variable } \\
\text { (RIME) }\end{array}$ & $\begin{array}{c}\text { Variable } \\
(\text { symbol) }\end{array}$ & Unit & Description \\
\hline Ref.0 & name & & - & Output Directory Name: 12 char. $\max$ \\
\hline 0 & key & & - & $0=$ Volume, $1=$ Surf., $2=$ Surf. no depos \\
\hline 1 & keyai & & - & Aging $=0 ;$ Irradiation $=1$ \\
\hline 0 & keyc & & - & Calculation: New $=0 ;$ Cont $=1$ \\
\hline 1 & $\mathrm{idm}$ & & - & $=1$ check cell size using criterion: \\
\hline $1.00 \mathrm{E}-06$ & ratmax & & - & max ratio bubble/cell size allowed \\
\hline 0 & keyNucl & & - & $=1$ no void nucleation: $\mathrm{v}+\mathrm{v}$ and $\mathrm{v}+\mathrm{Gas}$ \\
\hline 0 & keyNuclL & & - & $=1$ no $\mathrm{v}$-loop nucleation: $\mathrm{v}+\mathrm{v}$ \\
\hline $1.00 \mathrm{E}+10$ & $\mathrm{dttt} 1$ & & $\mathrm{~s}$ & LSODE INTERNAL max timestep \\
\hline $1.00 \mathrm{E}-06$ & $\mathrm{dt} 0$ & & $\mathrm{~s}$ & Initial timestep out \\
\hline $1.00 \mathrm{E}+03$ & time1 & & $\mathrm{s}$ & Time < time1: rio1 \\
\hline $5.00 \mathrm{E}+04$ & timem & & $\mathrm{s}$ & Time <timem: riom; if >: constant \\
\hline 1 & rio1 & & - & OUT timestep $\mathrm{dt}=\mathrm{dt} 0 \quad+\mathrm{t} /$ riol \\
\hline 10 & riom & & - & OUT Timestep dt=dt_previous $+\mathrm{t} /$ riom \\
\hline 1 & nscrn & & - & $\mathrm{N}$ steps for writing to screen $\&$ files \\
\hline 1 & nremm & & - & $\mathrm{N}$ steps for saving to continue \\
\hline 1 & nwrit & & - & N steps for writing SDs and 3-d graph \\
\hline 0 & mfeuler & & - & EULER $=1$, LSODE $=0$. Only 0 is used \\
\hline 1 & nround & & - & EULER \\
\hline \multirow[t]{2}{*}{1} & nsteps & & - & EULER $\mathrm{n}$ of steps $\mathrm{dt}$ integ. $=\mathrm{dt} / \mathrm{nsteps}$ \\
\hline & \multicolumn{4}{|c|}{-----------------Flags and Dimensions Defining System----------------- } \\
\hline 0 & $\mathrm{iHe}$ & & - & Flag for treatment of Helium gas \\
\hline 1 & iVac & & - & Flag for treatment of vacancies \\
\hline 1 & iInt & & - & Flag for treatment of interstitials \\
\hline 0 & iCrow & & - & Flag for treatment of crowdions \\
\hline 1 & iVoid & & - & Flag for treatment of voids/bubbles \\
\hline 0 & iVloop & & - & Flag for treatment of vacancy loops \\
\hline 0 & illoop & & - & Flag for treatment of interstitial loops \\
\hline 0 & iDisl & & - & Flag for treatment of dislocations \\
\hline 0 & iTrap & & - & Flag for explicit treatment of vacancy trapping \\
\hline 120 & $\mathrm{mvv}$ & & - & Initial \# of vacancy groups in voids/bubbles \\
\hline 120 & $\mathrm{mvb}$ & & - & Initial \# of helium groups in bubbles \\
\hline 120 & mil & & - & \# of interstitial loop groups \\
\hline 8 & mvl & & - & \# of vacancy loop groups \\
\hline \multirow[t]{2}{*}{10} & iexpnd & & - & \# of groups added when expanding cell \\
\hline & \multicolumn{4}{|c|}{ 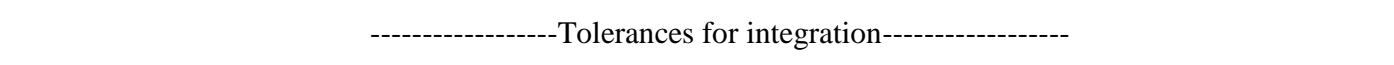 } \\
\hline 10 & ntol & & - & for all derivatives: atolD=atolV/ntol \\
\hline $1.0 \mathrm{~d}-4$ & rtol & & - & relative tolerance for all values \\
\hline $1.0 \mathrm{~d}^{-15}$ & atsia & & $\mathrm{at}^{-1}$ & initial abs tolerance for SIAs \\
\hline $1.0 \mathrm{~d}-7$ & atvac & & $\mathrm{at}^{-1}$ & initial abs tolerance for vacancies \\
\hline $1.0 \mathrm{~d}-7$ & atcro & & $\mathrm{at}^{-1}$ & initial abs tolerance for crowdions \\
\hline $1.0 \mathrm{~d}^{-17}$ & atgas & & $\mathrm{at}^{-1}$ & initial abs tolerance for gas atoms \\
\hline $1.0 \mathrm{~d}^{-1} 2$ & atbub & & $\mathrm{at}^{-1}$ & initial abs tolerance for bubbles \\
\hline $1.0 \mathrm{~d}^{-1} 6$ & atlps & & $\mathrm{at}^{-1}$ & initial abs tolerance for interstitial loops \\
\hline $1.0 \mathrm{~d}^{-1} 6$ & atVL & & $\mathrm{at}^{-1}$ & initial abs tolerance for vacancy loops \\
\hline $1.0 \mathrm{~d}+9$ & atdis & & $\mathrm{m}^{-2}$ & initial abs tolerance for dislocation \\
\hline \multirow[t]{2}{*}{$1.0 \mathrm{~d}+9$} & rspec & & $\mathrm{m}$ & bubble radius: > not used in fmax \\
\hline & \multicolumn{4}{|c|}{------------------Irradiation / Aging Conditions------------------ } \\
\hline
\end{tabular}

${ }^{7}$ Symbols included for variables used in the text. 
Table 6.2: Parameters used in a complete input file. Highlighted boxes in red are not used for the purpose of this work. Highlighted boxes in yellow have been adapted or changed.

\begin{tabular}{|c|c|c|c|c|}
\hline $\begin{array}{l}\text { Reference } \\
\text { Value }\end{array}$ & $\begin{array}{l}\text { Variable } \\
\text { (RIME) }\end{array}$ & $\begin{array}{c}\text { Variable } \\
\text { (symbol) }^{7}\end{array}$ & Unit & Description \\
\hline $4.50 \mathrm{E}+05$ & time & $t$ & $\mathrm{~s}$ & Irradiation/Aging TIME \\
\hline $1.00 \mathrm{E}-03$ & rknrt & $\dot{\Phi}$ & $\mathrm{dpa} / \mathrm{s}$ & Mean NRT generation rate \\
\hline 0.67 & Epsr & $\varepsilon$ & - & Defect fraction recombination in cascades \\
\hline 0 & epsis & & - & SIA fraction in sessile clusters \\
\hline 0 & epsig & & - & SIA fraction in crowdions \\
\hline 0 & epsv & & - & Vacancy fraction in sessile clusters \\
\hline 0.3 & epsw & & - & Vacancy fraction in micro-voids \\
\hline 460 & tc & $T$ & ${ }^{\circ} \mathrm{C}$ & Temperature, degree Celsius \\
\hline $0.00 \mathrm{E}+00$ & $\operatorname{cg} 0$ & & - & Initial gas-atom site fraction \\
\hline $3.0 \mathrm{~d}+14$ & $\operatorname{rod} 0$ & $\rho_{D}$ & $\mathrm{~m}^{-2}$ & Initial VOLUME dislocation density \\
\hline $1.0 \mathrm{~d}-8$ & rotrp & & $\mathrm{at}^{-1}$ & Density of vacancy trapping sites \\
\hline $1.0 \mathrm{~d}+19$ & rods & & $\mathrm{m}^{-2}$ & GB dislocation density (if key $>0$ ) \\
\hline $5.00 \mathrm{~d}-7$ & rgr & $d$ & $\mathrm{~m}$ & Grain radius \\
\hline $5.0 \mathrm{~d}-0$ & dfoil & & $\mathrm{M}$ & Foil thickness for crowdions \\
\hline & \multicolumn{4}{|c|}{------------------Thermal Neutrons------------------ } \\
\hline 0 & key_th & & & key: 0- exclude; 1 - include \\
\hline $1.132 \mathrm{~d}+9$ & time_th & & $\mathrm{s}$ & Irradiation time \\
\hline $5.38 \mathrm{~d}-8$ & rknrt & & $\mathrm{dpa} / \mathrm{s}$ & NRT generation rate for fast neutrons \\
\hline 0.9 & epsr_th & & - & Defect fraction recombination in cascades \\
\hline 0 & epsis_th & & - & SIA fraction in sessile clusters \\
\hline 0 & epsig_th & & - & SIA fraction in crowdions \\
\hline 0 & epsv_th & & - & Vacancy fraction in sessile clusters \\
\hline 0 & epsw-th & & - & Vacancy fraction in micro-voids \\
\hline & \multicolumn{4}{|c|}{----------------He atom production---------------- } \\
\hline $0.00 \mathrm{~d}-08$ & rkhe & & $\mathrm{s}^{-1}$ & He atom generation rate \\
\hline $0.00 \mathrm{~d}-13$ & rkhe_th & & $\mathrm{s}^{-1}$ & He atom gen' rate from thermal neutrons \\
\hline $0.00 \mathrm{~d}+18$ & Flux & & $\mathrm{n} / \mathrm{m}^{2}$ & Thermal neutron flux \\
\hline 0.7 & $\mathrm{CNi}$ & & - & Initial Ni58 fraction (0.68077 of all) \\
\hline $\begin{array}{c}8.254 d- \\
28 \\
\end{array}$ & SAlpha & & $\mathrm{m}^{-2}$ & 59Ni(n,alpha) cross section \\
\hline $\begin{array}{c}2.137 \mathrm{~d}- \\
28\end{array}$ & SGamma & & $\mathrm{m}^{-2}$ & $58 \mathrm{Ni}(\mathrm{n}$, gamma $)(1$ barn $=10-28 \mathrm{~m}-2)$ \\
\hline $\begin{array}{c}43.20 \mathrm{~d}- \\
28 \\
\end{array}$ & STotal & & $\mathrm{m}^{-2}$ & $59 \mathrm{Ni}$ total absorption cross section \\
\hline & \multicolumn{4}{|c|}{ 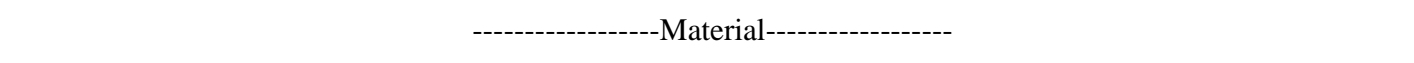 } \\
\hline 2 & type1 & & - & $\mathrm{N}$ at. in unit cell 2-BCC 4-FCC \\
\hline $2.86 \mathrm{~d}-10$ & $\mathrm{a}$ & $a$ & M & Lattice parameter: bcc $\mathrm{Fe}=2.87, \mathrm{fcc}=3.61$ \\
\hline 1 & $\mathrm{Nbv}$ & $b$ & - & Dislocation Burgers V: $1-\mathrm{S} 3 / 2 ; 2-0.5 ; 3^{-1} / \mathrm{S} 3$ \\
\hline 3 & NbvL & & - & Loop Burgers V: $1-\mathrm{S} 3 / 2 ; 2-0.5 ; 3^{-1} / \mathrm{S} 3$ \\
\hline 2.3 & gamma0 & $\gamma$ & $\mathrm{J} / \mathrm{m}^{2}$ & Surface energy defined as \\
\hline 1 & gama & & - & (gama*gamma0-gamtc*tc)/1.6e $\mathrm{e}^{-19}$ which is in $\mathrm{eV}$ \\
\hline $\begin{array}{c}0.0 \\
1.75 \mathrm{e}^{-3} \\
\end{array}$ & gamtc & $\gamma$ & $\mathrm{J} / \mathrm{m}^{2}$ & \\
\hline 0 & gammaVL & & $\mathrm{J} / \mathrm{m}^{2}$ & Stacking-fault energy for vacancy loops \\
\hline 0.33 & poisson & & - & Poisson ratio for vacancy loops \\
\hline 1 & rvinf & & $\mathrm{nm}$ & join radius for Evb for vacancy loops \\
\hline 1.79 & evf & $E_{f}^{v}$ & $\mathrm{eV}$ & Vacancy formation energy \\
\hline 0.63 & evm & $E_{m}^{v}$ & $\mathrm{eV}$ & Vacancy migration energy \\
\hline
\end{tabular}


Table 6.2: Parameters used in a complete input file. Highlighted boxes in red are not used for the purpose of this work. Highlighted boxes in yellow have been adapted or changed.

\begin{tabular}{|c|c|c|c|c|}
\hline $\begin{array}{c}\text { Reference } \\
\text { Value }\end{array}$ & $\begin{array}{l}\text { Variable } \\
\text { (RIME) }\end{array}$ & $\begin{array}{c}\text { Variable } \\
\text { (symbol) }^{7}\end{array}$ & Unit & Description \\
\hline 0.63 & evmeff & $E_{m, e f f}^{v}$ & $\mathrm{eV}$ & $\begin{array}{c}\text { Effective vacancy migration energy (same if no } \\
\text { traps) }\end{array}$ \\
\hline 0.22 & eim & $E_{m}^{i}$ & $\mathrm{eV}$ & SIA migration energy \\
\hline 0.22 & egm & & $\mathrm{eV}$ & Crowdion migration energy \\
\hline 0.07 & ehm & & $\mathrm{eV}$ & Gas-atom migration energy \\
\hline 1 & cve0 & $C_{0, f}^{v}$ & - & Pre-exp for vacancy formation \\
\hline $8.2 \mathrm{~d}-7$ & $\mathrm{c} 0 \mathrm{v}$ & $C_{0, m}^{v}$ & $\mathrm{~m}^{2} / \mathrm{s}$ & Pre-exp for vacancy migration \\
\hline $9.4 d-7$ & $\mathrm{cOi}$ & $C_{0, m}^{i}$ & $\mathrm{~m}^{2} / \mathrm{s}$ & Pre-exp for SIA migration \\
\hline $9.4 d-7$ & $\mathrm{c0g}$ & & $\mathrm{m}^{2} / \mathrm{s}$ & Pre-exp for crowdion migration \\
\hline $4.0 \mathrm{~d}-7$ & dh0 & & $\mathrm{m}^{2} / \mathrm{s}$ & Pre-exp for gas atom migration \\
\hline $0.0 \mathrm{~d}+22$ & Sdisl & & $\mathrm{m}^{-3}$ & Bardeen-Herring disl' source density \\
\hline 0 & Aprime & $A^{\prime}$ & - & Modified back stress term, Roger $=0.05$ \\
\hline 10.11 & & & $\mathrm{eV}$ & ik eim2 SIA 2-4i mig energy \\
\hline $16.5 \mathrm{~d}-8$ & & & $\mathrm{~m}^{2} / \mathrm{s}$ & ik c0i2 Pre-exp mig \\
\hline & \multicolumn{4}{|c|}{------------------Reaction cross-sections------------------ } \\
\hline 1.01 & zicl & $z_{L}^{i}$ & - & Loops capture efficiency for SIAs \\
\hline 1.01 & $\mathrm{zi}$ & $z_{N}^{i}$ & $z_{L}^{i}$ & Dislocation capture efficiency for SIAs \\
\hline 1 & $\mathrm{ZV}$ & $z_{N}^{v}$ & - & Dislocation capture efficiency for vacancies \\
\hline $0.0 \mathrm{~d}-9$ & rivoid & & $\mathrm{m}$ & Void capture distance for SIA \\
\hline 0 & zgd & & - & Dislocation capture efficiency for gas atoms \\
\hline 1 & zig & $z_{G B}^{i}$ & - & GB capture efficiency for SIAs \\
\hline 1 & zvg & $z_{G B}^{v}$ & - & GB capture efficiency for vacancies \\
\hline 0 & zgg & & - & GB capture efficiency for Gas atoms \\
\hline $1.00 \mathrm{E}+00$ & rava & & - & Fraction of sites for gas at dislocation \\
\hline 2.25 & edge & & $\mathrm{eV}$ & Emission energy for gas at from dislocation \\
\hline 1.6 & ebgbb & & $\mathrm{eV}$ & Emission energy for gas at from GBs \\
\hline 0.5 & ebtrv & $E_{b, t r}^{v}$ & $\mathrm{eV}$ & Emission energy for vacancy-trap complex \\
\hline $2.0 \mathrm{~d}+21$ & rec & $\eta$ & $\mathrm{m}^{2} / \mathrm{s}$ & Recombination constant \\
\hline 1 & rkk & & - & V+crow: =1-pure 1D; =0-preferential \\
\hline 1 & keybb & & - & EOS:1-Roger;(2 Carnahan-S;3?Manzke-T) $\quad *(1)$ \\
\hline $0.2 \mathrm{~d}-9$ & sigma & & - & Sigma for Carnahan-Starling EOS \\
\hline 0.3 & $\mathrm{e} 2 \mathrm{v}$ & & - & $\mathrm{v}-\mathrm{v}$ binding energy for Wolfer correct \\
\hline-1 & wolfn & & - & Wolfer corr: original \\
\hline 10000 & nevf & & - & i>nevf then Ev_binding=evf \\
\hline 1 & keyart & & - & $=1$ include punching of $\mathrm{i}$ from bubbles \\
\hline 2.3 & eeg & & $\mathrm{eV}$ & Gas atom-bubble binging energy \\
\hline $0.5 \mathrm{~d}-7$ & rro & & $\mathrm{m}$ & Dislocation capture radius for crowdions \\
\hline $3.0 \mathrm{~d}^{-1} 0$ & hilg & & $\mathrm{m}$ & SIA loop capture radius for crowdions \\
\hline $3.0 \mathrm{~d}^{-1} 0$ & hvlg & & $\mathrm{m}$ & Vacancy loop capture radius for crowdions \\
\hline $0.0 \mathrm{~d}-9$ & rcasc & & $\mathrm{m}$ & cascade size for restricting $C_{-} V_{-}$loops \\
\hline 0 & keyhd & & - & key for high-density corrections \\
\hline \multirow[t]{2}{*}{0} & key_rk & & - & key for generation rate correction \\
\hline & \multicolumn{4}{|c|}{ 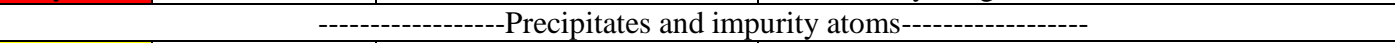 } \\
\hline 1 & iliz & & - & key: $=1$ change pre-existing microstructure !Eliz \\
\hline $0.0 \mathrm{~d}-2$ & Ccarbon & $C_{s}$ & $\mathrm{at}^{-1}$ & bulk concentration of impurity atoms !Eliz \\
\hline 0.5 & Ebcv & $E_{b}^{c, v}$ & $\mathrm{eV}$ & impurity-vacancy binding energy !Eliz \\
\hline 8 & znn & $z$ & - & coordination number !Eliz \\
\hline $1+0 \mathrm{e} 20$ & $\begin{array}{c}\text { N_U; } \\
\text { N_pptU }\end{array}$ & $\rho_{U}$ & & N_U: number of and N_pptU coefficients !Eliz \\
\hline $\begin{array}{c}2+0 \mathrm{e}-09 \\
+0 \mathrm{e}-12\end{array}$ & $\begin{array}{l}\text { R_U; } \\
\text { RpptU }\end{array}$ & $r_{U}$ & & _U: number of and R_pptU coefficients !Eliz \\
\hline
\end{tabular}


Table 6.2: Parameters used in a complete input file. Highlighted boxes in red are not used for the purpose of this work. Highlighted boxes in yellow have been adapted or changed.

\begin{tabular}{|c|c|c|c|c|}
\hline $\begin{array}{l}\text { Reference } \\
\text { Value }\end{array}$ & $\begin{array}{l}\text { Variable } \\
\text { (RIME) }\end{array}$ & $\begin{array}{c}\text { Variable } \\
{\text { (symbol })^{7}}^{7}\end{array}$ & Unit & Description \\
\hline $\begin{array}{c}4+0.0 \\
+0 \mathrm{e}+19- \\
0 \mathrm{e}+16+- \\
\mathrm{e} 13\end{array}$ & $\begin{array}{c}\text { N_B; } \\
\text { N_pptB }\end{array}$ & $\rho_{C P}$ & & N_B: number of and N_pptB coefficients !Eliz \\
\hline $\begin{array}{l}3+0.0 \\
+0 \mathrm{e}-1\end{array}$ & $\begin{array}{c}\text { R_B; } \\
\text { R_ppptB }\end{array}$ & $R_{C P}$ & & _B: number of and R_pptB coefficients !Eliz \\
\hline 1.0 & zipptB & & - & Ppts_B capture effic' for SIAs !Eliz \\
\hline 1 & rotrp & & & Trap effciency \\
\hline $\begin{array}{c}0+0 \mathrm{e}+20 \\
-0 \mathrm{e} 17\end{array}$ & $\begin{array}{l}\text { ilpnum, } \\
\text { num_lp }\end{array}$ & $\rho_{L}$ & & ilpnum: number of and num_lp coefficients !Eliz \\
\hline \multirow[t]{2}{*}{$\begin{array}{c}0+0 \mathrm{e}-8- \\
0 \mathrm{e}-10 \\
+0 \mathrm{e}-12- \\
0 \mathrm{e}-15\end{array}$} & Ilpr, r_lp & $r_{L}$ & & ilpr: number of and $r \_l p$ coefficients \\
\hline & \multicolumn{4}{|c|}{------------------Correlation-screening of voids from 1-d SIA clusters------------------ } \\
\hline $1.0 \mathrm{~d}+10$ & phics0 & & dpaFAS & T min damage for starting screening \\
\hline $1.0 \mathrm{~d}+01$ & phicsm & & dpaFAS & T max damage for complete screening \\
\hline \multirow[t]{2}{*}{6} & rwhcor & & $\mathrm{nm}$ & smallest void screened [nm] \\
\hline & \multicolumn{4}{|c|}{-------------------Voids: vacancies------------------ } \\
\hline 9 & mwm & & - & Max size for generation $(<\operatorname{mvxv})$ \\
\hline 1.94 & pnw & & - & Exponent: gener rate $\sim i^{* *}(-p n w)$ \\
\hline 30 & mvxv & & - & i > mvxv: groups \\
\hline 0.12 & drvv & & $\mathrm{nm}$ & $\mathrm{dr}$ or $\mathrm{dr} / \mathrm{r}$ for groups see below \\
\hline 2 & igrvac & & - & $=1-\mathrm{dr} ;=2-\mathrm{dr} / \mathrm{r}$ for above choice \\
\hline 1 & ifac & & - & Factor: increase size of last group \\
\hline \multirow[t]{2}{*}{358} & nvv & & - & Min i visible \\
\hline & \multicolumn{4}{|c|}{---------------Bubbles: gas atoms--------------- } \\
\hline 10 & mvxb & & - & $\mathrm{j}>$ mvxb: groups \\
\hline 0.12 & drvb & & $\mathrm{nm}$ & $\mathrm{dr}$ or $\mathrm{dr} / \mathrm{r}$ for groups see below \\
\hline 2 & igrgas & & - & $=1-\mathrm{dr} ;=2-\mathrm{dr} / \mathrm{r}$ for above choice \\
\hline 50 & $\mathrm{nvb}$ & & - & Min $\mathrm{j}$ visible \\
\hline 4.5 & g00 & & - & Definition for upper boundary \\
\hline 2 & g11 & & - & for Gas atom area: $\mathrm{g}<\mathrm{g} 00+\mathrm{g} 11^{*}\left(\mathrm{x}^{-1}\right)$ \\
\hline 100 & iimax & & - & $\mathrm{N}$ of vacancypoints to write $\mathrm{E} \_$bin \& emis \\
\hline \multirow[t]{2}{*}{100} & ijmax & & - & $\mathrm{N}$ of gas points to write $\mathrm{E} \_$bin $\&$ emis \\
\hline & \multicolumn{4}{|c|}{--------------------Interstitial loops-------------------- } \\
\hline 10 & $\operatorname{mim}$ & & - & Max size for generation $(<=\operatorname{mixl})$ \\
\hline 1 & pni & & - & Exponent: gener rate $\sim \mathrm{i}^{* *}(-$ pni) \\
\hline 20 & mixl & & - & i > mixl: groups \\
\hline $6.0 \mathrm{~d}-10$ & dri & & $\mathrm{m}$ & dr in groups \\
\hline \multirow[t]{2}{*}{20} & nli & & - & Min i visible \\
\hline & \multicolumn{4}{|c|}{-----------------Vacancy loops------------------- } \\
\hline 9 & $\mathrm{mvm}$ & & - & Max size for generation $(<\operatorname{mvxl})$ \\
\hline 1 & pnv & & - & Exponent: gener rate $\sim i^{* *}(-$ pnv $)$ \\
\hline 10 & mvxl & & - & i > mvxl: groups \\
\hline $1.0 \mathrm{~d}^{-1} 0$ & drvl & & $\mathrm{m}$ & dr in groups \\
\hline \multirow[t]{2}{*}{20} & $\mathrm{nlv}$ & & - & Min i visible \\
\hline & \multicolumn{4}{|c|}{------------------Hardening------------------- } \\
\hline $80.0 \mathrm{~d}+09$ & gshear & & $\mathrm{Pa}$ & Shear modulus for v-loop binding E \\
\hline 240 & sigma0 & & $\mathrm{MPa}$ & Initial stress yield \\
\hline 135 & hard0 & & Vikers & Initial hardness \\
\hline 2 & fsht & & - & Shmidt-Taylor factor \\
\hline 2.7 & sihard & & Vikers & Sigma to hardness coefficient \\
\hline 1 & ali & & - & Dimensionless coefficients \\
\hline 1 & alv & & - & for estimation of hardening \\
\hline
\end{tabular}


Table 6.2: Parameters used in a complete input file. Highlighted boxes in red are not used for the purpose of this work. Highlighted boxes in yellow have been adapted or changed.

\begin{tabular}{|c|c|c|c|c|}
\hline $\begin{array}{c}\text { Reference } \\
\text { Value }\end{array}$ & $\begin{array}{c}\text { Variable } \\
\text { (RIME) }\end{array}$ & $\begin{array}{c}\text { Variable } \\
\text { (symbol) }^{7}\end{array}$ & Unit & Description \\
\hline 1 & alc & & - & due to i and v loops and cavities \\
\hline
\end{tabular}


Table 6.3: Output file descriptions from RIME Model.

\begin{tabular}{|c|c|c|}
\hline File Name & Variable & Description \\
\hline Z_bevl.dat & vacancy - vacancy loop binding energy & \\
\hline Z_bewc.dat & vacancy - void binding energy & \\
\hline \multirow[t]{4}{*}{ z_bew3.dat } & vacancy-bubble binding energy for 3D graph & \\
\hline & xgas & number of gas atoms \\
\hline & xvac & number of vacancies \\
\hline & $\mathrm{Eb}(\mathrm{eV})$ & binding energy of a vacancy with a bubble \\
\hline \multirow[t]{3}{*}{ Z_bewb.dat } & vacancy-bubble binding energy $[\mathrm{eV}]$ & \\
\hline & vac & number of vacancies \\
\hline & $\mathrm{g} 0, \mathrm{~g} 1 \ldots$ & corresponding $0,1 \ldots$ gas atoms \\
\hline \multirow[t]{3}{*}{ Z_emig.dat } & gas emission rates from bubbles $\left[\mathrm{s}^{-1}\right]$ & \\
\hline & vac & number of vacancies \\
\hline & $\mathrm{g} 0, \mathrm{~g} 1 \ldots$ & corresponding $0,1 \ldots$ gas atoms \\
\hline \multirow[t]{3}{*}{ Z_emiv.dat } & vacancy emission rates from bubbles $\left[\mathrm{s}^{-1}\right]$ & \\
\hline & vac & number of vacancies \\
\hline & $\mathrm{g} 0, \mathrm{~g} 1 \ldots$ & corresponding $0,1 \ldots$ gas atoms \\
\hline \multirow[t]{4}{*}{ Z_grid-wg.dat } & gas axis grid for bubbles & \\
\hline & $\mathrm{N}$ & index of the array element (group) \\
\hline & x_def & number of defects (gas or vacancies) in bubble \\
\hline & R_nm & bubble radius $[\mathrm{nm}]$ \\
\hline \multirow[t]{4}{*}{ Z_grid-wv.dat } & vacancy axis grid for bubbles & \\
\hline & $\mathrm{N}$ & index of the array element (group) \\
\hline & x_def & number of defects (gas or vacancies) in bubble \\
\hline & R_nm & bubble radius $[\mathrm{nm}]$ \\
\hline Z_hard.dat & hardening & \\
\hline Z_harv.dat & hardening in Vickers & \\
\hline \multirow[t]{3}{*}{ Z_pcil.dat } & production rates of interstitial loops in cascades & \\
\hline & n_int & number of interstitials \\
\hline & Rate & production rate $\left[\mathrm{s}^{-1}\right]$ \\
\hline \multirow[t]{3}{*}{ z_pcvl.dat } & production rates of vacancy loops in cascades & \\
\hline & n_int & number of vacancies \\
\hline & Rate & production rate $\left[\mathrm{s}^{-1}\right]$ \\
\hline \multirow{3}{*}{ z_pcwv.dat } & production rates of micro-voids in cascades & \\
\hline & n_int & number of vacancies \\
\hline & Rate & production rate $\left[\mathrm{s}^{-1}\right]$ \\
\hline Z_rezz.dat & Input data read from input file to check reading & \\
\hline \multirow[t]{7}{*}{ Z_sdif.dat } & final size distribution of interstitial loops & \\
\hline & R_nm & interstitial loop radius [nm] \\
\hline & abs_f(R) & abs of size distribution to present in log scales \\
\hline & $\mathrm{f}(\mathrm{R})$ & size distribution in $\mathrm{R}$ space $\left[\mathrm{m}^{-3} \mathrm{~nm}^{-1}\right]$ \\
\hline & n_def & mean (over group) number of defects in a cluster \\
\hline & $\mathrm{f}\left(\mathrm{n} \_\mathrm{vac}\right)$ & size distribution in $\mathrm{x}$ space $\left[\mathrm{at}^{-1}\right]$ \\
\hline & slope & slope of $\mathrm{x}$-space size distribution in group $\left[\mathrm{at}^{-1}\right]$ \\
\hline \multirow[t]{7}{*}{ Z_sdil.dat } & all size distributions of interstitial loops & \\
\hline & R_nm & interstitial loop radius [nm] \\
\hline & abs_f(R) & abs of size distribution to present in log scales \\
\hline & $\mathrm{f}(\mathrm{R})$ & size distribution in $\mathrm{R}$ space $\left[\mathrm{m}^{-3} \mathrm{~nm}^{-1}\right]$ \\
\hline & n_def & mean (over group) number of defects in a cluster \\
\hline & $\mathrm{f}\left(\mathrm{n} \_\mathrm{vac}\right)$ & size distribution in $\mathrm{x}$ space $\left[\mathrm{at}^{-1}\right]$ \\
\hline & slope & slope of $\mathrm{x}$-space size distribution in group [at $\left.{ }^{-1}\right]$ \\
\hline \multirow[t]{4}{*}{ Z_sdw3.dat } & final size distribution of bubbles for 3D plot & \\
\hline & N_gas & number of gas atoms \\
\hline & R_nm & bubble radius $[\mathrm{nm}]$ \\
\hline & f(b) & bubble density in R-space $\left[\mathrm{m}^{-3 *} \mathrm{~nm}^{-1}\right]$ \\
\hline \multirow[t]{4}{*}{ Z_sdwf.dat } & R_nm & void/bubble radius [nm] \\
\hline & abs_f(R) & abs of size distribution to present in log scales \\
\hline & n_vac & mean (over group) number of vacancies in a cluster \\
\hline & $\mathrm{f}\left(\mathrm{n} \_\right.$vac $)$ & size distribution in $\mathrm{x}$ space $\left[\mathrm{at}^{-1}\right]$ \\
\hline
\end{tabular}


Table 6.3: Output file descriptions from RIME Model.

\begin{tabular}{|c|c|c|}
\hline File Name & Variable & Description \\
\hline & slope & slope of $\mathrm{x}$-space size distribution in group $\left[\mathrm{at}^{-1}\right]$ \\
\hline & $\mathrm{m} / \mathrm{x}$ & number of gas atoms to number of vacancies ratio \\
\hline & P_GPa & gas pressure $[\mathrm{GPa}]$ averaged over group \\
\hline & $\mathrm{Z}$ & gas compressibility, characteristic gas non-ideality \\
\hline & $\mathrm{r} / \mathrm{RWZ}$ & bubble radius/Wigner-Zeitz radius \\
\hline Z_sdws.dat & \multicolumn{2}{|c|}{ 2-D: gas-vacancy size distribution of bubbles for different damage levels } \\
\hline & \multicolumn{2}{|c|}{ two-dimensional size distributions in $\mathrm{x}$ space for different } \\
\hline & \multicolumn{2}{|c|}{ irradiation damage levels; the number of gas atoms increases from top to } \\
\hline & \multicolumn{2}{|c|}{ bottom and the vacancy numbers increases from left to right; the } \\
\hline & densities are in $\left[\mathrm{at}^{-1}\right]$ & \\
\hline \multirow[t]{10}{*}{ Z_sdwv.dat } & all size distributions of bubbles/voids & \\
\hline & R_nm & void/bubble radius $[\mathrm{nm}]$ \\
\hline & abs_f(R) & abs of size distribution to present in log scales \\
\hline & n_vac & mean (over group) number of vacancies in a cluster \\
\hline & $\mathrm{f}\left(\mathrm{n} \_\right.$vac $)$ & size distribution in $\mathrm{x}$ space $\left[\mathrm{at}^{-1}\right]$ \\
\hline & slope & slope of $\mathrm{x}$-space size distribution in group $\left[\mathrm{at}^{-1}\right]$ \\
\hline & $\mathrm{m} / \mathrm{x}$ & number of gas atoms to number of vacancies ratio \\
\hline & P_GPa & gas pressure [GPa] averaged over group \\
\hline & $\mathrm{Z}$ & gas compressibility, characteristic gas non-ideality \\
\hline & $\mathrm{r} / \mathrm{RWZ}$ & bubble radius/Wigner-Zeitz radius \\
\hline \multirow[t]{7}{*}{ Z_sdvf.dat } & final size distribution of vacancy loops & \\
\hline & R_nm & vacancy loop radius [nm] \\
\hline & abs_f(R) & abs of size distribution to present in log scales \\
\hline & $\mathrm{f}(\mathrm{R})$ & size distribution in $\mathrm{R}$ space $\left[\mathrm{m}^{-3} \mathrm{~nm}^{-1}\right]$ \\
\hline & n_def & mean (over group) number of defects in a cluster \\
\hline & $\mathrm{f}\left(\mathrm{n} \_\mathrm{vac}\right)$ & size distribution in $\mathrm{x}$ space $\left[\mathrm{at}^{-1}\right]$ \\
\hline & slope & slope of $\mathrm{x}$-space size distribution in group $\left[\mathrm{at}^{-1}\right]$ \\
\hline \multirow[t]{7}{*}{ Z_sdvl.dat } & all size distributions of vacancy loops & \\
\hline & R_nm & vacancy loop radius [nm] \\
\hline & abs_f(R) & abs of size distribution to present in log scales \\
\hline & $\mathrm{f}(\mathrm{R})$ & size distribution in $\mathrm{R}$ space $\left[\mathrm{m}^{-3} \mathrm{~nm}^{-1}\right]$ \\
\hline & n_def & mean (over group) number of defects in a cluster \\
\hline & $\mathrm{f}\left(\mathrm{n} \_\right.$vac $)$ & size distribution in $\mathrm{x}$ space $\left[\mathrm{at}^{-1}\right]$ \\
\hline & slope & slope of $\mathrm{x}$-space size distribution in group [at $\left.{ }^{-1}\right]$ \\
\hline \multirow[t]{6}{*}{ zm_conc.dat } & concentrations of mobile defects & \\
\hline & Damage /Time & either irradiation damage [dpa] or time in [s] \\
\hline & $\mathrm{Ci}$ & interstitial concentration $\left[\mathrm{at}^{-1}\right]$ \\
\hline & $\mathrm{Cv}$ & vacancy concentration $\left[\mathrm{at}^{-1}\right]$ \\
\hline & CGas & gas atom concentration $\left[\mathrm{at}^{-1}\right]$ \\
\hline & Ccrow & crowdion concentration $\left[\mathrm{at}^{-1}\right]$ \\
\hline \multirow[t]{8}{*}{ zm_dens.dat } & densities of extended defects $\left[\mathrm{m}^{-3}\right]$ & \\
\hline & Damage /Time & either irradiation damage $[\mathrm{dpa}]$ or time $[\mathrm{s}]$ \\
\hline & N_i_loop & number density of interstitial loops bigger than $2 \mathrm{i}$ \\
\hline & $\mathrm{Nn} \_$i_loop & number density of visible interstitial loops \\
\hline & N_v_loop & number density of vacancy loops bigger than $2 \mathrm{v}$ \\
\hline & Nn_v_loop & number density of visible vacancy loops \\
\hline & N_void & number density of voids bigger than $2 \mathrm{v}$ \\
\hline & Nn_void & number density of visible voids \\
\hline \multirow[t]{9}{*}{ zm_gasA.dat } & gas atoms at different sinks & \\
\hline & Damage /Time & either irradiation damage $[\mathrm{dpa}]$ or time $[\mathrm{s}]$ \\
\hline & CGas & freely migrating gas $\left[\mathrm{at}^{-1}\right]$ \\
\hline & Gas_bubb & in bubbles $\quad\left[\mathrm{at}^{-1}\right]$ \\
\hline & Gas_dsl & at dislocations $\quad\left[\mathrm{at}^{-1}\right]$ \\
\hline & Gas_GB & at grain boundaries $\left[\mathrm{at}^{-1}\right]$ \\
\hline & GB_Cover & grain boundary coverage with gas atoms, \\
\hline & G_Gas_s ${ }^{-1}$ & generation rate $\left[\mathrm{s}^{-1}\right]$ \\
\hline & <mgas $>$ & mean number of gas at per bubble \\
\hline
\end{tabular}


Table 6.3: Output file descriptions from RIME Model.

\begin{tabular}{|c|c|c|}
\hline File Name & Variable & Description \\
\hline \multirow[t]{10}{*}{ zm_gasB.dat } & all information on bubbles & \\
\hline & Damage /Time & either irradiation damage $[\mathrm{dpa}]$ or time $[\mathrm{s}]$ \\
\hline & N_m ${ }^{-3}$ & bubble number density $\left[\mathrm{m}^{-3}\right]$ \\
\hline & R_nm & bubble mean radius [nm] \\
\hline & Swelling_\% & swelling due to bubbles $[\%]=\left[100 . * \mathrm{at}^{-1}\right]$ \\
\hline & SS_m-2 & bubble sink strength for freely-migrating SIAs [m-2] \\
\hline & $\mathrm{Nn} \_\mathrm{m}^{-3}$ & number density of visible bubbles $\left[\mathrm{m}^{-3}\right]$ \\
\hline & Rn_nm & mean radius of visible bubbles [nm] \\
\hline & $\langle$ mgas $>$ & mean number of gas atoms at per bubble \\
\hline & DenZ_nm & mean distance between bubbles [nm] \\
\hline \multirow[t]{8}{*}{ zm_intL.dat } & all information on interstitial loops & \\
\hline & Damage /Time & either irradiation damage in dpa or time [s] \\
\hline & N_m ${ }^{-3}$ & loop number density $\left[\mathrm{m}^{-3}\right]$ \\
\hline & R_nm & loop mean radius [nm] \\
\hline & SNdeff & total defects accumulated in loops $\left[\mathrm{at}^{-1}\right]$ \\
\hline & SS_m-2 & sink strength for freely-migrating SIAs [m-2] \\
\hline & $\mathrm{Nn} \_\mathrm{m}^{-3}$ & number density of visible loops $\left[\mathrm{m}^{-3}\right]$ \\
\hline & Rn_nm & mean radius of visible loops [nm] \\
\hline \multirow[t]{9}{*}{ zm_radi.dat } & radii of extended defects [nm] & \\
\hline & Damage /Time & either irradiation damage in dpa or time [s] \\
\hline & R_i_loop & mean radius of interstitial loops bigger than $2 \mathrm{i}$ \\
\hline & Rn_i_loop & mean radius of visible interstitial loops \\
\hline & R_v_loop & mean radius of vacancy loops bigger than $2 \mathrm{v}$ \\
\hline & Rn_v_loop & mean radius of visible vacancy loops \\
\hline & R_void & mean radius of voids/bubbles bigger than $2 \mathrm{v}$ \\
\hline & Rn_void & mean radius of visible voids/bubbles \\
\hline & RWZ_void & Wigner-Zeitz cell radius for 3-D migrating defects \\
\hline \multirow[t]{12}{*}{ zm_swss.dat } & swelling and sink strengths & \\
\hline & Damage /Time & either irradiation damage in dpa or time [s] \\
\hline & I_in_loops & total number of interstitial in loops $\left[\mathrm{at}^{-1}\right]$ \\
\hline & V_in_loop & total number of vacancies in loops $\left[\mathrm{at}^{-1}\right]$ \\
\hline & Swelling_\% & swelling $[\%]=\left[100 . * \mathrm{at}^{-1}\right]$ \\
\hline & SW_GB & no description yet \\
\hline & SSi_i_lps & sink strength of interstitial loops for SIAs [m-2] \\
\hline & SSi_v_lps & sink strength of vacancy loops for SIAs [m-2] \\
\hline & SSi_voids & sink strength of voids/bubbles for SIAs [m-2] \\
\hline & rod_m-2 & dislocation density $[\mathrm{m}-2]$ \\
\hline & MFP3d_nm & mean-free path of freely-migrating defects [m] \\
\hline & S_dot_\%/dpa & swelling rate $\left[\mathrm{at}^{-1} / \mathrm{dpa}\right]$ \\
\hline \multirow[t]{8}{*}{ zm_vacL.dat } & all information on vacancy loops & \\
\hline & Damage /Time & either irradiation damage in dpa or time [s] \\
\hline & N_m ${ }^{-3}$ & loop number density $\left[\mathrm{m}^{-3}\right]$ \\
\hline & R_nm & loop mean radius [nm] \\
\hline & SNdeff & total defects accumulated in loops $\left[\mathrm{at}^{-1}\right]$ \\
\hline & SS_m-2 & sink strength for freely-migrating SIAs [m-2] \\
\hline & $\mathrm{Nn} \_\mathrm{m}^{-3}$ & number density of visible loops $\left[\mathrm{m}^{-3}\right]$ \\
\hline & Rn_nm & mean radius of visible loops [nm] \\
\hline \multirow[t]{10}{*}{ zm_liz.dat } & Dn_nm & mean diameter of visible loops [nm] \\
\hline & $\mathrm{Nn} \_\mathrm{m}^{-3}$ & number density of visible loops $\left[\mathrm{m}^{-3}\right]$ \\
\hline & Swelling_\% & swelling $[\%]=\left[100 . * \mathrm{at}^{-1}\right]$ \\
\hline & S_dot_\%/dpa & swelling rate $\left[\mathrm{at}^{-1} / \mathrm{dpa}\right]$ \\
\hline & SSi_voids & sink strength of voids/bubbles for SIAs [m-2] \\
\hline & Dn_i_loop & mean diameter of visible interstitial loops \\
\hline & Nn_i_loop & number density of visible interstitial loops \\
\hline & SSi_i_lps & sink strength of interstitial loops for SIAs [m-2] \\
\hline & rod_m-2 & dislocation density [m-2] \\
\hline & ss_pptU & Sink strength for $\mathrm{G}$ phase \\
\hline
\end{tabular}


Table 6.3: Output file descriptions from RIME Model.

\begin{tabular}{|l|l|l|}
\hline File Name & Variable & Description \\
\hline & ss_pptB & Sink strength for M2X \\
\hline & ss_loop_exp & Loop sink strength for explicit loop treatment \\
\hline zz_age_ct.dat & aging continuation & this file is needed to continue previous aging calculation \\
\hline zz_igr_ct.dat & $\begin{array}{l}\text { continuation of surface irradiation after bulk } \\
\text { irradiation (not in use) }\end{array}$ & $\begin{array}{l}\text { this file is needed to continue previously performed } \\
\text { irradiation }\end{array}$ \\
\hline zz_irr_ct.dat & \begin{tabular}{l} 
irradiation continuation \\
\hline
\end{tabular}
\end{tabular}


Table 6.4: Main input parameters used in sensitivity analysis. These inputs correspond to Ref.2 shown in Table 6.9.

\begin{tabular}{|l|l|l|}
\hline Parameter & Value & Reference \\
\hline Case Name & $100 \mathrm{dpa}$ & N.A \\
\hline Void Distribution & Input $(188 \mathrm{dpa})$ & From experiment \\
\hline Loop Distribution & Input $(188 \mathrm{dpa})$ & From experiment \\
\hline Number of Starting Void Groups & 120 & N.A \\
\hline Number of Loop Groups & 120 & N.A \\
\hline Precipitate Evolution? & No & N.A \\
\hline C-V traps? & No & N.A \\
\hline Initial Dislocation Density $\left[\mathrm{m}^{-2}\right]$ & $3 \times 10^{14}$ & N.A \\
\hline Grain Diameter [m] & $1 \times 10^{-6}$ & N.A \\
\hline Helium Generation Rate $[\mathrm{appm} / \mathrm{dpa}]$ & 0 & N.A \\
\hline Di-Vacancy Binding Energy[eV] & 0.3 & {$[106]$} \\
\hline Vacancy Formation Energy [eV] & 1.79 & {$[107]$} \\
\hline Vacancy Migration Energy $[\mathrm{eV}]$ & 0.63 & {$[109]$} \\
\hline Interstitial Migration Energy [eV] & 0.22 & {$[109]$} \\
\hline Helium Migration Energy $[\mathrm{eV}]$ & N.A & N.A \\
\hline Vac. Diffusion Pre-exponential $\left[\mathrm{m}^{2} / \mathrm{s}\right]$ & $8.2 \times 10^{-7}$ & {$[106]$} \\
\hline Int. Diffusion Pre-exponential $\left[\mathrm{m}^{2} / \mathrm{s}\right]$ & $9.4 \times 10^{-7}$ & {$[106]$} \\
\hline He Diffusion Pre-exponential $\left[\mathrm{m}^{2} / \mathrm{s}\right]$ & N.A & N.A \\
\hline $\begin{array}{l}\text { Dislocation Loop /Network Bias for } \\
\text { Interstitials }\end{array}$ & 1.01 & \\
\hline
\end{tabular}


Table 6.5: Significance calculated for swelling and swelling rate at $460^{\circ} \mathrm{C}, 21$ and $100 \mathrm{dpa}$ with and without the addition of unbiased precipitates at experimental levels observed for $\mathrm{G}$ phase. (Ref.2)

\begin{tabular}{|c|c|c|c|c|c|c|c|c|}
\hline & \multicolumn{4}{|c|}{ Swelling } & \multicolumn{3}{c|}{ Swelling Rate } \\
\hline & \multicolumn{2}{|c|}{$21 \mathrm{dpa}$} & \multicolumn{2}{c|}{$100 \mathrm{dpa}$} & \multicolumn{2}{c|}{$21 \mathrm{dpa}$} & \multicolumn{2}{c|}{100 dpa } \\
\hline Parameter & $\begin{array}{c}\text { Without } \\
\text { PPT }\end{array}$ & $\begin{array}{c}\text { With } \\
\text { PPT }\end{array}$ & $\begin{array}{c}\text { Without } \\
\text { PPT }\end{array}$ & $\begin{array}{c}\text { With } \\
\text { PPT }\end{array}$ & $\begin{array}{c}\text { Without } \\
\text { PPT }\end{array}$ & $\begin{array}{c}\text { With } \\
\text { PPT }\end{array}$ & $\begin{array}{c}\text { Without } \\
\text { PPT }\end{array}$ & $\begin{array}{c}\text { With } \\
\text { PPT }\end{array}$ \\
\hline $\begin{array}{c}\text { Bias } \\
\text { (Increase) }\end{array}$ & 1.5 & 1.4 & 62 & 61 & 108 & 108 & 120 & 119 \\
\hline $\begin{array}{c}\text { Grain Size } \\
\text { Increase) }\end{array}$ & 0.01 & 0.01 & 0.15 & 0.15 & 0.29 & 0.29 & 0.29 & 0.29 \\
\hline $\begin{array}{c}\text { Temperature } \\
\text { (Increase) }\end{array}$ & 0 & 0 & 0.33 & 0.22 & 0.392 & 0.366 & 0.47 & 0.43 \\
\hline $\begin{array}{c}\text { Damage } \\
\text { Rate } \\
\text { (Decrease) }\end{array}$ & -0.0087 & -0.00781 & -0.0829 & -0.0969 & -0.090 & -0.107 & -0.11 & -0.12 \\
\hline
\end{tabular}


Table 6.6: Significance of key input parameters in RIME model.

\begin{tabular}{|c|c|c|c|c|}
\hline & \multicolumn{2}{|c|}{ Swelling } & \multicolumn{2}{c|}{ Swelling Rate } \\
\hline Parameter & $21 \mathrm{dpa}$ & $100 \mathrm{dpa}$ & $21 \mathrm{dpa}$ & $100 \mathrm{dpa}$ \\
\hline $\begin{array}{c}\text { Cascade Efficiency } \\
(\varepsilon)\end{array}$ & -0.014 & -1.019 & -1.901 & -1.977 \\
\hline $\begin{array}{c}\text { Recombination } \\
\text { Coefficient }(\mu)\end{array}$ & 0.000 & -0.006 & -0.012 & -0.012 \\
\hline$E_{f}^{v}$ & 0.088 & 0.038 & 0.066 & 0.061 \\
\hline$E_{m}^{v}$ & 0.018 & 0.038 & 0.066 & 0.074 \\
\hline$E_{m}^{i}$ & 0.002 & -0.454 & -0.015 & -0.357 \\
\hline Bias $\left(z_{L}^{i}\right)$ & 1.51 & 108 & 62 & 119 \\
\hline
\end{tabular}


Table 6.7: Main changes in parameterization from FCC to BCC alloys.

\begin{tabular}{|c|c|c|}
\hline Parameter & Value & Reference \\
\hline Dislocation Density $\left[\mathrm{m}^{-2}\right]$ & $3 \times 10^{14}$ & Measured \\
\hline Grain Diameter [m] & $1 \times 10^{-6}$ & Measured \\
\hline Di-vacancy Binding Energy [eV] & 0.3 & [106] \\
\hline Vacancy Formation Energy [eV] & 1.79 & [107] \\
\hline Vacancy Migration Energy [eV] & 0.63 & [109] \\
\hline Interstitial Migration Energy [eV] & 0.22 & [109] \\
\hline Vac. Diffusion Pre-exponential [m²/s] & $8.2 \times 10^{-7}$ & [106] \\
\hline Int. Diffusion Pre-exponential $\left[\mathrm{m}^{2} / \mathrm{s}\right]$ & $1.1 \times 10^{-7}$ & [106] \\
\hline He Diffusion Pre-exponential $\left[\mathrm{m}^{2} / \mathrm{s}\right]$ & $4 \times 10^{-7}$ & {$[108]$} \\
\hline Cascade Efficiency & 0.3 & {$[110,111]$} \\
\hline Dislocation Bias for Interstitials & $1.01-1.25$ & $\begin{array}{c}{[8,41-} \\
43,114,115]\end{array}$ \\
\hline
\end{tabular}


Table 6.8: Concentration [10] and diffusion coefficients at $500^{\circ} \mathrm{C}$ for minor alloying elements in HT9.

\begin{tabular}{|c|c|r|r|}
\hline Element & wt\% & $\begin{array}{l}\text { Approximate } \\
\text { Diffusion } \\
\text { Coefficient }\end{array}$ & Reference \\
\hline $\mathrm{Cr}$ & 11.8 & $3.70 \mathrm{E}-20$ & {$[129]$} \\
\hline $\mathrm{Ni}$ & 0.51 & $1.00 \mathrm{E}-19$ & {$[130]$} \\
\hline $\mathrm{Si}$ & 0.21 & $1.00 \mathrm{E}-20$ & {$[131]$} \\
\hline $\mathrm{Mn}$ & 0.5 & $5.00 \mathrm{E}-18$ & {$[132]$} \\
\hline $\mathrm{Mo}$ & 1.03 & $2.70 \mathrm{E}-21$ & {$[133]$} \\
\hline $\mathrm{C}$ & 0.21 & $5.00 \mathrm{E}-13$ & {$[109,119]$} \\
\hline $\mathrm{P}$ & 0.008 & $5.00 \mathrm{E}-20$ & {$[134]$} \\
\hline $\mathrm{S}$ & 0.003 & $1.00 \mathrm{E}-17$ & {$[134]$} \\
\hline $\mathrm{N}$ & 0.01 & $1.00 \mathrm{E}-12$ & {$[109]$} \\
\hline $\mathrm{W}$ & 0.5 & $1.80 \mathrm{E}-21$ & {$[135]$} \\
\hline $\mathrm{V}$ & 0.33 & $2.00 \mathrm{E}-20$ & {$[136]$} \\
\hline
\end{tabular}


Table 6.9: Definition of reference case input. Changes between each reference case highlighted in yellow.

\begin{tabular}{|c|c|c|c|c|c|}
\hline Categories & Description & Variable & Ref.0 & Ref.1 & Ref.2 \\
\hline \multirow[t]{18}{*}{ Run Parameters } & Output Directory Name: 12 char. max & name & Ref.0 & Ref.1 & Ref.2 \\
\hline & $0=$ Volume, $1=$ Surf., $2=$ Surf. no depos & key & 0 & 0 & 0 \\
\hline & Aging $=0 ;$ Irradiation $=1$ & keyai & 1 & 1 & 1 \\
\hline & Calculation: $\mathrm{New}=0 ; \mathrm{Cont}=1$ & keyc & 0 & 0 & 0 \\
\hline & $=1$ check cell size using criterion: & idm & 1 & 1 & 1 \\
\hline & max ratio bubble/cell size allowed & ratmax & $1.00 \mathrm{E}-06$ & $1.00 \mathrm{E}-06$ & $1.00 \mathrm{E}-06$ \\
\hline & $=1$ no void nucleation: $\mathrm{v}+\mathrm{v}$ and $\mathrm{v}+\mathrm{Gas}$ & keyNucl & 0 & 0 & 0 \\
\hline & $=1$ no $\mathrm{v}$-loop nucleation: $\mathrm{v}+\mathrm{v}$ & keyNuclL & 0 & 0 & 0 \\
\hline & LSODE INTERNAL max timestep & dttt1 & $1.00 \mathrm{E}+10$ & $1.00 \mathrm{E}+10$ & $1.00 \mathrm{E}+10$ \\
\hline & Initial timestep out & $\mathrm{dt} 0$ & $1.00 \mathrm{E}-06$ & $1.00 \mathrm{E}-06$ & $1.00 \mathrm{E}-06$ \\
\hline & Time <time1: rio1 & time1 & $1.00 \mathrm{E}+03$ & $1.00 \mathrm{E}+03$ & $1.00 \mathrm{E}+03$ \\
\hline & Time <timem: riom; if >: constant & timem & $5.00 \mathrm{E}+04$ & $5.00 \mathrm{E}+04$ & $5.00 \mathrm{E}+04$ \\
\hline & OUT timestep $\mathrm{dt}=\mathrm{dt} 0 \quad+\mathrm{t} / \mathrm{rio} 1$ & rio1 & 1 & 1 & 1 \\
\hline & OUT Timestep $\mathrm{dt}=\mathrm{dt} \_$previous $+\mathrm{t} /$ riom & riom & 10 & 10 & 10 \\
\hline & $\mathrm{N}$ steps for writing to screen $\&$ files & nscrn & 1 & 1 & 1 \\
\hline & $\mathrm{N}$ steps for saving to continue & nremm & 1 & 1 & 1 \\
\hline & $\mathrm{N}$ steps for writing SDs and 3-d graph & nwrit & 1 & 1 & 1 \\
\hline & EULER $=1$, LSODE $=0$. Only 0 is used & mfeuler & 0 & 0 & 0 \\
\hline \multirow{10}{*}{$\begin{array}{c}\text { Flags and } \\
\text { Dimensions }\end{array}$} & Flag for treatment of vacancies & iVac & 1 & 1 & 1 \\
\hline & Flag for treatment of interstitials & int & 1 & 1 & 1 \\
\hline & Flag for treatment of voids/bubbles & iVoid & 1 & 1 & 1 \\
\hline & Flag for treatment of interstitial loops & illoop & 0 & 0 & 1 \\
\hline & Flag for treatment of dislocations & iDisl & 0 & 0 & 1 \\
\hline & Flag for explicit treatment of vacancy & iTrap & 0 & 0 & 0 \\
\hline & Initial \# of vacancy groups in voids/bubbles & mvv & 120 & 120 & 120 \\
\hline & Initial \# of helium groups in bubbles & $\mathrm{mvb}$ & 120 & 120 & 120 \\
\hline & \# of interstitial loop groups & mil & 120 & 120 & 120 \\
\hline & \# of groups added when expanding cell & iexpnd & 10 & 10 & 10 \\
\hline \multirow{6}{*}{$\begin{array}{l}\text { Tolerances for } \\
\text { integration }\end{array}$} & for all derivatives: atolD=atolV/ntol & ntol & 10 & 10 & 10 \\
\hline & relative tolerance for all values & rtol & $1.0 \mathrm{~d}-4$ & $1.0 \mathrm{~d}-4$ & $1.0 \mathrm{~d}-4$ \\
\hline & initial abs tolerance for SIAs & atsia & $1.0 \mathrm{~d}^{-15}$ & $1.0 \mathrm{~d}^{-15}$ & $1.0 \mathrm{~d}^{-15}$ \\
\hline & initial abs tolerance for vacancies & atvac & $1.0 \mathrm{~d}-7$ & $1.0 \mathrm{~d}-7$ & $1.0 \mathrm{~d}-7$ \\
\hline & initial abs tolerance for interstitial loops & atlps & $1.0 \mathrm{~d}^{-1} 6$ & $1.0 \mathrm{~d}^{-1} 6$ & $1.0 \mathrm{~d}^{-1} 6$ \\
\hline & initial abs tolerance for dislocation & atdis & $1.0 \mathrm{~d}+9$ & $1.0 \mathrm{~d}+9$ & $1.0 \mathrm{~d}+9$ \\
\hline \multirow[t]{12}{*}{ Irradiation } & Irradiation/Aging TIME & time & $4.50 \mathrm{E}+05$ & $4.50 \mathrm{E}+05$ & $4.50 \mathrm{E}+05$ \\
\hline & Mean NRT generation rate & rknrt & $1.00 \mathrm{E}-03$ & $1.00 \mathrm{E}-03$ & $1.00 \mathrm{E}-03$ \\
\hline & Defect fraction recombination in cascades & epsr & 0.67 & 0.67 & 0.67 \\
\hline & SIA fraction in sessile clusters & epsis & 0 & 0 & 0 \\
\hline & Vacancy fraction in sessile clusters & epsv & 0 & 0 & 0 \\
\hline & Vacancy fraction in micro-voids & epsw & 0.3 & 0.3 & 0.3 \\
\hline & Temperature, degree Celsius & tc & 460 & 460 & 460 \\
\hline & Initial gas-atom site fraction & $\operatorname{cg} 0$ & $0.00 \mathrm{E}+00$ & $0.00 \mathrm{E}+00$ & $0.00 \mathrm{E}+00$ \\
\hline & Initial VOLUME dislocation density & $\operatorname{rod} 0$ & $3.0 \mathrm{~d}+14$ & $3.0 \mathrm{~d}+14$ & $3.0 \mathrm{~d}+14$ \\
\hline & Density of vacancy trapping sites & rotrp & $1.0 \mathrm{~d}-8$ & $1.0 \mathrm{~d}-8$ & $1.0 \mathrm{~d}-8$ \\
\hline & GB dislocation density (if key>0) & rods & $1.0 \mathrm{~d}+19$ & $1.0 \mathrm{~d}+19$ & $1.0 \mathrm{~d}+19$ \\
\hline & Grain radius & rgr & $5.00 \mathrm{~d}-7$ & $5.00 \mathrm{~d}-7$ & $5.00 \mathrm{~d}-7$ \\
\hline \multirow[t]{7}{*}{ Material } & $\mathrm{N}$ at. in unit cell 2-BCC 4-FCC & type1 & 2 & 2 & 2 \\
\hline & Lattice parameter: bcc $\mathrm{Fe}=2.87, \mathrm{fcc}=3.61$ & a & $2.86 \mathrm{~d}-10$ & $2.86 \mathrm{~d}-10$ & $2.86 \mathrm{~d}-10$ \\
\hline & Dislocation Burgers V: $1-\mathrm{S} 3 / 2 ; 2-0.5 ; 3^{-1} / \mathrm{S} 3$ & $\mathrm{Nbv}$ & 1 & 1 & 1 \\
\hline & Loop Burgers V: 1-S3/2; 2-0.5; $3^{-1} / \mathrm{S} 3$ & NbvL & 3 & 3 & 3 \\
\hline & Surface energy & gamma0 & 2.3 & 2.3 & 2.3 \\
\hline & (gama*gamma0-gamtc*tc)/1.6e-19 & gama & 1 & 1 & 1 \\
\hline & gamma in $\mathrm{eV}$ & gamtc & $0.01 .75 \mathrm{e}^{-3}$ & $0.01 .75 \mathrm{e}^{-3}$ & $0.01 .75 \mathrm{e}^{-3}$ \\
\hline
\end{tabular}


Table 6.9: Definition of reference case input. Changes between each reference case highlighted in yellow.

\begin{tabular}{|c|c|c|c|c|c|}
\hline Categories & Description & Variable & Ref.0 & Ref.1 & Ref.2 \\
\hline & Vacancy formation energy & evf & 1.79 & 1.79 & 1.79 \\
\hline & Vacancy migration energy & evm & 0.63 & 0.63 & 0.63 \\
\hline & Effective vacancy migration energy (same if & evmeff & 0.63 & 0.63 & 0.63 \\
\hline & SIA migration energy & eim & 0.22 & 0.22 & 0.22 \\
\hline & Pre-exp for vacancy formation & cve0 & 1 & 1 & 1 \\
\hline & Pre-exp for vacancy migration & $\mathrm{c} 0 \mathrm{v}$ & $8.2 \mathrm{~d}-7$ & $8.2 \mathrm{~d}-7$ & $8.2 \mathrm{~d}-7$ \\
\hline & Pre-exp for SIA migration & $\mathrm{cOi}$ & $9.4 \mathrm{~d}-7$ & $9.4 \mathrm{~d}-7$ & $9.4 \mathrm{~d}-7$ \\
\hline & Bardeen-Herring disl' source density & Sdisl & $0.0 \mathrm{~d}+22$ & $0.0 \mathrm{~d}+22$ & $0.0 \mathrm{~d}+22$ \\
\hline & Modified back stress term, Roger $=0.05$ & Aprime & 0 & 0 & 0 \\
\hline & ik eim2 SIA 2-4i mig energy & & 10.11 & 10.11 & 10.11 \\
\hline & ik c0i2 Pre-exp mig & & $16.5 \mathrm{~d}-8$ & $16.5 \mathrm{~d}-8$ & $16.5 \mathrm{~d}-8$ \\
\hline \multirow{16}{*}{$\begin{array}{l}\text { Reaction cross } \\
\text { section }\end{array}$} & Loops capture efficiency for SIAs & zicl & 1.01 & 1.01 & 1.01 \\
\hline & Dislocation capture efficiency for SIAs & $\mathrm{zi}$ & 1.01 & 1.01 & 1.01 \\
\hline & Dislocation capture efficiency for vacancies & $\mathrm{ZV}$ & 1 & 1 & 1 \\
\hline & Void capture distance for SIA & rivoid & $0.0 \mathrm{~d}-9$ & $0.0 \mathrm{~d}-9$ & $0.0 \mathrm{~d}-9$ \\
\hline & Dislocation capture efficiency for gas atoms & $\mathrm{zgd}$ & 0 & 0 & 0 \\
\hline & GB capture efficiency for SIAs & zig & 1 & 1 & 1 \\
\hline & GB capture efficiency for vacancies & zvg & 1 & 1 & 1 \\
\hline & Emission energy for vacancy-trap complex & ebtrv & 0.5 & 0.5 & 0.5 \\
\hline & Recombination constant & rec & $2.0 \mathrm{~d}+21$ & $2.0 \mathrm{~d}+21$ & $2.0 \mathrm{~d}+21$ \\
\hline & V+crow: =1-pure 1D; =0-preferential & rkk & 1 & 1 & 1 \\
\hline & EOS:1-Roger;(2 Carnahan-S;3?Manzke-T) & keybb & 1 & 1 & 1 \\
\hline & Sigma for Carnahan-Starling EOS & sigma & $0.2 \mathrm{~d}-9$ & $0.2 \mathrm{~d}-9$ & $0.2 \mathrm{~d}-9$ \\
\hline & $\mathrm{v}$ - $\mathrm{v}$ binding energy for Wolfer correct & $\mathrm{e} 2 \mathrm{v}$ & 0.3 & 0.3 & 0.3 \\
\hline & Wolfer corr: original & wolfn & -1 & -1 & -1 \\
\hline & i>nevf then Ev_binding=evf & nevf & 10000 & 10000 & 10000 \\
\hline & $=1$ include punching of $\mathrm{i}$ from bubbles & keyart & 1 & 1 & 1 \\
\hline \multirow{12}{*}{$\begin{array}{l}\text { Precipitates and } \\
\text { impurity atoms }\end{array}$} & key: $=1$ change pre-existing microstructure & iliz & 1 & 1 & 1 \\
\hline & bulk concentration of impurity atoms !Eliz & Ccarbon & $0.0 \mathrm{~d}-2$ & $0.0 \mathrm{~d}-2$ & $0.0 \mathrm{~d}-2$ \\
\hline & impurity-vacancy binding energy !Eliz & Ebcv & 0.5 & 0.5 & 0.5 \\
\hline & coordination number !Eliz & znn & 8 & 8 & 8 \\
\hline & N_U: number of and N_pptU coefficients & N_U; N_pptU & $0+0 \mathrm{e} 20$ & $0+0 \mathrm{e} 20$ & $0+0 \mathrm{e} 20$ \\
\hline & _U: number of and R_pptU coefficients & R_U; RpptU & $0+0 \mathrm{e}-09+0 \mathrm{e}-$ & $0+0 \mathrm{e}-09+0 \mathrm{e}-$ & $0+0 \mathrm{e}-09+0 \mathrm{e}-$ \\
\hline & N_B: number of and N_pptB coefficients & N_B; N_pptB & $0+0.0$ & $0+0.0$ & $0+0.0$ \\
\hline & _B: number of and R_pptB coefficients & R_B; & $0+0.0+0 \mathrm{e}-1$ & $0+0.0+0 \mathrm{e}-1$ & $0+0.0+0 \mathrm{e}-1$ \\
\hline & Ppts_B capture effic' for SIAs !Eliz & zipptB & 1 & 1 & 1 \\
\hline & Trap effciency & rotrp & 1 & 1 & 1 \\
\hline & ilpnum: number of and num_lp & ilpnum, & $0+0 \mathrm{e}+20-$ & $23.13 \mathrm{e}+20-$ & $0+0 \mathrm{e}+20-$ \\
\hline & ilpr: number of and r_lp coefficients & Ilpr, r_lp & $\begin{array}{l}0+0 \mathrm{e}-8-0 \mathrm{e}-10 \\
+0 \mathrm{e}-12-0 \mathrm{e}-15\end{array}$ & $\begin{array}{c}4+1.68 \mathrm{e}-8- \\
1.58 \mathrm{e}-101.20 \mathrm{e}- \\
12-1.57 \mathrm{e}-15 \\
\end{array}$ & $\begin{array}{l}0+0 e-8-0 e-10 \\
+0 e-12-0 e-15\end{array}$ \\
\hline \multirow{3}{*}{$\begin{array}{l}\text { Correlation- } \\
\text { screening of voids } \\
\text { from 1-d SIA } \\
\text { clusters }\end{array}$} & T min damage for starting screening & phics0 & $1.0 \mathrm{~d}+10$ & $1.0 \mathrm{~d}+10$ & $1.0 \mathrm{~d}+10$ \\
\hline & T max damage for complete screening & phicsm & $1.0 \mathrm{~d}+01$ & $1.0 \mathrm{~d}+01$ & $1.0 \mathrm{~d}+01$ \\
\hline & smallest void screened [nm] & rwhcor & 6 & 6 & 6 \\
\hline \multirow[t]{7}{*}{ Voids: vacancies } & Max size for generation $(<\operatorname{mvxv})$ & mwm & 9 & 9 & 9 \\
\hline & Exponent: gener rate $\sim i^{* *}(-p n w)$ & pnw & 1.94 & 1.94 & 1.94 \\
\hline & i > mvxv: groups & mvxv & 30 & 30 & 30 \\
\hline & $\mathrm{dr}$ or $\mathrm{dr} / \mathrm{r}$ for groups see below & drvv & 0.12 & 0.12 & 0.12 \\
\hline & $=1-\mathrm{dr} ;=2-\mathrm{dr} / \mathrm{r}$ for above choice & igrvac & 2 & 2 & 2 \\
\hline & Factor: increase size of last group & ifac & 1 & 1 & 1 \\
\hline & Min i visible & nvv & 358 & 358 & 358 \\
\hline \multirow[t]{5}{*}{ Interstitial loops } & Max size for generation $(<=\operatorname{mixl})$ & $\operatorname{mim}$ & 10 & 10 & 10 \\
\hline & Exponent: gener rate $\sim i^{* *}(-$ pni) & pni & 1 & 1 & 1 \\
\hline & i > mixl: groups & $\operatorname{mixl}$ & 20 & 20 & 20 \\
\hline & $\mathrm{dr}$ in groups & dri & $6.0 \mathrm{~d}-10$ & $6.0 \mathrm{~d}-10$ & $6.0 \mathrm{~d}-10$ \\
\hline & Min i visible & nli & 20 & 20 & 20 \\
\hline
\end{tabular}




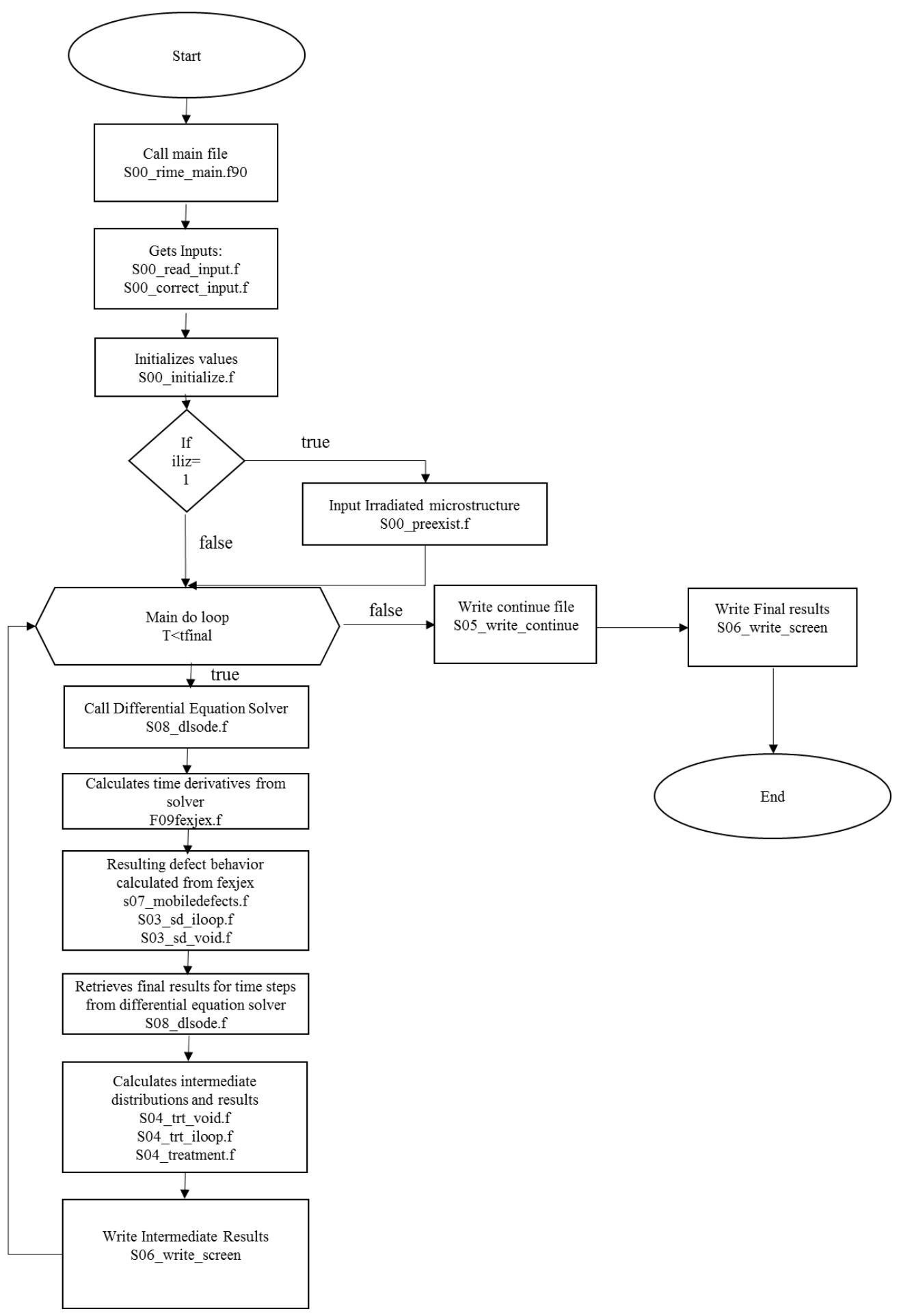

Figure 6.1: Main structure and sub routines in RIME model. 


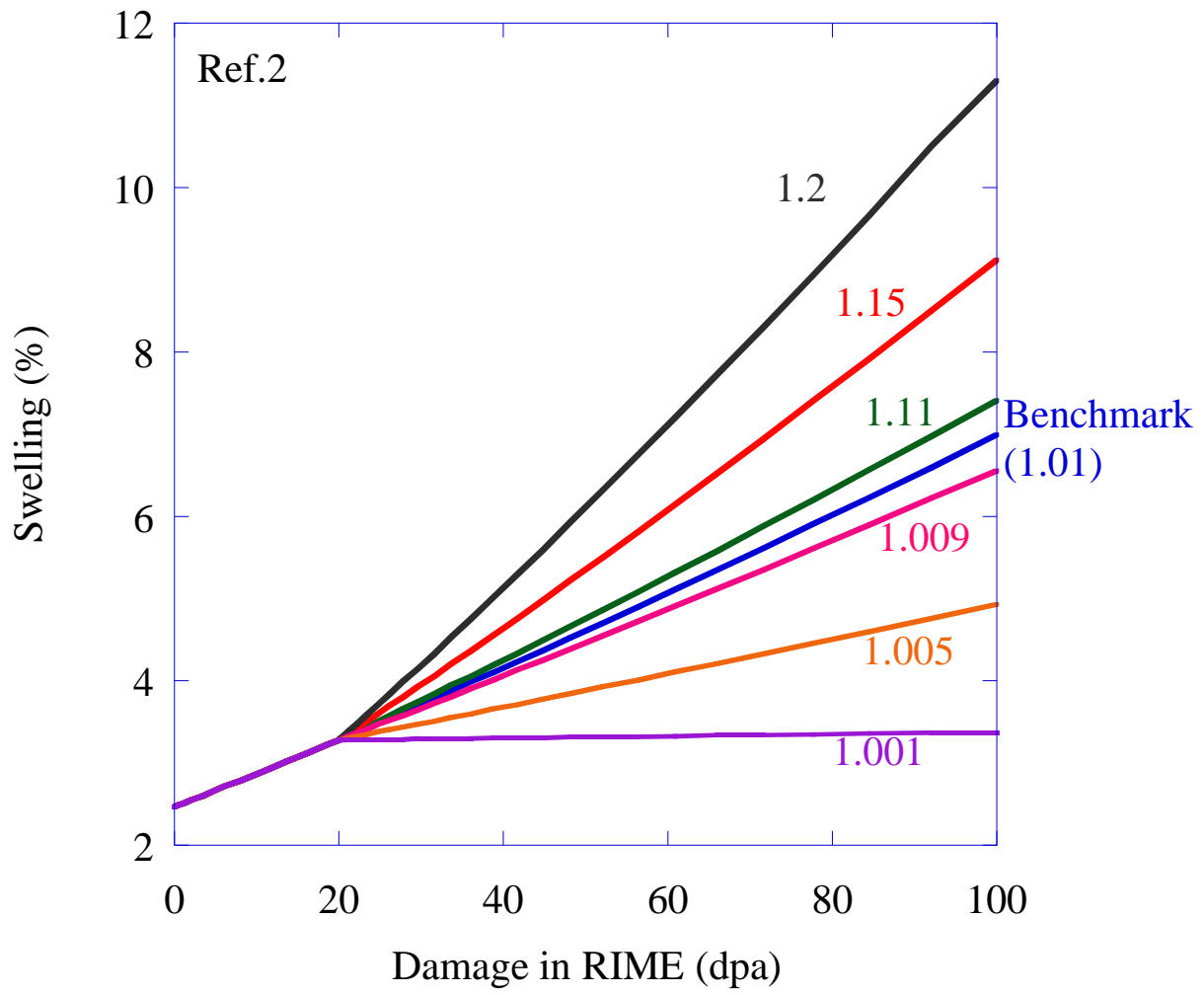

Figure 6.2: Effect of changing bias at $20 \mathrm{dpa}$ on swelling at $460^{\circ} \mathrm{C}$ using Ref.2. 


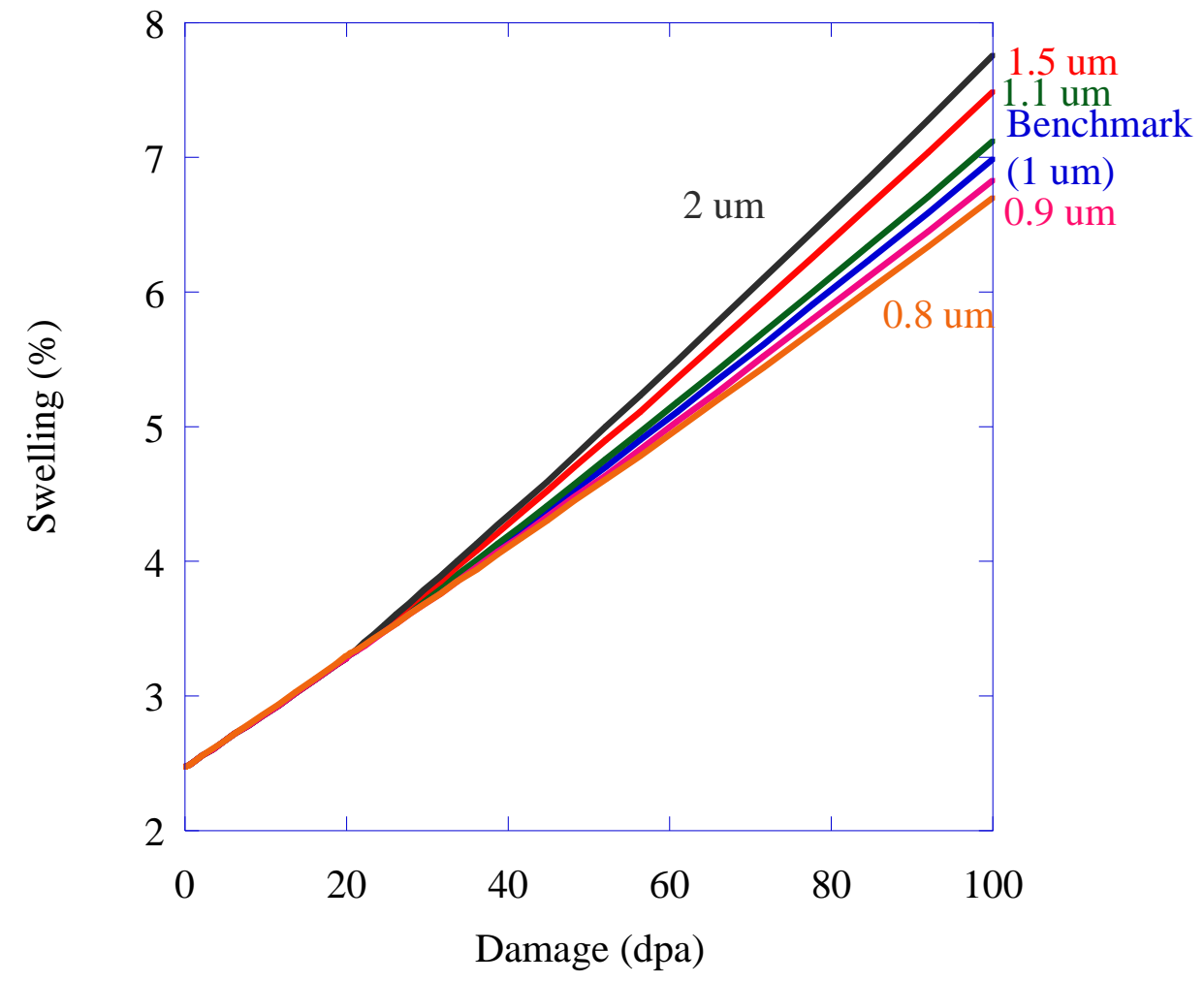

Figure 6.3: Effect of changing grain size at $20 \mathrm{dpa}$ on swelling at $460^{\circ} \mathrm{C}$ using Ref.2. 

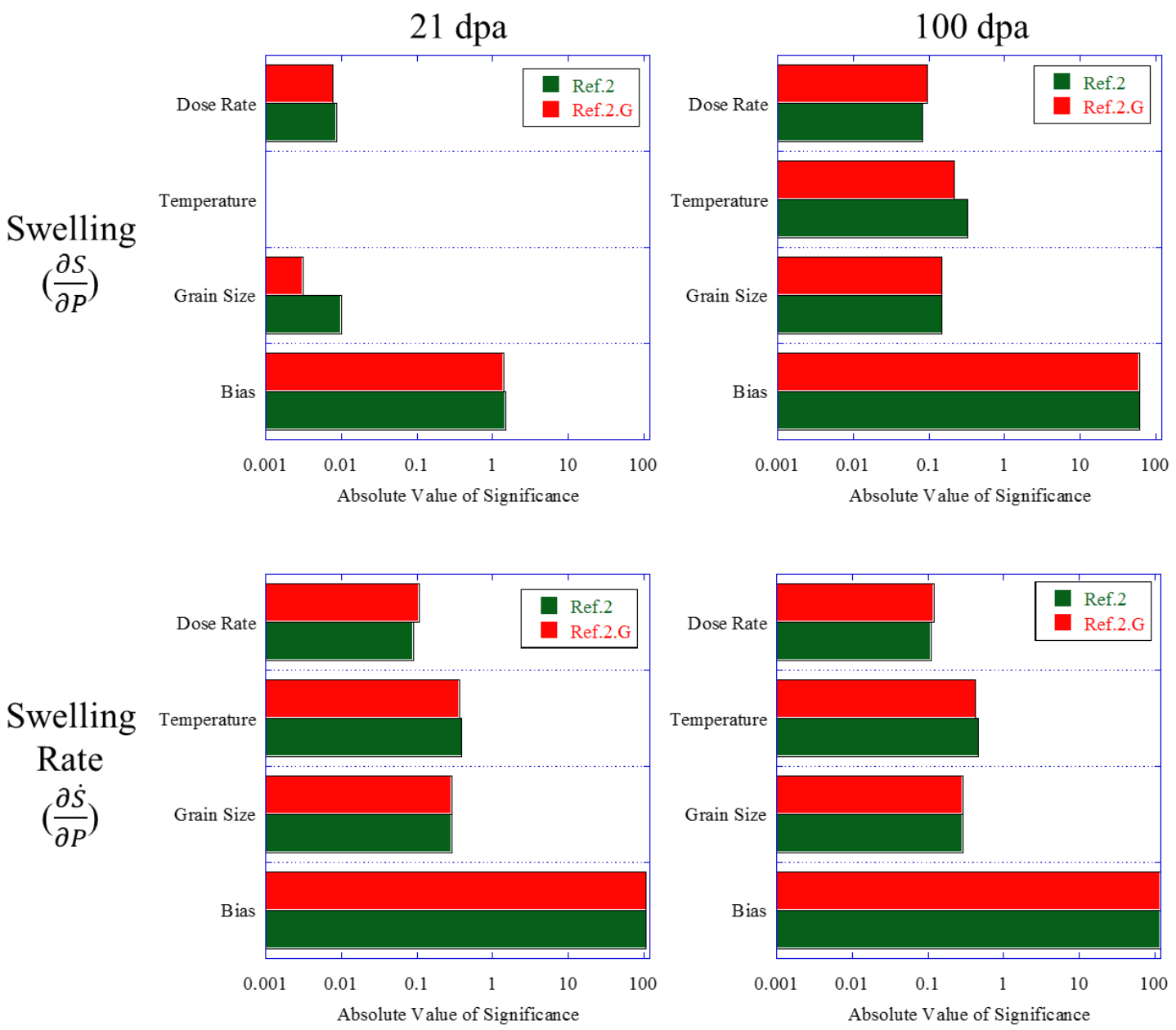

Figure 6.4: Absolute value of significance of damage rate, temperature, grain size and bias. Treatment including with (Ref.2) and without unbiased G phase (Ref.2.G) are both included as a function of damage in RIME at 21 and 100 dpa. 


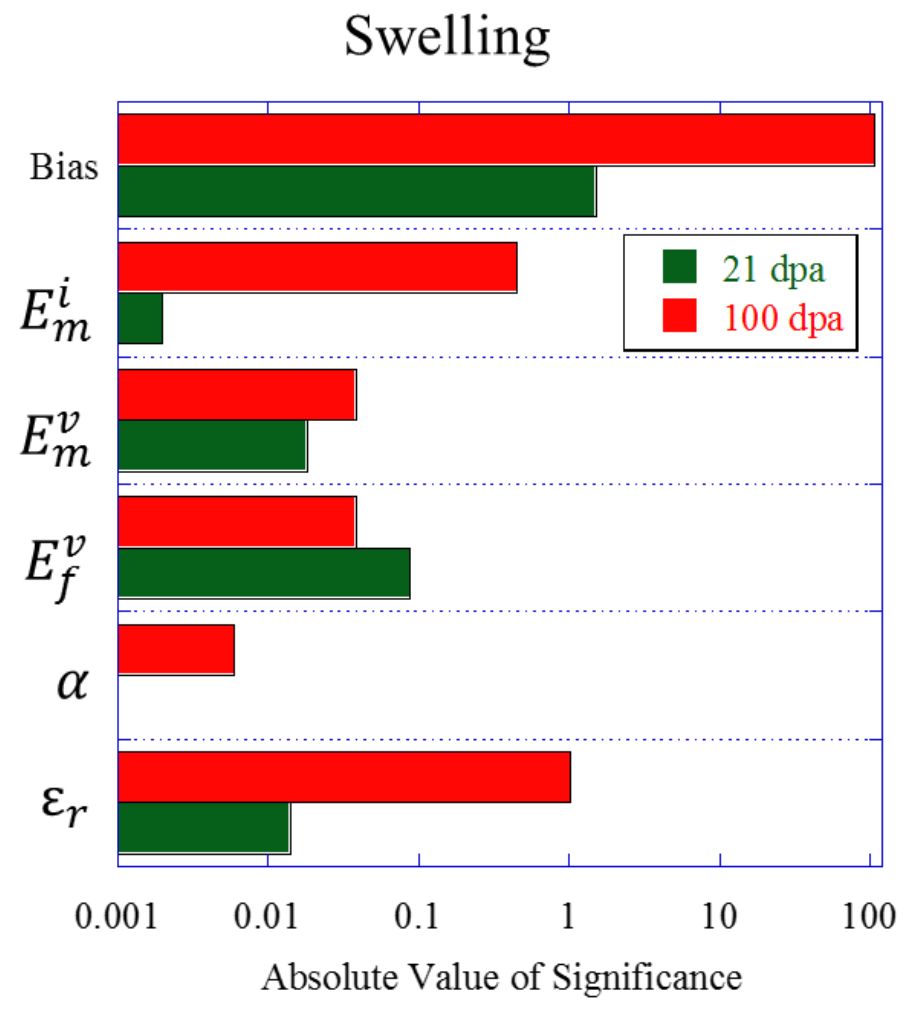

(a)

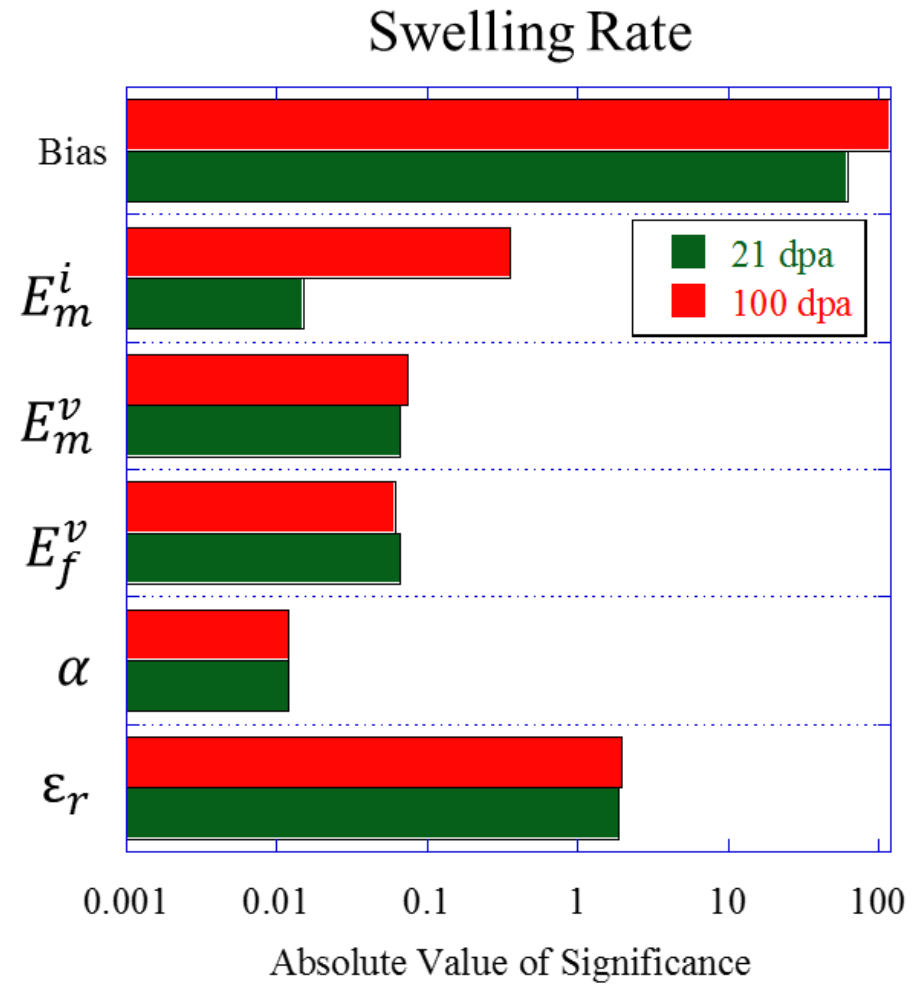

(b)

Figure 6.5: Absolute value of significance of bias, interstitial migration energy, vacancy migration and formation energy, recombination coefficient and cascade efficiency. DD treatment (Ref.2) used with significance reported as a function of damage in RIME at 21 and 100 dpa from the start of the case run. 


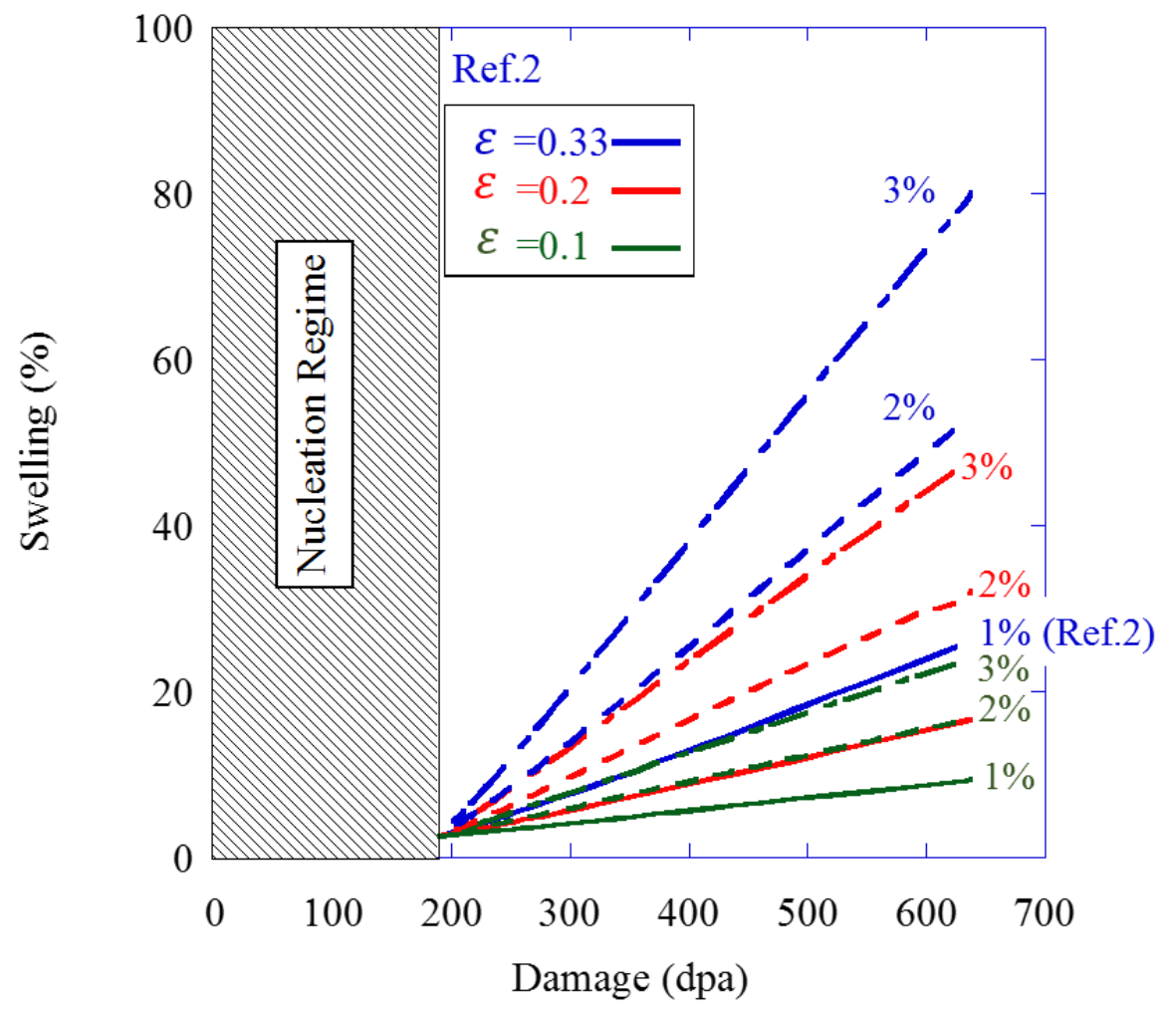

Figure 6.6: Comparison of cascade efficiency and dislocation loop and network bias for interstitials of 1\% (solid lines), 2\% (dashed lines) and 3\% (dotted lines). 


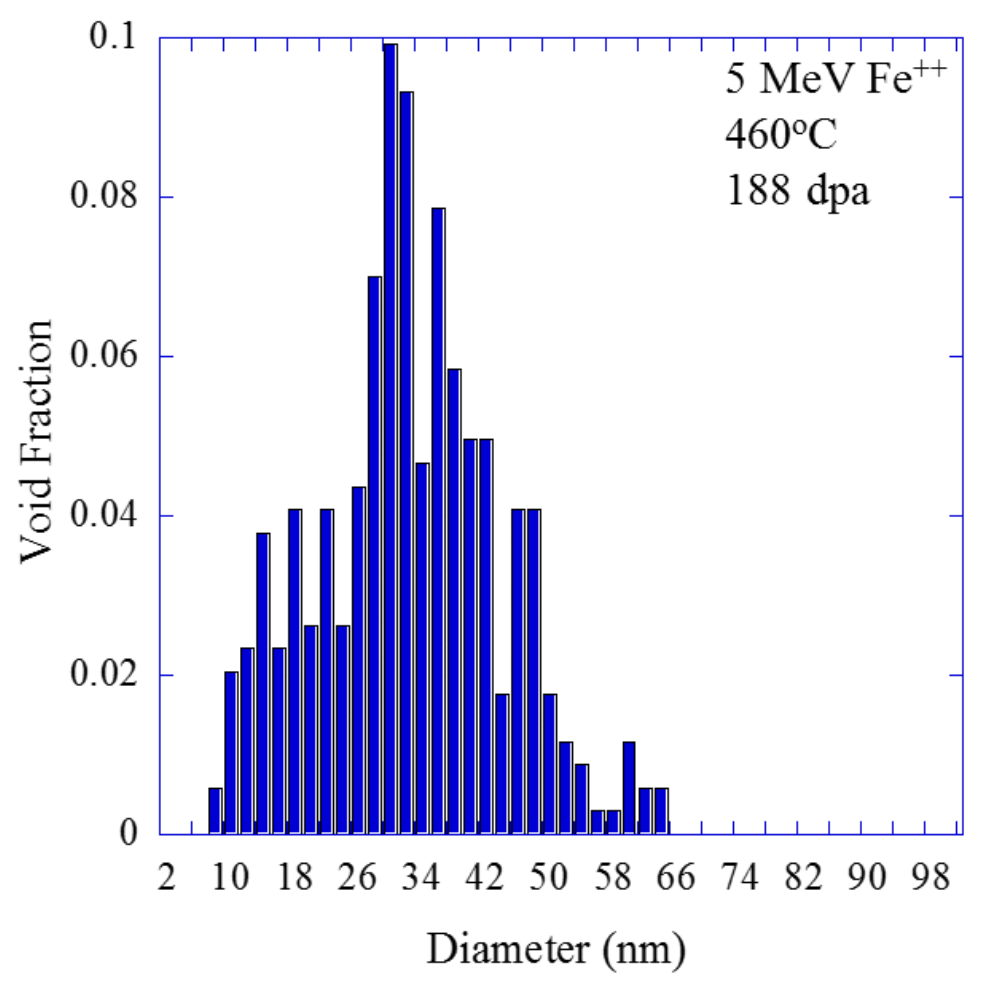

(a)

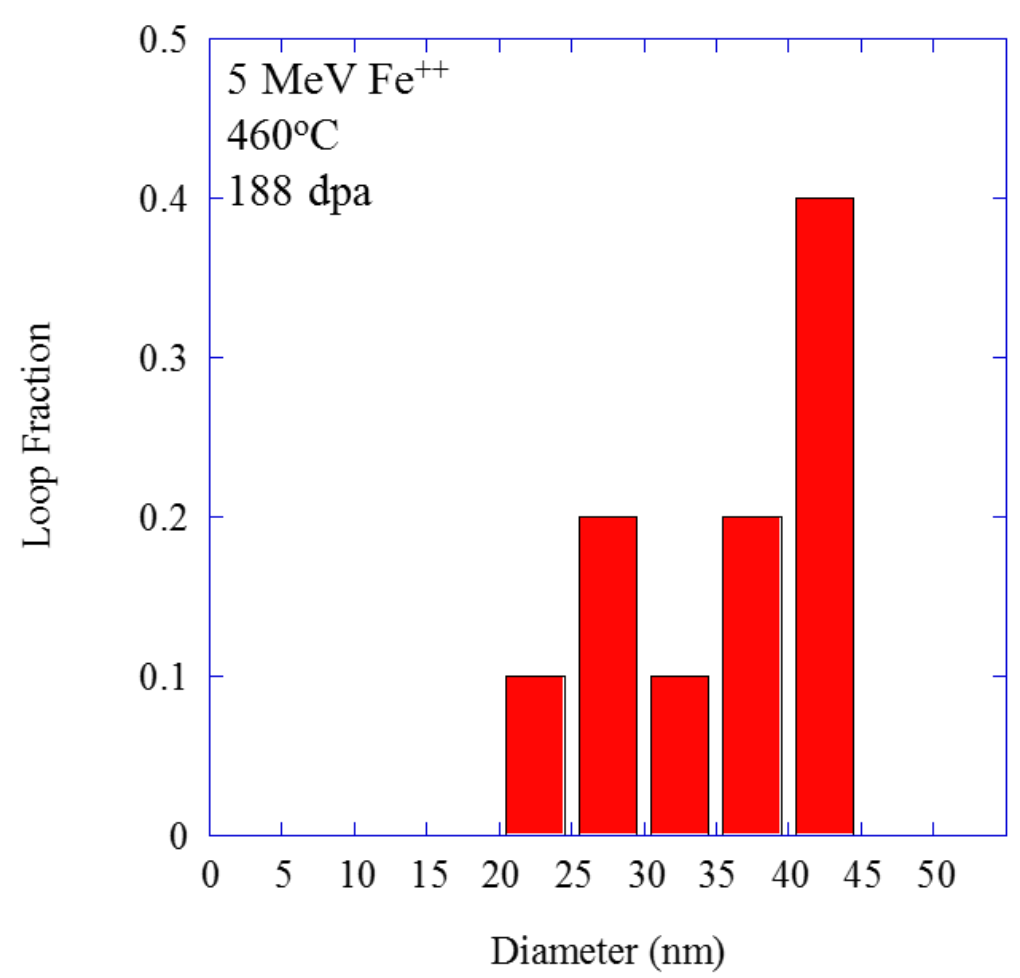

(b)

Figure 6.7: Measured a) void and b) loop fraction in HT9 irradiated with $5 \mathrm{MeV} \mathrm{Fe}^{++}$at $460^{\circ} \mathrm{C}$ to $188 \mathrm{dpa}$. 


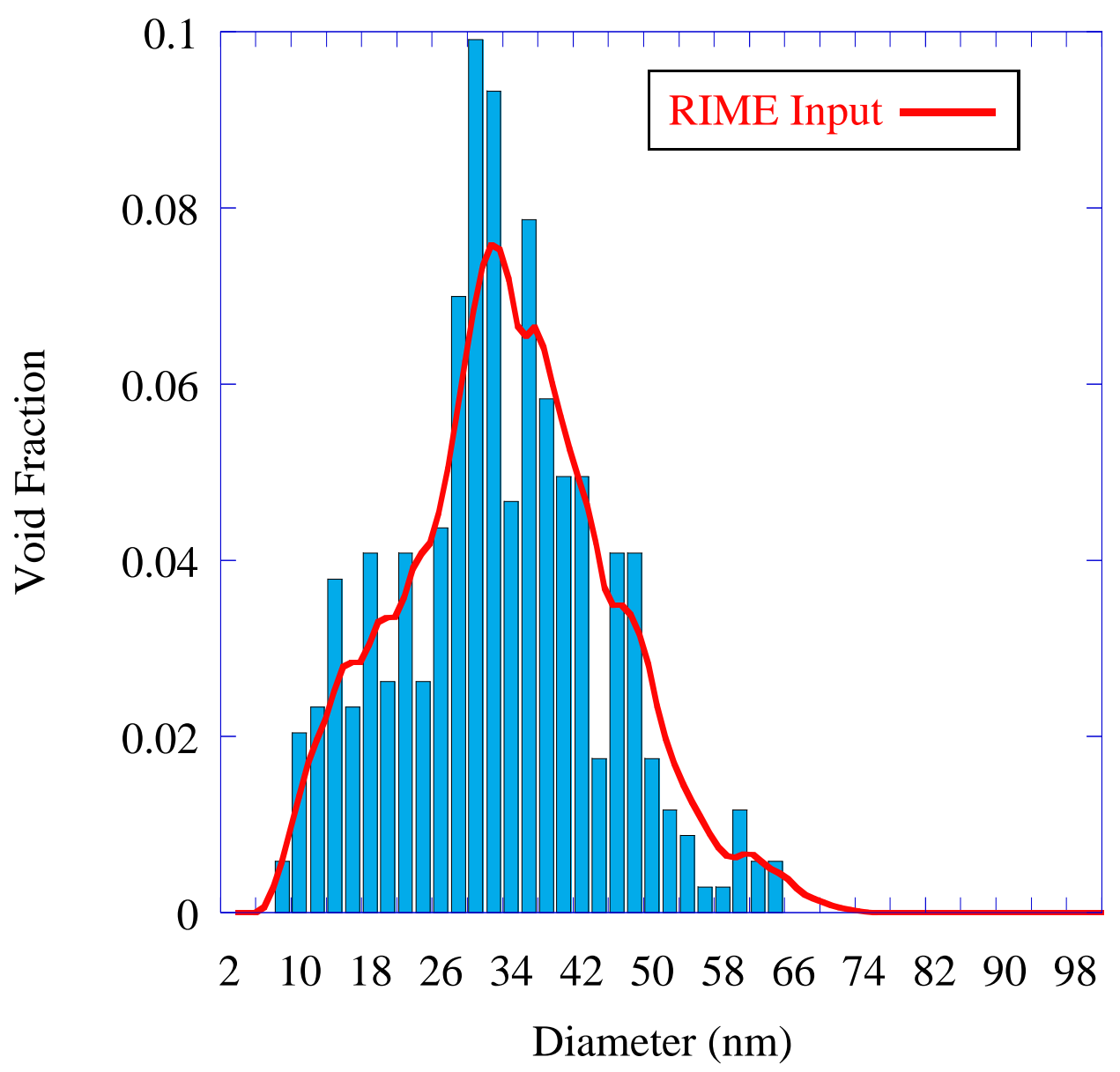

Figure 6.8: Experimental void distribution (blue columns) compared with RIME void distribution input (red line) into each case. 
Total Damage (dpa)

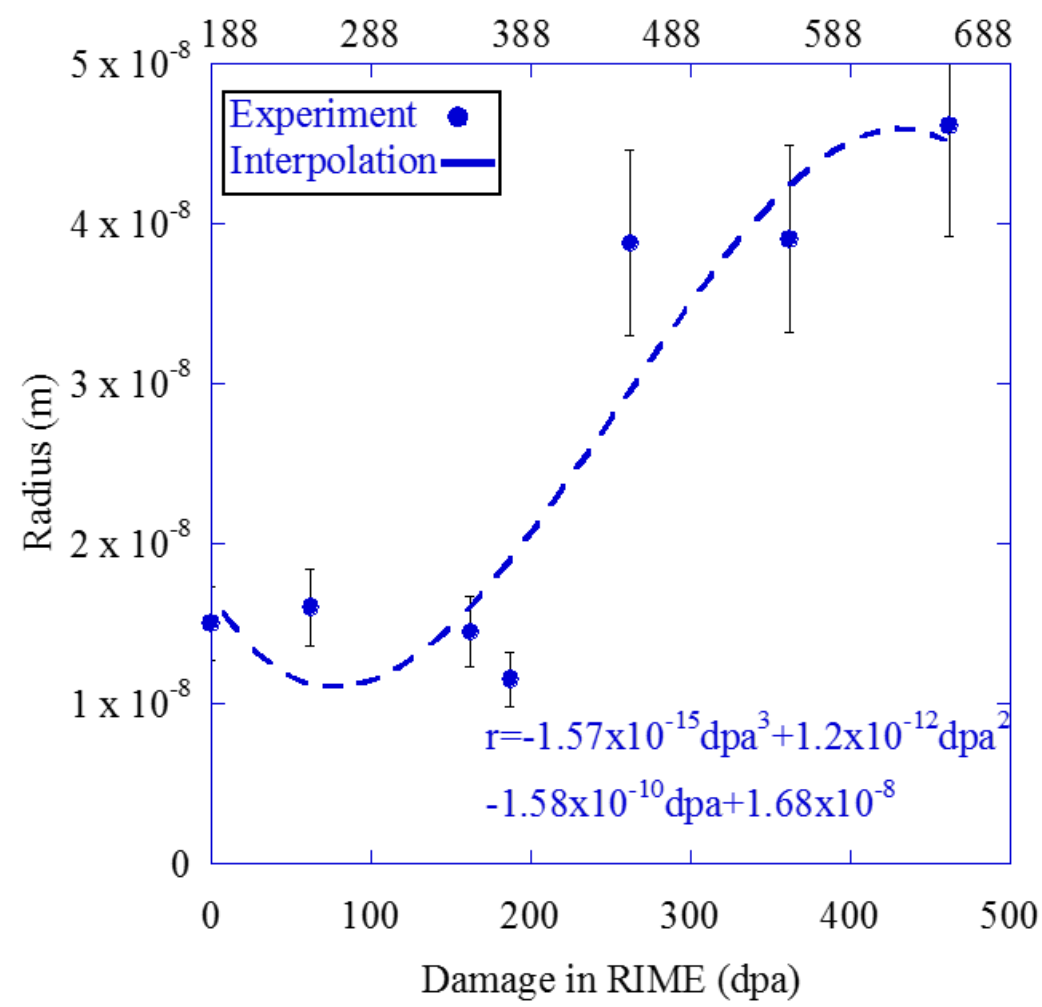

(a)
Total Damage (dpa)

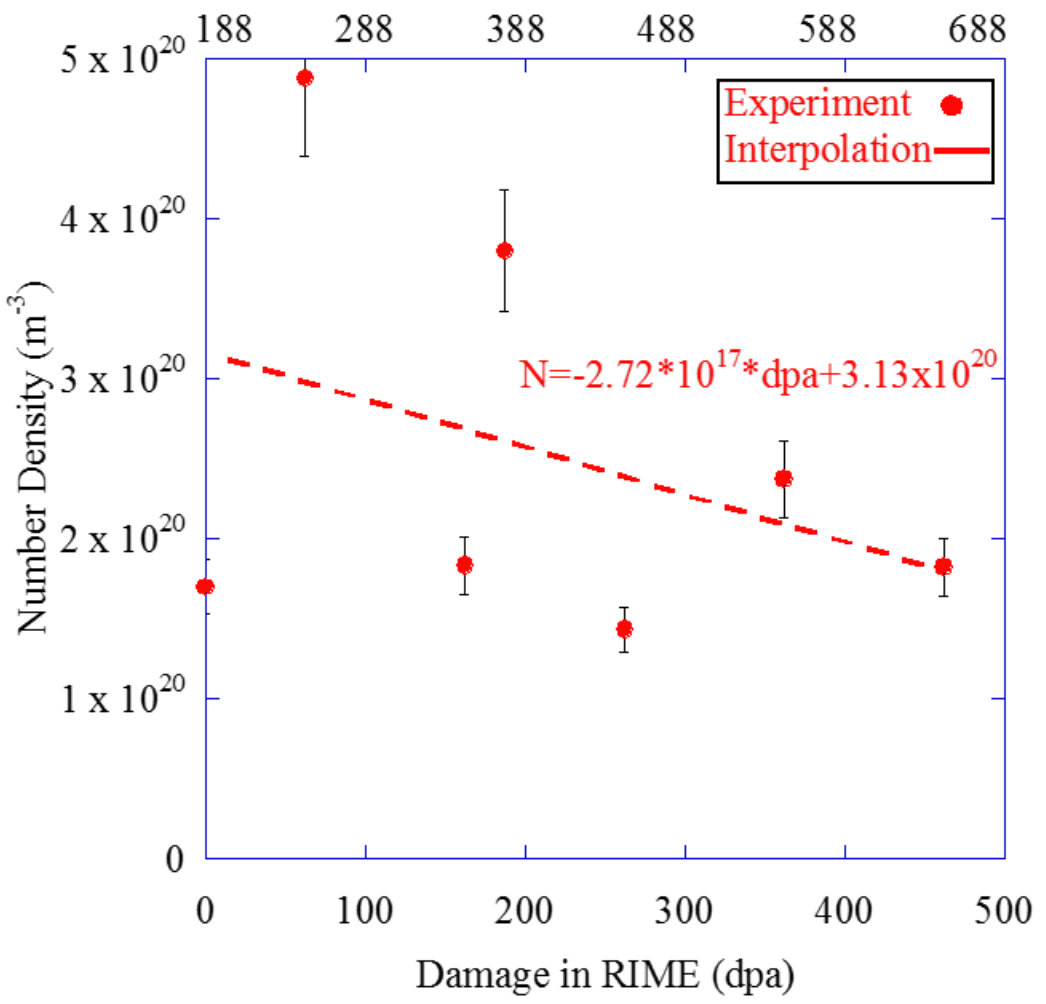

(b)

Figure 6.9: a) Radius and b) number density of dislocation loops in HT9 irradiated with $5 \mathrm{MeV} \mathrm{Fe}^{++}$from 188 to 650 dpa at $460^{\circ} \mathrm{C}$. Interpolation is from experimental data. 


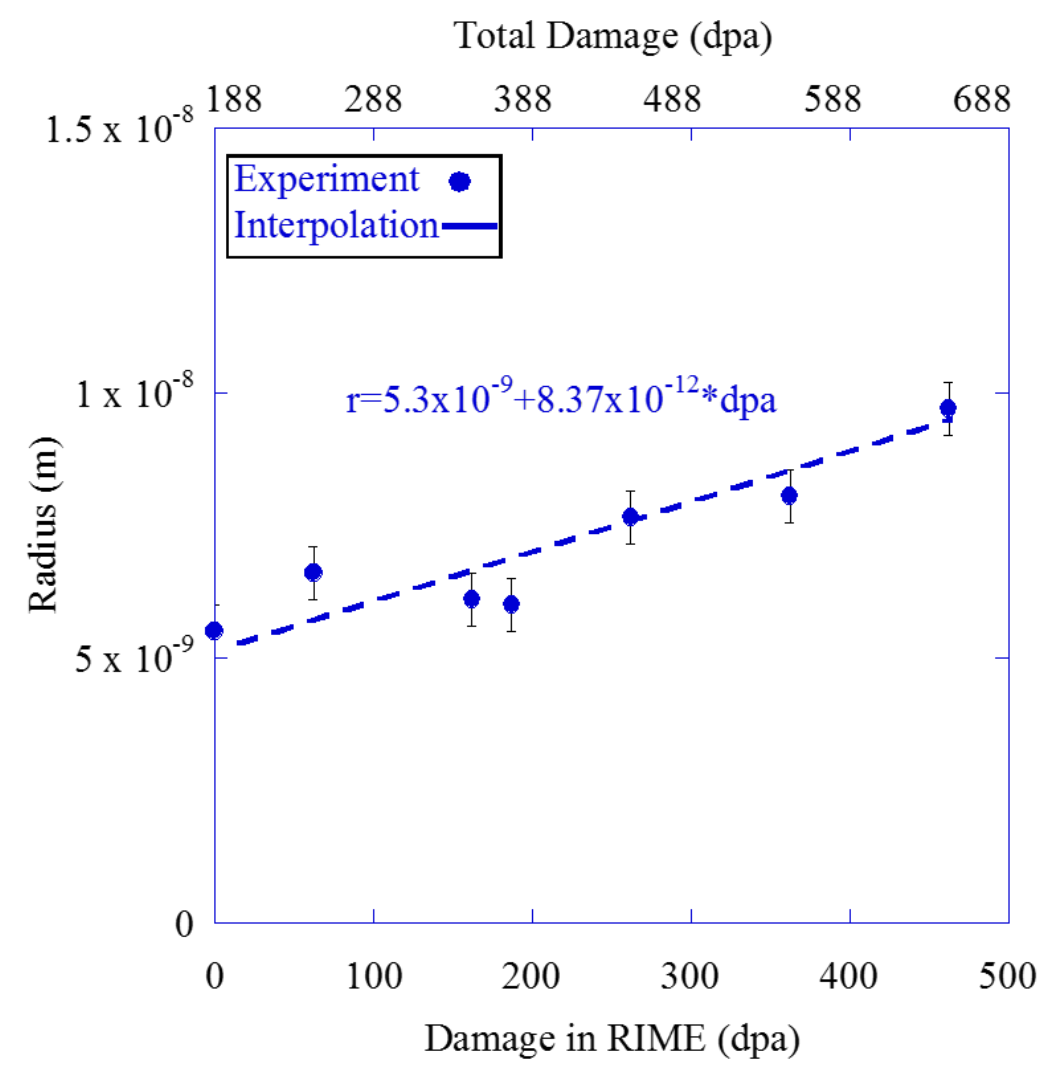

(a)
Total Damage (dpa)

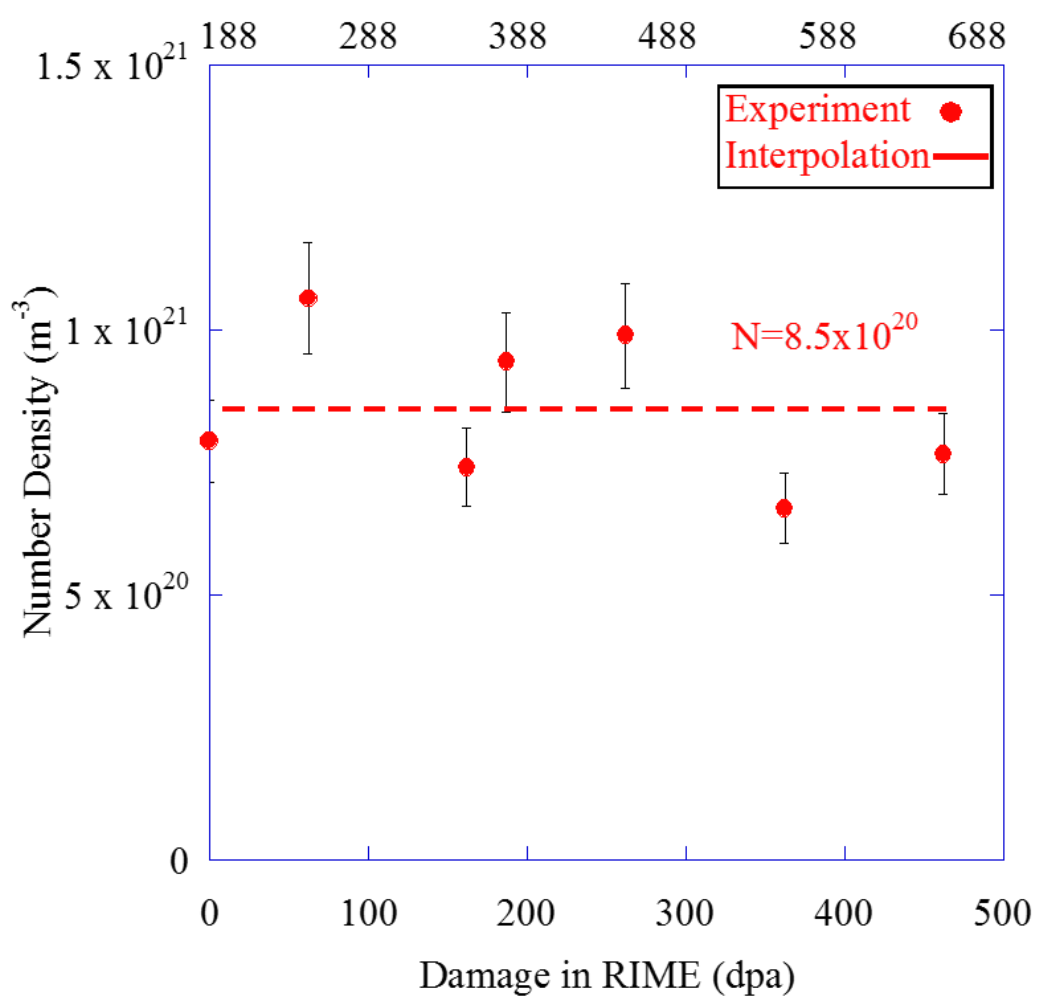

(b)

Figure 6.10: a) Radius and b) number density of G phase in HT9 irradiated with $5 \mathrm{MeV} \mathrm{Fe}{ }^{++}$from 188 to 650 dpa at $460^{\circ} \mathrm{C}$. Interpolation is from experimental data. 
Total Damage (dpa)

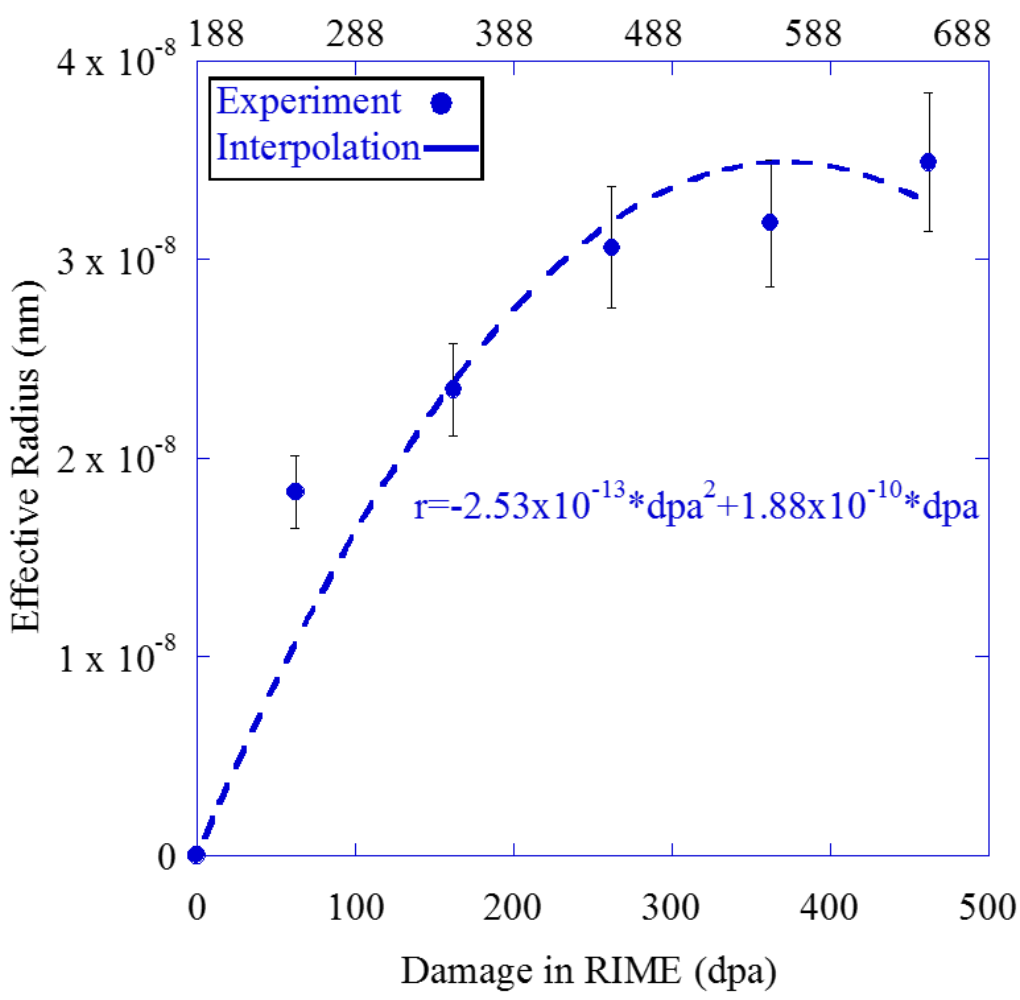

(a)
Total Damage (dpa)

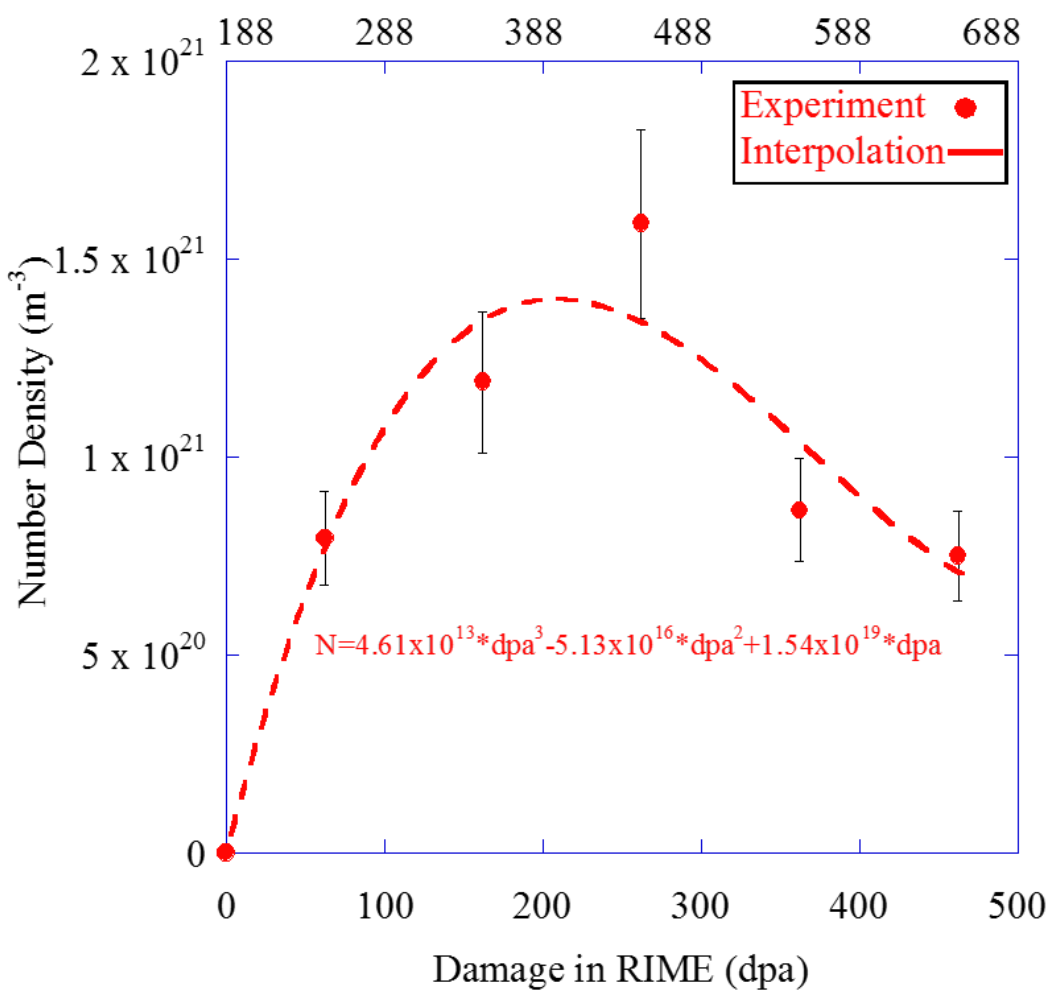

(b)

Figure 6.11: a) Effective radius and b) number density of $\mathrm{M}_{2} \mathrm{X}$-phase in HT9 irradiated with $5 \mathrm{MeV} \mathrm{Fe}^{++}$from 188 to $650 \mathrm{dpa}$ at $460^{\circ} \mathrm{C}$. Interpolation is from experimental data. 


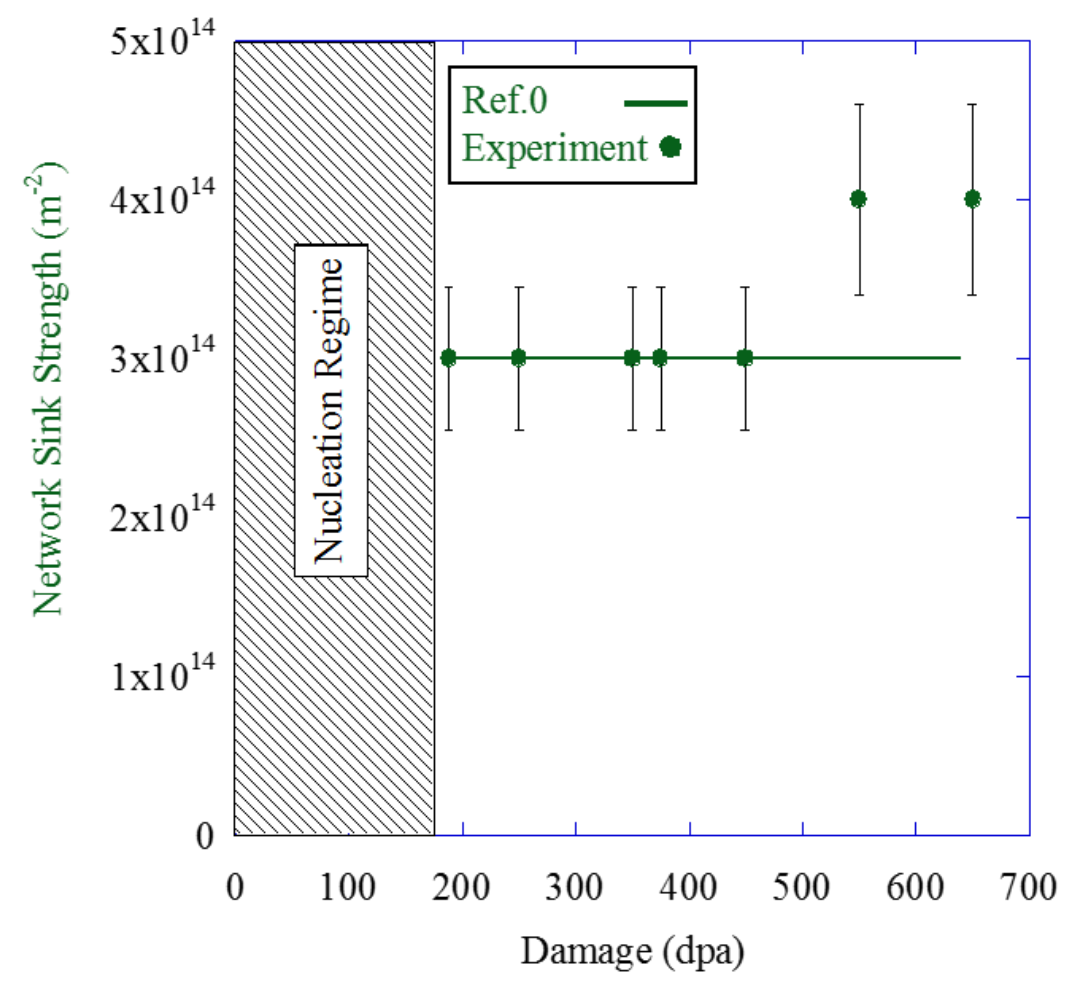

(a)

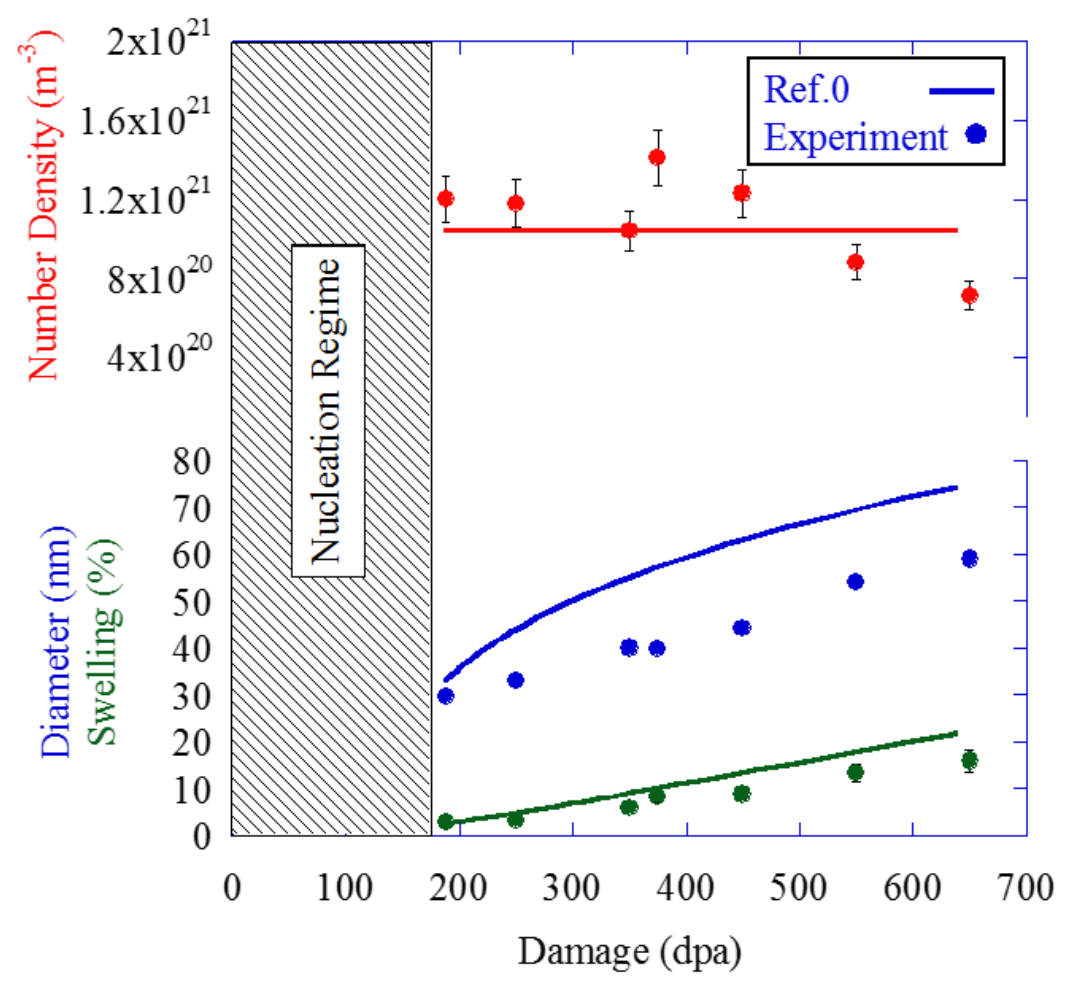

(b)

Figure 6.12: Comparison of Ref.0 treatment $\left(z_{i}^{\text {net }}=1.01\right)$ with experimental data in terms of a) network sink strength and $\left.b\right)$ void diameter, number density and swelling. 


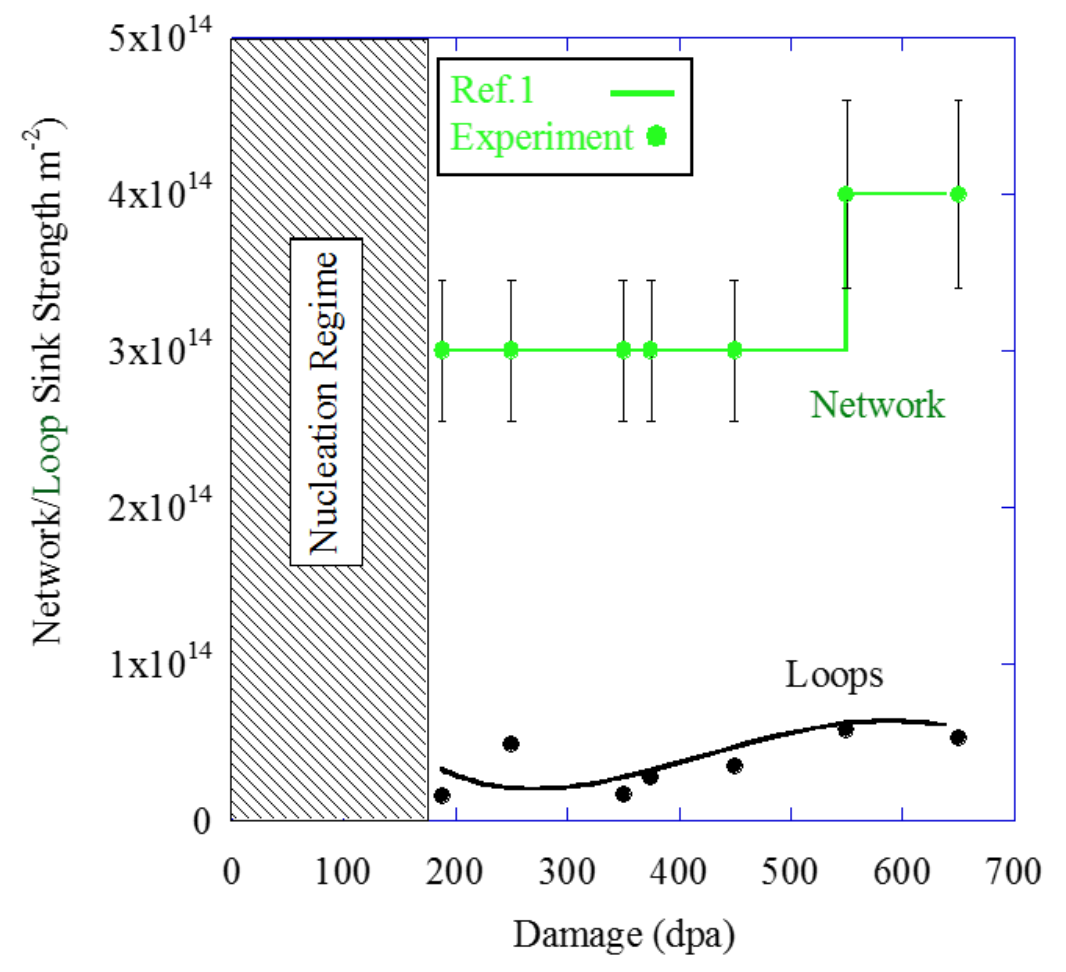

(a)

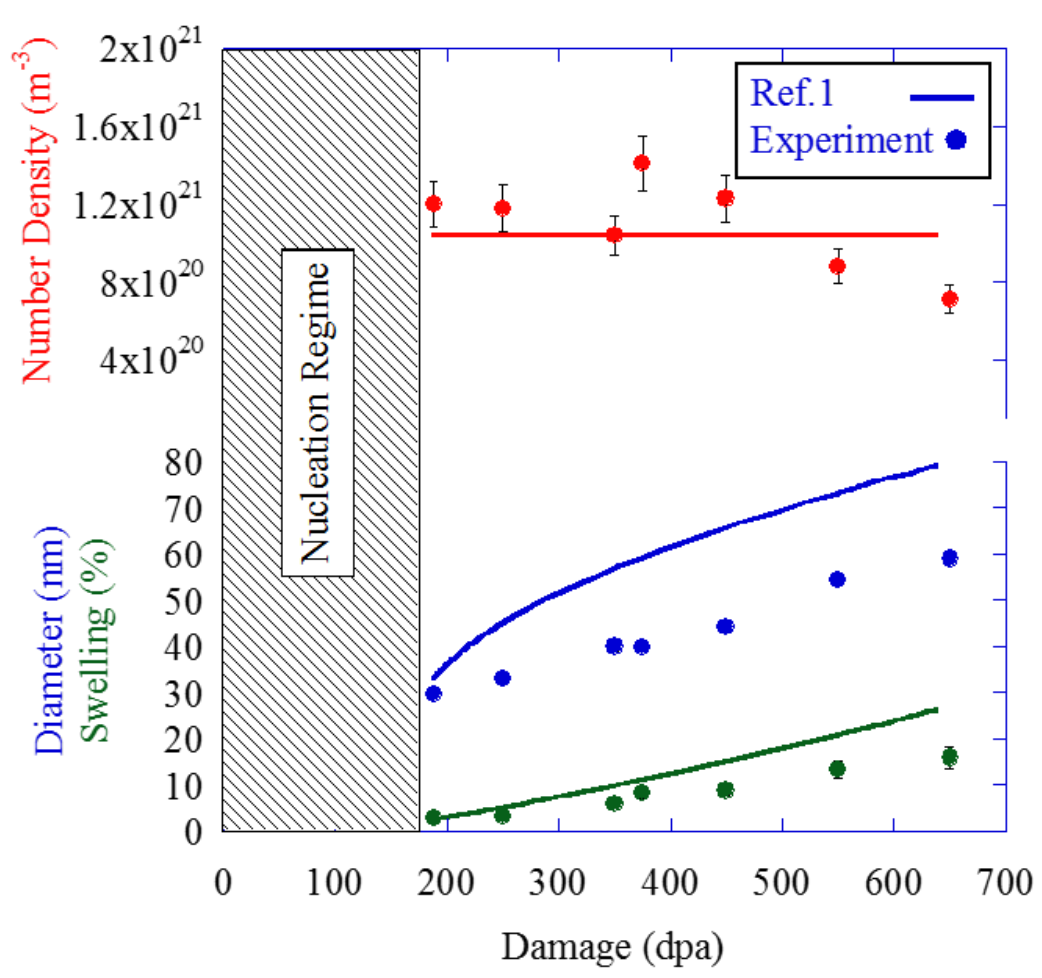

(b)

Figure 6.13: Comparison of Ref. 1 treatment $\left(z_{i}^{\text {loop }}=z_{i}^{\text {net }}=1.01\right)$ with experimental data in terms of a) network and loop sink strength and b) void diameter, number density and swelling. 


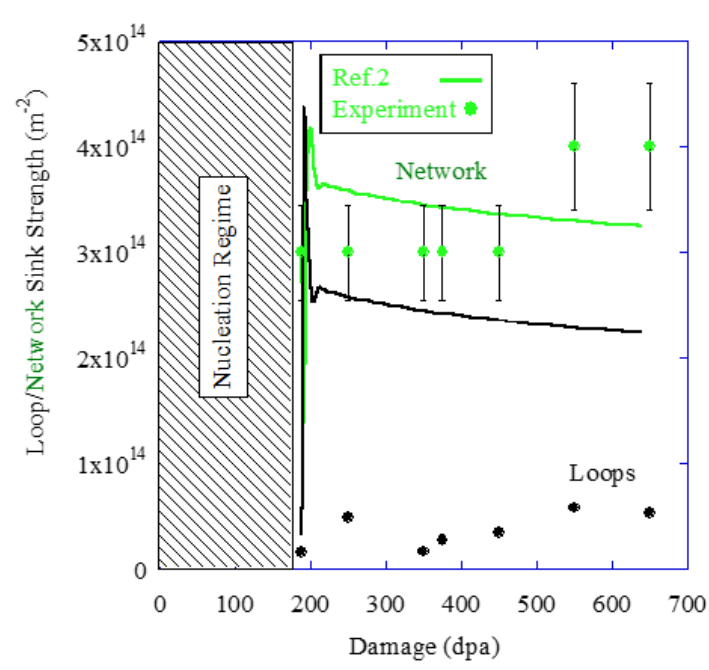

(a)

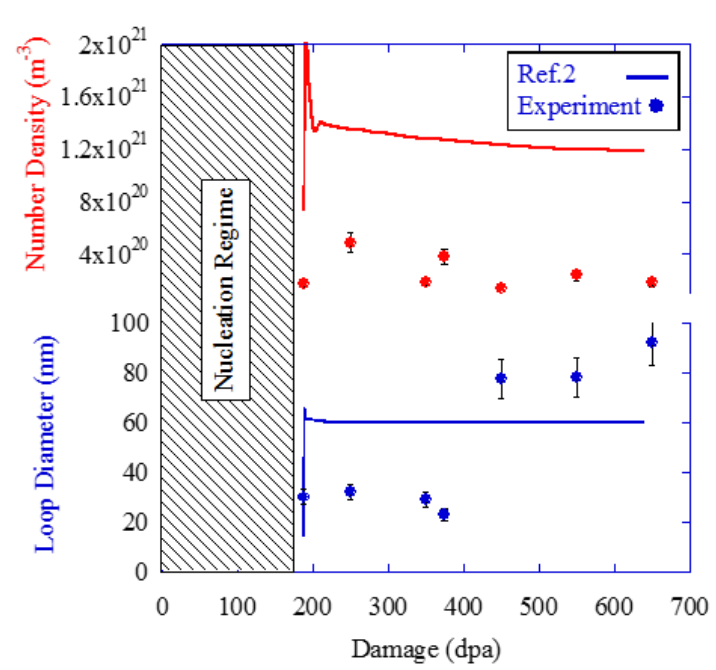

(b)

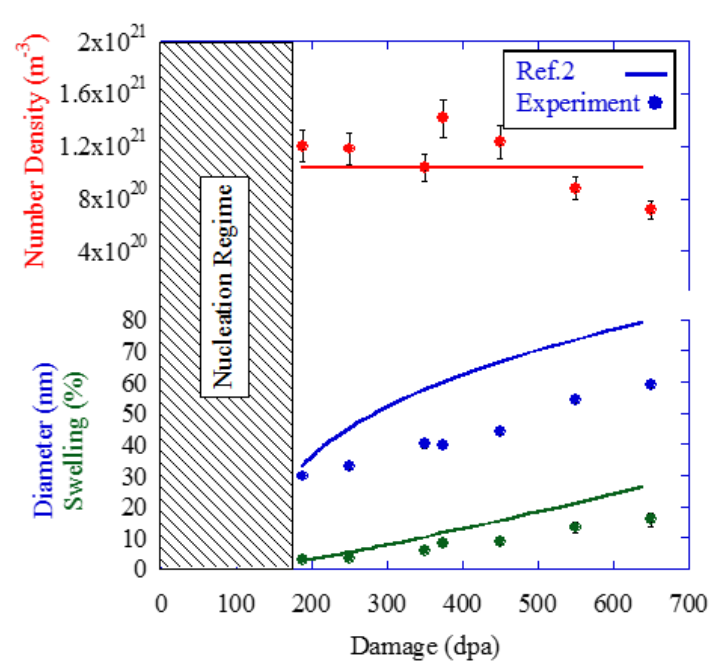

(c)

Figure 6.14: Comparison of Ref.2 treatment $\left(z_{i}^{\text {loop }}=z_{i}^{\text {net }}=1.01\right)$ with experimental data in terms of a) loop and network sink strength b) loop diameter and number density and c) void diameter, number density and swelling. 


\section{CHAPTER 7}

\section{DISCUSSION: CO-EVOLUTION OF THE IRRADIATED MICROSTRUCTURE}

The following analysis discusses the results from ion irradiation experiments in the high damage/void growth-dominated swelling regime within the context of the cases from the Radiation Induced Microstructure Evolution (RIME) cluster dynamics model to understand how dislocations, voids and precipitates co-evolve at very high damage levels beyond 188 dpa.

\subsection{Analysis Scope}

The RIME model itself and this analysis in particular include a number of inherent assumptions, which limit the understanding of the co-evolution of the microstructure. The first limitation of the RIME model is that the model utilizes a mean field approach, which means that it is unable to treat variations in the microstructure from grain to grain and instead presents mean or aggregate results from the overall microstructure. The microstructure data measured and reported in Chapter 5 is representative of an overall microstructure by examining a large number of grains in order to obtain average results. (For instance, at least 30-50 grains were examined for void data at a given damage level.) Thus, both RIME output and the reported measurements reflect the mean or aggregate microstructure features and do not capture local variations or heterogeneity that is common to FM alloys. 
Second, the code does not have the capability to model precipitate nucleation or growth; the experimentally determined evolution of the precipitates is provided as input to the code. The precipitate radius and density are parameterized by equations that describe their respective behaviors as a function of dpa. (Figure 6.10, Figure 6.11) The resulting sink strength is calculated in RIME and used to determine how defects are partitioned to the sinks according to the calculated sink strength, as well as how the defects at sinks are treated (as unbiased, biased or variable biased sinks or as recombination centers). However, the addition of defects to the sink does not alter the precipitate evolution, and they cannot be influenced by any of the other microstructure features in the model. Hence, precipitates can influence evolution of dislocations and voids, but not the reverse.

Third, the dislocation microstructure is treated as either experimentally input to the code (Ref.0 or Ref. 1) or is allowed to evolve dynamically by a model for dislocation loop and network growth provided in RIME (Ref.2). The experimentally input treatment can be described in two ways: either with network only or with network and loops as input. In one case, the dislocation network sink strength is set by the code and remains constant throughout the irradiation and the contribution of loops is neglected (Ref.0). In a second case, the loop radius and number density can be calculated from the experimental data and are included as an experimental input along with the network, thus prescribing the entire dislocation evolution (Ref.1). Therefore, Ref.1 allows the precisely observed dislocation loop and network behavior to be modeled. The third case, hereafter referred to as dynamic dislocation (DD) treatment, allows the dislocation loops and network to evolve according to models in the code $[18,100]$, with the microstructure at 188 dpa serving as input (Ref.2). Void evolution is modeled in the code and is able to respond to dislocation and precipitate evolution. 
Lastly, the microstructure evolution is only considered at damage levels from 188 to 650 dpa, where void growth dominates the swelling behavior, resulting in linear or near linear swelling. Starting with the already irradiated microstructure at a higher damage level avoids void nucleation effects reflecting void growth-dominated regime. Therefore, a sub routine "s01_preexist.f" was developed such that the network density, dislocation loop and void distributions, measured experimentally at $188 \mathrm{dpa}$ (Figure 6.7), could be used as the starting microstructure at time $t_{o}$. Thus, each model case starts at 188 dpa. Although cases are run from nominally " 0 dpa" in RIME, damage levels reported will reflect the total damage $\Phi_{T}$, which is consistent with the experimental damage reported.

Another way to understand the various relationships that can be explored is shown in Figure 7.1. Precipitates serve as the independent variable, voids as the dependent variable and dislocations can serve as either. Given these conditions and assumptions, the following relationships are explored in depth:

- effect of dislocations on voids,

- effect of precipitates on voids,

- effect of precipitates on dislocations and

- combined effect of dislocations and precipitates on voids.

The discussion is organized in the following way: 1) for each microstructure relationship, the experimental data will be summarized. 2) Each modification in RIME from the reference case will be compared to cases Ref.1 or Ref.2. 3) The resulting behavior will be compared back to the experimental observations to understand how the microstructure behavior influenced evolution of the other features being studied. 
The majority of this thesis has referred to voids and cavities interchangeably, with preference given to the "void" nomenclature. Given the high damage levels discussed, it is appropriate to describe all cavities as voids in this chapter, especially since there is no helium treatment included in the RIME cases.

\subsubsection{Definitions of Modifications to Reference Cases}

To aid in identifying specific cases, a system of flags for each microstructure treatment was created to denote modification from the reference cases. Inputs for each reference case were defined in Table 6.9. Table 7.1 presents a list of the flags and their meanings. Each flag is added to its respective reference case and multiple flags may be added to a single case. For instance, the inclusion of G phase to the experimentally input dislocation treatment is denoted "Ref.1.G." The inclusion of both $\mathrm{G}$ phase and $\mathrm{M}_{2} \mathrm{X}$ to the dynamic dislocation treatment is denoted "Ref.2.G.MV." Thus, each case's input can be quickly and easily identified.

\subsection{Effect of Dislocations on Voids}

Examining the effect of dislocations on voids in the absence of precipitates represents the simplest model system and the most fundamental microstructure interaction when considering void swelling, which is driven by the dislocation bias for interstitials. Identifying appropriate values for the dislocation bias for interstitials was not a straightforward task. To date, no experimental methodology has been developed to measure the dislocation bias for interstitials. Typically, there are two approaches to selecting an appropriate bias. There are a number of analytic solutions based upon a variety of methodologies, including, for example, the stationary drift-diffusion equation 
[137-139], atomistic interaction energy maps [48] or finite element analyses [137]. Second, and most commonly used in the literature, rate theory models tend to treat bias as an input parameter where it is estimated and used as a tuning parameter; the model results are then benchmarked to experimental results $[18,27,41,114]$. This approach is similar to the bias sensitivity study presented in Figure 6.2.

To further complicate bias selection, bias is expected to have a weak dependence on temperature, a stronger dependence on dislocation density and the strongest dependence on crystal structure [48]. In fact, it has been theorized that a lower dislocation bias is responsible for the swelling resistance of bcc steels versus fcc steels [41,140]. As such, results from the literature in the bcc iron system are given preference in this analysis.

Table 7.2 gives the ranges for each of the dislocation bias determination approaches. The largest variation in calculated bias is from analytic solutions which vary from 1.01-1.25. The wide range of bias values is due to bias calculated for different types of dislocation loops along different planes as was demonstrated by [48]. Chang et al. found bias for interstitials that ranged from negligible (nearly 1.0) for screw type dislocations up to 1.05 for edge $<111>$ dislocations. Finally, rate theory biases are lower than all first principles calculations and nearly all analytic solutions, in both ferritic and austenitic steels $[8,41-43,114,115]$. For this application, and based upon the experimental observation of predominantly <100> loops (Figure 5.45) and Chang's results, a bias input range between 1.01-1.04 was deemed to be reasonable.

Furthermore, a parametric analysis of bias (Figure 6.2) revealed that swelling was highly sensitive to the bias. With this consideration, a default bias of 1.01 was chosen because of its 
consistency with the literature, including the most relevant analytic solution from Chang et al. [48] as well as preliminary comparisons of the resulting swelling to observed experimental data (Figure $6.2)$.

\subsubsection{Summary of Dislocation and Void Evolution}

All experimental data for this analysis was performed at $460^{\circ} \mathrm{C}$ from 188 to $650 \mathrm{dpa}$ and was previously presented in Chapter 5. To summarize, both the loop (Figure 5.47) and void number density (Figure 5.17) were nearly constant from 188 to 650 dpa. The loop number density ( 2 to $\left.3 \times 10^{20} \mathrm{~m}^{-3}\right)$ was consistently lower than that of the voids $\left(\sim 10 \times 10^{20} \mathrm{~m}^{-3}\right)$. The diameter of the voids grew linearly $(\sim 0.06 \mathrm{~nm} / \mathrm{dpa}$ shown in Figure 5.16) as a function of damage whereas the loop diameter was nearly constant at approximately $25 \mathrm{~nm}$ from 188 to $375 \mathrm{dpa}$ and then underwent a period of rapid growth, tripling in size $(\sim 90 \mathrm{~nm})$ by the final damage level of 650 dpa (Figure 5.46). There appeared to be no direct correlation between loop and void diameter behavior. Loop sink strength was an order of magnitude $\left(10^{13} \mathrm{~m}^{-2}\right)$ lower than that for either the network or voids $\left(10^{14} \mathrm{~m}^{-2}\right)$, suggesting that loops might not be the controlling feature in terms of void behavior due to the low sink strength. The network and total dislocation sink strength (Figure 5.49) were very similar to the void sink strength, varying between 3 to $4 \times 10^{14} \mathrm{~m}^{-2}$ from 188 to $650 \mathrm{dpa}$. All three sink strengths exhibited very slow increases over the void growth-dominated swelling regime, indicating a very stable system in pseudo-equilibrium.

Before results for the simple system of dislocations and voids are analyzed, the pre-existing microstructure, which provides the overall swelling environment prior to irradiation, must be considered as more fundamental. The main features of the overall unirradiated microstructure, 
discussed in Section 5.1, are the preexisting dislocation network from processing, the grain boundaries and the $\mathrm{M}_{23} \mathrm{C}_{6}$ carbides, with corresponding sink strengths of $2 \times 10^{14}, 8 \times 10^{13}$ and $5 \times 10^{12} \mathrm{~m}^{-2}$, respectively. Clearly, the dislocation network is the dominant feature and will be analyzed in more detail in this section. The other feature to be considered from an overall microstructure view of swelling rate is the grain boundaries. (Carbides are not considered due to low sink strength.) Although changing the grain size and as a consequence, the grain sink strength, was shown to have a measurable impact on void behavior in the significance study in Figure 6.3, a change of grain size of $20 \%$ led to swelling decrease of less than $5 \%$ relative that from Ref.2. Furthermore, grain size is stable throughout the irradiation which precludes it from having an effect on evolution and the focus of this work is on how the microstructure co-evolves with damage level. For these reasons, grain size is not explored further to resolve overestimation of void swelling.

\subsubsection{Case Ref.0: Experimentally input network dislocations}

Analysis of model cases will begin with the most fundamental system of dislocations and voids in the absence of other features. The simplest treatment in RIME is case Ref.0, which includes only the network at a constant density of $3 \times 10^{14} \mathrm{~m}^{-2}$, which was the measured value at 188 dpa. The next most complex case, and incidentally, more experimentally accurate, incorporates the increase in network sink strength from 3 to $4 \times 10^{14} \mathrm{~m}^{-2}$ at $550 \mathrm{dpa}$. This increase was shown experimentally in Figure 5.44 and the case is referred to as "Ref.0.N."

The resulting comparison of Ref.0 and Ref.0.N is shown in Figure 7.2. In Figure 7.2a, it is shown that a modest increase in network sink strength resulted in a small increase in void sink strength. The resulting increase in void sink strength was due to an increase in void diameter. By 
$650 \mathrm{dpa}$, the final diameter was 75.1 versus $74.3 \mathrm{~nm}$ in the Ref.0.N and Ref.0 case, respectively. The resulting increase in void sink strength is also reflected in Figure 7.2b, where the void swelling rate slightly increased with respect to the reference case beyond $550 \mathrm{dpa}$.

This comparison suggests two conclusions. First, the simplest case, Ref.0, results in a reasonable approximation for swelling, despite considering only the network behavior. To fully model the microstructure, loops must be taken into account so that the model reflects the most accurate representation of the dislocation network behavior. Second, increasing the network sink strength by $33 \%$ resulted in an observable inflection point in the swelling curve at 550 dpa, demonstrating that void growth rates are very responsive to small changes in biased sink strengths.

\subsubsection{Case Ref.1: Experimentally input network and loops}

A further increase in both complexity and accuracy in representation of the experimental behavior in the dislocation treatment is case Ref.1. Ref.1 is simply Ref.0.N with the addition of experimentally input loop evolution, previously described in Section 6.2.2. Figure 7.3a compares the network, loop and total dislocation sink strength for Ref.0 and Ref.1. The red dashed line matched the experimentally input initial network density of $3 \times 10^{14} \mathrm{~m}^{-2}$ and the increase to $4 \times 10^{14}$ $\mathrm{m}^{-2}$ at 550 dpa (as in Ref.0.N). Loops are shown with blue dashed lines. The total dislocation network and loop sink strength is shown in green, and increases as a function of dpa. Overall, Ref.1 represents the experimentally observed dislocation loop and network behavior from Figure 5.49 .

The resulting swelling is compared in Figure 7.3b. With the addition of the network and loop sink strength, swelling increased relative to the constant network treatment (Ref.0) by about 
$15 \%$ (from 23 to $26 \%$ ) by 650 dpa. The addition of loops appreciably alters void behavior by promoting void growth, indicating that a biased sink with a sink strength an order of magnitude lower than for voids can still alter void behavior.

The simpler Ref.0 case was shown to be a reasonable approximation for void swelling behavior. However, since the experimentally input network and loop treatment in case Ref.1 is most representative of the experimental data, it will be used as the primary reference case for comparison with more complex cases and microstructure treatments later in this chapter.

\subsubsection{Case Ref.2: Dynamic Dislocation Treatment}

The final dislocation treatment is the case of dynamic dislocation (DD) evolution (Ref.2). The DD case is compared to Ref.1 in Figure 7.4. Figure 7.4a presents the sink strength behavior as a function of dpa from 188 to $650 \mathrm{dpa}$. Network sink strength (in red) was similar in magnitude between Ref.1 and Ref.2; however, the loop sink strength (in green) was higher in the DD

treatment by approximately an order of magnitude $\left(10^{13}\right.$ to $\left.10^{14} \mathrm{~m}^{-2}\right)$. The higher sink strength resulted in higher total dislocation sink strength in Ref.2 relative to Ref.1. Nevertheless, the void sink strength was quite similar between cases, which resulted in almost identical swelling behavior shown in Figure 7.4b.

The discrepancy between dislocation sink strengths is due to the increased defect flow of both vacancies and interstitials to loops in spite of the biased nature of the sinks. Even though there was higher biased sink strength in Ref.2, there was also a higher sink strength able to absorb vacancies as well as interstitials, even though interstitials are preferred at dislocations due to the $1 \%$ bias. The loops and network then respond to the increase in absorbed vacancies and 
interstitials, which decreases the total biased sink strength. The larger sink strength of loops in Ref.2, relative to Ref.1, also limited the vacancies available to diffuse to voids, but the overall flux of vacancies relative to interstitials at the voids was very similar for both cases. Figure 7.5 demonstrates this by plotting the net vacancies absorbed at voids as well as the total dislocation sink strength for Ref.1 and Ref.2. Figure 7.5 also shows that the total biased sink strength, i.e. loops and network in Ref.2 is greater than that in Ref.1 by approximately $75 \%$, but the net flux of vacancies absorbed at voids (measured as a percentage) is similar between Ref.1 and Ref.2 (0.4\% versus $0.5 \%$ ) prior to $450 \mathrm{dpa}$ and virtually indistinguishable after $450 \mathrm{dpa}$. Net flux of vacancies drives void growth, which in turn determines the swelling value or void sink strength, which was shown to be nearly identical in Figure 7.4. In other words, a 75\% increase in biased sink strength resulted in a $25 \%$ increase in net flux. The differing behavior between interstitial sink strength and net flux at voids demonstrates the subtle interaction between bias, sink strength of loops and sink strength of voids in the DD case. When the network or loops have prescribed increases in bias, swelling always increases, but when the system is allowed to respond, the higher sink strength does not always result in higher swelling, as loops and network respond.

Another way to interpret this data is that swelling response may be insensitive to dislocation microstructure, for certain ranges of sink strength. A range of initial dislocation densities input into the DD treatment in Figure 7.6a shows the insensitivity of the DD treatment to initial conditions. All three swelling curves were nearly identical regardless of whether $3 \times 10^{13} \mathrm{~m}^{-}$ ${ }^{2}, 3 \times 10^{14} \mathrm{~m}^{-2}$ or $3 \times 10^{15} \mathrm{~m}^{-2}$ was input as the dislocation microstructure responds to either grow or shrink the network to those observed in Ref.2, with a total dislocation network and loop density of $\sim 6-7 \times 10^{14} \mathrm{~m}^{-2}$. In contrast, when a similar range of dislocation network density $\left(3 \times 10^{13} \mathrm{~m}^{-2}\right.$ to $3 \times 10^{15} \mathrm{~m}^{-2}$ ) was into the experimentally input case, swelling is much more sensitive to the 
combined network and loop sink strength. Loop sink strength was kept at experimental levels. Below $8 \times 10^{13} \mathrm{~m}^{-2}$, there was not a large enough biased sink strength to cause swelling. As dislocation density increased up to $3 \times 14 \mathrm{~m}^{-2}$, the swelling increased in response. However, the swelling behavior was relatively insensitive to network density from $3 \times 10^{14} \mathrm{~m}^{-2}$ to $1 \times 10^{15} \mathrm{~m}^{-2}$. By $3 \times 10^{15} \mathrm{~m}^{-15}$, there was a decrease in swelling due to the dominance of the dislocation network. This is further evidence that it is reasonable that there was no change in swelling between experimentally input (Ref.1) and DD (Ref.2) treatments despite the increased network sink strength in Ref.2.

The similarity of the resulting swelling (Figure 7.4b) between the DD treatment (Ref.2) and the experimentally input (Ref.1) is significant for several reasons. First, the consistency of these two cases lends confidence to using either case. Second, DD is also necessary as a reference case in which the dislocations are allowed to respond to changes in microstructure, such as with the formation of precipitates, as Ref.1 does not have the capability.

\subsubsection{Comparison of Model to Experiment}

Ultimately, the goal is to achieve void and dislocation evolution in RIME similar to that which was observed experimentally. Rationalizing discrepancies between the observed dislocation and void behavior can lead to insights regarding interactions between various microstructure features. Figure 7.7 presents a comparison of Ref.1 and experimental data. (Ref.0 is not compared to experimental results here, as it does not include loop behavior). Figure 7.7 a demonstrates the accuracy of the experimentally input dislocation treatment, which acts as the independent variable

is this analysis. Voids are the only dependent variable. The resulting void diameter, number density 
and swelling resulting from this dislocation treatment are presented in Figure 7.7b. (Results from Ref.1 were previously compared to Ref.0 in Figure 7.3 and Ref.2 in Figure 7.4.) The void number density from RIME matched the experiment reasonably well, but the void growth rate and diameter were much higher, resulting in swelling that was overestimated by $62 \%$ (26\% swelling in model versus $16 \%$ swelling in experiment) by $650 \mathrm{dpa}$. This result suggests that the model is too simplistic in its current form. Rather, there must be another microstructure feature or mechanism diverting excess vacancy flow from the voids [34,82]. Thus, examining dislocation and void behavior may be too simplistic.

An alternative explanation is that the bias used in this case was too high. Bias directly impacts the number of vacancies free to migrate and then grow the void. In all reference cases considered to this point, the bias has been keep at $1 \%$ for loops and networks, regardless of dislocation treatment. Since swelling was overestimated by $62 \%$ in Figure 7.7 , a decreased bias case (Ref.1.1.007: $z_{i}^{l p}=z_{i}^{\text {net }}=1.007$ ) was run with the experimentally input network and loops to determine whether decreasing the bias would result in a better match of the void microstructure. A number of lower bias cases were run for comparison, but only $0.7 \%$ is presented here. (A bias of $0.7 \%$ was presented as it provided the best match of the swelling microstructure with the experimentally input dislocation treatment, in the absence of any other features.) Since the dislocation treatment was experimentally input, the dislocation behavior was identical to that in Figure 7.7a and so was not included again. The swelling resulting from bias factors of 1.01 and 1.007 is shown in Figure 7.8. With lower bias $(0.7 \%)$, the void diameter and swelling were much more consistent with experimentally observed void behavior. In particular, the diameter growth rate from 188 to 650 dpa was $0.067 \mathrm{~nm} /$ dpa relative to $0.082 \mathrm{~nm} / \mathrm{dpa}$ in Ref.1, whereas the number density did not change with bias. The excellent matching with lower bias suggests one of two 
things; first, the actual bias of the dislocations was lower than expected from literature. Previously mentioned, measuring biases is extremely difficult, but most literature suggests that biases are somewhere between 1-5\% for ferritic-martensitic alloys; no references were found with bias below $1 \%[41,48,114,115,137]$.

Assuming dislocation bias in the absence of any other effects remains at $1 \%$, an alternative explanation is that there was another feature or mechanism that lowers the effective bias below the expected levels. One similar mechanism suggested in the literature by Little [121,122,141] was that interstitial solutes such as $\mathrm{C}$ and $\mathrm{N}$ may form Cottrell atmospheres around dislocation core. Cottrell atmospheres can relax the dislocation strain field, which thus decreased the dislocations' effective bias. In extreme cases, it was also hypothesized that eventually the bias would be reversed as interstitials were trapped at the dislocations [122,142] creating an artificial preference for vacancies. There is no mechanism in RIME to account for such a phenomenon and there is no experimental technique to confirm this interaction mechanism directly.

It was previously observed that void results from the experimentally input reference and the DD reference were very similar (Figure 7.4). For completeness, the DD reference (Ref.2) is also compared to experimental data in Figure 7.9. (Results from Ref.2 were also compared to Ref.1 in Figure 7.4) Figure 7.9a shows the resulting dislocation loop and network behavior. The dislocation network sink strength was quite reasonable (remains between 3 to $4 \times 10^{14} \mathrm{~m}^{-2}$ ). The loop sink strength is overestimated, which was due to the higher than expected loop number density from RIME $\left(\sim 1 \times 10^{21} \mathrm{~m}^{-3}\right.$ Figure $\left.7.9 \mathrm{~b}\right)$ compared to experiment $\left(\sim 2-3 \times 10^{20} \mathrm{~m}^{-3}\right)$. The loop diameter reflects approximately the average of what was experimentally observed as a function of dpa either $\sim 25$ or $80 \mathrm{~nm}$ at low and high damage levels, respectively. Since the diameter is a direct result of 
RIME inputs, this was deemed a reasonable assumption, but the consequences of this will be discussed further in Section 7.4.2.

The void results are plotted in Figure 7.9c. Similar to Ref.1 (Figure 7.7b), the number density was accurately captured but the diameter/diameter growth rate was too high $(0.080 \mathrm{~nm} / \mathrm{dpa}$, which was consistent with Ref.1). Again, the resulting void swelling is higher than expected.

Table 7.3 compares the various treatments presented in Section 7.2 and how well they match observed experimental behavior. Both experimentally input Ref.1 and DD treatment (Ref.2) results in overestimation of void swelling in general. The high swelling was mitigated by lowering loop and network bias to $0.7 \%$ (Ref.1.1.007) to yield more realistic void results. However, $0.7 \%$ is below most suggested biases even for low swelling FM alloys.

The major conclusion of this analysis of dislocations and voids only is that there is a systemic overestimation of final swelling value at $650 \mathrm{dpa}$ in the model relative to experiment by $63 \%$ (26\% swelling was observed in model relative to $16 \%$ in the experiment), in both Ref. 1 and Ref.2. Thus, it follows that there is some other mechanism inhibiting the effective bias of the system or acting as a sink to divert defect flow away from the voids. Although lowering the effective bias is an attractive option in matching the void swelling behavior, the bias required is below any reported in the literature and is therefore not convincing as a possible mechanism. Thus, the effect of other sinks is likely the cause. Since the other major features in the microstructure that are continuing to evolve at high damage are precipitates; precipitates will be analyzed next to determine if they provide a sufficiently effective alternate sink for defects. 


\subsection{Effect of Precipitates on Voids}

For the purpose of this analysis, $G$ phase and $M_{2} X$ are treated separately. $G$ phase and $M_{2} X$ vary in terms of both their sink character (incoherent and coherent, respectively) and their evolution with dpa. First, G phase and its effect on voids will be addressed.

\subsection{1 $\underline{\mathrm{G} \text { Phase }}$}

$\mathrm{G}$ phase is analyzed first as the behavior of $\mathrm{G}$ phase in the irradiated microstructure is better understood as a result of being a commonly observed irradiation-induced precipitate [7,9,10,31,51,52]. In addition, incoherency [88,143] and that effect on precipitate-defect interactions [20] is better understood for $\mathrm{G}$ phase than for coherent $\mathrm{M}_{2} \mathrm{X}$.

G phase was confirmed to be an incoherent precipitate from HRTEM analysis presented in Section 5.3.1 in Figure 5.29, which is consistent with what is reported in the literature $[88,143,144]$. The evolution of $\mathrm{G}$ phase diameter and number density were presented in Figure 5.26 , but a brief summary and comparison to voids is included here. The diameter increased at a nominally linear rate over this dpa interval, similar to voids, although the linear rate was nearly an order of magnitude lower than the void growth rate $(\sim 0.06 \mathrm{~nm} / \mathrm{dpa}$ versus $\sim 0.01 \mathrm{~nm} / \mathrm{dpa}$ for $\mathrm{G}$ phase and voids, respectively). G phase and voids had roughly constant number density from 188 to 650 dpa (Figure 5.9 and Figure 5.30) leading to an approximately linear volume fraction increase, which is reflected in Figure 5.26. Thus, G phase volume fraction tracked void swelling albeit at an order of magnitude lower, with a corresponding sink strength an order of magnitude lower (4 to $9 \times 10^{13} \mathrm{~m}^{-2}$ relative to 3 to $4 \times 10^{14} \mathrm{~m}^{-2}$ for voids). The correlation of the diameter and number density 
behavior are additional supporting evidence that the interaction of both of these sinks with defects is identical i.e. they are both incoherent, unbiased sinks, which have no preference for either type of defects. For these reasons, $\mathrm{G}$ phase is treated as an unbiased sink in RIME.

The effect of $\mathrm{G}$ phase on void sink strength and swelling is compared to Ref.1 in Figure 7.10. With the addition of $\mathrm{G}$ phase, void sink strength decreased due to decreased void diameter. (Figure 7.10a) In the presence of $G$ phase, void swelling reflected the lower void sink strength (Figure 7.10b). Although overall swelling/swelling rate decreased visibly with the addition of $\mathrm{G}$ phase, the overall effect was minimal. The final swelling at 650 dpa was $24.7 \%$ relative to $26.1 \%$ without $\mathrm{G}$ phase, which is a decrease of about $5 \%$, which is not judged to be statistically significant, especially since the swelling was overestimated by $55 \%$ relative to experiment. The lack of effect is likely due to a combination of both the low sink strength and the fact that $\mathrm{G}$ phase is an unbiased sink unlike biased loops, which were still effective at a lower sink strength.

Figure 7.11 presents a comparison of the results of Ref.1.G with the experimental results. (The results from case Ref.1.G were also included in Figure 7.10.) Figure 7.11a shows the sink strength of the loops, network and G phase as a function of dpa. All sink strengths match experimental data as they are experimental inputs. Figure $7.11 \mathrm{~b}$ presents the resulting void diameter, number density and swelling with the addition of the $G$ phase treatment. The void number density is unaffected, but the diameter growth rate and swelling remained overpredicted relative to that observed in experiment. The combination of Figure 7.10b and Figure 7.11b show that although $G$ phase decreases the final swelling value by $\sim 5 \%$, it is not a strong enough effect to resolve the overestimation of void swelling consistently observed in Ref.1. 
Thus, G phase had a minimal effect in resolving the overestimation of swelling due to its low sink density. It was found to decrease overall swelling by $5 \%$ of the final swelling from $26.1 \%$ to $24.7 \%$, which was insufficient to serve as an alternate sink in a meaningful capacity. Therefore, the effect of $\mathrm{M}_{2} \mathrm{X}$ is now considered as a possible alternate sink to resolve the overestimation of void swelling.

\subsection{2 $\underline{\mathrm{M}_{2} \mathrm{X}}$}

The formation and evolution of $\mathrm{M}_{2} \mathrm{X}$ in this material is significant as irradiation-induced $\mathrm{M}_{2} \mathrm{X}$ has been observed in only a few studies to date $[38,53,90,145]$. Furthermore, the interaction of coherent sinks or interfaces with irradiation induced defects is much less well understood than with incoherent sinks.

The evolution of $\mathrm{M}_{2} \mathrm{X}$ with increasing dpa was previously presented in Figure 5.41 but is compared directly to void evolution in Figure 7.12. Voids demonstrated nearly linear growth from 188 to 650 dpa whereas $M_{2} X$ grew rapidly up to 450 dpa then saturated. $M_{2} X$ number density peaks at $450 \mathrm{dpa}$, then drops leading to a corresponding decrease in volume fraction. (Figure 7.12) The drop in number density has been attributed to loss of coherency leading to destabilization of the $\mathrm{M}_{2} \mathrm{X}$, which has been observed in the literature [38,52]. From 188 to $650 \mathrm{dpa}$, swelling rate appeared to be independent of the formation and growth of $\mathrm{M}_{2} \mathrm{X}$, which is consistent with some reports in the literature [126,127], despite the large sink strength $\left(10^{14} \mathrm{~m}^{-2}\right)$, which was the same order of magnitude as both void and dislocation network. 
$\mathrm{M}_{2} \mathrm{X}$ was verified to be a semi-coherent sink up through $650 \mathrm{dpa}$, using HRTEM analysis presented in Section 5.3.2 (Figure 5.39), consistent with recent results from Wang et al. in a Fe12Cr HT9 analogue [90] and from Borodin et al. in a Fe-13Cr FM alloy [38]. There are several competing theories regarding the behavior of semi-coherent or coherent sinks $[20,122,146]$ in the microstructure. Having established the semi-coherency of $\mathrm{M}_{2} \mathrm{X}$, there are two possible precipitate treatments that are appropriate: as a variable biased sink or as a recombination center. The formalism for each of these treatments was compared in Section 6.2.4, but each will be summarized here.

The first treatment is that of a variable biased sink (MV). The bias for either vacancies $\left(Y_{v}\right)$ or interstitials $\left(Y_{i}\right)$ changes as a function of damage based upon $d_{i} c_{i}$ and $d_{v} c_{v}$ (Equation 6.39) and $Y_{V}$ is calculated from Equation 6.40 using a first order approximation of recombination at the sink $(\delta f)$ from Equation 6.41. $Y_{i}, Y_{v}$ evolve as a function of dpa unlike the loop bias for interstitials $\left(z_{i}^{L}\right)$. Thus, the sink strength for either vacancies or interstitials adjusts as a function of damage level; the $\mathrm{M}_{2} \mathrm{X}$ sink strength has an affinity for whichever defect is dominant at any given time so that sink strength for either vacancies or interstitials is responsive to the overall defect population at any given time. This formalism was developed from theoretical work performed by Brailsford and Bullough [127,146].

A more rigorous treatment of $\mathrm{M}_{2} \mathrm{X}$ treats precipitate surfaces as partially or fully efficient recombination centers $(\mathrm{MR})$ that form at the precipitate-matrix interface due to the strain at the interface, which traps vacancies that are then annihilate by the more mobile interstitials. $\mathrm{M}_{2} \mathrm{X}$ has a slightly smaller lattice parameter than the matrix ( 0.279 versus $0.286 \mathrm{~nm}$, respectively). This puts the matrix near the interface in a state of compressive stress, making a vacancy trap. 
Measurement of the strain energy in the ferrite due to $\mathrm{M}_{2} \mathrm{X}$ ranges from 0.06 to $0.16 \mathrm{eV}$ from [147,148]. Alternatively, the misfit strain energy, calculated from the strain and lattice

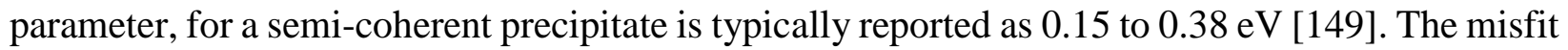
strain energy for a semi-coherent precipitate represents an upper bound on vacancy-trap emission energy for a coherent or semi-coherent precipitate. With these ranges providing context, a comparison of swelling with increasing emission energy for vacancy-trap complex $\left(E_{b}^{v, t r}\right)$ is shown in Figure 7.13. With increasing trap binding energy, the swelling decreased as expected as the drive to emit vacancies decreases. Based primarily upon the results from the literature and also considering the results of Figure 7.13, a trap energy of $E_{b}^{v, t r}=0.1 \mathrm{eV}$ was chosen as default energy for comparison to the variably biased treatment. Since the range reported in the literature of $\mathrm{M}_{2} \mathrm{X}$ in ferrite is 0.06 to $0.16 \mathrm{eV}$ and the upper bound imposed on coherent precipitates of the strain energy of a semi-coherent precipitate is from 0.15 to $0.38 \mathrm{eV}, 0.1 \mathrm{eV}$ is reasonable.

Using the chosen trap energy of $E_{b}^{v, t r}=0.1 \mathrm{eV}$, the recombination center treatment (Ref.1.MR) and variable biased treatment (Ref.1.MV) are compared in Figure 7.14 with an explicit dislocation network and loop treatment. The swelling behavior is nearly identical, indicating the reasonability of using either sink treatments, which was not unexpected as they have very similar underlying mechanisms with a recombination component. The Brailsford formalism that was incorporated into the MV treatment is based upon a combination of the balance between $d_{i} c_{i}$ and $d_{v} c_{v}$, and the term $f_{v}$, is a first order approximation for recombination expected at variable biased sink. Furthermore, both corrections are based upon the surface area and sink strength. So, the interaction mode is perhaps less important than the high sink strength of $\mathrm{M}_{2} \mathrm{X}$. Thus, the similarity of results lends confidence to 1) the chosen trap energy and 2) both precipitate treatments. 
Since the recombination center and variable biased treatments are so similar for the $E_{b}^{v, t r}$ considered, Ref.1.MV treatment was chosen as the default $\mathrm{M}_{2} \mathrm{X}$ precipitate treatment for continued analysis and is compared to the experimental results in Figure 7.15. (The case presented here was the same as that shown in Figure 7.14.) The sink strength for $M_{2} X$, loops and network is plotted in Figure $7.15 \mathrm{a}$ and the resulting void diameter, number density and swelling is plotted in Figure 7.15b. The void number density remains reasonable $\left(10^{21} \mathrm{~m}^{-3}\right)$ and the growth rate and swelling are still overpredicted with respect to swelling. However; compared to the Ref.1 case, the void behavior is trending in the correct direction with the addition of $\mathrm{M}_{2} \mathrm{X}$. For Ref.1, swelling was overpredicted by $62 \%$ ( $26.1 \%$ versus $16 \%$ observed in experiment) whereas for Ref.1.MV swelling was overpredicted by $25 \%$ ( $18.8 \%$ versus $16 \%$ observed in experiment), indicating that the $\mathrm{M}_{2} \mathrm{X}$ precipitate is a significant feature in the overall defect partitioning.

Some studies suggest that coherent sinks should not have an effect on void growth at effective radii larger than $10 \mathrm{~nm}$ [128]. For comparison, the effective radius of $\mathrm{M}_{2} \mathrm{X}$ is $18 \mathrm{~nm}$ at $250 \mathrm{dpa}$, where they are first observed. The results from the modeling are exactly the opposite; Ref.1.MV shows that $\mathrm{M}_{2} \mathrm{X}$ does have an effect on void growth, which is proportional with precipitate size. In other words, there was no $\mathrm{M}_{2} \mathrm{X}$ size observed above which the effect on void growth became negligible.

$\mathrm{M}_{2} \mathrm{X}$ was shown to have a strong impact on the overall void swelling by serving as a variable biased sink or recombination center, either of which have been result in very similar swelling behavior. The primary effect of $\mathrm{M}_{2} \mathrm{X}$ was to serve as an alternate sink for vacancies which limited the amount of vacancies free to diffuse to and grow the void. The ultimate decrease in 
swelling was from $26.1 \%$ to $18.8 \%$, a $21 \%$ decrease in swelling relative to final swelling from Ref.1.

\subsection{Effect of Precipitates on Dislocations}

The effect of precipitates on dislocations will be discussed first for $\mathrm{G}$ phase followed by $\mathrm{M}_{2} \mathrm{X}$.

\subsection{1 $\underline{\mathrm{G} \text { Phase }}$}

G phase is treated as an unbiased sink and Ref.2 will be used as a reference case for this section. Ref. 2 is used as dislocations are responsive to changes in microstructure unlike in the case of Ref.1. The evolution of $\mathrm{G}$ phase with dpa was previously presented in Figure 5.30 and the evolution of dislocation loops and network were shown in Figure 5.46, Figure 5.47 and Figure 5.49. G phase diameter exhibited nearly linear growth with dpa, whereas loop diameter exhibited sudden growth from 375 to 450 dpa ( 23 to $78 \mathrm{~nm}$, respectively). G phase diameter was also considerably smaller (11 to $20 \mathrm{~nm})$ than loop diameter $(23-92 \mathrm{~nm})$ at any given dpa. Number density of both $\mathrm{G}$ phase and loops was stable from 188 to $650 \mathrm{dpa}$, indicating that nucleation of new $\mathrm{G}$ phase or loops was minimal in this regime, which was consistent with the high damage levels considered. There was no correlation in diameter or volume fraction, indicating that there was likely no effect of $\mathrm{G}$ phase on dislocation network or loops, especially considering the low $\mathrm{G}$ phase sink strength.

Figure 7.16 shows the effect of $\mathrm{G}$ phase on dislocation loops with the dynamic dislocation treatment. There was a decrease in both loop and in network sink strength (Figure 7.16a) with the 
addition of $\mathrm{G}$ phase as an alternate sink. The decrease in loop strength was a consequence of lower loop density (Figure 7.16b). Furthermore, the lower loop density provided a lower overall network source term, so there were fewer loops available to grow and join the network leading to the lower network sink strength shown in Figure 7.16a. Loop diameter was not affected as the loop diameter was set by the number of interstitial groups, the consequence of this will be discussed in Section 7.4.2. As was the case with the effect of $G$ phase on voids (Figure 7.10), there was an insignificant impact on overall dislocation loop or network behavior, which was attributed to the low sink strength relative to the other features in the microstructure [20].

For completeness, Figure 7.17 compares the results of Ref.1.G (case previously presented in Figure 7.16) with the experimental observations. The overall network sink strength is very similar to that observed experimentally (Figure 7.17a), but the loop sink strength remained too high, which is a consequence of the overestimated loop density (Figure 7.17b). The loop sink strength but was marginally closer (5\%) to the experimental observations than in the case of Ref.2 for both loop number density and sink strength. The improvement on loop sink strength was a decrease of $5 \%$, which was especially insignificant considering that an improvement of $250 \%$ is required to match the results seen experimentally.

The addition of $\mathrm{G}$ phase had an insignificant impact (5\% decrease) on loop and network sink strengths and was therefore not sufficient to resolve discrepancies between model and experiment. Furthermore, there is no observed correlation in terms of the experimental behavior i.e. loop and $\mathrm{G}$ phase growth behavior at the high dpa levels considered. Therefore, $\mathrm{G}$ phase has little effect on dislocation loop or network behavior, due to its low sink strength and it can be concluded that $\mathrm{G}$ phase has minimal effect on co-evolution of the microstructure. 


\subsection{2 $\underline{\mathrm{M}_{2}} \underline{\mathrm{X}}$}

A direct comparison of loop and $\mathrm{M}_{2} \mathrm{X}$ diameter and length and number density from experiment are shown in Figure 7.18. The results are striking; the period of sudden growth in loop diameter corresponds with the period of large growth and nucleation of $\mathrm{M}_{2} \mathrm{X}$ by $450 \mathrm{dpa}$, indicating there may be some correlation between the two features. After this sudden growth, both $\mathrm{M}_{2} \mathrm{X}$ and loops saturated. Loop number density slowly decreased from 188 to 650 dpa, but $\mathrm{M}_{2} \mathrm{X}$ number density peaked at 450 dpa before decreasing.

The correlation between loop growth and the increase in $\mathrm{M}_{2} \mathrm{X}$ length and density suggests that they may be linked. If the formation of $\mathrm{M}_{2} \mathrm{X}$ is considered from a microchemical perspective, precipitation results in both chromium and carbon being pulled out of solution. Both chromium and carbon have been observed to suppress loop growth relative to that in pure iron [122,150,151]. Suppressed growth is due to enrichment of either species at the dislocations pinning the loops limiting growth. To further explore linkages between the $\mathrm{M}_{2} \mathrm{X}$ and dislocations, Ref.2.MV will be compared to Ref.2.

$\mathrm{M}_{2} \mathrm{X}$ is treated as a variable biased sink in this section (Ref.2.MV) and all dislocation behavior will be compared to Ref.2. The addition of the variable biased $\mathrm{M}_{2} \mathrm{X}$ is compared to Ref.2. in Figure 7.19. By $450 \mathrm{dpa}$, the sink strength of $\mathrm{M}_{2} \mathrm{X}$ is the same order of magnitude as the network and loops and remained similar to the total dislocation sink strength at its peak concentration at 450 dpa (Figure 7.19a). As $\mathrm{M}_{2} \mathrm{X}$ grows in sink strength, a corresponding drop in network and loop sink strength was observed relative to the reference case, which was a consequence of the decreased loop number density shown in Figure 7.19b. $\mathrm{M}_{2} \mathrm{X}$ and loop density are inversely proportional. Since network sink strength is a function of increasing loop sink strength as loops 
grow, there was also a decrease in network sink strength as a result of the high sink strength of $\mathrm{M}_{2} \mathrm{X}$.

Figure $7.19 \mathrm{~b}$ showed that there was no effect of $\mathrm{M}_{2} \mathrm{X}$ on the loop diameter. It was previously observed that a limitation of the RIME model is the insensitivity of loop diameter to either dpa or other microstructure interactions, after a period of initial loop and network instability from the beginning of the case run (Figure 7.17). This insensitivity is a consequence of the loop diameter being defined by the number of interstitial cluster groups and the maximum group size before loops "join" the network; both of which are model inputs. The reference number of interstitial cluster groups is 120 , which resulted in an average loop diameter of $60 \mathrm{~nm}$. The number of interstitial cluster groups cannot be changed mid irradiation without causing major instabilities in the loop, network and void behavior. As such, there was no way to include a mechanism such that precipitates could affect diameter, which was postulated earlier based upon experimental observations. As an alternative analysis, two different cases were compared to determine how changing diameter by changing number of interstitial cluster groups would affect the dislocation number density with the assumption that carbon precipitating into $\mathrm{M}_{2} \mathrm{X}$ was the cause of the dislocation diameter increase. $\mathrm{M}_{2} \mathrm{X}$ is still treated as a variable biased sink (Ref.2.MV) for these cases.

Figure 7.20a shows the corresponding change in loop and network density using 60 or 160 interstitial cluster groups. The number of groups was chosen to reflect the average diameter of the loops from 188 to $375 \mathrm{dpa}$ ( $25 \mathrm{~nm}$ : 60 interstitial groups) and from 450 to $650 \mathrm{dpa}$ (80 nm: 160 interstitial groups). Loop sink strength was much higher in the 160 group case $\left(4 \times 10^{14} \mathrm{~m}^{-2}\right.$ versus $5 \times 10^{13} \mathrm{~m}^{-2}$, respectively) and the network was comparable $\left(3 \times 10^{14} \mathrm{~m}^{-2}\right.$ versus $2 \times 10^{14} \mathrm{~m}^{-2}$ in 160 
and 60 group cases, respectively). With 60 groups (Figure 7.20b), the loops had a smaller diameter $(\sim 25 \mathrm{~nm})$ and a lower number density. With the increase in number of interstitial cluster groups, both the diameter and number density increases. Thus, diameter and number density of loops are strongly correlated.

Overall, Figure 7.20 demonstrates two significant limitations of the RIME model. First, dislocation diameter was more or less stagnant with increasing damage level. This assumption is consistent with literature results from FM alloys suggesting that there was little overall evolution in loops beyond 10 or 20 dpa [16,17]; however, this was not reflected in these new experimental results at very high damage levels. (Figure 5.46) As such, adding an additional treatment to model the loop diameter evolution is outside the scope of this thesis, but would improve the quality of dislocation modeling. Moreover, the loop growth at high dpa is one of the most significant findings of the ion irradiation experiments and is not captured in RIME.

The second limitation highlighted by these cases is the artificial interdependence of dislocation loop diameter, number density and network density. As diameter increases, the number density is forced to increase as a result of more loops growing before joining the network, since the maximum radius $\left(r_{\max }\right)$ before loops "join" the network is a function of the number of interstitial cluster groups. $r_{\max }$ is set by the number of interstitial groups $(\mathrm{mil})$ and the group size $(d r i)$. Thus, as loops become larger, the radius/diameter at which they join the network is a function of this $r_{\max }$ since at $r_{\max }$, the cluster group changes from being defined as an interstitial loop of radius $r_{\max }$ to being part of the network. Since the overall sink strength due to interstitial clusters is more or less the same regardless of $r_{\max }$ since the bias for loops or network is the same (minus an additional annihilation term for the network, shown in Equation 6.19), then $r_{\max }$ is the point at which 
interstitial clusters change from being defined as loops or network. In general, as $r_{\max }$ increases the loop density, diameter and sink strength increases but the network sink strength decreases.

A more realistic view of dislocation evolution is that which was observed experimentally. Loop number density decreases slowly while loops grow to sizes large enough to join the network, evidenced by the increased network density at $550 \mathrm{dpa}$. This is consistent with the void behavior in the high damage regime; constant void number density with the growth of voids causing increased void sink strength/swelling.

Returning to the Ref.2.MV case with the reference number (120) of interstitial cluster groups for consistency (previously presented and compared to Ref .2 in Figure 7.19), Figure 7.21 compares results from the model to experimental results. Figure 7.21a presents the loop, network and $\mathrm{M}_{2} \mathrm{X}$ sink strengths as a function of dpa. Similar to previous cases, the network behavior in both model and experiment were in reasonable agreement $\left(\sim 3 \times 10^{14} \mathrm{~m}^{-2}\right)$. With the addition of $\mathrm{M}_{2} \mathrm{X}$, the overall loop sink strength $\left(2 \times 10^{14} \mathrm{~m}^{-2}\right)$ was closer to the experimentally observed values $\left(\sim 3\right.$ to $\left.5 \times 10^{13} \mathrm{~m}^{-2}\right)$. The sink strength was an order of magnitude too high, but the loop sink strength decreased by a maximum of $75 \%$ from the Ref. 2 case, relative to $250 \%$ decrease required to match experiment. The lowered loop sink strength was a result of the decreased loop number density with the addition of $\mathrm{M}_{2} \mathrm{X}$ (Figure 7.21b). Again, the loop number density was closer to the experimentally observed values but remained too high $\left(9 \times 10^{20} \mathrm{~m}^{-3}\right.$ versus $\left.2-3 \times 10^{20} \mathrm{~m}^{-3}\right)$. Therefore, overall dislocation behavior is more realistic with $\mathrm{M}_{2} \mathrm{X}$, but exact matching with the experimental data was still not achievable. 
The analysis of the effect of $\mathrm{M}_{2} \mathrm{X}$ on dislocations has two overall conclusions. First, $\mathrm{M}_{2} \mathrm{X}$, when treated as a variably biased sink, has a measurable effect on loop and network behavior indicating that $\mathrm{M}_{2} \mathrm{X}$ not only suppresses void swelling, but also dislocation loop formation. Second, the limitations of the RIME treatment of dislocations preclude development of a mechanism that explains the period of enhanced growth from 375 to $450 \mathrm{dpa}$ on the basis of the variable biased treatment, but the experimental observations are strong evidence of an indirect effect of $\mathrm{M}_{2} \mathrm{X}$ precipitation that pulls carbon out of solution, which will be discussed next.

\subsection{Carbon Trapping}

The behavior of carbon in the microstructure of FM alloys is one of the least well understood topics in radiation effects and presents a significant challenge. The precipitation of $\mathrm{M}_{2} \mathrm{X}$ is intimately linked with carbon and its behavior both in solution and as a secondary phase. The precipitation of $\mathrm{M}_{2} \mathrm{X}$ will pull carbon out of solution. Carbon can act as a solute trap for defects, and solute traps have been shown to inhibit swelling and loop growth $[150,151]$. Solute traps can also cause an indirect interaction mechanism between $\mathrm{M}_{2} \mathrm{X}$ and voids/dislocations. Thus, $\mathrm{M}_{2} \mathrm{X}$ can interact both directly, by acting as a sink or recombination center for defects, and indirectly, by removing carbon from solution and reducing the number of traps in the bulk. Both effects are incorporated into the overall model of the system through the addition of solute traps, modeled as a change in the effective vacancy migration energy in Section 6.2.3.

The removal of $\mathrm{C}$ from solution to form $\mathrm{M}_{2} \mathrm{X}$ is predicated on the detrapping of $\mathrm{C}$ from Cottrell atmospheres i.e. the solutes that diffuse to the dislocation core, which inhibit dislocation movement [122]. The binding energy of a $\mathrm{C}$ atom to a dislocation was determined using a 
molecular statics approach by Veiga et al. to be approximately 0.41 or $0.65 \mathrm{eV}$ for edge and screw type, respectively. Since it is difficult to determine the loop character in ferritic-martensitic alloys especially after irradiation, $0.5 \mathrm{eV}$ was chosen as an approximate trap energy. A carbon-dislocation binding energy of $0.5 \mathrm{eV}$ is very similar to the $\mathrm{C}$ vacancy trap energy, which has been estimated to range anywhere from 0.36 to $0.57 \mathrm{eV}[123-125,152]$. While the trap strength is the same, still lacking is a mechanism for removal of $\mathrm{C}$ from the loops.

An alternate interpretation of the system is that of dynamic dislocation nucleation and growth. Although number density of loops is slowly decreasing in the 188 to 650 dpa range, there are likely new loops nucleating and growing till they join the network at large enough sizes, characteristic of a pseudo steady state. As carbon is pulled out of solution that was not already formed into solute atmospheres, more of these newly nucleated loops are free to grow without the restraint of carbon to trap them. Thus, even if the $\mathrm{C}$ is unable to be de-trapped from the existing loops, the overall loop distribution expands in terms of length and the average diameter increases.

HT9 has $0.2 \mathrm{wt} \% \mathrm{C}$, which corresponds to 0.92 at\%, or 9200 appm C. The solubility of carbon at $460^{\circ} \mathrm{C}$ is 855 appm C. A calculation was performed to account for the amount of carbon precipitated into $\mathrm{M}_{2} \mathrm{X}$ at $450 \mathrm{dpa}$, where peak volume fraction of carbides was observed. $\mathrm{M}_{2} \mathrm{X}$ has a hexagonal crystal structure with $a=0.272 \mathrm{~nm}$ and $c=0.452 \mathrm{~nm}$. By calculating the number of unit cells contained in the observed $\mathrm{M}_{2} \mathrm{X}$, the $\mathrm{C}$ number density can be obtained $\left(3.71 \times 10^{26} \mathrm{~m}^{-3}\right)$ and compared to the number density of HT9 $\left(8.34 \times 10^{28} \mathrm{~m}^{-3}\right)$. This results in a maximum of $\mathrm{C}$ concentration of 3940 appm retained in $\mathrm{M}_{2} \mathrm{X}$. A comparison of the accounting for carbon in HT9 in the as-received condition and at $450 \mathrm{dpa}$ is compared in Figure 7.22. Of the 9200 appm C total in the microstructure, a majority of the carbon is unaccounted for in the as 
received condition and nearly half of the carbon is unaccounted for even at the peak volume fraction of $\mathrm{M}_{2} \mathrm{X}$ at $450 \mathrm{dpa}$. The unaccounted carbon may be at grain boundaries, segregated to voids or other interfaces, in $\mathrm{M}_{23} \mathrm{C}_{6}$ carbides and also at small carbides at high density, such as vanadium carbides $(\mathrm{MX})$ or small $\mathrm{M}_{2} \mathrm{X}$, which are more difficult to image or quantify. Furthermore, it is not possible to determine source of the $\mathrm{C}$ in the $\mathrm{M}_{2} \mathrm{X}$ experimentally, so $\mathrm{C}$ may diffuse from any or all of these sources mentioned.

To determine the approximate amount of carbon removed from solution, the range 0 to 3940 appm was examined in Figure 7.23. But first, the effect of carbon on dislocation and swelling behavior must be determined by adding in its effect on vacancy mobility. Figure 7.23 a reveals that increasing the amount of carbon in solution above 100 appm affects dislocation network and loop sink strength. It was assumed in the model that carbon was only allowed to trap vacancies causing decreased effective vacancy diffusion coefficient. Above 500 appm, the network sink strength remained constant or slightly increased, which was not consistent with results from Ref.2. Furthermore, above 500 appm, the simulation failed entirely at 380 and 260 dpa with 1000 and 3940 appm C, respectively. The simulation failure was due to a negative swelling rate which caused voids to shrink (Figure 7.23b), and was attributed to such a high trap strength that vacancy diffusion decreased to the point where voids began to shrink.

The effect of carbon in solution from 0 to 3940 appm on void swelling is presented in Figure 7.23b. There was no major effect on swelling until at least $100 \mathrm{appm} \mathrm{C}$ is included in the model. Beyond 100 appm, there was significant decrease in void swelling rate, by an order of magnitude from approximately 0.05 to $0.001 \% / \mathrm{dpa}$ and even resulted in a negative swelling rate previously mentioned with 1000 and 3940 appm C. 
While there is no way to directly measure or characterize the amount of carbon in solution contributing to vacancy trapping, the effect of $\mathrm{C}$ addition or removal can be modeled to assess its effect of vacancy mobility and therefore, dislocation and void evolution. The failure of the cases run with 1000 or more appm $\mathrm{C}$ means that these are not appropriate $\mathrm{C}$ amounts of carbon. With 500 appm C, the dislocation behavior diverges significantly from what is observed in the reference cases. Furthermore, the decrease in swelling rate is an order of magnitude, which is not consistent with experimental swelling results (Figure 7.23b). At $100 \mathrm{appm}$, there is only a minor effect on the network and loop behavior and a measurable effect on swelling. Below $100 \mathrm{appm}$, there is a negligible effect on the swelling behavior. Thus. 100 appm was chosen as an appropriate amount of $\mathrm{C}$ removed from solution by precipitation of $\mathrm{M}_{2} \mathrm{X}$.

Furthermore, an even more realistic treatment of carbon is to assume that the $100 \mathrm{appm}$ decreases as a function of dpa until $450 \mathrm{dpa}$, at which point, the carbon is completely removed by the precipitation of $\mathrm{M}_{2} \mathrm{X}$. The resulting treatment is presented in Figure 7.24. Physically, this represents the case of carbon gradually removed from solution with the precipitation of $\mathrm{M}_{2} \mathrm{X}$. There was a modest decrease in swelling rate as expected, but after the gradual removal of $\mathrm{C}$ from the system, swelling rate increases to its nominal rate represented by Ref.2 in blue. Note that there is a clear parabolic shape of the curve as a result of removal of carbon from solution indicating a monotonic increase in swelling rate, that accelerates after the full removal of carbon from solution. For comparison, the instantaneous swelling at 300 dpa was $0.043 \% / \mathrm{dpa}$ compared to the instantaneous swelling rate at $500 \mathrm{dpa}$, which was $0.055 \% / \mathrm{dpa}$ (measured from 450 to $650 \mathrm{dpa}$ ). This can also be approximated as two regions of linear swelling: 0.042 (measured from 188 to 450 dpa) to $0.055 \% / \mathrm{dpa}$. As noted before in Figure 5.19, either linear regression or calculation of an 
instantaneous swelling rate from the parabolic regression results in very similar swelling rate values.

A comparison between experimentally input and DD treatments with 100 appm carbon removed in a stepwise fashion from 188 to $450 \mathrm{dpa}$ is given in Figure 7.25. The effect of carbon is much stronger in the case of the experimentally input case (Ref.1.C) resulting in final swelling of $21.2 \%$ versus $24.3 \%$ in the DD treatment (Ref.2), which is only a modest decrease from the reference case $(26.1 \%)$. The suppression is mitigated in Ref.2 because the carbon also has a small impact on loop and network sink strength. The loop and network sink strength are actually 8 and $5 \%$ lower than the Ref.2, which effectively frees up more vacancies to diffuse to the voids. In contrast, the experimentally input case (Ref.1.C) does not respond to the changes in $d_{i} c_{i}$ and $d_{v} c_{v}$, and the loop and network remains the same as in Ref.1. The carbon treatment is the only microstructure treatment that has a major difference in response between Ref.1 and Ref.2 unlike in precipitate treatments where the difference between treatments is negligible (below 5\%), such as the case of $\mathrm{G}$ phase and $\mathrm{M}_{2} \mathrm{X}$. It can also be seen in Figure 7.25 that the parabolic shape is exaggerated.

Figure 7.24 demonstrated the effect of accounting for carbon in solution from 188 to 450 dpa, which suppressed void growth prior to the removal of the C in Ref.1.C consistent with mechanisms suggested in $[122,141]$. If carbon works to suppress swelling both as a solute trap and formed into a sink, this dynamic process could explain why no obvious decrease in void swelling with $\mathrm{M}_{2} \mathrm{X}$ was observed in experiment. In other words, the suppression of swelling by $\mathrm{M}_{2} \mathrm{X}$ was compensated by the loss of suppression of $\mathrm{C}$ in solution. 
A more comprehensive description of $\mathrm{M}_{2} \mathrm{X}$ in the microstructure is as both a sink and corresponding microchemical effect. Thus, $\mathrm{M}_{2} \mathrm{X}$ should act as a sink for the entire growthdominated regime as well as a consumer of carbon form solution; this interpretation is presented (Ref.1.MV.C) in Figure 7.26 and compared to the effect of $\mathrm{M}_{2} \mathrm{X}$ alone (Ref.1.MV) and Ref.1. Compared to either Ref.1 or Ref.1.MV, accounting for the presence of carbon below 450 dpa suppresses swelling by an additional factor. The swelling rate is visibly reduced in the range of 188 to $450 \mathrm{dpa}(0.025 \% / \mathrm{dpa}$ measured instantaneously at $300 \mathrm{dpa})$; the swelling rate increased to $0.038 \% / \mathrm{dpa}$ measured instantaneous at $500 \mathrm{dpa}$. However, the average swelling rate measured by linear regression from 188 to $650 \mathrm{dpa}$, is $0.03 \% / \mathrm{dpa}$, which is consistent with that observed experimentally in Figure 5.18. The agreement in overall swelling rate from 188 to 650 dpa between computational result and experiment provides confidence in the modeling that major interactions are being captured.

For completeness, the simple case of only carbon (Ref.2.C) is compared to experiment in Figure 7.27. (Results from this case were previously presented in Figure 7.25.) Network behavior remains reasonable $\left(3-4 \times 10^{14} \mathrm{~m}^{-2}\right)$ and the loop sink strength is still an order of magnitude too high (Figure 7.27a). Again, as with all cases analyzed, the loop sink strength is a consequence of the higher than experimentally observed loop number density in Figure 7.27b. Finally, void diameter and swelling are more consistent with experiment until $450 \mathrm{dpa}$ (Figure 7.27c), but the swelling and growth rate increases once carbon is fully taken out of solution, leading to a final swelling values of $24.3 \%$ relative to the expected $16 \%$, which represents a decrease in swelling from the reference case (Ref.2) of $7 \%$. 
The behavior of carbon has been linked to formation of $\mathrm{M}_{2} \mathrm{X}$ as an indirect microchemical effect. Carbon in solution has been shown to have a strong effect on the swelling, decreasing the swelling from $26.1 \%$ to $21.1 \%$ for Ref.1 and $24.3 \%$ for Ref.2, when 100 appm of C in solution were accounted for up to $450 \mathrm{dpa}$. The discrepancy between cases was resolved by the observation of a modest decrease in dislocation loop and network sink strength, which decreased the amount of biased sink strength available in the system relative to Ref.2. The implementation of this mechanism was supported by experimental observations of the precipitation of large amount of $\mathrm{M}_{2} \mathrm{X}$, which was correlated with increased loop growth.

\subsection{Combined Impact of Microstructure Features on Voids and Dislocations}

Previous analyses in this discussion have shown the behavior of various microstructure features and treatments relative to the reference cases. On their own, no single interaction was able to completely resolve the discrepancy between the expected void behavior in experiment and model, regardless of whether dislocation microstructure was experimentally input (Ref.1) or was dynamically developed (Ref.2). Since void swelling was overestimated in all cases, it is reasonable to assume that there is a combination of interaction mechanisms in play. Both the precipitate $(\mathrm{G}$ phase $+\mathrm{M}_{2} \mathrm{X}$ ) and the carbon treatment will be included to determine whether the combined treatments can account for the dislocation/void discrepancy or if there is an additional process that was not captured in this version of the RIME model. G phase will continue to be treated as an unbiased sink. $\mathrm{M}_{2} \mathrm{X}$ will be treated as a variable biased sink (MV) according to Brailsford formalism, since the recombination center treatment was shown (Figure 7.14) to be nearly identical for the trap energy considered. 
A case with the full microstructure treatment and experimentally input dislocation (Ref.1.G.MV.C) is shown in Figure 7.28. Figure 7.28a shows the sink strength evolution of the network, loops, $\mathrm{G}$ phase and $\mathrm{M}_{2} \mathrm{X}$. There is a small discontinuity in the $\mathrm{M}_{2} \mathrm{X}$ sink strength at 450 dpa due to the sudden increase in $d_{v} c_{v}$ when carbon in solution is removed as the effective sink strength responds to the change in defect kinetics. Figure $7.28 \mathrm{~b}$ shows near perfect match of experimentally observed swelling, with $16.4 \%$ from the model case Ref.1.G.MV.C and $16 \%$ experimentally, indicating that $\mathrm{M}_{2} \mathrm{X}, \mathrm{G}$ phase and carbon in solution are all needed to resolve excess vacancy flux from the dislocation loops and network. Most importantly, this result indicates the robustness of this model; when all microstructure features are accounted for, the swelling behavior is nearly identical between experiment and computation.

Figure 7.28 demonstrated the excellent matching of void behavior when all alternate microstructure treatments are included. To confirm this result, and also to determine if the dislocation behavior can be likewise matched with all three treatments included, the full microstructure treatment was applied to Ref.2 in Figure 7.29 (Ref.2.G.MV.C). The network density, even with the addition of all microstructure treatments, remained similar to experiment, but the loop density was still too high by an order of magnitude relative to the experiment $\left(\sim 9 \times 10^{20}\right.$ versus $2-3 \times 10^{20} \mathrm{~m}^{-3}$, respectively, shown in Figure $7.29 \mathrm{~b}$ ) leading to a loop sink strength that was still an order of magnitude too high $\left(\sim 1-2 \times 10^{14} \mathrm{~m}^{-2}\right.$ shown in Figure $\left.7.29 \mathrm{a}\right)$. However, the addition of the other microstructure treatments was an improvement in terms of trends over the simpler Ref.2 reference case, which was shown in Figure 7.9. The resulting swelling at 650 dpa was 18.0\%, which is within experimental error measured value of $16 \%$, indicating that a combination of both the precipitate treatments along with the stepwise removal of carbon in solution from 188 to 450 
dpa is capable of resolving the dislocation and void behavior, in the DD treatment as well as in the experimentally input case.

A summary of model cases is provided in Table 7.4, which also includes cases not explicitly presented here. The closest match for all conditions was from either the experimentally input network and loop or DD treatment with full precipitate treatment including carbon. Regardless of dislocation treatment, the addition of $\mathrm{M}_{2} \mathrm{X}$ using either variable biased treatment is required to resolve the swelling to more appropriate levels, indicating that $\mathrm{M}_{2} \mathrm{X}$ formation does influence swelling, despite the apparent linear swelling rate from 188 to $650 \mathrm{dpa}$. These results demonstrate the effectiveness of the RIME model as a method of deconvoluting the complex interactions inherent in the irradiated microstructure.

A direct comparison of the deconvolution of the four treatments including Ref.1 is shown in Figure 7.30. It is clearly demonstrated that $\mathrm{M}_{2} \mathrm{X}$ and carbon in solution have the largest impact and the effect of $\mathrm{G}$ phase is negligible. To quantify the relative impact of the treatments, Table 7.5 presents the relative change in final swelling value with the addition of each microstructure treatment to either reference case. G phase has the most minor effect, a final swelling reduction of about $5 \%$ of the final swelling value, regardless of dislocation treatment. Carbon in solution and $\mathrm{M}_{2} \mathrm{X}$ had the largest effect on final swelling. With the addition of $\mathrm{M}_{2} \mathrm{X}$ only, the overall swelling was reduced by $21 \%$. The stepwise removal of 100 appm of carbon from 188 to 450 dpa decreased the final swelling value by $19 \%$, in the case of Ref.1 and 7\% for Ref.2. (The difference in carbon response for each dislocation treatment was noted in Figure 7.25 and addressed in Section 7.5.) However, since carbon precipitation is considered as an indirect effect of $\mathrm{M}_{2} \mathrm{X}$. If $\mathrm{M}_{2} \mathrm{X}$ is 
considered as the sum of its direct (as a sink) and indirect (microchemical) effects, $\mathrm{M}_{2} \mathrm{X}$ has by far the most significant effect on void behavior.

\subsection{Alternate Considerations Regarding Overall Microstructure Co-evolution}

A strong case has been made for including the combined effects of the irradiated microstructure in modeling the void and dislocation evolution. The final section will discuss implications of these results.

\subsubsection{Discussion of Other Possible Interaction Mechanisms}

Figure 7.28 and Figure 7.29 offers convincing evidence that the primary interactions are between the dislocation and voids with $\mathrm{M}_{2} \mathrm{X}$, which served as both an alternate sink for defects

and as a consumer of carbon out of solution. However, to support this statement, other alternative explanations and compelling evidence supporting the final chosen treatment are considered here.

\subsubsection{Lower Effective Bias}

The results of Figure 7.8 suggested that the swelling behavior could be explained in the absence of any features that were not dislocations, if a lower bias for interstitials with the experimentally input case were used (Ref.1.1.007). It was not pursued initially because this was a lower bias then those reported in the literature. However, disregarding this for the sake of argument, the case could be made that $0.7 \%$ bias is not unreasonable, as it is within $30 \%$ of $1 \%$ bias. Carrying this line of reasoning a step further, the lower biased case was run with the addition of the full microstructure treatment including $G$ phase, $M_{2} X$ and 
carbon in solution; results are presented in Figure 7.31a. Figure 7.31a is identical to Figure 7.24a, as bias does not change the experimentally input evolution, but a resulting decrease in void diameter and swelling is shown in Figure 7.31b. The addition of the precipitates decreased the void diameter enough to underpredicts swelling relative to the experiment by $43 \%$ (10 versus $16 \%$, respectively). Thus, when all the possible microstructure interaction mechanisms are considered, including lowered effective bias $(0.7 \%$ vs $1 \%)$ is not an appropriate treatment explaining the overall system's behavior. This demonstrates how the view of understanding void swelling as simply being influenced by dislocations is overly simplistic. A full understanding of void swelling needs to take into account other features that have sink strengths on the same order of magnitude. In fact, one of the strongest supports for this including all treatments is that it is unreasonable to assume a feature with sink strength $10^{14} \mathrm{~m}^{-2}$ is not significant. Thus, lowered effective bias as a mechanism is not pursued further.

\subsubsection{Reconsidering Swelling Rates from Experiment}

The addition of, and subsequent removal of $\mathrm{C}$ in a stepwise fashion highlighted the slightly parabolic shape of the swelling curve, which was observed in cases with carbon only (Ref.1.C: Figure 7.24), carbon and $\mathrm{M}_{2} \mathrm{X}$ as a variable sink (Ref.1.MV.C: Figure 7.26) and with the full microstructural treatment including $G$ phase (Ref.1.G.MV.C: Figure 7.28). It was also demonstrated in Figure 7.28 that there was excellent matching of the overall swelling rate from 188 to 650 dpa from both the experimental data and model swelling curve. The question then becomes: can the slowly monotonically increasing swelling rate due to the parabolic shape be predicted in RIME be reasonably measured from the experimental data? 
Figure 7.32 plots the swelling only as a function of damage from Ref.1.G.MV.C. Lines guiding the eye have been drawn to draw attention to the swelling rate which clearly increases with the removal of carbon and as a function of damage. The origin of this increase is that carbon suppresses void growth from 188 to $450 \mathrm{dpa}$, and then is removed from the model which, by its absence, promotes growth, despite $\mathrm{M}_{2} \mathrm{X}$ acting as a sink for defects in the higher damage regime beyond 450 dpa.

To examine whether a case can be made experimentally for a slowly increasing swelling rate, the swelling rate was calculated at $300 \mathrm{dpa}$ and at $500 \mathrm{dpa}$ using the instantaneous swelling rate calculated from the parabolic regression least squares method described in Section 4.5.1. To make the analysis more thorough, it was also repeated using the fixed depth correction from Section 5.2.5, which gives an even more accurate swelling rate, taking into account void-induced extension of the ion damage curve. Furthermore, linear regression from 188 to 450 dpa and 450 to 650 dpa was used to confirm this as well. The results from all of these calculations are in Table 7.6 and Figure 7.33. The instantaneous swelling rate, calculated from the parabolic fit, increased from $0.025 \pm 0.004 \% / \mathrm{dpa}$ to $0.032 \pm 0.005 \% / \mathrm{dpa}$ at 300 and $500 \mathrm{dpa}$, respectively, which was an increase of $29 \%$. For comparison, a linear regression was also calculated from 188 to 450 dpa and 450 to $650 \mathrm{dpa}$. Considering the first case with the nominal swelling calculation with no correction (Figure 7.33a), the swelling from 188 to $450 \mathrm{dpa}$ is $0.025 \pm 0.004 \% / \mathrm{dpa}$ whereas the swelling rate calculated from 450 to $650 \mathrm{dpa}$ is $0.036 \pm 0.005 \% / \mathrm{dpa}$, which represents an increase of $44 \%$ that is outside the error bars.

Furthermore, considering the more accurate fixed depth corrected swelling (Figure 7.33b), the swelling rate increases from $0.027 \pm 0.004$ to $0.035 \pm 0.006 \% / \mathrm{dpa}$, from 300 and $500 \mathrm{dpa}$, 
respectively, which represents a swelling rate increase of $30 \%$ as well. This is comparable to the increase in swelling rate predicted from RIME of $44 \%$ from the lower $(0.025 \% / \mathrm{dpa})$ to higher $(0.036 \% / \mathrm{dpa})$ swelling rate. Thus, a strong case can be made for the observation of this slowly increasing swelling rate.

Practically speaking, the observation of the monotonically swelling increasing swelling rate demonstrates the fidelity of the experimental observations; the irradiation and characterization procedures were consistent enough to capture a swelling rate of change of less than a factor of 2. More importantly, this demonstrates the robustness of the model and is convincing evidence that the underlying mechanism treatments applied were appropriate.

The significance of this work is in that it highlights the importance of microstructure coevolution in terms of interactions between sinks in the microstructure, regardless of whether the precise co-evolution reflected in the model was that observed exactly. A case can always be made for other potential interaction mechanisms, but the overall shape and trajectory of the co-evolving microstructure is strong evidence of the suitability of this type of analysis for unfolding radiation damage interactions.

In a larger context, this has significant ramifications for how void swelling evolution is interpreted at very high damage levels in the void growth-dominated regime. Much emphasis has been placed on the concept of a "steady state" swelling rate and determining what that rate is; less emphasis has been on how the entire microstructure as a whole evolves and how this can influence swelling rates. This work demonstrates the necessity of considering the evolution of the entire microstructure, insofar as this is feasible, as the continual change in dislocation, precipitates and 
solutes that continue to affect void swelling at damage levels beyond any observed in the literature until now.

Furthermore, this work also demonstrates that the concept of "steady state" or linear swelling regime is likely overstated in the literature. A better interpretation of the void swelling evolution is that of a nucleation dominated regime, followed by a transition regime with both nucleation and growth followed by a growth-dominated regime, which can still be influenced by any changes in the microstructure. For the specific case examined here of HT9 irradiated with 5 $\mathrm{MeV} \mathrm{Fe}{ }^{++}$, the swelling behavior in the void growth-dominated region is characterized by a nearly monotonically increasing linear swelling rate around $\sim 0.03 \% / \mathrm{dpa}$ for the damage levels examined. For the purposes of reactor design applications, taking the growth regime as a whole (188 to 650 dpa), $0.033 \% /$ dpa can serve as an excellent approximation. Reactor designs, in general, are limited to $10 \%$ swelling, which was within the swelling levels observed here. Beyond $10 \%$, determining the swelling rate is more or less an academic exercise.

This work is particularly significant for the Gen IV reactor community as candidate materials are selected for the newest reactor design. As new reactor designs are proposed with more extreme environments in terms of high temperature, pressure or damage levels, the ability to understand how void swelling is expected to evolve beyond damage levels reached in reactor irradiations is very useful. More importantly, the continuous microstructure evolution at high damage levels provides a context for alloy developers working on improving the radiation tolerance of advanced materials, particularly in the area of void swelling. It also provides confidence for pursing alternate materials with high precipitate densities to mitigate void swelling, such as oxide dispersed steels, as a method of improving swelling resistance. Finally, the combined 
approach of a set of systematic, well-controlled ion experiments with a rigorous modeling program demonstrates a powerful technique for understanding the fundamental mechanistic interactions between microstructure features. 
Table 7.1: Description of flags denoting modification of reference cases with various microstructure treatments.

\begin{tabular}{|c|c|c|}
\hline Flag & $\begin{array}{c}\text { Description of } \\
\text { Microstructure/Treatment }\end{array}$ & Notes \\
\hline $\mathrm{N}$ & Network & $\begin{array}{c}\text { Change network density from } 3 \\
\text { to } 4 \times 10^{14} \mathrm{~m}^{-2} \text { at } 550 \mathrm{dpa}\end{array}$ \\
\hline 'bias' & Changed bias from 1.01 & $\begin{array}{c}\text { Reflects any bias that is NOT } \\
1.01 \text {. i.e. new bias is in flag } \\
\text { ' } 1.007 \text { ' }\end{array}$ \\
\hline $\mathrm{G}$ & G phase & $\begin{array}{l}\text { G phase always unbiased } \\
\text { treatment }\end{array}$ \\
\hline MU & $\mathrm{M}_{2} \mathrm{X}$-Unbiased & $\begin{array}{l}\mathrm{M} \text { refers to } \mathrm{M}_{2} \mathrm{X} \text {; second letter } \\
\quad \text { refers to ppt treatment }\end{array}$ \\
\hline MB & $\mathrm{M}_{2} \mathrm{X}$-Biased & $\begin{array}{l}\mathrm{M} \text { refers to } \mathrm{M}_{2} \mathrm{X} \text {; second letter } \\
\quad \text { refers to ppt treatment }\end{array}$ \\
\hline MV & $\mathrm{M}_{2} \mathrm{X}$-Variable Biased & $\begin{array}{l}\mathrm{M} \text { refers to } \mathrm{M}_{2} \mathrm{X} \text {; second letter } \\
\quad \text { refers to ppt treatment }\end{array}$ \\
\hline MR & $\begin{array}{l}\mathrm{M}_{2} \mathrm{X} \text {-Recombination } \\
\text { Center }\end{array}$ & $\begin{array}{l}\mathrm{M} \text { refers to } \mathrm{M}_{2} \mathrm{X} \text {; second letter } \\
\quad \text { refers to ppt treatment }\end{array}$ \\
\hline $\mathrm{C}$ & Carbon in solution & Solute-vacancy traps in solution \\
\hline
\end{tabular}


Table 7.2: Dislocation biases for interstitials reported in literature using a variety of approaches.

\begin{tabular}{|c|c|c|}
\hline Bias Range & Approach & Reference \\
\hline $1.01-1.25$ & Analytic Solutions & {$[48,137-139]$} \\
\hline $1.01-1.05$ & Rate Theory & {$[9,41,42,115,154]$} \\
\hline
\end{tabular}


Table 7.3: Description of reference cases with modifications used to match void and dislocation behavior to experiment.

\begin{tabular}{|c|c|c|c|c|c|c|c|}
\hline Case & \multicolumn{1}{|c|}{ Bias } & Void & Network & Loop & $\mathrm{M}_{2} \mathrm{X}$ & G Phase & $\begin{array}{c}\text { Carbon in } \\
\text { Solution }\end{array}$ \\
\hline Ref.0 & 1.01 network & Too high & Input & Not included & N/A & N/A & N/A \\
\hline Ref.0.N & 1.01 network & Too high & Input & Not included & N/A & N/A & N/A \\
\hline Ref.1 & 1.01 network and loop & Too high & Input & Input & N/A & N/A & N/A \\
\hline Ref.1.1.007 & 1.007 network and loop & Match & Input & Input & N/A & N/A & N/A \\
\hline Ref.2 & 1.01 network and loop & Too high & Match & Too high & N/A & N/A & N/A \\
\hline
\end{tabular}


Table 7.4: Summary of attempts to match microstructure behavior with precipitate and carbon treatments.

\begin{tabular}{|c|c|c|c|c|c|c|c|}
\hline Case & Bias & Void & Network & Loop & $\mathrm{M}_{2} \mathrm{X}$ & G Phase & $\begin{array}{l}\text { Carbon in } \\
\text { Solution }\end{array}$ \\
\hline Ref.1 & 1.01 network and loop & Too high & Input & Input & N/A & N/A & N/A \\
\hline Ref.1.1.007 & 1.007 network and loop & Match & Input & Input & N/A & N/A & N/A \\
\hline Ref.2 & 1.01 network and loop & Too high & Match & Too high & N/A & N/A & N/A \\
\hline Ref.1.G.MV & 1.01 network and loop & Too high & Input & Input & Input & Input & N/A \\
\hline Ref.2.G.MV & 1.01 network and loop & Too high & Match & Too high & Input & Input & N/A \\
\hline Ref.1.G.MV.C & 1.01 network and loop & Match & Match & Input & Input & Input & $\begin{array}{c}\text { Stepwise } \\
\text { decrease } 100 \\
\text { appm from } 188 \\
\text { to } 450 \mathrm{dpa} \\
\end{array}$ \\
\hline Ref.2.G.MV.C & 1.01 network and loop & Match & Match & Too high & Input & Input & $\begin{array}{c}\text { Stepwise } \\
\text { decrease } 100 \\
\text { appm from } 188 \\
\text { to } 450 \mathrm{dpa}\end{array}$ \\
\hline Ref.1.1.007.G.MV.C & 1.007 network and loop & Too low & Input & Input & Input & Input & N/A \\
\hline
\end{tabular}


Table 7.5: Comparison of relative effect on final swelling values at $650 \mathrm{dpa}$ of each microstructure treatment.

\begin{tabular}{|c|c|c|c|c|}
\hline \multirow{2}{*}{ Microstructure Treatment } & \multicolumn{2}{|c|}{ Swelling (\%) } & \multicolumn{2}{c|}{$\begin{array}{c}\text { Change in Final } \\
\text { Swelling }\end{array}$} \\
\cline { 2 - 5 } & Ref.1 & Ref.2 & Ref.1 & Ref.2 \\
\cline { 2 - 5 } & 26.1 & 26.1 & N.A. & N.A. \\
\hline G & 24.7 & 24.6 & $-5.4 \%$ & $-5.7 \%$ \\
\hline MV & 20.6 & 20.5 & $-21.1 \%$ & $-21.5 \%$ \\
\hline $\begin{array}{c}\text { C (100 appm removed } \\
\text { stepwise from 188 to 450 dpa) }\end{array}$ & 21.2 & 24.3 & $-18.8 \%$ & $-6.9 \%$ \\
\hline
\end{tabular}


Table 7.6: Comparison of swelling rates calculated in the range of 188 to $650 \mathrm{dpa}$ using nominal or fixed depth method [85].

\begin{tabular}{|c|c|c|c|c|}
\hline & \multicolumn{2}{|c|}{ Nominal } & \multicolumn{2}{|c|}{ Fixed Depth Correction } \\
\hline Damage Range & $\begin{array}{c}\text { Swelling Rate } \\
\text { (\%/dpa) }\end{array}$ & $\mathrm{R}^{2}$ & $\begin{array}{l}\text { Swelling } \\
\text { Rate } \\
(\% / \mathrm{dpa})\end{array}$ & $\mathrm{R}^{2}$ \\
\hline \multicolumn{5}{|c|}{ Parabolic Regression } \\
\hline $\begin{array}{c}\text { Instantaneous } \\
\text { Swelling at } 300 \mathrm{dpa}\end{array}$ & $0.025 \pm 0.004$ & N/A & $0.027 \pm 0.004$ & N/A \\
\hline $\begin{array}{c}\text { Instantaneous } \\
\text { Swelling at } 500 \mathrm{dpa}\end{array}$ & $0.032 \pm 0.005$ & N/A & $0.035 \pm 0.006$ & N/A \\
\hline \multicolumn{5}{|c|}{ Linear Regression } \\
\hline $188-450 \mathrm{dpa}$ & $0.025 \pm 0.004$ & 0.94 & $0.027 \pm 0.004$ & 0.93 \\
\hline $450-650 \mathrm{dpa}$ & $0.036 \pm 0.005$ & 0.98 & $0.042 \pm 0.006$ & 0.98 \\
\hline $188-650 \mathrm{dpa}$ & $0.033 \pm 0.005$ & 0.98 & $0.036 \pm 0.005$ & 0.98 \\
\hline
\end{tabular}




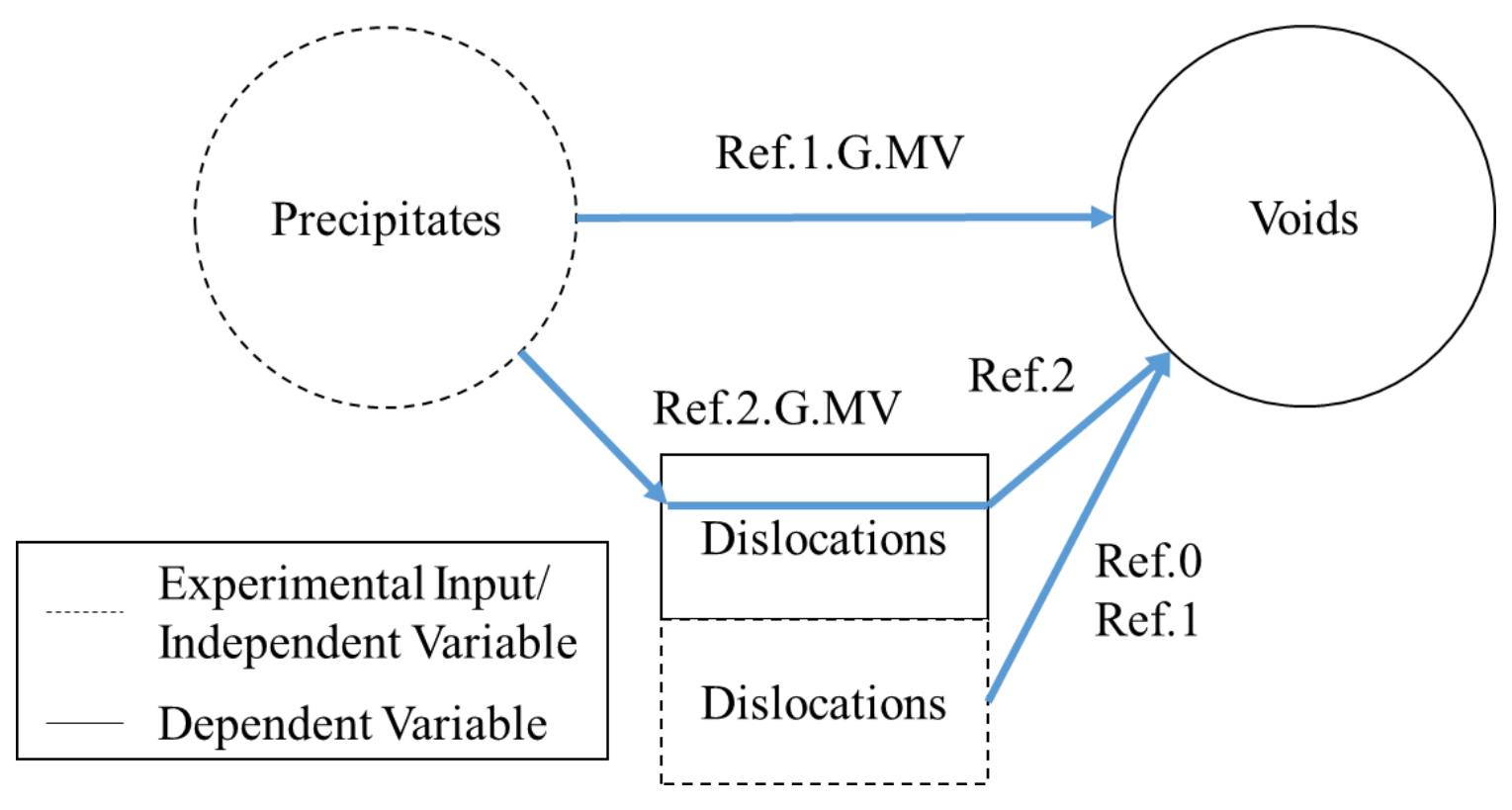

Figure 7.1: Schematic of relationships between precipitates, dislocations and voids explored in depth using RIME. 


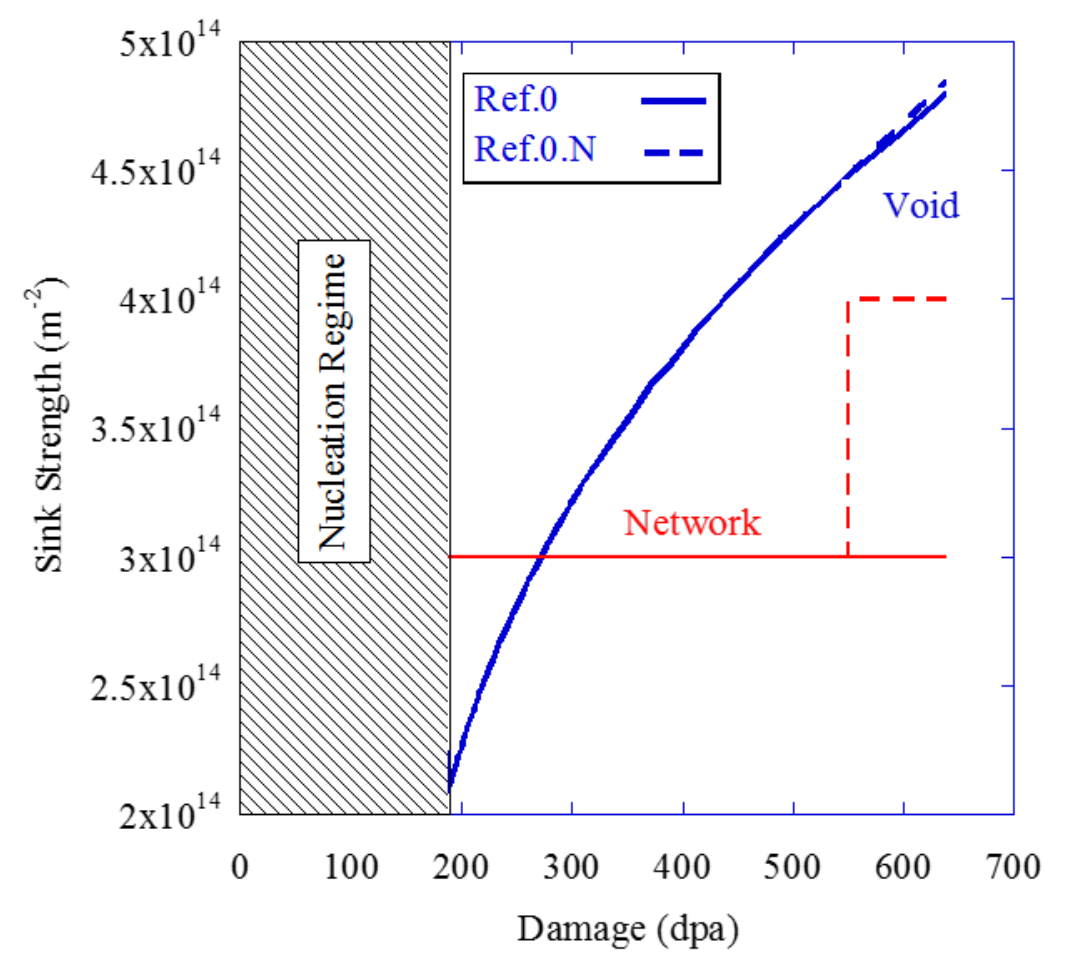

(a)

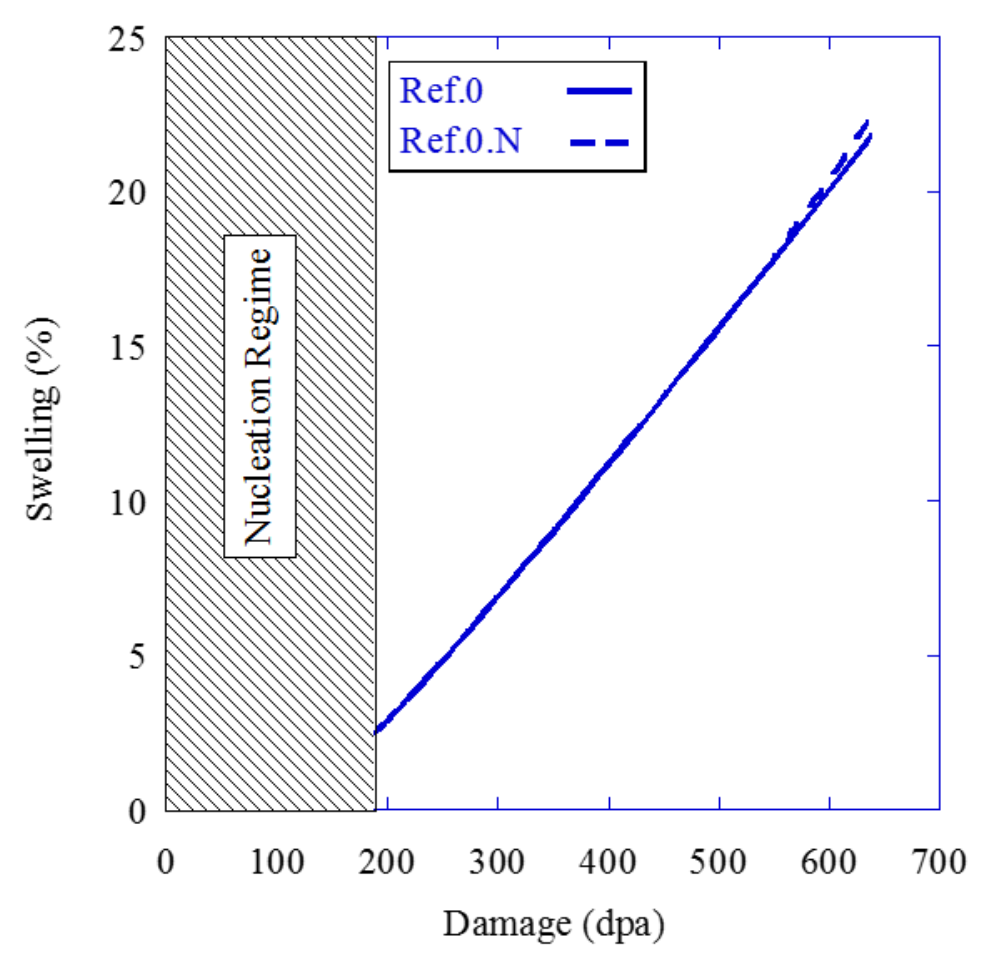

(b)

Figure 7.2: Comparison of Ref.0 (solid) and Ref.0.N (dashed) treatments $\left(z_{i}^{\text {net }}=1.01\right)$ with experimental data in terms of a) void and network sink strength and $b$ ) void swelling. 


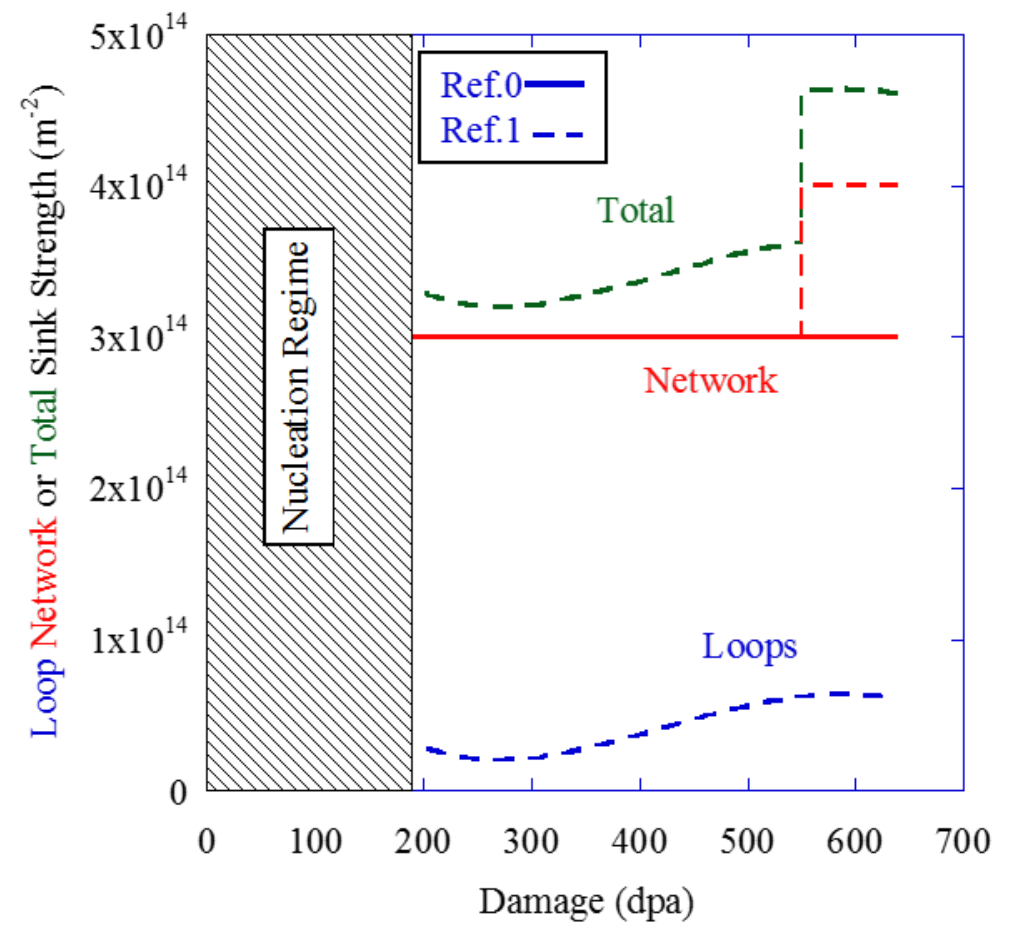

(a)

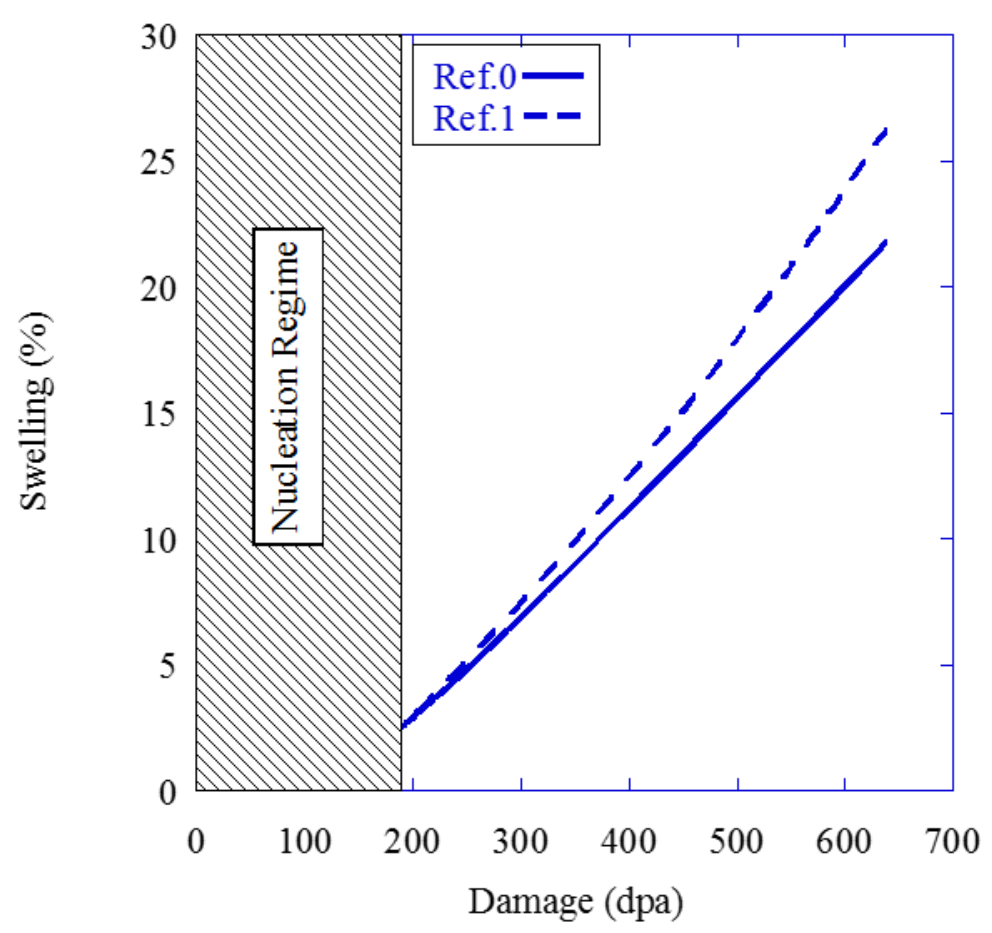

(b)

Figure 7.3: Comparison of Ref.0 (solid) and Ref.1 (dashed) treatments ( $\mathrm{z}_{\mathrm{i}}^{\text {lp }}=\mathrm{z}_{\mathrm{i}}^{\text {net }}=1.01$ ) with experimental data in terms of a) network, loop and total dislocation sink strength and b) void swelling. 


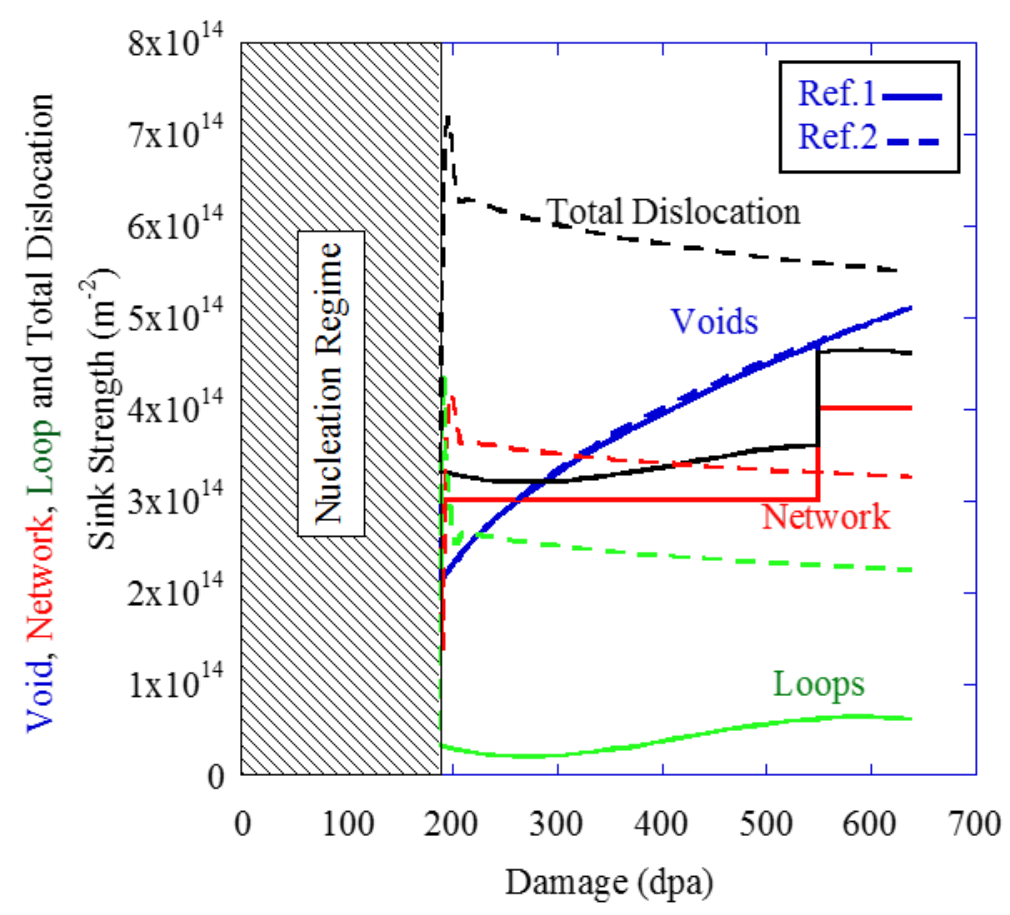

(a)

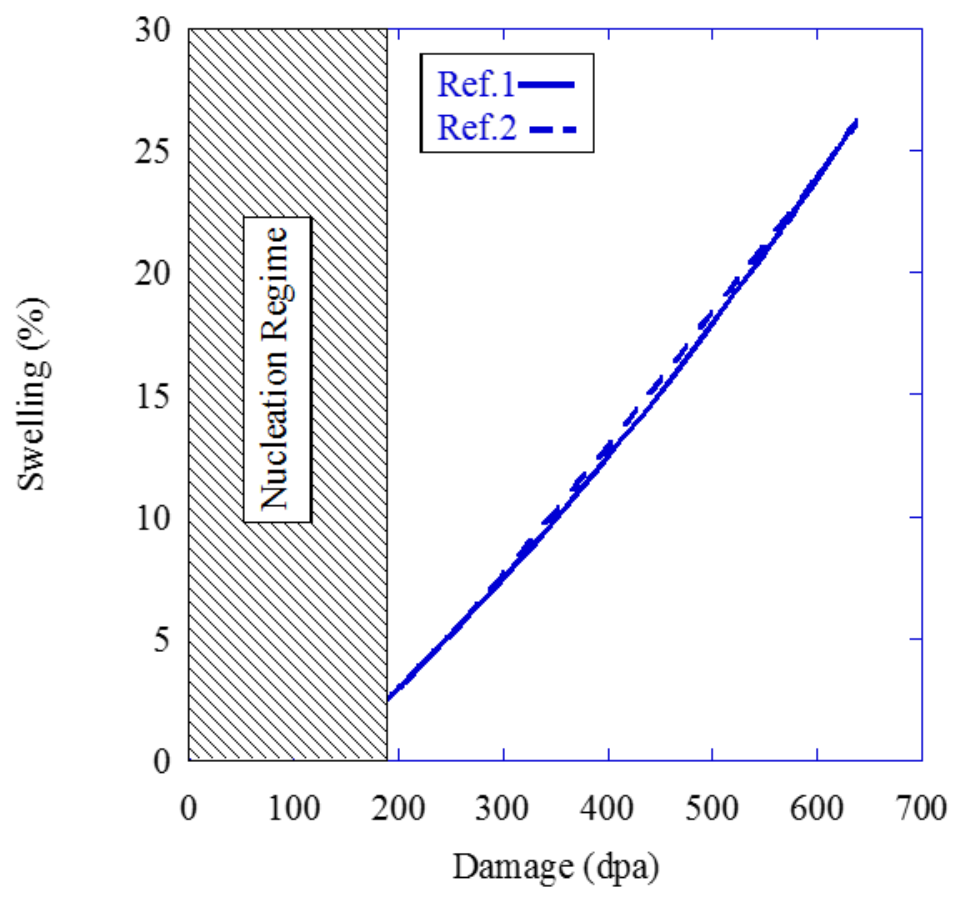

(b)

Figure 7.4: Comparison of Ref.1 (solid) and Ref.2 (dashed) treatments $\left(z_{i}^{l p}=z_{i}^{\text {net }}=1.01\right.$ ) in terms of a) void, network, loop and total dislocation sink strength and $\mathrm{b}$ ) void swelling. 


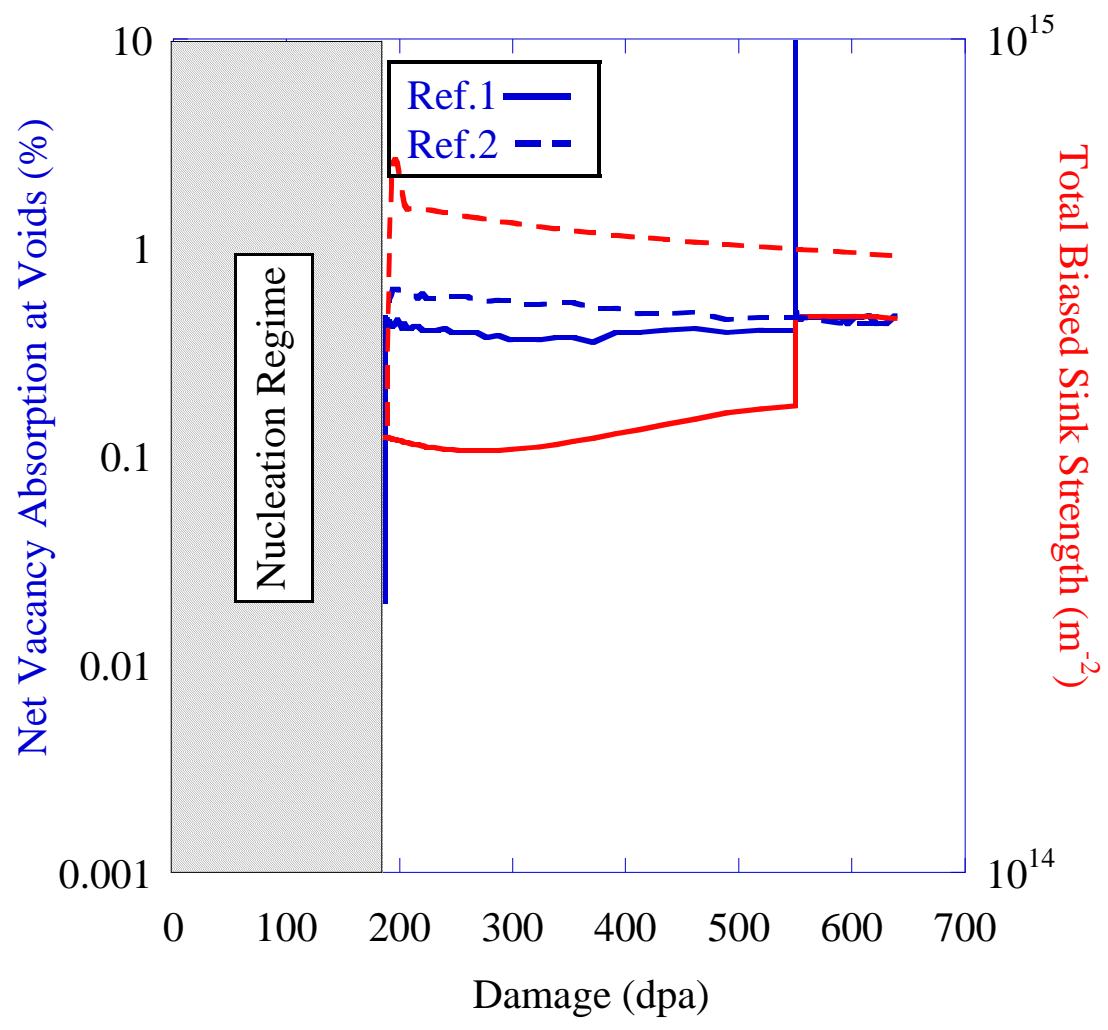

Figure 7.5: Comparison of net vacancies absorbed at voids in Ref.1 (solid) and Ref.2 (dashed). Total dislocation sink strength is included in red. Discontinuity in Ref. 1 case at 550 dpa is due to the manual increase in network sink strength which decreases $d_{i} c_{i}$ and $d_{v} c_{v}$. 


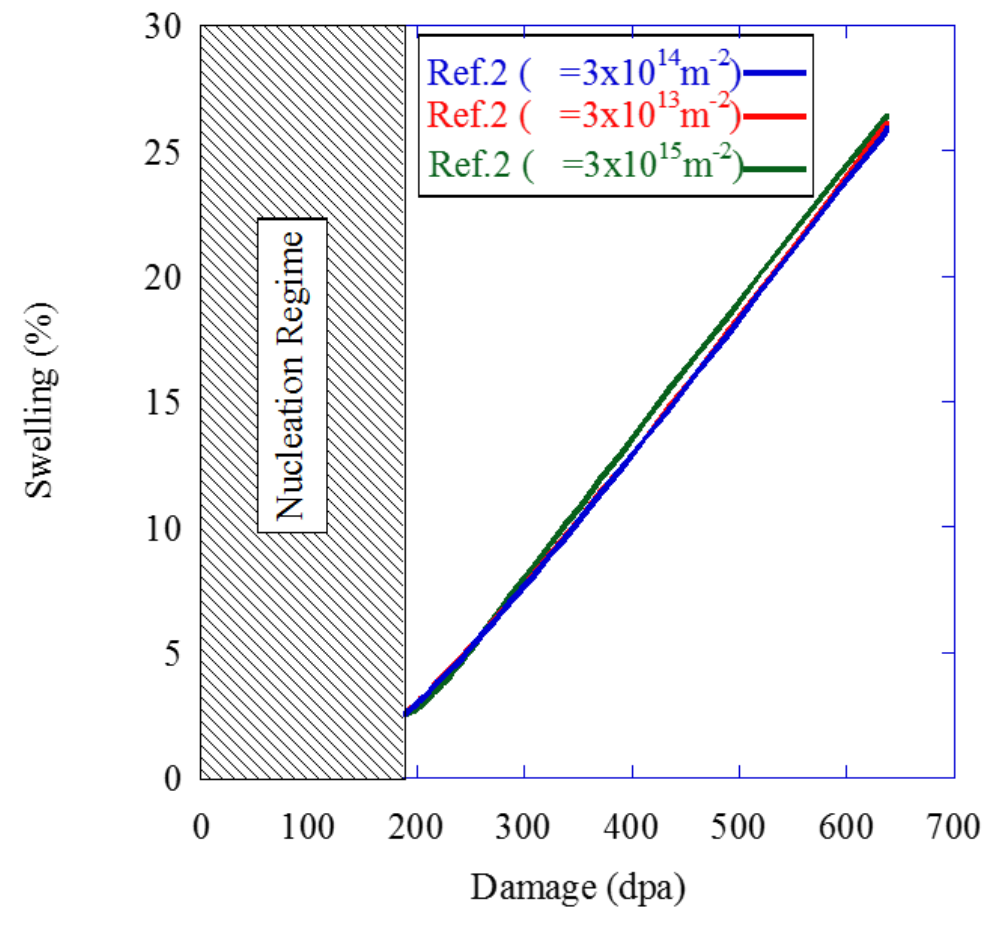

(a)

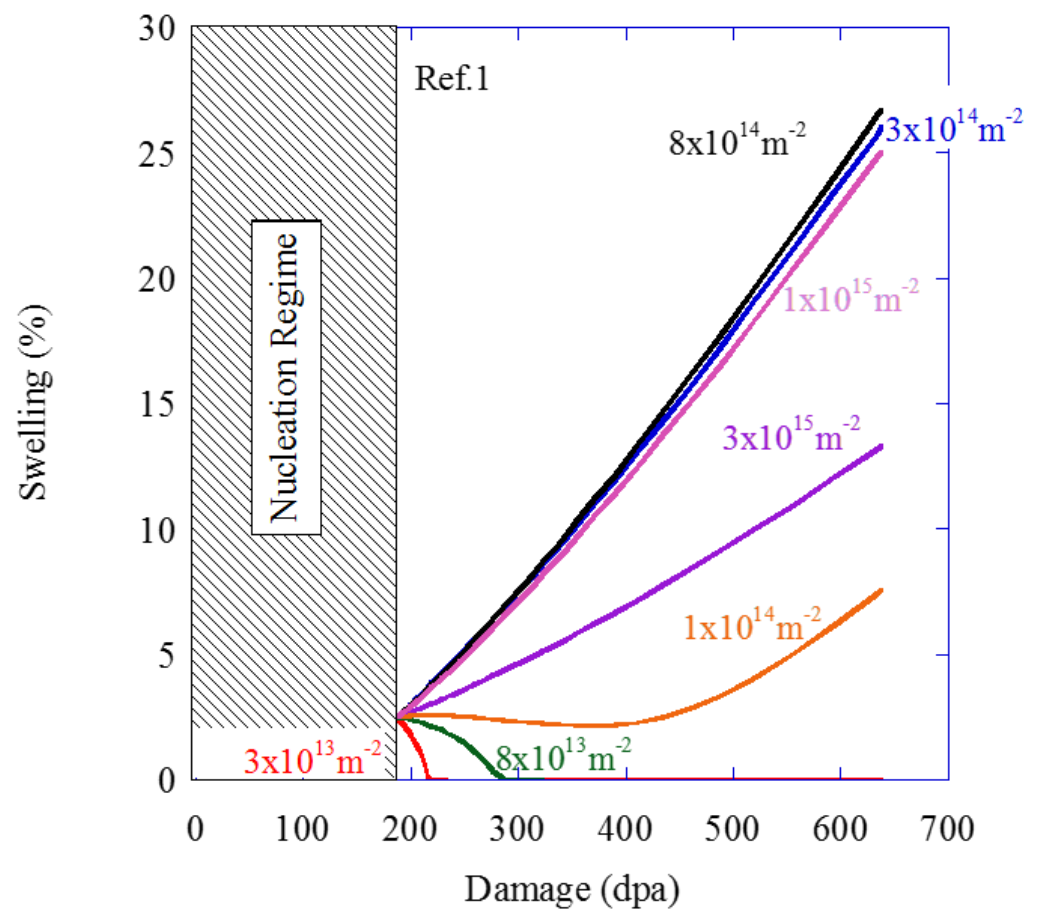

(b)

Figure 7.6: Comparison of swelling response to initial dislocation network density varied from 0.3 to $30 \times 10^{14} \mathrm{~m}^{-2}$ in a) Ref.2 and b) Ref.1. 


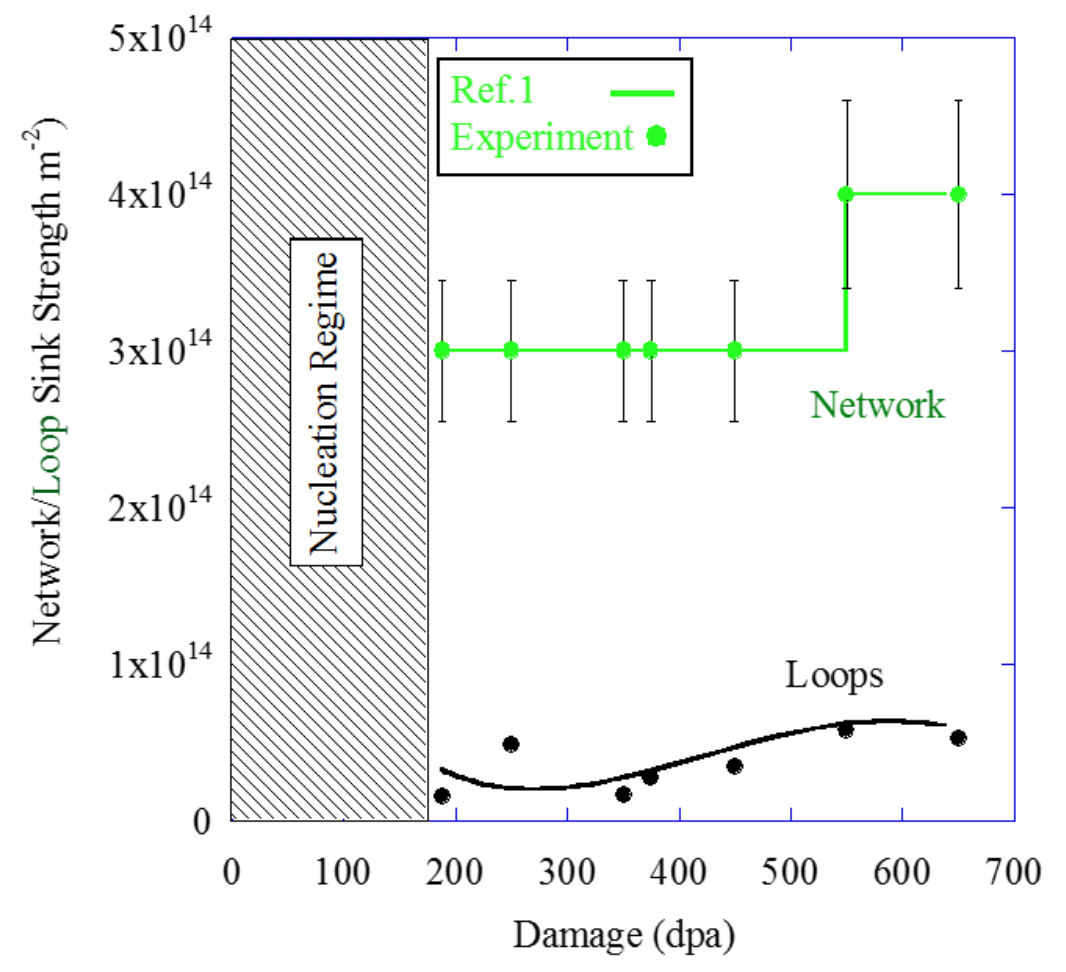

(a)

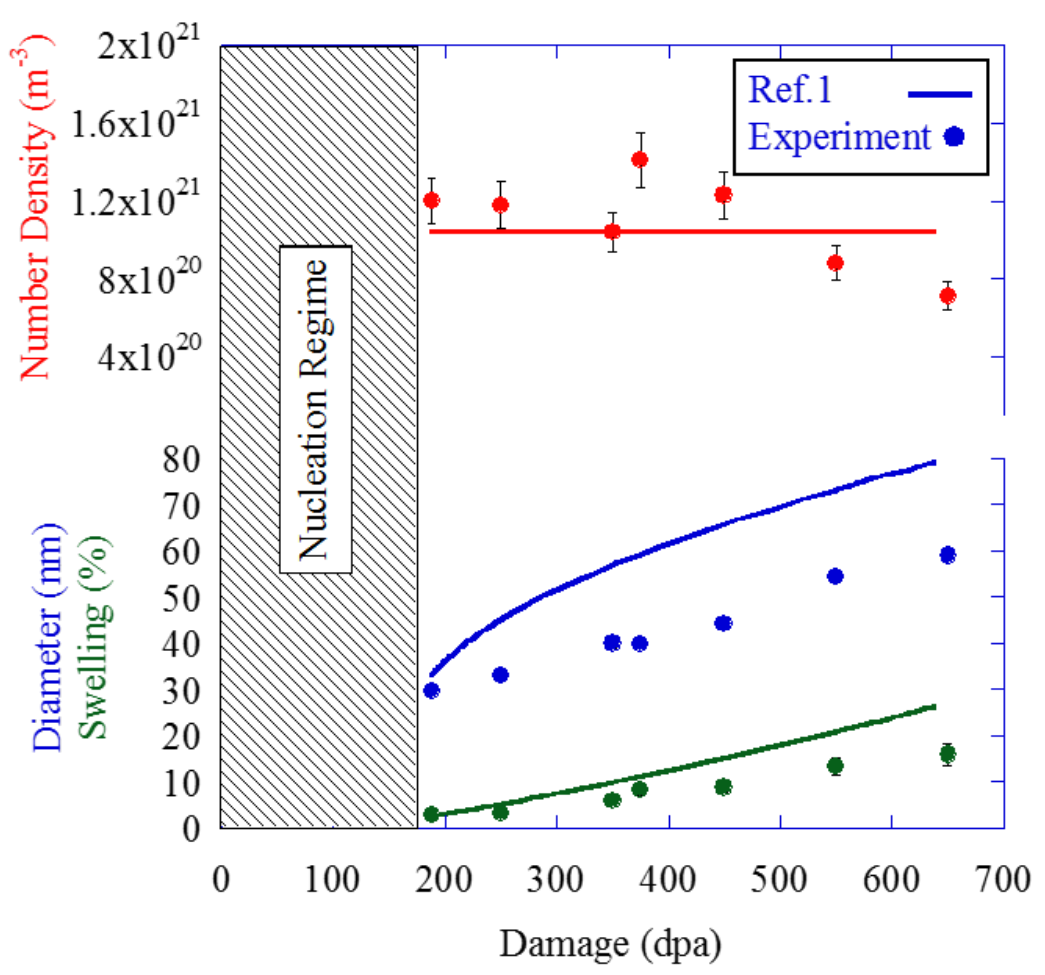

(b)

Figure 7.7: Comparison of experimentally input loop and network treatment (Ref.1: $z_{i}^{l p}=z_{i}^{\text {net }}=1.01$ ) with experimental data in terms of a) network/loop sink strength and b) void diameter, number density and swelling. 


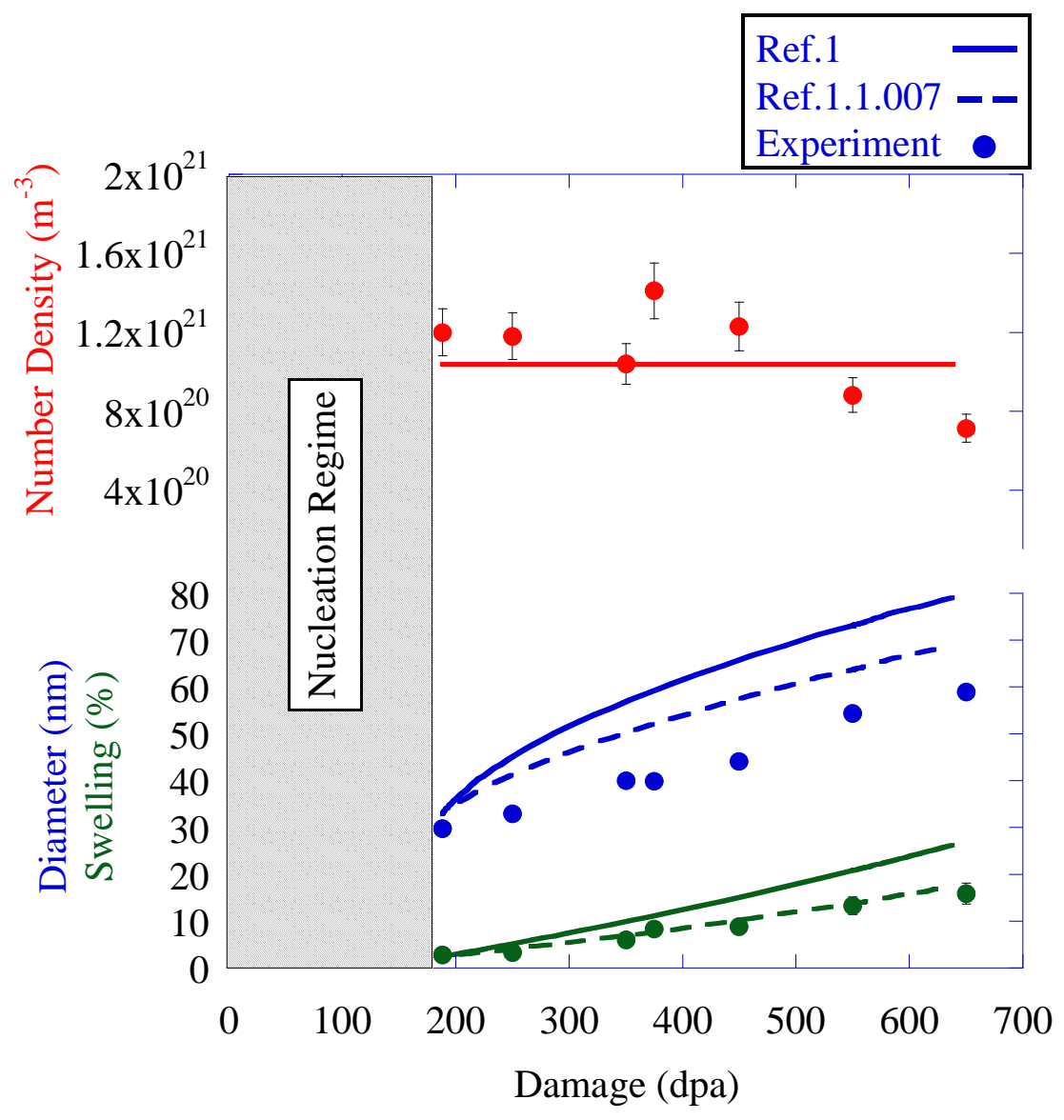

Figure 7.8: Comparison of 1\% (solid lines: Ref.1) and 0.7\% biased (dashed lines: Ref.1.1.007) with experimental data in terms of void diameter, number density and swelling. 


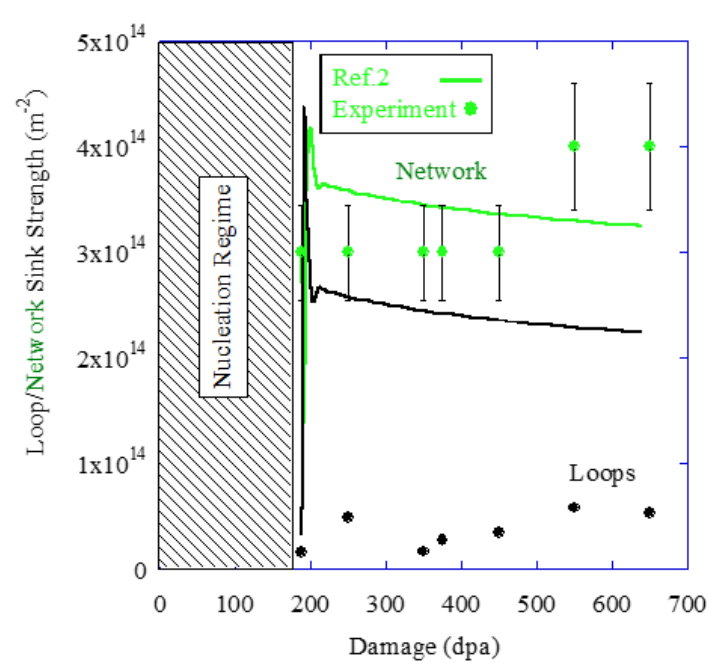

(a)

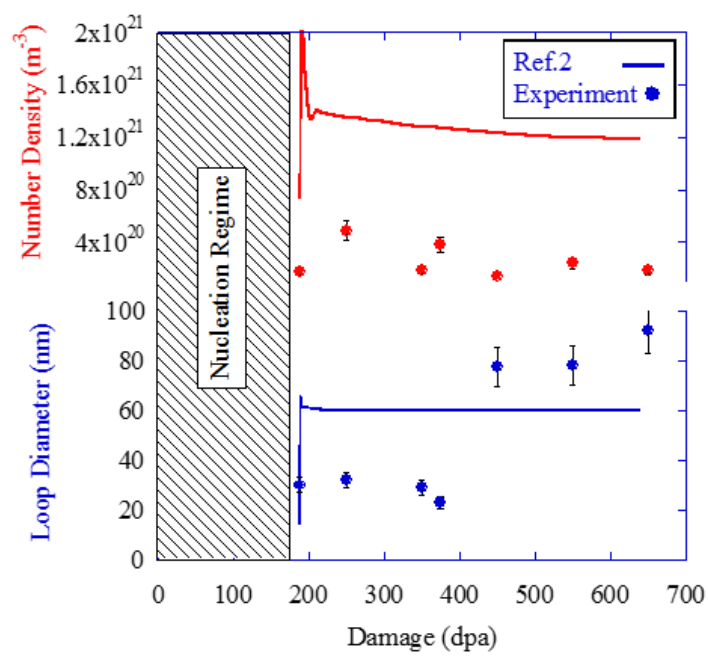

(b)

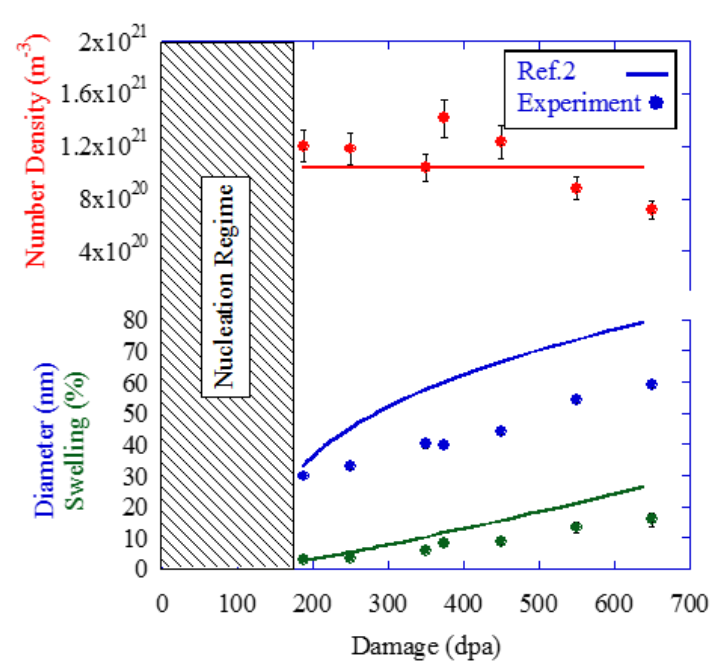

(c)

Figure 7.9: Comparison of DD treatment (Ref.2: $\left.\mathrm{z}_{\mathrm{i}}^{\mathrm{lp}}=\mathrm{z}_{\mathrm{i}}^{\text {net }}=1.01\right)$ with experimental data in terms of a) loop and network sink strength b) loop diameter and number density and c) void diameter, number density and swelling. 


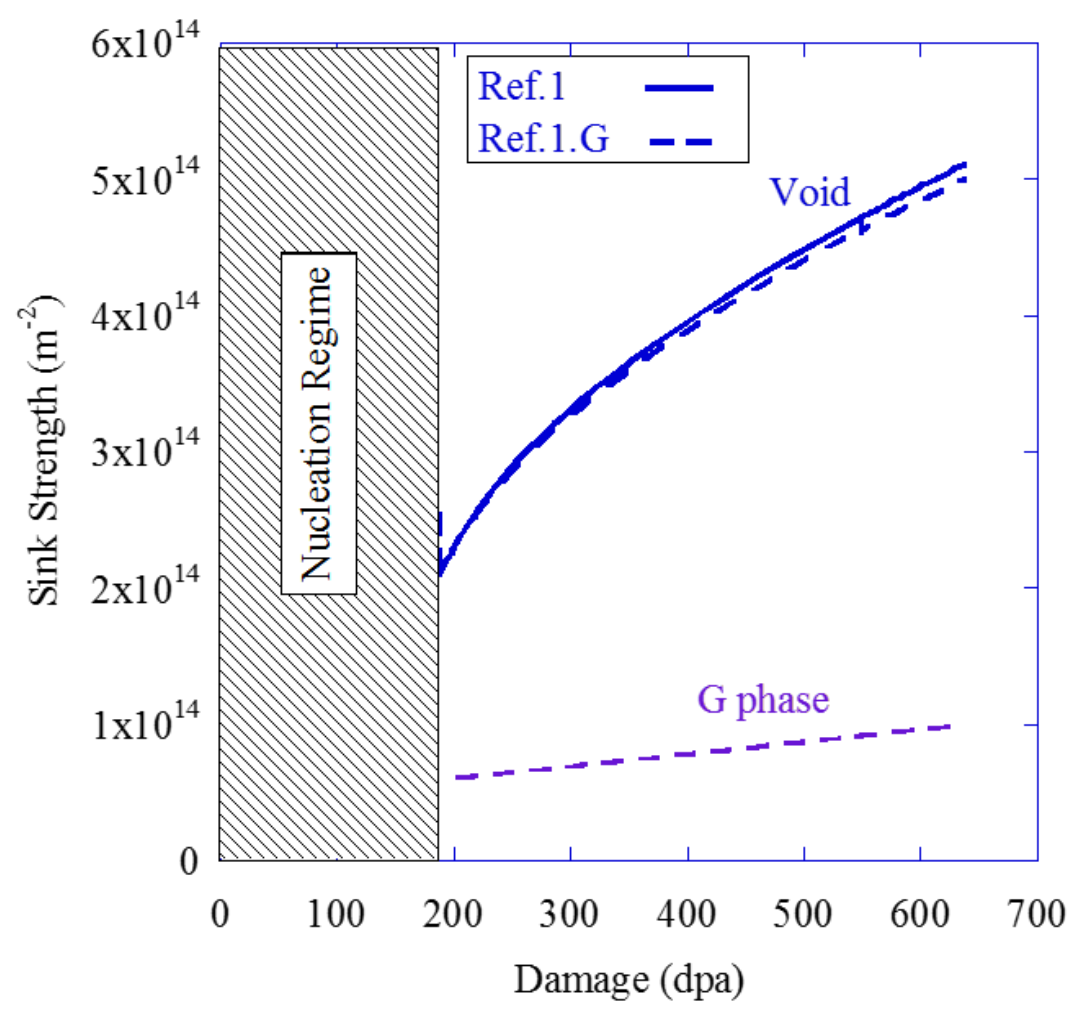

(a)

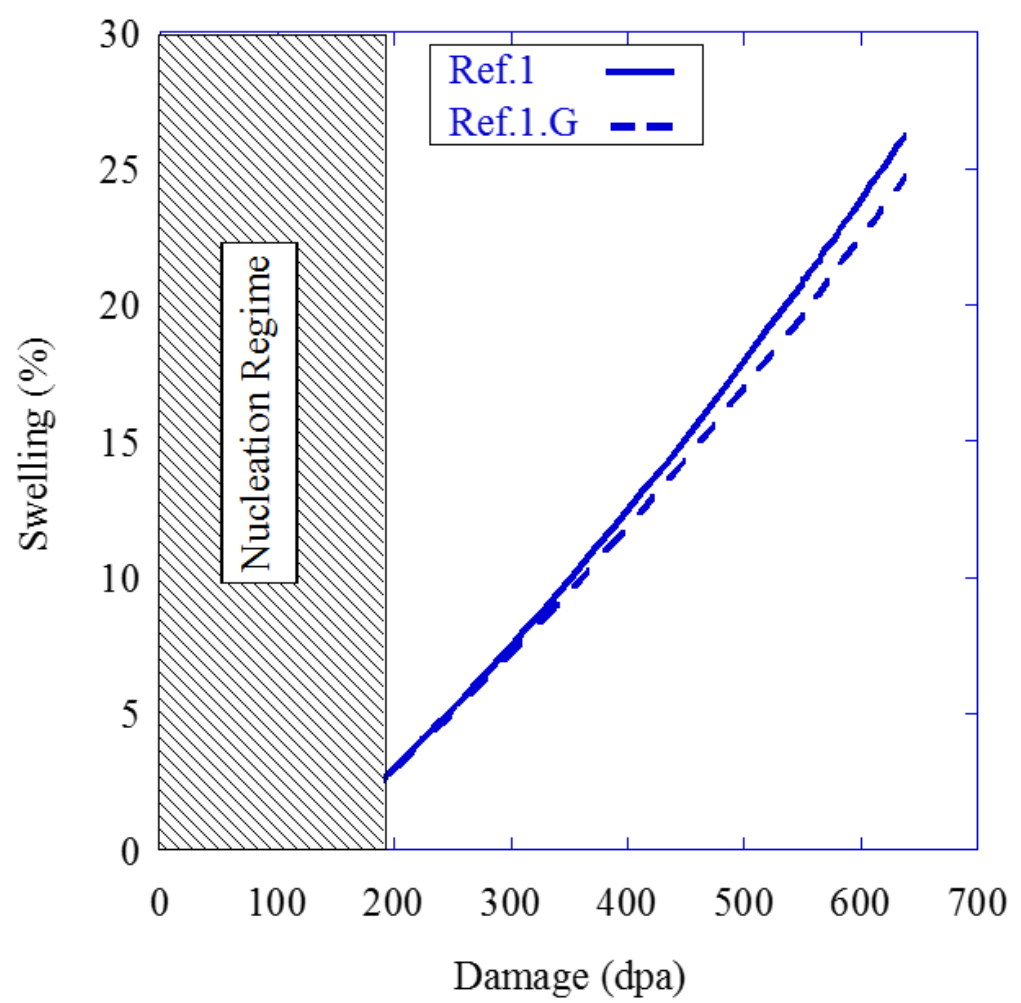

(b)

Figure 7.10: Effect of $G$ phase on void a) sink strength and b) swelling when included in experimentally determined levels as an unbiased sink. Dislocations are treated as experimentally input network and loops with (Ref.1.G: dashed lines) and without G phase (Ref.1: solid) with $\mathrm{z}_{\mathrm{i}}^{\mathrm{lp}}=\mathrm{z}_{\mathrm{i}}^{\text {net }}=1.01$. 


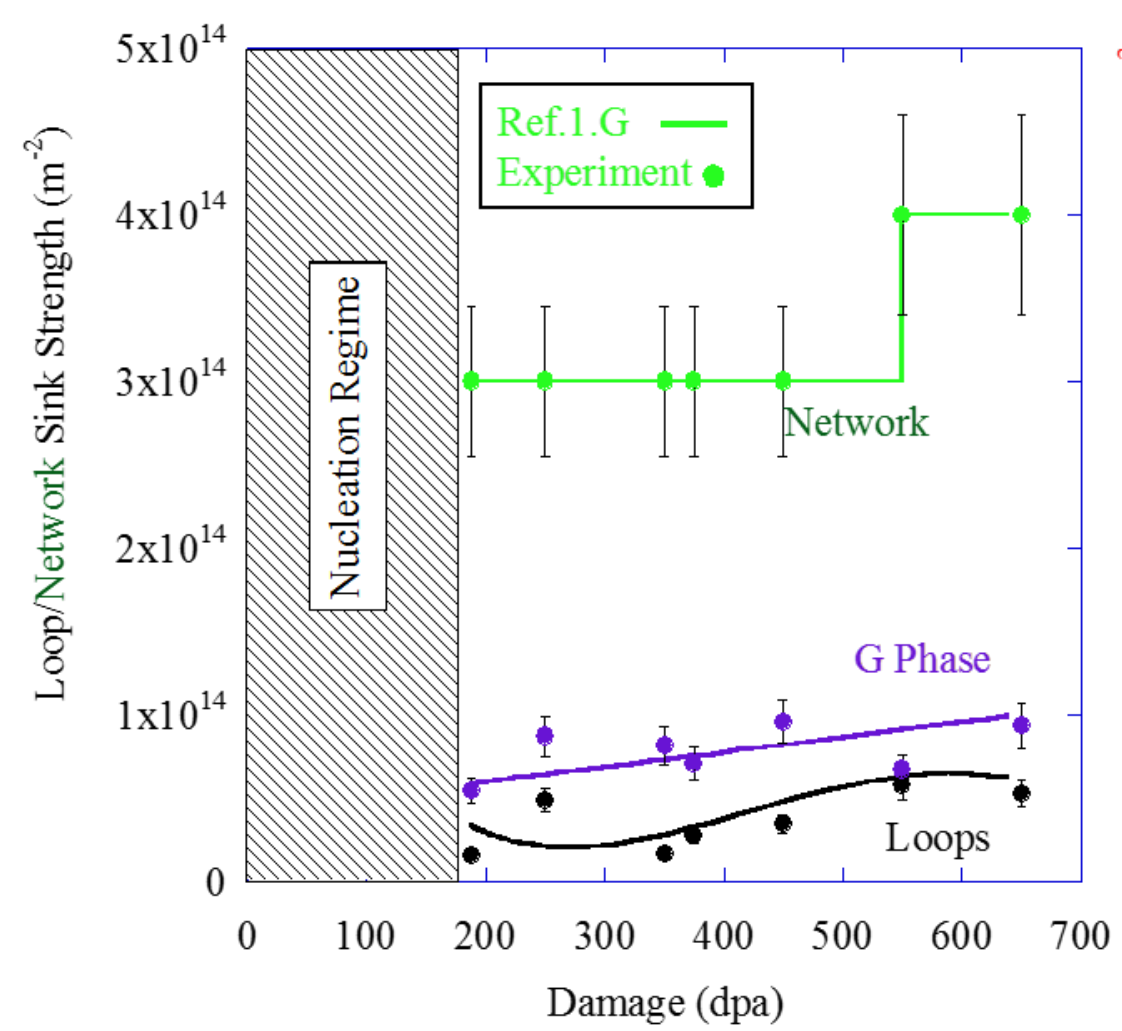

(a)

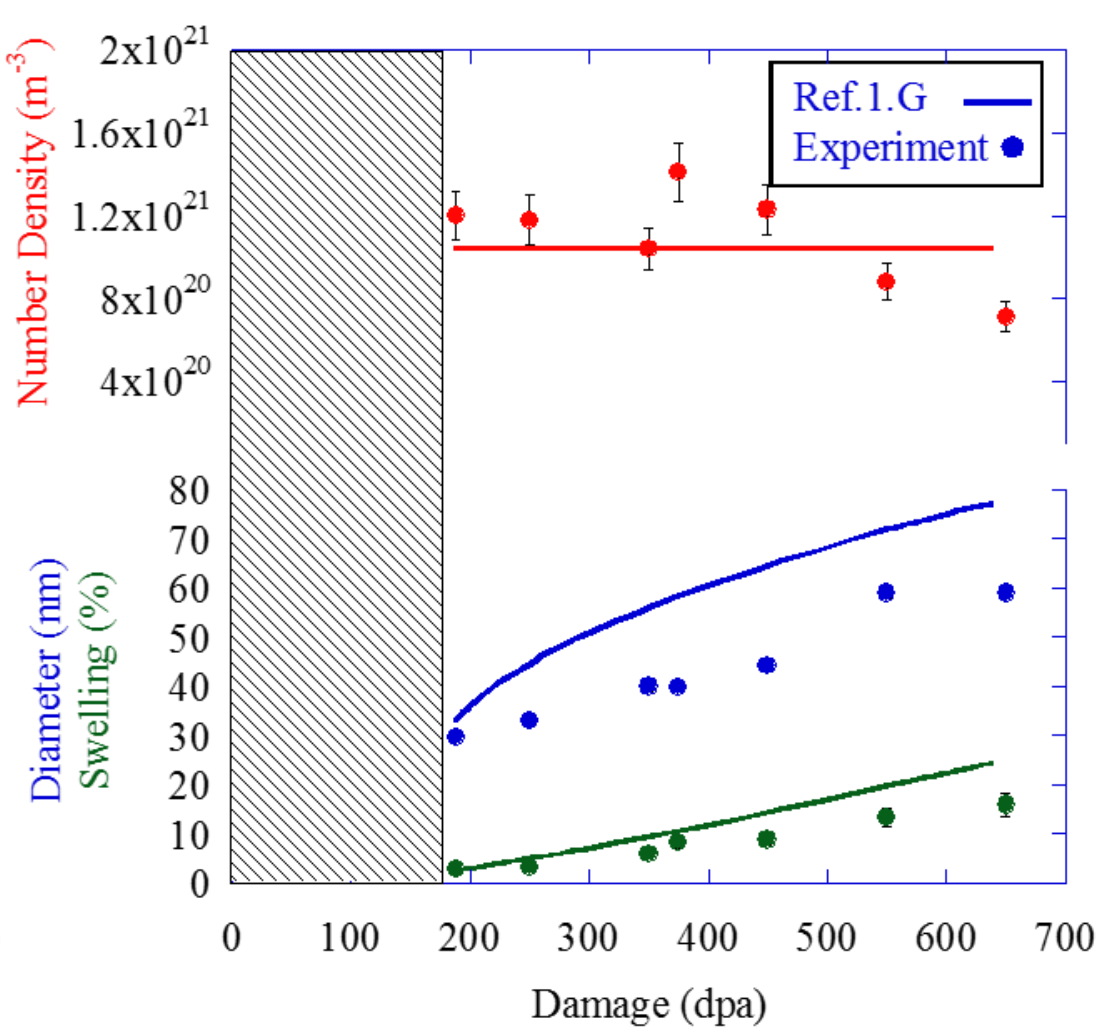

(b)

Figure 7.11: Comparison of experimentally input loop and network treatment with $G$ phase included $\left(\operatorname{Ref} .1 \mathrm{G}: \mathrm{z}_{i}^{\mathrm{lp}}=\mathrm{z}_{\mathrm{i}}^{\text {net }}=1.01\right)$ with experimental data in terms of a) G phase, loop and network sink strength and b) void diameter, number density and swelling. 


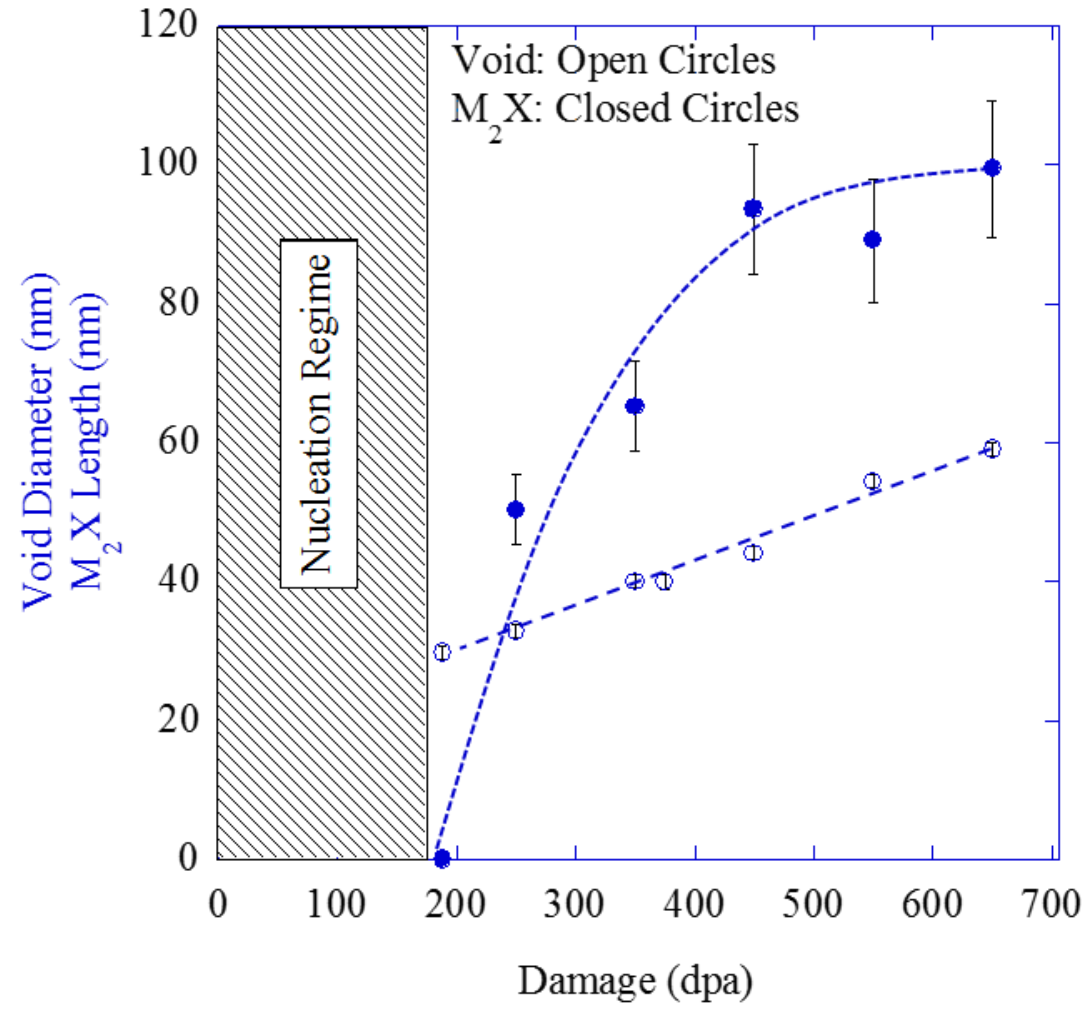

(a)

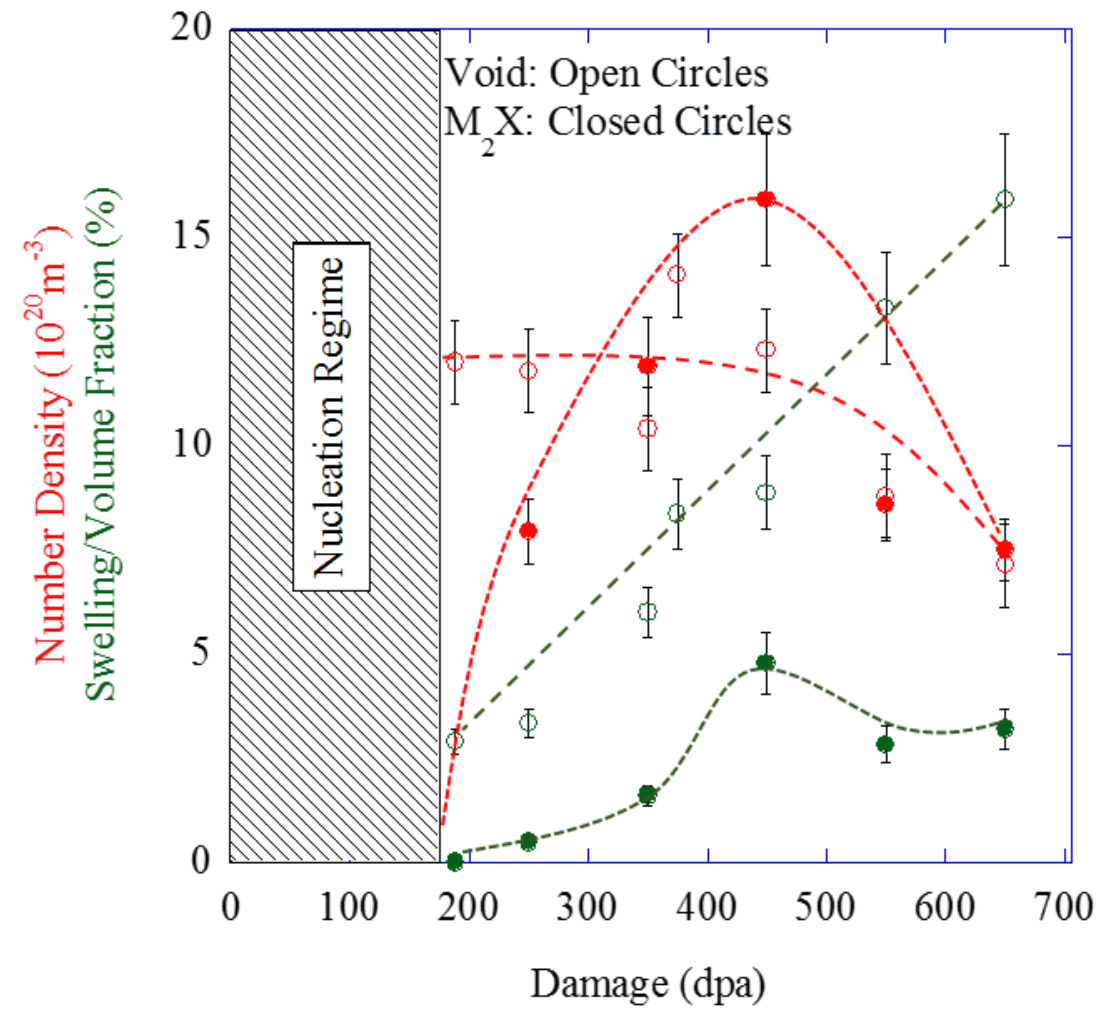

(b)

Figure 7.12: Comparison of evolution of void and $\mathrm{M}_{2} \mathrm{X}$ a) diameter and length and b) number density and volume fraction/swelling from 188 to $650 \mathrm{dpa}$. All irradiations performed with $5 \mathrm{MeV} \mathrm{Fe}^{++}$at $460^{\circ} \mathrm{C}$ with $10 \mathrm{appm} \mathrm{He}$ preimplanted. Lines guide the eye. 


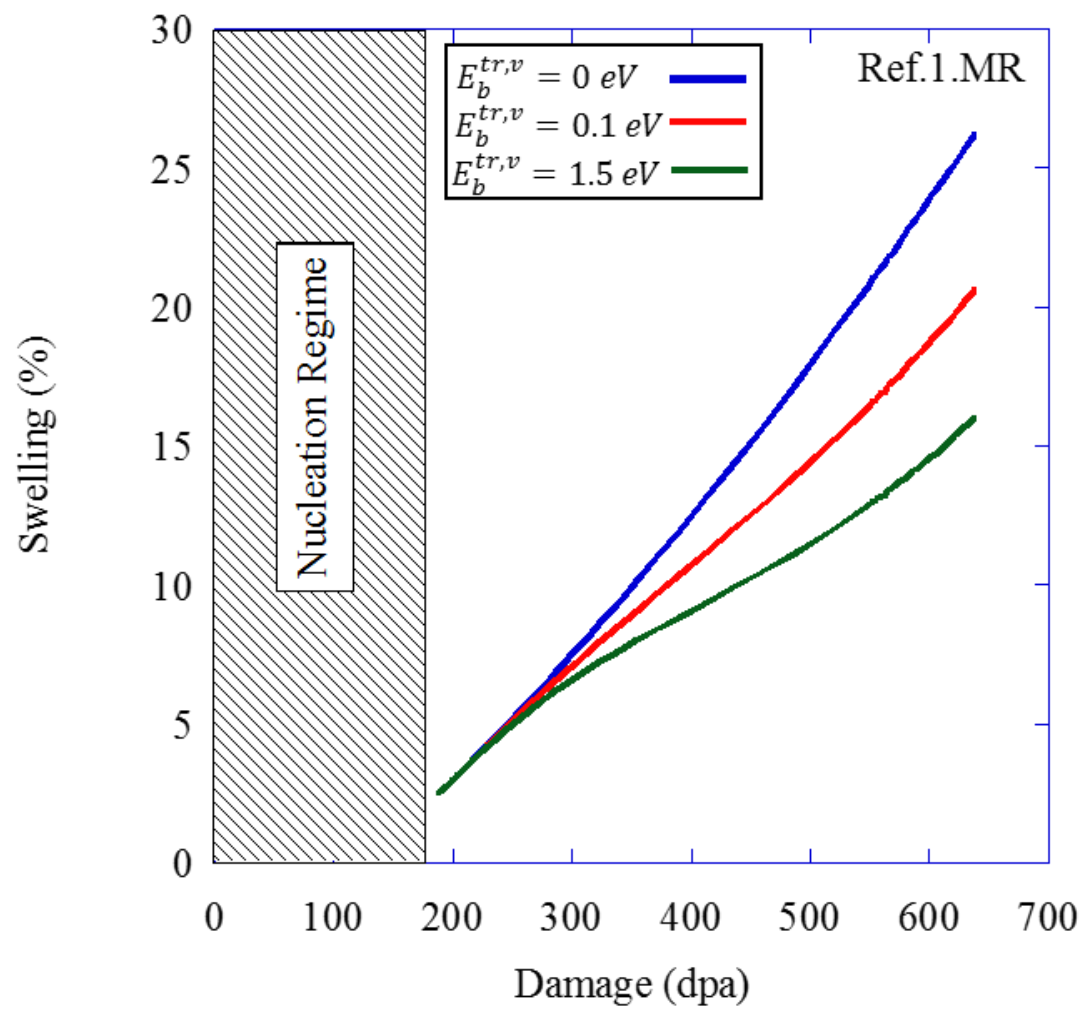

Figure 7.13: Comparison of swelling behavior with increasing $E_{b}^{v, t r}$ using Ref.1.MR with $\mathrm{z}_{\mathrm{i}}^{\mathrm{lp}}=\mathrm{z}_{\mathrm{i}}^{\mathrm{net}}=1.01$. 


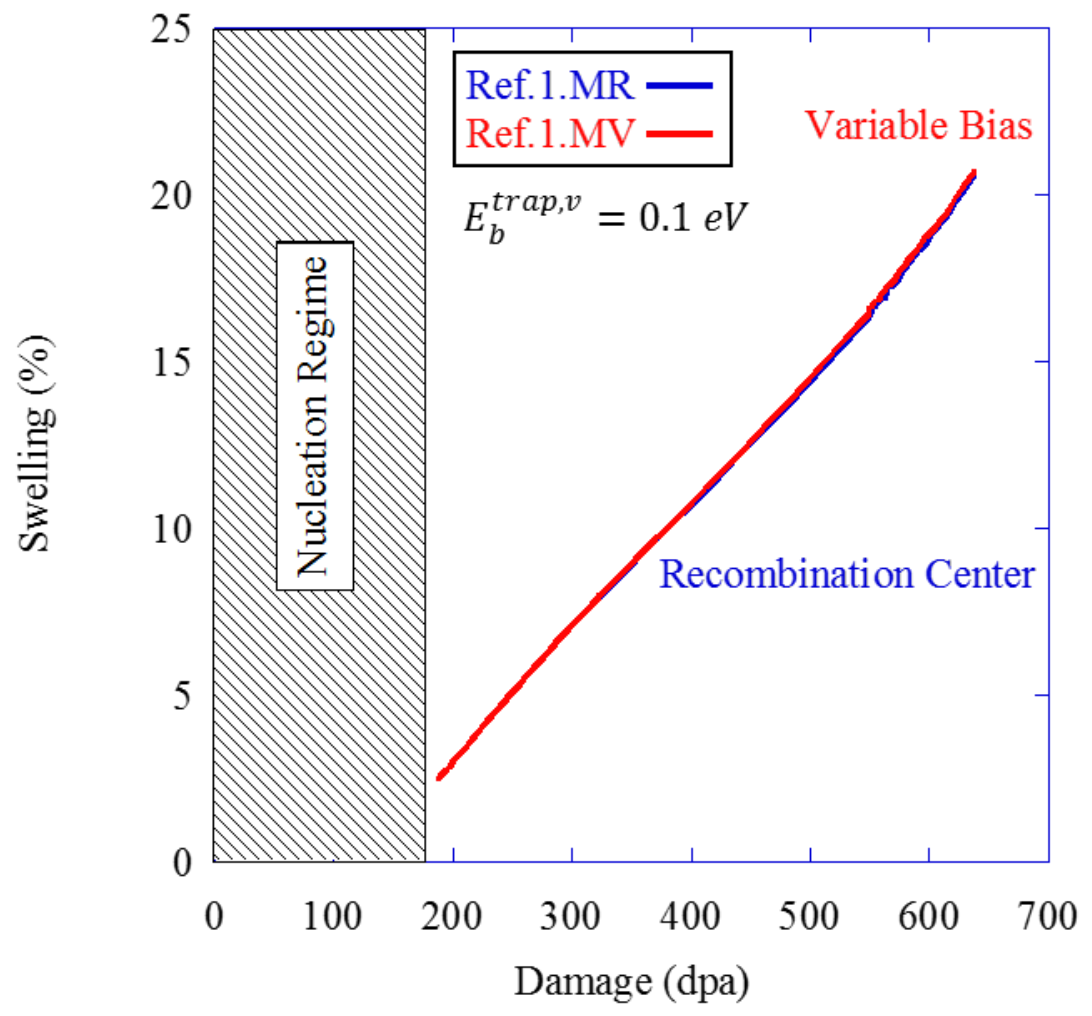

Figure 7.14: Comparison of swelling with $\mathrm{M}_{2} \mathrm{X}$ treated as either variable bias (red) or recombination centers (blue) with experimentally input network and loop with $\mathrm{z}_{\mathrm{i}}^{\mathrm{lp}}=\mathrm{z}_{\mathrm{i}}^{\text {net }}=1.01$. 


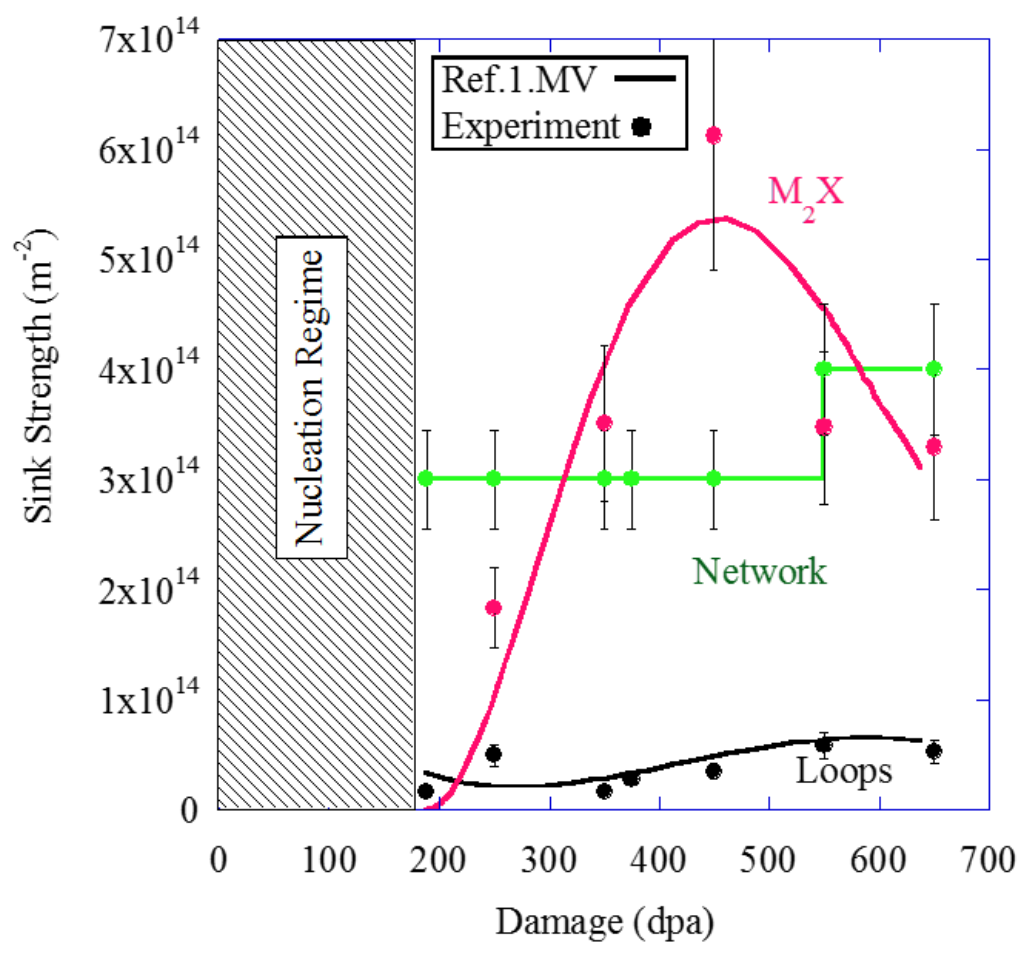

(a)

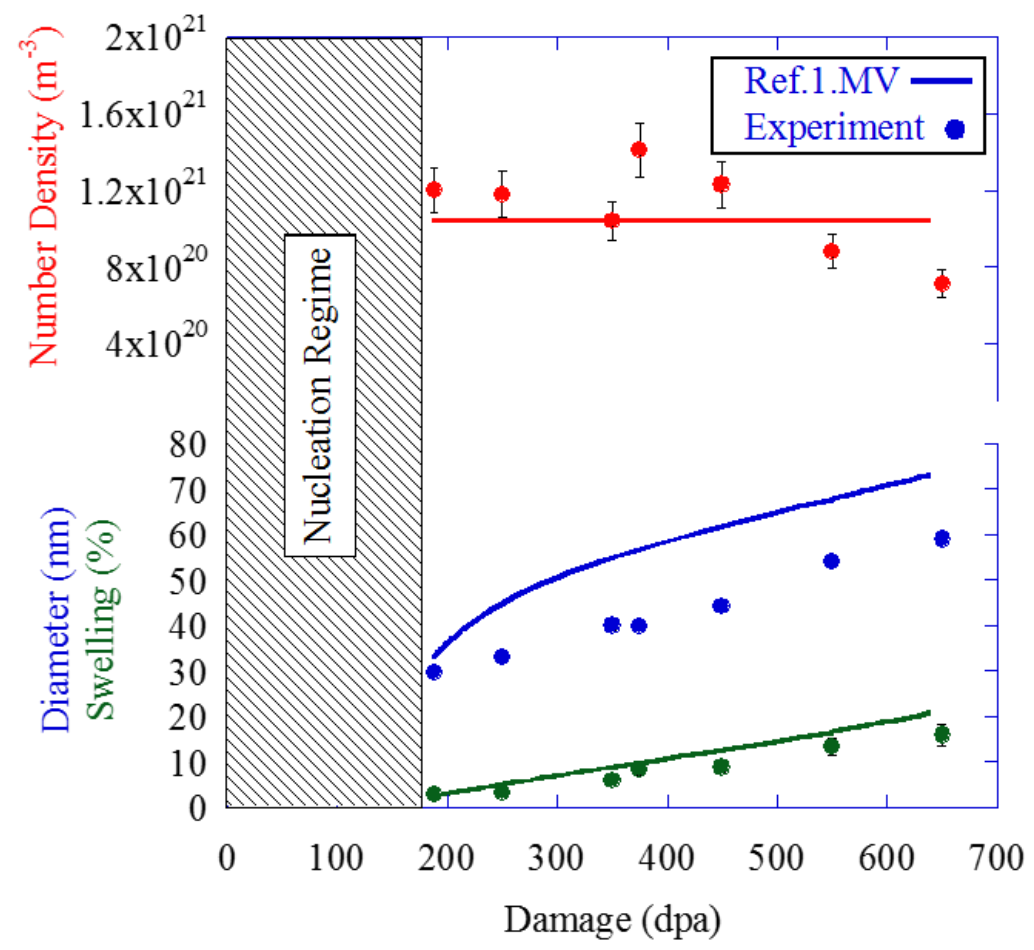

(b)

Figure 7.15: Comparison of Ref.1.MV $\left(z_{i}^{l p}=z_{i}^{n e t}=1.01\right)$ with experimental data in terms of a) $\mathrm{M}_{2} \mathrm{X}$, loop and network sink strength and b) void diameter, number density and swelling. All irradiations performed with $5 \mathrm{MeV} \mathrm{Fe}^{++}$at $460^{\circ} \mathrm{C}$ with 10 appm $\mathrm{He}$ preimplanted. 


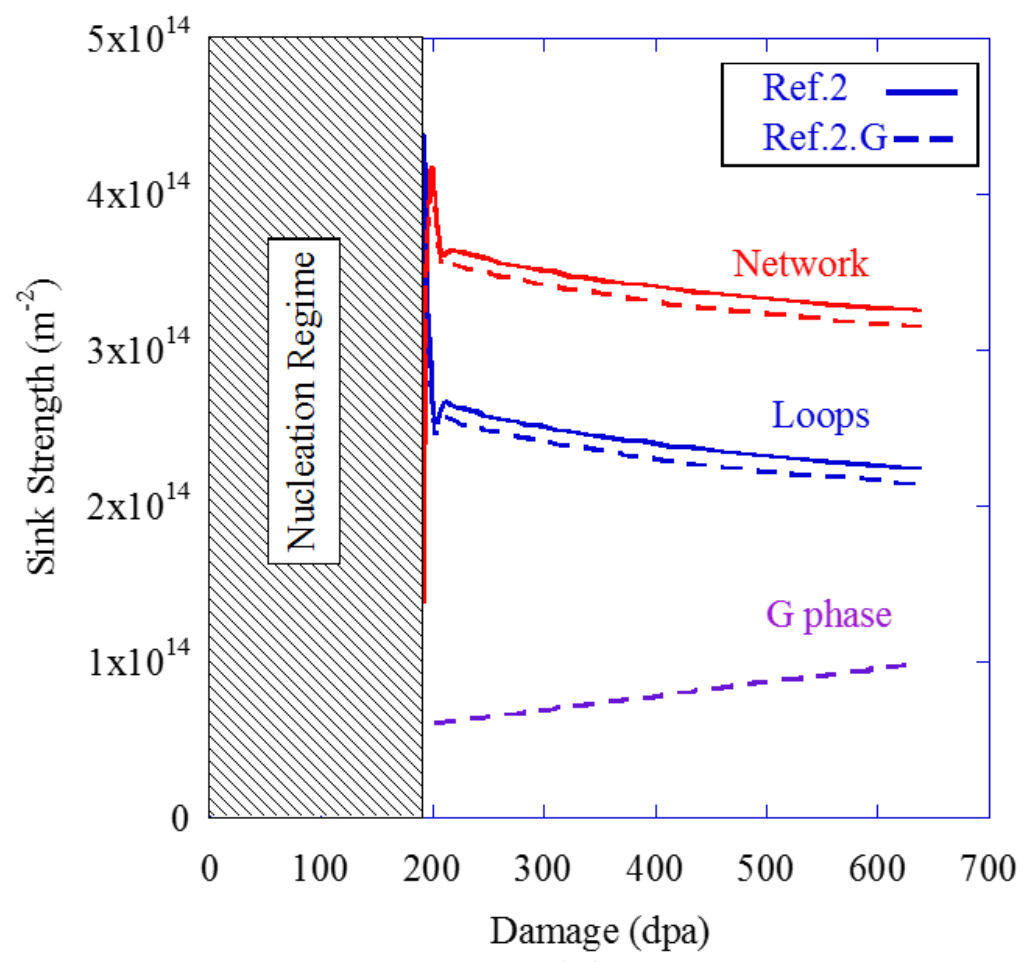

(a)

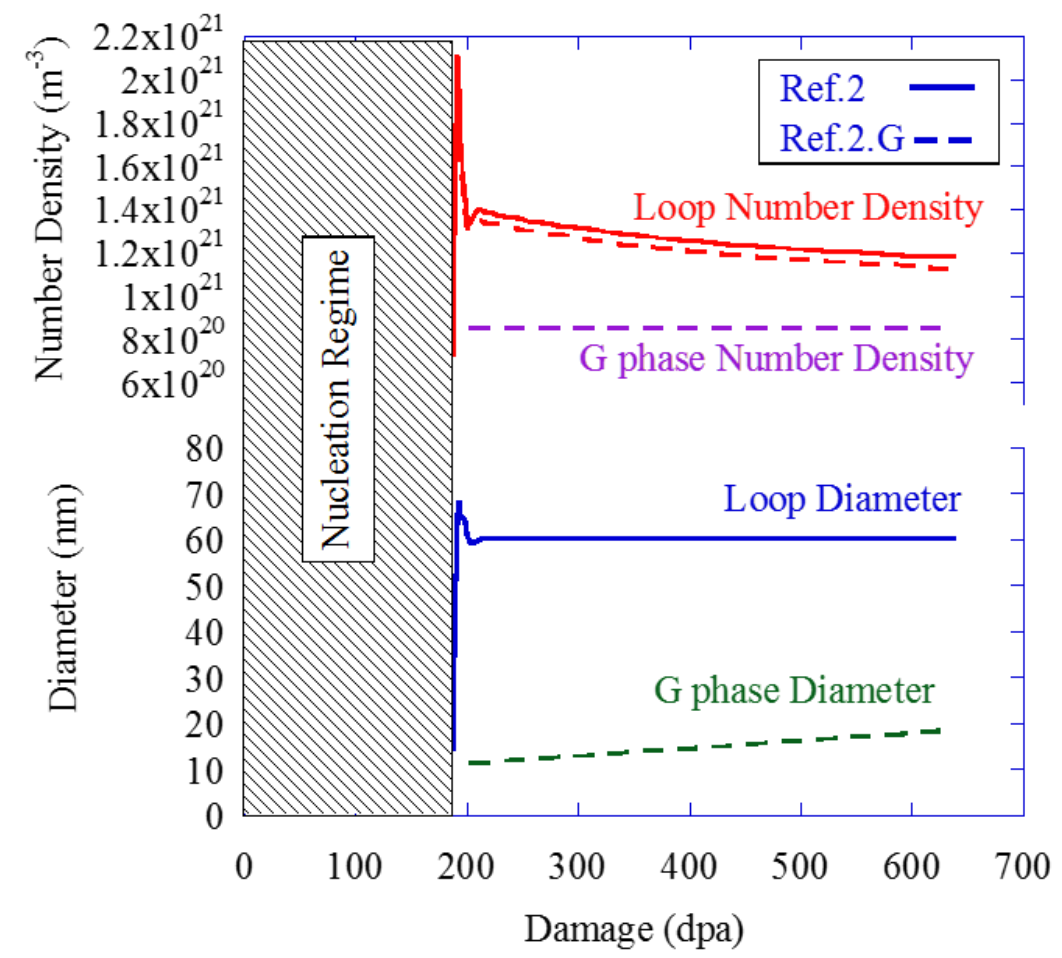

(b)

Figure 7.16: Effect of G phase on network and loop a) sink strength and b) loop number density and diameter when included in experimentally determined levels as an unbiased sink. Dynamic dislocation treatment used both with (Ref.2.G: dashed lines) and without G phase (Ref.2: solid lines) with $\mathrm{z}_{\mathrm{i}}^{\mathrm{lp}}=\mathrm{z}_{\mathrm{i}}^{\text {net }}=1.01$. 


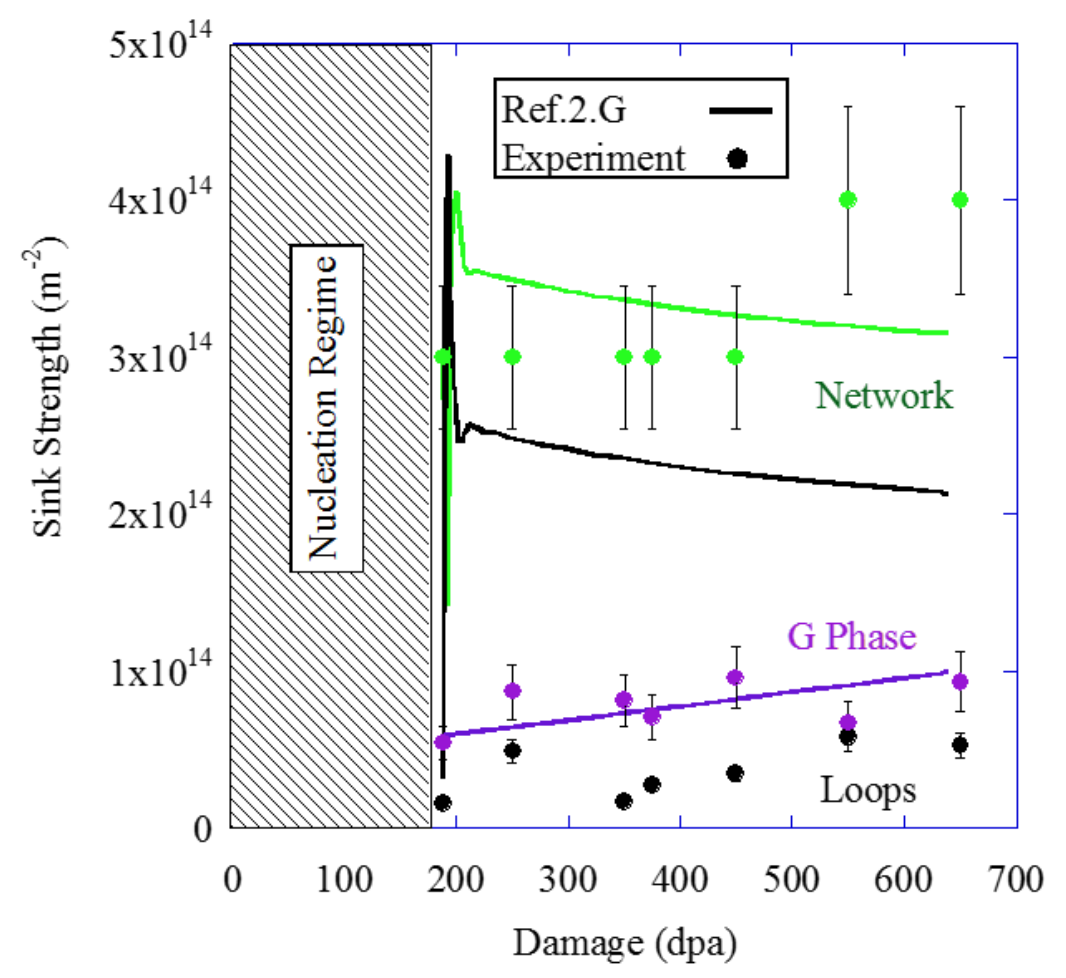

(a)

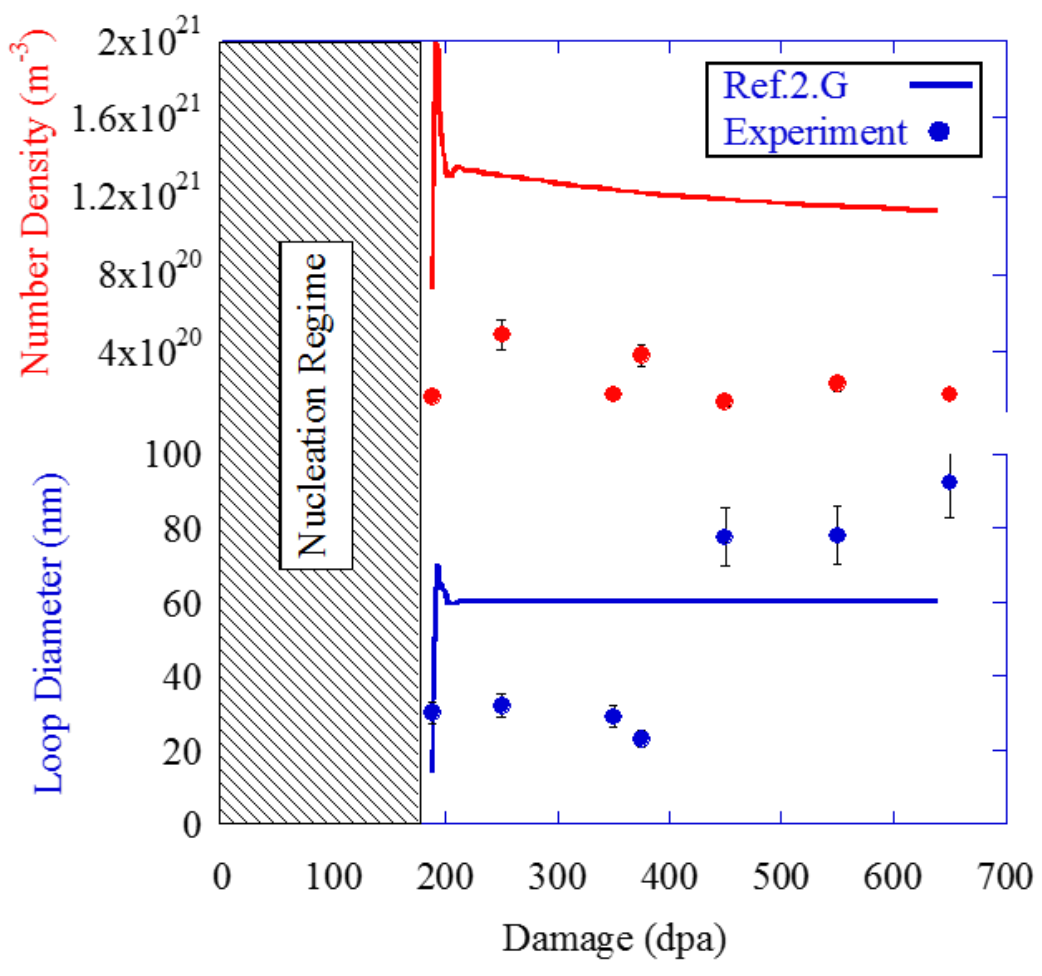

(b)

Figure 7.17: Comparison of Ref.2.G $\left(z_{i}^{l p}=z_{i}^{\text {net }}=1.01\right)$ with experimental data in terms of a) G phase, loop and network sink strength and b) dislocation loop diameter and number density. 


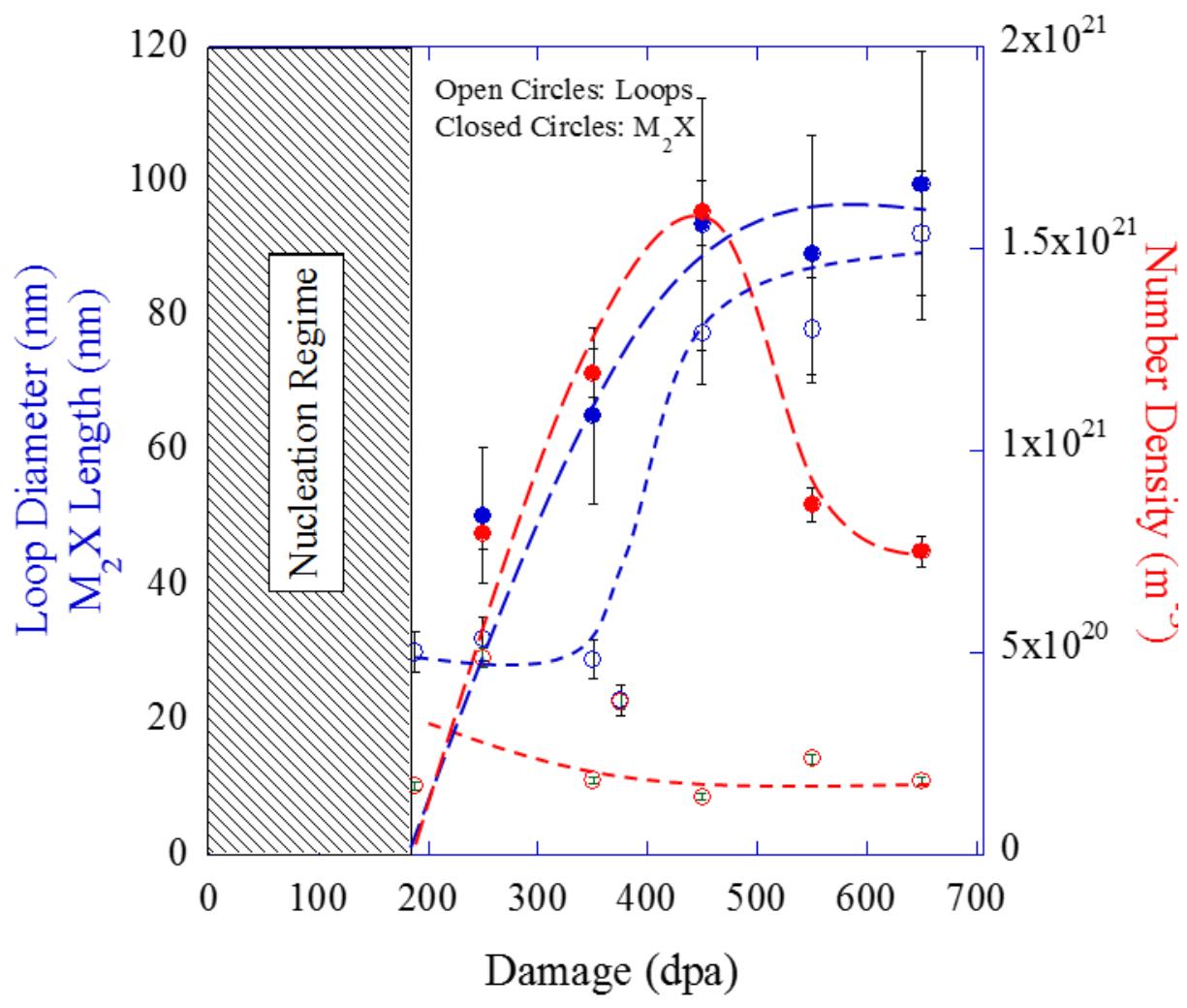

Figure 7.18: Loop and $\mathrm{M}_{2} \mathrm{X}$ diameter/length compared with number density as a function of damage. All irradiations performed with $5 \mathrm{MeV} \mathrm{Fe}^{++}$at $460^{\circ} \mathrm{C}$ with 10 appm He preimplanted. Lines guide the eye. 


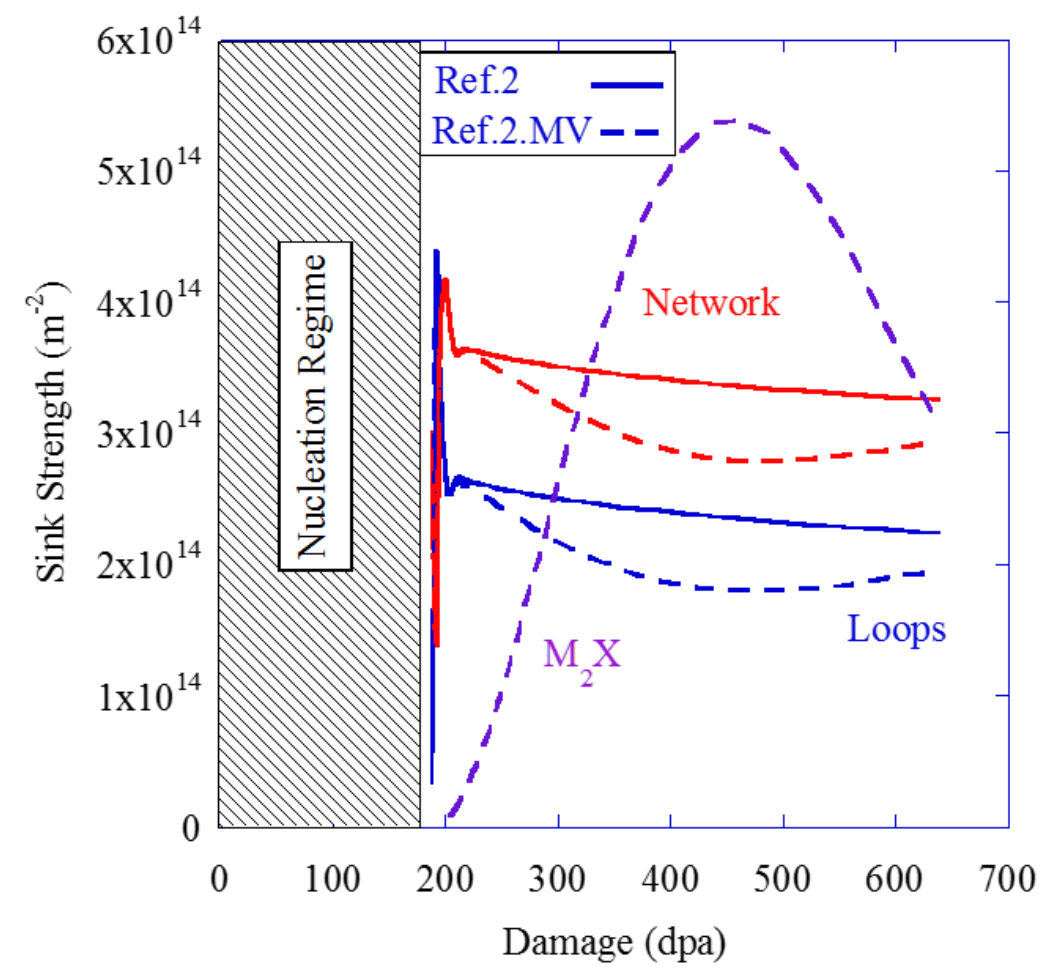

(a)

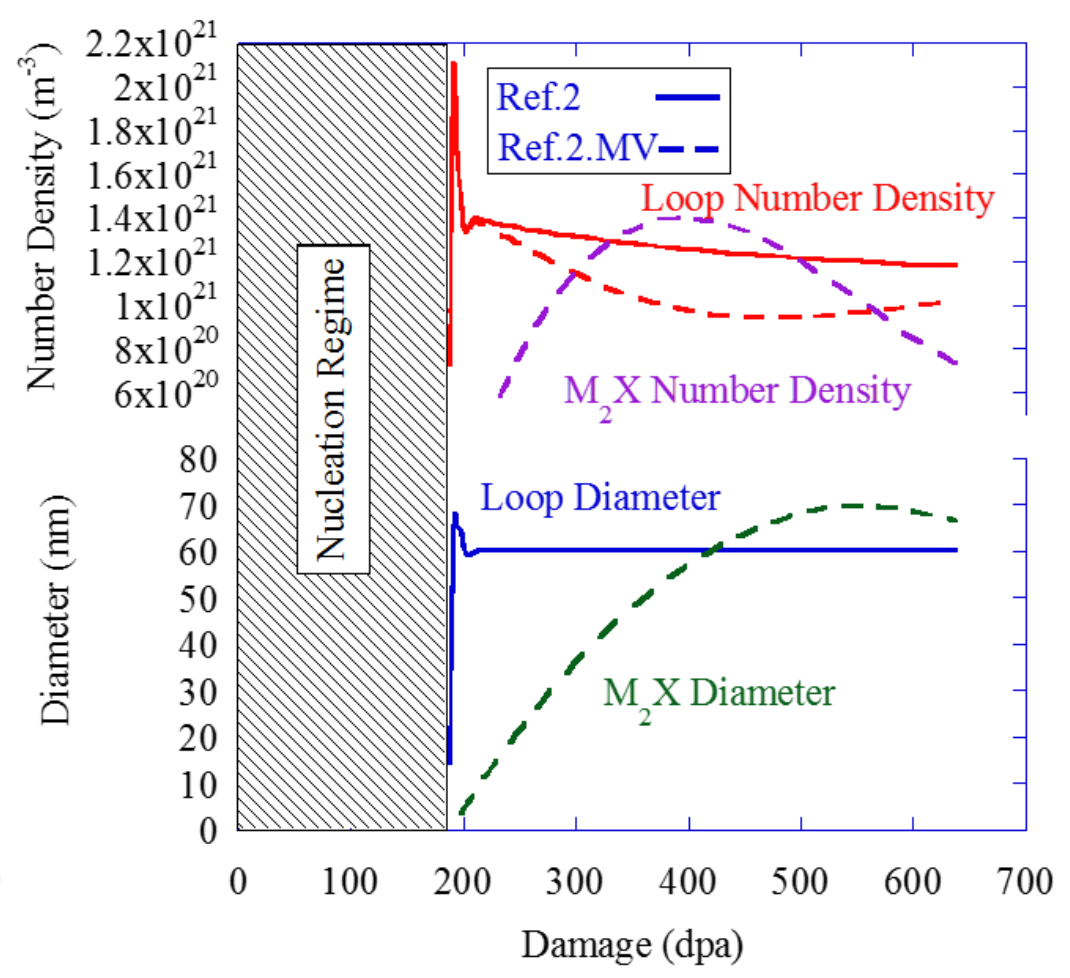

(b)

Figure 7.19: Effect of $\mathrm{M}_{2} \mathrm{X}$ phase on network and loop a) sink strength and b) loop number density and diameter when included in experimentally determined levels as a variably biased sink. Dynamic dislocation treatment used with (Ref.2.MV: dashed lines) and without $\mathrm{M}_{2} \mathrm{X}$ phase (Ref.2: solid lines) with $\mathrm{z}_{\mathrm{i}}^{\mathrm{lp}}=\mathrm{z}_{\mathrm{i}}^{\text {net }}=1.01$. 


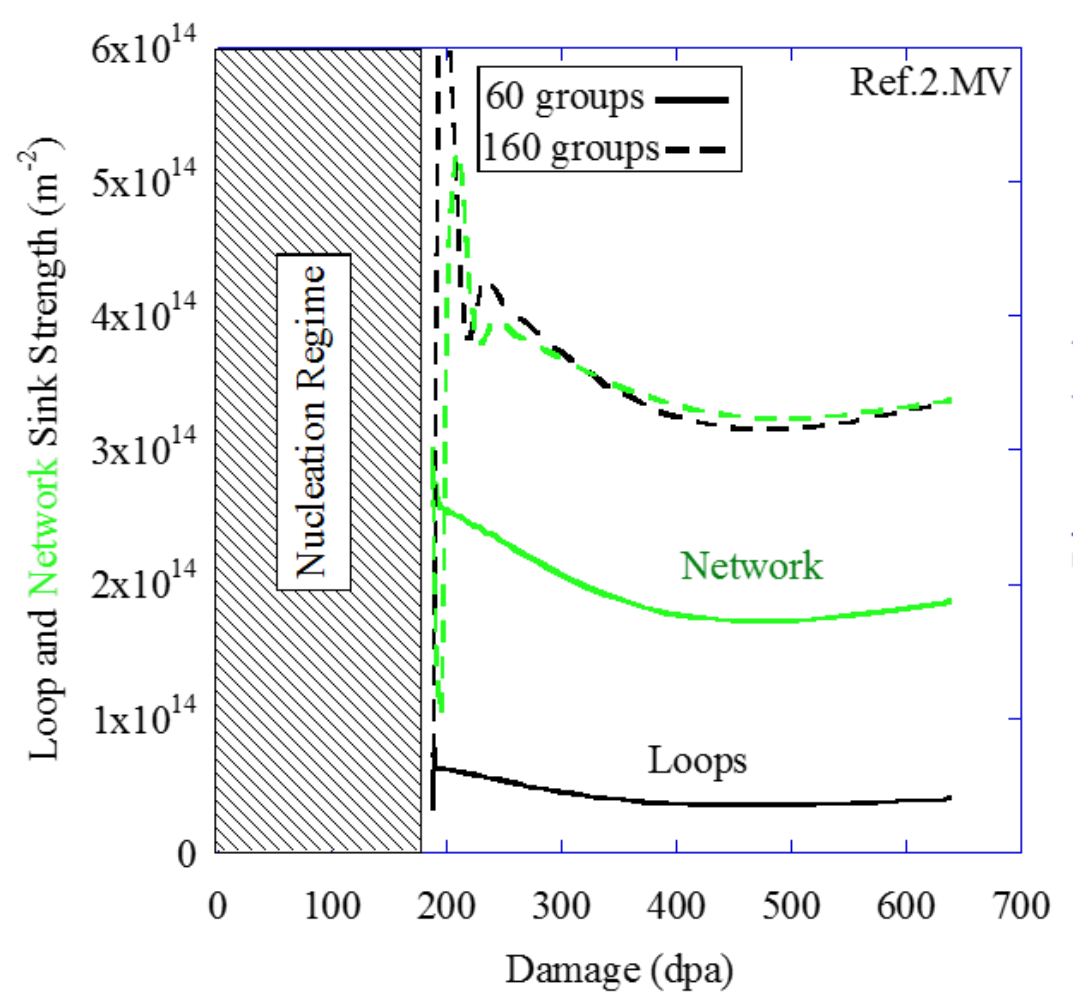

(a)

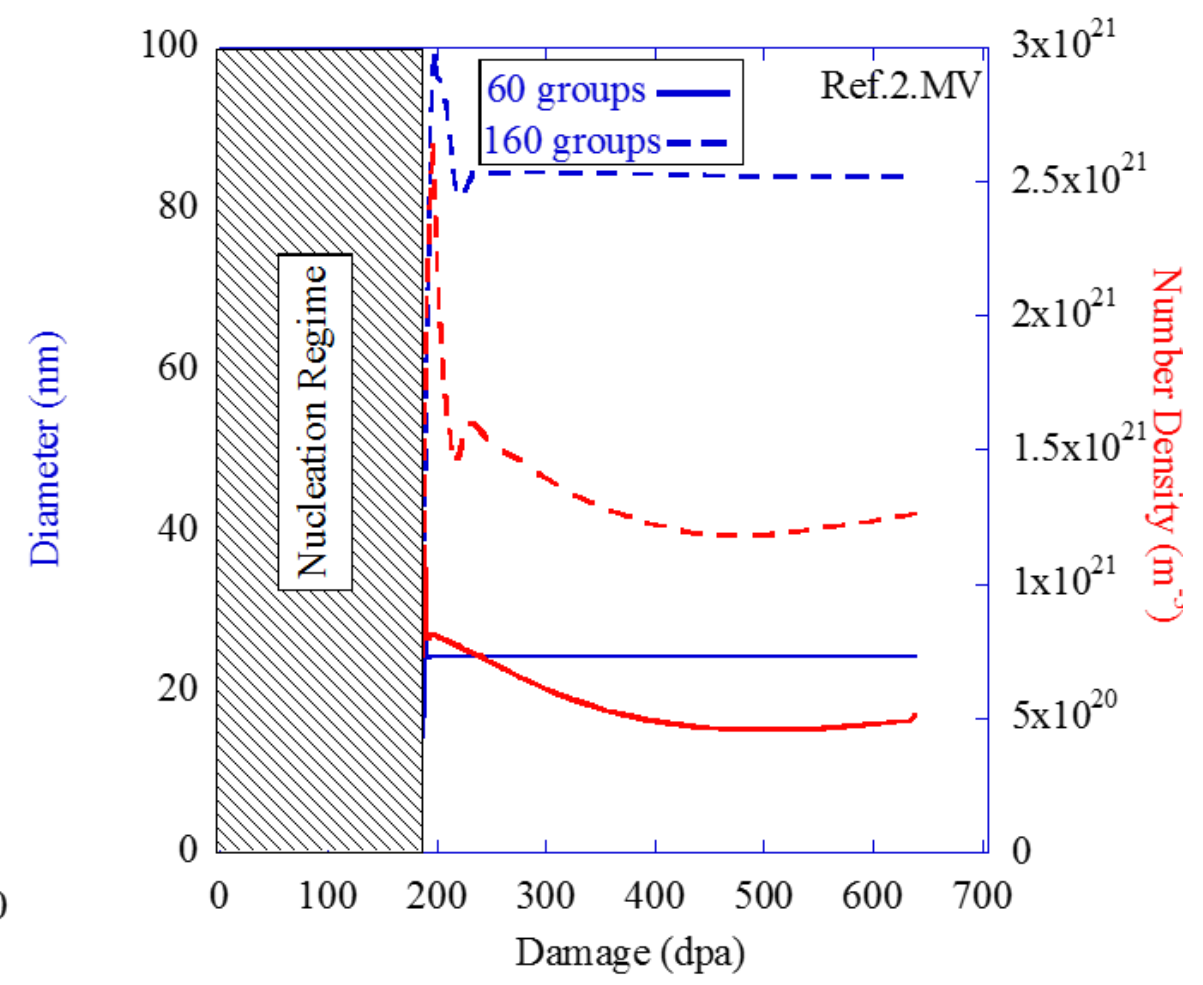

(b)

Figure 7.20: Effect of changing number of interstitial cluster groups from 60 (solid) to 160 (dashed) with DD treatment (Ref.2.MV: $\left.\mathrm{z}_{\mathrm{i}}^{\mathrm{lp}}=\mathrm{z}_{\mathrm{i}}^{\text {net }}=1.01\right) . \mathrm{M}_{2} \mathrm{X}$ treatment as variable bias sink was used. 


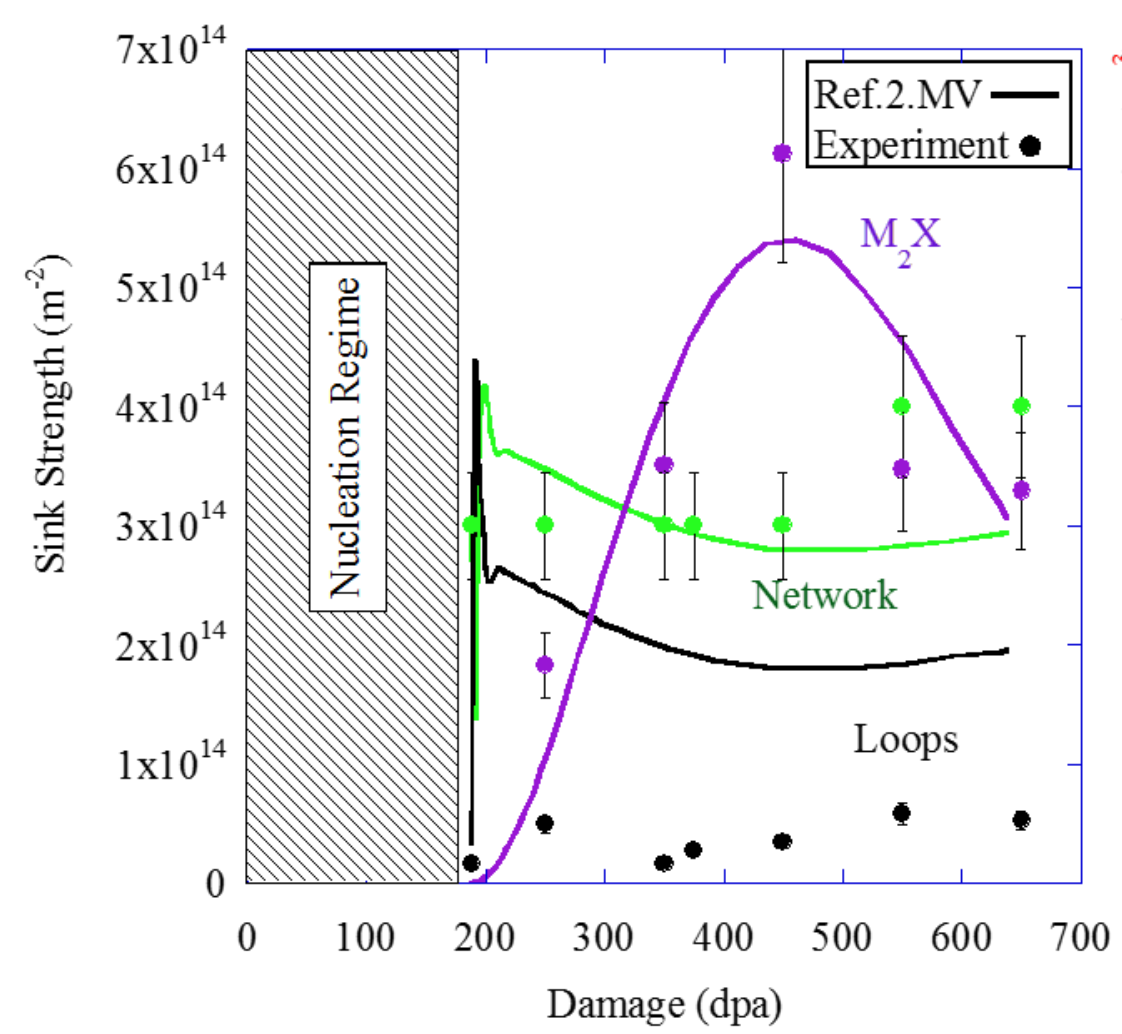

(a)

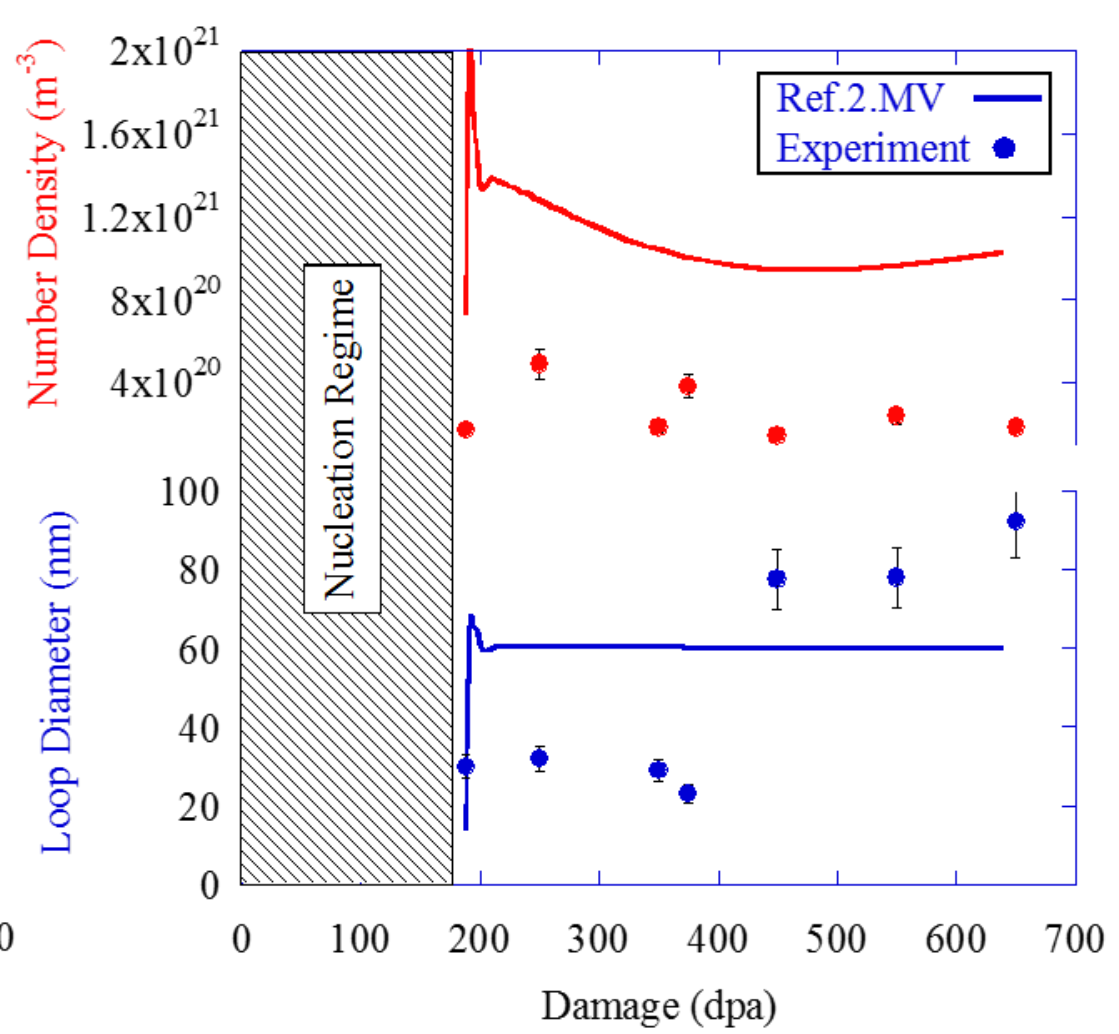

(b)

Figure 7.21: Comparison of Ref.2.MV $\left(z_{i}^{l p}=z_{i}^{n e t}=1.01\right)$ with experimental data in terms of a) $\mathrm{M}_{2} \mathrm{X}$, loop and network sink strength and $b$ ) dislocation loop diameter and number density. 


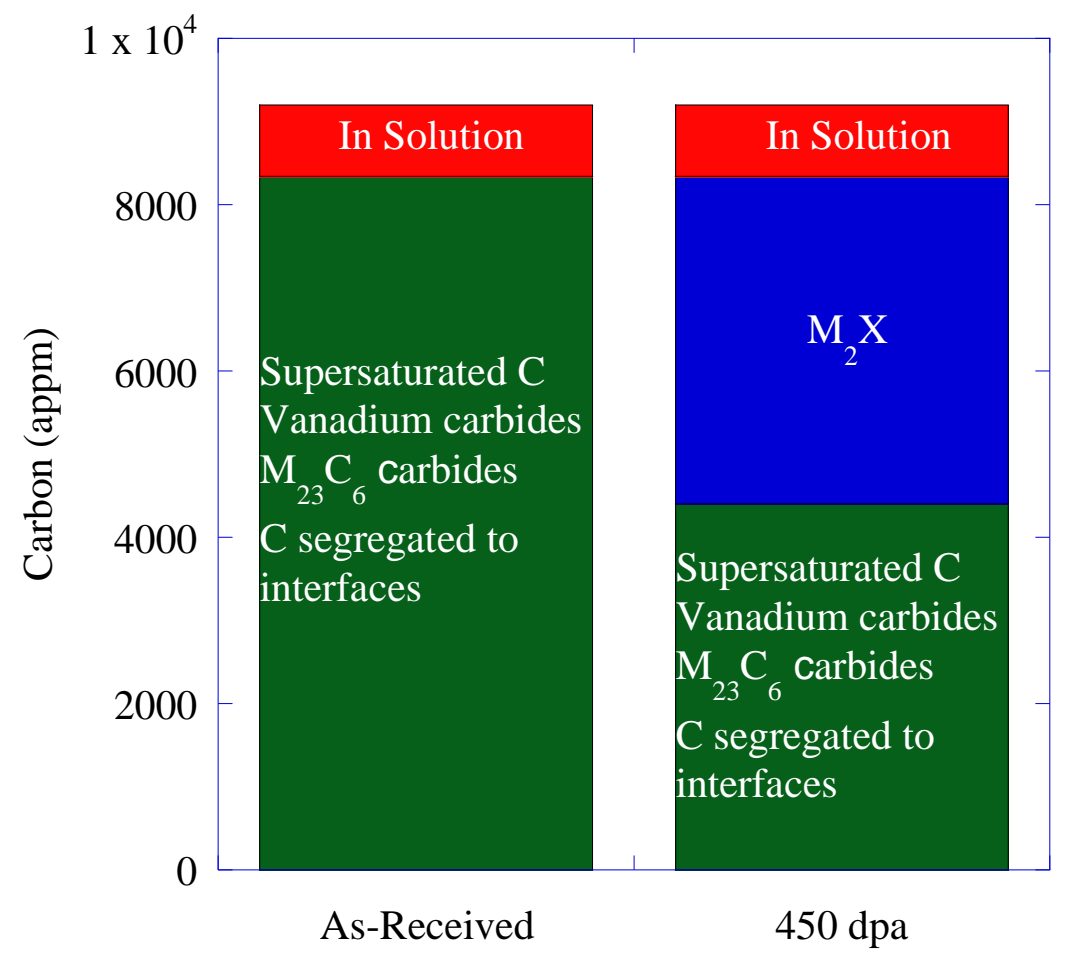

Figure 7.22: Accounting for carbon in HT9 in the as-received condition and at 450 dpa. 


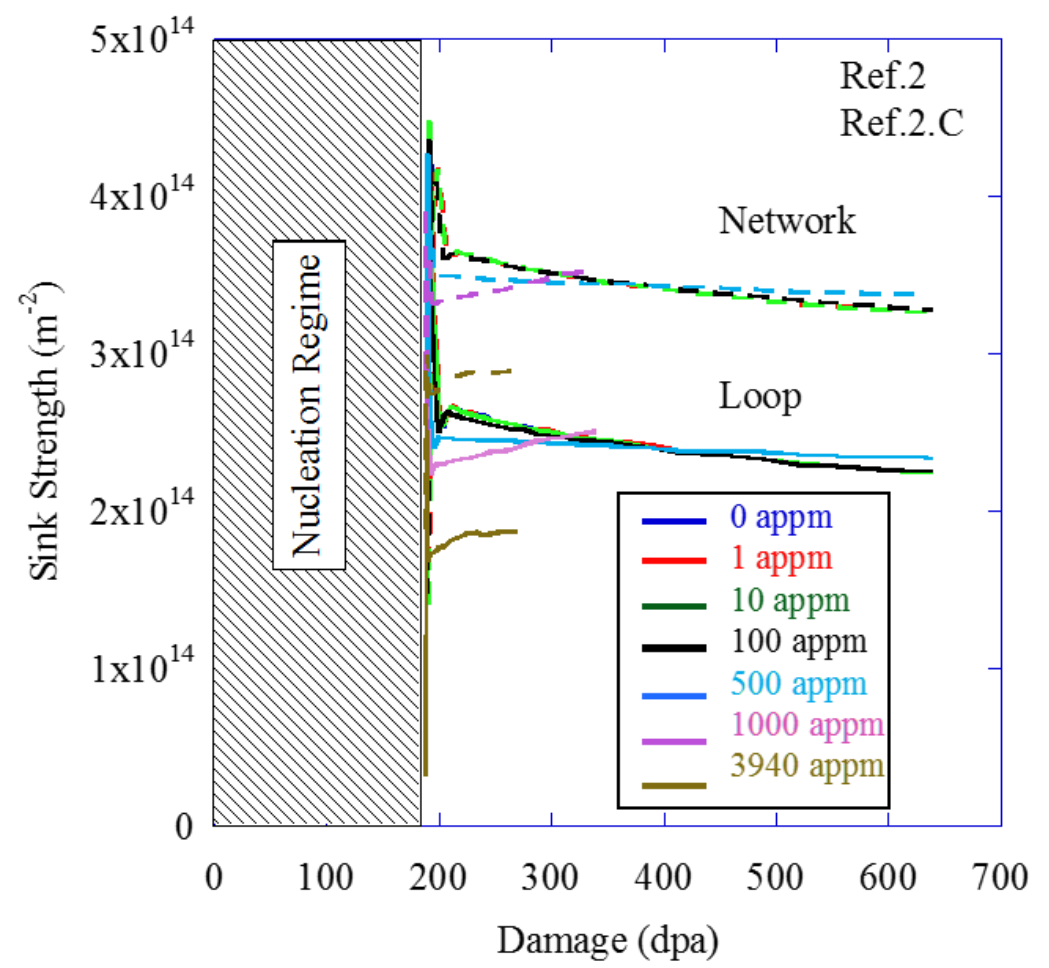

(a)

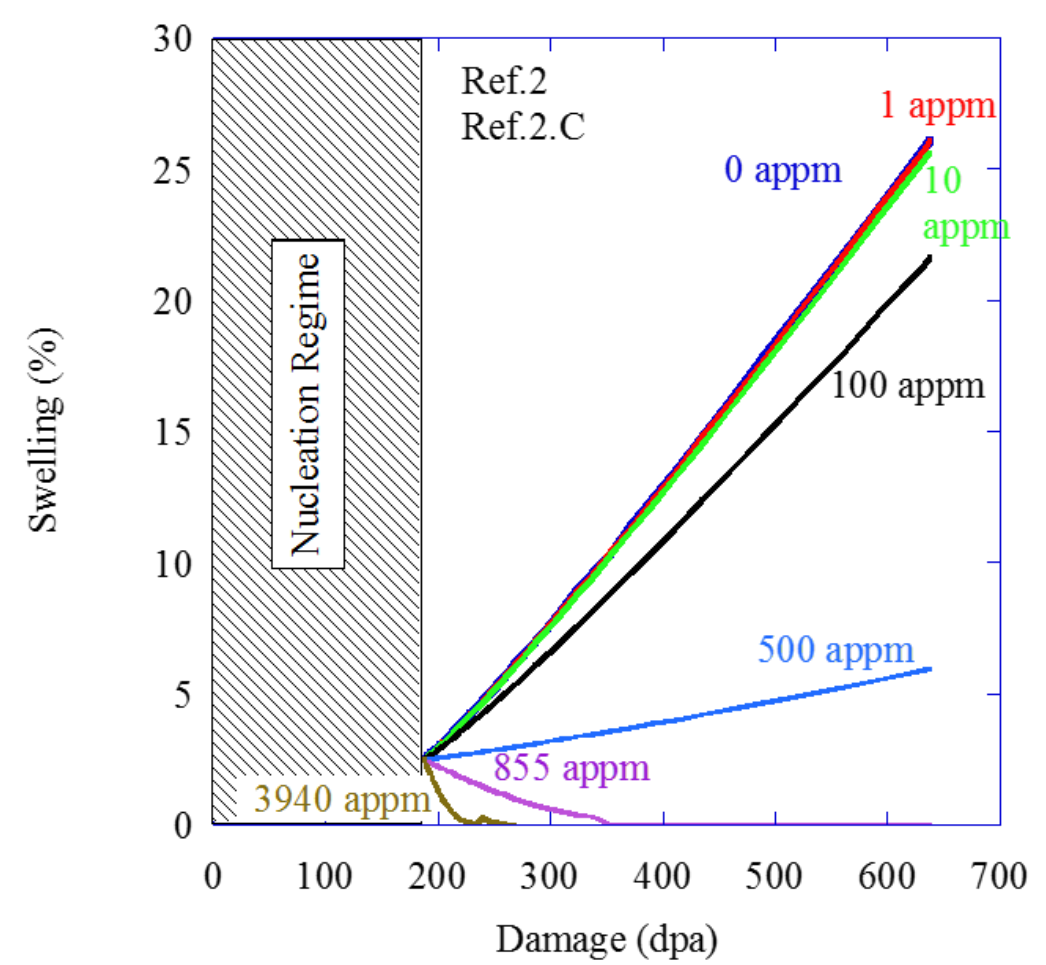

(b)

Figure 7.23: Comparison of Ref.2 with Ref.2.C with 0 to 855 appm for a) network and loop sink strength and b) swelling. Case run with 3940 appm terminated at 400 dpa. 0 and 1 appm lines are indistinguishable in b). 


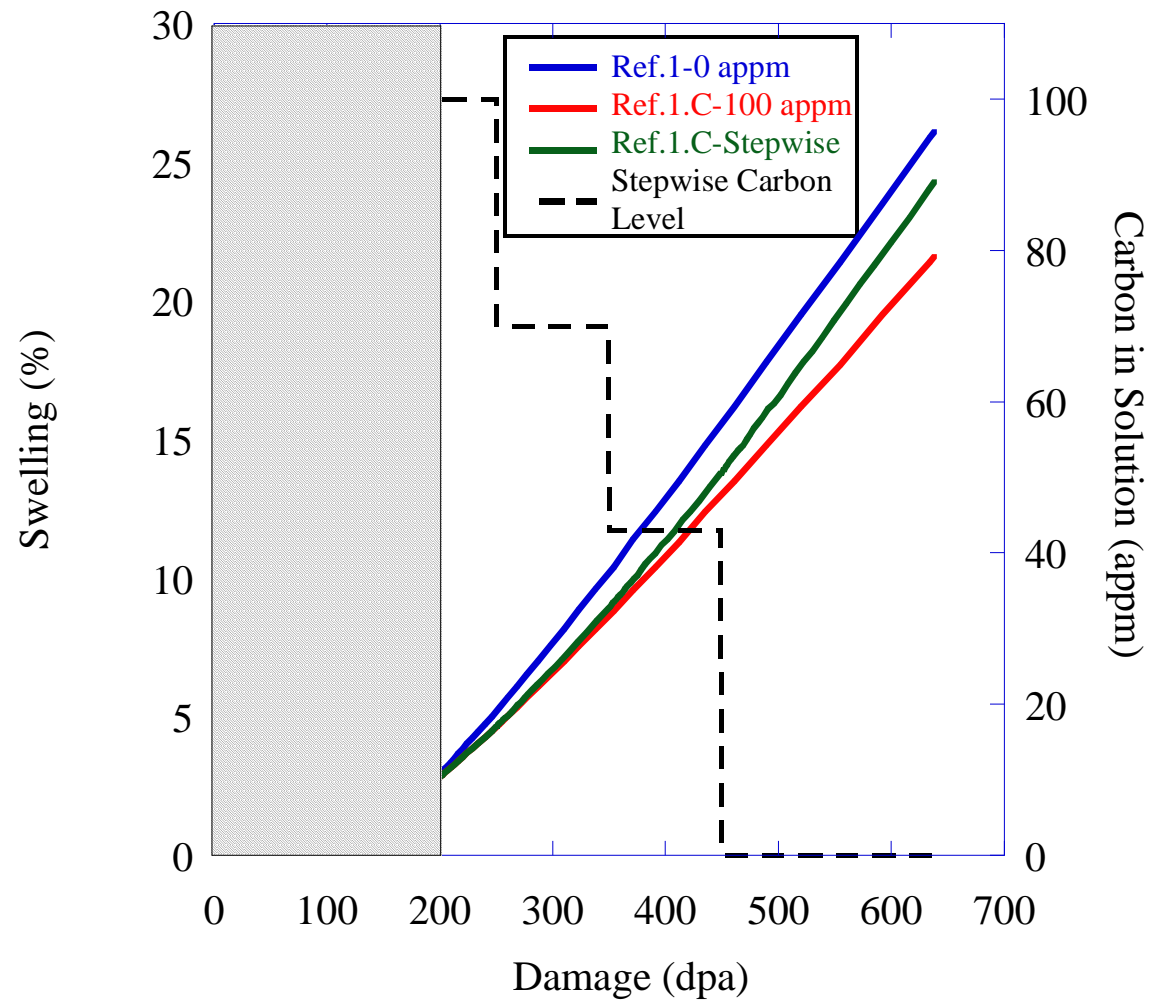

Figure 7.24: Comparison of Ref.1 (blue), Ref.1.C with 100 appm in solution (red) and 100 appm in solution removed stepwise from 188 to $450 \mathrm{dpa}$ (green) representing the precipitation of $\mathrm{M}_{2} \mathrm{X}$. 


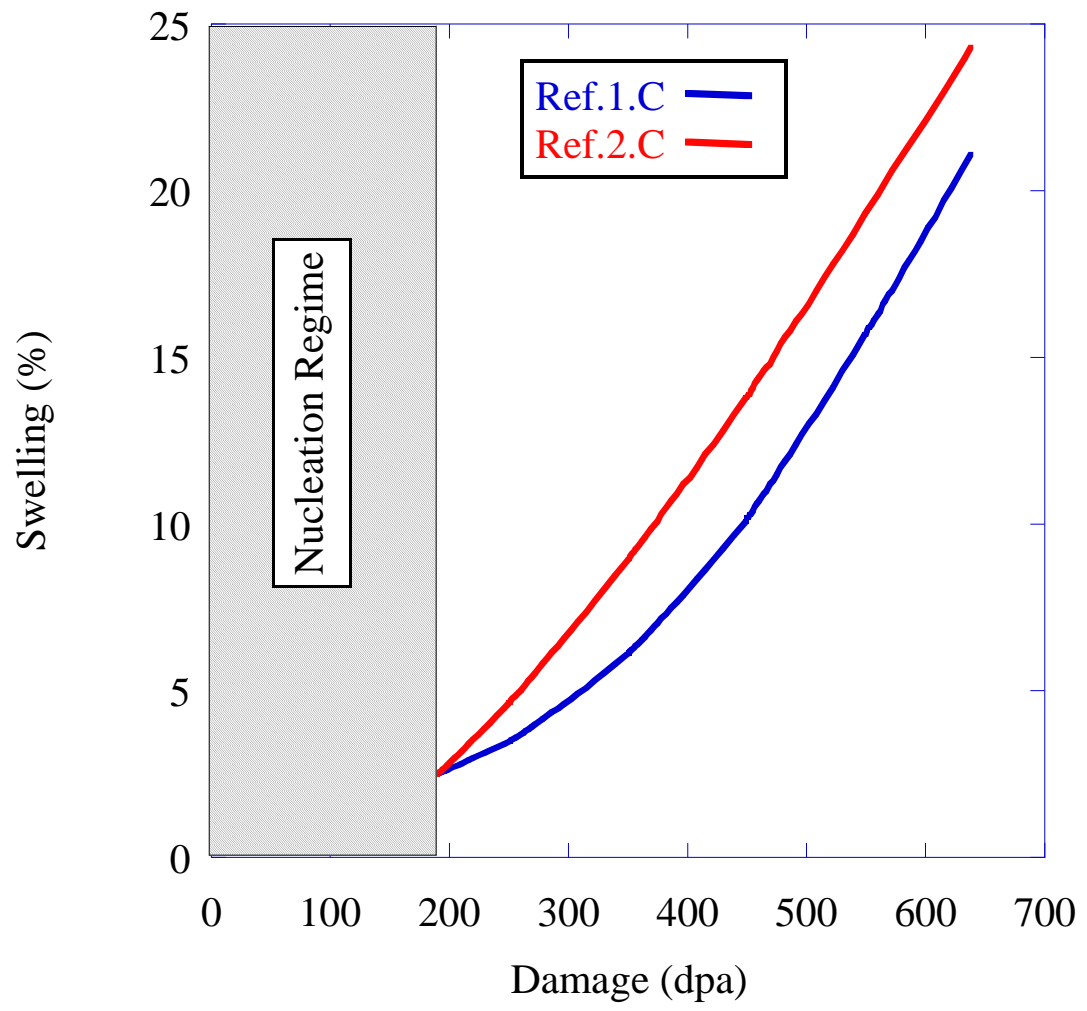

Figure 7.25: Comparison of Ref.1.C (blue) and Ref.2.C (red) with 100 appm of carbon in solution removed stepwise from 188 to 450 dpa representing the precipitation of $\mathrm{M}_{2} \mathrm{X}$. 


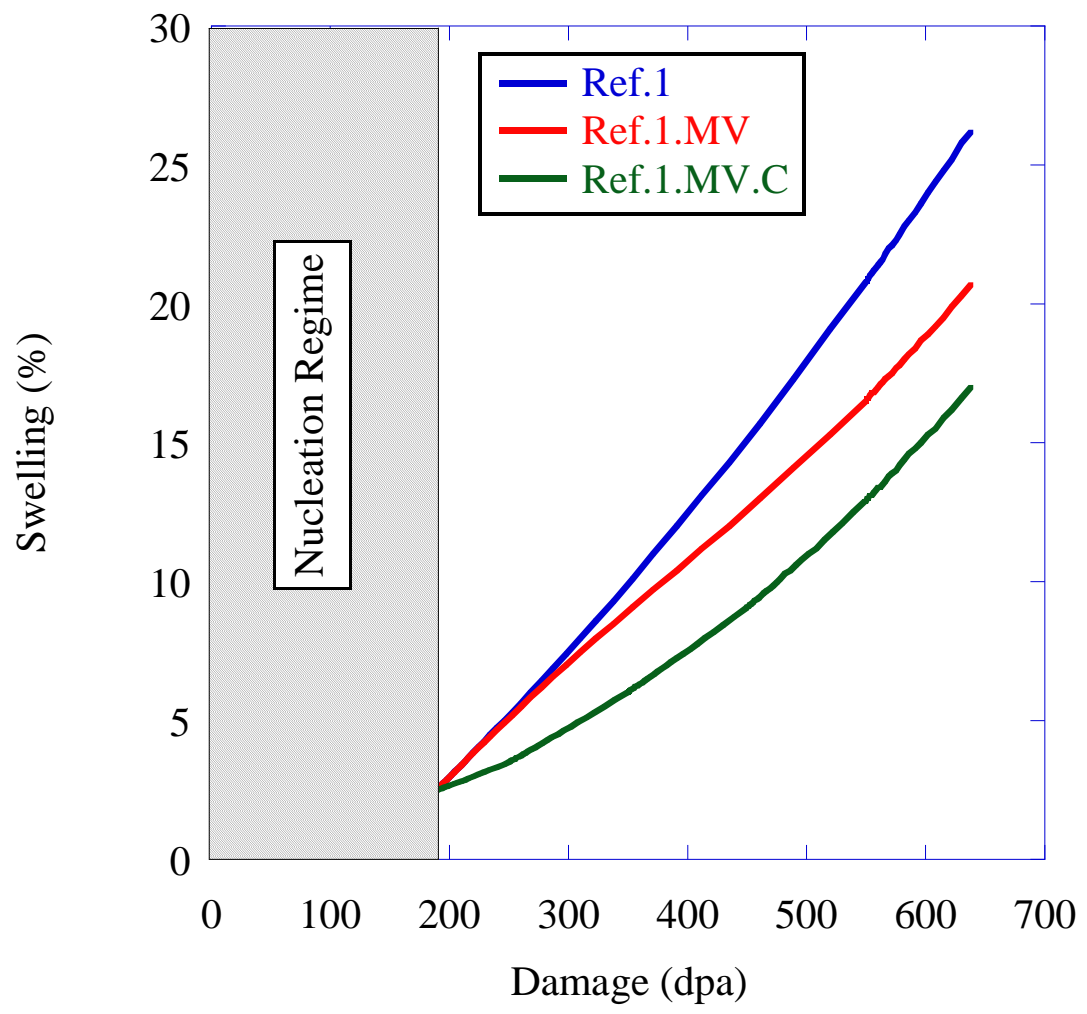

Figure 7.26: Comparison of void swelling with $\mathrm{M}_{2} \mathrm{X}$ plus 100 appm of carbon in solution removed stepwise from 188 to 450 dpa (Ref.1.MV.C: green), $\mathrm{M}_{2} \mathrm{X}$ (Ref.1.MV: red) and Ref.1 (blue). All $\mathrm{M}_{2} \mathrm{X}$ treated as variably biased with experimentally input dislocations. 


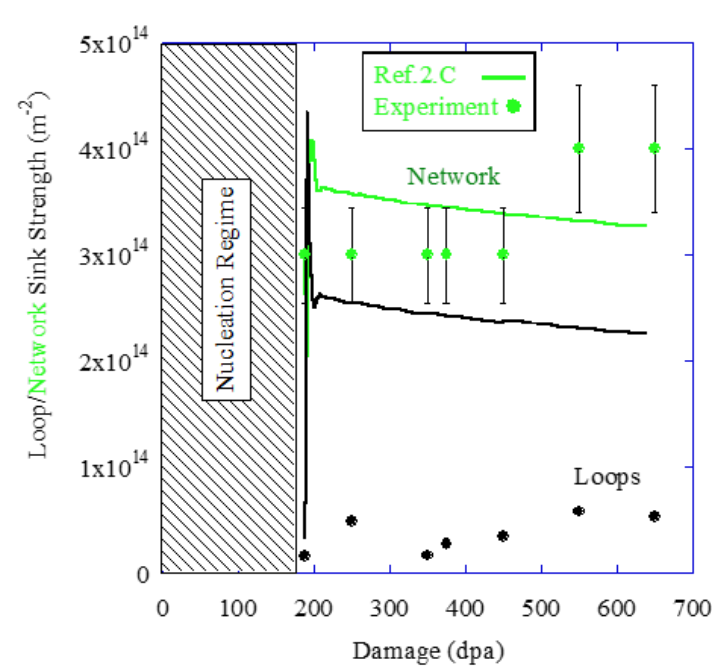

(a)

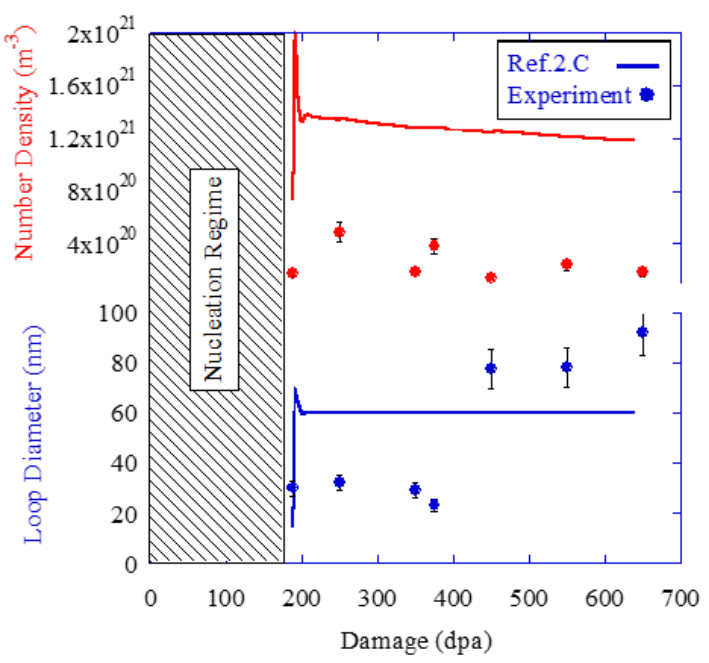

(b)

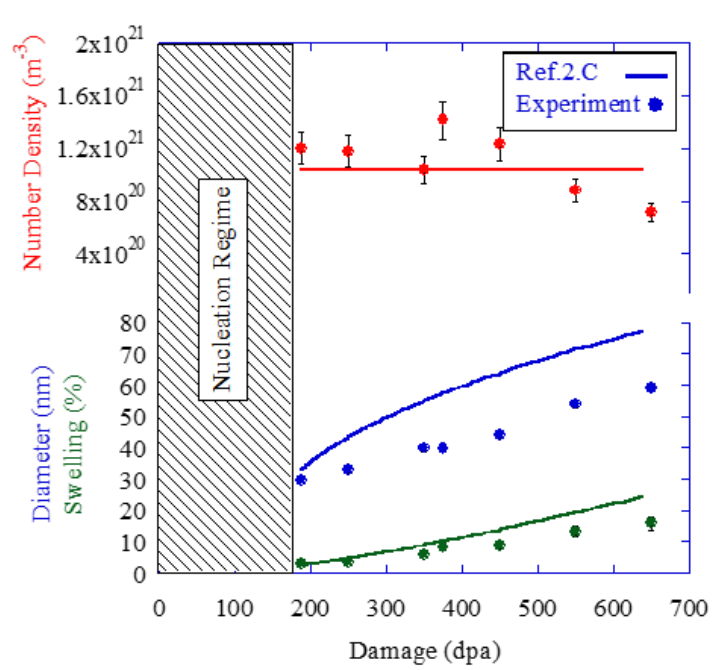

(c)

Figure 7.27: Comparison of DD treatment (Ref.2.C: $z_{i}^{l p}=z_{i}^{\text {net }}=1.01$ ) with experimental data in terms of a) loop and network sink strength b) loop diameter and number density and c) void diameter, number density and swelling including 100 appm of carbon in solution removed stepwise from 188 to $450 \mathrm{dpa}$. 


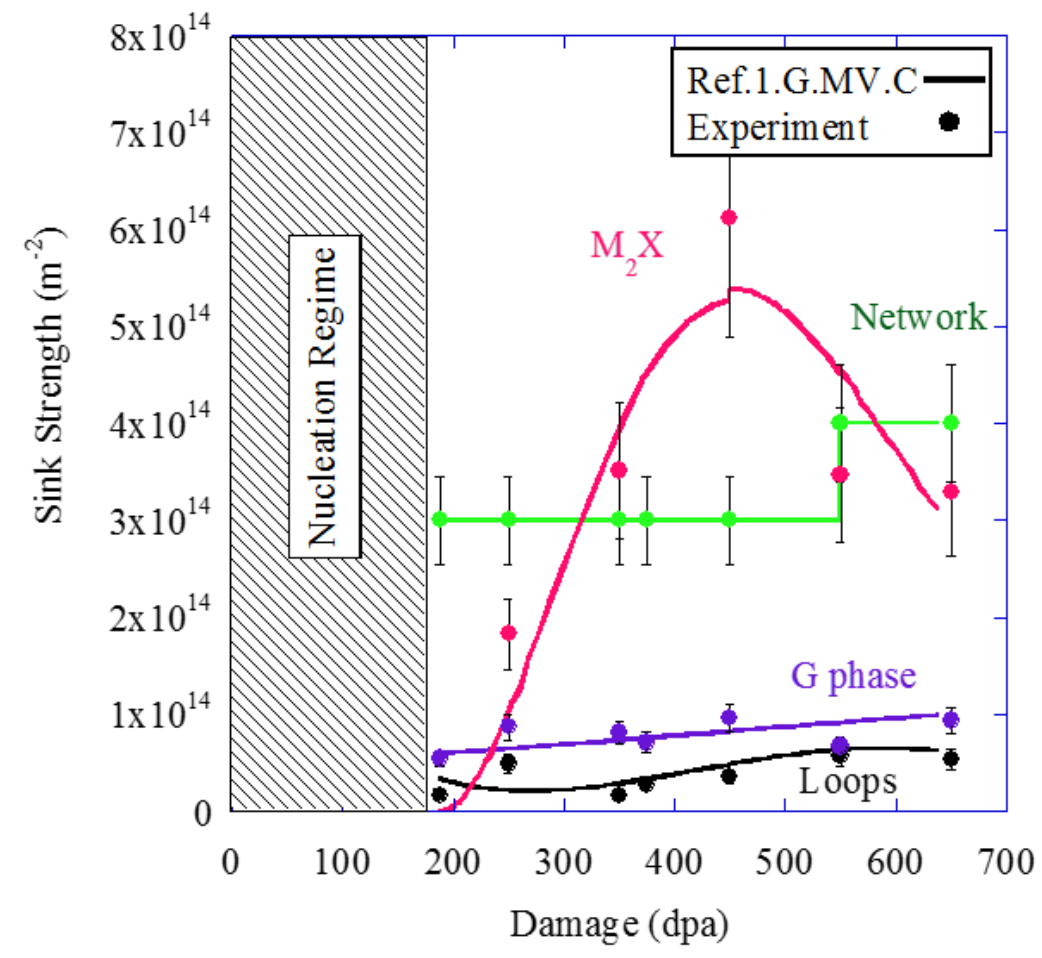

(a)

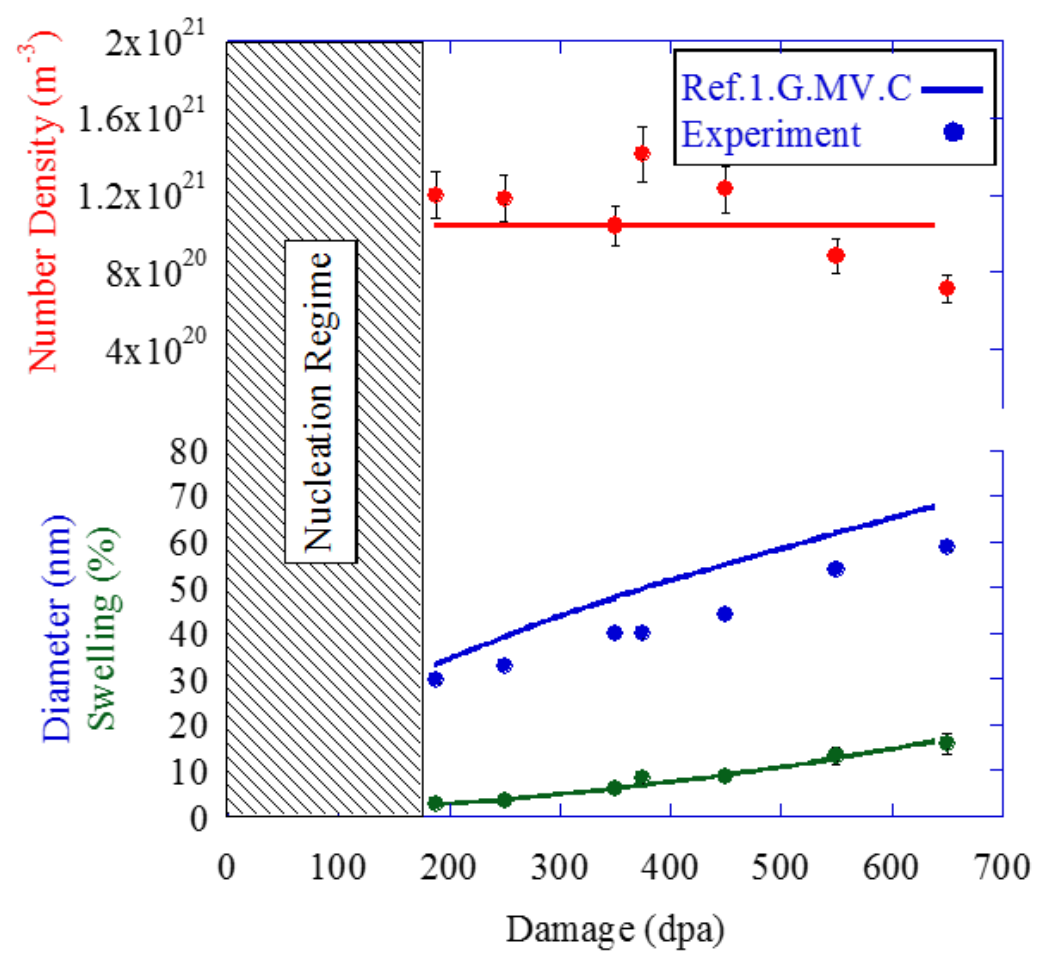

(b)

Figure 7.28: Comparison of experimentally input loop and network treatment (Ref.1.G.MV.C: $z_{i}^{\text {lp }}=z_{i}^{\text {net }}=1.01$ ) with experimental data in terms of a) network, loop, $G$ phase and $M_{2} X$ sink strength and $b$ ) void diameter, number density and swelling including $\mathrm{M}_{2} \mathrm{X}$ (variable biased), $\mathrm{G}$ phase and stepwise removal of 100 appm of carbon in solution from 188 to 450 dpa. 


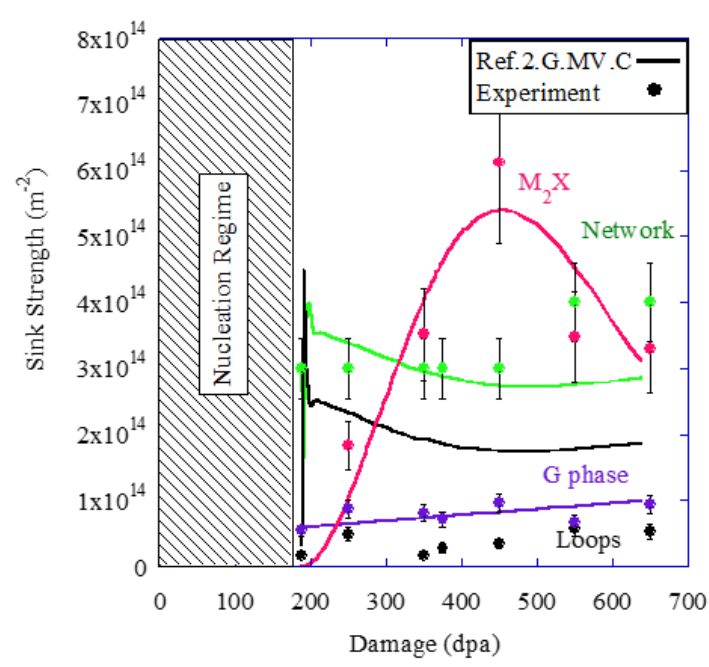

(a)

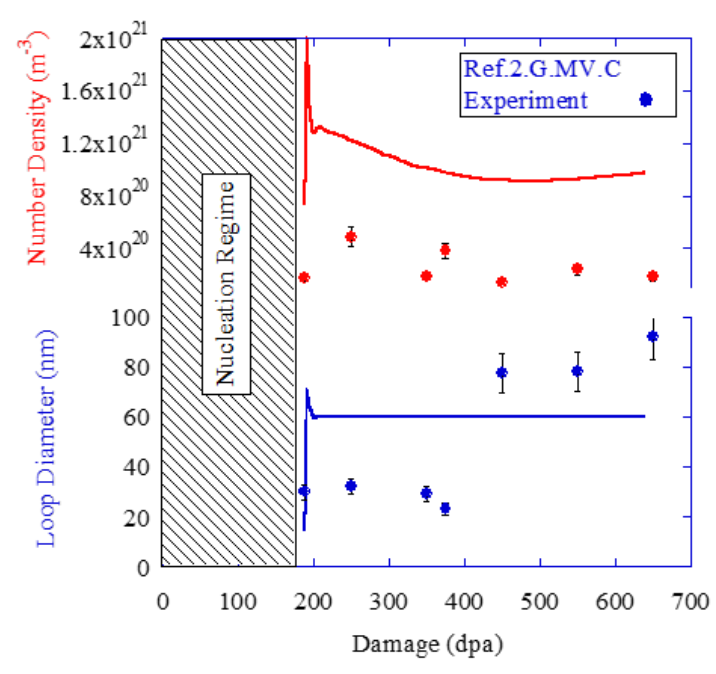

(b)

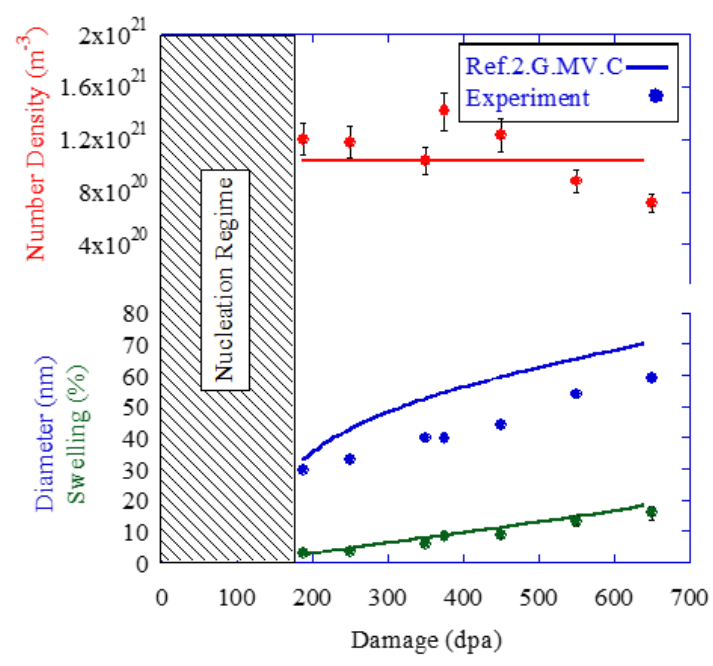

(c)

Figure 7.29: Comparison of DD treatment (Ref.2.G.MV.C: $z_{i}^{l p}=z_{i}^{\text {net }}=1.01$ ) with experimental data in terms of a) network, loop, $\mathrm{G}$ phase and $\mathrm{M}_{2} \mathrm{X}$ sink strength $\mathrm{b}$ ) loop diameter and number density and $\mathrm{c}$ ) void diameter, number density and swelling including $\mathrm{M}_{2} \mathrm{X}$ (variable biased), $\mathrm{G}$ phase and stepwise removal of 100 appm of carbon in solution from 188 to $450 \mathrm{dpa}$. 


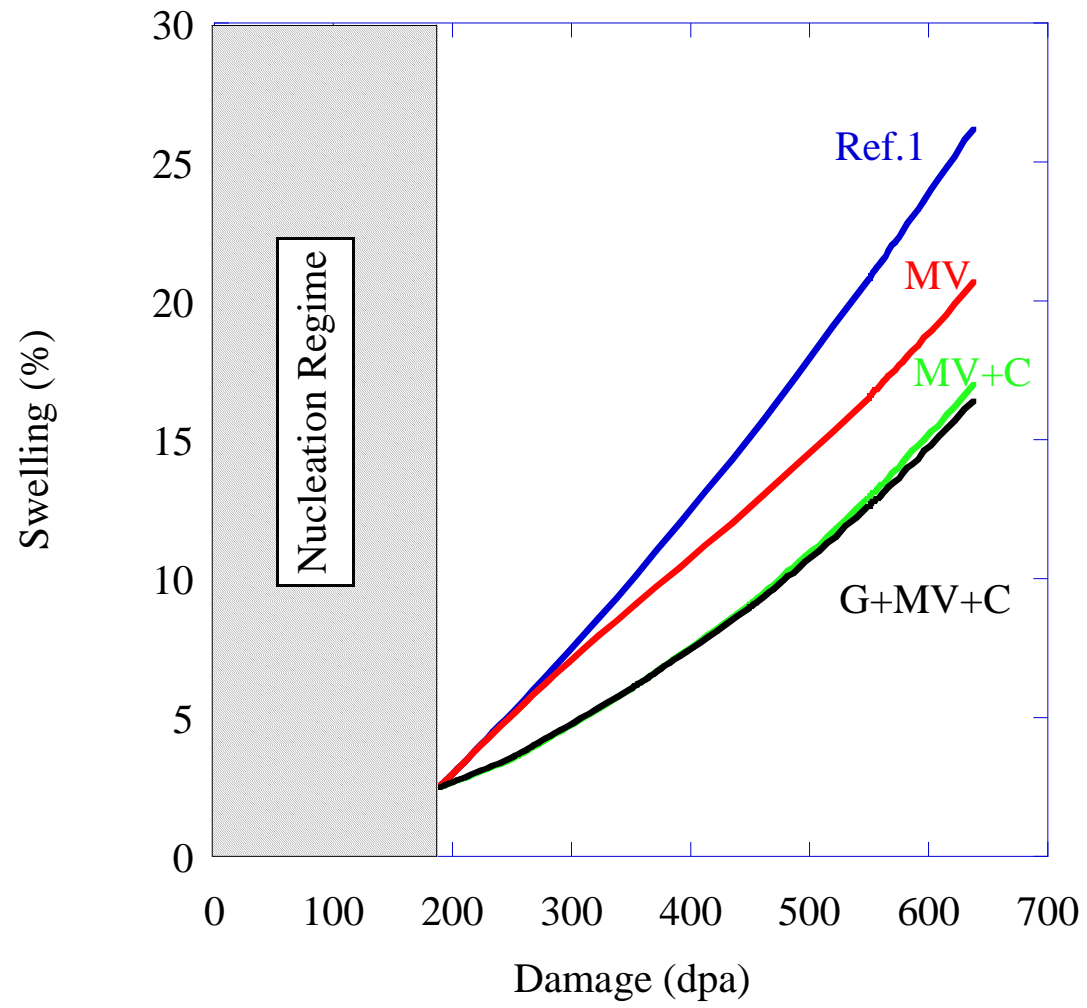

Figure 7.30: Comparison of swelling curves with various microstructure treatments. All cases run with experimentally input dislocations (Ref.1:

$$
\left.z_{i}^{l p}=z_{i}^{\text {net }}=1.01\right) \text {. }
$$




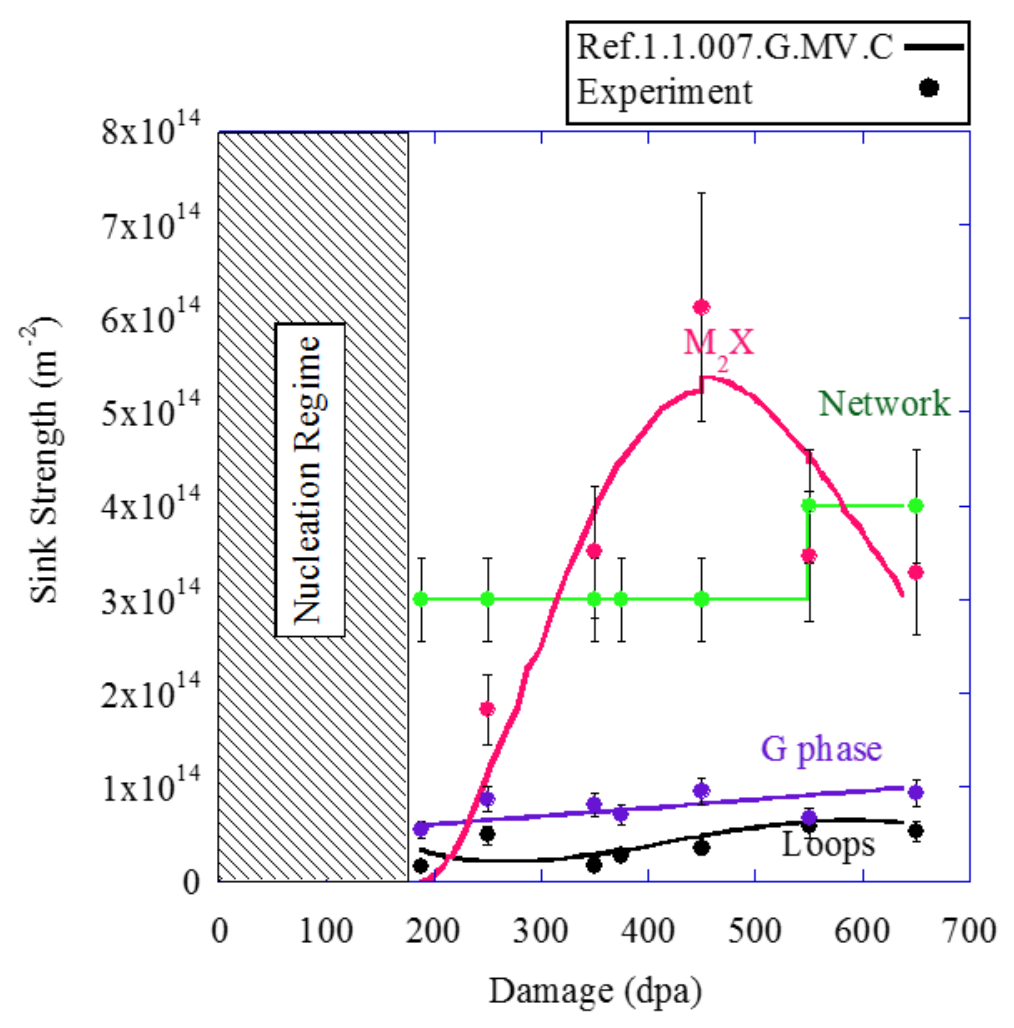

(a)

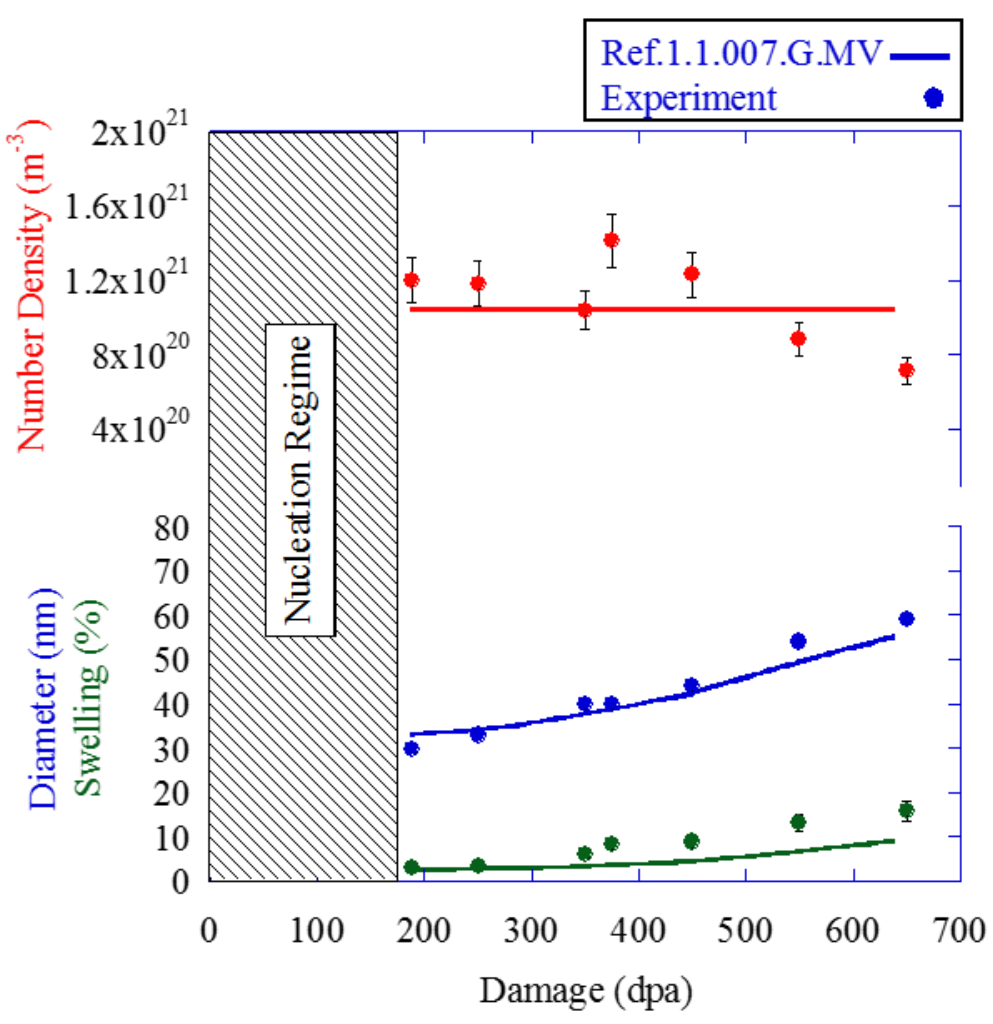

(b)

Figure 7.31: Comparison of experimentally input loop and network treatment with lower bias (Ref.1.1.007.G.MV.C: $\mathrm{z}_{\mathrm{i}}^{\mathrm{lp}}=\mathrm{z}_{\mathrm{i}}^{\text {net }}=1.01$ ) with experimental data in terms of a) network, loop, $\mathrm{G}$ phase and $\mathrm{M}_{2} \mathrm{X}$ sink strength and $\mathrm{b}$ ) void diameter, number density and swelling including $\mathrm{M}_{2} \mathrm{X}$ (variable biased), $\mathrm{G}$ phase and 100 appm $\mathrm{C}$ in solution removed stepwise from 188 to $450 \mathrm{dpa}$. 


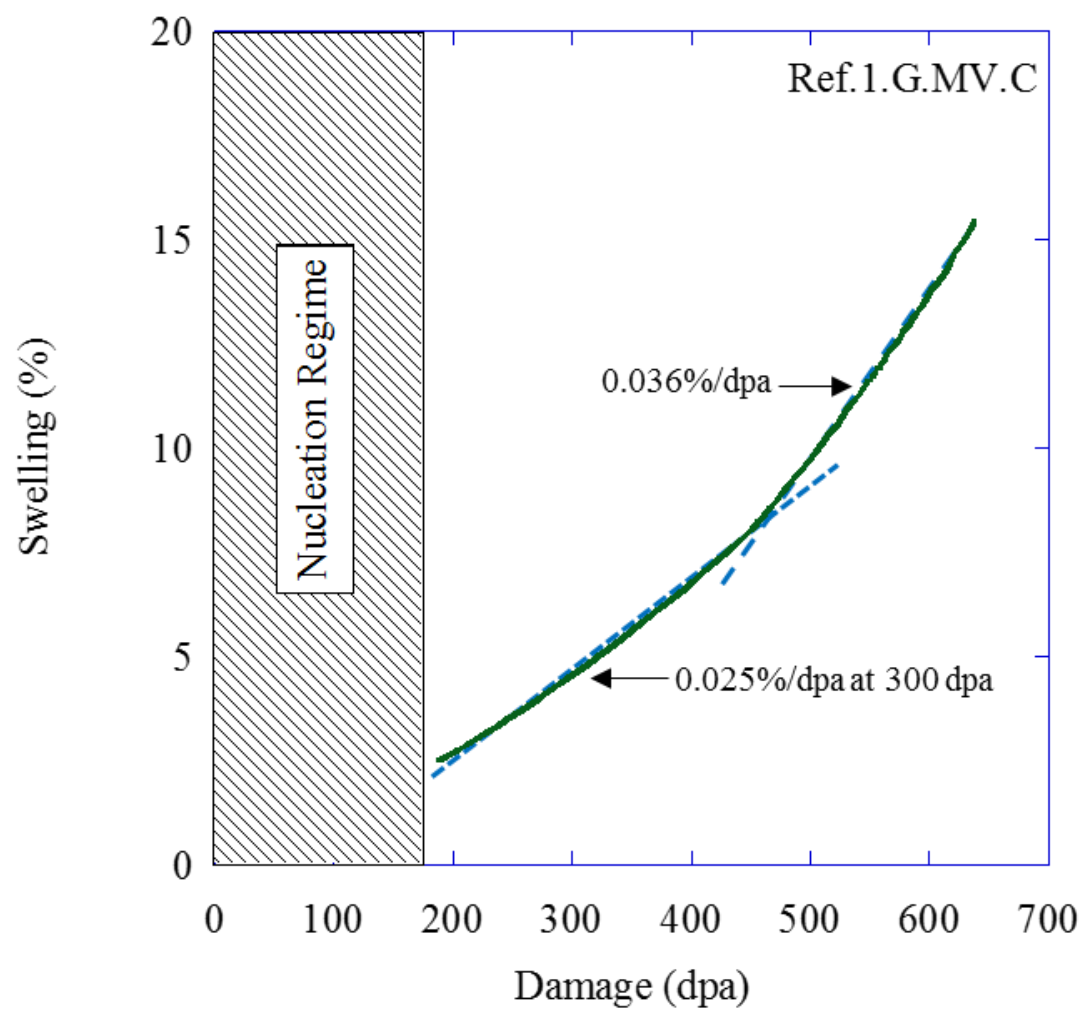

Figure 7.32: Comparison between swelling rate before and after $450 \mathrm{dpa}$ (Ref.1.G.MV.C: $\mathrm{z}_{\mathrm{i}}^{\mathrm{lp}}=\mathrm{z}_{\mathrm{i}}^{\text {net }}=1.01$ ). Dashed lines guide the eye to approximate swelling rates calculated from RIME. 


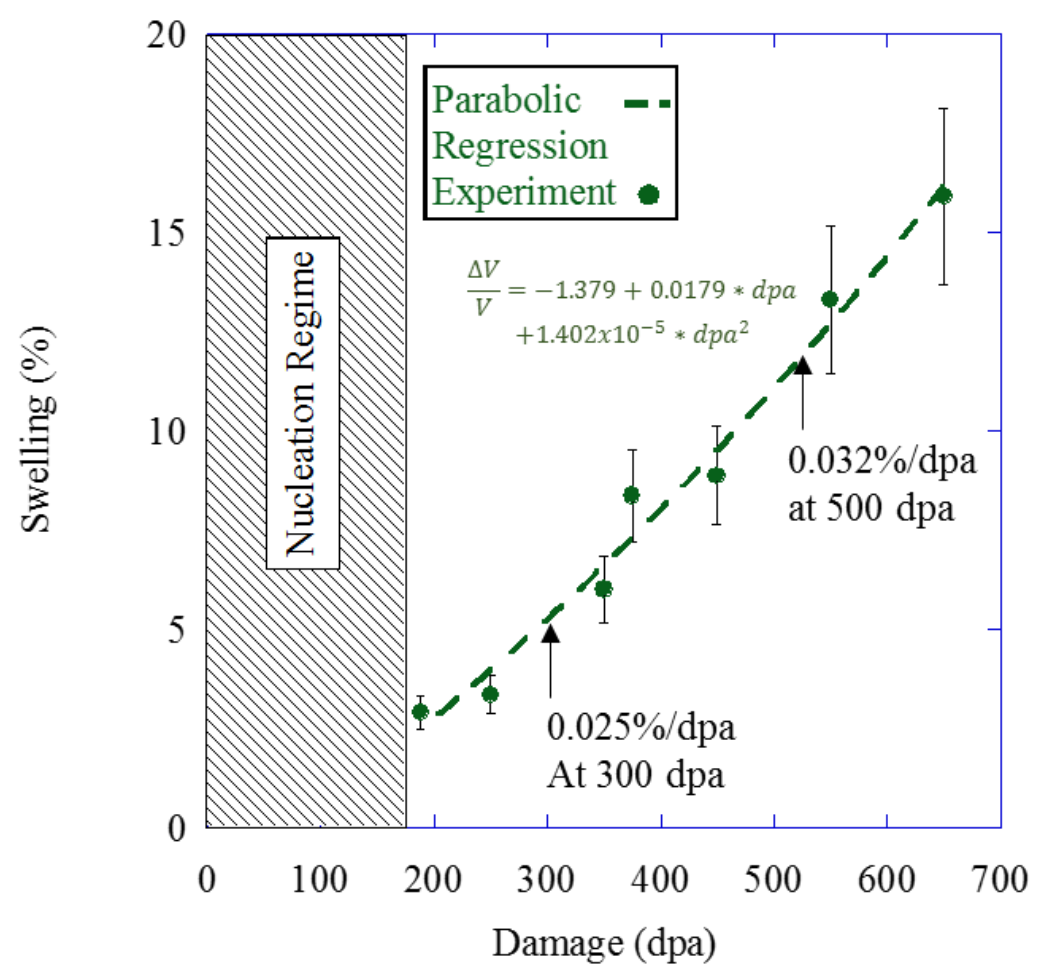

(a)

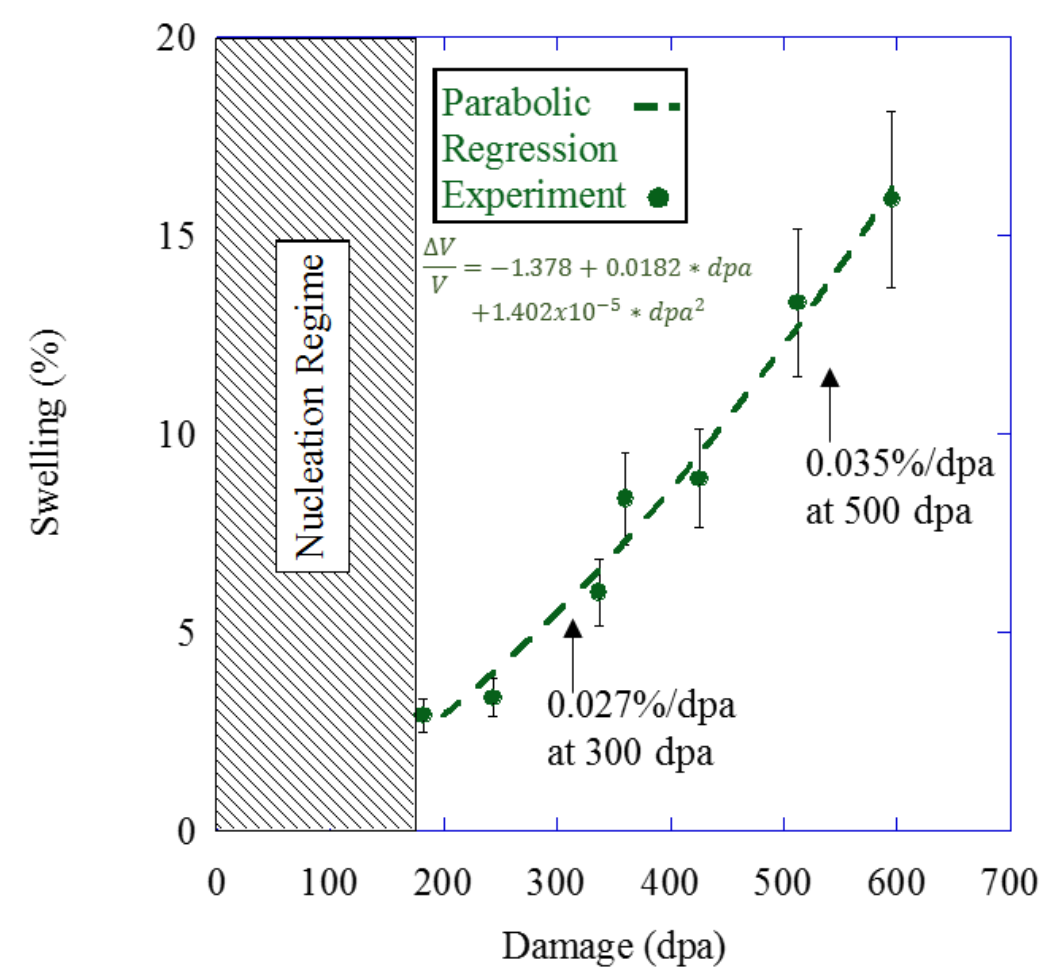

(b)

Figure 7.33: Comparison of instantaneous swelling rate calculated from experiment using parabolic regression before and after 450 dpa using a) uncorrected swelling and b) fixed depth method [85]. 


\section{CHAPTER 8}

\section{CONCLUSIONS}

The following conclusions have been reached regarding the interactions between voids, dislocations and precipitates in self-ion irradiated HT9 at very high damage levels.

A simple system of voids and dislocations was modeled using RIME in which the dislocations measured from experiment were used as input, or the dislocations were allowed to evolve dynamically. The result was that swelling was overestimated by $63 \%$ relative to that observed in experiment at 650 dpa for both treatments. The large discrepancy in measured vs. predicted swelling suggests that other sinks were acting to divert defect flow away from the voids. The consistency of the two dislocation treatments indicates the suitability of either method for analysis of more complex microstructure systems.

$G$ phase had limited effect on the void or dislocation behavior. The addition of $\mathrm{G}$ phase decreased final swelling by about $5 \%$ from 26.1 to $24.7 \%$, which was insufficient to serve as an alternate sink in a meaningful capacity. Thus, $\mathrm{G}$ phase was determined not to have a strong enough effect on voids as expected from its low sink density. $G$ phase also had an insignificant impact (5\% decrease) on loop and network sink strengths; it was not sufficient to resolve discrepancies between model and experiment. Therefore, $\mathrm{G}$ phase is minimal in terms of the coevolution of the overall microstructure. 
The behavior of $M_{2} X$ within the microstructure was characterized by a direct effect as a coherent sink, and an indirect effect in consuming carbon from the matrix. The primary effect of $\mathrm{M}_{2} \mathrm{X}$ was to serve as alternate sink for vacancies which limited the amount of vacancies free to diffuse and grow the voids, decreasing swelling from $26.1 \%$ to $20.7 \%$. The growth of $\mathrm{M}_{2} \mathrm{X}$ also resulted in the removal of carbon in solution from the matrix. Carbon trapping of vacancies was shown to have a strong effect on the swelling, decreasing the swelling from $26.1 \%$ to $21.1 \%$ for Ref.1 and to $24.3 \%$ for Ref.2, when 100 appm of C was removed is a stepwise fashion from 188 to $450 \mathrm{dpa}$. The removal of carbon due to growth of $\mathrm{M}_{2} \mathrm{X}$ resulted in an increase in instantaneous swelling rate from approximately $0.029 \% / \mathrm{dpa}$ to $0.056 \% / \mathrm{dpa}$ before and after $450 \mathrm{dpa}$ in the model.

Dislocation loops continued to evolve up through 650 dpa and underwent a step change in diameter between 375 and 450 dpa. $M_{2} X$ had the largest impact on dislocation behavior. Loop growth beyond tens of dpa has not been observed to this point and this data set represents the most systematic data set at the highest damage levels. $\mathrm{M}_{2} \mathrm{X}$, when treated as a variably biased sink or recombination center, has a measurable effect ( $75 \%$ decrease) on loop and network behavior by suppressing the dislocation loop number density, and by extension, the loop and network sink strength.

An inflection point at 450 dpa was observed both experimentally and computationally, with instantaneous swelling rates measured at 300 dpa of $\sim 0.025 \% / d p a$ and $\sim 0.032 \% /$ dpa at 500 dpa. The inflection point, which occurred due to loss of matrix carbon by $\mathrm{M}_{2} \mathrm{X}$ formation, demonstrates swelling cannot be at a true steady state as long as the surrounding microstructure features continue to evolve with dpa. Since microstructure evolution has been shown to occur out 
to several hundred dpa, "steady state" swelling will not be achieved. A better interpretation of the void swelling evolution is that of a nucleation dominated regime, a transition regime where both void nucleation and growth are important, and a growth-dominated regime characterized by a monotonic, slowly increasing swelling rate.

The agreement in void behavior between measurement and model when all microstructure effects (loops, network, $G$ phase, $M_{2} X$ formation and growth, and removal of carbon) are accounted for demonstrates the importance of characterizing the evolution of the full microstructure over the entire dpa range. The continuing evolution of the entire microstructure was a significant finding and was able to resolve the RIME model to better match experiment with wither Ref.1 or Ref.2. 


\section{CHAPTER 9}

\section{FUTURE WORK}

The results from this study are significant, but there are some unanswered questions that merit further study.

Confining the analysis to the void-growth dominated regime limits the applicability of this methodology in understanding the nucleation-dominated void swelling regime.

Understanding when and how voids nucleate is an even more challenging question and arguably, a more useful analysis for reactor applications. The majority of ion irradiation data to this point has been in the nucleation dominated regime, but no modeling efforts to date have been able to satisfactorily explain the variations in the length of incubation period of swelling, as nucleation of voids is a much more complex phenomenon when compared to modeling of void growthdominated behavior. Although the current treatment of void nucleation in RIME represents an improvement over previous nucleation modeling, it has yet to capture the variation in length of a nucleation period observed in different FM alloys.

The formation and growth mechanisms of precipitates in the high damage level regime have yet to be fully understood. The formation, growth and saturation of $\mathrm{M}_{2} \mathrm{X}$ was observed, but not understood in a mechanistic way in this study. Neither experimental nor computation work was advanced enough to understand the full precipitate evolution from nucleation to growth. To 
fully integrate a precipitate model into RIME that could respond to defect flows would represent a large improvement in capability, and also an increase in complexity which could further explain or confirm the defect-precipitate mechanisms utilized in this version of the model. Furthermore, no satisfactory explanation for the $250 \mathrm{dpa}$ incubation period before the appearance of $\mathrm{M}_{2} \mathrm{X}$.

The continuing evolution of dislocation loops and network at very high damage level was a significant result that was not captured by the model. A significant finding of the ion irradiations in this thesis was the continuing evolution of the loops and network at high damage levels, which greatly influenced void behavior. Neither the loop growth observed experimentally between 375 to 450 dpa nor the increase in loop network was observed. An interaction mechanism was proposed based upon the literature results, but further study is required to understand that complex interaction between precipitates, dislocations and carbon in solution in solution.

This study did not address the effect of helium implantation method on microstructure evolution. Dual ion irradiation is far more representative of damage in-reactor when compared to preimplanted helium, which was used in this study to approximate the effect of $(n, \alpha)$ reactors. Continuous helium implantation is expected to primarily affect void formation and growth, but the possible effect on other microstructure features cannot be discounted. In addition, this study has shown that microstructure continues to evolve as a function of dpa, therefore the continuing addition of helium is very likely to strongly influence the overall microstructure evolution either directly or indirectly. 
Highly heterogeneous void nucleation behavior was observed from grain to grain but was not explained by either the experiment or model. The heterogeneous void nucleation behavior has been well documented in FM alloys, and has not been sufficiently explained either using microscopy techniques or modeling, though some studies have suggested that retained $\delta$ ferrite may promote void swelling. For the purpose of this thesis, the average swelling of many grains was used in the final analysis. Results from this study suggest that the grain microchemistry, especially of $\mathrm{C}$, may be important, but again, further work is needed to understand what grain environments promote or suppress void swelling. A study of this would be highly relevant to future alloy development programs. 


\section{APPENDICES}

\section{APPENDIX A - TEMPERATURE HISTOGRAMS}

Temperature histograms are included for all irradiations presented in this thesis. 

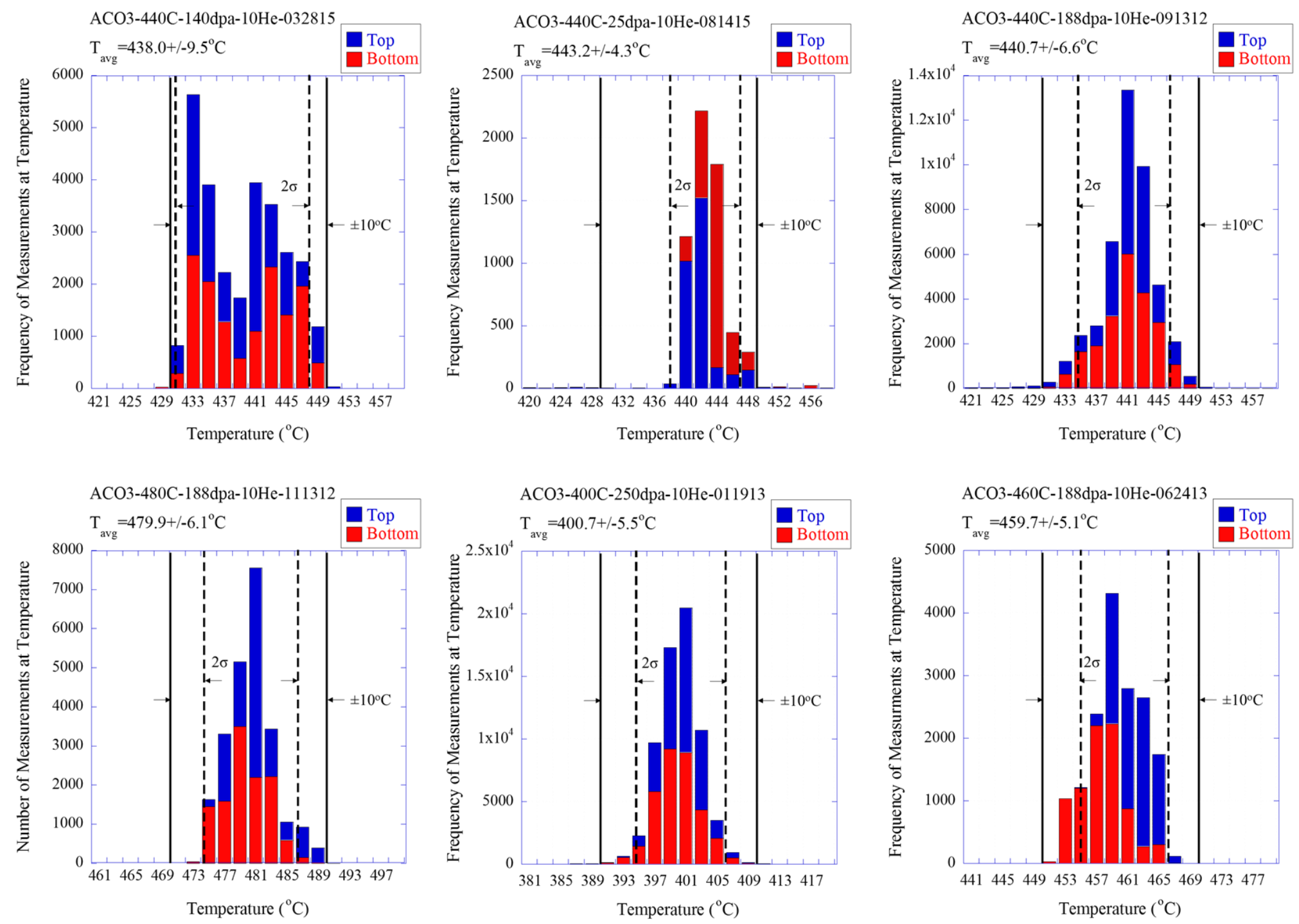

Figure A. 1: Temperature histograms for HT9 (heat 84425: ACO3) irradiated at $400^{\circ} \mathrm{C}: 250 \mathrm{dpa} ; 440^{\circ} \mathrm{C}: 25,140,188$; $460^{\circ} \mathrm{C}$ : $188 \mathrm{dpa}$ and $480^{\circ} \mathrm{C}: 188 \mathrm{dpa}$ 

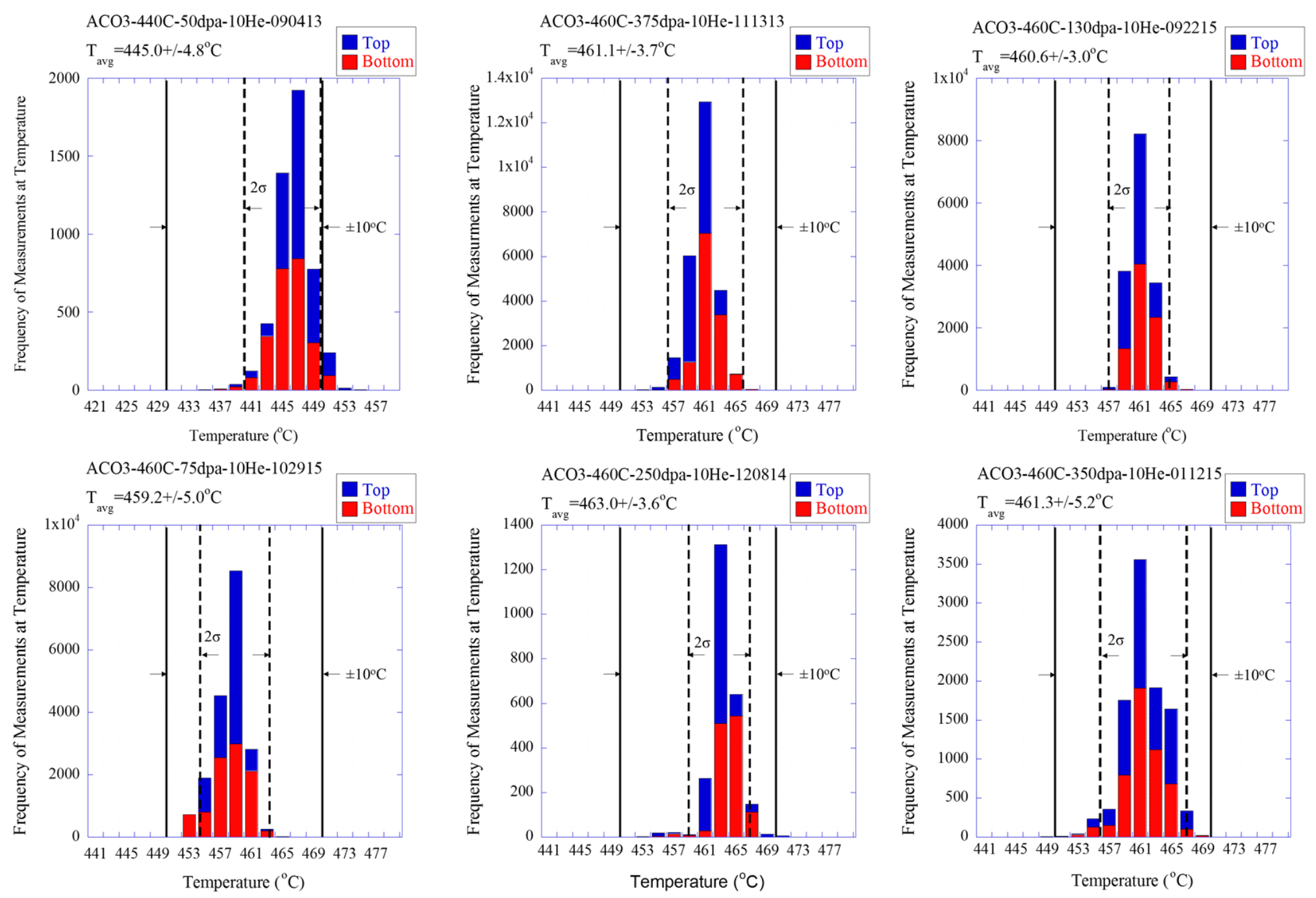

Figure A. 2: Temperature histograms for HT9 (heat 84425 : ACO3) irradiated at $440^{\circ} \mathrm{C}: 50$ dpa and $460^{\circ} \mathrm{C}: 75,130$, 250, 350, 375 dpa. 

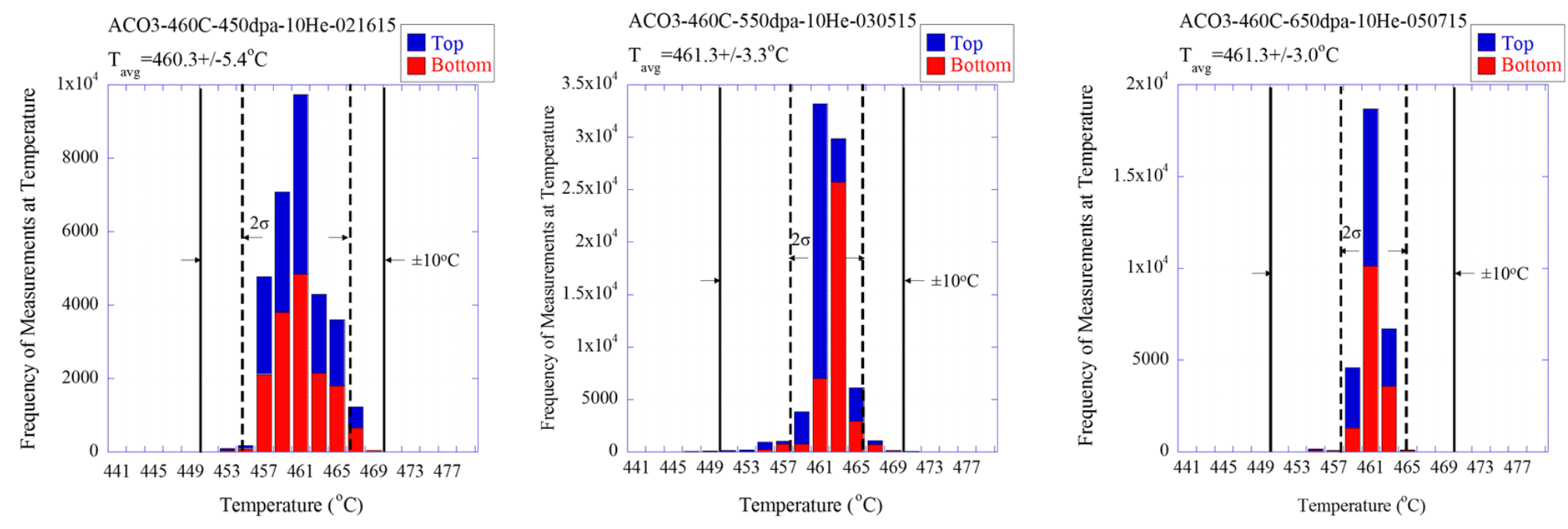

Figure A. 3: Temperature histograms for HT9 (heat 84425: ACO3) irradiated at $460^{\circ} \mathrm{C}: 450,550,650 \mathrm{dpa}$. 


\section{APPENDIX B - VOID IMAGES}

The following are images for a liftout of each of the irradiated conditions. 


\section{ACO3-440C-140dpa-10.10He-032812-TEM-Grid A-071113}
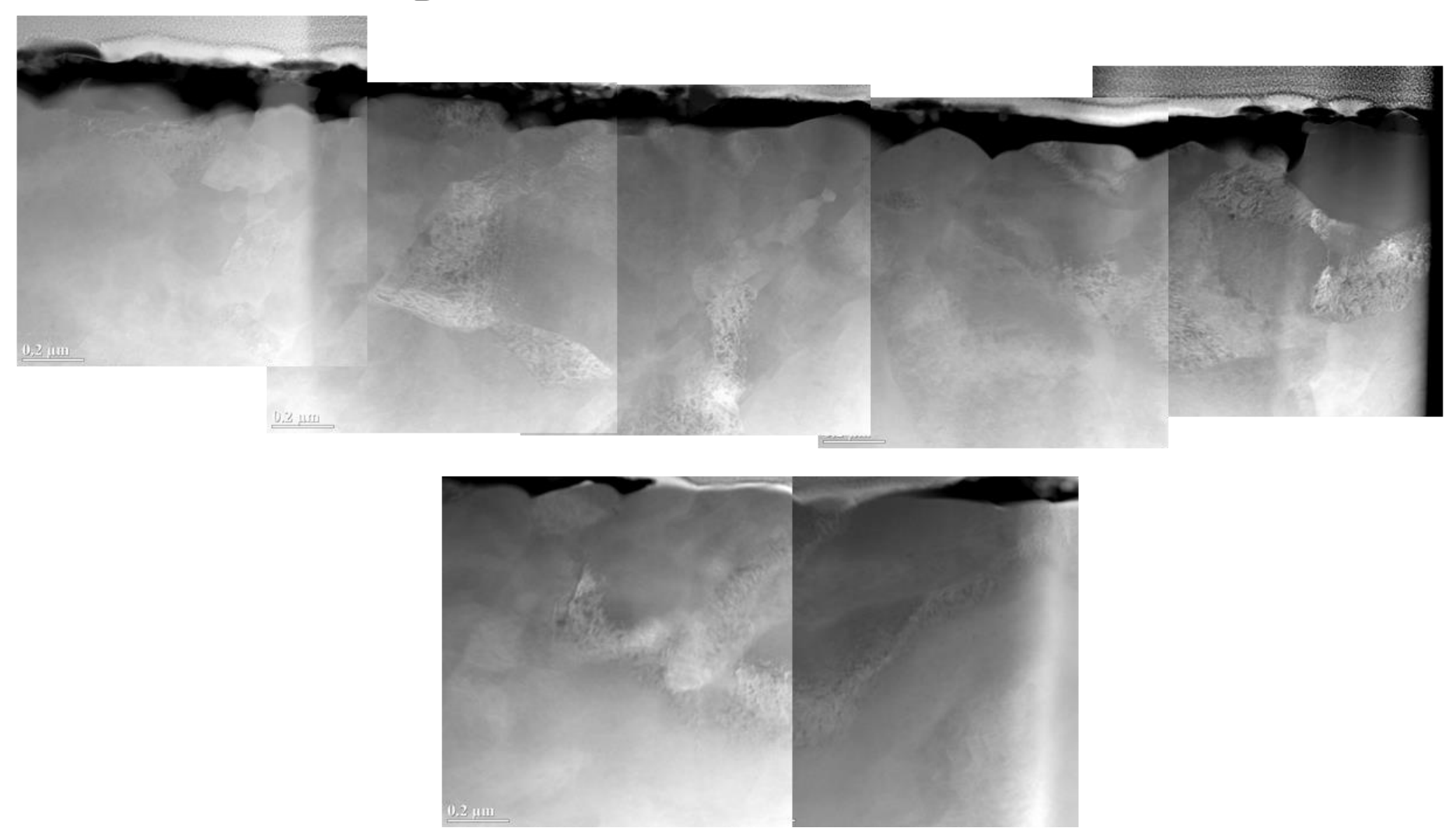

Figure B. 1: $\mathrm{HT} 9$ (ACO3) irradiated with $5 \mathrm{MeV} \mathrm{Fe}^{++}$at $440^{\circ} \mathrm{C}$ up to 140 dpa with $10 \mathrm{appm} \mathrm{He}$ preimplanted. 


\section{ACO3-440C-25dpa-10.10He-081412-TEM-Grid B-050114}
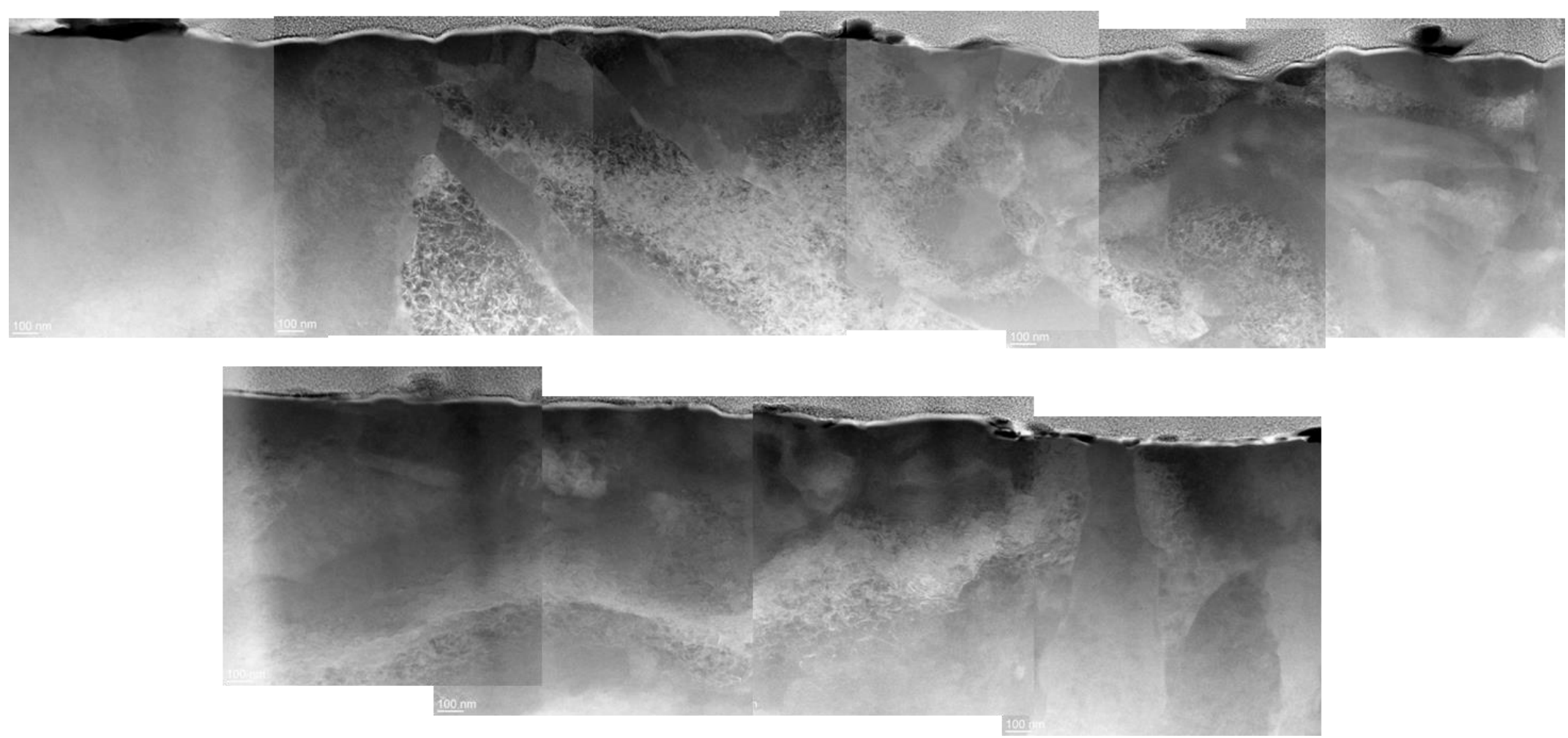

Figure B. 2: HT9 (ACO3) irradiated with $5 \mathrm{MeV} \mathrm{Fe}^{++}$at $440^{\circ} \mathrm{C}$ up to 25 dpa with 10 appm He preimplanted. 


\section{ACO3-440C-188dpa-10.10He-091312-TEM-Grid B-101812}

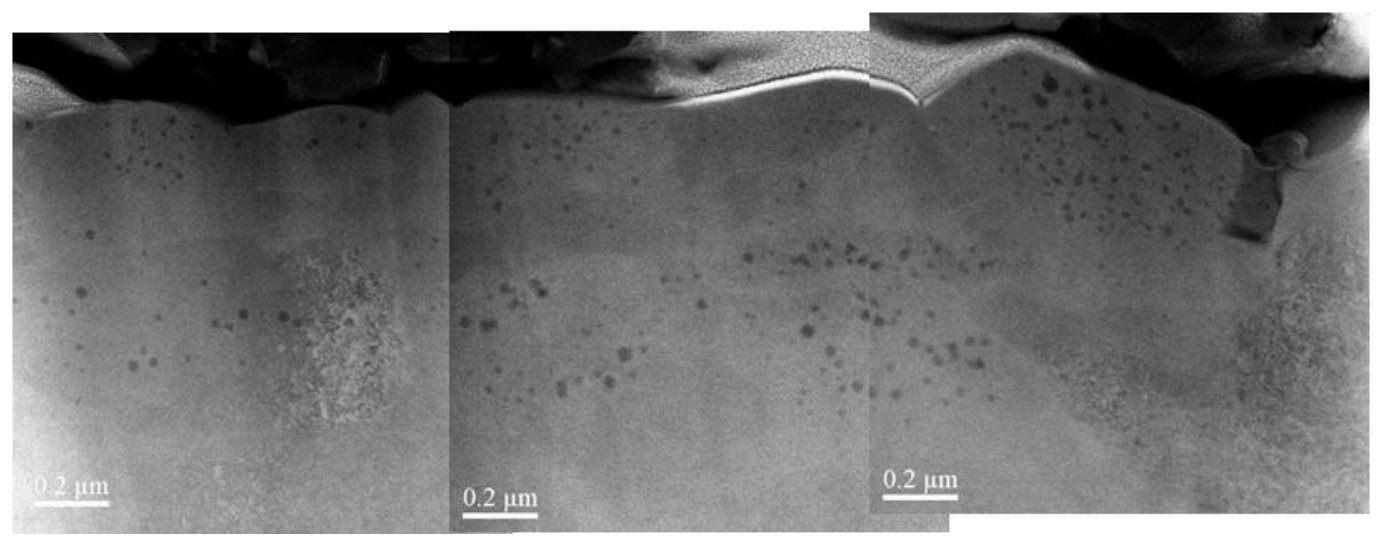

Figure B. 3: $\mathrm{HT} 9$ (ACO3) irradiated with $5 \mathrm{MeV} \mathrm{Fe}^{++}$at $440^{\circ} \mathrm{C}$ up to 188 dpa with 10 appm He preimplanted. 


\section{ACO3-480C-188dpa-10.10He-111312-TEM-Grid B-012813}
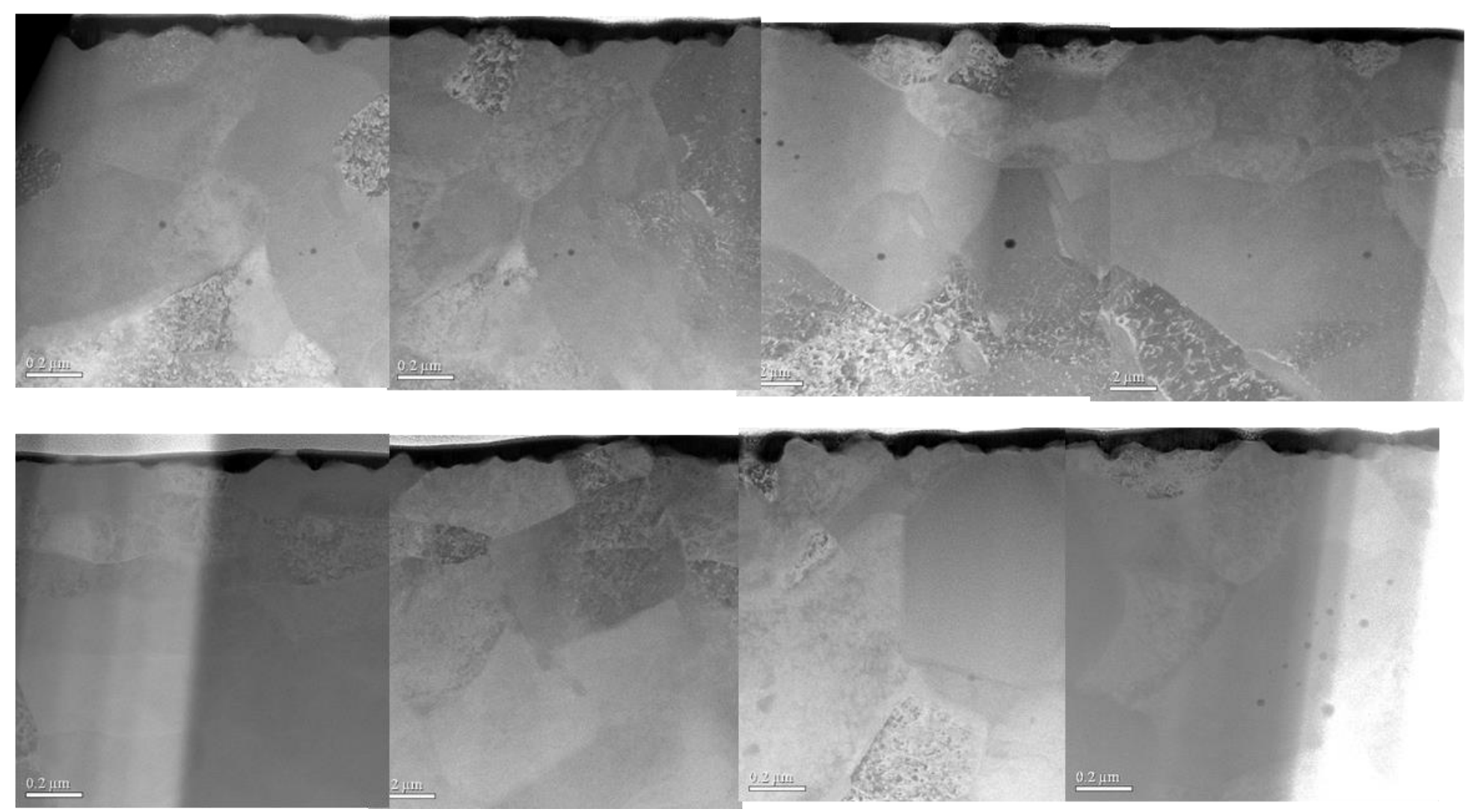

Figure B. 4: HT9 (ACO3) irradiated with $5 \mathrm{MeV} \mathrm{Fe}^{++}$at $480^{\circ} \mathrm{C}$ up to 188 dpa with 10 appm He preimplanted. 


\section{ACO3-400C-250dpa-0He-011913-TEM-Grid C-052113}
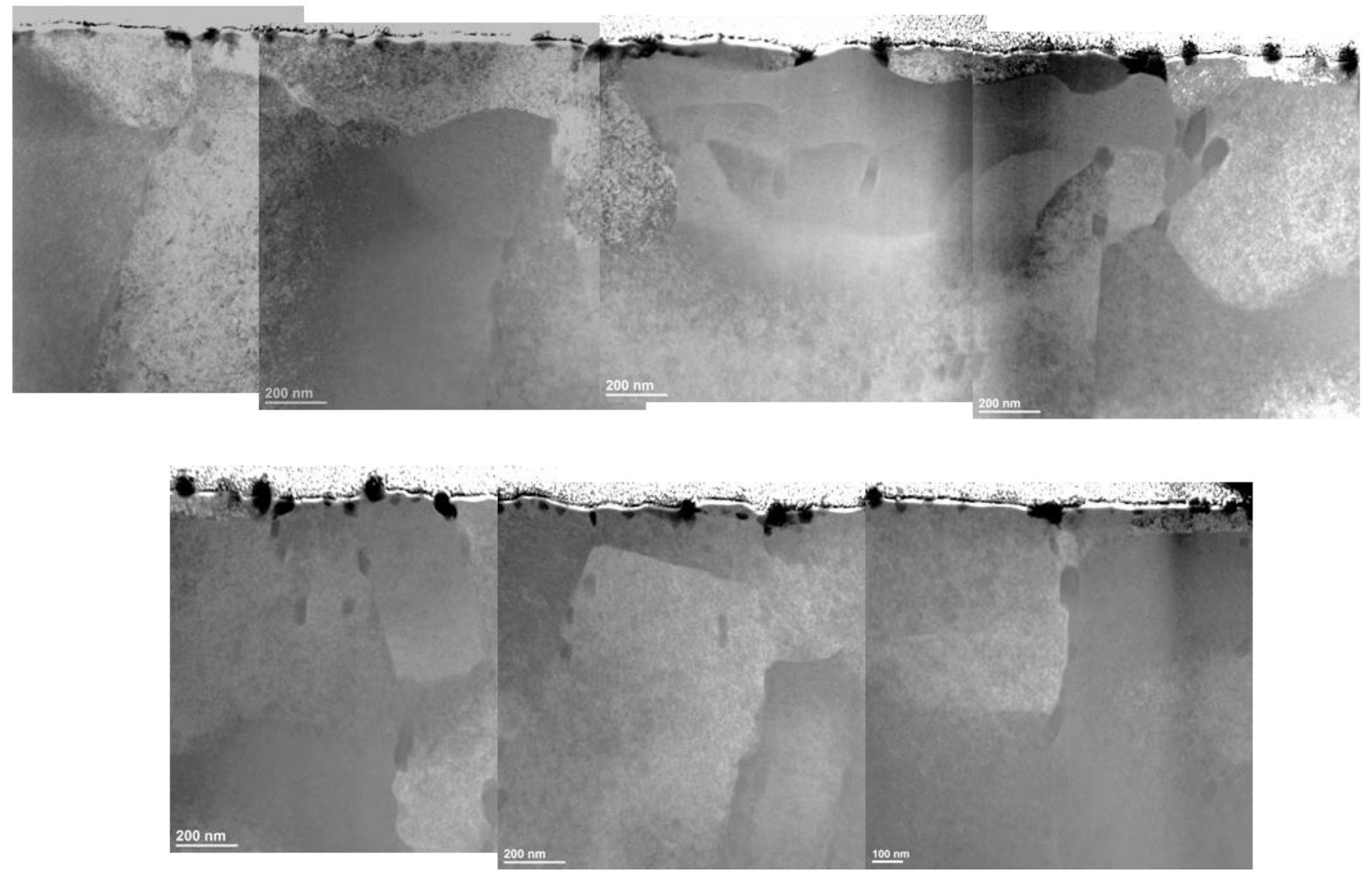

Figure B. 5: HT9 (ACO3) irradiated with $5 \mathrm{MeV} \mathrm{Fe}^{++}$at $400^{\circ} \mathrm{C}$ up to 250 dpa with 0 appm $\mathrm{He}$ preimplanted. 


\section{ACO3-460C-188dpa-10.10He-062413-TEM-Grid C-072013}

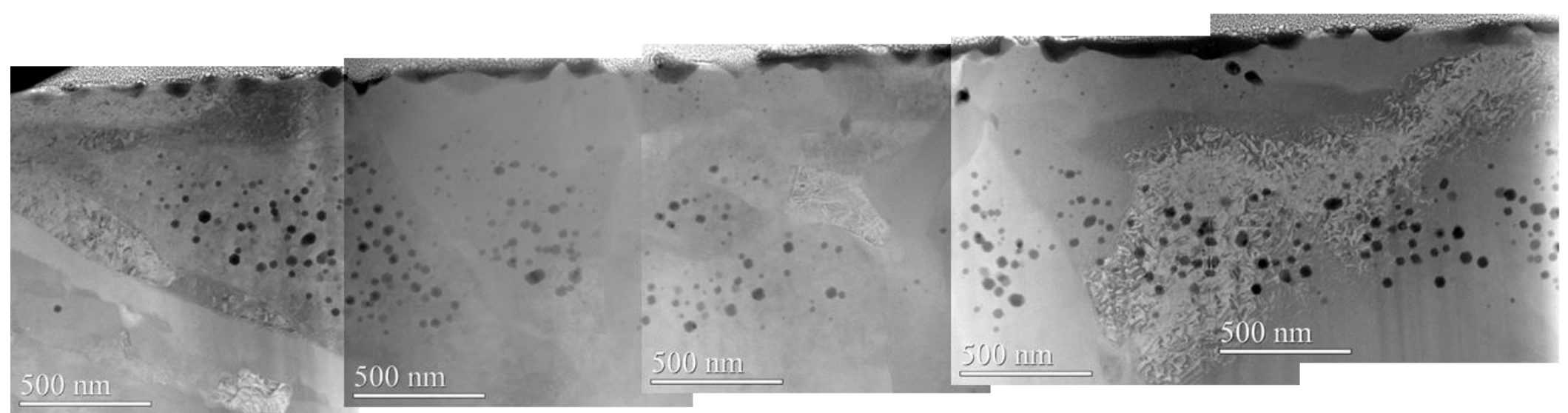

Figure B. 6: HT9 (ACO3) irradiated with $5 \mathrm{MeV} \mathrm{Fe}^{++}$at $460^{\circ} \mathrm{C}$ up to 188 dpa with 10 appm He preimplanted. 


\section{ACO3-440C-50dpa-10.10He-090413-TEM-Grid C-050114}
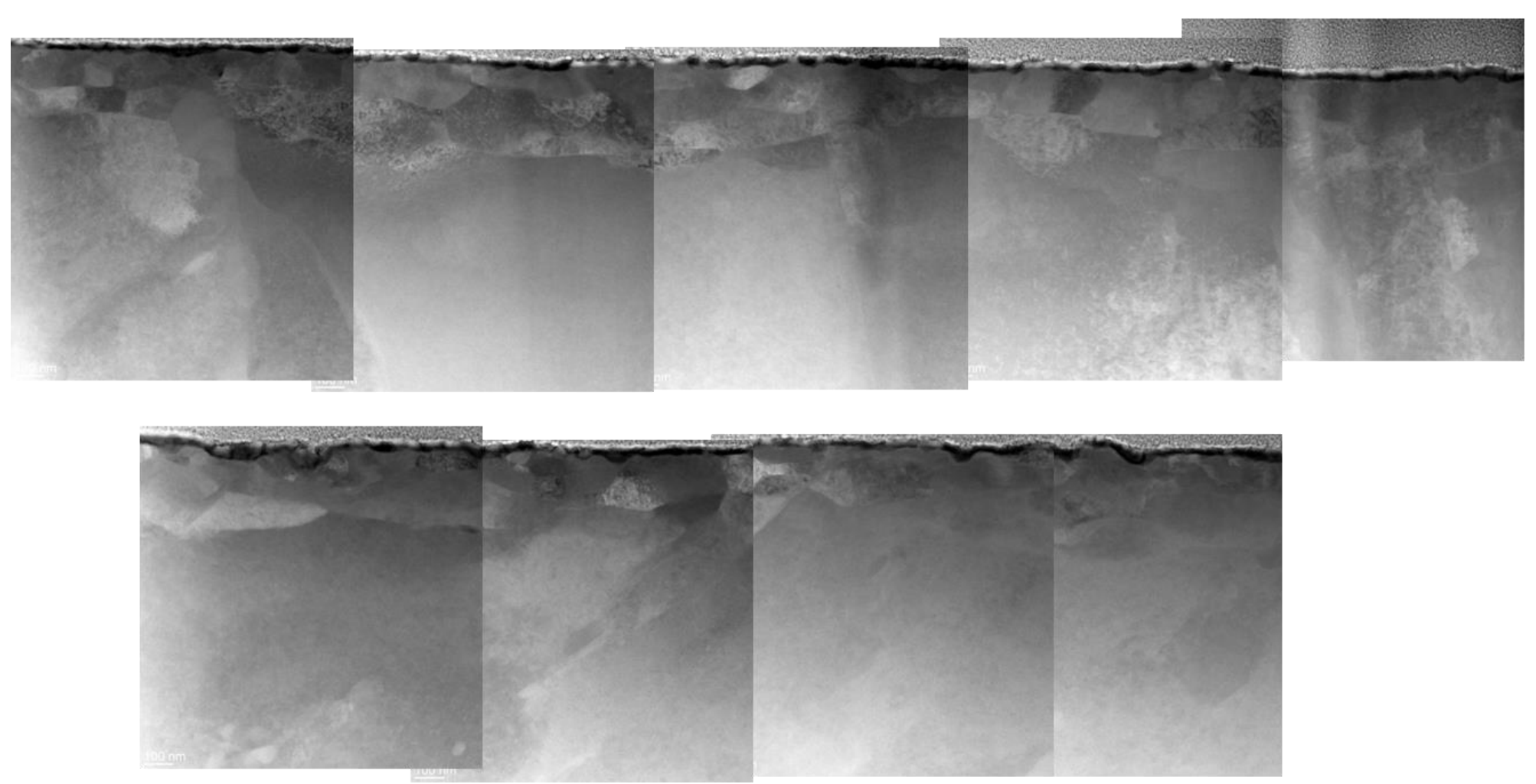

Figure B. 7: $\mathrm{HT} 9$ (ACO3) irradiated with $5 \mathrm{MeV} \mathrm{Fe}^{++}$at $440^{\circ} \mathrm{C}$ up to 50 dpa with 10 appm He preimplanted. 


\section{ACO3-460C-375dpa-10.10He-111313-TEM-Grid B-120213}

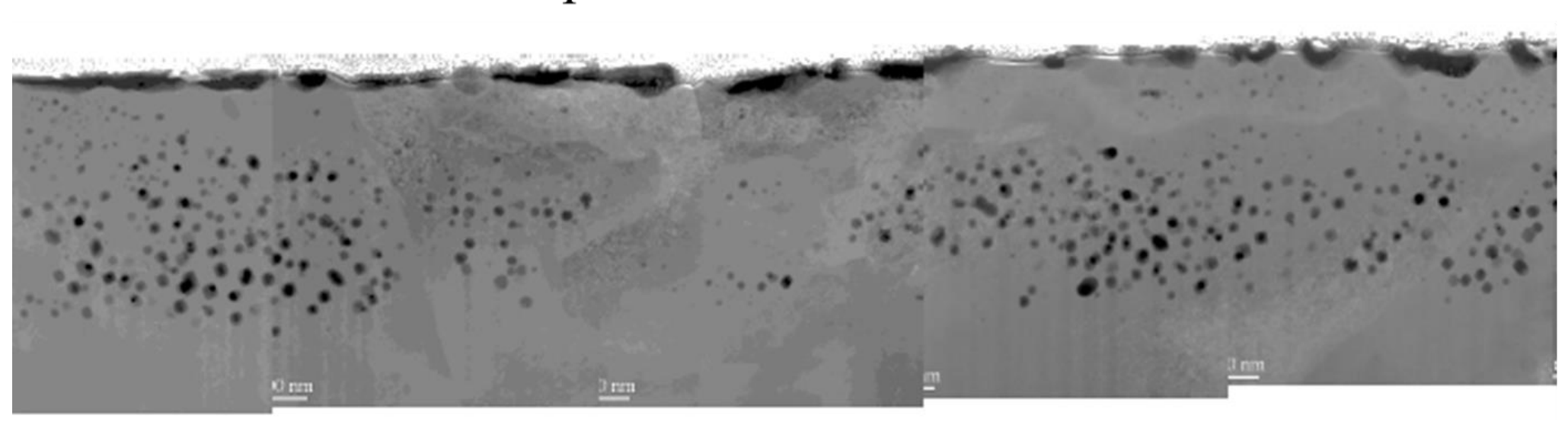

Figure B. 8: HT9 (ACO3) irradiated with $5 \mathrm{MeV} \mathrm{Fe}^{++}$at $460^{\circ} \mathrm{C}$ up to 375 dpa with 10 appm He preimplanted. 


\section{ACO3-460C-130dpa-10.10He-092214-TEM-Grid C-100314}

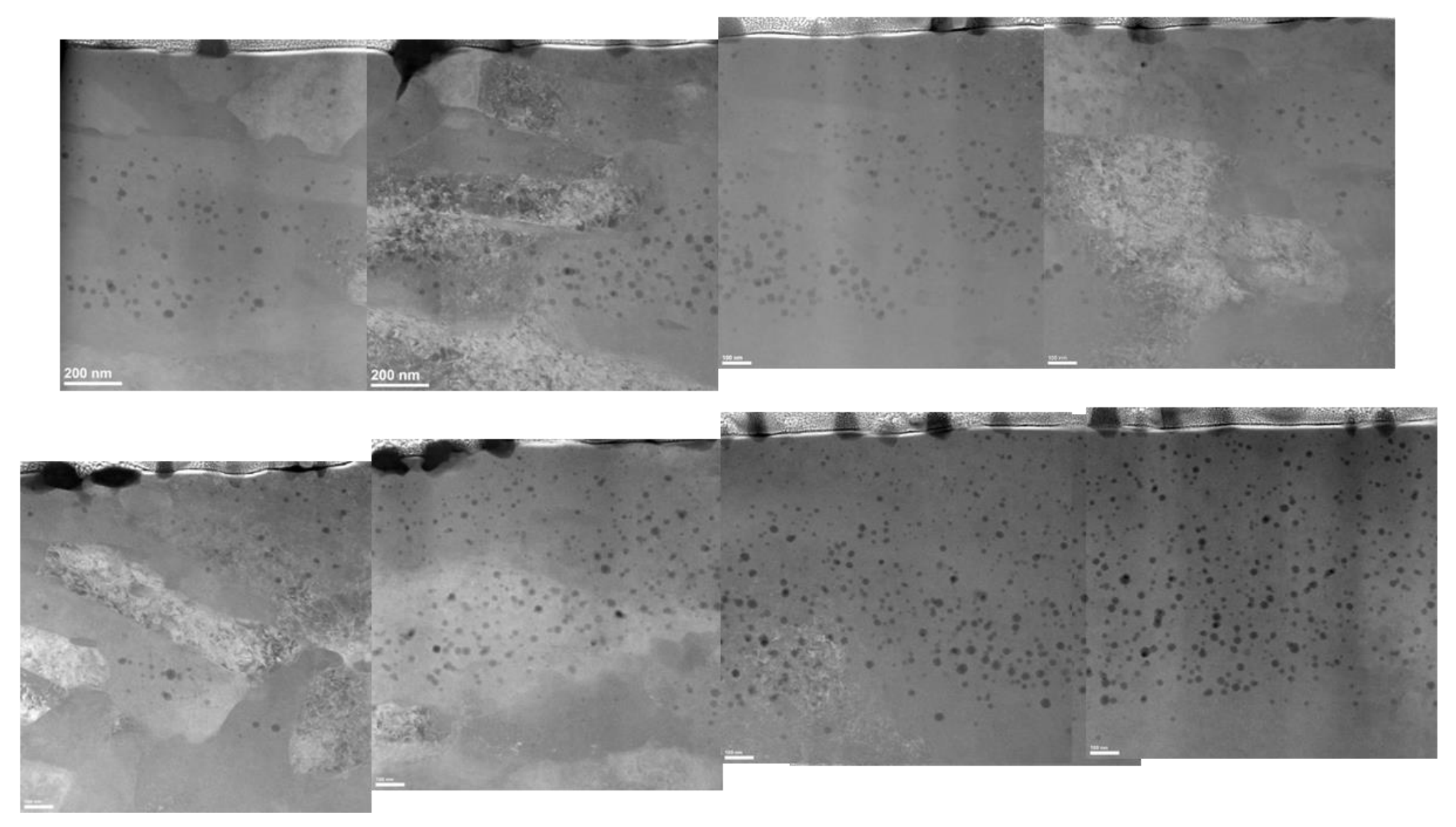

Figure B. 9: HT9 (ACO3) irradiated with $5 \mathrm{MeV} \mathrm{Fe}^{++}$at $460^{\circ} \mathrm{C}$ up to 130 dpa with 10 appm He preimplanted. 


\section{ACO3-460C-75dpa-10.10He-102814-TEM-Grid C-033115}

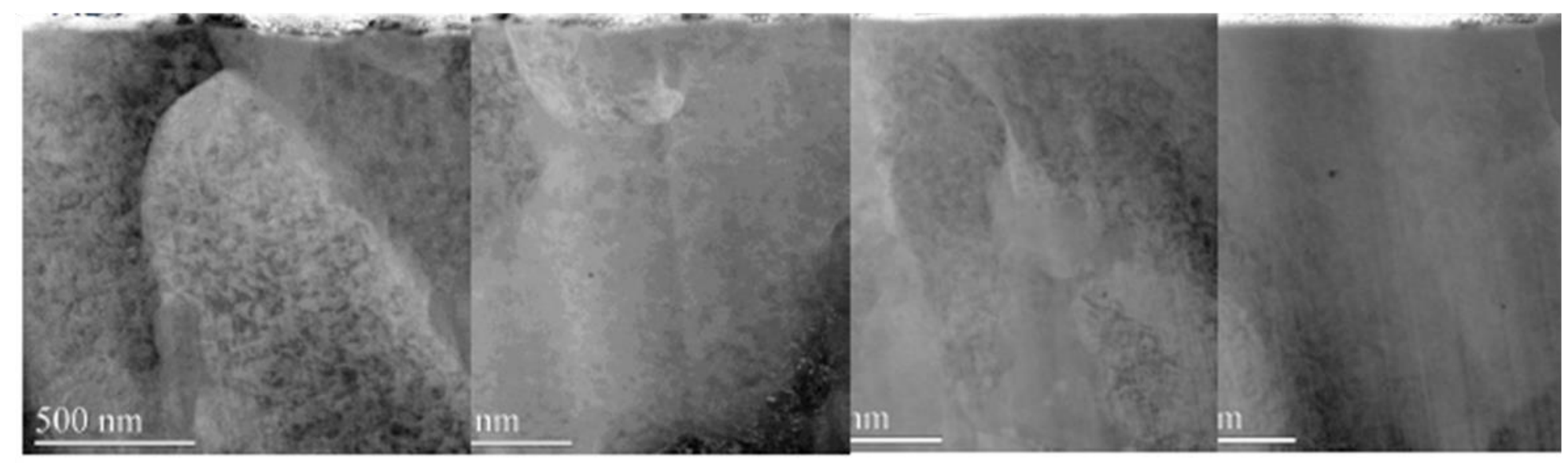

Figure B. 10: HT9 (ACO3) irradiated with $5 \mathrm{MeV} \mathrm{Fe}^{++}$at $460^{\circ} \mathrm{C}$ up to 75 dpa with 10 appm He preimplanted. 


\section{ACO3-460C-250dpa-10.10He-120814-TEM-Grid A-121214}
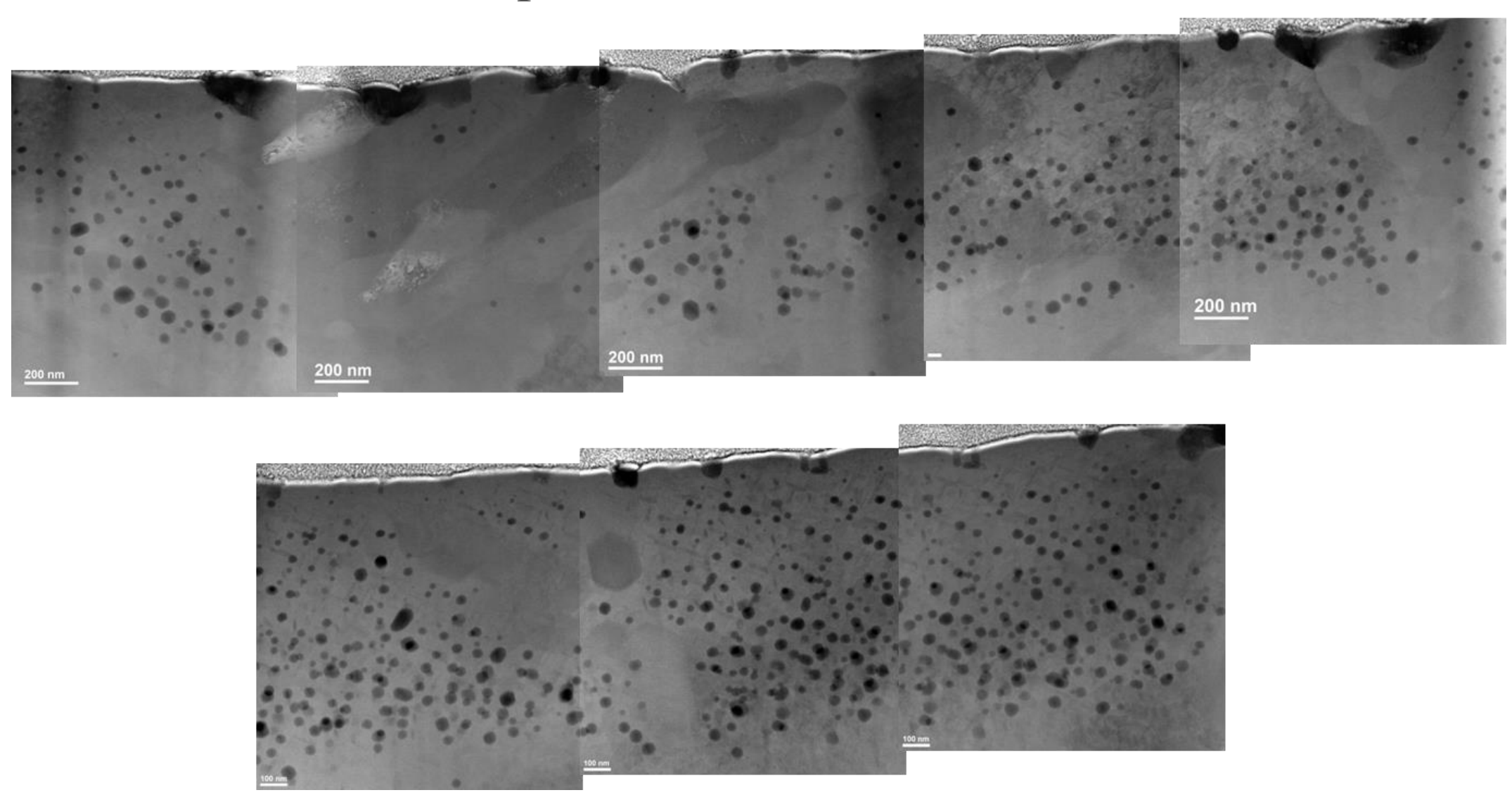

Figure B. 11: $\mathrm{HT} 9$ (ACO3) irradiated with $5 \mathrm{MeV} \mathrm{Fe}^{++}$at $460^{\circ} \mathrm{C}$ up to 250 dpa with 10 appm He preimplanted. 


\section{ACO3-460C-350dpa-10.10He-011214-TEM-Grid B-011615}
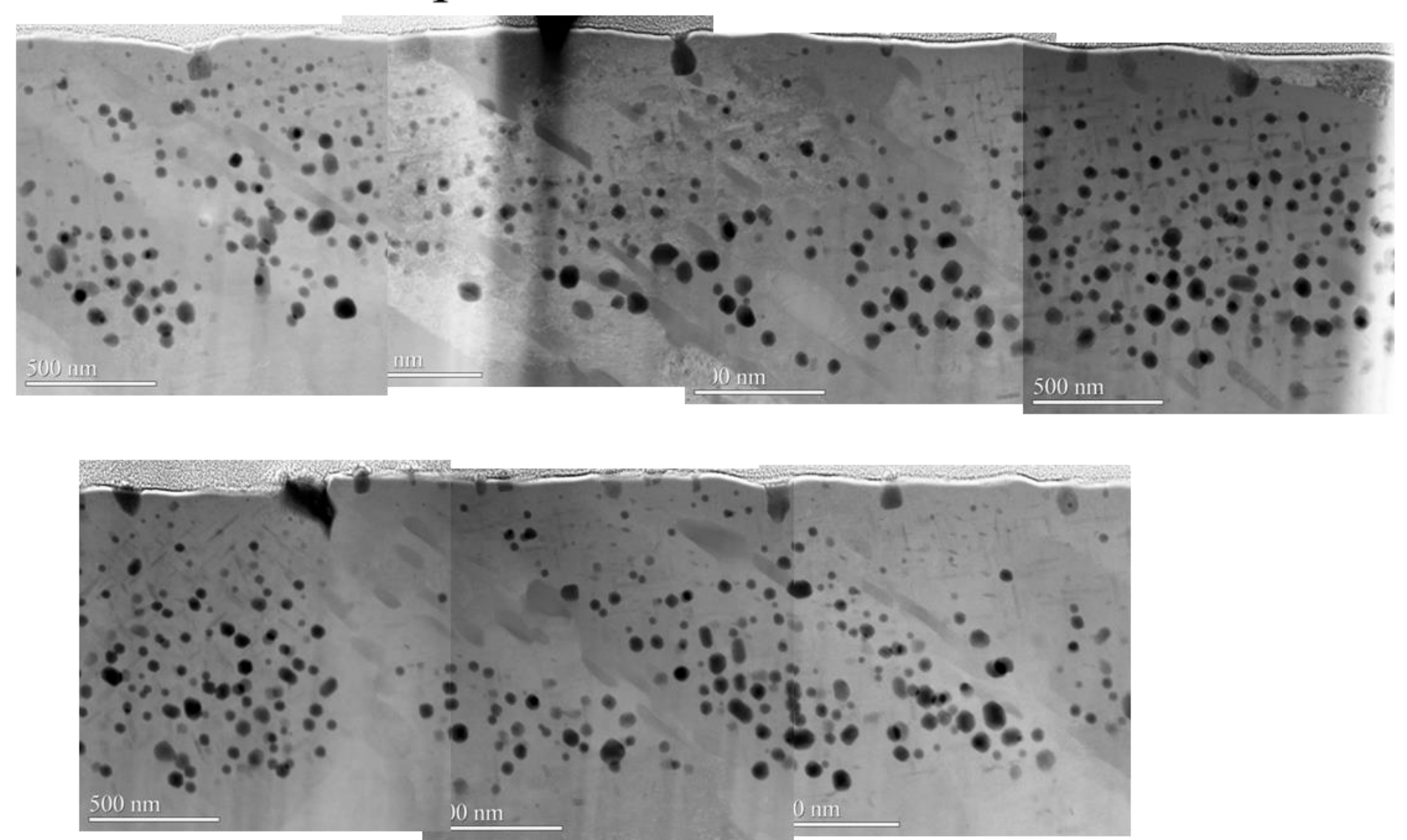

Figure B. 12: HT9 (ACO3) irradiated with $5 \mathrm{MeV} \mathrm{Fe}^{++}$at $460^{\circ} \mathrm{C}$ up to 350 dpa with 10 appm He preimplanted. 


\section{ACO3-460C-450dpa-10He-021615-Grid B-021915}
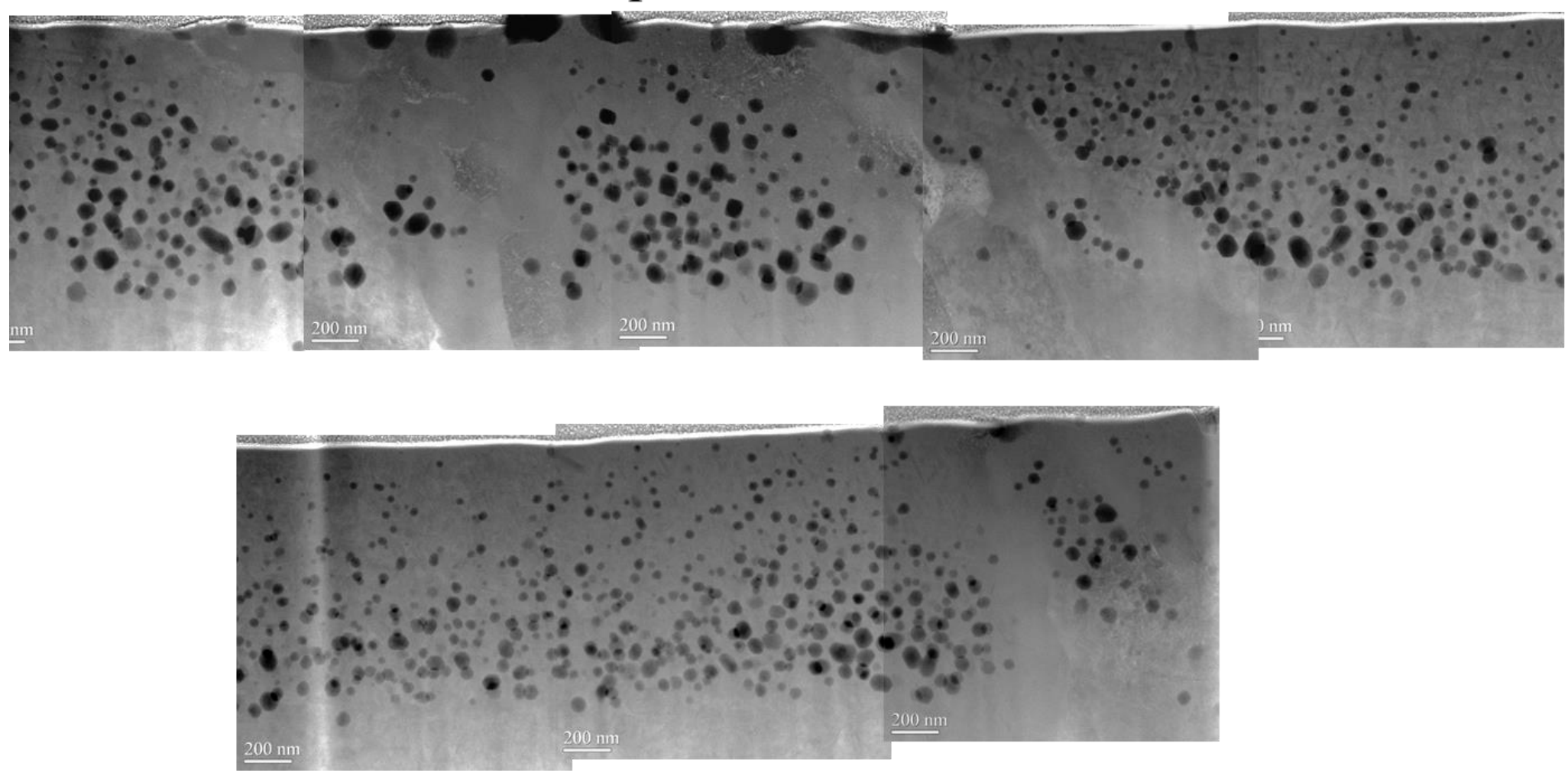

Figure B. 13: $\mathrm{HT} 9$ (ACO3) irradiated with $5 \mathrm{MeV} \mathrm{Fe}^{++}$at $460^{\circ} \mathrm{C}$ up to 450 dpa with 10 appm He preimplanted. 
ACO3-460C-550dpa-10.10He-030515-TEM-Grid B-031315

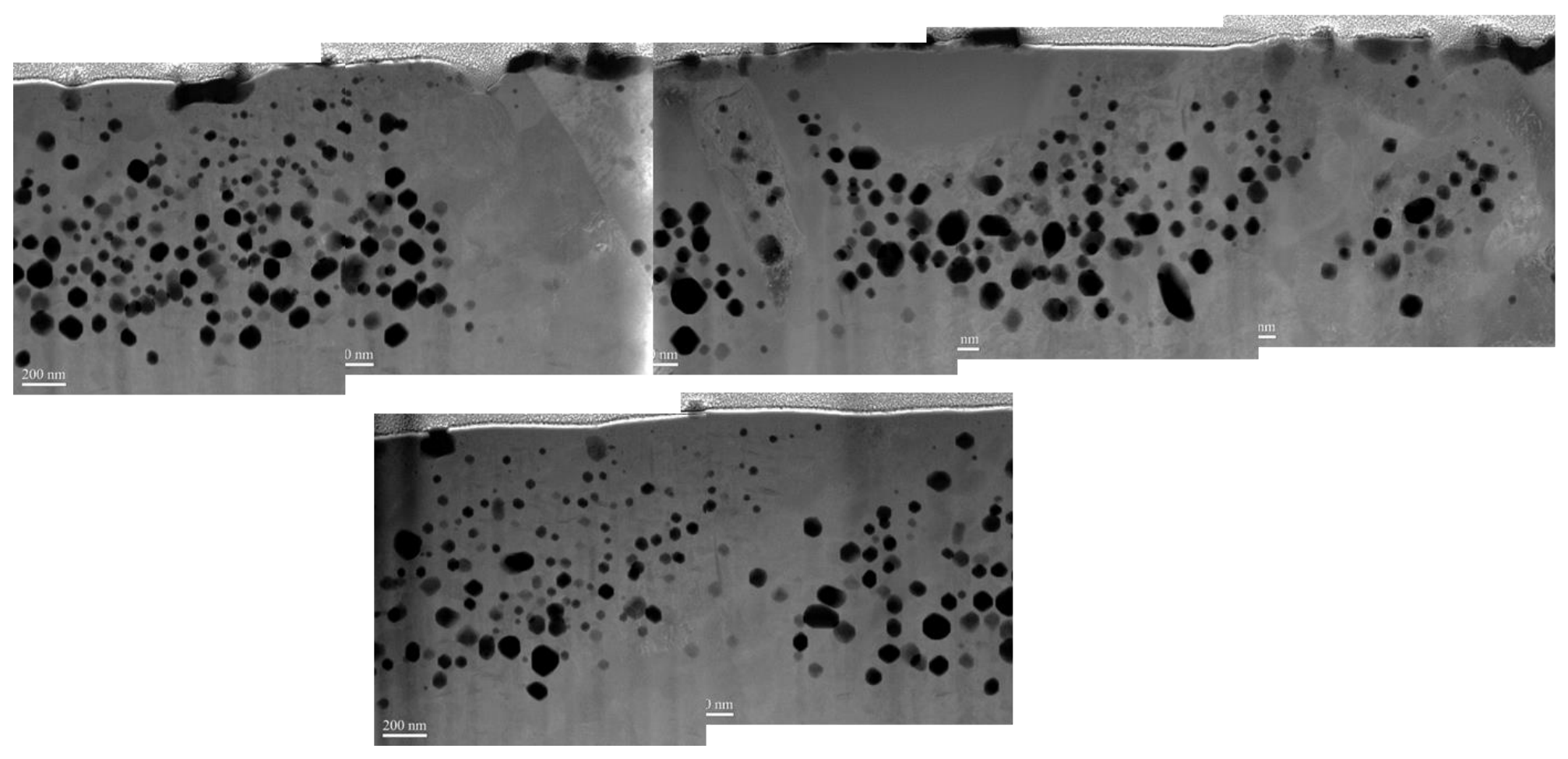

Figure B. 14: $\mathrm{HT} 9$ (ACO3) irradiated with $5 \mathrm{MeV} \mathrm{Fe}^{++}$at $460^{\circ} \mathrm{C}$ up to 550 dpa with 10 appm He preimplanted. 
ACO3-460C-650dpa-10.10He-050714-TEM-Grid B-051515

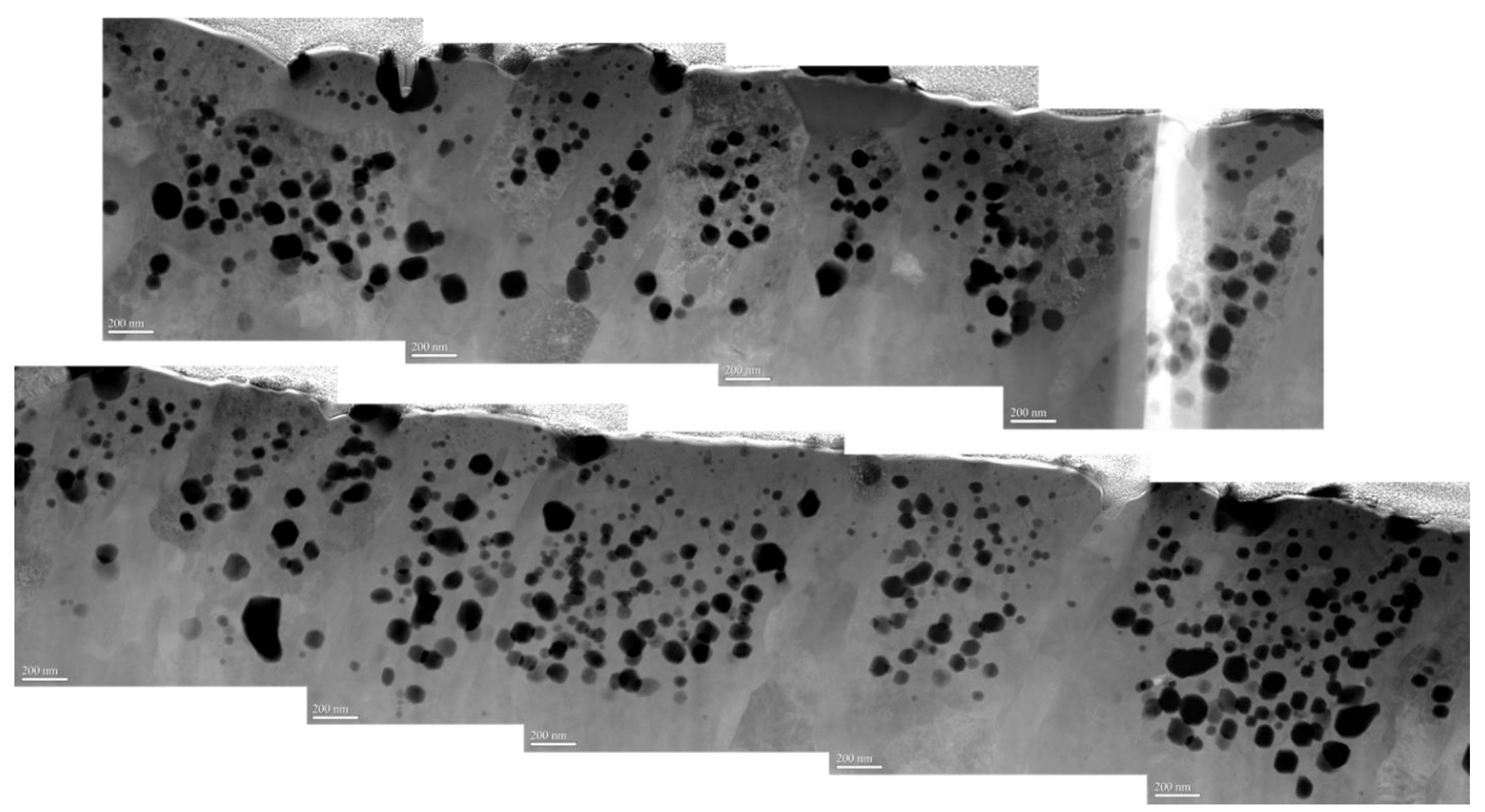

Figure B. 15: HT9 (ACO3) irradiated with $5 \mathrm{MeV} \mathrm{Fe}^{++}$at $460^{\circ} \mathrm{C}$ up to 650 dpa with 10 appm He preimplanted. 


\section{APPENDIX C - VOID DEPTH PROFILES AND DISTRIBUTIONS}

The following are void depth profiles and distributions for all irradiation conditions considered in this thesis. 

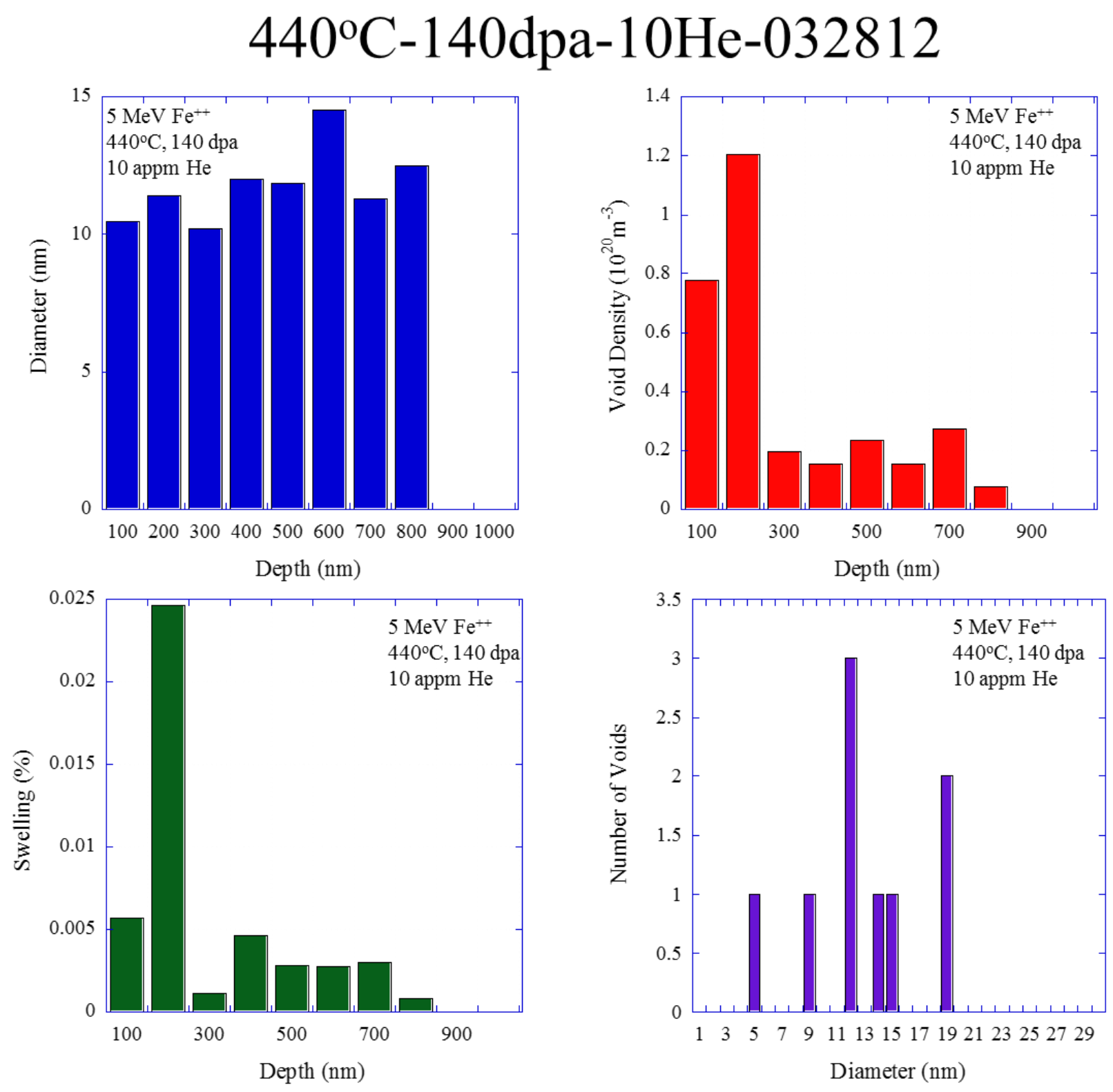

Figure C. 1: Void diameter, number density and swelling depth profiles as well as void distribution in the 500 to $700 \mathrm{~nm}$ depth region of HT9 irradiated with $5 \mathrm{MeV}$ $\mathrm{Fe}^{++}(\mathrm{ACO} 3)$ at $440^{\circ} \mathrm{C}$, to 140 dpa with 10 appm He. 

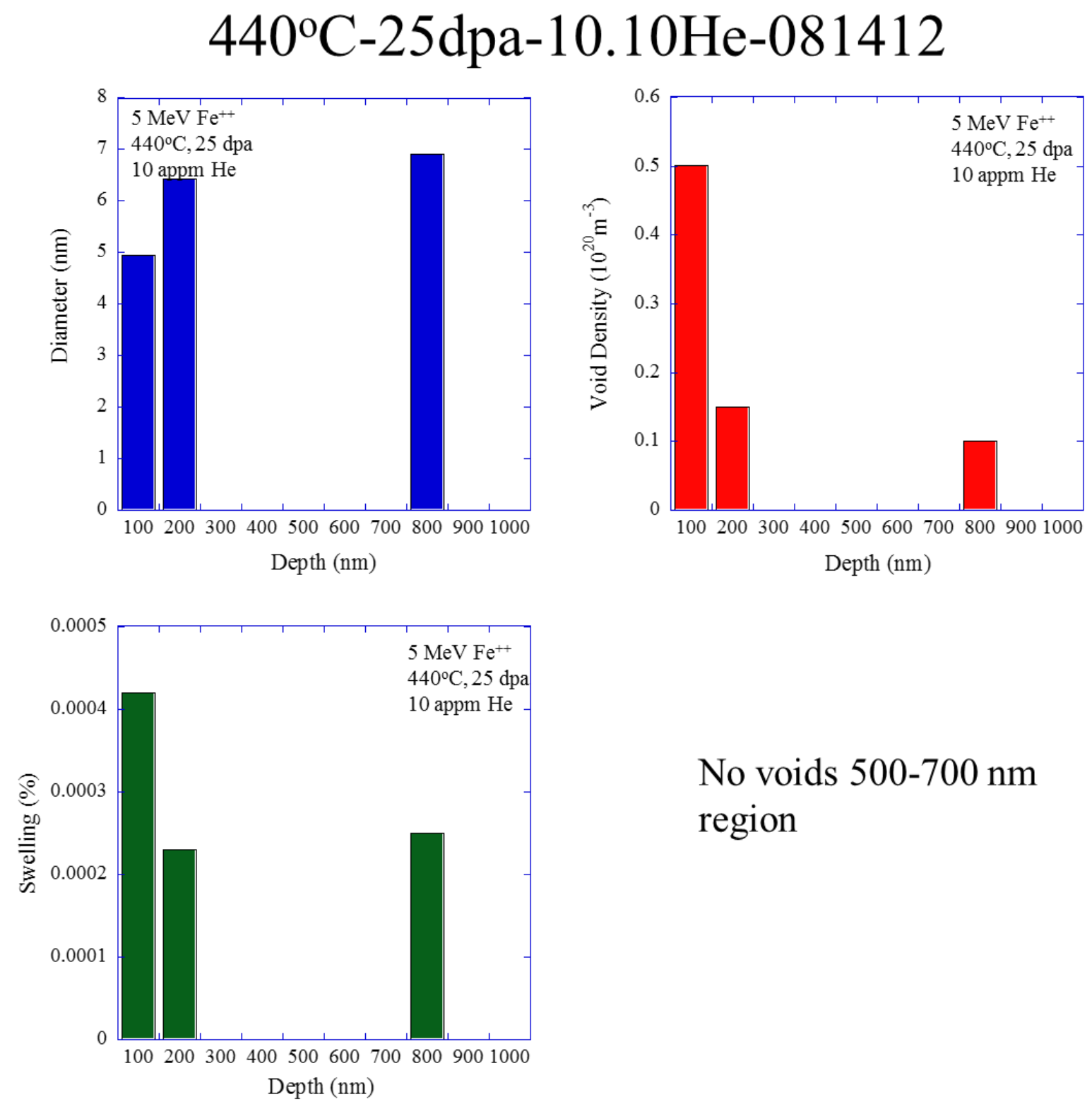

No voids $500-700 \mathrm{~nm}$ region

Figure C. 2: Void diameter, number density and swelling depth profiles as well as void distribution in the 500 to $700 \mathrm{~nm}$ depth region of HT9 irradiated with $5 \mathrm{MeV}$ $\mathrm{Fe}^{++}(\mathrm{ACO} 3)$ at $440^{\circ} \mathrm{C}$, to 25 dpa with 10 appm He. 

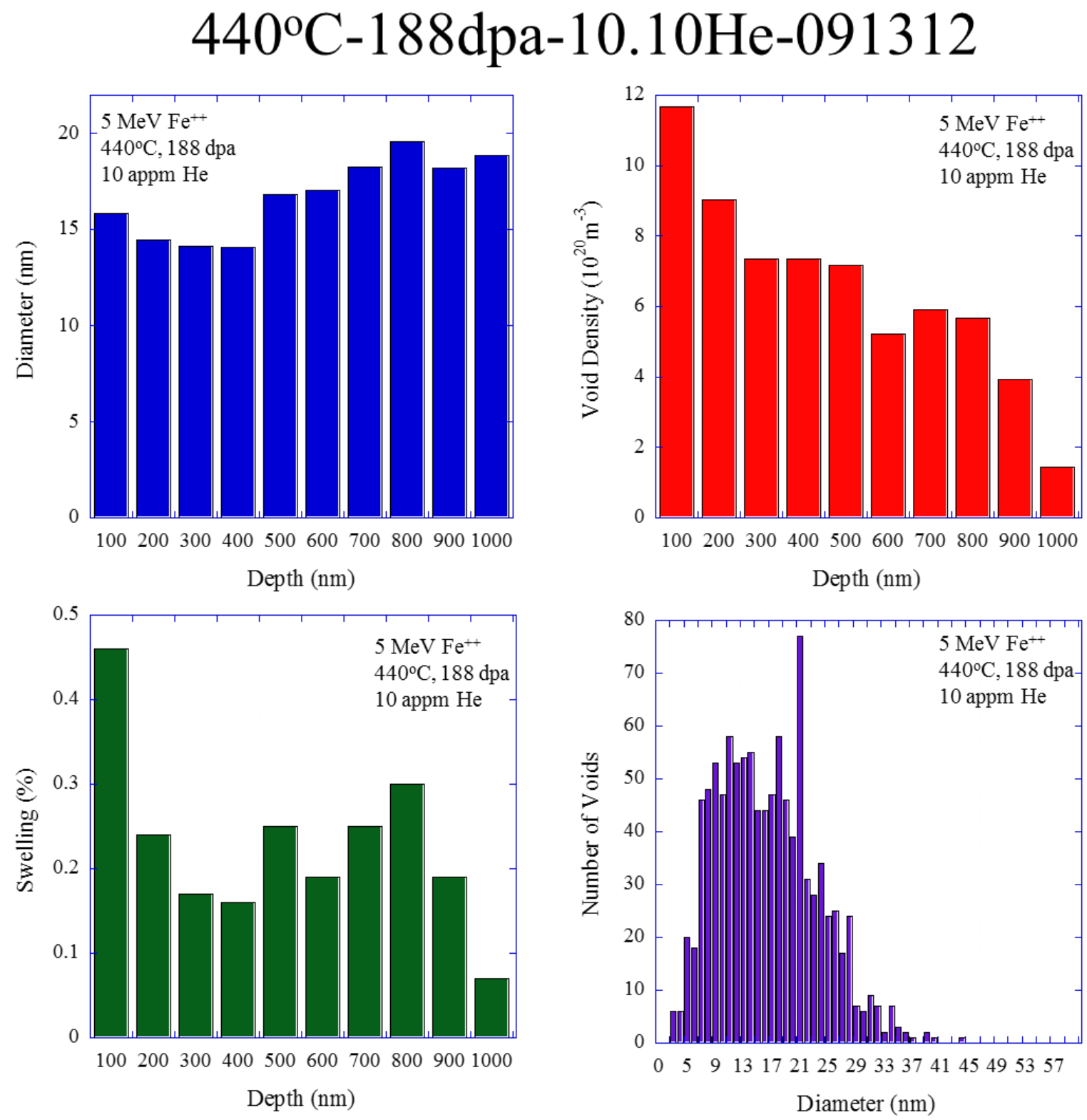

Figure C. 3: Void diameter, number density and swelling depth profiles as well as void distribution in the 500 to $700 \mathrm{~nm}$ depth region of HT9 irradiated with $5 \mathrm{MeV}$ $\mathrm{Fe}^{++}(\mathrm{ACO} 3)$ at $440^{\circ} \mathrm{C}$, to $188 \mathrm{dpa}$ with $10 \mathrm{appm} \mathrm{He}$. 

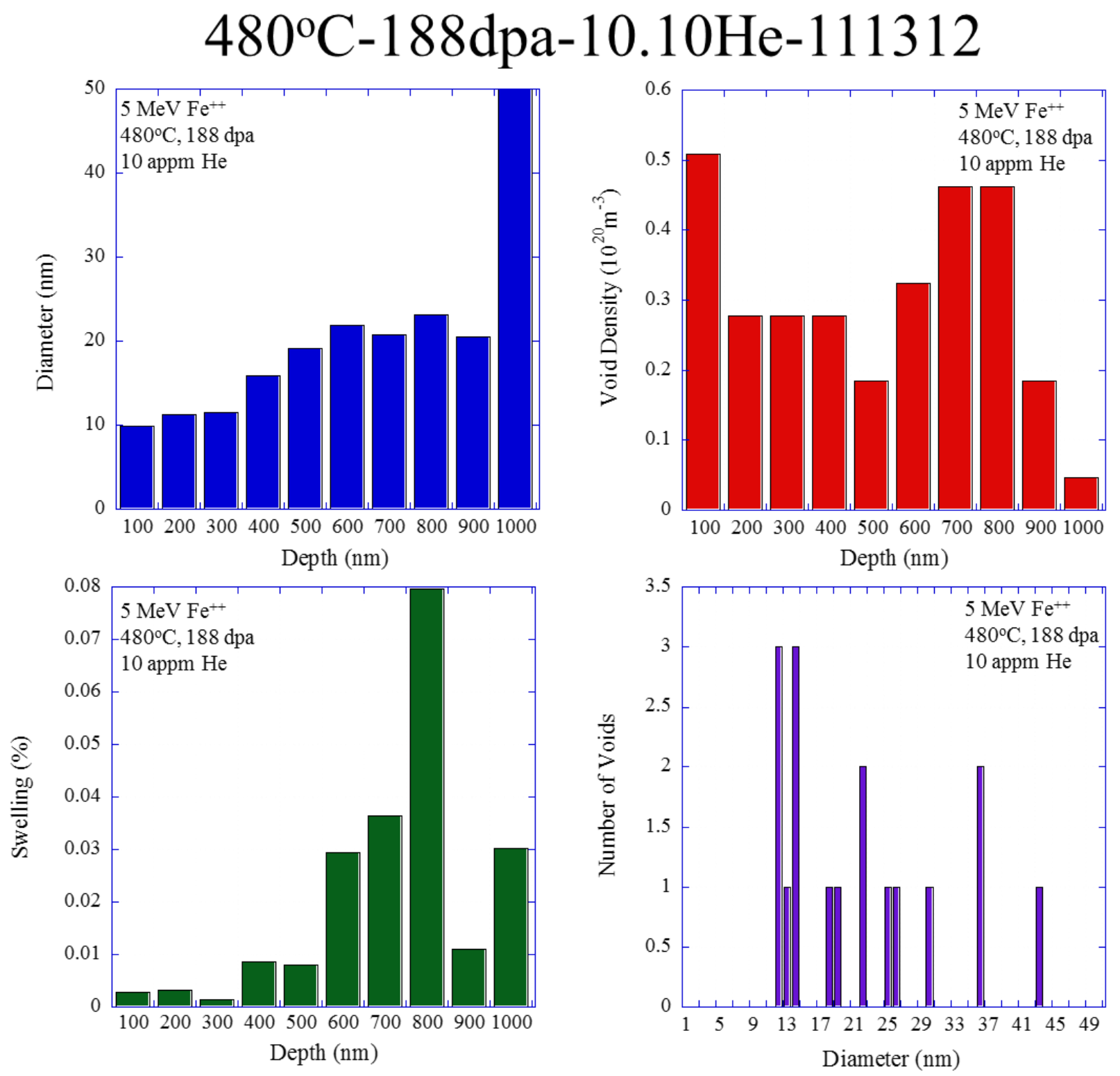

Figure C. 4: Void diameter, number density and swelling depth profiles as well as void distribution in the 500 to $700 \mathrm{~nm}$ depth region of HT9 irradiated with $5 \mathrm{MeV}$ $\mathrm{Fe}^{++}(\mathrm{ACO} 3)$ at $480^{\circ} \mathrm{C}$, to $188 \mathrm{dpa}$ with $10 \mathrm{appm} \mathrm{He}$. 


\section{$400^{\circ} \mathrm{C}-250 \mathrm{dpa}-0.0 \mathrm{He}-011913$}

No voids observed

No voids observed

No voids observed

No voids observed

Figure C. 5: Void diameter, number density and swelling depth profiles as well as void distribution in the 500 to $700 \mathrm{~nm}$ depth region of HT9 irradiated with $5 \mathrm{MeV}$ $\mathrm{Fe}^{++}(\mathrm{ACO} 3)$ at $400^{\circ} \mathrm{C}$, to $250 \mathrm{dpa}$ with $10 \mathrm{appm} \mathrm{He}$. 


\section{$460^{\circ} \mathrm{C}-188 \mathrm{dpa}-10.10 \mathrm{He}-062413$}
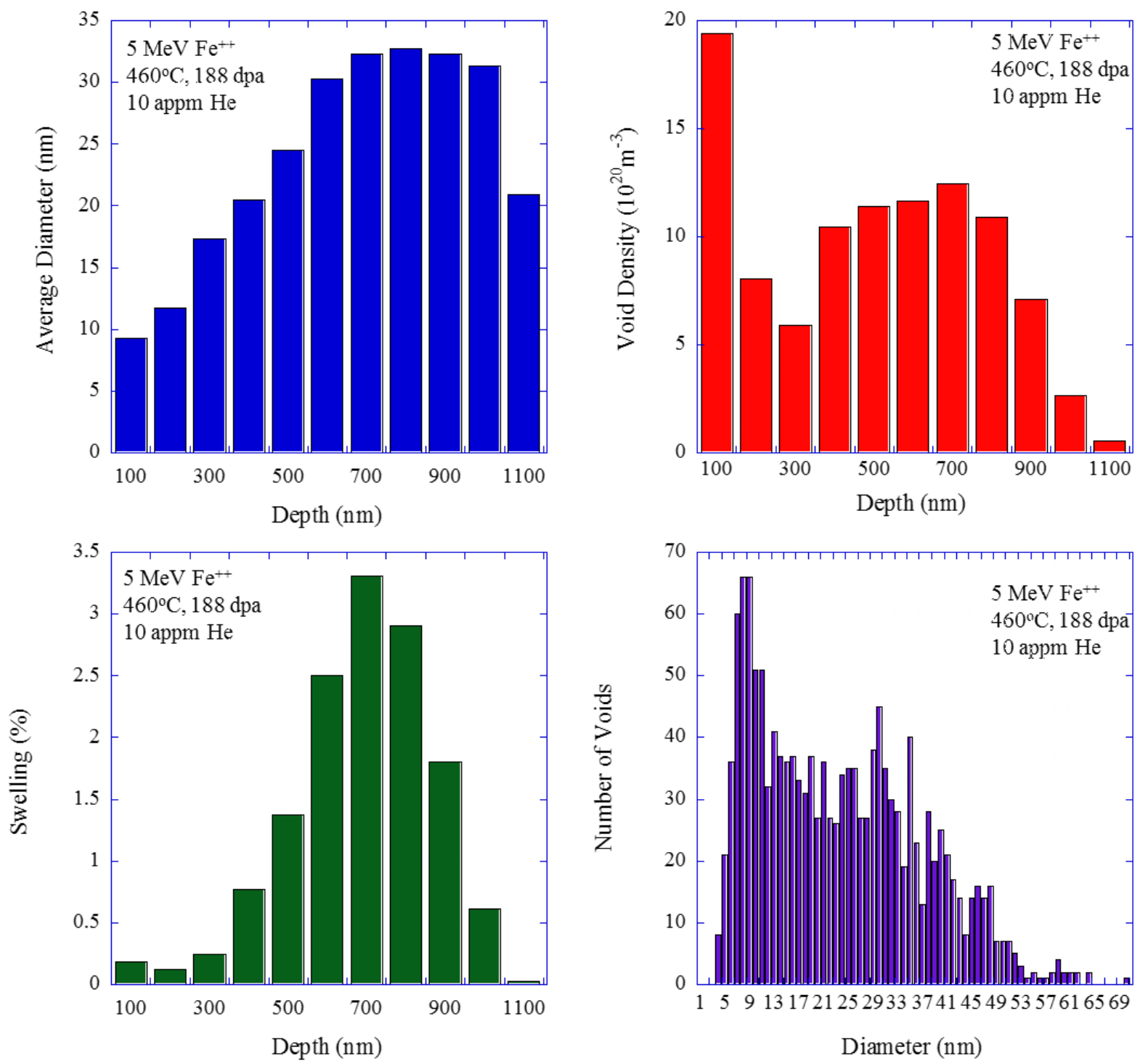

Figure C. 6: Void diameter, number density and swelling depth profiles as well as void distribution in the 500 to $700 \mathrm{~nm}$ depth region of HT9 irradiated with $5 \mathrm{MeV}$ $\mathrm{Fe}^{++}(\mathrm{ACO} 3)$ at $460^{\circ} \mathrm{C}$, to $188 \mathrm{dpa}$ with $10 \mathrm{appm} \mathrm{He}$. 


\section{$440^{\circ} \mathrm{C}-50 \mathrm{dpa}-10.10 \mathrm{He}-090413$}
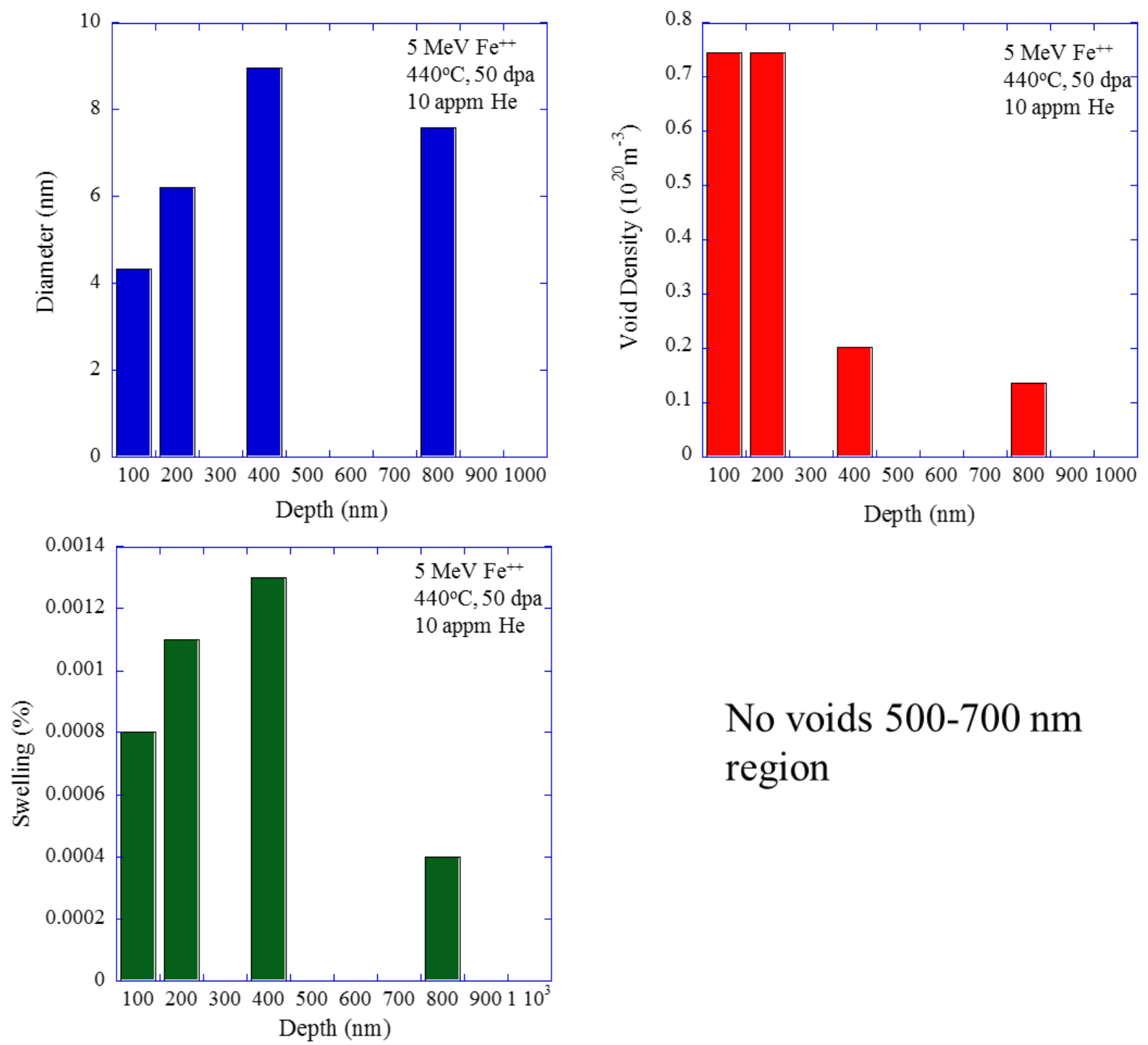

No voids $500-700 \mathrm{~nm}$ region

Figure C. 7: Void diameter, number density and swelling depth profiles as well as void distribution in the 500 to $700 \mathrm{~nm}$ depth region of HT9 irradiated with $5 \mathrm{MeV}$ $\mathrm{Fe}^{++}(\mathrm{ACO} 3)$ at $440^{\circ} \mathrm{C}$, to $50 \mathrm{dpa}$ with 10 appm He. 

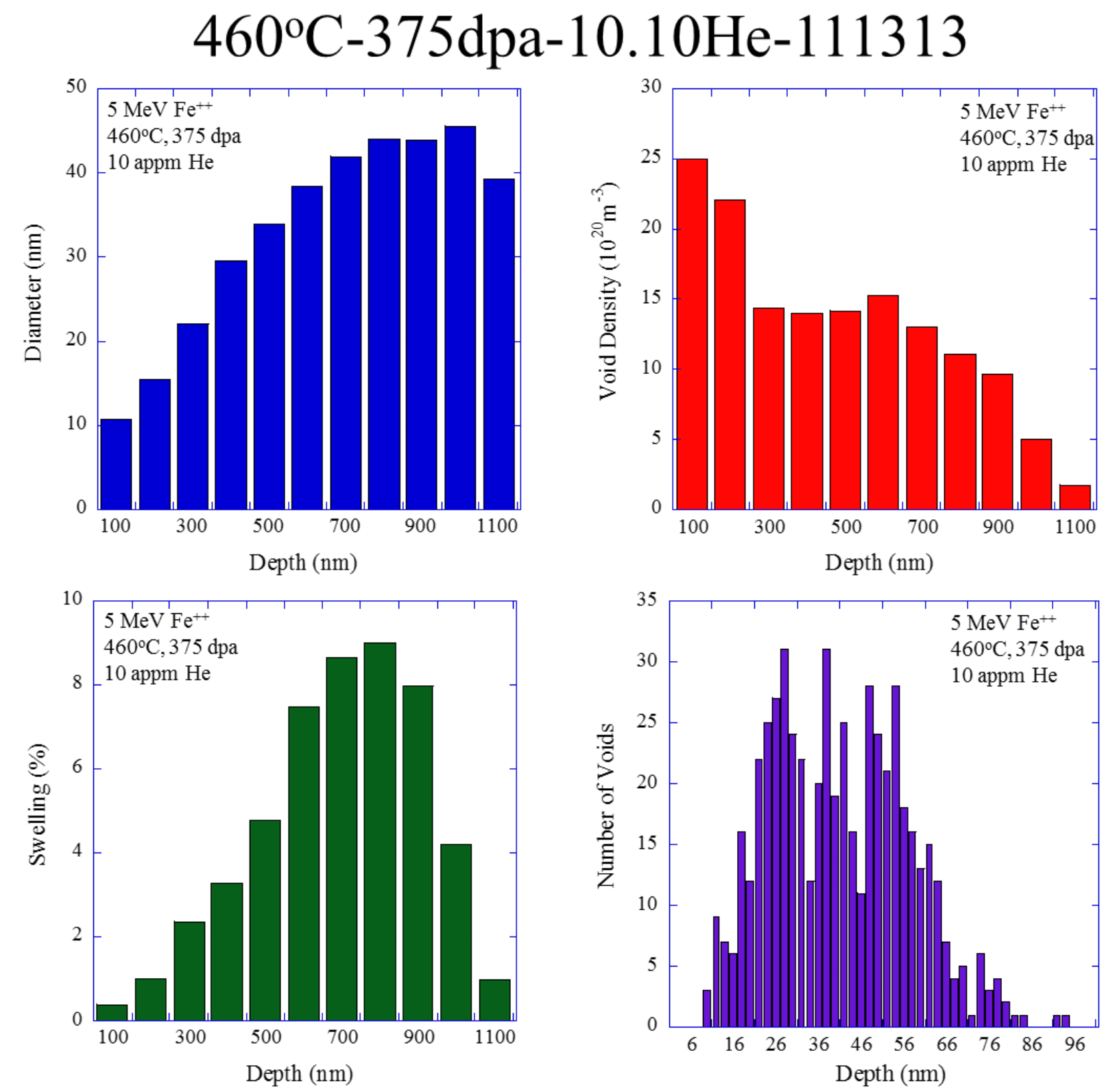

Figure C. 8: Void diameter, number density and swelling depth profiles as well as void distribution in the 500 to $700 \mathrm{~nm}$ depth region of HT9 irradiated with $5 \mathrm{MeV}$ $\mathrm{Fe}^{++}(\mathrm{ACO} 3)$ at $460^{\circ} \mathrm{C}$, to $375 \mathrm{dpa}$ with $10 \mathrm{appm} \mathrm{He}$. 

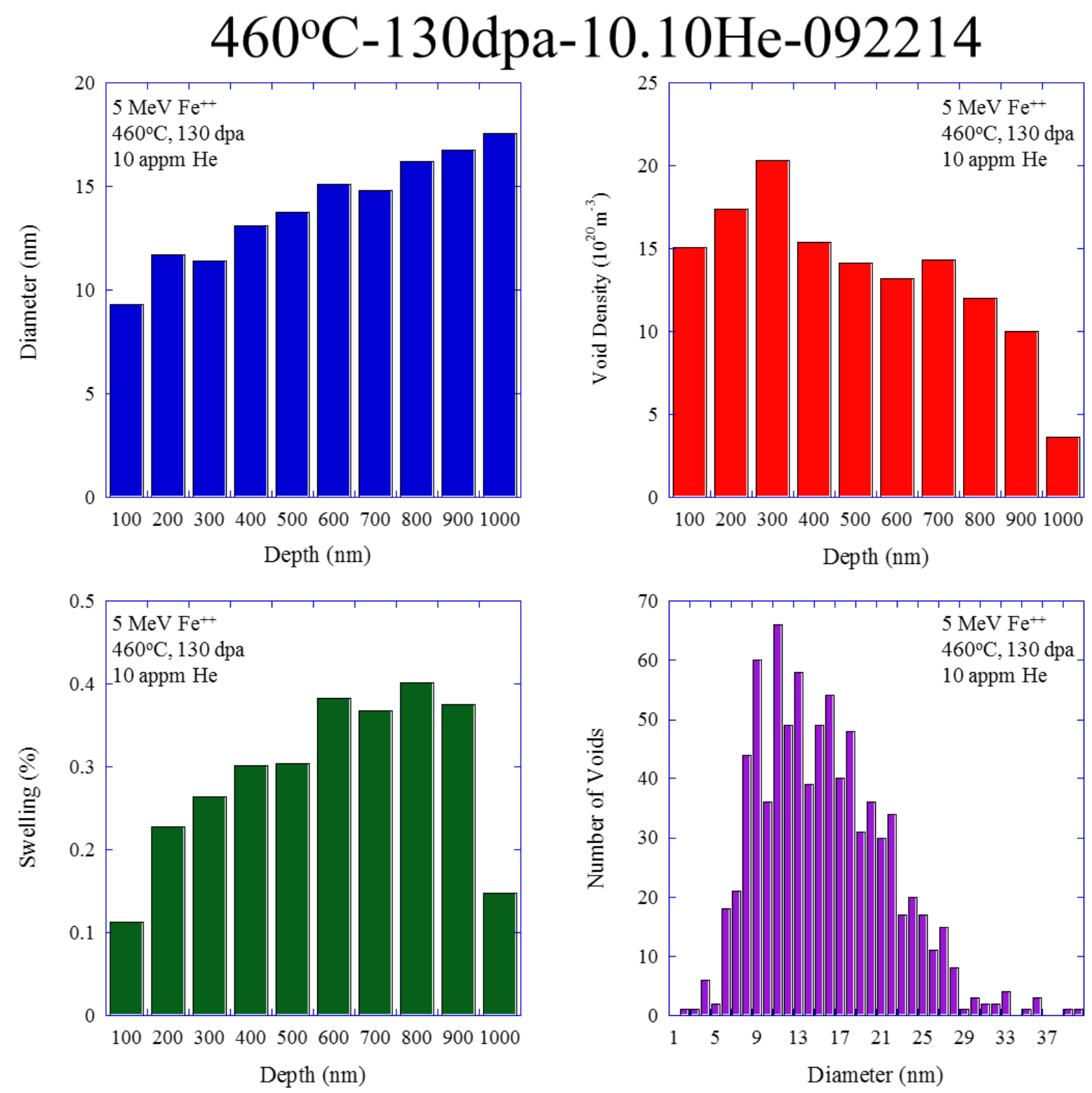

Figure C. 9: Void diameter, number density and swelling depth profiles as well as void distribution in the 500 to $700 \mathrm{~nm}$ depth region of $\mathrm{HT} 9$ irradiated with $5 \mathrm{MeV}$ $\mathrm{Fe}^{++}(\mathrm{ACO} 3)$ at $460^{\circ} \mathrm{C}$, to $130 \mathrm{dpa}$ with $10 \mathrm{appm} \mathrm{He}$. 

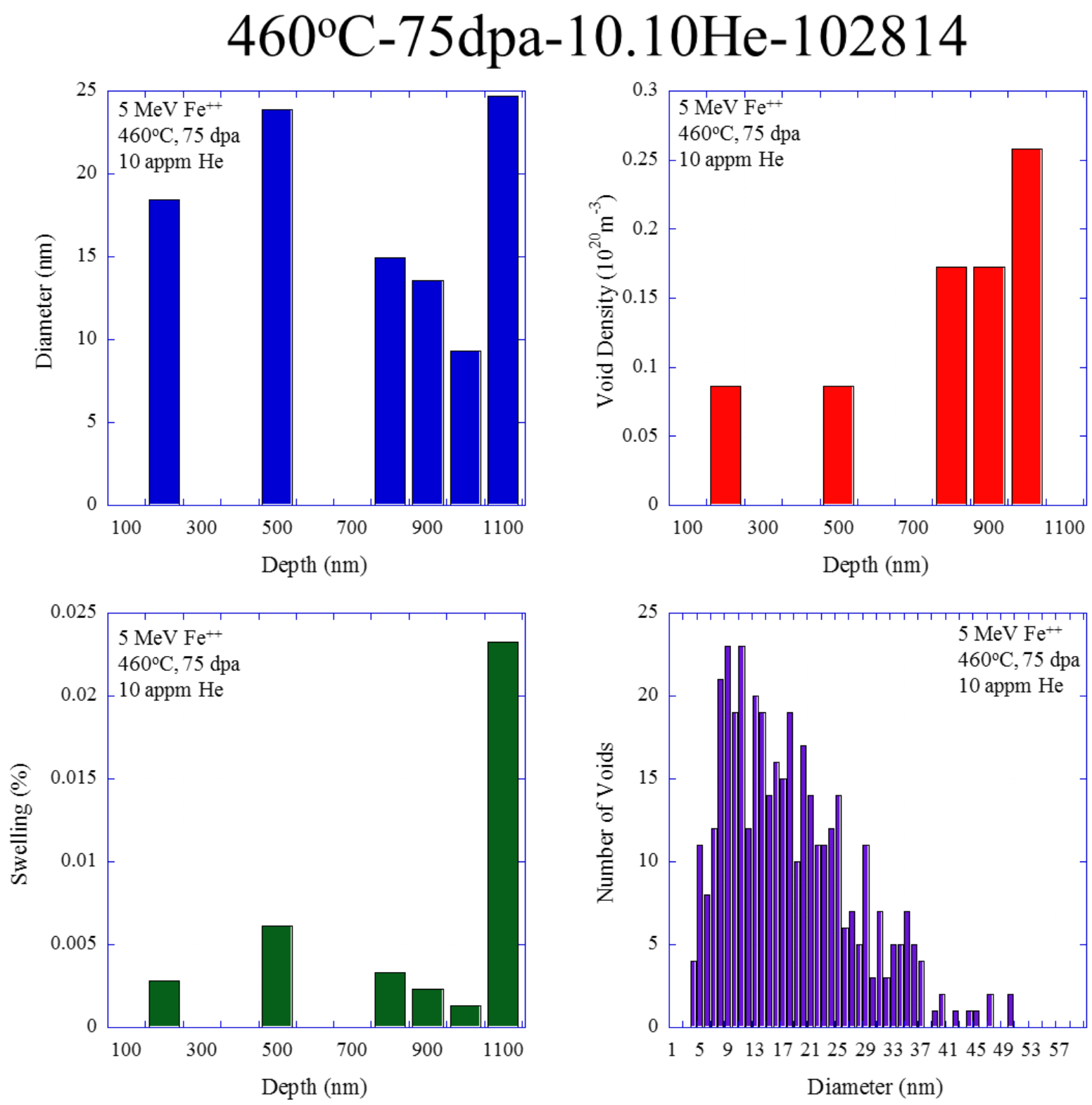

Figure C. 10: Void diameter, number density and swelling depth profiles as well as void distribution in the 500 to $700 \mathrm{~nm}$ depth region of HT9 irradiated with 5 $\mathrm{MeV} \mathrm{Fe}^{++}(\mathrm{ACO} 3)$ at $460^{\circ} \mathrm{C}$, to $75 \mathrm{dpa}$ with $10 \mathrm{appm} \mathrm{He}$. 

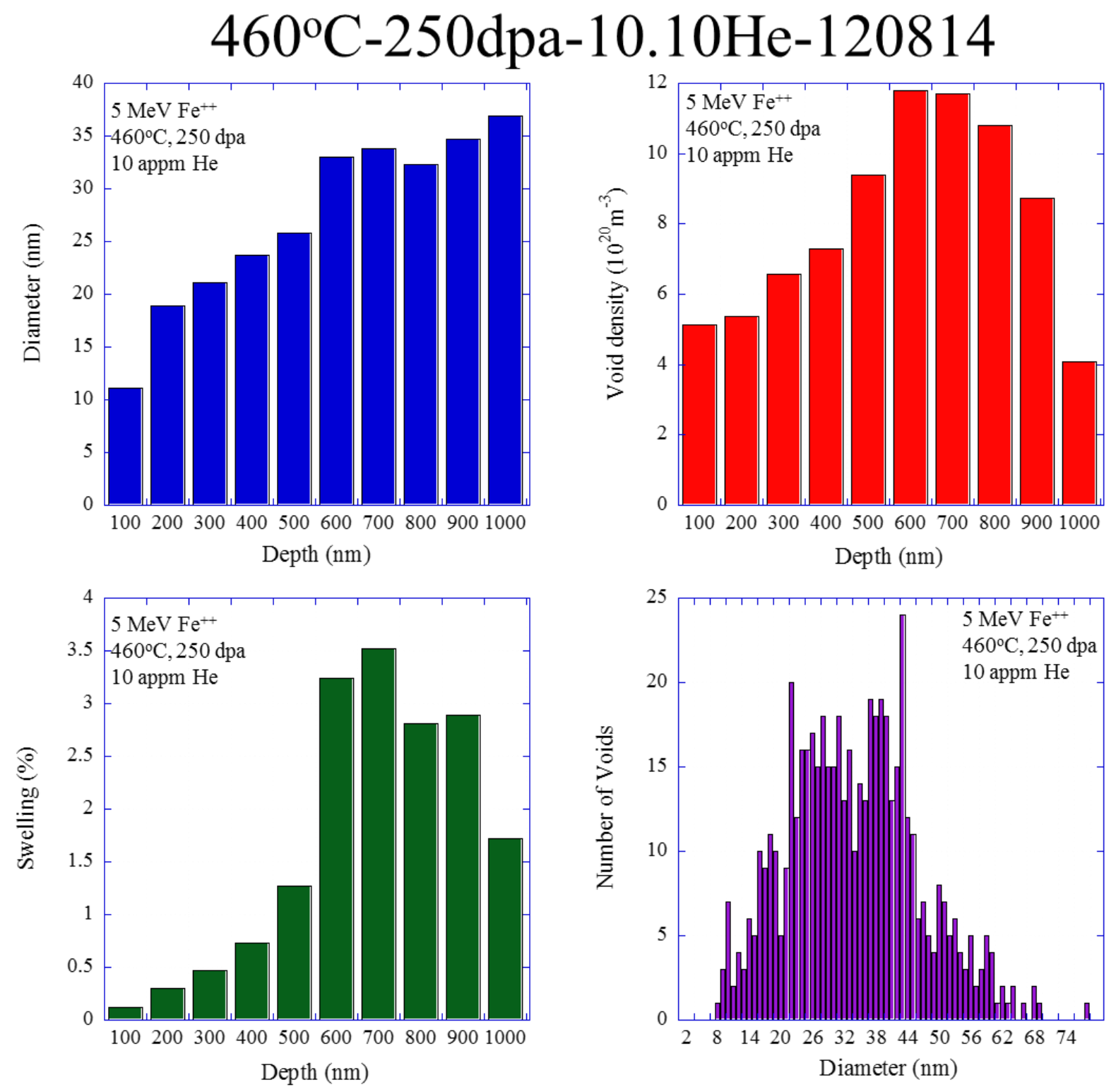

Figure C. 11: Void diameter, number density and swelling depth profiles as well as void distribution in the 500 to $700 \mathrm{~nm}$ depth region of HT9 irradiated with 5 $\mathrm{MeV} \mathrm{Fe}{ }^{++}(\mathrm{ACO} 3)$ at $460^{\circ} \mathrm{C}$, to $250 \mathrm{dpa}$ with $10 \mathrm{appm} \mathrm{He}$. 

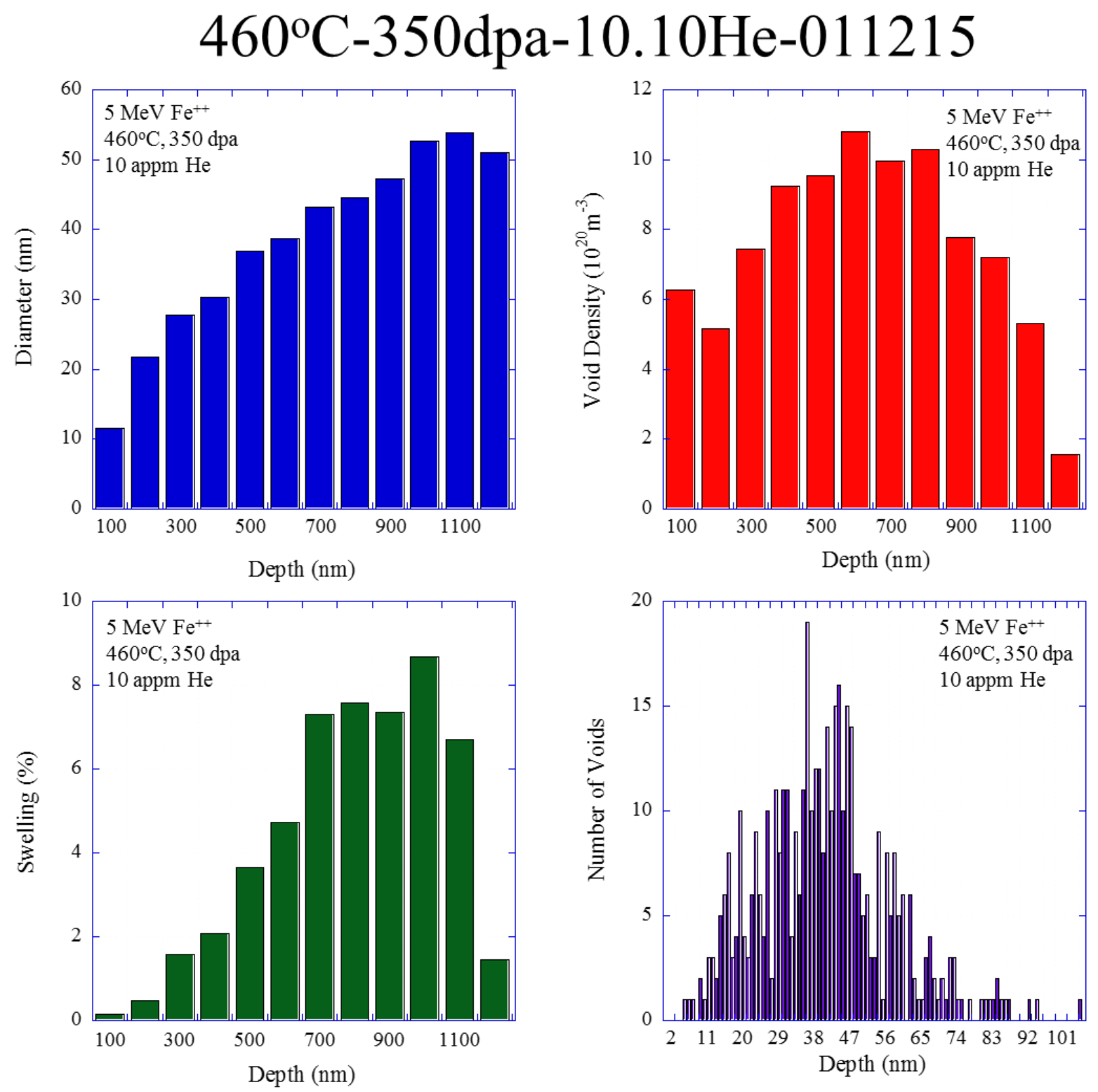

Figure C. 12 Void diameter, number density and swelling depth profiles as well as void distribution in the 500 to $700 \mathrm{~nm}$ depth region of HT9 irradiated with $5 \mathrm{MeV}$ $\mathrm{Fe}^{++}(\mathrm{ACO} 3)$ at $460^{\circ} \mathrm{C}$, to 350 dpa with 10 appm He. 


\section{$460^{\circ} \mathrm{C}-450 \mathrm{dpa}-10.10 \mathrm{He}-021615$}
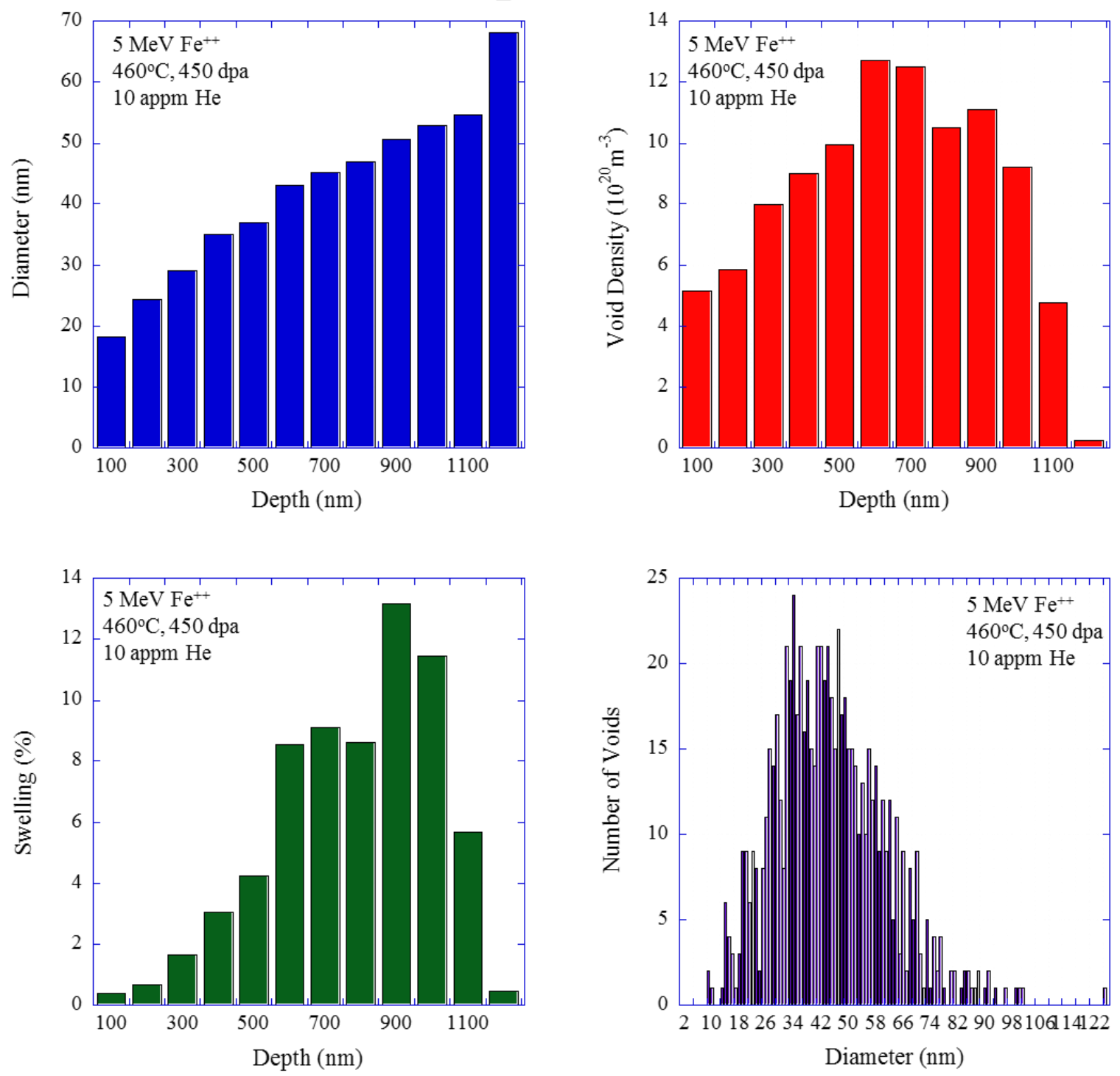

Figure C. 13: Void diameter, number density and swelling depth profiles as well as void distribution in the 500 to $700 \mathrm{~nm}$ depth region of HT9 irradiated with 5 $\mathrm{MeV} \mathrm{Fe}{ }^{++}(\mathrm{ACO} 3)$ at $460^{\circ} \mathrm{C}$, to $450 \mathrm{dpa}$ with $10 \mathrm{appm} \mathrm{He}$. 

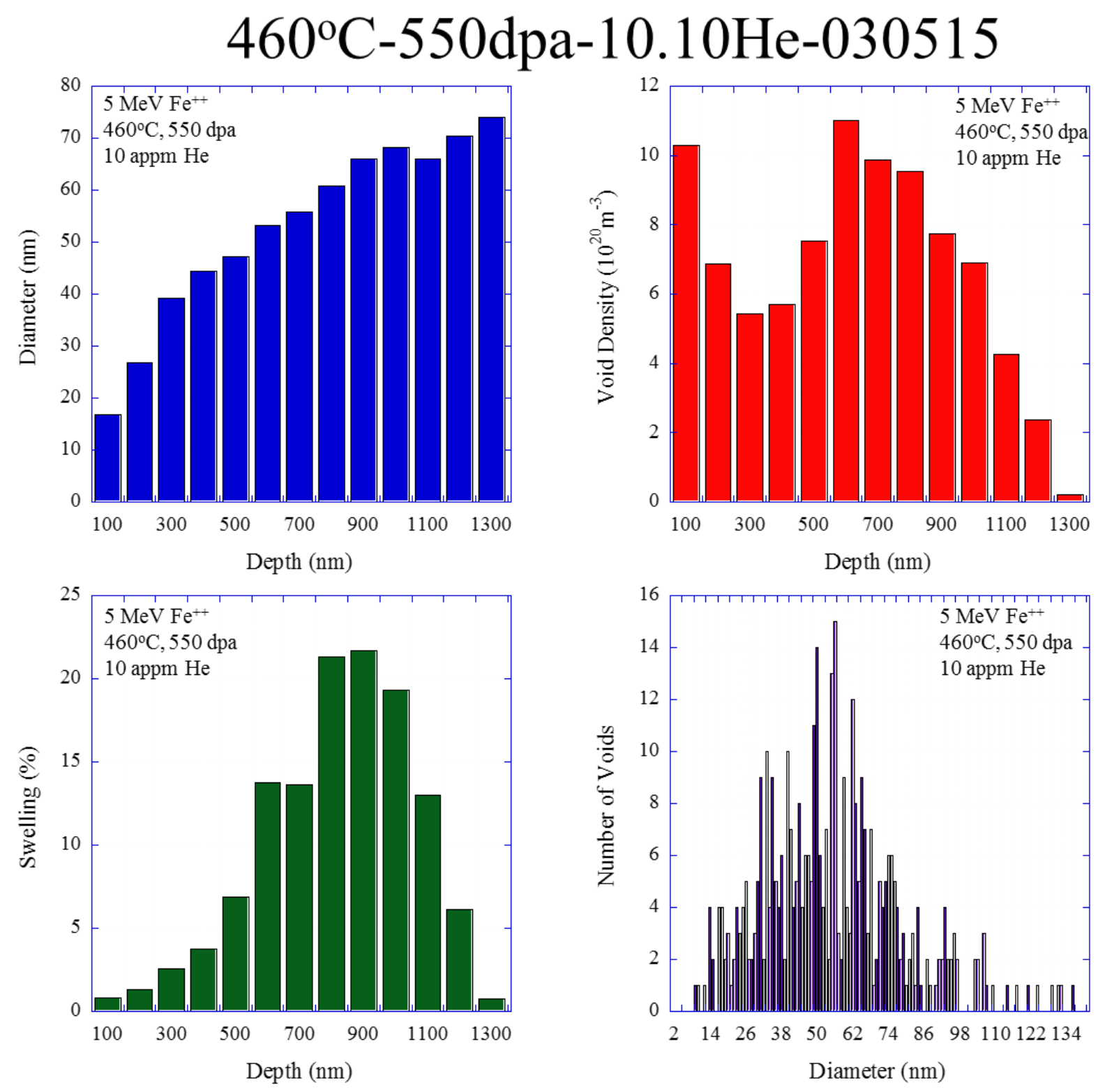

Figure C. 14: Void diameter, number density and swelling depth profiles as well as void distribution in the 500 to $700 \mathrm{~nm}$ depth region of HT9 irradiated with 5 $\mathrm{MeV} \mathrm{Fe}^{++}(\mathrm{ACO} 3)$ at $460^{\circ} \mathrm{C}$, to $550 \mathrm{dpa}$ with $10 \mathrm{appm} \mathrm{He}$. 

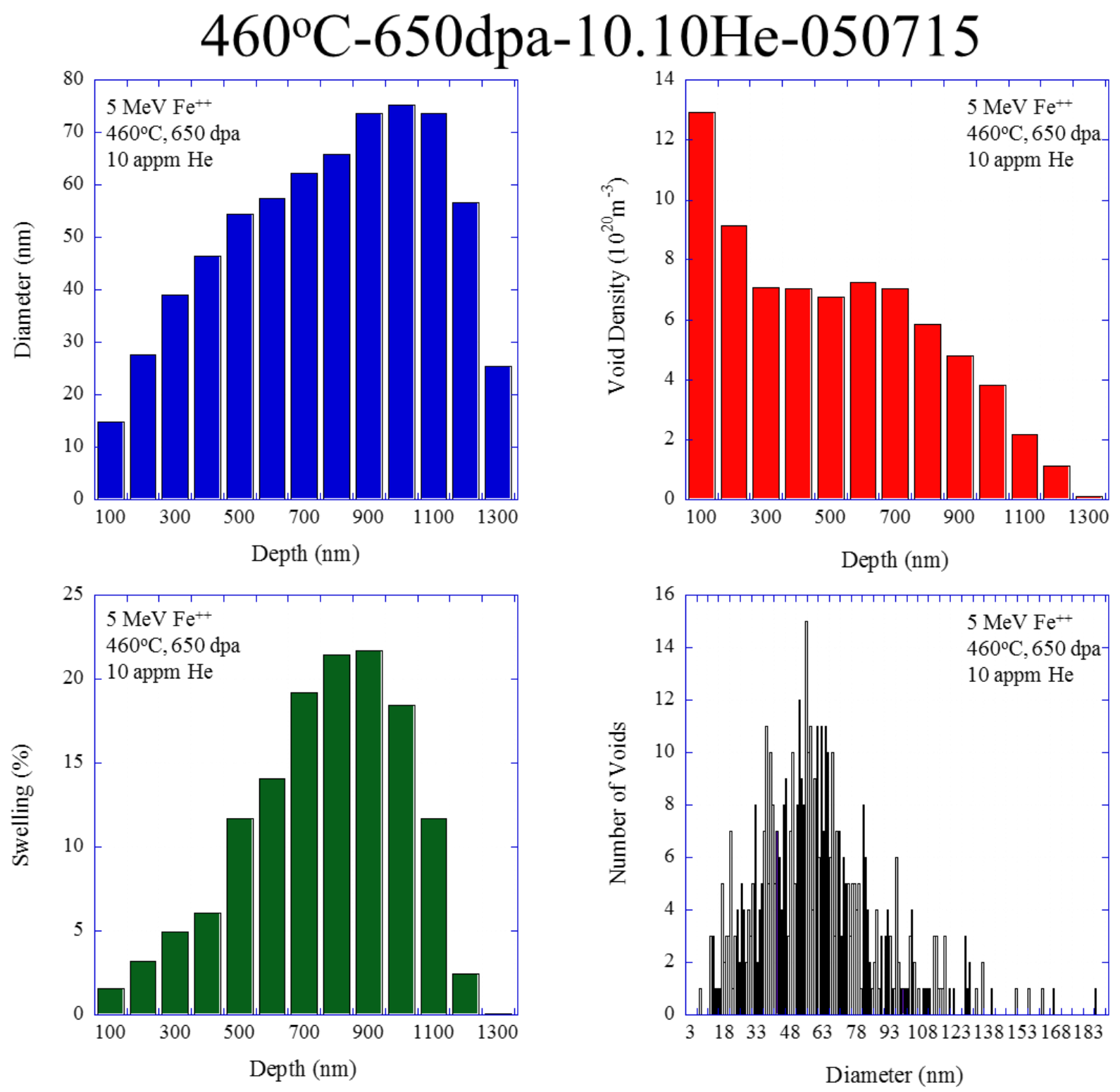

Figure C. 15: Void diameter, number density and swelling depth profiles as well as void distribution in the 500 to $700 \mathrm{~nm}$ depth region of HT9 irradiated with 5 $\mathrm{MeV} \mathrm{Fe}{ }^{++}(\mathrm{ACO} 3)$ at $460^{\circ} \mathrm{C}$, to $650 \mathrm{dpa}$ with $10 \mathrm{appm} \mathrm{He}$. 


\section{REFERENCES}

[1] T. Ellis, R. Petroski, P. Hejzlar, G. Zimmerman, D. Mcalees, C. Whitmer, et al., Traveling-wave reactors : A truly sustainable and full-scale resource for global energy needs, in: Int. Congr. Adv. Nucl. Power Plants, 2010.

[2] G.S. Was, Z. Jiao, E. Getto, K. Sun, A.M. Monterrosa, S.A. Maloy, et al., Emulation of reactor irradiation damage using ion beams, Scr. Mater. 88 (2014) 33-36. doi:10.1016/j.scriptamat.2014.06.003.

[3] R.L. Klueh, D.R. Harries, High-Chromium Ferritic and Martensitic Steels for Nuclear Applications, 2001. doi:10.1520/MONO3-EB.

[4] M.B. Toloczko, F.A. Garner, Variability of Irradiation Creep and Swelling of HT9 Irradiated to High Neutron Fluence at 400-600C, Eff. Radiat. Mater. ASTM STP 1325. (1999).

[5] M.B. Toloczko, F.A. Garner, C.R. Eiholzer, Irradiation creep and swelling of the US fusion heats of HT9 and 9Cr-1Mo to 208 dpa at - 400C, J. Nucl. Mater. 215 (1994) 604607.

[6] M.B. Toloczko, F.A. Garner, Irradiation creep and void swelling of two LMR heats of HT9 at 400 C and 165 dpa, J. Nucl. Mater. 237 (1996) 289-292.

[7] D. Gelles, Microstructural examination of commercial ferritic alloys at 200 dpa, J. Nucl. Mater. 237 (1996) 293-298. http://www.sciencedirect.com/science/article/pii/S002231159600222X (accessed August 
19, 2011).

[8] F. Garner, M.B. Toloczko, B.H. Sencer, Comparison of swelling and irradiation creep behavior of fcc-austenitic and bcc-ferritic/martensitic alloys at high neutron exposure, $\mathrm{J}$. Nucl. Mater. 276 (2000) 123-142. doi:10.1016/S0022-3115(99)00225-1.

[9] B.H. Sencer, J.R. Kennedy, J.I. Cole, S.A. Maloy, F.A. Garner, Microstructural analysis of an HT9 fuel assembly duct irradiated in FFTF to $155 \mathrm{dpa}$ at $443^{\circ} \mathrm{C}$, J. Nucl. Mater. 393 (2009) 235-241. doi:10.1016/j.jnucmat.2009.06.010.

[10] B.H. Sencer, J.R. Kennedy, J.I. Cole, S.A. Maloy, F.A. Garner, Microstructural stability of an HT-9 fuel assembly duct irradiated in FFTF, J. Nucl. Mater. 414 (2011) 237-242. doi:10.1016/j.jnucmat.2011.03.050.

[11] D.S. Gelles, Microstructural development in reduced activation ferritic alloys irradiated to 200 dpa at $420^{\circ} \mathrm{C}$, J. Nucl. Mater. 212-215 (1994) 714-719. doi:10.1016/00223115(94)90150-3.

[12] E. Getto, Z. Jiao, A.M. Monterrosa, K. Sun, G.S. Was, Effect of pre-implanted helium on void swelling evolution in self-ion irradiated HT9, J. Nucl. Mater. 462 (2015) 458-469. doi:10.1016/j.jnucmat.2015.01.045.

[13] E. Getto, Z. Jiao, A.M. Monterrosa, K. Sun, G.S. Was, Effect of irradiation mode on the microstructure of self-ion irradiated ferritic-martensitic alloys, J. Nucl. Mater. 465 (2015) 116-126. doi:10.1016/j.jnucmat.2015.05.016.

[14] F.A. Smidt, P.R. Malmberg, J.A. Sprague, J.E. Westmoreland, Swelling behavior of commercial alloys, EM-12 and HT-9, as assessed by heavy ion bombardment, Am. Soc. 
Test. Mater. (1976) 227-241.

[15] M.B. Toloczko, F.A. Garner, V.N. Voyevodin, V.V. Bryk, O.V. Borodin, V.V. Mel'nychenko, et al., Ion-induced swelling of ODS ferritic alloy MA957 tubing to 500 dpa, J. Nucl. Mater. 453 (2014) 323-333. doi:10.1016/j.jnucmat.2014.06.011.

[16] G. Gupta, Z. Jiao, A.N. Ham, J.T. Busby, G.S. Was, Microstructural evolution of proton irradiated T91, J. Nucl. Mater. 351 (2006) 162-173. doi:10.1016/j.jnucmat.2006.02.028.

[17] Z. Jiao, N. Ham, G.S. Was, Microstructure of helium-implanted and proton-irradiated T91 ferritic/martensitic steel, J. Nucl. Mater. 367-370 (2007) 440-445. doi:10.1016/j.jnucmat.2007.03.118.

[18] R. Stoller, G.R. Odette, A Composite Model of Microstructural Evolution in Austenitic Stainless Steel Under Fast Neutron Irradiation, Am. Soc. Test. Mater. (1987) 371-392.

[19] J.J. Kai, Effects of thermal annealing and ion irradiation on the microstructures of HT-9 ferritic steel (precipitates, cavities, TEM, dislocation Loops, cross-Section), 1986.

[20] G.S. Was, Fundamentals of Radiation Materials Science, 2007.

[21] R.E. Stoller, G.R. Odette, A comparison of the relative importance of helium and vacancy accumulation in void nucleation, Radiation-Induced Chang. Microstruct. Int. Symp. (1987) 358-370.

http://books.google.com/books?hl=en\&lr=\&id=k5j2P1xKKqEC\&oi=fnd\&pg=PA358\&dq $=$ Zinkle+Helium+Void\&ots=PLOM9BhLCV\&sig=JiHIISfUYfJNTnSbouH8ihLkWAk.

[22] A.S. Kalchenko, V.V. Bryk, N.P. Lazarev, I.M. Neklyudov, V.N. Voyevodin, F.A. Garner, Prediction of swelling of $18 \mathrm{Cr} 10 \mathrm{NiTi}$ austenitic steel over a wide range of 
displacement rates, J. Nucl. Mater. 399 (2010) 114-121.

doi:10.1016/j.jnucmat.2010.01.010.

[23] F.A. Garner, T. Okita, N. Sekimura, Swelling of pure vanadium and $\mathrm{V}-5 \mathrm{Cr}$ at $\sim 430^{\circ} \mathrm{C}$ in response to variations in neutron flux-spectra in FFTF, J. Nucl. Mater. 417 (2011) 314318. doi:10.1016/j.jnucmat.2010.12.072.

[24] A.M. Dvoriashin, S.I. Porollo, Y. V Konobeev, F.A. Garner, Influence of cold work to increase swelling of pure iron irradiated in the BR-10 reactor to $\sim 6$ and $\sim 25$ dpa at $\sim 400^{\circ}$ C, J. Nucl. Mater. 287 (2000) 157-160.

[25] A. Kumar, F.A. Garner, Saturation of proton-induced swelling in AISI 316, J. Nucl. Mater. 117 (1983) 234-238.

[26] Y.V. Konobeev, a. M. Dvoriashin, S.I. Porollo, F. a. Garner, Swelling and microstructure of pure $\mathrm{Fe}$ and $\mathrm{Fe}-\mathrm{Cr}$ alloys after neutron irradiation to $\sim 26 \mathrm{dpa}$ at $400^{\circ} \mathrm{C}$, J. Nucl. Mater. 355 (2006) 124-130. doi:10.1016/j.jnucmat.2006.04.011.

[27] B.H. Sencer, F.A. Garner, Compositional and temperature dependence of void swelling in model $\mathrm{Fe}-\mathrm{Cr}$ base alloys irradiated in the EBR-II fast reactor, J. Nucl. Mater. 283-287 (2000) 164-168. doi:10.1016/S0022-3115(00)00338-X.

[28] R. Viswanathan, W.T. Bakker, Materials for Boilers in Ultra Supercritical Power Plants, in: Int. Jt. Power Gener. Conf., 2000: pp. 1-22. http://www.scribd.com/doc/37295180/Boiler-Materials-for-USC-Plants-IJPGC-2000.

[29] T.S. Byun, J.H. Baek, O. Anderoglu, S.A. Maloy, M.B. Toloczko, Thermal annealing recovery of fracture toughness in HT9 steel after irradiation to high doses, J. Nucl. Mater. 
449 (2014) 263-272. doi:10.1016/j.jnucmat.2013.07.064.

[30] J. Pešička, A. Aghajani, C. Somsen, A. Hartmaier, G. Eggeler, How dislocation substructures evolve during long-term creep of a $12 \% \mathrm{Cr}$ tempered martensitic ferritic steel, Scr. Mater. 62 (2010) 353-356. doi:10.1016/j.scriptamat.2009.10.037.

[31] O. Anderoglu, J. Van den Bosch, P. Hosemann, E. Stergar, B.H. Sencer, D. Bhattacharyya, et al., Phase stability of an HT-9 duct irradiated in FFTF, J. Nucl. Mater. 430 (2012) 194-204. doi:10.1016/j.jnucmat.2012.06.038.

[32] J.J. Penisten, The Mechanism of Radiation-Induced Segregation in Ferritic-Martensitic Steels, 2012.

[33] D.S. Gelles, Void swelling in binary FeCr alloys at 200 dpa, J. Nucl. Mater. 225 (1995) 163-174. doi:10.1016/0022-3115(95)00053-4.

[34] L. Mansur, Theory and experimental background on dimensional changes in irradiated alloys方, J. Nucl. Mater. 216 (1994) 97-123. doi:10.1016/0022-3115(94)90009-4.

[35] J.J. Kai, G.L. Kulcinski, $14 \mathrm{MeV}$ nickel-ion irradiated HT-9 ferritic steel with and without helium pre-implantation, J. Nucl. Mater. 175 (1990) 227-236.

[36] D.L. Plumton, W.G. Wolfer, Supression of void nucleation by injected interstitials during heavy ion bombardment, J. Nucl. Mater. 120 (1984) 245-253.

[37] G. Ayrault, Cavity formation during single and dual-ion 9Cr-1Mo ferritic alloy irradiation, J. Nucl. Mater. 114 (1983) 34-40.

[38] O.V. Borodin, V.N. Voyevodin, V.F. Zelenskij, I.M. Neklyudov, P.V. Platonov, Radiation 
damage studies of the 10 to $13 \%$ chromium-containing steels and alloys irradiated with heavy ions, in: Eff. Radiat. Mater. 15th Int. Symp., 1992: pp. 1157-1166. http://books.google.fi/books?id=SuRjaV0MVdEC.

[39] A. Ward, S. Fisher, A comparison of heavy ion-irradiated ferritic and austenitic steels in the early stages of damage, in: Eff. Radiat. Mater. 15th Int. Symp., 1992: pp. 1167-1179.

[40] M. Suzuki, N. Sekimura, N. Fukuya, H. Kusangi, H. Taguchi, M. Satake, et al., Microstructural change in ferritic steels under heavy ion irradiation, in: Eff. Radiat. Mater. 14th Annu. Symp. ASTM STP 1046, 1989: p. 160. doi:10.1017/CBO9781107415324.004.

[41] G.R. Odette, On mechanisms controlling swelling in ferritic and martensitic alloys, J. Nucl. Mater. 157 (1988) 921-927.

[42] G.P. Walters, The electron irradiation of pure $\mathrm{FeCrNi}$ alloys in the temperature range 400 to 700C, J. Nucl. Mater. 136 (1985) 263-279. doi:10.1016/0022-3115(85)90014-5.

[43] R. Bullough, R.C. Perrin, The Mechanism and Kinetics of Void Growth During Neutron Irradiation, ASTM STP 484. (n.d.) 317-331.

[44] C. Xu, G.S. Was, Anisotropic dislocation loop distribution in alloy T91 during irradiation creep, J. Nucl. Mater. 454 (2014) 255-264. doi:10.1016/j.jnucmat.2014.07.062.

[45] M.N. Batista, S. Hereñú, I. Alvarez-Armas, The Role of Microstructure in Fatigue Crack Initiation and Propagation in 9-12Cr Ferritic-martensitic Steels, Procedia Eng. 74 (2014) 228-231. doi:10.1016/j.proeng.2014.06.253.

[46] M. Li, L. Wang, J.D. Almer, Dislocation evolution during tensile deformation in ferriticmartensitic steels revealed by high-energy X-rays, Acta Mater. 76 (2014) 381-393. 
doi:10.1016/j.actamat.2014.05.026.

[47] R.. Faulkner, S. Song, P.E.. Flewitt, Radiation-induced inter-granular segregation in first wall fusion reactor materials, J. Nucl. Mater. 283-287 (2000) 147-151. doi:10.1016/S0022-3115(00)00353-6.

[48] Z. Chang, D. Terentyev, N. Sandberg, K. Samuelsson, P. Olsson, Anomalous bias factors of dislocations in bcc iron, J. Nucl. Mater. 461 (2015) 221-229. doi:10.1016/j.jnucmat.2015.03.025.

[49] P. Dubuisson, D. Gilbon, J.L. Séran, Microstructural evolution of ferritic-martensitic steels irradiated in the fast breeder reactor Phénix, J. Nucl. Mater. 205 (1993) 178-189. doi:10.1016/0022-3115(93)90080-I.

[50] A.F. Padilha, R.L. Plaut, Stainless steel heat treatment, in: Steel Heat Treat. Handb., 2006: pp. $695-735$.

[51] Z. Jiao, G.S. Was, Precipitate evolution in ion-irradiated HCM12A, J. Nucl. Mater. 425 (2012) 105-111. doi:10.1016/j.jnucmat.2011.12.017.

[52] O.V. Borodin, V.V. Bryk, V.N. Voyevodin, I.M. Neklyudov, V.K. Shamardin, Investigation of microstructure of ferritic-martensitic steels containing 9 and $13 \% \mathrm{Cr}$ irradiated with fast neutrons, J. Nucl. Mater. 207 (1993) 295-302. doi:10.1016/00223115(93)90272-Z.

[53] P.J. Maziasz, Formation and stability of radiation-induced phases in neutron-irradiated austenitic and ferritic steels, J. Nucl. Mater. 169 (2008) 95-115.

[54] Z. Jiao, G.S. Was, Segregation behavior in proton- and heavy-ion-irradiated ferritic- 
martensitic alloys, Acta Mater. 59 (2011) 4467-4481. doi:10.1016/j.actamat.2011.03.070.

[55] J.P. Wharry, G.S. Was, A systematic study of radiation-induced segregation in ferriticmartensitic alloys, J. Nucl. Mater. 442 (2013) 7-16. doi:10.1016/j.jnucmat.2013.07.071.

[56] S.A. Maloy, M.R. James, G. Willcutt, W.F. Sommer, M. Sokolov, L.L. Snead, et al., The mechanical properties of 316L / 304L stainless steels , Alloy 718 and $\mathrm{Mod} 9 \mathrm{Cr} \pm 1 \mathrm{Mo}$ after irradiation in a spallation environment, J. Nucl. Mater. 296 (2001) 119-128.

[57] J.T. Busby, M.C. Hash, G.S. Was, The relationship between hardness and yield stress in irradiated austenitic and ferritic steels, J. Nucl. Mater. 336 (2005) 267-278. doi:10.1016/j.jnucmat.2004.09.024.

[58] D.S. Gelles, R. Schaublin, Examination of postirradiation deformation microstructures in F82H, in: Eff. Radiat. Mater. 21st Int. Symp., 2004: pp. 401-407.

[59] Y. de Carlan, X. Avery, J.-C. Brachet, J.-L. Bertin, F. Rozenblum, O. Rabouille, et al., Post-irradiation tensile behavior and residual activity of several ferritic/martensitic and austenitic steels irradiated in Osiris reactor at 325C up to 9 dpa, in: Eff. Radiat. Mater. 14th Annu. Symp. ASTM STP 1475, 2006: pp. 67-85.

[60] S.A. Maloy, M. Toloczko, J. Cole, T.S. Byun, Core materials development for the fuel cycle R\&D program, J. Nucl. Mater. 415 (2011) 302-305. doi:10.1016/j.jnucmat.2011.04.027.

[61] T.S. Byun, W. Daniel Lewis, M.B. Toloczko, S.A. Maloy, Impact properties of irradiated HT9 from the fuel duct of FFTF, J. Nucl. Mater. 421 (2012) 104-111. doi:10.1016/j.jnucmat.2011.11.059. 
[62] T.S. Byun, M.B. Toloczko, T.A. Saleh, S.A. Maloy, Irradiation dose and temperature dependence of fracture toughness in high dose HT9 steel from the fuel duct of FFTF, J. Nucl. Mater. 432 (2013) 1-8. doi:10.1016/j.jnucmat.2012.07.019.

[63] M.J. Hackett, Personal communication, 2015.

[64] J.F. Ziegler, M.D. Ziegler, J.P. Biersack, SRIM - The stopping and range of ions in matter (2010), Nucl. Instruments Methods Phys. Res. Sect. B Beam Interact. with Mater. Atoms. 268 (2010) 1818-1823. doi:10.1016/j.nimb.2010.02.091.

[65] R.E. Stoller, M.B. Toloczko, G.S. Was, a. G. Certain, S. Dwaraknath, F. a. Garner, On the use of SRIM for computing radiation damage exposure, Nucl. Instruments Methods Phys. Res. Sect. B Beam Interact. with Mater. Atoms. 310 (2013) 75-80. doi:10.1016/j.nimb.2013.05.008.

[66] M.J. Norgett, M.T. Robinson, I.M. Torrens, A proposed method of calculating displacement dose rates, Nucl. Eng. Des. 33 (1975) 50-54. doi:10.1016/00295493(75)90035-7.

[67] ASTM E521, Standard practice for neutron radiation damage simulation by chargedparticle, Annu. B. ASTM Stand. 12.02 (2009) 1-21. doi:10.1520/E0521-96R09E01.

[68] W.K. T. Csörgô, S. Hegyi, From e+e- to Heavy Ion Collisions, in: 30th Int. Symp. Multiparticle Dyn., 2001: p. 241.

[69] G.S. Was, J.T. Busby, T. Allen, E.A. Kenik, A. Jenssen, S.M. Bruemmer, et al., Emulation of neutron irradiation effects with protons: validation of principle, J. Nucl. Mater. 300 (2002) 198-216. doi:10.1016/S0022-3115(01)00751-6. 
[70] C. Xu, In-Situ Proton Irradiation Creep of FM Steel T91, 2014.

[71] T. Malis, S.C. Cheng, R.F. Egerton, EELS log-ratio technique for specimen-thickness measurement in the TEM, J. Electron Microsc. Tech. 193-200 (1988).

[72] L. Shao, C.-C. Wei, J. Gigax, A. Aitkaliyeva, D. Chen, B.H. Sencer, et al., Effect of defect imbalance on void swelling distributions produced in pure iron irradiated with $3.5 \mathrm{MeV}$ self-ions, J. Nucl. Mater. 453 (2014) 176-181. doi:10.1016/j.jnucmat.2014.06.002.

[73] A.S. Kumar, F.A. Garner, Dual-ion irradiation: Impact of the conflicting roles of helium on void nucleation, Eff. Radiat. Mater. ASTM STP 870. (1985) 493-506.

[74] C.S. Smith, L. Guttman, Measurement of Internal Boundaries in Three Dimensional Structures by Random Sectioning, J. Met. Trans. (1953) 81-87.

[75] W.L. Fink, E.D. Campbell, Campbell and Campbell, Am. Soc. Heat. Treat. 1926 (1926) $717-752$.

[76] O.D. Sherby, J. Wadsworth, D.R. Lesuer, C.K. Syn, The c/a Ratio in Quenched Fe-C and Fe-N Steels - A Heuristic Story, Mater. Sci. Forum. 539-543 (2007) 215-222. doi:10.4028/www.scientific.net/MSF.539-543.215.

[77] O.D. Sherby, J. Wadsworth, D.R. Lesuer, C.K. Syn, Revisiting the Structure of Martensite in Iron-Carbon Steels, Mater. Trans. 49 (2008) 2016-2027. doi:10.2320/matertrans.MRA2007338.

[78] D.-H. Ping, Understanding Solid-Solid ( $\mathrm{fcc} \rightarrow \omega+\mathrm{bcc}$ ) Transition at Atomic Scale, Acta Metall. Sin. (English Lett. 28 (2015) 663-670. doi:10.1007/s40195-015-0283-Z. 
[79] D. Kaoumi, Personal communication, (2016).

[80] D. Xu, B.D. Wirth, M. Li, M.A. Kirk, Combining in situ transmission electron microscopy irradiation experiments with cluster dynamics modeling to study nanoscale defect agglomeration in structural metals, Acta Mater. 60 (2012) 4286-4302. doi:10.1016/j.actamat.2012.03.055.

[81] R.E. Stoller, The effect of free surfaces on cascade damage production in iron, J. Nucl. Mater. 311 (2008) 935-940.

[82] A.D. Brailsford, L.K. Mansur, Effect of self-ion injection in simulation studies of void swelling, J. Nucl. Mater. 71 (1977) 110-116.

[83] T. Yamamoto, Y. Wu, G. Robert Odette, K. Yabuuchi, S. Kondo, A. Kimura, A dual ion irradiation study of helium-dpa interactions on cavity evolution in tempered martensitic steels and nanostructured ferritic alloys, J. Nucl. Mater. 449 (2014) 190-199. doi:10.1016/j.jnucmat.2014.01.040.

[84] T. Chen, E. Aydogan, J.G. Gigax, D. Chen, J. Wang, X. Wang, et al., Microstructural changes and void swelling of a 12Cr ODS ferritic-martensitic alloy after high-dpa self-ion irradiation, J. Nucl. Mater. 467 (2015) 42-49. doi:10.1016/j.jnucmat.2015.09.016.

[85] E. Getto, K. Sun, S. Taller, A.M. Monterrosa, Z. Jiao, G.S. Was, Methodology for determining void swelling at very high damage under ion irradiation, J. Nucl. Mater. 477 (2016) 273-279. doi:10.1016/j.jnucmat.2016.05.026.

[86] G.R. Odette, D.M. Schwartz, A.G. Ardell, Particle Range and Energy Deposition in Materials Containing Voids, Radiat. Eff. 22 (1974) 217-223. 
[87] W.G. Johnston, J.H. Rosolowski, A.M. Turkalo, T. Lauritzen, The depth distribution of void swelling produced by 5 MeV Ni ions, J. Nucl. Mater. 62 (1976) 167-180. doi:10.1016/0022-3115(76)90014-3.

[88] A. Mateo, L. Llanes, M. Anglada, A. Redjaimia, G. Metauer, Characterization of the intermetallic G-phase in an AISI 329 duplex stainless steel, J. Mater. Sci. 32 (1997) 45334540. doi:10.1023/A:1018669217124.

[89] J.M. Vitek, S.A. David, D.J. Alexander, J.R. Keiser, R.K. Nanstad, Low temperature aging behavior of type 308 stainless steel weld metal, Acta Metall. Mater. 39 (1991) 503516. doi:10.1016/0956-7151(91)90118-K.

[90] X. Wang, Q. Yan, G.S. Was, L. Wang, Void swelling in ferritic-martensitic steels under high dose ion irradiation: Exploring possible contributions to swelling resistance, Scr. Mater. 112 (2016) 9-14. doi:10.1016/j.scriptamat.2015.08.032.

[91] T.R. Allen, J.I. Cole, E.A. Kenik, G.S. Was, Analyzing the effect of displacement rate on radiation-induced segregation in 304 and 316 stainless steels by examining irradiated EBR-II components and samples irradiated with protons, J. Nucl. Mater. 376 (2008) 169173. doi:10.1016/j.jnucmat.2008.01.025.

[92] T.R. Allen, G.S. Was, E. a. Kenik, The effect of alloy composition on radiation-induced segregation in Fe-Cr-Ni alloys, J. Nucl. Mater. 244 (1997) 278-294. doi:10.1016/S00223115(96)00744-1.

[93] X. Wang, Q. Yan, G.S. Was, L. Wang, Void swelling in ferritic-martensitic steels under high dose ion irradiation: Exploring possible contributions to swelling resistance, Scr. 
Mater. (2015) 6-11. doi:10.1016/j.scriptamat.2015.08.032.

[94] D.S. Gelles, Neutron Irradiation Damage in Ferritic Fe-Cr Alloys, in: Eff. Radiat. Mater. 14th Int. Symp. Vol. 1, ASTM STP 1046, 1989: pp. 73-97. doi:10.1017/CBO9781107415324.004.

[95] B.L. Eyre, R. Bullough, On the formation of interstitial loops in b.c.c. metals, Philos. Mag. 12 (1965) 31-39. doi:10.1080/14786436508224943.

[96] Y. Katoh, D.S. Gelles, Swelling and dislocation evolution in simple ferritic alloys irradiated to high fluence in FFTF / MOTA Fe-3Cr I Fe-12Cr Fe-15Cr, J. Nucl. Mater. 225 (1995) 154-162.

[97] S.I. Golubov, R.E. Stoller, S.J. Zinkle, A.M. Ovcharenko, Kinetics of coarsening of helium bubbles during implantation and post-implantation annealing, J. Nucl. Mater. 361 (2007) 149-159. doi:10.1016/j.jnucmat.2006.12.032.

[98] S.I. Golubov, A. V. Barashev, R.E. Stoller, Radiation damage theory, 1st ed., Elsevier Inc., 2012. doi:10.1016/B978-0-08-056033-5.00029-X.

[99] M. Kiritani, Analysis of the clustering process of supersaturated lattice vacancies, J. Phys. Soc. Japan. 35 (1973) 95-107. doi:10.1143/JPSJ.37.1532.

[100] R.E. Stoller, Ph.D. Thesis, 1987.

[101] A.C. Hindmarsh, LSODE and LSODI, Two new initial value ordinary differential equation solvers, ACM SIGNUM Newsl. 15 (1980) 10. doi:10.1017/CBO9781107415324.004. 
[102] B.N. Singh, A.J. Foreman, Production bias and void swelling in the transient regime under cascade damage conditions., Philos. Mag. A. 66 (1992) 975-990.

[103] A.V. Barashev, S.I. Golubov, H. Trinkaus, Reaction kinetics of glissile interstitial clusters in a crystal containing voids and dislocations, Philos. Mag. A. 81 (2001) 2515-2532. doi:10.1080/01418610108217161.

[104] A. V Barashev, Y.N. Osetsky, D.J. Bacon, A. V Barashevt, A Mechanism of onedimensional glide of self-interstitial atom clusters in $\alpha$-iron, Philos. Mag. a. 80 (2000) 2709-2720. doi:10.1080/01418610008216500.

[105] R.S. Nelson, D.J. Mazey, J.A. Hudson, The use of ion accelerators to simulate fast neutron-induced voidage in metals, J. Nucl. Mater. 37 (1970) 1-12. doi:10.1016/00223115(70)90176-5.

[106] C.-C. Fu, J.D. Torre, F. Willaime, J.-L. Bocquet, A. Barbu, Multiscale modelling of defect kinetics in irradiated iron, Nat. Mater. 4 (2005) 68-74. doi:10.1038/nmat1286.

[107] E. Del Rio, J.M. Sampedro, H. Dogo, M.J. Caturla, M. Caro, A. Caro, et al., Formation energy of vacancies in FeCr alloys: Dependence on Cr concentration, J. Nucl. Mater. 408 (2011) 18-24. doi:10.1016/j.jnucmat.2010.10.021.

[108] D. Terentyev, N. Juslin, K. Nordlund, N. Sandberg, Fast three dimensional migration of He clusters in bcc Fe and Fe-Cr alloys, J. Appl. Phys. 105 (2009). doi:10.1063/1.3126709.

[109] J.R.G. da Silva, R.B. McLellan, Diffusion of carbon and nitrogen in B.C.C. iron, Mater. Sci. Eng. 26 (1976) 83-87. doi:10.1016/0025-5416(76)90229-9.

[110] R.E. Stoller, Role of cascade energy and temperature in primary defect formation in iron, 
J. Nucl. Mater. 276 (2000) 22-32. doi:10.1016/S0022-3115(99)00204-4.

[111] R.E. Stoller, G.R. Odette, B.D. Wirth, Primary damage formation in bcc iron, J. Nucl. Mater. 251 (1997) 49-60. doi:10.1016/S0022-3115(97)00256-0.

[112] H. Xu, Y.N. Osetsky, R.E. Stoller, Cascade annealing simulations of bcc iron using object kinetic Monte Carlo, J. Nucl. Mater. 423 (2012) 102-109. doi:10.1016/j.jnucmat.2012.01.020.

[113] H. Xu, R.E. Stoller, Y.N. Osetsky, Cascade defect evolution processes: Comparison of atomistic methods, J. Nucl. Mater. 443 (2013) 66-70. doi:10.1016/j.jnucmat.2013.07.001.

[114] D. Brimbal, L. Fournier, A. Barbu, Cluster dynamics modeling of the effect of high dose irradiation and helium on the microstructure of austenitic stainless steels, J. Nucl. Mater. 468 (2015) 124-139. doi:10.1016/j.jnucmat.2015.11.007.

[115] S. I. Golubov, B. N. Singh, H. Trinkau, On recoil-energy-dependent defect accumulation in pure copper Part II. Theoretical treatment, Philos. Mag. A. 81 (2001) 2533-2552. doi:10.1080/01418610110043163.

[116] R.M. Mayer, L.M. Brown, Nucleation and growth of voids by radiation VI. Role of alloying atoms, J. Nucl. Mater. 95 (1980) 92-99. doi:10.1016/0022-3115(80)90078-1.

[117] F.A. Smidt, J.A. Sprague, Suppression of void nucleation by a vacancy trapping mechanism, Scr. Metall. 7 (1973) 495-501. doi:10.1016/0036-9748(73)90102-6.

[118] L.K. Mansur, M.H. Yoo, The effects of impurity trapping on irradiation-induced swelling and creep, J. Nucl. Mater. 74 (1978) 228-241. 
[119] D. Jiang, E. Carter, Carbon dissolution and diffusion in ferrite and austenite from first principles, Phys. Rev. B. 67 (2003) 214103. doi:10.1103/PhysRevB.67.214103.

[120] P.R. Okamoto, N.. Lam, H. Wierdersich, R.. Johnson, Effects of defect trapping and solute segregation on defect recombination rates and void swelling in irradiated alloys, J. Nucl. Mater. 70 (1978) 821-824.

[121] E.A. Little, Microstructural evolution in irradiated transitions to high dose behaviour steels :, J. Nucl. Mater. 206 (1993) 324-334.

[122] E.A. Little, Void swelling in irons and ferritic steels I. Mechanisms of swelling suppression, J. Nucl. Mater. 87 (1979) 11-24.

[123] A. Bakaev, D. Terentyev, X. He, E.E. Zhurkin, D. Van Neck, Interaction of carbonvacancy complex with minor alloying elements of ferritic steels, J. Nucl. Mater. 451 (2014) 82-87. doi:10.1016/j.jnucmat.2014.03.031.

[124] N. Anento, A. Serra, Carbon-vacancy complexes as traps for self-interstitial clusters in Fe-C alloys, J. Nucl. Mater. 440 (2013) 236-242. doi:10.1016/j.jnucmat.2013.04.087.

[125] A.B. Lidiard, The influence of solutes on self-diffusion in metals, Philos. Mag. 5 (1960) 1171-1180. doi:10.1080/14786436008238325.

[126] A.D. Brailsford, L.K. Mansur, The effect of precipitate-matrix interface sinks on the growth of voids in the matrix, J. Nucl. Mater. 104 (1981) 1403-1408. doi:10.1016/00223115(82)90796-6.

[127] A.D. Brailsford, R. Bullough, The rate theory of swelling in irradiated metals, J. Nucl. Mater. 44 (1972) 121-135. 
[128] A.D. Brailsford, Point defect trapping and void growth, J. Nucl. Mater. 78 (1978) 354361. doi:10.1016/0022-3115(78)90457-9.

[129] C.-G. Lee, Y. Iijima, T. Hiratani, K. Hirano, Diffusion of Chromium in alpha-Iron, Mater. Trans. JIM. 31 (1990) 255-261. doi:10.2320/matertrans1989.31.255.

[130] K. Hirano, M. Cohen, B.L. Averbach, Diffusion of Nickel into Iron, Acta Metall. 9 (1961) 440-445.

[131] A. Galerie, G. Dearnaley, Radiation enhanced diffusion of silicon into iron for high temperature oxidation improvement, Nucl. Instruments Methods Phys. Res. 210 (1983) 823-829.

[132] V. Irmer, M. Feller-Kniepmeier, Diffusion of manganese in alpha-iron single crystals of different purity, J. Phys. Chem. Solids. 33 (1972) 2141-2148. doi:10.1016/S00223697(72)80244-0.

[133] H. Nitta, K. Miura, Y. Iijima, Self-diffusion in iron-based Fe-Mo alloys, Acta Mater. 54 (2006) 2833-2847. doi:10.1016/j.actamat.2006.02.020.

[134] H. Oikama, Review of Lattice Diffusion of Substitutional Impurities in Iron, Summ. Rep. Tech Reports. 47 (1982) 1982.

[135] S. Takemoto†, H. Nittał, Y. Iijimaß, Y. Yamazaki, Diffusion of tungsten in $\alpha$-iron, Philos. Mag. 87 (2007) 1619-1629. doi:10.1080/14786430600732093.

[136] A.W. Bowen, G.M. Leak, Solute diffusion in alpha- and gamma-iron, Metall. Trans. 1 (1970) 1695-1700. doi:10.1007/BF02642019. 
[137] T. Jourdan, Influence of dislocation and dislocation loop biases on microstructures simulated by rate equation cluster dynamics, J. Nucl. Mater. 467 (2015) 286-301. doi:10.1016/j.jnucmat.2015.09.046.

[138] W.G. Wolfer, The dislocation bias, J. Comput. Mater. Des. 14 (2007) 403-417. doi:10.1007/s10820-007-9051-3.

[139] F.S. Ham, Stress-assisted precipitation on dislocations, J. Appl. Phys. 30 (1959) 915-926. doi:10.1063/1.1735262.

[140] J.J. Sniegowski, W.G. Wolfer, Sniegowski, J.J. Wolfer, Proc. Top. Conf. Ferritic Alloy. Use Nucl. Energy Technol. (1984) 1984.

[141] E. Little, D. Stow, Void-swelling in irons and ferritic steels II. An experimental survey of materials irradiated in a fast reactor, J. Nucl. Mater. 87 (1979) 25-39. http://www.sciencedirect.com/science/article/pii/0022311579901235 (accessed August 4, 2011).

[142] J. Weertman, W.V. Green, Irradiation Effects on the Microstructure and Properties of Metals, Irradiat. Eff. Microstruct. Prop. Met. ASTM STP 6 (1976) 256.

[143] V.. Voyevodin, I.. Neklyudov, V.V. Bryk, O.V. Borodin, Microstructural evolution and radiation stability of steels and alloys, J. Nucl. Mater. 271-272 (1999) 290-295. doi:10.1016/S0022-3115(98)00785-5.

[144] J. Van Den Bosch, O. Anderoglu, R. Dickerson, M. Hartl, P. Dickerson, J.A. Aguiar, et al., SANS and TEM of ferritic-martensitic steel T91 irradiated in FFTF up to 184 dpa at $413{ }^{\circ} \mathrm{C}$, J. Nucl. Mater. 440 (2013) 91-97. doi:10.1016/j.jnucmat.2013.04.025. 
[145] E. Getto, K. Sun, A.M. Monterrosa, Z. Jiao, G.S. Was, Void swelling and microstructure evolution at very high damage level in self-ion irradiated ferritic-martensitic steels, J. Nucl. Mater. (2016) accepted.

[146] A.D. Brailsford, R. Bullough, Void growth and its relation to intrinsic point defect properties, J. Nucl. Mater. 70 (1978) 434-450.

[147] K.A. Bywater, D.J. Dyson, The Precipitation of Cr 2 N in 17\%Cr Steels, Met. Sci. 9 (1975) 155-162. doi:10.1179/030634575790444685.

[148] R. Lagneborg, Metallography of the 475 C embrittlement in an Iron-30\% chromium alloy, Trans. Am. Soc. Met. 60 (1967).

[149] D.A. Porter, K.E. Easterling, M.Y. Sherif, Phase Transformations in Metals and Alloys, 2008. doi:10.1017/CBO9781107415324.004.

[150] A. Bhattacharya, E. Meslin, J. Henry, C. Pareige, B. Décamps, C. Genevois, et al., Chromium enrichment on the habit plane of dislocation loops in ion-irradiated high-purity Fe-Cr alloys, Acta Mater. 78 (2014) 394-403. doi:10.1016/j.actamat.2014.06.050.

[151] K. Arakawa, M. Hatanaka, H. Mori, K. Ono, Effects of chromium on the one-dimensional motion of interstitial-type dislocation loops in iron, J. Nucl. Mater. 329-333 (2004) 11941198. doi:10.1016/j.jnucmat.2004.04.263.

[152] D. Terentyev, K. Heinola, a. Bakaev, E.E. Zhurkin, Carbon-vacancy interaction controls lattice damage recovery in Iron, Scr. Mater. (2014). doi:10.1016/j.scriptamat.2014.04.003.

[153] W.D. Callister, Materials Science and Engineering An Introduction, 2007. doi:10.1126/science.1213003. 
[154] J.L. Brimhall, L.A. Charlot, E.P. Simonen, Effect of Pulsed Irradiation on Void Swelling in Nickel, J. Nucl. Mater. 104 (1981) 1147-1150.

[155] J.M. Vitek, R.L. Klueh, Microstructure of $9 \mathrm{Cr}-1 \mathrm{MoVNb}$ steel irradiated to 36 dpa at elevated temperatures in HFIR, J. Nucl. Mater. 122 (1984) 254-259. doi:10.1016/00223115(84)90606-8.

[156] J.J. Kai, R.L. Klueh, Microstructural analysis of neutron-irradiated martensitic steels, J. Nucl. Mater. 230 (1996) 116-123. doi:10.1016/0022-3115(96)00165-1. 